\title{
TRUST AND RISK IN WEBSITE LEGITIMACY AND \\ SOFTWARE APPLICATIONS: DELVING INTO USER \\ UNDERSTANDING OF INTERNET SECURITY MECHANISMS
}

by

\section{DANIEL JEAN LEBLANC}

A thesis submitted to

the Faculty of Graduate and Postdoctoral Affairs

in partial fulfillment of the requirements for the degree of

Doctor of Philosophy

in

Psychology

Carleton University

Ottawa, Ontario

(C)2016 Daniel Jean LeBlanc 


\begin{abstract}
When people choose to visit a given website, they make a trust decision about the supplier and source. It appears that a large majority of users commonly place their trust in most, if not all, websites they encounter, and this causes significant security problems. Any solutions proposed to reduce the threat of malicious websites must include a consideration of the psychological processes of the end users. This thesis presents several studies with the aim of understanding how people interpret the available information when making a trust decision. This understanding will better support users in making appropriate decisions and should inform better design of security mechanisms. It was found that users show some understanding of some of the key concepts in Internet security, and often make reasonable decisions. However, there are important anomalies. For example, many users had important misunderstandings about malware, suggesting they had poor mental models about the capabilities of malware and the capabilities of antivirus software applications in protecting them from threats online. Moreover, participants showed lack of confidence across a range of issues, but in practice they were still willing to make decisions even with this uncertainty. Some evidence was found which suggests that users employ heuristics in making such decisions and judgments under uncertainty. Potential solutions to address this would include closed software markets with certificates, or improved design to help users build better mental models.
\end{abstract}




\section{Acknowledgements}

There were definitely many people and organizations involved in helping me reach the completion stages of my PhD program. I would like to thank my supervisor, Dr. Robert Biddle, who spent an enormous amount of time with me going through the different sections that make up a thesis, mainly the planning, research, and writing of the thesis. He also devoted a lot of time helping me towards the end of the final write-up - including weekends and holidays - in order to meet the final deadline for final submission and defence. I am thankful to my committee members, who provided me guidance during the research phases of my degree: Dr. Warren Thorngate, Professor Emeritus in Psychology at Carleton University, and Dr. Andrew Patrick, Adjunct Research Professor in Computer Science and Cognitive Science. I am also grateful for the insightful comments and questions during my PhD defence from Dr. Kirstie Hawkey at Dalhousie University, and Dr. Gabriel Wainer in Systems and Computer Engineering.

I would not have been able to complete my degree had it not been for the ongoing support of various organizations, including the NSERC Strategic Network on Internetworked Systems Security, and the Industry Canada Network of Centers of Excellence on Graphics, Animation, and New Media. I am very grateful for the funding received from these sources that have allowed me to continue my doctoral degree courses and thesis write-up without interruptions.

I would like to thank my wife, Jacynthe, who has been patient, encouraging, and loving throughout the entire process. She was definitely one the main reasons that kept me

going all these years, keeping my wits and sanity intact throughout the long thesis-writing process. I do - and will continue to - love you forever, Jacynthe.

To my son, who kept asking me towards the end of my degree, "Daddy, did your presentation go well today?", even though I still had weeks to go before defending. You know what they say, "Kids say the funniest things sometimes". Even though he was too young to fully understand the magnitude of such an accomplishment, I would still like to thank him for being patient with me and his mother - my wife - when I had to work long hours wrapping up the final stages of my thesis.

To my parents who, over the years, have shown nothing but respect, admiration, and love for me and my work. They have helped me both emotionally and financially throughout the years. Without their help and support, completing this degree would have been many, many times more difficult. This being said, I would like to dedicate this thesis and all the hard work it represents, to my mother and father, Phyllis E. LeBlanc and Jean-Valmond LeBlanc. I love you both so very much. 


\section{Contents}

Introduction 1

Psychology Background . . . . . . . . . . . . . . . . . . . . . . 2

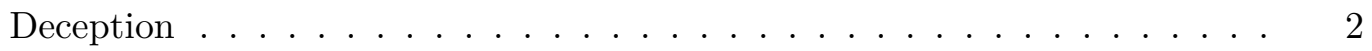

Distraction principle . . . . . . . . . . . . . . . . . 3

Social compliance principle . . . . . . . . . . . . . . . . 3

Herd principle . . . . . . . . . . . . . . . . . . . 3

Dishonesty principle . . . . . . . . . . . . . . . . . 4

Need and greed principle . . . . . . . . . . . . . . . . . . . 4

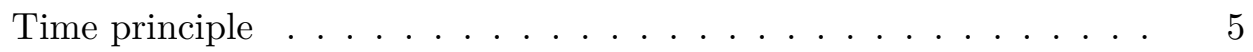

Deception principle. . . . . . . . . . . . . . . 5

Social engineering . . . . . . . . . . . . . . . 5

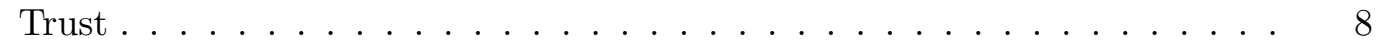

Understanding trust . . . . . . . . . . . . . . . . . . . 9

Satisficing . . . . . . . . . . . . . . . . . . . . . . . . . . . . . 12

Riegelsberger's trust framework . . . . . . . . . . . . . . . . . . . . . . . . . . . . . .

Egger's trust framework . . . . . . . . . . . . . . . . . 17

Computer Security Background . . . . . . . . . . . . . . . . . . . 20

Security threats and attacks . . . . . . . . . . . . . . 20

Phishing. ......................... 20

Malicious software download websites . . . . . . . . . . . . . . 24

Usable security . . . . . . . . . . . . . . . . . . . . . . . . . 26

Technical aspects of Internet security . . . . . . . . . . . . . . . 30

Web certificates. . . . . . . . . . . . . . . 31

Symmetric key cryptography . . . . . . . . . . . . . . . . 31

Asymmetric key cryptography . . . . . . . . . . . . . . . . 31

Software signatures . . . . . . . . . . . . . . . . . . . . . . . . . . . . . . . . . . . . . . . 32

Hardware signatures . . . . . . . . . . . . . . . . . . . . . . . . . . . . . . . . . . . . . . . . .

Proposed solutions . . . . . . . . . . . . . . . . . . . . . 33

Web of trust . . . . . . . . . . . . . . . . . 34

Browser chrome security information . . . . . . . . . . . . . 35

External devices . . . . . . . . . . . . . . . . . 37

External authorities . . . . . . . . . . . . . . . . . . . . . . . . . . . . . . . . . . . .

Software markets . . . . . . . . . . . . . . . . . 40

Main Research Question . . . . . . . . . . . . . . . . . . . . . . . . 42

Study Summaries . . . . . . . . . . . . . . . . . . . . . . . 43

Study one: Determining website legitimacy . . . . . . . . . . . . . . 43

Study two: Risk perception of Internet-related activities . . . . . . . . . . . 43

Study three: Understanding of Internet software abilities . . . . . . . . . . . . . . . 44

Study four: Exploring understanding and confidence . . . . . . . . . . . . . . . 44

Study five: Heuristics, knowledge, and self-perception . . . . . . . . . . . 45 
Study One: Determining Website Legitimacy 46

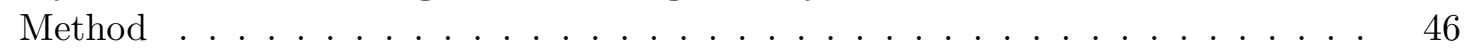

Study design . . . . . . . . . . . . . . . . . . . 46

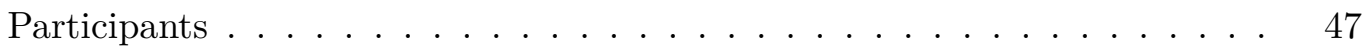

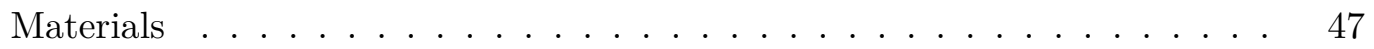

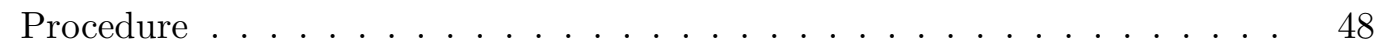

Results . . . . . . . . . . . . . . . . . . . . . 49

Qualitative interview response themes . . . . . . . . . . . . 51

Internet addresses $\ldots \ldots \ldots \ldots \ldots \ldots \ldots \ldots \ldots$

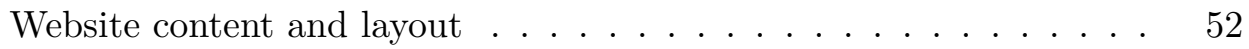

Security indicators $\ldots \ldots \ldots \ldots \ldots \ldots \ldots \ldots \ldots$

Browser behaviour . . . . . . . . . . . . . . . . . 54

Responsibility and identity . . . . . . . . . . . . 54

Trust influences . . . . . . . . . . . . . . . . . . . 55

Discussion . . . . . . . . . . . . . . . . . . 56

Limitations of the present study $\ldots \ldots \ldots \ldots \ldots \ldots$

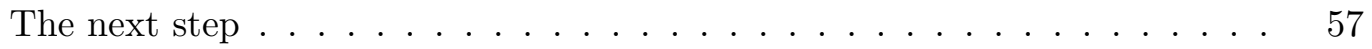

\begin{tabular}{ll}
\hline Study Two: Risk Perception of Internet-Related Activities & 58
\end{tabular}

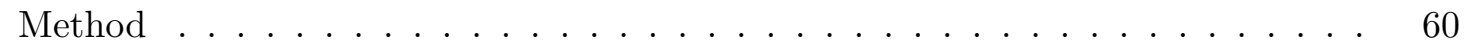

Experimental design . . . . . . . . . . . . . . . . . . 60

Activities . . . . . . . . . . . . . . . 60

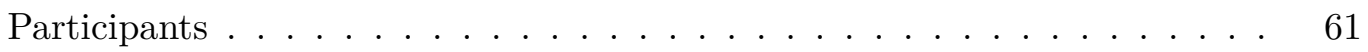

Materials . . . . . . . . . . . . . . . . . . . 62

Procedure . . . . . . . . . . . . . . . . . . . . . 62

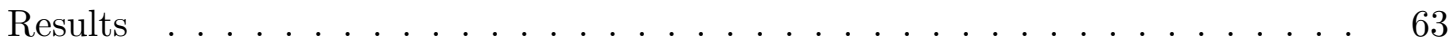

Data distributions . . . . . . . . . . . . . . . 63

Relationships between rating scales . . . . . . . . . . . . . . . . . 68

Factor analysis $\ldots \ldots \ldots \ldots \ldots \ldots \ldots \ldots \ldots \ldots \ldots$

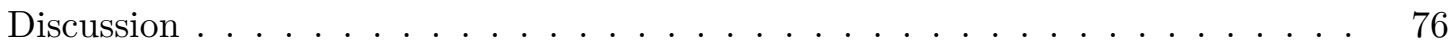

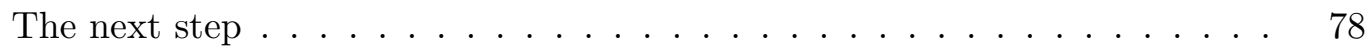

\begin{tabular}{|l|l|}
\hline Study Three: Understanding of Internet Software Abilities & $\mathbf{8 0}$
\end{tabular}

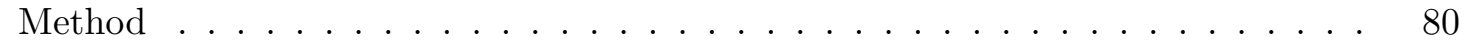

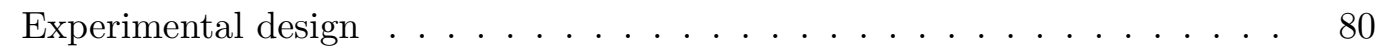

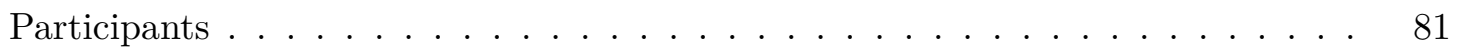

Hypotheses $\ldots \ldots \ldots \ldots \ldots$. . . . . . . . . . . . . . . . . 82

Materials . . . . . . . . . . . . . . . . . . . . 82

Procedure . . . . . . . . . . . . . . . . . . . . . . . 82

Activity scenario: Doing your banking online (Bank of America) . . 84

Activity scenario: Making an online purchase (Amazon) $\ldots$. . . . . . 84

Activity scenario: Installing a software application downloaded from the Internet (CNet Download) $\ldots \ldots \ldots \ldots$. . . . . . . . . 84

Activity scenario: Using a search engine (Google) . . . . . . . . . 85

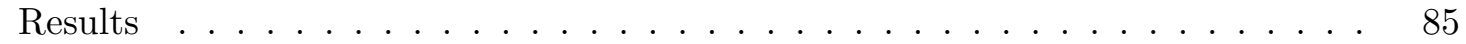




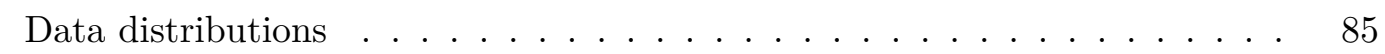

Observations of activity 1 responses: Doing your banking online . . 85

Observations of activity 2 responses: Making an online purchase . . 88

Observations of activity 3 responses: Installing a software application downloaded from the Internet . . . . . . . . . 90

Observations of activity 4 responses: Using a search engine . . . . . 92

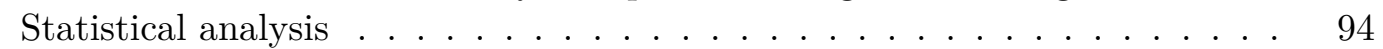

Notable responses . . . . . . . . . . . . . . . . . . . . . . . . 96

Discussion . . . . . . . . . . . . . . . . . . . . . . . . . . . . . . 98

The next step . . . . . . . . . . . . . . . . . . . . 102

Study Four: Exploring Understanding and Confidence 103

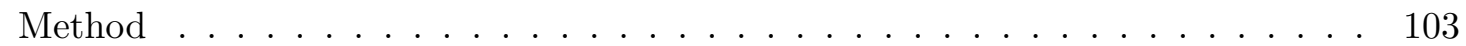

Experimental design . . . . . . . . . . . . . . . . . . . 103

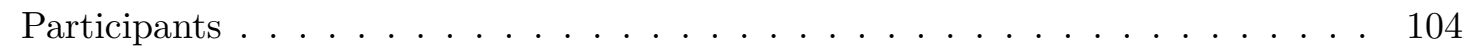

Materials . . . . . . . . . . . . . . . . . . . . . . . . . . 104

Procedure . . . . . . . . . . . . . . . . . . . . . . 104

Demographics data . . . . . . . . . . . . . . . . . . 104

Task . . . . . . . . . . . . . . . . . . . . . . . . 104

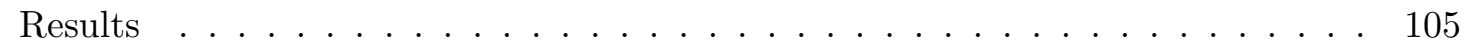

Exploratory analysis . . . . . . . . . . . . . . . . . 105

Website/browser . . . . . . . . . . . . . . . . . . . 105

Communications . . . . . . . . . . . . . . . . . . 107

Malware . . . . . . . . . . . . . . . . . . . . . . . . 110

Discussion . . . . . . . . . . . . . . . . . . . . . . . . . 114

The next step . . . . . . . . . . . . . . . . . 116

\begin{tabular}{ll|}
\hline Study Five: Heuristics, Knowledge, and Self-Perception & 117
\end{tabular}

Keyword heuristic . . . . . . . . . . . . . . . . . . . 118

Functionality heuristic . . . . . . . . . . . . . . . . . . . . . . . . . . . . . . . . . . . . . . . . . . . .

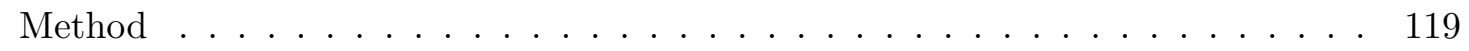

Experimental design . . . . . . . . . . . . . . . . . . . 119

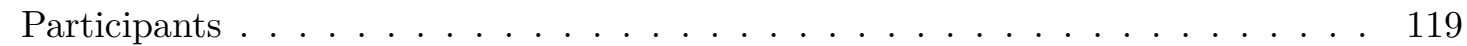

Hypotheses . . . . . . . . . . . . . . . . . . . . . . . . . . . 120

Materials . . . . . . . . . . . . . . . . . . . . . . . . 120

Testing interface . . . . . . . . . . . . . . . . . . . . . . . . . . . . . . . . . 120

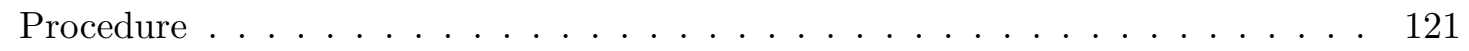

Results for Section One: Domain Names . . . . . . . . . . . . . . . . . 121

Analysis . . . . . . . . . . . . . . . . . . 121

Interpretation . . . . . . . . . . . . . . . . . . . . . . 125

Results for Section One: Malware . . . . . . . . . . . . . . . . . . . 127

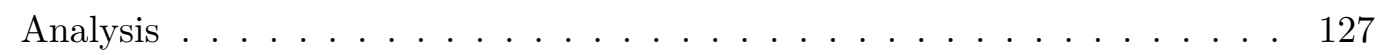

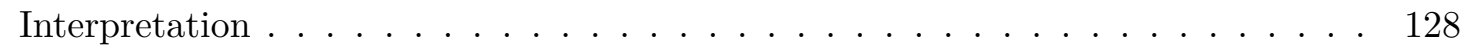

Results for Section Two: Knowledge and Risk . . . . . . . . . . . . . . . 135

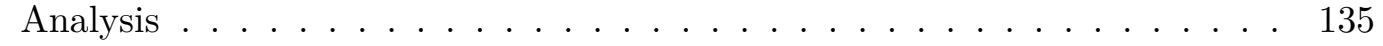


Interpretation . . . . . . . . . . . . . . . . . . . . . . 140

Results for Section Three: Attitudes and Beliefs . . . . . . . . . . . . . . . . . . 143

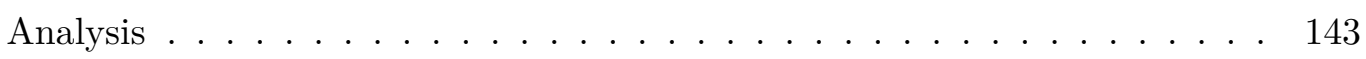

Responsibility . . . . . . . . . . . . . . . 146

Experience . . . . . . . . . . . . . . . . . . . . . 146

Interpretation . . . . . . . . . . . . . . . . . . . 148

$\begin{array}{ll}\text { General Discussion } & 150\end{array}$

Study One: Determining Website Legitimacy . . . . . . . . . . . . . . . . . . . . . 150

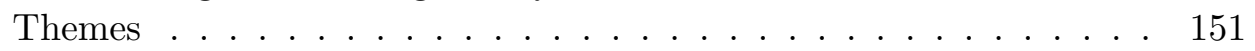

Study Two: Risk Perception of Internet-Related Activities . . . . . . . . . . . . . 152

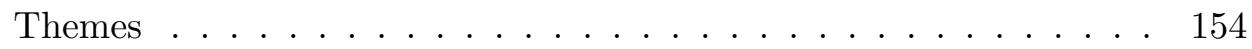

Study Three: Understanding of Internet Software Abilities. . . . . . . . . . . . . 154

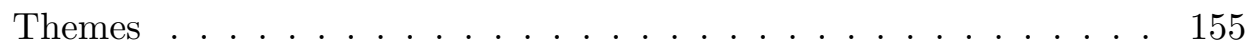

Study Four: Exploring Understanding and Confidence . . . . . . . . . . . . . . . 155

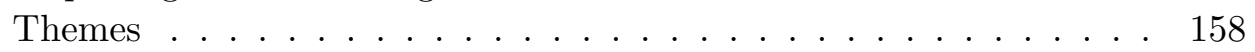

Study Five: Heuristics, Knowledge, and Self-Perception . . . . . . . . . . . . . 159

Themes ............................ 161

Mental Models . . . . . . . . . . . . . . . . . . . . . . . . . . . . . 161

$\begin{array}{ll}\text { Conclusion } & 165\end{array}$

Limitations . . . . . . . . . . . . . . . . . . . . . . . . . 166

Future Work . . . . . . . . . . . . . . . . . . . . 167

\begin{tabular}{ll}
\hline References & 168
\end{tabular} 


\section{List of Figures}

$1 \quad$ Riegelsberger's trust framework . . . . . . . . . . . . . . . . . . . 14

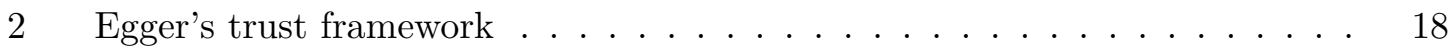

3 Sample phishing website . . . . . . . . . . . . . . . . . . 21

$4 \quad$ Sample phishing email . . . . . . . . . . . . . . . . . . . . . . . . . . . . . . . . . . . .

5 Sample malicious software download website. . . . . . . . . . . . . 25

6 Cranor's Human-in-the-loop Framework . . . . . . . . . . . . . . . . . . . . 30

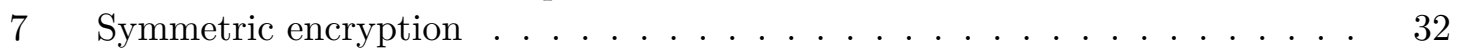

8 \begin{tabular}{lll}
\hline & Asymmetric encryption $\ldots \ldots \ldots \ldots$ & $\ldots$
\end{tabular}

9 Screenshot of the Bank of America website depicted on the Safari Web browser. 37

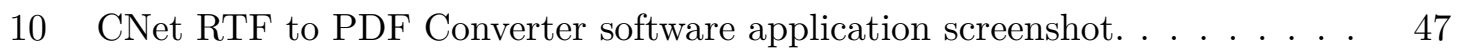

11 DuplicateFinder software application screenshot. . . . . . . . . . . . . 48

12 Boxplots of the 20 activities for the rating scale "Benefits". . . . . . . . . . 64

13 Boxplots of the 20 activities for the rating scale "Likelihood". . . . . . . . . 64

14 Boxplots of the 20 activities for the rating scale "Severity". . . . . . . . . . 65

15 Histograms representing Likert scale responses for the six different rating scales for the activity "Using a search engine". . . . . . . . . . . . . 66

16 Histograms representing Likert scale responses for the six different rating scales for the activity "Sending and receiving email". . . . . . . . . . . 67

17 Histograms representing Likert scale responses for the six different rating scales for the activity "Doing online banking". . . . . . . . . . . . . 68

18 Histograms representing Likert scale responses for the six different rating scales for the activity "Making an online purchase". . . . . . . . . . . . 69

19 Figure showing the relationships between rating scales. . . . . . . . . . . . . 70

$20 \quad$ Figure showing the relationship between the perceived likelihood of information loss rating scale and the severity of loss rating scale. . . . . . . . . . 71

21 Multidimensional scaling graph showing the similarities and dissimilarities between the activities. . . . . . . . . . . . . . . 74

22 Figure depicting a hierarchical clustering dendrogram illustrating the ar-

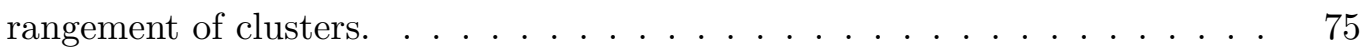

23 Questionnaire page demonstrating the activity scenario, website image screenshot, and rating scale statements found within the survey. $\ldots . . . .884$

$24 \quad$ Study participants distribution of age and gender. $\ldots \ldots \ldots$. . . . . . . 86

$25 \quad$ Study participants distribution of education and expertise. . . . . . . . . . 86

26 Histograms of the rating scales for the activity "Doing Your Banking Online". 88

27 Histograms of the rating scales for the activity "Making an Online Purchase". 91

28 Histograms of the rating scales for the activity "Downloading and Installing

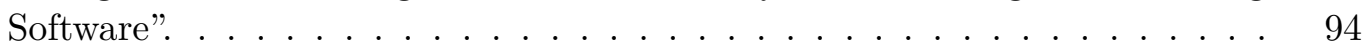

29 Histograms of the rating scales for the activity "Using a Search Engine". . . $\quad 97$

$30 \quad$ Figure showing gender differences in participant responses to activities. . . 101

31 Distribution of participant responses for the high risk and low risk domain

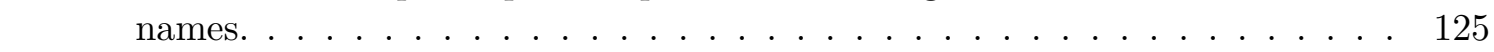

32 Rating scale responses for each of the six funcionality-related and functionality-unrelated events of the photo software application. . . . . . . . 130 
33 Rating scale responses for each of the six functionality-related and functionality-unrelated events of the game software application. . . . . . . . 133

34 Correlation matrix of knowledge and risk assessment for the "download" theme. 141

35 Correlation matrix of knowledge and risk assessment for the "browser" theme. 142

36 Correlation matrix of assessed knowledge by responsible dimension rating

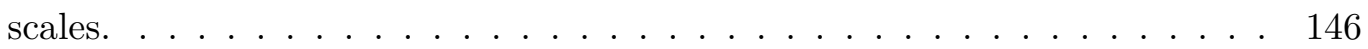

37 Correlation matrix of assessed knowledge by the agree/disagree rating scales. 148

38 CNet RTF to PDF Converter website screenshot. . . . . . . . . . . . . . . 193

$39 \quad$ 1ClickSpyClean website screenshot. . . . . . . . . . . . . . . . . 193

$40 \mathrm{XP} /$ Vista Antivirus website screenshot. . . . . . . . . . . . . . . . . 194

$41 \quad$ Ashisoft Duplicate File Finder 2009 website screenshot. . . . . . . . . . . . . . 194

42 Tucows SpeedUpMyPC website screenshot. . . . . . . . . . . . . . . 195

43 CNet Photoshop website screenshot. . . . . . . . . . . . . . . . . . . . . . . . . . . . . . . . . . .

44 Bank of America website screenshot. . . . . . . . . . . . . . . . . 280

45 Amazon website screenshot. . . . . . . . . . . . . . . . . . 280

46 CNet website screenshot. . . . . . . . . . . . . . . . . . . . . . . . . . 281

47 Google website screenshot. . . . . . . . . . . . . . . . . . . . . 281

48 Correlation matrix of knowledge by knowledgeable dimension rating scales. 430

$49 \quad$ Correlation matrix of knowledge by capable dimension rating scales. . . . . 431

$50 \quad$ Correlation matrix of knowledge by difficult dimension rating scales. . . . . 432

51 Correlation matrix of knowledge by time dimension rating scales. . . . . . . 433

52 Correlation matrix of knowledge by motivated dimension rating scales. . . . 434

53 Correlation matrix of the "How the Internet Works" rating scale. . . . . . . 435

54 Correlation matrix of the "How to protect your security and privacy on the Internet" rating scale. . . . . . . . . . . . . . . . . 436

55 Correlation matrix of the "How to avoid malicious software applications being installed on your computer" rating scale. . . . . . . . . . . . . . . . 437

56 Correlation matrix of the "How to identify potentially harmful websites" rating scale. . . . . . . . . . . . . . . . . . . . . 438 


\section{List of Tables}

$1 \quad$ Contextual Scenarios Used in the Main Task Survey . . . . . . . . . . . . . 50

2 Questions Used in the Main Task Survey . . . . . . . . . . . . . . . . . 51

3 Responses to the Post-Test Multiple Choice Questions. . . . . . . . . . . . . 51

$4 \quad$ Ordering of perceived risk for 30 activities and technologies. . . . . . . . . . 59

$5 \quad$ Mean Scores of Benefit, Risk, and Usage from the 20 Activities Used in the

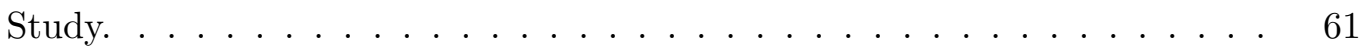

$6 \quad$ Questions Used in the Main Task Limesurvey Questionnaire. . . . . . . . . 63

7 Rating Scales Used in the Main Task Survey for the Activity "Doing Your Banking Online". . . . . . . . . . . . . . . . . 87

8 Participant Responses to the Rating Scales Used in the Main Task Survey for the Activity "Doing Your Banking Online". . . . . . . . . . . . . . . . . 89

9 Rating Scales Used in the Main Task Survey for the Activity "Making An Online Purchase". . . . . . . . . . . . . . . . . . . . 90

10 Participant Responses to the Rating Scales Used in the Main Task Survey for the Activity "Making an Online Purchase". . . . . . . . . . . . . . . . . 92

11 Rating Scales Used in the Main Task Survey for the Activity "Installing a Software Application Downloaded from the Internet". . . . . . . . . . . . . . 93

12 Participant Responses to the Rating Scales Used in the Main Task Survey for the Activity "Downloading and Installing Software". . . . . . . . . . . . 95

13 Rating Scales Used in the Main Task Survey for the Activity "Using a Search

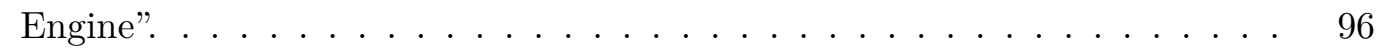

14 Participant Responses to the Rating Scales Used in the Main Task Survey for the Activity "Using a Search Engine". . . . . . . . . . . . . . . . . . . . 98

15 Participant Responses to the Rating Scales Used in the Post-Test Survey. . $\quad 99$

16 Statistical Tests Conducted on the Study Data . . . . . . . . . . . . . 100

17 Website/Browser Category Question 8 . . . . . . . . . . . . . . . . 106

18 Website/Browser Category Question 12 . . . . . . . . . . . . . . . . . 107

19 Communications Category Question 1 . . . . . . . . . . . . . . . . . . 108

20 Communications Category Question 3 . . . . . . . . . . . . . . . . . . . . . . . . . . . . . . . . .

21 Communications Category Question 6 . . . . . . . . . . . . . . . . . . . . . . . . . . . . . . . .

22 Malware Category Question 2 . . . . . . . . . . . . . . . . . . . 112

23 Malware Category Question 4 . . . . . . . . . . . . . . . . . . . . . 113

24 Malware Category Question 5 . . . . . . . . . . . . . . . . . . . . . 114

25 Part 1-Domain Names Statistical Analyses. . . . . . . . . . . . . . . . . . 122

26 Part 1-Domain Names. . . . . . . . . . . . . . . . . . 124

27 Malware: Events relating to the download and installation of photo editing

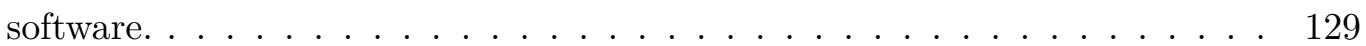

28 Part 1 - Malware Scenario 1 (Photo Editing Software). . . . . . . . . . . . . 131

29 Malware: Events relating to the download and installation of game software. 132

30 Part 1 - Malware Scenario 2 (Game Software). . . . . . . . . . . . . . 134

31 Knowledge Test Questions. . . . . . . . . . . . . . . . . . . . . . 136

32 Perception of Risk Rating Scale Statements. . . . . . . . . . . . . . . . . 137 
33 Perception of Risk Rating Scales: Rating the Risk Involved in Terms of the Severity of Consequences. . . . . . . . . . . . . . . 138

34 Knowledge and Risk Statistics. . . . . . . . . . . . . . . . . . . . . . . 139

35 Rating scales used in each of the five dimensions. . . . . . . . . . . . . . . . 143

36 Part 3-Post Test Question 6. . . . . . . . . . . . . . . . . . . . . . . 144

37 Part 3-Post Test Question 7. . . . . . . . . . . . . . . . . . . . . . 145

38 Website/Browser Category Question 1 . . . . . . . . . . . . . . . . . . . 332

39 Website/Browser Category Question 2 . . . . . . . . . . . . . . . . . . . 333

$40 \quad$ Website/Browser Category Question 3 . . . . . . . . . . . . . . . . . . 334

41 Website/Browser Category Question $4 \ldots \ldots$. . . . . . . . . . 335

42 Website/Browser Category Question $5 \ldots \ldots \ldots$. . . . . . . . . 336

43 Website/Browser Category Question 6 . . . . . . . . . . . . . . . . . 337

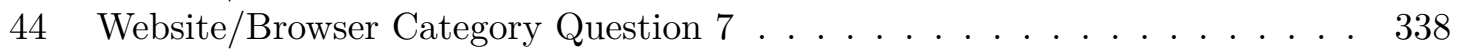

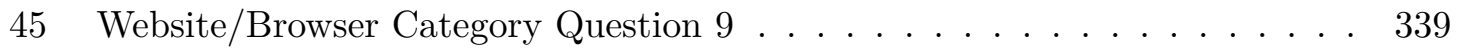

$46 \quad$ Website/Browser Category Question 10 . . . . . . . . . . . . . . . . . . 340

$47 \quad$ Website/Browser Category Question 11 . . . . . . . . . . . . . . . . . . 341

48 Website/Browser Category Question 13 . . . . . . . . . . . . . . . . . . 342

49 Website/Browser Category Question 14 . . . . . . . . . . . . . . . . . . 343

$50 \quad$ Website/Browser Category Question 15 . . . . . . . . . . . . . . . . . . . . . . . . . . . . . . . . . . .

$51 \quad$ Website/Browser Category Question 16 . . . . . . . . . . . . . . . . . . . . . . . . . . . . . . . . . . . .

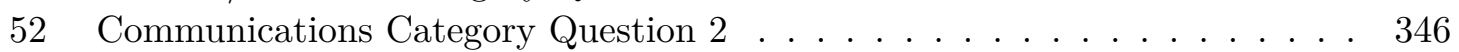

53 Communications Category Question $4 \ldots \ldots$. . . . . . . . . . 347

54 Communications Category Question 5 . . . . . . . . . . . . . . . . . 348

55 Communications Category Question 7 . . . . . . . . . . . . . . . . . . . 349

56 Communications Category Question 8 . . . . . . . . . . . . . . . 350

57 Communications Category Question 8 (continued...) . . . . . . . . . . . . . 351

58 Malware Category Question 1 . . . . . . . . . . . . . . . . . . . . . . . . . . . . . . . . 352

59 Malware Category Question 3 . . . . . . . . . . . . . . . . . . . . . 353

60 Malware Category Question 6 . . . . . . . . . . . . . . . . . . . 354

61 Part 2-Knowledge Question 1. . . . . . . . . . . . . . . . . . . . 415

62 Part 2-Knowledge Question 2. . . . . . . . . . . . . . . . . . . . . . . . . . . . . . . . . . . . . .

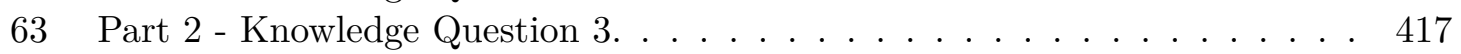

64 Part 2 - Knowledge Question 4. . . . . . . . . . . . . . . . . . . . . . 418

65 Part 2-Knowledge Question 5. . . . . . . . . . . . . . . . . . . . . . . . 419

66 Part 2 - Knowledge Question 6. . . . . . . . . . . . . . . . . . . . 420

67 Part 2-Knowledge Question 7. . . . . . . . . . . . . . . . . . . . . . 421

68 Part 2 - Knowledge Question 8. . . . . . . . . . . . . . . . . . . . . 422

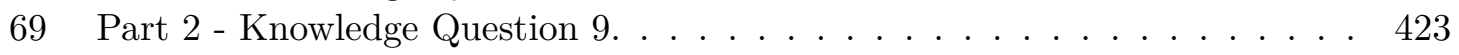

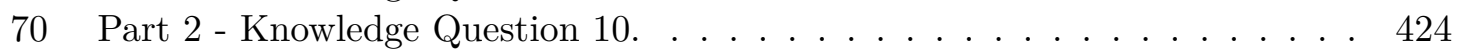

71 Part 3-Post Test Question 1. . . . . . . . . . . . . . . . . . . . . . 425

72 Part 3-Post Test Question 2. . . . . . . . . . . . . . . . . . . . 426

73 Part 3-Post Test Question 3. . . . . . . . . . . . . . . . . . . . . . . 427

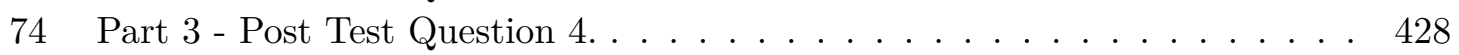

75 Part 3-Post Test Question 5. . . . . . . . . . . . . . . . . . . . 429 


\section{List of Appendices}

Appendix A . . . . . . . . . . . . . . . . . . 177

Appendix B . . . . . . . . . . . . . . . . . . . . . . . . . 185

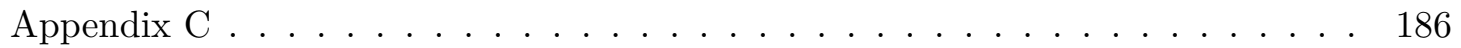

Appendix D . . . . . . . . . . . . . . . . . . . . . . . . 189

Appendix E . . . . . . . . . . . . . . . . . . . . . . . 193

Appendix F . . . . . . . . . . . . . . . . . . 196

Appendix G. . . . . . . . . . . . . . . . . . . . . . . 2233

Appendix $\mathrm{H} . \ldots \ldots \ldots$

Appendix 1 . . . . . . . . . . . . . . . . . . . . . . . . . . . . 2 253

Appendix J . . . . . . . . . . . . . . . . . . . . . . 255

Appendix K. . . . . . . . . . . . . . . . . . . 256

Appendix L . . . . . . . . . . . . . . . . . . . . . . . . . 258

Appendix M . . . . . . . . . . . . . . . . . . . . . . . . 259

Appendix N . . . . . . . . . . . . . . . . . . . . . . . . . . . . . . 268

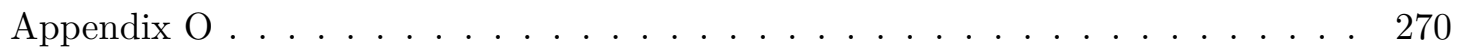

Appendix P. . . . . . . . . . . . . . . . . . . . . . 271

Appendix Q . . . . . . . . . . . . . . . . . . . . . 272

Appendix R . . . . . . . . . . . . . . . . . . . . . . . 280

Appendix S . . . . . . . . . . . . . . . . . . . . . . . . . . . . 282

Appendix T . . . . . . . . . . . . . . . . . . . . . . . 284

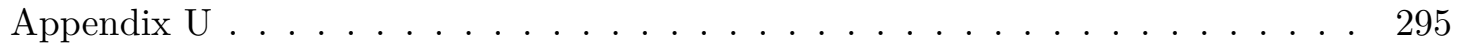

Appendix V . . . . . . . . . . . . . . . . . . . . . . . . . . 2297

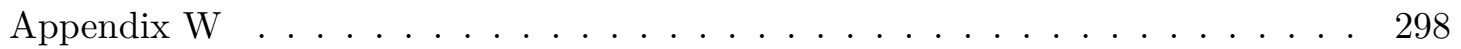

Appendix X . . . . . . . . . . . . . . . . . . . . . . . . 332

Appendix Y . . . . . . . . . . . . . . . . . . . . . 355

Appendix Z . . . . . . . . . . . . . . . . . . . 385

Appendix AA . . . . . . . . . . . . . . . . . . . . . . 395

Appendix AB . . . . . . . . . . . . . . . . . . . . . . . 397

Appendix AC . . . . . . . . . . . . . . . . . . . . 398

Appendix AD . . . . . . . . . . . . . . . . . . . . . 415

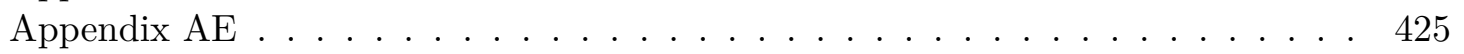




\section{Introduction}

Security is an increasingly important topic when dealing with everyday interactions on the Internet. When people choose to visit a given website, they make decisions about whether to trust the source of the website in question. There exist many different methods for attackers to trick users into trusting their website and agreeing to enter private information or to download and install a piece of software (Dhamija, Tygar, \& Hearst, 2006). It appears that a large majority of users commonly place their trust in most, if not all, websites they encounter, and this can lead to significant security problems. Once an attacker has breached a person's defences (or lack thereof), they now have access to the user's personal information, which they are then able to exploit to their advantage. Additionally, if malicious software is downloaded and installed on the user's computer, any other security mechanisms can be defeated. Any solutions proposed to reduce the threat of these malicious websites should include a consideration of the psychological processes of the users.

The motivation of this thesis deals with one primary issue. We need to better understand how users come to a decision about whether to trust or distrust a given website. Once a better understanding of users' decision-making processes has been reached, this understanding can then be used to develop better strategies, materials, and tools to help users identify malicious websites for their own protection.

In order to study this issue, the research question identified was "How do people perceive, understand, and make decisions regarding the dangers associated with malicious websites, and are they able to identify and understand the various levels of risks associated with engaging in online activities?" Overall, five studies are presented that attempt to find out more information regarding users' understanding of the security aspects of online interactions, including software download websites, secure websites (e.g., online banking websites, online electronic commerce websites), and so forth.

This thesis comprises this introduction, an overview of the present research, the five studies, and a discussion and conclusion. The remainder of the Introduction is divided into two sections. The next section, background in psychology, presents summaries of relevant 
psychological literature. Following this, the section on background in computer security presents and discusses computer security threats that are commonplace over the Internet, especially "phishing" websites (illegitimate websites that look like the legitimate site it is emulating) and malicious software download websites. Then, technical aspects of Internet security, such as Web certificates and signatures of software and hardware, are reviewed. That section concludes with a discussion of the solutions proposed in the research literature to some of the security threats prominent on the Internet.

\section{Psychology Background}

In order to better understand the issues related to trust decisions and their security implications in an online environment, background material specific to some of the research literature available in the areas of computer security and psychology is introduced. Some of the topics covered in this section discuss deception, social engineering, phishing, trust, and informational content.

Deception. Deception has been linked to a large portion of the fraudulent activities

that take place in many online environments, including phishing and malicious software websites (Jagatic, Johnson, Jakobsson, \& Menczer, 2007).

Research on the understanding of scam victims, conducted by Stajano and Wilson (2011), proposes that users are often vulnerable to the types of attacks mentioned above because the behaviours they exhibit do not follow a specific pattern that allows them to better protect themselves against potentially deceiving actions on the part of an attacker. This behavioural pattern can often lead users to make the wrong decision regarding a specific interaction. Based on their observations in an experimental setting, the researchers identified seven key principles of behaviour that users demonstrate when they are in a given situation requiring them to make a trust decision. Each of the seven principles identified in their research demonstrates how users are often deceived into making a trust decision without having examined all of the critical information regarding the interaction beforehand (Stajano \& Wilson, 2011). In the paragraphs below, each of Stajano \& Wilson's principles 
is discussed. Several of these principles are based on the earlier work by Cialdini (2009).

Distraction principle. The distraction principle claims that users will focus on the most attention-grabbing aspect of a given stimulus while interacting with a given system. In relation to online interactions, this behaviour allows attackers to present information to users in a way that steers their attention away from the true purpose of the system. A fake antivirus software application can be used as an example. When a user navigates to a given website, the application is presented to the user in an attempt to persuade them to purchase and download it, the attention of the user is directed to a screen supposedly depicting several critical flaws currently affecting their computer. The real purpose of this attack is to persuade users to purchase the fraudulent software, but the method used by attackers in order to achieve this is to distract users from realizing that they are being scammed, since the software application appears to be fulfilling its purpose by alerting them to other, more crucial — though fraudulent — information.

Social compliance principle. The social compliance principle suggests that people follow the advice of authorities without question. Authors of malicious software make use of this principle when designing their distribution website by posting banners of third-party authorities claiming the association with the Better Business Bureau, VeriSign, TrustE, and various others. By doing this, attackers are leveraging the trust users have in authorities and the likelihood that users will decide in favor of trusting the advice of the authority and downloading the software application found on the website. The reason why this presents a problem in online interactions is that users often do not follow through with their investigation of the feedback of the third-party authorities regarding the website in question. They usually simply take the presence of the authorities' banners as proof that the website has been approved by these organizations and that it is safe to use. By taking the information at face value, users have not considered that the banners can be duplicated and placed on any website with little to no effort on the part of the attackers.

Herd principle. The herd principle states that users will make decisions regarding interacting with a given system based on the actions and opinions of their friends, family, 
and co-workers, among others. If people from one or more of these groups gives the user in question positive feedback regarding a given website, then it is highly likely that the user will decide in favor of trusting the website as well. The explanation of the behaviour represented by this principle is that users will blindly follow the opinions of their friends and family because they let their guard down when others like them behave in a similar way. Attackers rely on this principle by depicting positive feedback scores and testimonials on their websites as a way of enticing other users to interact with their website. Furthermore, the feedback scores and testimonials could easily have been fabricated by the attackers.

Dishonesty principle. The dishonesty principle operates on the assumption that users will make decisions regarding illegitimate activity, providing that they should reap some sort of benefit in the end. An example of this could be a person, working for a certain organization, who falls victim to a computer attack of some kind. The user could have inadvertently installed a Trojan horse program when browsing the Internet for something specific, such as music files, or movies. Knowing that they engaged in illegitimate activity over the Internet, the user would then have strong incentives not to divulge any of the activity that took place when the malicious program was installed, for fear that an investigation would reveal their lack of vigilance, and appropriate action would be taken against them. By displaying this behaviour, users can easily become victims because they are easily lured into thinking they will profit from an activity. However, because the malicious program had been installed on the machine in question, it would be the attacker who would benefit from the user's participation in the illegitimate activity.

Need and greed principle. The need and greed principle works on the assumption that, once users have a pressing need for something in particular, they will make an immediate trust decision instead of an informed one regarding a given website or product. Attackers can easily make use of this behaviour by manipulating the users into performing specific actions that they normally would not be willing to do if the desire for something in particular wasn't present in the equation. 
Time principle. The time principle operates on the assumption that users use different decision strategies when they are under pressure to make a quick decision regarding a given interaction. Much like the distraction principle, attackers make use of the time principle by integrating a large amount of information directly onto their website. Information can be in the form of unnecessarily lengthy explanations of the product or service offered, or pop-up windows that provide no additional functionality to the website. These and other methods have the effect of cutting short the amount of time users have to become more informed on the product or service offered by the website, which will lead them to make a hasty decision based on the information they have gathered during this time.

Deception principle. The deception principle suggests that users can easily be fooled by something that appears to be real, but is in fact a replica. In terms of computer security research, this principle is most often used by phishing websites, where the website in question represents a mirror image of the legitimate website. As an example, if a user receives an email that appears to be from the security team working for the PayPal company, they will read the message and make a judgment about trustability. If the user is convinced of the veracity of the email message, then he or she will click on the provided link. This link would then redirect them to the fake website, where the user must make another trust decision. However, as the fake website is a mirror image of the real website, users would be likely to trust the website in question and interact with it, thus being drawn in by the phishing attack.

These seven principles have helped outline some of the behaviours that users exhibit while engaging in interactions over the Internet. Some of the problems users have in detecting the deception methods used in conjunction with these online interactions are discussed in the next section.

Social engineering. Research conducted by Qin (2007) investigated the difficulties users have in detecting deception, and the ramifications this can have on the victimization of users by way of social engineering attacks. A social engineering attack can be defined as being a non-technical (i.e., human-oriented) method of obtaining information (Bacon, 2014). 
This method relies heavily on the use of human interaction, and it can also involve deceiving other users to break normal security procedures in order to obtain the desired information. A common phishing technique, which is a form of social engineering attack, is to send out an email containing a subject line displaying an urgent matter that needs the user's immediate attention. A subject line such as "Your PayPal account will be suspended if action is not taken immediately" would be likely to gain immediate attention from some users. Within the message itself are explicit warnings along with official logos of the company. Once the user has opened the email message, the content of the message would give them information regarding the status of their account, and require that they click on a link provided in the email message in order to enter their account information and resolve the problem with their account. However, once the user has entered their information into the form found on the website, the attack has succeeded and the user's personal account information has been obtained, and can be used for malicious purposes by the attackers (Workman, 2008).

Research on social engineering discusses truth-bias, stereotypical thinking, and processing ability as factors that influence users' ability to detect deception Millar \& Millar, 1997). Qin (2007) proposes that attackers find social engineering as being a more simple way of discovering information, while this intrusion is often less detectable by users than are other types of attacks (e.g., malicious software). In order to counteract this, he proposes using an automated method of detecting deception in order to help users avoid potentially dangerous online interactions. The method proposed uses automatic deception detection techniques in order to determine whether machines can detect deception more easily than humans. The machine system takes inputs (messages in audio, text, or video format), then parses each input and looks for the presence of deceptive indicators. Next, the machine system takes the parsed cues and applies statistical and classification methods in order to tell deception apart from truth. Finally, the machine system outputs its decision as to the veracity of each input cue (Qin, 2007).

The automatic system used in Qin's research is useful in detecting deception cues because it doesn't judge information the same way human users do. Human users make 
judgments on appearance, stereotypical thinking, ratings of honesty, and so forth. These findings suggest that human users are less motivated to expend the additional effort required in order to determine with a certain degree of accuracy whether the information presented to them carries legitimate or malicious intent. For this reason, social engineering can be a useful tool that attackers can use in order to gain access to a user's private information. From the user's perspective, this type of deception is therefore especially dangerous.

Further research on social engineering was conducted by Huber, Kowalski, Nohlberg, and Tjoa (2009) using social networking sites as the medium for gathering information from users. In their experiment, the researchers were interested in determining how deception could be used as a tool for gathering users' personal information through these types of websites. They conducted simulated attacks on various users using Automated Social Engineering (ASE) bots — which are automated programs — as their primary method of gaining information. The social networking website Facebook was selected to conduct the automated social engineering attacks. The goal of the ASE bot's attack on Facebook was to obtain other users' account information. In order to achieve this, the researchers programmed the bot to follow a specific order of actions when interacting with potential victims on the social networking site. The bot would gather basic information from potential victims in various groups on Facebook. Once the desired users had been identified through a series of parameters (e.g., geographical network, open profiles, and so forth), the bot began to build relationships with the users through online chatting. Once the bot had gained a certain degree of trust from the user in question, it then began its attack by either providing a link to the user containing a malware program, or simply asking the user for personal information. This indicated a successful attack and ended the bot's interactions with the user in question.

The results from the study showed that a large proportion of users did not realize that the person they were chatting with on Facebook was in fact an automated bot. This means that automated social engineering programs can be used as tools by attackers to easily obtain information from users, because the latter do not easily recognize deceptive 
clues when engaged in online interactions in a social networking setting.

Trust. The notion of trust in human-computer interaction has become a widely researched topic, in view of the fact that an increasing number of technologies are based on users transacting with each other without ever actually meeting face to face. In cases of interactions through computing systems, the notion of trust can be defined as the following: "trust concerns a positive expectation regarding the behaviour of someone or something in a situation that entails risk to the trusting party" (A. S. Patrick, Briggs, \& Marsh, 2005, p. 77). According to the researchers, this definition of trust can be further broken down into three separate layers of trust: dispositional trust, which is the psychological disposition or personality trait which one decides to either trust or not trust; learned trust, which refers to someone's tendency to trust, or not to trust, another person or entity based on previous experience; and situational trust, which refers to the basic tendencies that are adjusted in response to situational cues. These three layers or trust work together in an effort to produce sensible trusting behaviour in specific situations where a person may or may not feel familiar.

Adding to this definition, there are two distinct categories in which trust can be classified: trustworthiness, and trustability. Trustworthiness refers to the technical aspects of trust. An organization can be seen as trustworthy if they have the right credentials, the ability to provide stated services, and if they can provide proof of authenticity via a certificate or third party recommendation (e.g., the Better Business Bureau).

Trustability refers to a person's perceived trust in regards to the organization or person who is at the receiving end of trust. These perceptions of trust can be ascertained by the presentation of information to the trustor (e.g., in guarantee statements, privacy policies, and so forth), benevolence towards the individual, and previous experience in dealing with the trustee.

There will always be people wanting to take advantage of others for personal gain. As such, trustworthiness and trustability can be faked in an attempt to lure someone to make a trusting decision based on presented information and eventually transact with the 
deceiving party. Fraudulent trustability is discussed in later sections.

It is important to note that something can be trustworthy but not trustable. Bad design of online content can be an example of this. If an organization creates a website in order to attract clients, and it turns out the website is severely lacking in proper design components, they could be viewed as untrustworthy. In contrast, something can be trustable but not trustworthy. An example of this would be a website attempting to attract users to download its software application. The website content could be done in a very graphically appealing manner, but the reputation of the vendor or organization could be questioned due to a lack of security certificates placed on the website.

Understanding trust. In order to better understand the trust decisions that are made by users regarding their interactions on the Internet, it is important to understand what brings them to make these decisions. This section examines some of the research literature looking into issues such as trust, risk perception, and decision-making.

Humans are known to have the ability to sense and avoid environmental conditions that can be harmful (Slovic, 1987). People learn from past experiences and are thus able to avoid repeat scenarios that can be considered dangerous. This leads people to be able to perceive different levels of risk. But how does this perception of risk translate to interactions between humans and websites on the Internet? Do people perceive online threats as being harmful to them, or more as a minor annoyance? It is known that phishing and malware attacks can lead to the theft of private information, which is itself a harmful experience, though not in a physical sense. Previous sections dealing with the dangers of the Internet show evidence demonstrating that people do not behave in similar ways when faced with online threats than they do when faced with real-life situations that can bring them physical harm (Schechter, Dhamija, Ozment, \& Fischer, 2007)).

The perception of risk. This brings the discussion to some of the theories regarding the perception of risk and the probabilities associated with various kinds of activities. Research has been conducted in various fields relating to the perception of risk. Research in sociology has shown that the perception of risk is influenced by social and cultural factors 
(Douglas \& Wildavsky, 1982) as well as by friends, family, and individual authorities (e.g., doctors, government officials) (Short, 1984). Research concerning geographical elements have shown the understanding of human behaviours in conjunction with natural hazards (e.g., earthquakes, tsunamis) as well as technical hazards (e.g., nuclear reactors) (Burton, Kates, \& White, 1978).

The risks involved with various activities and technologies can mean different things to different people (Slovic, 1987). What is meant by the differentiation of people's perception of risk is the level of expertise that each carries in regards to a given technology or activity. Examples of these include nuclear power, aviation, medical technologies, sports, and so forth. When experts of a given topic talk about the risks involved, they generally discuss the dangers involved with the activity or technology, while non-experts will make judgments about the risks involved in another fashion. They tend to correlate risk with the potential for harm to themselves or other people.

To address misunderstandings about risk, it has been proposed to use a mental models approach to understand what people know and need to know concerning risks and appropriate responses (Morgan, Fischhoff, Bostrom, \& Atman, 2002). The researchers propose that mental models are unlikely to involve a strict correspondence between phenomena already understood and the unknown. The authors instead suggest that:

By definition, the audience for a communication lacks a complete understanding of its subject matter. Yet for most risks, people have at least some relevant beliefs, which they will use in interpreting the communication. They may have heard some things about the risk in question. It may remind them of related phenomena. Its very name may invoke some associations. If they must make inferences about the risk, such as how big it is, how it can be controlled, or who manages it, they will assemble their fragmentary beliefs into a mental model, which they will then use to reach their conclusions. (Morgan et al., 2002, p. 21)

In other work, Camp (2009) reviews research exploring a variety of metaphors that 
may play roles in users' mental models for understanding computer privacy and security. For example, the author suggests a crime metaphor entails belief that a central authority is involved, whereas warfare suggests tight controls, economic metaphors suggest litigation, and public health metaphors imply coordinated public action. Overall, the idea is that different metaphors may play a role in shaping user understanding of risks associated with privacy and security in an online setting.

Most recently, Rafalak, Abramczuk, and Wierzbicki (2014) conducted a large study relating personality characteristics to risk taking in an Internet context. Their results showed a wide range of behaviour, where propensity to take risk distinguishes different kinds of behaviour. Some people overestimate risk, others underestimate risk, and these affect determination of website legitimacy.

Decision-making. People are faced with the task of making decisions on a daily basis. Some of these decisions made might concern a problem that has to be resolved, or a choice between a set of alternatives. When people are faced with problem-solving or decision-making, they must choose how to act in accordance with each scenario. Research shows that people often decide in favor of options that carry superior value, even when that selection is paired with another option that has a much inferior value (Slovic, 1975 . Tversky, Slovic, \& Sattath, 1988). If offered the choice, a person would be more likely to select a pair of hockey teams in an office betting pool that stood at opposite ends of the points standings than they would be likely to select a pair of teams that are much closer to each other in the points standings.

In previous gambling research, it has been found that people tend to base gambling decisions on whether they are actually betting their own money, or simply discussing betting their own money. It has been found that people are more willing to wager their money when their chances of winning a small amount are low to moderate. Conversely, people are less willing to wager their money when their chances of losing are high (Slovic \& Lichtenstein, 1968). In this research, they found that people stated they would risk their money when discussing various odds of winning and losing. These people never actually placed any 
gambling bets. In contrast, persons asked to place bets on similar odds were more likely to be conservative with their money when the odds of losing were high, than they were likely to place a bet when the odds of winning were low to moderate. This means that they paid more attention to the odds of losing their money when the odds were high. These findings represent an important discrepancy between people's decisions when faced with discussing the risks associated with a given activity, and their perceived risks when actually engaging in the activity. It is likely that these findings could be generalized to other research in risk perception, and are not only found in gambling research. For example, a "Threat Avoidance" model has been proposed for user behaviour in Internet security challenges (Liang \& Xue, $2009,2010)$.

Satisficing. From a decision-making point of view, research has shown that people would rather devote less energy to finding an adequate solution to a given problem than expending more energy in order to find the optimal solution to the problem (Prabha, Connaway, Olszewski, \& Jenkins, 2007). It has been argued that humans do not possess the cognitive resources to optimize their decision-making skills, and that they usually do not know the information concerning the probabilities of specific outcomes. As such, they are less likely to evaluate all possible outcomes with sufficient precision (Simon, 1956). Simon called this process satisficing.

People rely on heuristic cues, such as representativeness, availability, and the truth bias (Tversky \& Kahneman, 1974), when making decisions about whether deception is or is not involved in a specific scenario (Burgoon, Blair, \& Strom, 2005). Truth bias refers to a person's judgment of information as being truthful, regardless of the veracity of the information provided. People rely on these heuristics in order to expend a small amount of cognitive effort when faced with a given scenario that involves some sort of choice between alternatives in order to come to a decision. It was found that people rely heavily on the truth-bias heuristic to determine whether information presented to them is truthful or deceitful. Because people are willing to expend less energy than is necessary to analyze all of the information presented to them, they are more likely to decide in favor of trusting the 
information presented rather than be distrustful of it.

When dealing with an online setting, users are presented with various types of information for any given website. Based on the decision-making strategy of satisficing information needs, it would be possible that users are searching for information pertinent to what they hope to achieve during their online session, and are much less concerned with any other information they are not aware of or interested in that might be important to the protection of their private information. Crucial information such as security indicators and warning pages may be ignored simply in order to save time in an effort to complete a transaction, or log in to an email account, as well as many other types of online activities.

The theory behind satisficing information needs could be a valid one to study in conjunction with users and their behaviours in an online environment, but it begs the question as to whether the problems plaguing users stem from issues in decision-making. It could be possible that users don't even realize they have a decision to make when faced with their choices concerning online interactions. Some of these issues are discussed in conjunction with the results from some preliminary work that was conducted in this area.

Riegelsberger's trust framework. Riegelsberger's model of trust has been designed for the purposes of improving our understanding of the factors that support trust in various online interactions, including call centre interactions, e-commerce transactions, and online gaming, to name a few (Riegelsberger, Sasse, \& McCarthy, 2005). This can be used to understand the process involved in users making trust decisions, how websites can be designed to appear trustworthy, and also how illegitimate websites can mislead users. The framework is depicted in Figure 1 .

In Riegelsberger's trust framework there are two main participants in an interaction: the trustor, and the trustee. In most computer interactions, the trustor refers to the party — in most cases a person - who is engaged in a particular activity (e.g., shopping on amazon.com for a DVD). The trustee is the person, or system, on the receiving end of the interaction (i.e., the amazon. com transaction processing servers).

The foundation of this trust framework is based on three key factors that support 


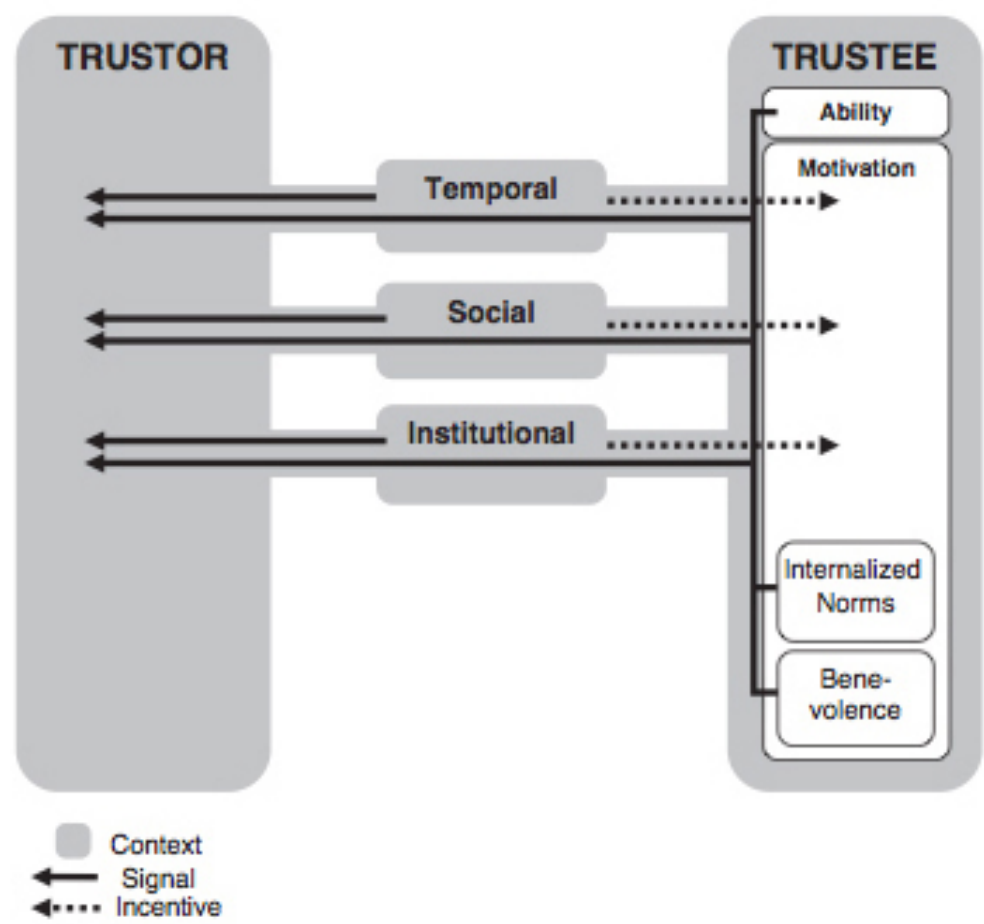

Figure 1. Riegelsberger's trust framework (Riegelsberger et al., 2005).

trusting behaviours on the part of both the trustor and the trustee. Each of these three factors, temporal embeddedness, social embeddedness, and institutional embeddedness, fits into the category of contextual-properties. These contextual properties provide a situational reference to allow the trustee to behave in a trusting manner.

The relevance of this framework to the thesis is that it might help understand how a trustor might assess a website. However, much of the framework is targeted at prospective trustees (i.e., the company represented by the website).

Temporal embeddedness. Temporal embeddedness refers to the information available which would allow for future interactions between the trustor and the trustee. The prospect of future transactions provides the trustee with a significant incentive to act in a trustworthy manner. The example of amazon. com can once again be used in order to illustrate temporal embeddedness. As the trustee in the relationship, amazon.com needs to provide the context that gains the trust of their perspective clients. As amazon.com is an online-only merchant, 
it has developed a number of methods that attract clients, such as special sales, coupons, and discounts for multiple item purchases. This initial investment, whose sole purpose is to secure a client base, will prove rewarding to Amazon in the long run. Prospective clients will go through the process of choosing merchandise from the selection of items, ask for help from the customer service staff, read up on the past customer reviews for various products, and eventually order items online and have them delivered to their home. This process will help clients get acquainted with Amazon as an online store, how they operate, what services they offer, and so forth, while creating a trusting relationship between trustor and trustee in the process.

It is important to note that, as interactions become more frequent between the trustor and trustee, the trustor will learn to predict the behaviours of the trustee, resulting in an increase - or decrease if there were problems — in trust between both parties. This shows how the principle of temporal embeddedness may be used by prospective trustees to help gain the trust of trustors. In contrast, prospective trustors should be aware of this strategy in making their assessment. These two perspectives work the same way for social embeddedness and institutional embeddedness as well.

Social embeddedness. Social embeddedness refers to the information gained on the part of the trustor in regards to the trustee's past performance (i.e., the trustee's reputation). It can be argued that the more reputable a given organization is, the more trustable it is. Honesty, reliability, and dependability come into play when determining the degree of social embeddedness of any given trustee. What other people have to say regarding a given organization can prove effective in helping others make decisions regarding their relationship with a given merchant.

As an example, online marketplaces such as eBay make use of feedback scores in an attempt to alleviate the burden on buyers when they are deciding whether the seller involved in the item auction is trustworthy. Previous buyers are given the option of rating the seller once a transaction between the two have has been completed. The buyer can provide positive or negative feedback to the seller, relating to the seller's shipping price 
and speed, and the overall transaction experience. The buyer is also able to leave a short comment regarding the transaction. The seller is also able to leave feedback regarding the buyer. This constitutes an anomaly compared to normal everyday transactions, since most online marketplace websites provide feedback regarding only the seller, or trustee. eBay allows sellers to leave feedback to buyers in order to give other prospective sellers information regarding the trustworthiness of the buyer. eBay only allows sellers to leave positive feedback to buyers, or no feedback at all, and the "unpaid-item strike" mechanism is used to record on the buyer's account that items were not paid for.

Institutional embeddedness. Institutional embeddedness refers to the trustee's direct involvement with one or more institutions. In this case, institutions are defined as being an organization that influences the behaviour of trustors in an interaction setting. These institutions often create an image (i.e., seal) in order to attribute trustworthiness to a given organization. Examples of such seals include the Canadian government's listing of the 50 best managed Canadian companies, or the inclusion of one's organization in the Better Business Bureau list of companies. Trust seals, such as eTrust (www.etrust.org) and VeriSign (www.verisign.com), also contribute to the institutional embeddedness of an organization's website by allowing users to click on the links provided by the seal image, and find out more information on why the organization qualified for the trust seal in question.

An organization that is able to receive one or more of these seals will be regarded as a very trustworthy company, even though the trustor does not necessarily know why by what criteria - the company was able to achieve such a status. The idea is that an organization, corporation or government states that a company is trustworthy is sufficient to allow the trustor to have a high degree of confidence in the company in question, although the effectiveness of this strategy is unclear (Kirlappos, Sasse, \& Harvey, 2012).

The three contextual properties discussed above relate to both the trustor and the trustee. There are three intrinsic properties that are significant only to the trustee's involvement in the framework. These are: ability, benevolence, and motivation for internalized norms. 
Ability. The intrinsic property ability refers to the competence, skill, and expertise that a trustee has over his or her specific areas of interaction. A company's ability to provide the service or goods they offer will significantly increase the trust it receives from its customers (i.e., trustors). Consequently, any deviation in quality of any of these factors will impact the company's trustworthiness. A company that is not able to fulfill the needs of the trustor will likely be seen as untrustworthy.

Motivation for internalized norms. Internalized norms refer to the motivation the trustee has in conforming to the social norms as these are defined by the geo-cultural milieu where the particular business is based. These norms are an important part of the social fabric, and trustees benefit from adapting their business model to reflect some or all of these. A number of companies have been known to include a description of their values and standards while displaying their mission statement on the company website. This has the effect of informing others (i.e., trustors) that the company believes in these social norms, and has taken the necessary steps to ensure that they are reflected in their business model and practices.

Benevolence. Benevolence refers to one person's positive action towards another person. In the context of this framework, the benevolence is an attribute of the trustee, where the interaction between trustor and trustee determines how the trustee will act towards the trustor, while keeping his or her best interests in mind. In some cases, the trustee may act benevolently towards one specific trustor but will act differently towards another, depending on a number of factors affecting their relationship (e.g., length of the relationship, positive experiences in the past between trustor and trustee, and so forth).

Egger's trust framework. Egger's model of trust was constructed with user interactions in e-commerce transactions in mind (the MoTEC model: [M]odel [o]f [T]rust for $[$ E]lectronic $[\mathrm{C}]$ ommerce) Egger, 2000). There are three principal components to this model: a user's pre-purchase knowledge of the vendor, the interface properties of the vendor's system, and informational content of the vendor's system. The framework is depicted in Figure 2 . 


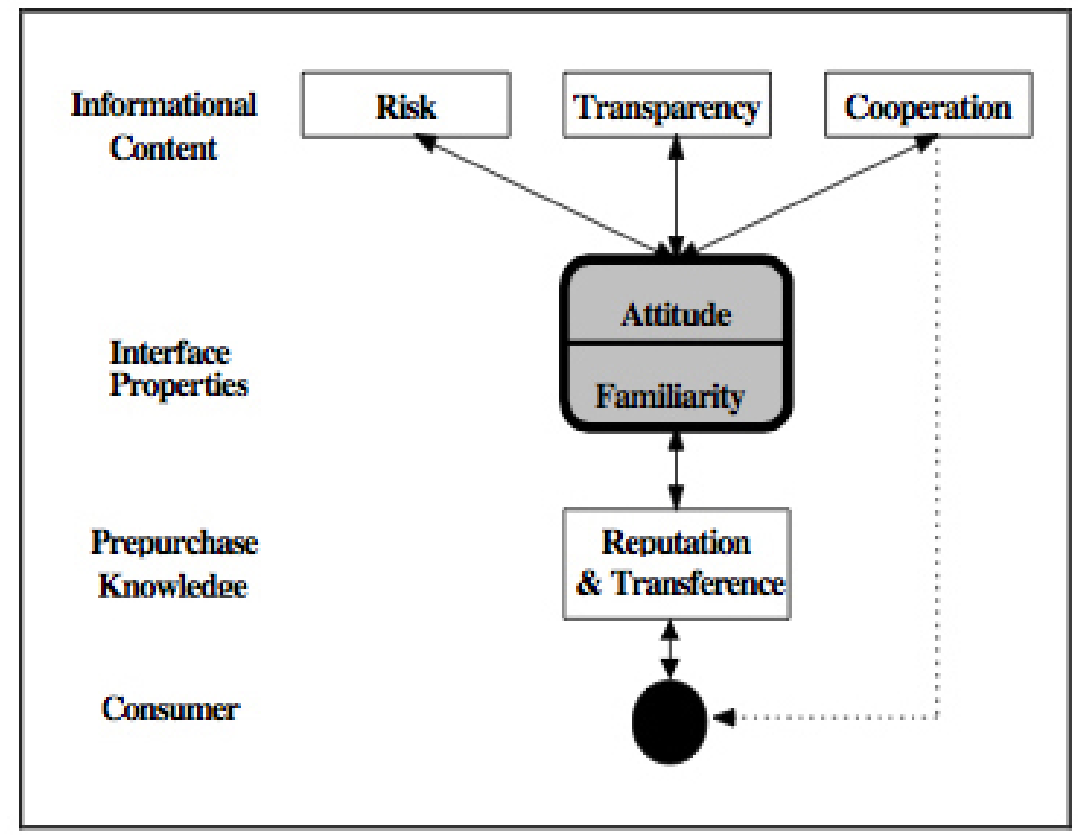

Figure 2. Egger's trust framework (Egger, 2000).

Pre-purchase knowledge. Pre-purchase knowledge refers to the opinions, or mindset, that a customer already has regarding the trustworthiness of the merchant in question. This can include - but is not limited to - the vendor's reputation, a previous experience the customer has had in dealing with that merchant in the past, or reports from third party companies (e.g., the Better Business Bureau). This last is regarded as the transference of trust from one company or organization to another.

Interface properties. Interface properties are broken down into two components: first, the familiarity (e.g., a user's previous experience in navigating the website) of the user regarding the system as well as its usability (e.g., how it operates, how easy the site is to navigate, and so forth) and domain model (e.g., a conceptual model of the system which describes the various concepts or constructs relevant to that system and the relationships between them) come into play in the framework; and second, the attitude of the user in regards to his or her first impression of the domain as a whole - the impression is based on the way the information is presented, the overall architecture of the website, the graphical 
content and design, how quickly the site loads, among other factors.

Informational content. Informational content is broken down into three separate components: risk, transparency, and cooperation. Risk refers to the information provided by the merchant to the buyer regarding risks of any kind (i.e., financial, privacy) and the guarantees made available to the user when transacting with the merchant. Transparency is the vendor's openness regarding their business policies and privacy policies. This extends somewhat the definition of the risk component, where a user is given information regarding the inherent risks in transacting through the merchant's system. Cooperation refers to the willingness of both parties (buyer and merchant) to interact within the transaction in question via the merchant's online system.

The Internet allows users access to an overwhelming amount of information, which can sometimes appear daunting. Rieh and Belkin (1998) propose that, in order for this amount of information to make sense to the user, it has to be filtered in some way as to allow the pertinent information to be extracted from the source. The quality of the information presented by the source is paramount to being able to filter it and make sense of it. As such, in order to filter the information, people must make judgments and decisions based on its quality. The authors identify seven important aspects that contribute to information filtering, such as the source of the information, its presentation, as well as its accuracy in relation to the context, and so forth. In order to learn more about the methods people use in order to filter information using these facets, the authors conducted a study which looked at authority and quality of information on websites. Their research demonstrated that users differentiated significantly between institutional authorities (e.g., universities and governmental institutions) and individual authorities (e.g., doctors, lawyers), giving the former authority more credibility. They also found that there was a general consensus that the quality of the information presented and the authorities responsible for its existence on the Internet generally contributed to people's favorable trusting decisions regarding the content in question. 


\section{Computer Security Background}

As mentioned in the introductory section, the focus of this thesis is on attempting to understand the decisions users make regarding various kinds of websites, in order to improve the methods used to relay crucial information to these users regarding the characteristics of a given website that identify it as being legitimate or illegitimate. For this, an understanding

of two main issues related to computer security is needed: security threats and security defence. First, the different threats that are prominent on the Internet are examined. Second, the different methods organizations use in order to identify themselves and their websites as being secure are examined. Then, the technical aspects of Internet security related to website identification and server communications are reviewed.

Security threats and attacks. There are several different methods of gaining access to someone's private information over the Internet. This section presents and discusses phishing websites as well as malicious software download websites. Both types of attacks attempt to mask the real intent of the website in question.

Phishing. Phishing sites are imitation websites made to look like their real counterparts. Phishing sites are created to lure users into a false sense of security and provide their personal information, such as login and identity information (Dhamija et al., 2006). Authors of phishing websites rely on the user's perceived trust of the legitimate source in question, whether it is a bank (e.g., Royal Bank of Canada), an e-commerce or trading website (e.g., eBay, E-Trade), third-party merchant websites (e.g., PayPal), or websites that hold one's private information such as email (e.g., Gmail) or social websites (e.g., Facebook). An example of a phishing website can be seen in Figure 3. This example demonstrates how users could be lured into interacting with the website in question, as it looks just like the real banking website. However, there is one important difference: the URL of the malicious website is spelled with two V's instead of one W (Dhamija et al., 2006).

Usually, links for phishing websites are sent to a user's email address, carrying messages of urgency regarding the compromise of their private information. The message could imply that a user's account has been compromised, and they should immediately login to 


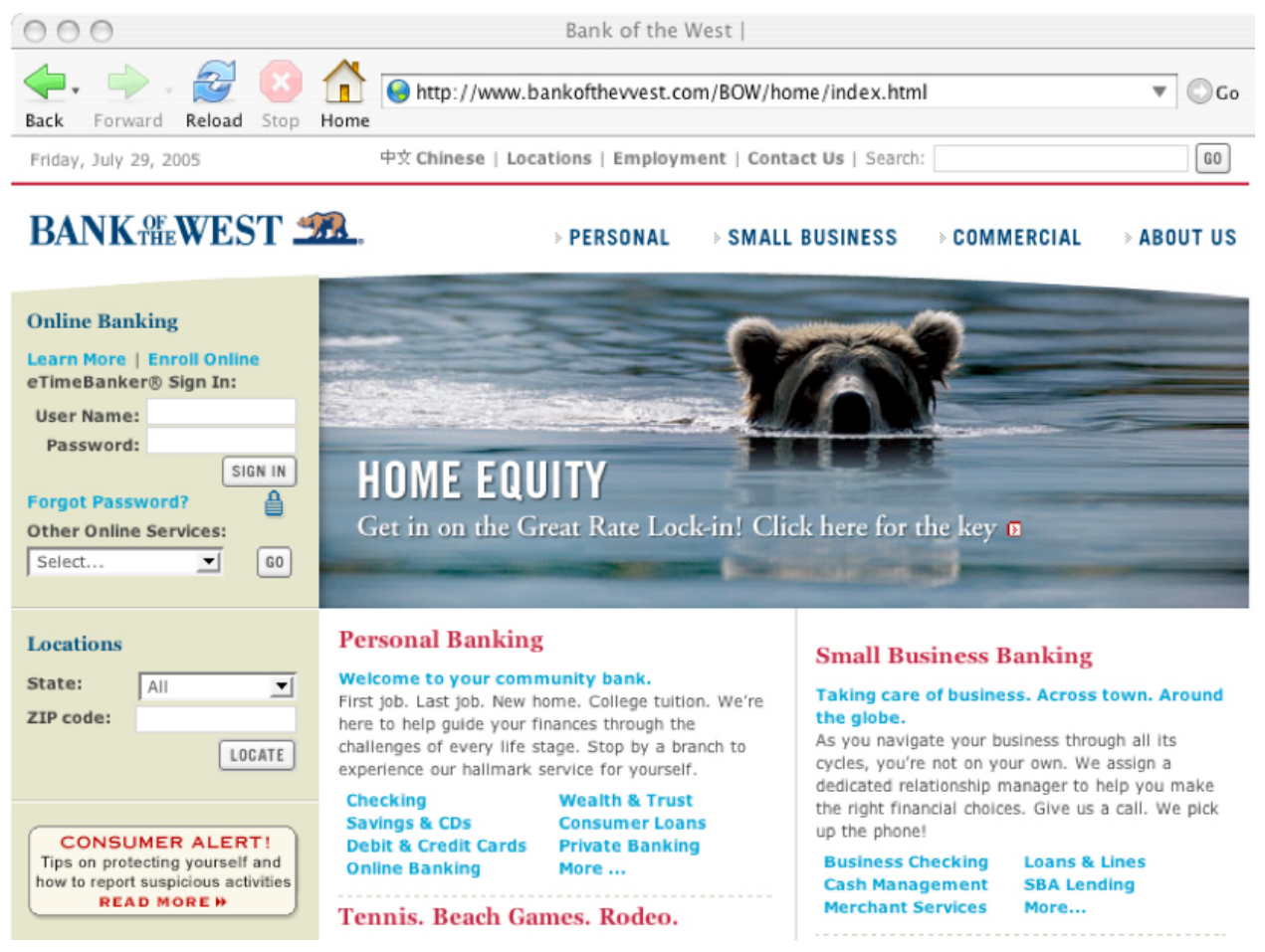

Figure 3. Sample phishing website (Dhamija et al., 2006).

their account and change their password, credit card numbers, or other types of personal information. The attack is accomplished by redirecting the user from the email message to a website that looks the same as its legitimate counterpart. However, unknown to the user, the website is actually a fake representation of the real one. Once the user inputs the private information for their real account, the attack has succeeded in gaining access to that information. The user is then left without access to the real website, puzzled as to what has occurred during the authentication phase (Dodge, Carver, \& Ferguson, 2007). Moreover, the attackers can then act in place of the owner, transferring funds, making commitments, and so forth. An example of a phishing email message is shown in Figure 4. This example demonstrates how a user might be lured into providing their private information upon having clicked on the link provided.

Research conducted by Dhamija et al. (2006) demonstrated empirical evidence that shows some of the malicious strategies often used by attackers in order to make phishing attacks successful. In this research, the authors analyzed a large number of phishing attacks 


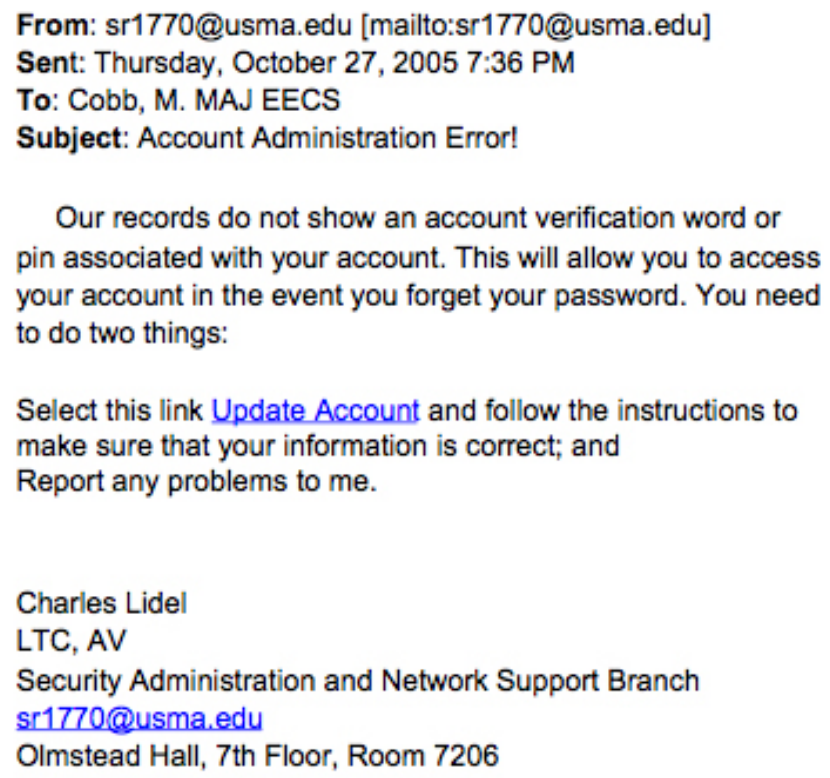

Figure 4. Sample phishing email (Dodge et al. 2007).

and developed an experiment with the goal of providing them with vital information that would explain why the strategies used by attackers in phishing attacks often lure users into giving up their personal information. Their results showed that an important proportion of users cannot distinguish a legitimate website from a fake website (Dhamija et al., 2006). Website indicators used in order to signal trustability (e.g., https, Web certificates, and so forth) are often not completely understood or even noticed by participants. Security indicators presented directly in the content of the website could easily be faked by a phishing website. An example of this would be a lock icon placed directly on the website itself. The lock icon is often present on the website and not on the browser chrome, the surrounding border which is not under control by the website. This might provide users with a false sense of security, where they may decide in favor of trusting the website in question and provide their personal information, or download a specific piece of software. Most users are not aware that the Web security indicators are not part of the website itself, but part of the chrome of the browser. Neither a legitimate website nor a malicious website can alter the chrome's security indicators in a way that would provide more or less information 
about the securities inherent with the website in question. In order to make the security indicators present on the website, the organization or person responsible for the website must go through specific processes in order to obtain each specific security indicator. As an example, the lock icon present on the browser chrome is only present when a valid Web certificate has been added in conjunction with the website address by an external authority (e.g., VeriSign). The lock icon is automatically placed on the browser chrome when the Web certificate is present and valid (i.e., not yet expired).

Several strategies for successful phishing attacks have been identified by the Anti Phishing Working Group (APWG). This group defines three dimensions used within these strategies in order to perform a successful phishing attack, and these have been identified through experimental research (Rasmussen, Aaron, \& Routt, 2011). These dimensions are: lack of knowledge, visual deception, and lack of attention.

Lack of knowledge. The APWG proposes that many users lack the knowledge of how most of the technology behind computer systems works, such as operating systems, software applications, email applications, and the Internet. As such, most users are unable to distinguish between a website and its domain name, which leads them to accept fraudulent URLs (Uniform Ressource Locators) as belonging to a legitimate website. Furthermore, users lack knowledge concerning website security indicators, which often leads them to make a bad decision regarding a website's legitimacy. Most users don't understand secure-socket layer (SSL) connection indicators, which indicate that the information is sent back and forth to the server using a secure connection. This problem is compounded by the fact that the different browsers available to the public (e.g., Chrome, Firefox, Internet Explorer, Safari, among others) do not depict secure connections using the same methods.

Visual deception. Attackers make use of visual deception in order to trick users into trusting the website in question. Some of the methods used include: substituting letters within a domain name so that the fake name closely resembles the real name (e.g., www.roya1bank.com instead of www.royalbank.com); using an image of a legitimate Internet address with a functional link to a malicious website; and, cloning a legitimate website 
and substituting the legitimate programming code of a given form on the website with programming code created for malicious purposes.

Lack of attention. As the third dimension of the strategies used in deceiving users into giving up their personal information, lack of attention involves the notion that users may have knowledge of the various security indicators prevalent in website design and operation, but may make trusting decisions when they are not fully attentive as to the veracity of these indicators. As an example, even if a user knows about SSL connections, they may not necessarily make an effort to find the SSL security indicators on the website in question if they feel they have browsed to the website that they strongly believe is the legitimate version. Also, if a user mistypes a given URL in the address bar, and are then redirected to a malicious website with a domain name closely resembling that of the legitimate website, the user may mistakenly believe that the website shown is in fact the real one, and make a trusting decision in favor of interacting with it.

Malicious software download websites. Malicious software download sites operate somewhat differently from phishing websites (Verizon, 2012). In many cases, malicious software download sites attempt to lure users with the promise of a software application that will help the user in some way, such as an antivirus or a speed-boosting program, or a similar type of application. Websites that fall into the category of malicious intent often attempt to mirror legitimate, professional-looking websites (e.g., http://www.microsoft.com). By designing their websites in a way that mimics the more reputable websites, the attackers are hoping that users will treat their website as comparable, in terms of professional design and implementation of content, to the other legitimate websites currently on the Internet. If users find that the website in question fits well with their idea of a legitimate website, they may be more likely to trust the website in question without conducting any further investigation in regards to its legitimacy. Once users download and install malicious software applications, these then have access to the user's computer. Furthermore, with these privileges, attackers can collect and use the user's personal information for malicious purposes. Figure 5 provides an example of a malicious software download website. 


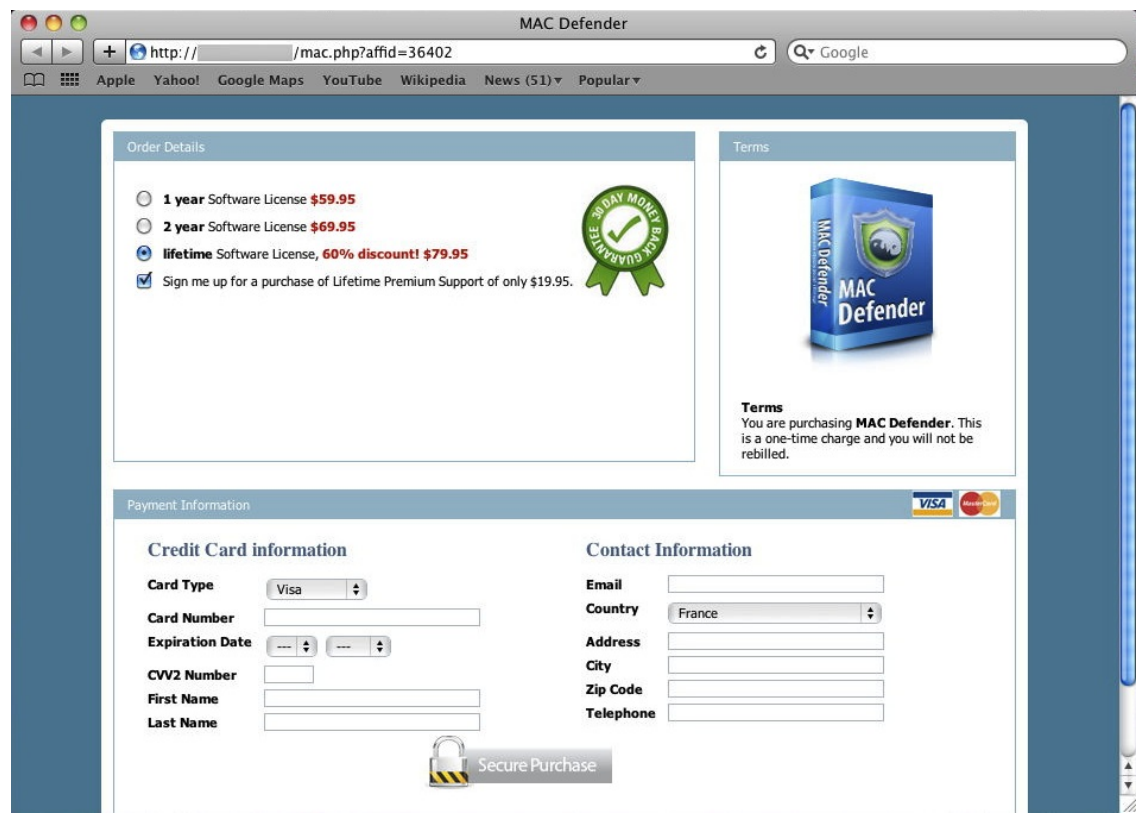

Figure 5. Sample malicious software download website (from www.howtogeek.com).

Nowadays, malicious software often involves a variety of characteristics relating to installation, propagation, and harm done. Taxonomies of malware are continually being developed and updated to capture a variety of important aspects to aid tracking and defence (Bontchev, 2005; Kirillov, Beck, Chase, \& Martin, 2011). In the security community, there continues to be a lexicon of commonly used names for some kinds of malicious software. Some of the most commonly discussed are described below.

- Virus: A computer virus is a type of malware that propagates by inserting a copy of itself into and becoming part of another program. It spreads from one computer to another, leaving infections as it travels. Viruses can range in severity from causing mildly annoying effects to damaging data or software and causing denial-of-service (DoS) conditions (Operations, 2015).

- Worm: Computer worms are similar to viruses in that they replicate functional copies of themselves and can cause the same type of damage. In contrast to viruses, which require the spreading of an infected host file, worms are standalone software and do not require a host program or human help to propagate (Operations, 2015). 
- Trojan: A Trojan is another type of malware named after the wooden horse the Greeks used to infiltrate Troy. It is a harmful piece of software that looks legitimate. Users are typically tricked into loading and executing it on their systems. After it is activated, it can achieve any number of attacks on the host, from irritating the user (popping up windows or changing desktops) to damaging the host (deleting files, stealing data, or activating and spreading other malware, such as viruses). Trojans are also known to create back doors to give malicious users access to the system. Unlike viruses and worms, Trojans do not reproduce by infecting other files nor do they self-replicate. Trojans must spread through user interaction such as opening an e-mail attachment or downloading and running a file from the Internet (Operations, 2015).

- Bot: "Bot"' is derived from the word "robot" and is an automated process that interacts with other network services. Bots often automate tasks and provide information or services that would otherwise be conducted by a human being. A typical use of bots is to gather information (such as web crawlers), or interact automatically with instant messaging (IM), Internet Relay Chat (IRC), or other web interfaces. They may also be used to interact dynamically with websites. A malicious bot is selfpropagating malware designed to infect a host and connect back to a central server or servers that act as a command and control $(\mathrm{C} \& \mathrm{C})$ center for an entire network of compromised devices, or "botnet." With a botnet, attackers can launch broad-based, "remote-control", flood-type attacks against their target(s) (Operations, 2015).

- Keylogger: Software keyloggers track systems that collect keystroke data within the target operating system, store them on disk or in remote locations, and send them to the attacker who installed the keylogger (Sagiroglu \& Canbek, 2009).

Usable security. Usable security is a research area that has merged over the last decade to address human factors challenges in computer security. A prominent goal is to offer a system that provides ease of use for the computer user, as well as security, simultaneously. Influential early papers in this area were published in the 1990s. The paper 
by Zurko and Simon, "User-Centered Security", outlined three major categories of work: making secure systems usable, developing user-centered security models, and considering user security needs (Zurko \& Simon, 1996). The paper "Why Johnny Can’t Encrypt" showed the critical importance of empirical study in the area, demonstrating that even the most usable email encryption software allowed devastating errors to be made (Whitten \& Tygar, 1999). Adams and Sasse's paper "Users are not the Enemy" highlighted another important factor whereby legitimate users were often seen as antagonists by the security community (Adams \& Sasse, 1999). In 2005, Cranor and Garfinkel published the first book in the area, including a wide range of early work by multiple researchers (Cranor \& Garfinkel, 2005). More recently, in 2014, Garfinkel and Lipford published an extensive overview of the area (Garfinkel \& Lipford, 2014).

One set of literature in the area addresses ways of helping users avoid phishing. Some projects at Carnegie-Melon University have emphasized ways of training users to identify and avoid phishing websites (Sheng et al., 2007, Kumaraguru et al., 2009). This approach teaches several rules of thumb that can help users recognize phishing websites. However, several of these rules, such as spelling mistakes and poor website design, have become unreliable as attackers have become more sophisticated. Other research explores how Web browsers might help users avoid phishing websites. An early study by Wu, Miller, and Garfinkel (2006) showed the difficulty of this approach because users' understanding of phishing was poor, and they often failed to inspect or understand the browser warnings. A later study by Egelman, Cranor, and Hong (2008) investigated users' reaction to a range of phishing strategies, and showed that most people were deceived by at least one. More recent research studied the effectiveness of domain highlighting, a strategy adopted by several popular Web browsers, that draws user attention to a website's domain name (Lin, Greenberg, Trotter, Ma, \& Aycock, 2011). This approach was shown to have some benefits, but was of limited effectiveness because users still chose to trust fraudulent websites. Other research has focused on deeper understanding of user behaviour. Early studies of user reasoning about phishing suggested people might avoid risks they understood well, but not risks with 
which they were unfamiliar (Downs, Holbrook, \& Cranor, 2006); moreover, factors such as severity of consequences was not found to be a reliable indicator of behaviour (Downs, Holbrook, \& Cranor, 2007). More recently, neuro-physiological studies of phishing have been conducted using electroencephalograms and eye gaze patterns (Neupane, Rahman, Saxena, \& Hirshfield, 2015). These studies suggested that users are often engaged in determining website legitimacy, but often fail to take enough time to adequately inspect and process the available information. Although all this research primarily concerns phishing and capture of user credentials, these results all also relate to the problem of malware, software created with malicious intent. One of the main ways in which users are persuaded to install malware is from fraudulent software download websites, where apparently well-known software is offered for download. If the user perceives the website to be legitimate, they will download and install the software, and suffer the consequences. Baxter, Malahy, and Lubin (2013) conducted a study where users were shown search engine results; users tended to trust what was shown by the search engine, falling prey to both phishing attacks and malware download websites.

Another set of literature relevant to this thesis concerns browser warnings. Early work by Whalen and Inkpen (2005) studied the use of visual security cues in Web browsers using eye tracking to determine user attention. They found very little attention paid to certificate information and found that, once people have made a brief initial assessment, they rarely consider the browser information again. Herzberg (2009) presents review of SSL and how it might be used to detect phishing, finding that the elements involved are complex and likely misunderstood. The authors conclude that the mechanisms are not sufficient to support users in avoiding phishing. A study by Sunshine, Egelman, Almuhimedi, Atri, and Cranor (2009) explored user understanding of browser SSL warnings, and suggested potential improvements. The study found poor understanding of SSL and the browser warnings, and showed the suggested improvements did have better results when tested with a computer science student participant group. However, even with the improvements, too many participants exhibited dangerous behaviours, and the researchers speculated whether it might 
be better to block access to websites giving rise to SSL warnings rather than letting the user decide. Some work has explored better user interface design for browsers and warnings (Sobey, Biddle, Oorschot, \& Patrick, 2008, Biddle, van Oorschot, Patrick, Sobey, \& Whalen, 2009: Shi, Xu, \& Zhang, 2011). More generally, other research focused on browser warnings specifically — rather than on security certificates — including warnings from external sources (i.e., search engines) (Akhawe \& Felt, 2013). The researchers found that these helped users avoid malicious websites; however, many users ignored these warnings. The researchers suggested that, by reducing the weight of user attention, as well as reducing the steps needed in order to accomplish a certain task, would thereby improve user amenability to the security mechanisms in place. In follow-up research, Felt, Reeder, Almuhimedi, and Consolvo (2014) investigated users' lack of observation of browser warnings. The researchers found that users often ignored the browser warnings, and did not understand the differences between warnings, specifically if these warnings were found on websites familiar to them. Finally, Almuhimedi, Felt, Reeder, and Consolvo (2014) reported on their attempts to apply safety warning literature in order to make browser warnings more understandable to users. Though their results were not as significant as the researchers had hoped, their efforts did make an impact in the research community, contributing to the changing of the design of the Google Chrome Web browser. Further discussion of security certificates is given in the next section, on technical aspects of Internet security.

Finally, there are some proposals of relevance in the usable security literature that proposed general frameworks of relevance to this thesis. Bravo-Lillo, Cranor, Downs, and Komanduri (2010) proposed a framework based on supporting development of better mental models. The idea was that, if users better understood the components involved in identifying malicious websites, then they would be more vigilant and able to make better decisions. The concept of mental models was discussed in consideration of perception of risk earlier in this thesis, and later in the third study, as well as in the General Discussion. Cranor (2008) articulated the human-in-the-loop security framework to address the issue of where human reasoning should be employed in security decision making. The model identifies critical 


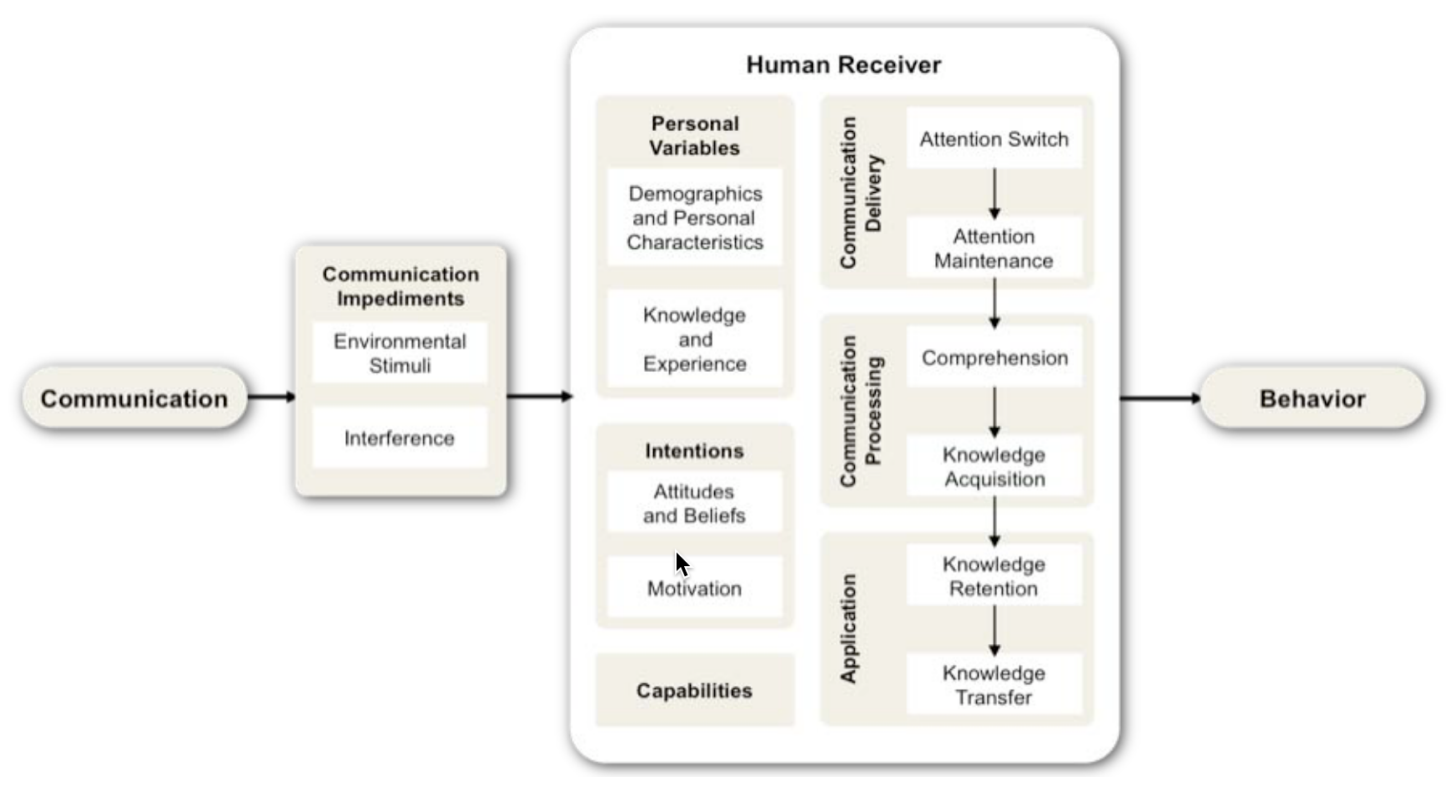

Figure 6. Cranor's Human-in-the-loop Framework (Cranor, 2008).

elements, such as intentions and capabilities as well as factors relating to attention and learning (see Figure 6). This framework is considered later in the thesis in the discussion of results.

All empirical studies in usable security face great difficulties relating to ecological validity. These issues are described in detail by Motiee, Hawkey, and Beznosov (2010) and Sotirakopoulos, Hawkey, and Beznosov (2011). The key problem is reproducing the relevant context addressing considerations such as users perceiving real risk, their personal responsibility, and the relative rarity of dangerous situations amid long periods of day to day activity.

Technical aspects of Internet security. Internet security can be quite complex for the average user. Computer scientists deal with issues ranging from digital signatures, secure-socket layer communications, and certificates on a daily basis. In order to provide more insight into how to deal with users being able to identify more secure websites, an understanding of the types of security support are present while browsing the Internet is needed. 
Web certificates. Web certificates originate from cryptography (the practice and study of hiding information) (Coron, 2006). Internet websites make use of a public key certificate (also known as a digital certificate or identity certificate) in order to identify its source. A public key certificate is an electronic document which uses a digital signature to bind a public key with the identity of its source. In this signature is often found information pertaining to the name of a person or organization responsible for the website and/or certificate, as well as their contact information and other relevant information. The Web certificate can be interpreted by the user in order to verify that the public key actually belongs to the source identified therein.

In most public key infrastructure (PKI) schemes, the signature present in the certificate will have been issued by a certificate authority (Brayton, Finneman, Turajski, \& Wiltsey, 2006). These authorities are organizations that issue the Web certificates (e.g., VeriSign). In a Web of trust scheme, as will be discussed in more detail in later sections, the signature found in the certificate will be signed either by the user responsible for the website (referred to as a self-signed certificate) or other users (referred to as endorsements). In either of these cases, the signatures found on the certificate are an affirmation by the certificate signer that the information pertaining to the source matches the information found in the public key.

Symmetric key cryptography. Symmetric-key cryptography refers to encryption methods in which both the sender and recipient share a key. The key is used in order to perform two separate tasks: encryption and decryption (Menezes, Vanstone, \& Oorschot, 1996). Figure 7 depicts the process of symmetric encryption and decryption.

Asymmetric key cryptography. Asymmetric key cryptography, also known as public-key cryptography, refers to a system that requires two separate keys in order to function. The first key is used to encrypt the plaintext, while the other key is used to decrypt the cyphertext. Neither of the two separate keys is able to do both the encryption and the decryption functions. One of the keys is referred to as being the public key, while the other one is referred to as being the private key (Menezes et al., 1996). Figure 8 demonstrates the 


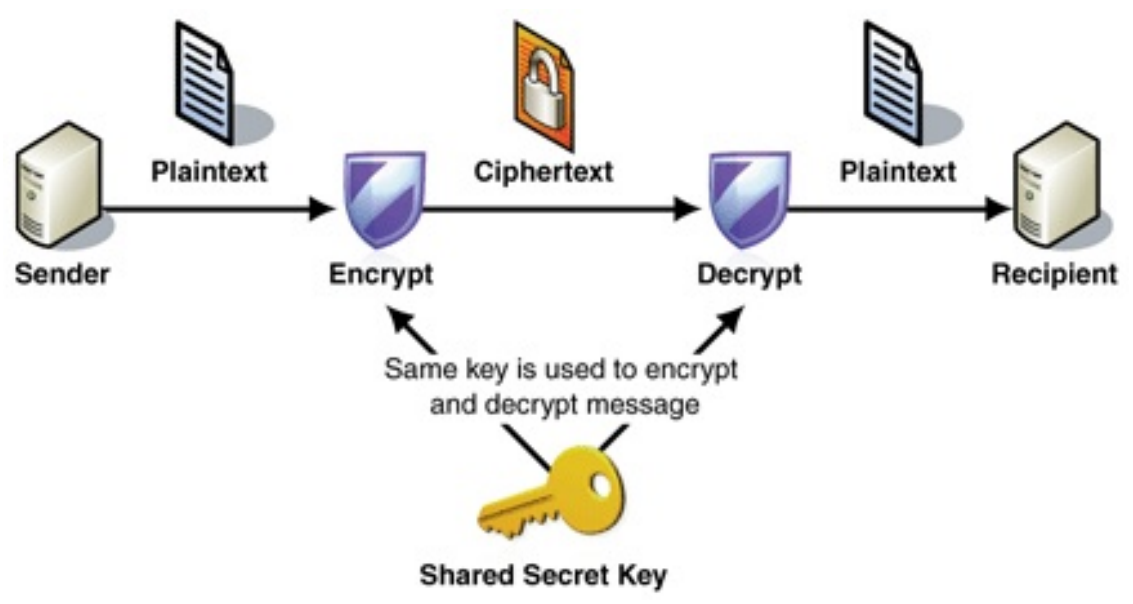

Figure 7. Symmetric encryption (Microsoft, 2005).

process of asymmetric encryption and asymmetric decryption. Because the private key is never distributed, holders of the public key can infer that only the legitimate sender holds the private key needed for encryption. This therefore can be used in order to determine the identity of the sender.

Software signatures. A software signature is similar to a Web certificate, in the way that it is used to identify software installed on a computer (Shinder, 2005). Software signatures are customizable by the organization or user responsible for the creation of the product, and identify that it has been digitally signed by the organization responsible for its creation.

When importing software packages, the software signature is extracted from the package file and checked automatically. For example, the Adobe Photoshop installer package will extract the signature directly from the installer package, and check it with the Adobe website. This means that the source of the software product will know where and when the application was installed on the system, and the creator and the user will be in a position to know if anyone has tampered with the product. These are the same advantages that a user can rely on, since they can verify the identity of the source of the software product before deciding whether to install it or not on their machine. This makes software signatures similar to Web certificates, where the identity of the source can be readily identified 


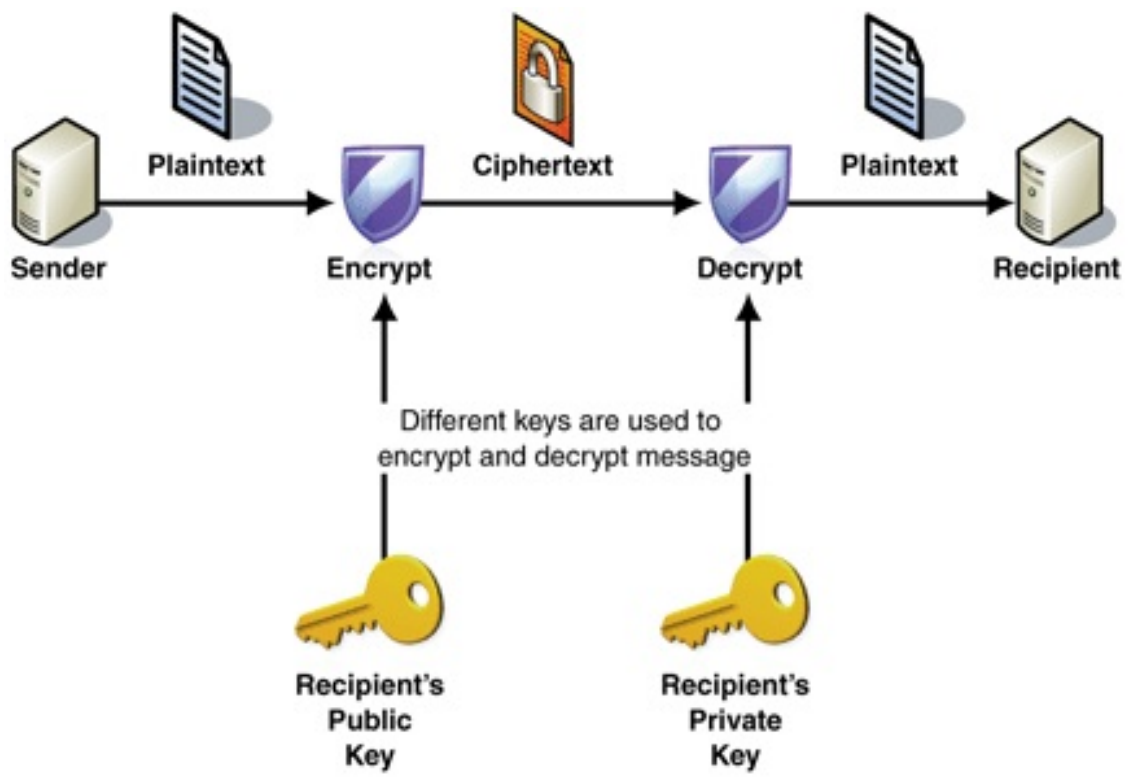

Figure 8. Asymmetric encryption (Microsoft, 2005).

by simply verifying the certificate signature authenticity and issuing authority.

Hardware signatures. Hardware signatures are provided by trusted platform modules (TPM). A TPM is a highly specialized computer chip that is installed on the motherboard of a computer for the purposes of authenticating the hardware installed on the system in question (Mellor, 2005). This chip then authenticates the system on which it is installed. In order for this process to work, the chip module stores the information pertaining specifically to the system on which it is installed; such information can be in the form of encryption keys, digital certificates, and user-generated passwords. Having such a module on one's personal computer has some distinct advantages: the risk that important information stored on the system will not fall into the wrong hands, should the computer be stolen or hacked by an attacker, is minimized; a secure subsystem runs the system's authentication processes; and, software-based attacks are less likely to succeed because of the hardware protection.

Proposed solutions. Having discussed the security threats of illegitimate websites and the defensive technical infrastructure available for computer security, some of the proposed solutions to the problems in Internet security are presented and discussed. The goal 
of this thesis is to make sense of the knowledge that will be gained regarding users' decisionmaking processes concerning trust in Internet websites and software download sites. Doing so will secure a position to better understand how users make trust decisions, which will in turn allow for the design and implementation of improved security techniques in determining legitimate versus illegitimate websites and online content. Reviewing these proposed solutions will inform what kind of understanding might facilitate their effectiveness.

Web of trust. A Web of trust is a concept that was first described in conjunction with cryptography (Coron, 2006). The web of trust establishes a trusted authenticity of the relationship between a public key and its owner. Contrasting the public key infrastructure, the web of trust was conceptualized using a decentralized model, where independent users are tasked with determining the legitimacy of the relationship between owner and key.

A Web of trust can be extended to the identification of good versus bad websites on the Internet. A community-powered tool, the Web of Trust (www.mywot.com), allows registered users to search the Internet and report comments and feedback regarding any of the websites they browse. Other users interested in finding out about a specific website's reputation can browse to the Web of Trust website (http://www.mywot.com), type in a requested URL, and read up on feedback given in regards to the website in question by other users from all over the world.

Of course, such a system can have negative impacts on the Internet community as a whole. This particular web of trust allows users to provide feedback in either of two forms: feedback ratings (on a 100-point scale), and free-form comments. One of the disadvantages to this system is that users are not forced to provide both types of feedback. They are allowed to choose to submit only one type of feedback, while leaving out the other type of feedback. This has a tendency to bias the feedback received by the website that is being rated. If a user has a positive experience regarding a website, they are likely to leave positive feedback, but leave out any additional free-form comments. However, when another user has a bad experience, they are likely to provide comments via the free form only. As an example, the website www.tucows.com, an established software download website, received 
an $87 \%$ rating on the four categories of point-scale feedback (i.e., trustworthiness, vendor reliability, privacy, and child safety). These are very high feedback ratings. Conversely, www. tucows.com received several different negative free-form comments, which discuss issues dealing with malicious software, adware, browser exploits, and other various problems. Allowing users to leave either type of feedback results in users who have had good experiences leaving good point-scale scores, while users who have had bad experiences leave only negative free-form feedback for the website in question. This has the effect of causing uneven feedback for the same website, thus confusing users who are looking to find out more information regarding a given website.

Browser chrome security information. A browser's chrome is defined as being the functional frame of the browser itself. This frame provides independent functionality from the information provided from the website itself. This means that the information provided to the browser via the incoming server surrounds the website's content. This information is then presented to the user as cues, or security indicators, for them to be able to assess the legitimacy of the website. An example of such a security cue is the lock icon on the top or bottom of the browser chrome, which indicates that the communication between the browser and website server is encrypted using a digital Web certificate.

The chrome provides us with many advantages. The chrome displays objects like the toolbar and the status bar, which are in plain view of the user, providing critical information at all times regarding the website displayed at the moment. When an attacker creates a malicious website, they are able to add whatever content and functionality they want within the website coding itself (limited only by their programming and design abilities), but they are unable to change the functionality of the browser chrome. By preventing website authors from having access to the chrome, the browser adds a layer of protection since the end-user will always be able to take advantage of the security cues — if any — presented by the chrome, in order to find out more information about the source of the website, its communications with the server, and whether any security certificates are present and valid.

Of course, not all Internet users are knowledgeable in detecting these security cues. 
As later sections of this thesis will discuss, users are not always adept in detecting browser cues, such as locks in the status bar denoting Web certificates, secure hyper-text transfer protocols (i.e., https) denoting secure socket layer connections with the server, and so forth. In order for the average user to recognize these security cues, it may be useful to provide them with more visually salient information on the browser chrome - and within the browser content window itself to invite users to check the chrome - for them to be able to identify and interpret these security cues. Several different kinds of certificates are available. Self-signed certificates can be created by the website administrators themselves; domain validated certificates are granted by certificate authorities to anyone who can demonstrate they control the domain; and, extended validation certificates are only granted to those who can satisfy a list of organizational requirements. How browsers show these varies, and changes over time. Studies have shown that users show little awareness of and difficulty interpreting such browser cues (Sobey et al., 2008). Other studies were discussed in the Usable Security section of this thesis.

Indicators that denote https connections, site-authentication images, and lock icons are present within the browser chrome to indicate secure connections. Research shows that, when users are presented with these security indicators while interacting with websites, they often do not notice their presence (Schechter et al., 2007). The researchers conducted a study looking at these authentication measures which are put in place in order to protect users from fraudulent websites, such as phishing sites. The users involved in their study were asked to log in to their own bank account over several different banking sessions. On each subsequent visit, the security indicators (e.g., https, site-authentication images, lock icons) were removed from the user's banking website. Their findings demonstrated that a significant amount of users chose to continue with the login process when one or more of these security indicators was removed by the researchers from the banking website. On several occasions, users were presented with a warning page informing them that there was a problem with the website's security certificate, and that it was recommended that they not proceed with the session. Their findings showed that a large proportion of users 


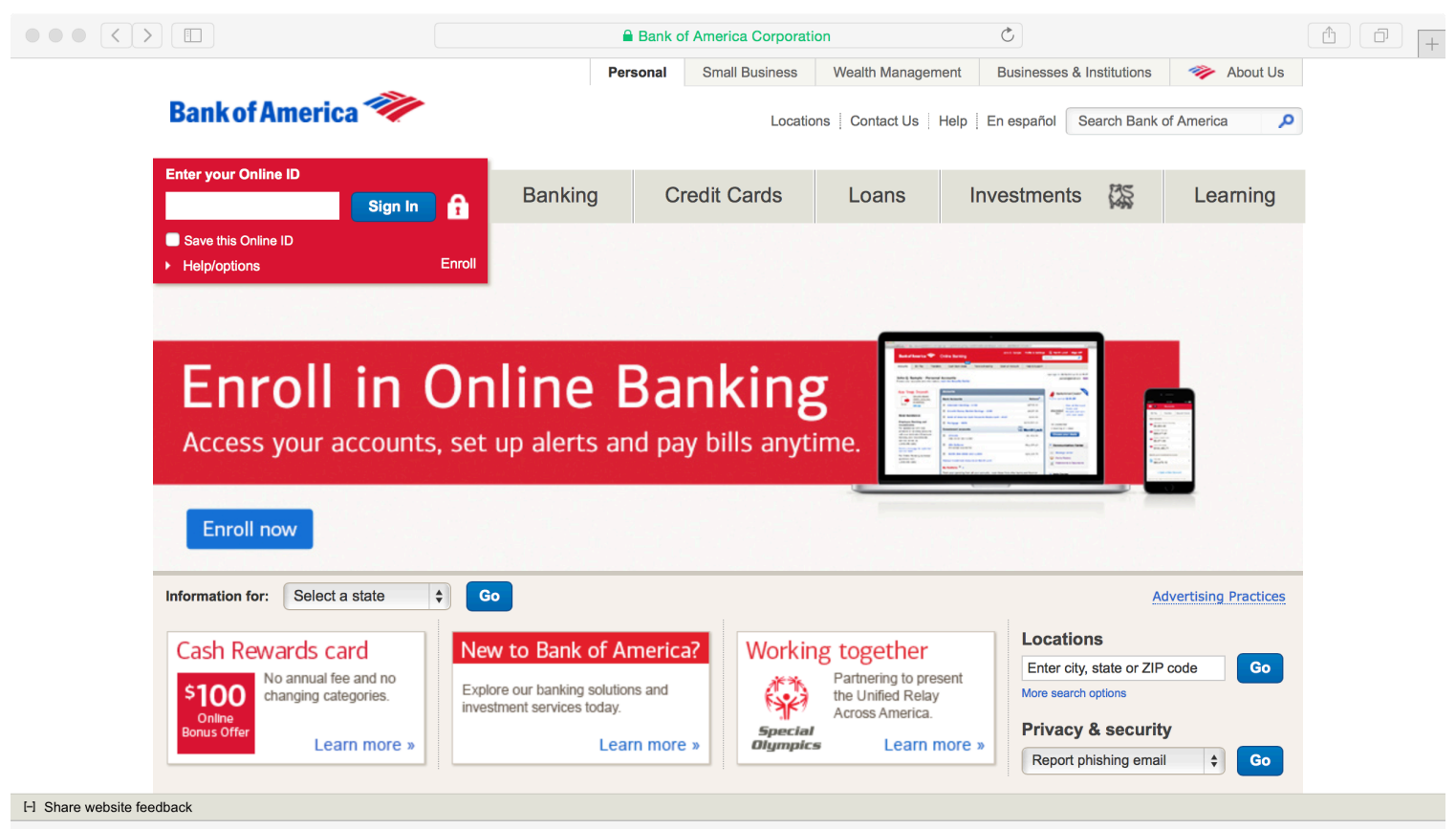

Figure 9. Screenshot of the Bank of America website depicted on the Safari Web browser.

decided to ignore the warning and continue with the banking session. While acknowledging the importance of the work, there has been some suggestion that the research methodology could have been stronger (A. Patrick, 2007).

The findings of this study show surprising behaviours on the part of everyday users regarding online interactions. Adding to the fact that one of the problems with online security is the lack of user education regarding security indicators, it is now known that users sometimes don't take into account the presence of security indicators and warning messages when deciding whether to proceed or not with an online interaction.

External devices. External devices are currently in use as crucial components to improving security over the Internet. Some devices have been created specifically for this function, such as USB tokens manufactured by companies such as RSA. Alternatively, more 
general devices, such as cellular phones, tablet computers, among others, can be used as third-party confirmation tools. The way this works is by having the external device identify the user to the system in question. If a user attempts to log in to a given website using their username and password, the server could then send a query to their external device (e.g., an iPhone), and request that the user perform an additional verification task on their device in order to confirm their identity. Using this method of additional validation would increase security in password systems, and lend more protection to users. The idea of identity confirmation through the use of external devices is the extra layer of protection that is added to the interaction between user and software download websites. Much like Web certificates that identify the source of a website, external devices can help in identifying them as well. If a user were to browse to a website that they have not encountered before, the website could not validate its identity by sending a message to the user's external device. This communication is typically accomplished out of band instead of through the same channel, significantly raising the difficulty of any attack.

A two-factor authentication system has been implemented by Google. When a user attempts to login to their Google account (i.e., Google Mail), he or she needs to enter their passcode currently associated with their account, and a special second passcode. This second passcode is generated by a mobile device application, "Google Authenticator", which is available for users to download on various applications stores (e.g., iPhone App Store, Google Play, and so forth). Once the application is launched, it randomly generates the secondary password in order to authenticate the user attempting to gain access to the Google account in question. Users have the choice to use either the software application, a text message, or an automated telephone call in order to complete the authentication process (Kincaid, 2011).

The use of external devices in conjunction with identity confirmation does have some disadvantages. First, not all users possess some form of external device, such as a smartphone, which could be used as a method of confirmation of identity. For the users that do possess these devices, they may not be willing to provide their device's information to an 
unknown source (e.g., telephone number). Second, implementing this added layer of security could give rise to longer waiting times before interacting with the website in question. The time needed for the server to send a confirmation request to the external device could potentially be high, depending on the system in question and its communication load at the time of the request. Finally, some users may not want to make use of the external device for validation purposes. If the system requires additional validation via the external device, some users may decide not to visit the website on subsequent browsing sessions.

External authorities. In an Internet setting, external authorities are defined as organizations that provide identification information for a given website. In North America, the Better Business Bureau is a good example of such an authority which has extended its original service to include organizations present on the Internet. The BBB gathers information regarding businesses throughout Canada and the United States, and then creates reports regarding their business practices in order to inform the general population about their past dealings with customers. In these reports, the BBB provides information regarding the type of business in question, their duration of operation, any customer feedback ratings, and the number of complaints received in a given time frame.

The advantages of using external authorities in an Internet setting is that they make it difficult for attackers to lure users to their websites. Organizations can opt to pay for an external authority's services. This is usually done to make prospective customers feel more trusting towards organizations when they have good track records with the external authority in question. External authorities provide graphical images as well as banners depicting their organization's crest, which can be used as a link from the organization's website to the external authority's website, where information regarding the organization in question is depicted. However, since the graphics used are simply images (e.g., jpeg images), attackers are also able to show these images depicting the symbols of external authorities in the content of their website. But, they are not able to provide direct links to the external authority's website that show pertinent information regarding their organization. Attackers would then be able to provide users with a link to an imitation external authority website, 
where the user can be lured to believe the information posted about the website in question.

Of course, much like the browser chrome, this once again falls into the category of added protection for more educated users. Some people will undoubtedly take the presence of the external authority's symbol as evidence of the website's credibility, and subsequently decide in favor of trusting it without verifying that the link to the external authority's website is legitimate. The goal should be to provide users with the knowledge necessary to detect the real external authorities from the ones provided for malicious activities.

Software markets. With the increasing popularity of smartphones and tablet computers, software marketplaces are quickly becoming the place to go for compatible software applications. Such market places, also known as "app stores", are now widely available. In this section, the differences between open market app stores, and closed market app stores are discussed (Tekedia, 2011).

On the Internet, an open market refers to a collection of software applications available from one or multiple sources, where there is little to no control over the software products available for users to download. An example of an open software market is the Android marketplace, which is administered by Google. Google allows software developers to create and submit their software applications to the Android marketplace while providing little to no supervision and verification of these applications. This means that developers are able to submit just about any type of software product, regardless of functionality or subject matter. As the marketplace operates more on an open-source platform, the permissions required in order to access personal information, such as a user's contact lists and email addresses, are managed automatically and may be easier to subvert.

This type of market does have its advantages and disadvantages. An open market platform may leave users open to attacks while downloading applications. The software they choose to download from the open market may appear to be legitimate, while the real intent of the application could be to obtain personal information from the user's device. On the other hand, an advantage is that developers have a lot of freedom when it comes to the types of applications they are allowed to develop and upload to the marketplace for users 
to purchase and download.

A closed market refers to a collection of software applications available usually in one location, which is tightly controlled by an organization. In the area of computer security, this type of marketplace is often referred to as a walled garden; this description refers to a garden surrounded by walls, enclosing its perimeter so nothing is permitted in or out. The most widely known example of a closed market is the Apple App Store. In this particular market, every developer must be registered and pay a fee to Apple, and each of the software applications is closely monitored by Apple. Strict guidelines are enforced regarding the types of applications that can be made available to the users of the marketplace, such as the programming languages used.

This type of software marketplace is much less open to attacks than is its open market counterpart. Since the governing company, Apple in this case, claims to have put in place rigorous regulations for the developers to follow in order to be able to upload and sell their applications on the market in question, the software products developed by individuals and companies are much less likely to contain malicious code. On the other hand, the most notable disadvantage of this type of marketplace is the freedom which developers have when creating software applications. Since they have to abide by the regulations put forth by the governing company, which are present to ensure that the applications available on the marketplace remain free of malicious code, this negatively impacts the freedom developers have in creating software applications that cater to all different types of users. As an example, Apple does not allow developers to make use of applications that run the Adobe Flash plug-in in their software products. The company claims that this third-party application provides too many opportunities for malicious code to be introduced into the final product and, as such, has disallowed its use on their marketplace. This puts users who want to use the Flash plug-in to either accept not being able to use it with Apple marketplace applications, or switch over to another software marketplace that allows the use of this third-party plug-in, such as the Android marketplace.

Building upon both types of marketplace models is the Amazon software application 
marketplace. Amazon makes use of both types of marketplaces by offering software applications designed for the Android-based cellular telephones and tablet PCs, while controlling for the type of software products made available to users for purchase. The Amazon marketplace claims to have tighter controls for things like subject matter and malicious code submitted by developers. This makes their marketplace safer and more orderly for their users. However, Amazon does not control the development platform, while Apple does control its own development platform.

Having examined open and closed markets, it can be agreed upon that both types have distinct advantages. By creating a marketplace that fully encompasses the advantages of open and closed markets, organizations may be able to more tightly control for the presence of malicious code. This would also allow developers more freedom to create and share their applications to end-users with a minimal amount of constraints imposed on the software products.

\section{Main Research Question}

The primary purpose of the present research is to examine and analyze how people determine whether websites on the Internet are legitimate or illegitimate, which psychological processes they use in order to arrive at this decision, and whether they can identify and make use of security mechanisms in order to protect themselves from malicious attacks. Furthermore, the research attempts to understand whether people perceive the appropriate levels of risk involved in engaging in various types of online activities.

The main research question of this thesis work is "How do people perceive, understand, and make decisions regarding the dangers associated with malicious websites, and are they able to identify and understand the various levels of risks associated with engaging in online activities?"

Five studies were conducted in order to find out more information regarding users' understanding of the security aspects of online interactions, including software download websites, secure websites (e.g., online banking websites, online electronic commerce web- 
sites), and so forth.

The focus of the first study was to explore participants' abilities to make appropriate decisions regarding trustworthiness of websites.

The second study attempted to determine participants' perception of risk related to online activities.

The third experiment was to explore participants' understanding of Internet structure

in general, particularly in relation to malware, communications between computers and servers, and website security mechanisms.

The fourth study was to conduct exploratory analysis on participants' understanding of Internet structure in general, based on findings from the previous studies.

And, the fifth study focused on exploring the basis on which users make decisions regarding domain names and malware. This study also explored relationships between knowledge, perception of risk, attitudes, and beliefs about various aspects of the Internet.

\section{Study Summaries}

Study one: Determining website legitimacy. The first study was to explore participants' abilities to make appropriate decisions regarding trustworthiness of websites. The results showed a tendency for participants to distrust each of the websites shown when questioned, whether they recognized them from previous browsing experience or not. The general understanding is that users are usually willing to make trusting decisions in an online interaction, but the participants were clearly not confident in explaining their choices. It was concluded that users are not able to determine, with any confidence, website legitimacy by locating and identifying security mechanisms embedded within the webpage.

Study two: Risk perception of Internet-related activities. The second study was to examine how users perceive the benefits and risks associated with various Internetrelated activities. The research approach was based on earlier work on risk perception by Fischhoff, Slovic, Lichtenstein, Read, and Combs (1978). The study method involved asking participants to fill out a questionnaire about their beliefs across 6 rating scales in 
regards to different activities. The dimensions included their perceived benefits to the general population, the risks involved with engaging in the activities, and their usage of the activities. Various analyses were conducted on the resulting data, including description of data distributions, relationships between scales, and factor analysis. Overall results showed that financially-linked activities were attributed to carry a high level of severity of consequences should something go wrong.

Study three: Understanding of Internet software abilities. The third study was to examine the knowledge users possess about how to identify potential threats that exist in online activities. The research approach was based on earlier work on risk communication by Morgan et al. (2002). The study method involved asking participants to fill out a questionnaire about their knowledge across 40 rating scales in regards to four different online activities: online banking, online purchasing, installing software, and using a search engine. The rating scale dimensions included subject matter in accordance with particular threats relating to each of the four online activities in the study. It was determined that users' main misunderstanding of Internet structure relates to the dangers of what malware can do once it has been installed on their computer. They also don't completely understand the mechanisms inherent in domain names and Web addresses. This could be attributed to users not understanding the series of events that can lead to their computer being attacked, such as downloading a software application from the Internet could potentially lead to a user's online bank account username and password being copied.

Study four: Exploring understanding and confidence. The fourth study was to explore participants' understanding of Internet structure in general, particularly in relation to malware, communications between computers and servers, and website security mechanisms. A confidence question was included after each question or rating scale situation group. The analysis was exploratory in nature, looking at the knowledge users possess in regards to Internet security mechanisms. Findings included a general misunderstanding of domain name structure and sub-domains, and problems understanding communications via the http and https protocols. Perhaps most importantly there was a misunderstanding 
of malware capability and immediacy of effect, as well as incorrect assumptions regarding the abilities of antivirus software applications.

Study five: Heuristics, knowledge, and self-perception. The fifth study was to identify the basis on which users make decisions regarding domain names and malware. The research approach was based on earlier work on heuristics by Tversky and Kahneman (1974). The study also explored relationships between knowledge, perception of risk, attitudes, and beliefs about various aspects of the Internet. This study specifically looked at decisions made regarding the legitimacy of domain names and software applications, and how users make these judgments. The research targeted user understanding of domain names and software applications via heuristics. Specifically, the study investigated whether a keyword heuristic was used in assessing domain names, and a functionality heuristic was used in assessing capability of malware. Evidence was unclear as to whether users make use of the keyword heuristic when determining risks associated with domain names. However, they do make use of the functionality heuristic when determining risks associated with downloading and installing certain types of software applications.

Furthermore, users' knowledge scores do not correlate very highly with their perception of risk associated with engaging in various online activities. Evidence about users' attitudes, beliefs, and self-reporting suggests that correlations exist between some of the self-rated attitude dimensions. Some of these correlations offer more insight into people's beliefs about their understanding of the Internet, as well as who they believe is responsible in protecting them when engaging in online activities. Finally, evidence from the study suggests that the less someone knows, the more they think it's someone else's responsibility to keep them secure while engaging in online activities. 


\section{Study One: Determining Website Legitimacy}

The purpose of study one was to gain insight into users' knowledge about the many facets that co-exist during their daily interactions on the Internet, especially those facets having to do with malicious websites. The study targeted specific characteristics of the Internet: the source of a website, and the browser security cues that are present during browsing sessions. The study set out to determine to what extent users are able to identify and explain these different facets of online interactions and to what extent they understand the mechanisms of security involved in these interactions.

This study examined the steps Internet users follow in order to determine the legitimacy of websites and/or online content. Some malicious websites may try to lure users to download malicious software, or to fraudulently obtain users' credentials. By better understanding how users determine which websites they trust, better software development techniques might be created in order to help end-users identify illegitimate content, and avoid the danger it represents.

\section{Method}

Study design. A qualitative research design was used for this study. This design offered a systematic approach used to describe participant experiences through the testing methods, thus giving them meaning based on their thoughts and insights regarding the legitimacy of websites. In this design, participants were shown six different website screenshots, and asked several questions about various topics concerning these websites. Questions included information about security indicators present on the browser chrome or embedded within the website image, potential deceptive cues found within the website image, and so forth. During testing, the think aloud protocol (Fonteyn, Kuipers, \& Grobe, 1993) was used, where each participant was encouraged to speak out loud while absorbing the information found on each of the website images, thus allowing them to offer in-depth explanations to each of the questions posed within the testing period. The study was approved by the Carleton University Psychology Research Ethics Board (Appendix A). 
Participants. Twelve Carleton University students were invited to participate in this study. Ten participants were female, and two were male. Ages ranged from 18 to 23 years. Educational backgrounds were varied, and included areas such as biology, law, criminology, psychology, business. All participants were studying at the undergraduate level. Six were first year undergraduates, five were second year, and one was fifth year.

Materials. The study made use of a Dell personal computer, running Windows 7 on a seventeen inch LCD monitor displaying a 1280 by 1024 pixel resolution. This computer was used to allow participants access to the demographics form. Two of the website screenshots used in the study can be seen in Figures 10 and 11. The complete list of images can be found in Appendix E

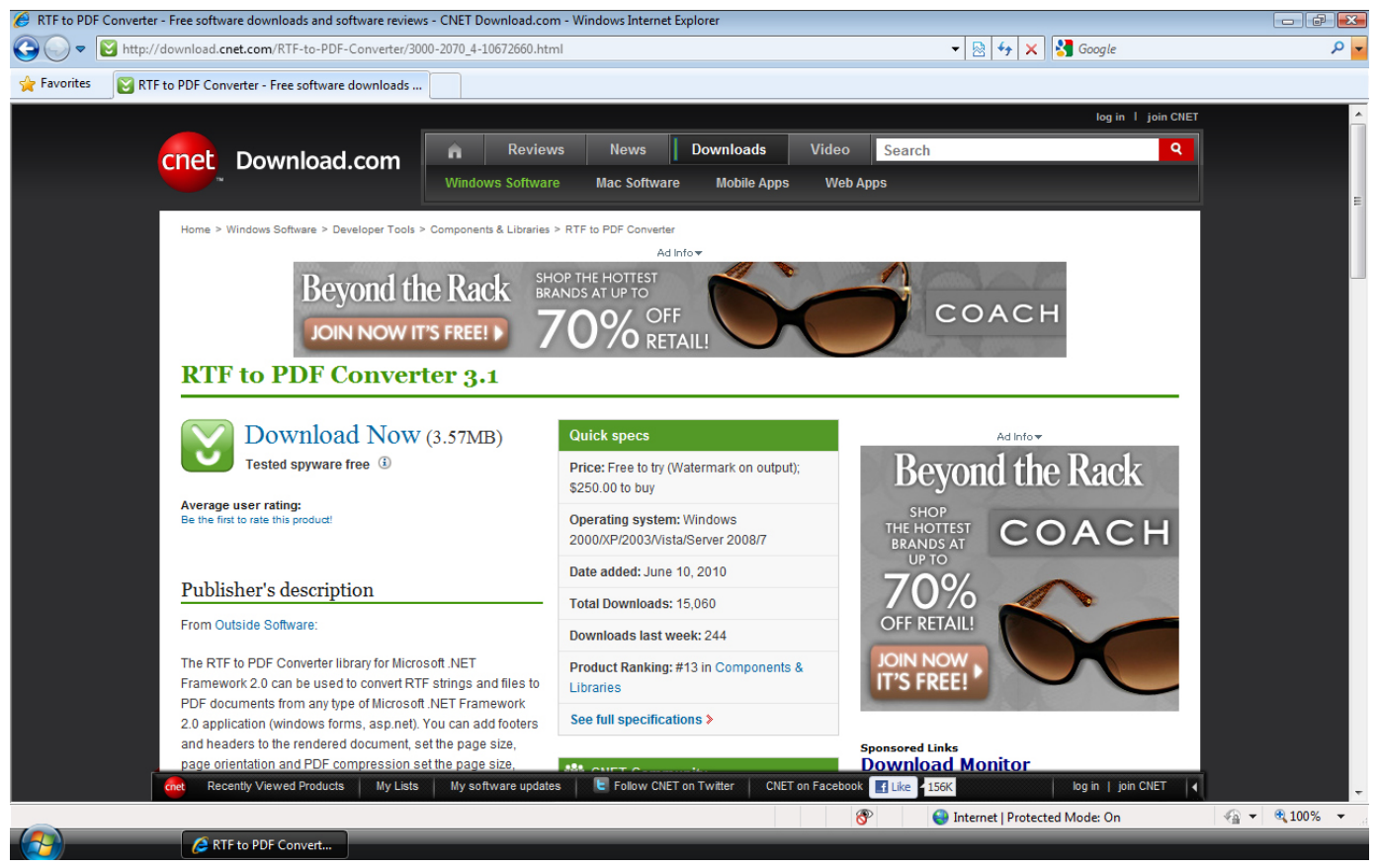

Figure 10. CNet RTF to PDF Converter software application screenshot.

A demographics questionnaire was given to each participant prior to the main task, and asked questions regarding their gender, age, occupation or student status, their Inter- 


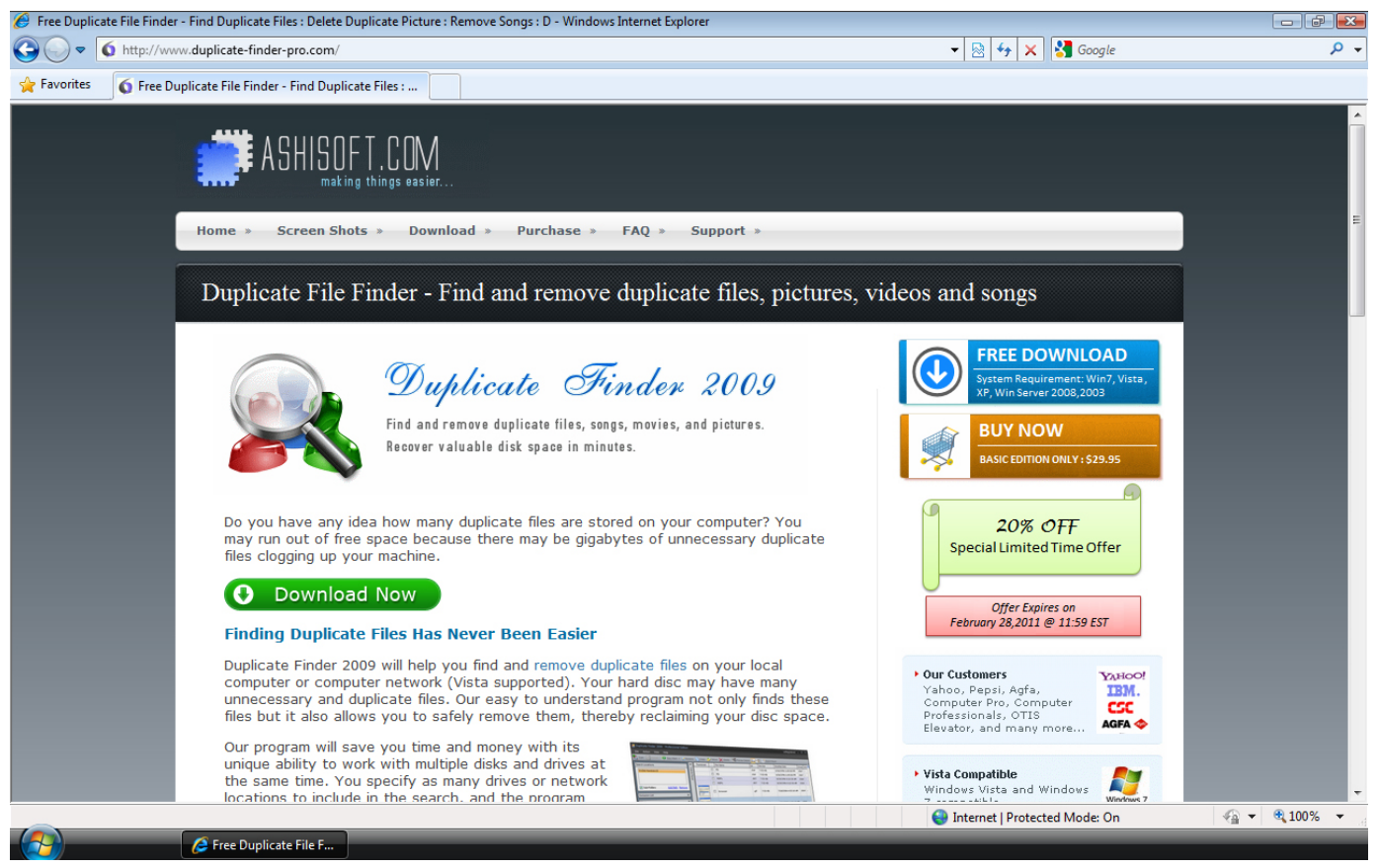

Figure 11. DuplicateFinder software application screenshot.

net usage and self-reported expertise in computer skills. The questionnaire can be found in Appendix B. A post-test questionnaire was also administered after the main task had been completed. This questionnaire included multiple-choice questions as well as long-form questions, designed to test the participant's general knowledge about various components of the Internet. The post-test questionnaire can be found in Appendix D Responses to this questionnaire can be found in Table 3.

Procedure. For the study's main task, each participant was shown, over a one hour session, six different images of websites, one at a time, in the same order. A contextual scenario was given to the participants for each of the six different website images. These can be found in Table 1. Participants were instructed to look at each website image for a duration of approximately 30 seconds, after which a series of eight questions were posed regarding the website in question while they continue to look at the image. The questions 
are listed in Table 2.

It was decided prior to the experiment that static images of the websites should be used, as opposed to their dynamic states. The reasoning for this was to obtain users' feedback on each website without them having the ability to browse other sections of the website. Doing so allowed follow-up questions to each participant regarding what they saw on each website image specifically, instead of the website in its entirety. The six website images gathered for the purposes of the study reflected some legitimate websites and some illegitimate websites. Some of the websites used in the study were CNet's www.downloads.com, www.1clickspyclean.com, and www.tucows.com. A complete list of the website images used in the study can be found in Appendix E. The legitimacy and appropriateness of each website was determined by the researchers.

\section{Results}

Participants responded to questions regarding their computer skills and Web activity while filling out the demographics form prior to beginning the main task in the study. Regarding their computer skills, where 1 was "Novice" and 10 was "Expert", one participant answered 5, three answered 6, five answered 7, two answered 8, and one answered 9. In regards to how often they browsed the Web, one participant responded "less than once a week", two responded "once a week", four responded "several times a week", and five responded "daily".

Participants were also asked to answer a post-test questionnaire after the main task was completed. There were five multiple choice questions, eight open-ended questions, two yes or no questions, and one confidence self-rating question. Table 3 shows participant responses to the multiple choice questions, the yes or no questions, and the confidence self-rating question. This table shows some surprising results: the highest percentage of responses answered correctly was in question one, with $50 \%$ having answered correctly. The rest of the responses fell much below the $50 \%$ mark, indicating that the participants in the study lacked a lot of knowledge regarding Web browsing and security indicators 
Table 1

Contextual Scenarios Used in the Main Task Survey

\begin{tabular}{|c|c|c|c|}
\hline \# & Website & Contextual Scenario & Software \\
\hline 1 & $\begin{array}{l}\text { CNet RTF to } \\
\text { PDF Converter }\end{array}$ & $\begin{array}{l}\text { Imagine you are a student, and your professor has sent } \\
\text { you a document in "rtf" format. You are looking on } \\
\text { the Internet for a method to read this file, and you } \\
\text { come upon this website. How do you decide whether or } \\
\text { not to download and install this software application? }\end{array}$ & Legitimate \\
\hline 2 & Click SpyClean & $\begin{array}{l}\text { Based on the previous scenario, you've downloaded the } \\
\text { document your professor sent you via email, but are } \\
\text { now concerned about the possibility that a virus has } \\
\text { been integrated into this file. You decide to browse } \\
\text { Google for an antivirus software program, and come } \\
\text { upon this screen. How do you decide whether or not } \\
\text { to download and install this software application? }\end{array}$ & Illegitimate \\
\hline 3 & $\begin{array}{l}\text { Windows An- } \\
\text { tivirus Software }\end{array}$ & $\begin{array}{l}\text { You're not completely satisfied that the } 1 \text { Click Spy } \\
\text { Clean program will fix your computer properly. You } \\
\text { decide to browse Google once more and come upon } \\
\text { this software application. How do you decide whether } \\
\text { or not to download and install this program? }\end{array}$ & Illegitimate \\
\hline 4 & $\begin{array}{l}\text { Ashisoft Dupli- } \\
\text { cate File Finder } \\
2009\end{array}$ & $\begin{array}{l}\text { After having downloaded the .rtf file from your profes- } \\
\text { sor, you've discovered that the file has been duplicated } \\
\text { on your hard drive. You find the application "Dupli- } \\
\text { cate Finder } 2009 \text { ", which will help you find and remove } \\
\text { duplicate files on your computer. How do you decide } \\
\text { whether or not to download and install this program? }\end{array}$ & Legitimate \\
\hline 5 & $\begin{array}{l}\text { Tucows } \\
\text { SpeedUpMyPC } \\
\text { Software }\end{array}$ & $\begin{array}{l}\text { After having cleaned your computer of duplicate files, } \\
\text { you find that the computer speed is slower than usual. } \\
\text { You decide to browse Google once more and you come } \\
\text { upon this website that is offering a downloadable ver- } \\
\text { sion of the SpeedUpMyPC software. How do you de- } \\
\text { cide whether or not to download and install this pro- } \\
\text { gram? }\end{array}$ & Illegitimate \\
\hline 6 & $\begin{array}{l}\text { CNet } \\
\text { shop }\end{array}$ & $\begin{array}{l}\text { After having spent time looking for various computer } \\
\text { fix-it programs, you decide to browse Google in search } \\
\text { of the Adobe Photoshop application, in order to im- } \\
\text { prove your photo enhancing skills. You come upon } \\
\text { the CNet website once more, where this program is } \\
\text { advertised for download. How do you decide whether } \\
\text { or not to download and install this program? }\end{array}$ & Legitimate \\
\hline
\end{tabular}


Table 2

Questions Used in the Main Task Survey

\begin{tabular}{|c|c|}
\hline $\mathrm{Q} \#$ & Question \\
\hline 1 & Would you recommend this webpage to anyone you know? \\
\hline 2 & $\begin{array}{l}\text { How does this webpage compare to another webpage you trust and use con- } \\
\text { sistently? }\end{array}$ \\
\hline 3 & $\begin{array}{l}\text { Is there a particular aspect of the webpage that makes you trusting or mis- } \\
\text { trusting of it? }\end{array}$ \\
\hline 4 & Do you think the page looks professional? If yes, how? If not, why? \\
\hline 5 & With the information provided, can you tell who is responsible for the website? \\
\hline 6 & Did you look at the Internet address? If so, does it tell you anything? \\
\hline 7 & Was there a lock icon present on the webpage? If so, what does this tell you? \\
\hline 8 & $\begin{array}{l}\text { Now that you've had the time to assess this webpage, would you decide in } \\
\text { favor of trusting or distrusting this website? }\end{array}$ \\
\hline
\end{tabular}

Table 3

Responses to the Post-Test Multiple Choice Questions.

\begin{tabular}{lr}
\hline Question & \% Correct \\
\hline What is a Web browser? & 50.00 \\
What does a picture of a lock icon in a Web browser indicate? & 41.67 \\
What is a website security certificate? & 16.67 \\
What does "https://" mean at the beginning of a website address? & 41.67 \\
What does this seal by the Better Business Bureau mean? & 16.67 \\
\hline
\end{tabular}

in general. For a full list of the responses provided by participants for the open-ended questions, please refer to Appendix G.

Qualitative interview response themes. For the purposes of qualitative analysis, this section is divided into themes which were identified based on the comments and feedback received from participants - using the think aloud protocol — during testing. A complete transcript of all participant responses can be found in Appendix F

Internet addresses. A large proportion of the participants that were tested in the study expressed confusion when it came to their understanding of the components of an Internet or Web address, particularly in terms of the domain name extensions and the file extensions associated with Web addresses. When asked whether the website address meant anything to them, most participants reported that the URL contained the name and/or path of the product or website concerned. However, none of them mentioned anything regarding the possibility of security information being present within the URL address bar 
on the chrome of the browser, such as the website's security certificate or evidence of a secure-sockets layer (SSL) connection.

Additionally, participants stated that, for some of the website screenshots shown, there were missing parts to the URL representing the Web address. There was some confusion regarding the Web address top level file or directory represented within the Web address, where one user in particular was under the impression that ".html" was an image file, and not the actual website hyper-text markup language (HTML) code used to create the webpage itself. For the most part, participants stated that they were unsure what the Web address was, what it meant, or how it was related directly to the webpage they were currently viewing on the image screenshot.

Website content and layout. Most users offered comments and feedback concerning advertising material embedded within the website screenshots they were shown during testing. When making reference to these, some users stated that their mere presence on the website made the website more trustable, while other users stated the complete opposite, that the presence of ads on any website or webpage indicates that it is not to be trusted because ads make the website appear "unprofessional". This led the researchers to conclude that the declaration of an "unprofessional" website also meant that it was untrustworthy. This theme is supported by various articles published in the literature on the importance of Web content and visual appeal in the eyes of users (Fogg et al., 2003, Lindgaard, Fernandes, Dudek, \& Brown, 2006, Song \& Zahedi, 2005).

Website template and layout was also an emphasized topic during discussion. Most users commented that the professional layout, ease of navigation, and presence of company logos made the website seem trustworthy. In contrast, many participants discussed the presence of testimonials embedded within the website itself, making the website appear to be untrustworthy. Their reasoning behind this judgment was that the testimonials made the website in question appear to be attempting to lure users into a false sense of security while providing them with other users' positive experiences while browsing the website and taking advantage of the products and services available. Also, users stated that testimonials 
can be easily faked, making them practically worthless on any website, whether its intent is legitimate or illegitimate. The fact that many users found testimonials a bad thing in terms of the legitimacy of a website came as a surprise, as the literature suggests that people rely on others factors in order to make a decision in favour of the product or service offered online (Metzger, Flanagin, \& Medders, 2010). This may indicate a change in user attitudes over time.

The information found on the website in question was also mentioned. Some users believe that the presence of various descriptions of products and services makes the websites concerned appear more reputable and less deceptive. Users who were able to identify a website in question due to the content being shown (e.g., products, logos, templates, and so forth) would report that they had heard about and/or used the website before, which would lead them to make a positive decision regarding trusting the website in the future. These factors are supported by Riegelsberger's framework, discussed earlier, where the concept is called temporal embeddedness.

Security indicators. When asked to locate and identify a lock icon present on the website screenshot, most participants were unable to find it on five of the six website screenshots presented during the testing session. Once the question related to these lock icons was posed, the participant stated that they could see it, but wasn't exactly sure what the lock icon itself meant. On the Windows antivirus website screenshot, all participants noticed the larger lock icon placed directly on the webpage. Furthermore, none of the participants mentioned anything regarding the possibility of security information being present within the Web address found within the address bar in the browser chrome.

In contrast to users being unable to locate and identify the lock icon on the websites shown, when prompted further, some participants noted that the presence of a lock icon in general means that the browser is protecting users from phishing scams and information theft. This comment was only mentioned by a few participants, making it a notable finding that all users were unable to locate the lock icons mentioned during testing. 
Browser behaviour. Participants often noted that some websites seemed to have pop-up windows embedded within the screenshot, or that the website itself had the characteristics of a pop-up window. This comment was usually followed by a declaration of mistrust on the part of the user concerning the website shown at the time. Some participants attributed this to browser behaviour, in which they believed that the browser had been responsible for the appearance of the pop-up window. Hence, they commented that these particular websites should not be trusted, as pop-up windows are a bad thing in general, in terms of security over the Internet during browsing sessions.

This particular theme seen within testing was surprising to the researchers, as this implies that most users - if a broad generalization of this feedback is applied - rely on a lack of pop-up windows in order to make a positive trust decision concerning particular websites, and the presence of pop-up windows in order to make a negative trusting decision concerning these same websites.

Responsibility and identity. When questioned about the responsible party involved in the creation and maintenance of the website in question, participants felt unable — in large part — to determine with certainty who was responsible for each of the websites used in the study. Some participants simply repeated the website's name as the responsible party. For example, participants would often state that "1 Click Spy Clean" was the name of the company responsible for the website where this product was advertised, when in reality "1 Click Spy Clean" isn’t a company, organization, or government department. Even when a company name was identified on the part of the participant, such as CNet or Adobe, the responses to the questions still projected uncertainty on their part, in terms of the party responsible for the website or webpage shown.

During this part of the study, participants didn't appear to be actively looking directly on the websites for any clues to the company or organization in charge of the website. This wasn't very surprising for the websites that were classified by the researchers as illegitimate, but for the websites that were considered legitimate by the researchers, such as CNet and Ashisoft, these results seemed surprising and offered insight into participants' browsing 
habits.

Trust influences. The results of the study showed that users claimed to distrust most of the websites shown in the project, whether they had previous knowledge of the website or not, even if they suspected the website in question was in fact legitimate. Most of the answers provided by the participants pertained to how they viewed the website in question, and not how the website compared to other ones they trust and use consistently.

Most participants were not familiar with the five websites shown. Overall, the most familiar of the websites was in fact CNet. The CNet website was shown twice during testing, with a different software application available for download on each website screenshot. Some participants said they recognized some of the websites shown during testing as looking very similar to actual viruses and spyware programs they had seen before (e.g., Windows antivirus software application and 1 Click SpyClean software application).

Participants would liken the website in question to the other websites shown during testing. Often, participants would change their decision from wanting to trust the website to distrusting it, once the final question for each of the website images had been asked. The reasons that were stated to substantiate this claim were that ads present on the webpage made the website in question look cheap and desperately looking to increase revenue. Some users felt more secure when the ads they saw were from trusted companies (e.g., the Toronto Dominion Bank). This again reflects the framework of Riegelsberger et al. (2005). In this case, institutional embeddedness is the element of the framework involved in user reasoning.

From a professional design point of view, some participants regarded the presence of Facebook and Twitter icons as a good addition to a website's content, while others regarded this as a bad thing. The former participants stated that these social networking sites made them trust the website more, while the latter participants stated they would be less likely to trust the website because of the presence of these links to social networking websites.

Many participants stated that they would likely ask someone regarding their concerns for a particular website and its software application before agreeing to download it. 


\section{Discussion}

The study's design allowed a mixture of closed and opened questions in the study's post-test questionnaire. Using a mixture of both types of questions allowed for the gathering of more pertinent information about what participants' views are regarding website legitimacy, and their overall knowledge of various aspects of the Internet and online security. What emerged, however, was a lack of understanding of the elements of security involved in Internet activity. This reflects what was found in other studies discussed in the Usable Security section, such as those of Downs et al. (2006) and Shi et al. (2011). More importantly, it appeared that participants were hesitant to claim that they would trust any of the websites presented. The directness of the questions and the context of security could possibly have influenced the participants in reporting their decision to trust or distrust the website in question, and in revealing the depth of their knowledge in Internet security. This exact result does not appear to be discussed in the literature, although it relates to the studies showing lack of user knowledge and the success of phishing and malware. The clear lack of confidence but continued activity suggests users do somehow make decisions despite their lack of confidence; this may relate to the findings of Tversky and Kahneman (1974).

Limitations of the present study. Various study limitations are involved in the study. It was noted by the researcher that most participants were guarded in providing their true thoughts about the websites shown during testing. Oftentimes, they would declare having used one or more of the websites in question, but would state their lack of willingness to trust the website shown after having been asked questions about its trustworthiness. They would also often change their mind about the trustworthiness of a website during testing, based on which question was asked at a specific time during the interview. Furthermore, as the websites shown to the participants were simply image screenshots, participants were not able to click on links embedded within the website, nor were they able to scroll up or down on the webpage itself. Participants indicated that they were hesitant to trust a given website because they felt they were missing critical information which they could not obtain due to each website being composed of a single image screenshot. 
The next step. Evidence from this study suggests that users have a difficult time in recognizing security indicators in relation to the websites they frequently visit. Their uncertainty regarding identifying and understanding browser chrome security cues plays a large part in their decisions to trust or distrust a given website. They tend to rely more on superficial cues in order to decide the trustworthiness of a given website. It is believed that the next logical step in delving further into user understanding is to find out about users' perception of risk related to engaging in online activities, and their willingness to trust websites. 


\section{Study Two: Risk Perception of Internet-Related Activities}

The risks involved with various activities and technologies can mean different things to different people and this has been the focus of much earlier research in social psychology, for example the work of Slovic (Slovic, 1987), discussed in the earlier section on psychology background, especially the subsection on understanding risk. Some researchers have suggested that risk has a greater role than trust in user decisions-making about Internet security (Lacohée, Phippen, \& Furnell, 2006). People's perception of risk differs according to the level of expertise about technology. Experts generally discuss the dangers involved with activities and technologies, but non-experts make judgments differently. Experts in online security, as well as in computer science, advise that one of the primary threats to users is malware. For example, the Verizon data breach investigations report Verizon, 2012 ) singles out the dangers of malware, especially in conjunction with phishing.

Previous research conducted by Fischhoff et al. appears to offer guidance in learning how people perceive risk in activities and technologies (Fischhoff et al., 1978, Fischhoff, Slovic, Lichtenstein, Read, \& Combs, 2000). In their early study, the researchers put together a list of thirty technologies and activities that participants were tasked to rank order in regards to several different dimensions that were hypothesized to influence perceptions of actual or acceptable risk. The list of rank ordered risks of technologies and activities can be seen in Table 4. Participants were placed in either expert or non-expert categories in relation to the technology or activity in question. The table shows one row for each technology or activity, and columns for different participant categories. The researchers used psychometric techniques in order to identify similarities and differences among groups with regard to risk perceptions and attitudes (Slovic, 1987). The study design was based on the previous work of Starr (Starr, 1969) which dealt with the balance of the risks and benefits attributed to various activities in everyday life. The findings of Fischoff et al. showed that people perceived the risks associated with various activities and technologies in view of different factors, mainly "dread risk" and "unknown risk". The former factor generalizes activities and technologies that people are afraid of and possess some knowledge regarding 
its risks, while the latter factor encompasses activities and technologies that people know much less about the risks involved.

Building on the early study of Fischhoff et al. (1978), the purpose of the second study in the thesis work was to explore participants' perception of risk related to online activities.

Table 4

Ordering of perceived risk for 30 activities and technologies (Slovic, 1987).

\begin{tabular}{|c|c|c|c|c|}
\hline & League of $\mathrm{WV}^{*}$ & $\mathrm{C}^{*}$ Students & Active CM* & Experts \\
\hline Nuclear power & 1 & 1 & 8 & 20 \\
\hline Motor vehicles & 2 & 5 & 3 & 1 \\
\hline Handguns & 3 & 2 & 1 & 4 \\
\hline Smoking & 4 & 3 & 4 & 2 \\
\hline Motorcycles & 5 & 6 & 2 & 6 \\
\hline Alcoholic beverages & 6 & 7 & 5 & 3 \\
\hline General (private) aviation & 7 & 15 & 11 & 12 \\
\hline Police work & 8 & 8 & 7 & 17 \\
\hline Pesticides & 9 & 4 & 15 & 8 \\
\hline Surgery & 10 & 11 & 9 & 5 \\
\hline Fire fighting & 11 & 10 & 6 & 18 \\
\hline Large construction & 12 & 14 & 13 & 13 \\
\hline Hunting & 13 & 18 & 10 & 23 \\
\hline Spray cans & 14 & 13 & 23 & 26 \\
\hline Mountain climbing & 15 & 22 & 12 & 29 \\
\hline Bicycles & 16 & 24 & 14 & 15 \\
\hline Commercial aviation & 17 & 16 & 18 & 16 \\
\hline Electric power & 18 & 19 & 19 & 9 \\
\hline Swimming & 19 & 30 & 17 & 10 \\
\hline Contraceptives & 20 & 9 & 22 & 11 \\
\hline Skiiing & 21 & 25 & 16 & 30 \\
\hline $\mathrm{X}$-rays & 22 & 17 & 24 & 7 \\
\hline High-school and college football & 23 & 26 & 21 & 27 \\
\hline Railroads & 24 & 23 & 20 & 19 \\
\hline Food preservatives & 25 & 12 & 28 & 14 \\
\hline Food colouring & 26 & 20 & 30 & 21 \\
\hline Power mowers & 27 & 28 & 25 & 28 \\
\hline Prescriptions antibiotics & 28 & 21 & 26 & 24 \\
\hline Home appliances & 29 & 27 & 27 & 22 \\
\hline Vaccinations & 30 & 29 & 29 & 25 \\
\hline
\end{tabular}

$*$ Notes. $\mathrm{WV}=$ Women Voters. $\mathrm{C}=$ College. $\mathrm{CM}=$ Club Members. 


\section{Method}

The study followed the example of Fischhoff et al., focusing on finding out more information about how users perceive the risks involved in online interactions. In the study, the earlier approach was followed, while substituting the technologies and activities that were used previously with various Internet-related activities, such as sending and receiving email, engaging in online banking, and making an online purchase. Some of the rating scales used by Fischhoff et al., such as likelihood of occurence, were used in the study, while others were not replicated as they did not target the same types of risks involved in the study. The study was conducted in order to find out how users perceive the risks involved with online activities, and also how they rank them in order of importance when measured against more tangible technologies and activities, such as using an automated transaction machine (ATM), or paying bills through postal mail. Five such activities were added to the list.

Experimental design. A commonly used web-based survey tool, Limesurvey, was used in order to obtain participant data in this study. The survey contained questions in the following five categories: benefits of activity, likelihood of something going wrong, severity of consequences, immediacy of effect, delay of effect, and usage of activity. There were twenty questions for each category. Using the format of the Likert Scale, answers were rated from 1 to 7 . The participants selected the appropriate rating according to the direction given (e.g., $1=$ No benefits whatsoever, $7=$ Extremely beneficial) in the "benefits" scale. Participants were asked to respond, to the best of their abilities, to each of the 20 rating scale questions for each of the five questions, in a specified order. The study was approved by the Carleton University Psychology Research Ethics Board (Appendix H).

Activities. The list of 20 activities comprises five non Internet-related activities, and 15 Internet-related activities. They appear in Table 5. The list of six rating scales is comprised of a perceived benefit rating scale, four perceived risk rating scales, and an amount of usage rating scale. They appear in Table 6. The item descriptions in both the activities list and rating scales list have been abbreviated in the tables in order to save 
space.

Table 5

Mean Scores of Benefit, Risk, and Usage from the 20 Activities Used in the Study.

\begin{tabular}{lllllll}
\hline & \multicolumn{6}{c}{$\bar{c}$ Ratings } \\
Activity & B & L & Benefits, Risk, Usage \\
\hline Using an (ATM) & 5.5 & 3.2 & 4.6 & 3.4 & 5.9 & 4.1 \\
Having your computer fixed & 4.9 & 4.0 & 4.3 & 4.0 & 4.6 & 1.6 \\
Giving your CC number over the phone & 3.6 & 5.0 & 5.7 & 3.0 & 6.4 & 1.9 \\
Belonging to a loyalty program & 4.8 & 3.2 & 3.6 & 4.7 & 3.2 & 3.8 \\
Paying bills by postal mail & 4.6 & 3.5 & 3.0 & 4.8 & 4.9 & 2.6 \\
Installing downloaded software & 5.0 & 4.6 & 5.3 & 3.1 & 4.9 & 4.0 \\
Sending and receiving email & 6.2 & 3.9 & 4.7 & 3.5 & 4.2 & 6.5 \\
Making an online purchase & 5.9 & 3.8 & 4.9 & 3.4 & 5.3 & 4.4 \\
Doing your banking online & 5.9 & 3.8 & 4.9 & 3.3 & 6.3 & 5.3 \\
Using a search engine & 6.4 & 3.1 & 3.8 & 4.2 & 3.1 & 6.7 \\
Searching for adult content online & 3.1 & 4.6 & 5.0 & 3.4 & 4.3 & 2.7 \\
Downloading pirated movies or music & 2.8 & 4.4 & 4.8 & 3.1 & 4.7 & 2.1 \\
Using social networking websites & 4.9 & 5.0 & 4.9 & 3.4 & 4.1 & 5.5 \\
Using a webcam through a chat program & 4.4 & 3.3 & 4.4 & 3.5 & 3.5 & 2.6 \\
Using an online dating service & 3.8 & 4.2 & 4.2 & 4.2 & 3.8 & 1.6 \\
Making a purchase on an auction website & 5.2 & 3.7 & 4.9 & 3.6 & 5.1 & 3.2 \\
Paying through a payment website & 5.4 & 3.7 & 5.1 & 3.2 & 5.9 & 3.9 \\
Selling through an online ad website & 5.3 & 3.8 & 4.4 & 3.7 & 4.3 & 2.2 \\
Responding to an online classified ad & 4.8 & 3.9 & 4.5 & 3.8 & 4.0 & 2.6 \\
Playing gambling games online & 2.5 & 4.2 & 5.0 & 3.0 & 5.1 & 1.5 \\
\hline
\end{tabular}

*Notes. $\mathrm{B}=$ Benefits. $\mathrm{L}=$ Likelihood. $\mathrm{I}=$ Immediacy. $\mathrm{D}=$ Delay. $\mathrm{S}=$ Severity. $\mathrm{O}=$ Often.

Participants. Participants were recruited from the Amazon Mechanical Turk online work marketplace (https://www.mturk.com/mturk/). If anything, MTurk users may be a little bit more knowledgeable than average Internet users. Past research has shown that conducting studies on MTurk allows for reliable and quick participant samples, though care must be taken to avoid or screen out participants who try to take advantage of the system by not providing complete or thought-out answers. In general, MTurk as a crowd sourcing online marketplace has proven to be reliable and efficient (Buhrmester, Kwang, \& Gosling, 2011; Kittur, Chi, \& Suh, 2008, Kelley, 2010, Crump, McDonnell, \& Gureckis, 2013). As Buhrmester et al. (2011) states, "Overall, MTurk can be used to obtain high-quality data inexpensively and rapidly." 
Invitations were limited to users who resided in the United States, to increase the consistency of knowledge of website activities. In all, 94 individuals were recruited. Ages ranged from 18 to 71 years. Of the 94 participants, 44 were male and 50 were female. Educational backgrounds varied from high school to university graduate degrees. Occupations also varied, from students to medical doctors. The pre-requisites of eligibility for participating in the study were for participants to be at least 18 years of age, not an expert or student in Computer Security, possess a minimum of 2 years of experience with the Internet, and be regular users (i.e., use the Internet at least 2 times each week).

To assure the validity of participant responses, several aspects were verified before analysis. In particular, the following characteristics were ensured: participants made varied responses to the questions, rather than uniform responses throughout the questionnaire; participants responded throughout the entire questionnaire rather than stopping at an arbitrary point; each participant only completed the survey one time; and, each participant provided coherent responses to the open-ended questions. Participant responses that did not meet these controls were excluded. This totaled approximately $5 \%$ of the responses. This same process was also followed for studies three, four, and five.

Materials. The materials used in the study included task instructions Appendix J), a demographics questionnaire Appendix I , the list of activities Appendix K, and the rating scale questions Appendix L.

Procedure. Participants answered a short demographics questionnaire which included questions on age, gender, occupation, education, and frequency of Internet use. This data was collected for data analysis to explore different responses from demographic categories. The next step was to respond to the main task questionnaire. Each of these surveys was located on the university laboratory servers, using the Limesurvey Web-based survey tool.

Participants were informed that the task was designed to study people's beliefs about losing personal information from their computers. In this case, the loss of personal information was described as "the loss of financial information", "the loss of confidential information 
Table 6

Questions Used in the Main Task Limesurvey Questionnaire.

\begin{tabular}{ll}
\hline Scale Type & Question \\
\hline Perceived Benefit & $\begin{array}{l}\text { For each activity, how beneficial is this activity to the general } \\
\text { population? }\end{array}$ \\
Perceived Risk - Likelihood & $\begin{array}{l}\text { How likely is it that the loss of personal information would } \\
\text { occur as a result of this activity? }\end{array}$ \\
Perceived Risk - Immediacy & When information loss occurs, how immediate is the loss? \\
Perceived Risk - Delay & When information loss occurs, after how much time would you \\
& be affected by the consequences? \\
Perceived Risk - Severity & When personal information is lost, how severe are the conse- \\
& quences? \\
Usage & How often do you engage in each of the activities? \\
\hline
\end{tabular}

that could prove embarrassing to you", and "the loss of personal information that could prove harmful". They were then given a few example situations where someone's information could potentially be compromised, such as having their personal information copied from their office computer, or their credit card number being copied by their waiter at a restaurant. The examples given to participants were not included in the list of 20 activities. The main task then requested participants to complete a questionnaire containing six different Likert-scale questions, on a scale of 1 to 7 . The questions are shown in Table 6 and each was followed by a randomized list of the 20 activities.

\section{Results}

Data distributions. Table 5 presents the mean Likert-scale scores of the benefit, risk, and usage responses for each activity. Figures 12 to 14 show the distributions of responses to several of our rating scales as box plots. These show each distribution, the center line is the median, the box represents the middle quartiles, and the whiskers the outer quartiles; the notch represents the $95 \%$ confidence interval and circles represent outliers. The rating scales for benefits, likelihood, and severity were emphasized in order to save space, and because these scales show the most important perceptions that may affect decisionmaking. The colours representing the three information loss categories that were offered as examples in the study instructions, including red (financial), green (embarrassment), and 
Rating Scale 1 : How beneficial is this activity?

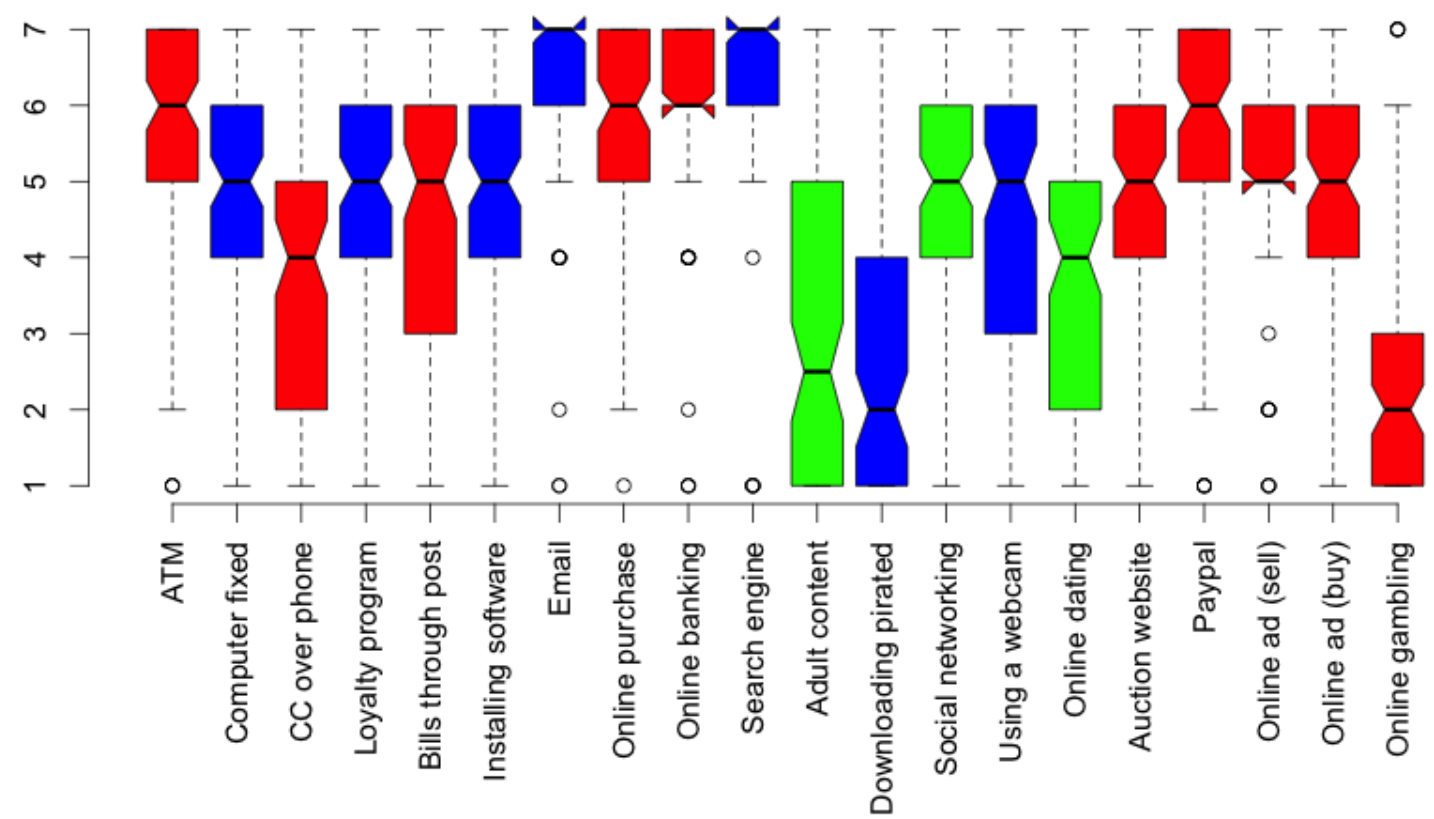

Figure 12. Boxplots of the 20 activities for the rating scale "Benefits".

Rating Scale 2 : How likely is the loss of personal information?

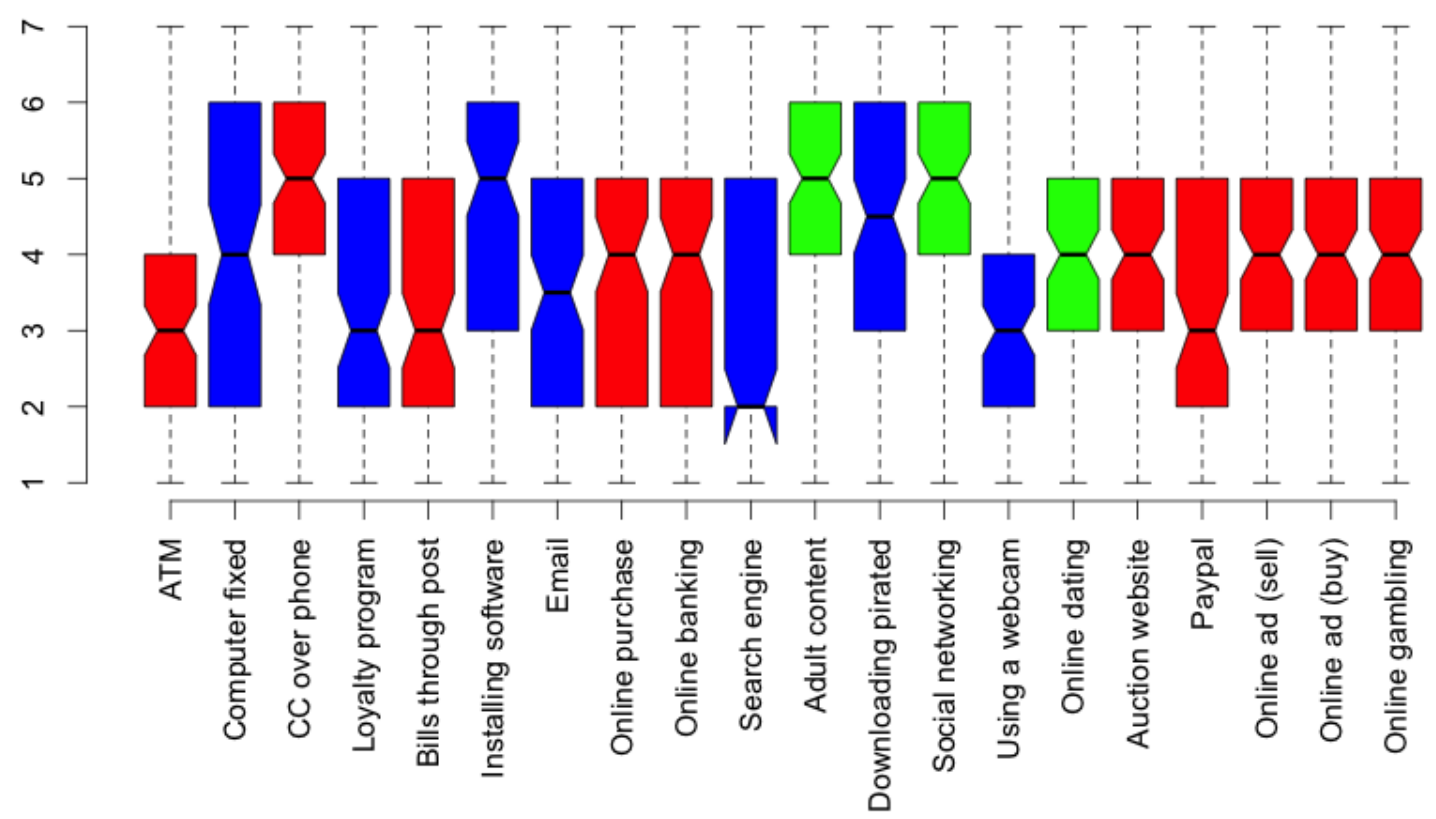

Figure 13. Boxplots of the 20 activities for the rating scale "Likelihood". 
Rating Scale 5 : How severe are the consequences?

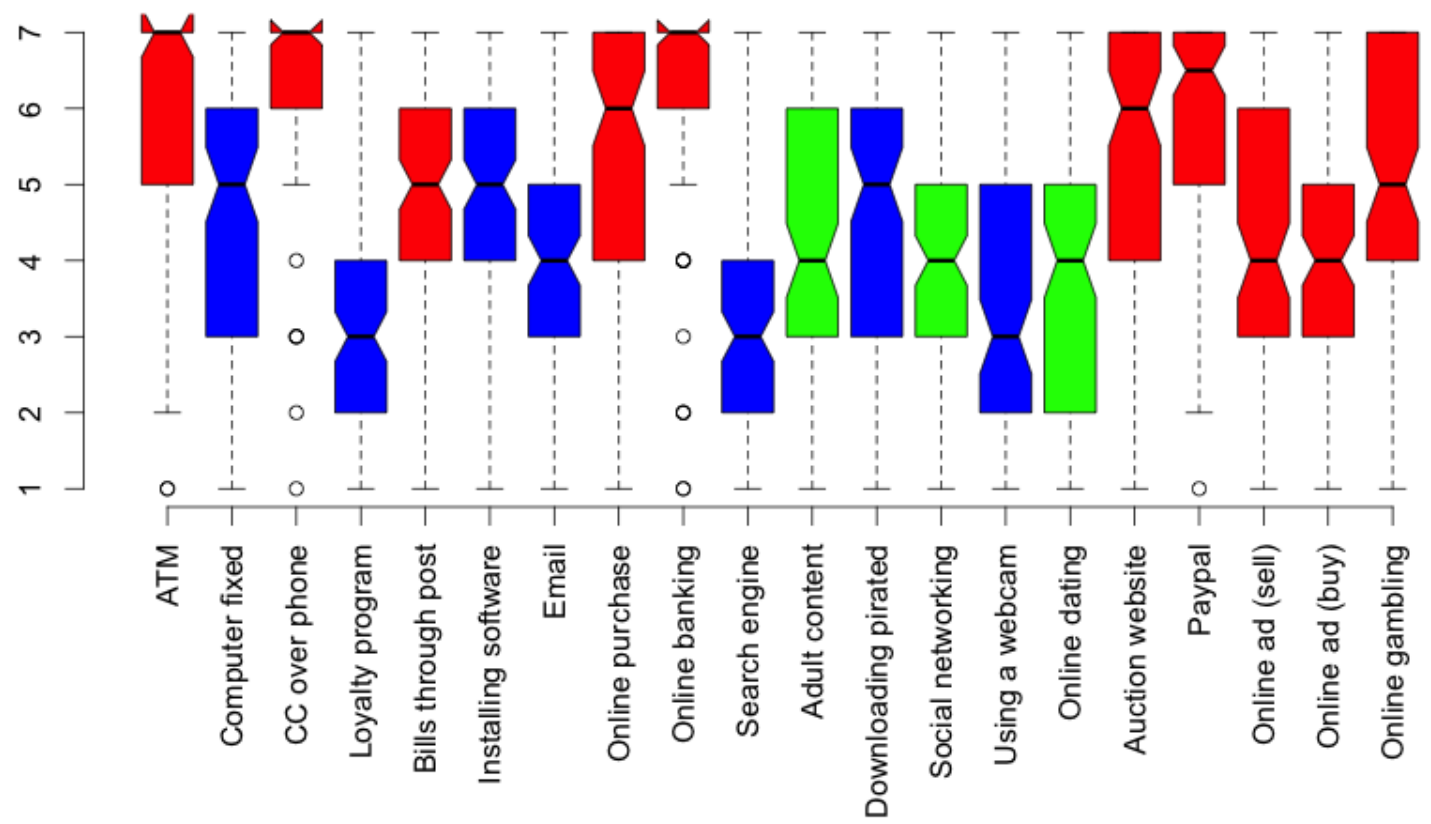

Figure 14. Boxplots of the 20 activities for the rating scale "Severity".

blue (other) are reflected in the box plots. Many box plots indicate that the responses to the rating scales for many of the activities in the study were widely distributed. For example, Figure 13 shows that there was a lack of consensus between participants when rating the likelihood of the loss of personal information occurring as a result of engaging in some of the activities used in the experiment.

Of the 20 activities used in the study, four of them were selected to demonstrate the data distribution between the six different rating scales. These activities are shown because, out of the list of items, these are activities people often engage in on a daily basis; also, these activities are especially prone to online attacks. These are "using a search engine", "sending and receiving email", "doing online banking", and "making an online purchase". These results are depicted in Figures 15 through 18 . Each of the four figures depicts histograms for each of the six rating scales. The distribution of Likert-scale responses are shown by bars ranging from red (low) to yellow (high). A blue line is superimposed on each histogram, showing a fitted normal curve. However, it can be seen that many distributions 


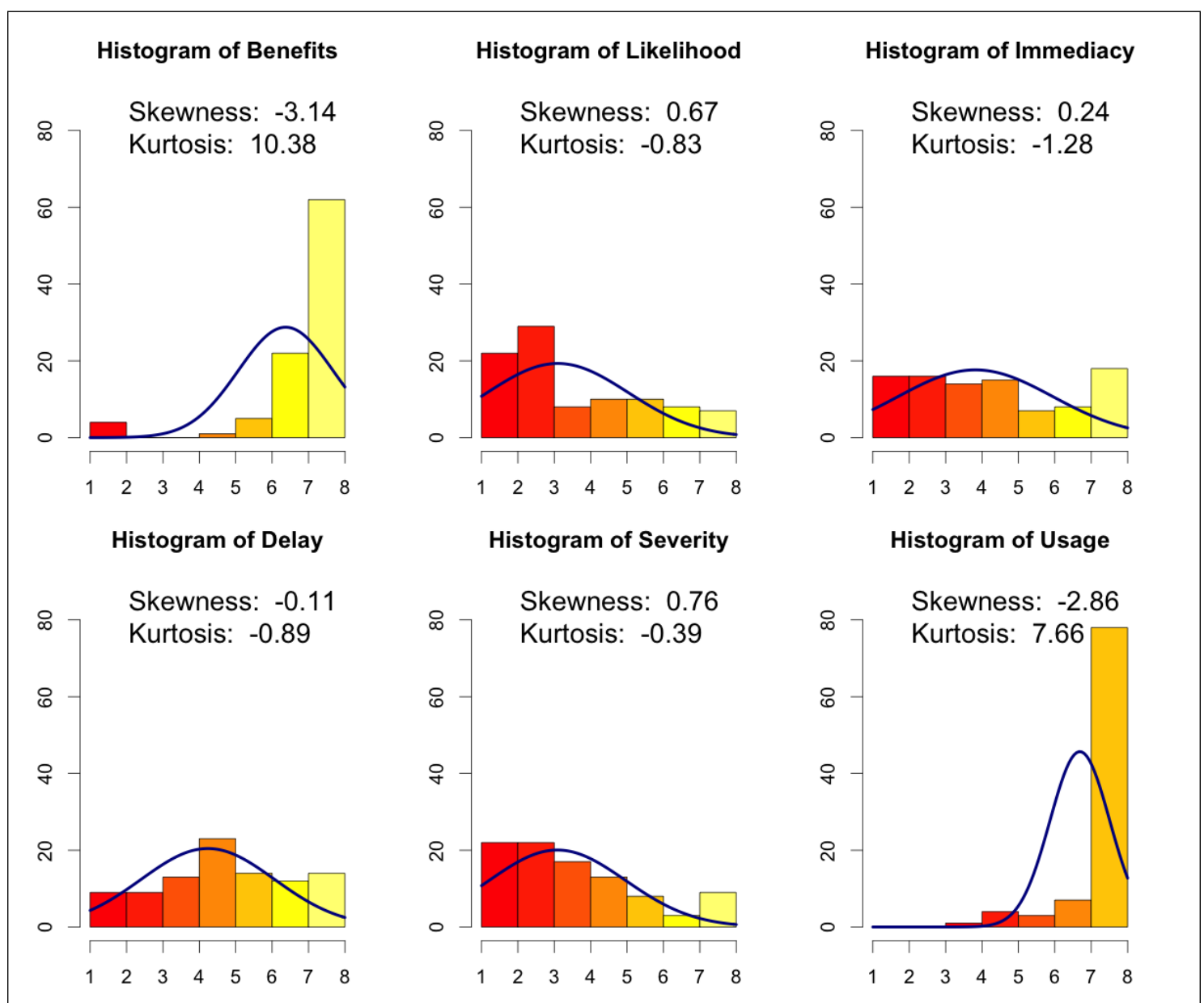

Figure 15. Histograms representing Likert scale responses for the six different rating scales for the activity "Using a search engine".

are not normal, and skewness and kurtosis values are shown on each.

Looking at the distributions, users claim to frequently use search engines and email, and less frequently use online banking and make purchases online. The reported likelihood of personal information being stolen or copied by way of these activities was pretty much evenly distributed, except for using a search engine, which many participants reported as an activity where the loss of personal information is highly unlikely. Participants reported the activities of making an online purchase and doing banking online as having high severity of consequences, should the loss of personal information occur as a result of engaging in one of these activities. In contrast, they also reported that the severity of consequences for the activities of sending and receiving email and using a search engine are much lower than the 


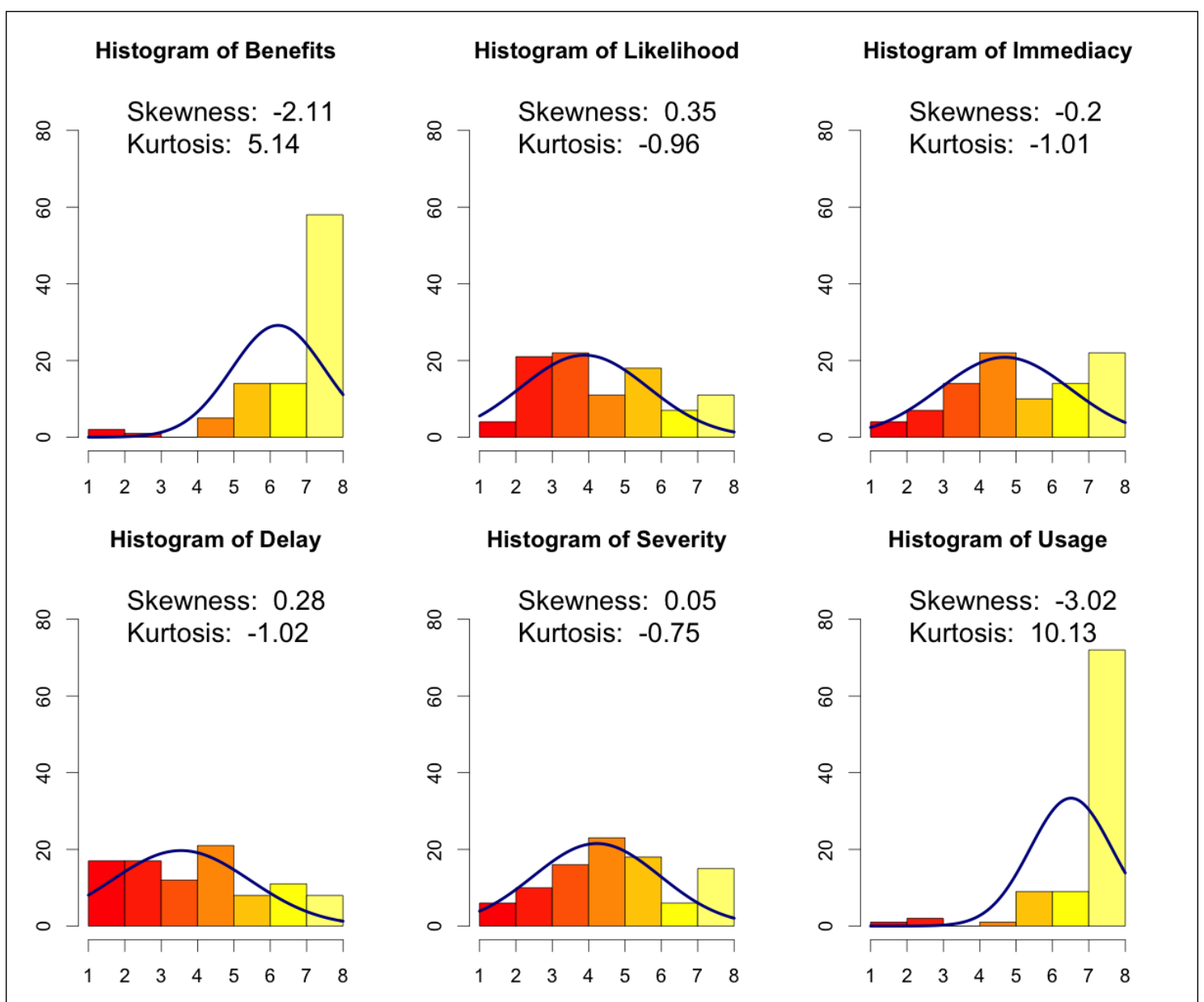

Figure 16. Histograms representing Likert scale responses for the six different rating scales for the activity "Sending and receiving email".

former activities.

The results suggest that many Internet users are unaware of, or believe they will not be affected by, some of the risks involved in these Internet-related activities. In addition, there were no significant differences observed between participants based on gender, age, and level of education, in regards to the perception of risk attributed to each activity. In particular, over-reliance on search engines leaves users vulnerable to malicious redirection, or careless reliance on a bank's website can increase the risk of falling victim to a phishing attack. Doing one's banking online and making a purchase online do carry risks as well, while mostly of a financial nature, but users have more protection from the security guarantees in place by the providers of their banking or credit card institutions. 


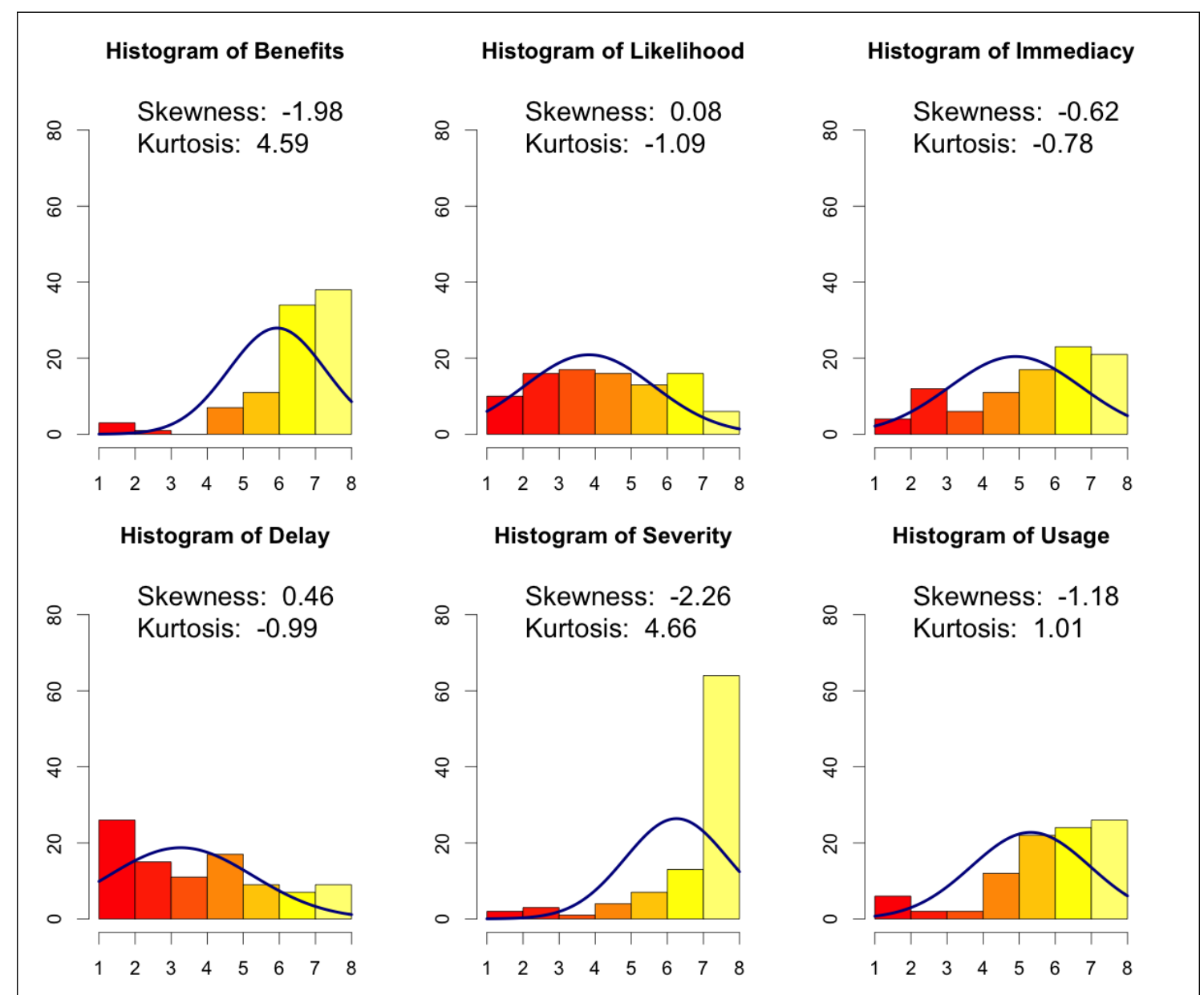

Figure 17. Histograms representing Likert scale responses for the six different rating scales for the activity "Doing online banking".

Relationships between rating scales. Correlations between the six different rating scales are shown in Figure 19. This figure shows a correlation matrix of all scales compared to each other. The diagonal shows histograms of the data distribution for each scale. The lower triangle in the figure shows the scatterplots of these relationships. The upper triangle shows the Spearman correlation $(\rho)$ statistic. This figure shows strong positive correlations between the perceived benefits of a given activity, and the amount of times the user in question engages in the activity. This makes sense because it is expected that users will attribute benefits to anything they have decided to adopt as a behaviour. A very strong negative correlation exists between the immediacy of effect and the immediacy of consequences rating scales, which suggests that participants reported that if the loss of personal 


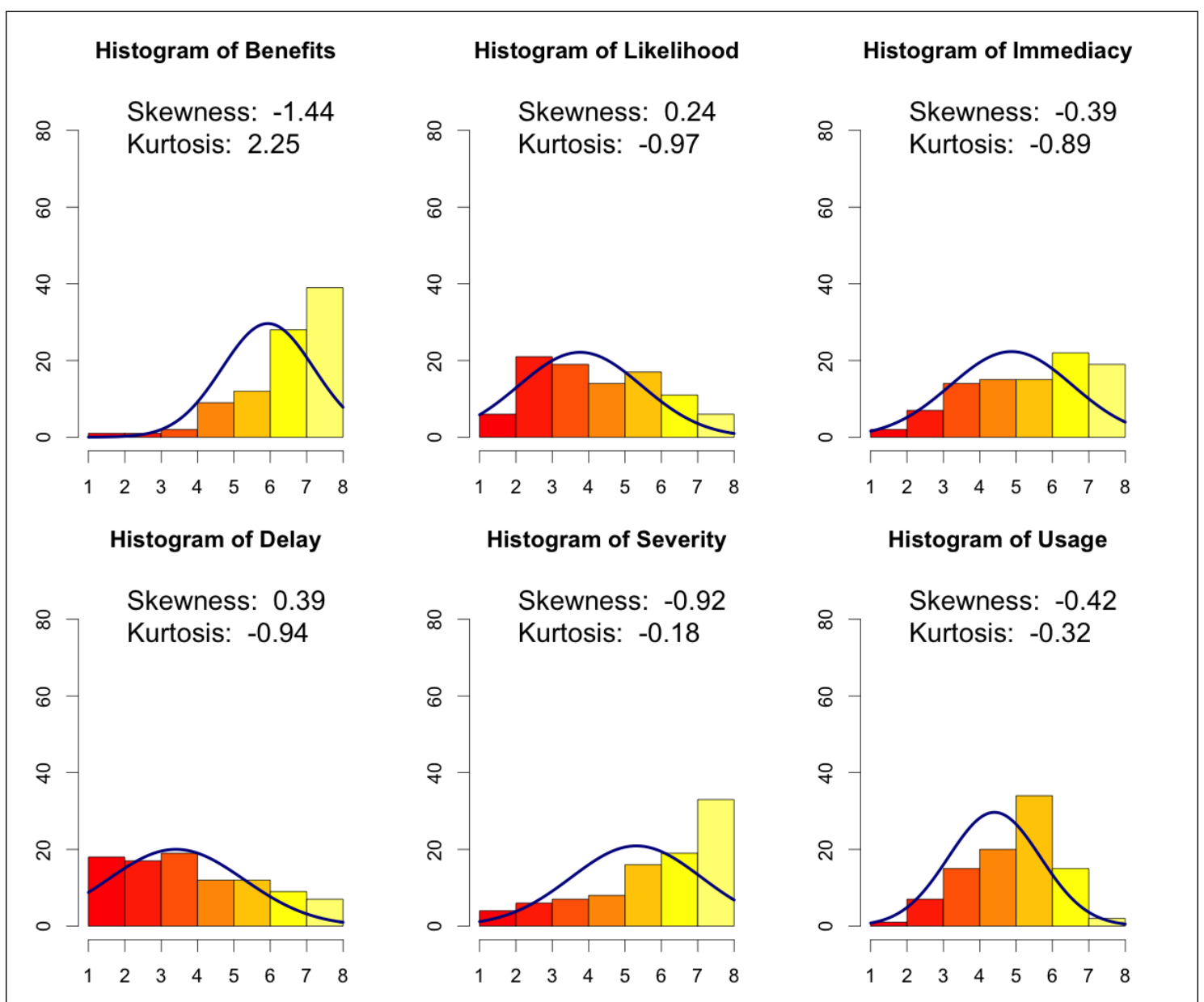

Figure 18. Histograms representing Likert scale responses for the six different rating scales for the activity "Making an online purchase".

information were to occur immediately, the severity of that loss would also be seen immediately. It is important to note that the two scales were reversed in their wording to help participants distinguish the two questions from each other. It appears the participants did make this distinction, but still felt that immediacy of effect and immediacy of consequences were aligned. A moderate to strong relationship exists between the immediacy and the likelihood rating scales. This result suggests that participants reported that activities with a certain amount of risk would be paired with a relatively quick loss of personal information in the event that an attack took place.

Figure 20 shows the relationship between perceived likelihood of information loss rating, severity of loss, and benefit for the 20 activities in the study. These scales were 


\section{Relationships Between Rating Scales}
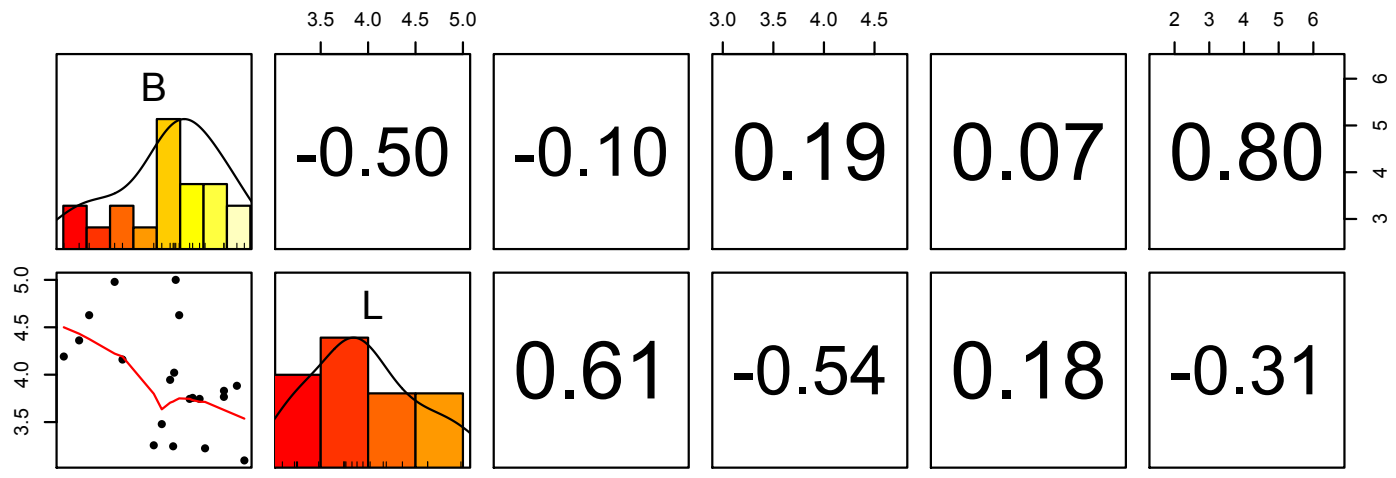

0.61
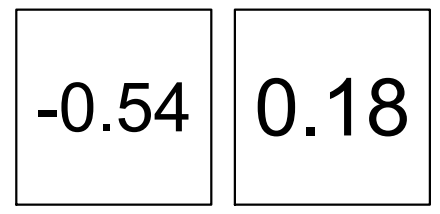

$-0.31$
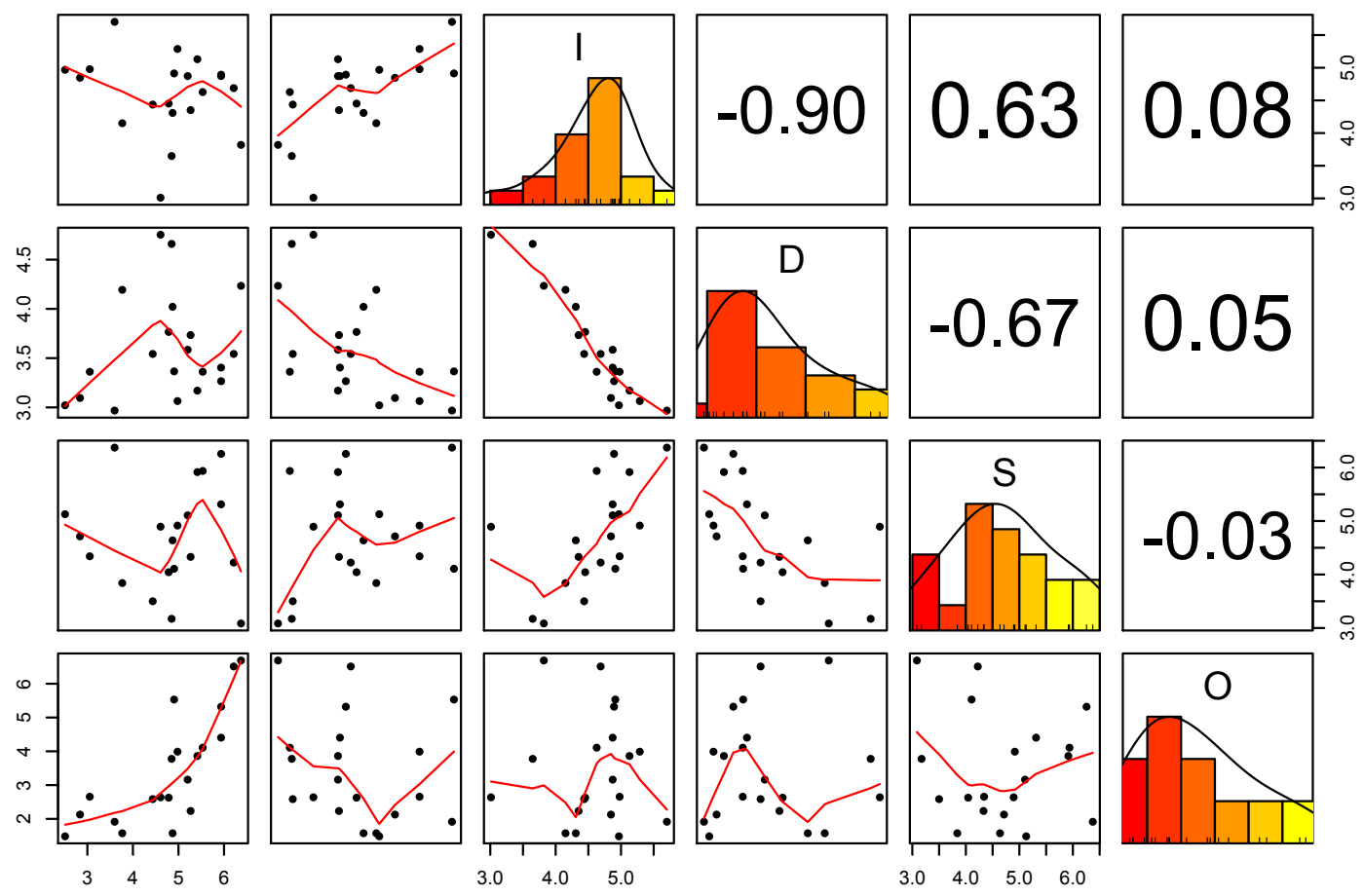

Figure 19. Figure showing the relationships between rating scales. $\mathrm{B}=$ Benefit. $\mathrm{L}=$ Likelihood. I = Immediacy. $\mathrm{D}=$ Delay. $\mathrm{S}=$ Severity. $\mathrm{O}=$ Usage. 


\section{Activities Plotted in Relation to Rating Scales}

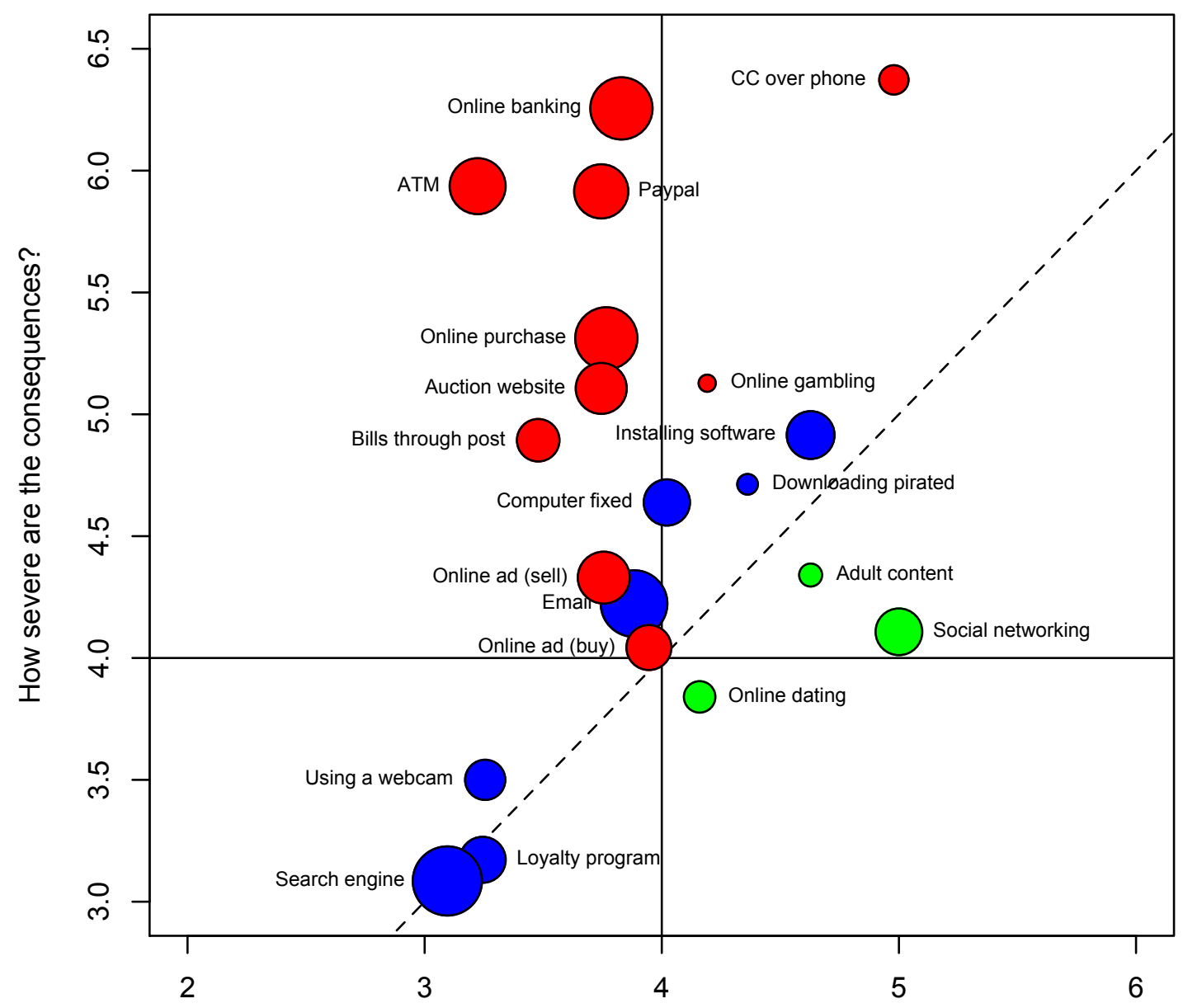

How likely is the loss of personal information?

Figure 20. Figure showing the relationship between the perceived likelihood of information loss rating scale and the severity of loss rating scale. The size of the circles demonstrates the level of benefits attributed to each activity. The dotted line represents the line of symmetry between the likelihood and severity scales. 
selected for this graph as these same scales that were shown as box plots in the data distribution section, and these scales represent the key decision-making attributes. The $\mathrm{X}$ axis represents the likelihood of information loss rating scale and the $\mathrm{Y}$ axis shows the severity of information loss rating scale. The perceived benefit rating scale was plotted as a third dimension on top of the other two rating scales. The size of the circles represents the level of perceived benefits attributed to each activity by the participants, where a larger circle entails a higher benefit rating. The dotted line in the graph represents the line of symmetry between the likelihood and severity rating scales. Activities above this line indicate a greater severity than likelihood, and activities below the line indicate a greater likelihood than severity.

Before plotting these relationships, it was speculated that the nature of the activity might be reflected in the relationships between rating scales. Three broad categories of activity were distinguished as they might relate to risk, following the earlier examples. The first category includes financial activities, where the risk involves the loss of money. The second category includes activities that might lead to embarrassment. The third category encompasses a range of activities which might carry diverse risks. When plotting the data, these categories were identified by color: financial activities are shown in red, potentially embarrassing activities are shown in green, and the other activities are shown in blue.

In the figure, it is apparent that the relationship between the different activities varies on the likelihood and severity rating scales. In particular, the categories of activities are strikingly distinct in their placement on the graph. It appears that activities which carry the risk of loss of financial information are above the line of symmetry, showing that the participants felt that the severity was greater than the likelihood of loss. Several financial activities had very high severity ratings, including several that also had high benefit ratings: online banking, online payment websites (e.g., PayPal), and using an automated transaction machine (ATM). Interestingly, the activity with both the highest severity and likelihood was giving a credit card over the phone, which was associated with a low benefit rating. Activities which carry a risk of potential embarrassment nature are below the line of 
symmetry, which suggests that participants felt that the likelihood of loss was greater than the severity. Participants felt that two of these activities, searching for adult content and using online dating websites, were far less beneficial than belonging to a social networking website. However, it was the social networking activity that was seen as most beneficial and carrying the most likely risk. The benefit ratings for each of the activities varied between all the various types of risks (financial, embarrassing, etc.).

Factor analysis. To explore deeper relationships between the scales, exploratory factor analysis was conducted on the data. This method of analysis was used in the hopes that the resulting data would demonstrate which activities clustered together in terms of risk. This would allow the inference of the existence of different factors, such as knowledge of risk and dread of said risk, in conjunction with the various activities.

Classical multidimensional scaling, also known as principal factor analysis, was conducted first. The results are shown in Figure 21, where the graph may be helpful in better visualizing the relationships between activities. The figure shows the activities arranged in relation to two yet undetermined factors, which is characteristic of the multidimensional process. In their earlier work, Fischhoff et al. were able to interpret the results of multidimensional scaling as identifying factors such as "dread risk" and "unknown risk". In this study's results, it is apparent that some of the activities that carry the risk of losing information of a personal nature (i.e., embarrassment) and those activities dealing with the risk of losing financial information are close to one another within the two-factor space. However, the results were not able to provide any confident identification as to the nature of the two factors that emerge.

Next, hierarchical clustering using complete agglomeration was conducted. This method yields a result in the form of a dendrogram, which is a tree diagram used to illustrate the arrangement of the clusters produced by hierarchical clustering. Figure 22 shows the results where the activities are arranged together in terms of distance between their respective rating scale responses. The reasoning behind constructing this type of graph is to help determine to which type of group each activity belongs. As an example, 


\section{Multidimensional Scaling of Activities}

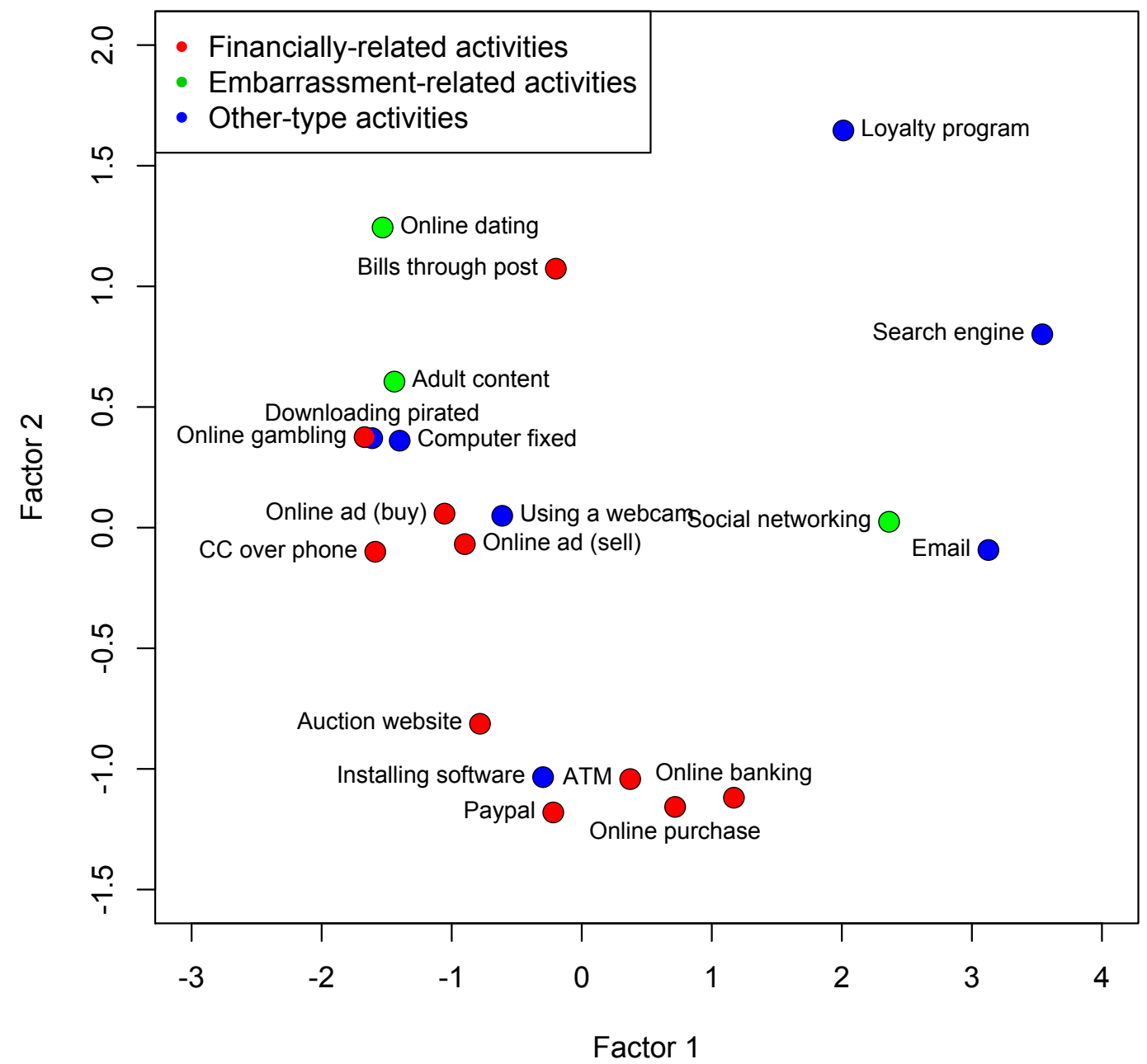

Figure 21. Multidimensional scaling graph showing the similarities and dissimilarities between the activities. 


\section{Hierarchical Clustering Dendrogram}

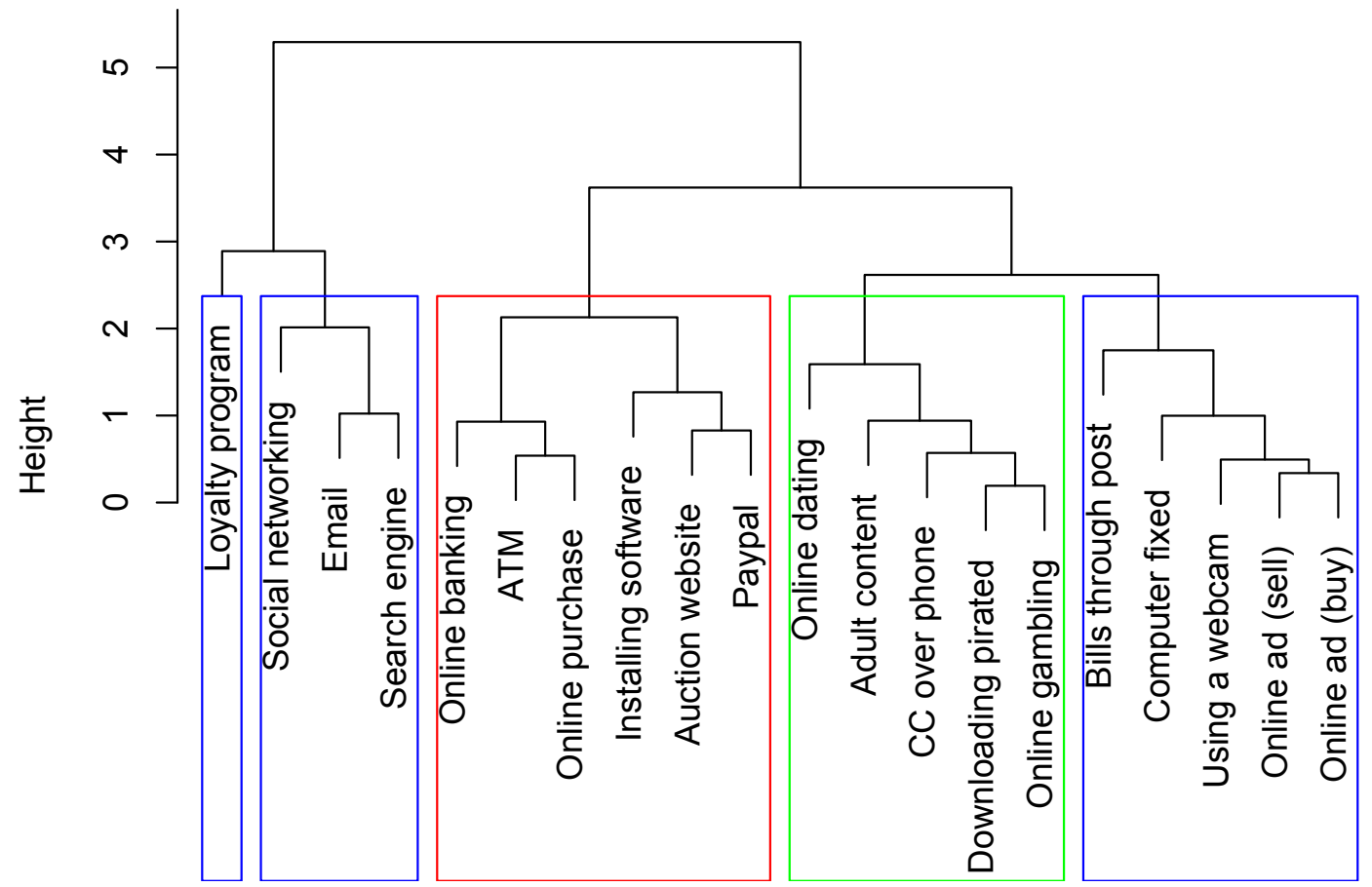

\section{Activities}

Figure 22. Figure depicting a hierarchical clustering dendrogram illustrating the arrangement of clusters.

it appears that the activities of using an ATM, online banking, and making a purchase online cluster closely together. As such, it could be inferred that these activities share some sort of strong relationship; a grouping such as this one might imply that users attribute similar benefits, risk, and degree of usage to activities that share similar characteristics of personal information, financial information, or other type of information. It is striking how the groups ressemble the initial speculation about categories of activities (i.e., financial, embarrassment, etc.). Of the activities in the study, belonging to a loyalty program was distinguished by itself. Giving a credit card over the phone was clustered together with the activities belonging to the potentially embarrassing group, rather than the financial group. 
Also, installing software was clustered together with the activities belonging to the financial group, rather than the various activities group. This cluster also includes downloading pirated software and online gambling, which were not previously grouped along with the potentially embarrassing activities. However, it is important to note that online gambling is illegal in the United States, as well as pirating software.

\section{Discussion}

It is known that people's interactions in an online environment are influenced by the risks people perceive. It isn't apparent how people associate risks with various tasks and activities. In particular, though people perceive ranges of risk, it is not clear how well their perceptions align with actual current online threats. This study focused on exploring how users perceive risks across a range of tasks and activities to gain insight that may help in improving the ways that online security is communicated and supported.

Fischhoff et al., beginning in the 1970s, studied people's general perception of risks involving various technologies (e.g., nuclear power, pesticides, transportation). They experimented with technologies and activities that their participants had been tasked to respond to in regards to several different dimensions that were hypothesized to influence perceptions of actual or acceptable risk. They found that participants attributed a high level of benefits to high-risk activities that were perceived as being an important part of people's daily lives, such as driving a car (Fischhoff et al., 1978). On the other hand, they appeared to overemphasize the likelihood of "dread risk", meaning severe large scale events involving highly technological processes.

The study in this thesis research was modeled on the methods of Fischhoff et al., and explored perception of risk and various online activities. The reasoning behind designing the study to test participants using a Likert-scale response scheme was that, asking participants to provide data in this respect, allowed them to make judgments about the complexities inherent in Internet-related activities in a way that was not overly complicated and perfectly understandable to the average computer user. The various analyses used to make sense of 
the data allow for different interpretations of the results. For many activities, the reported levels of the likelihood of personal information loss, immediacy of loss, and severity of consequences were high. These results were expected for some of the activities in the study, such as online gambling, online dating, and viewing adult content online, which were hypothesized to carry an unacceptable level of risk to some users due to the general knowledge about the risks related to such subject matter. However, for some of the other activities that users engage in on a daily basis, such as sending and receiving email, making a purchase online, and using a search engine, it was surprising to see that users reported high benefits and high severity of consequences, but attributed low likelihood of the loss of personal information occurring as a result of engaging in the activity. These findings go against what researchers in computer security might suspect, based on computer security and industry literature (e.g., (Verizon, 2012, Anderson, 2008)).

There was a relatively high relationship between the perception of benefits of a given activity and the amount of times users reported engaging in that activity. This did not come as a surprise; it was expected that participants would attribute a high level of benefits to activities they engaged in, and a low level of benefits to activities they do not actively engage in. Activities such as using a search engine, sending and receiving email, and social networking, were seen to carry a high level of perceived benefits.

The exploration of the relationships between the response scales and efforts at factor analysis appear to reveal some patterns. Activities appeared to fall into natural categories, reflecting financial involvement or potential risk for embarrassment. For severity, financial activities were rated highest, but in likelihood activities with potential for embarrassment were rated highest. Some activities stood out among the rest. For example, giving a credit card number over the phone was reported amongst the most severe and likely risks, while social networking was reported among the most likely risks but was also seen as very beneficial.

In contrast to the high level of benefits attributed to some activities, activities such as online gambling, viewing adult content online, and online dating were found to be in the 
low range of perceived benefits. These results were not surprising; it was anticipated that these activities, which might be seen as embarrassing to admit, would fall within the low range of perceived benefits.

The study of Fischhoff et al. used factor analysis to gain insight into people's thought processes, but the present study did not show any equivalent result. However, the patterns seen in the results, as well as some of the anomalies, appear to suggest some potential insight into users' understanding of the risks in online activities. In particular, financial risks are seen as more severe, and embarrassment risks are seen as more likely. However, because embarrassment risks may lead to loss of personal information and the installation of malware, they may lead to loss from financial activities at a later time. It appears users may not understand this relationship. These findings could be due to users' lack of awareness of the risks involved with these Internet-related activities, such as search engine redirection, dictionary attacks on passwords, and hacking a merchant's server and copying the entire credit card information database. The fact that users attributed a higher likelihood of the loss of personal information to activities such as giving a credit card number over the phone and using an automated transaction machine (ATM) shows that they appear to be unaware of, or less worried about, the risks involved with these types of transactions over the Internet. Many activities may result in malware installation on the user's machine, which could then undermine every other kind of activity.

As an overall finding — and perhaps the most significant to this study's results — is that users generally misunderstand the threats posed by malware, as well as the potential for the loss of personal and financial information that can occur as a result of the installation of malware on their computer. There do not appear to be other uses of the work of Fischhoff et al. in the study of computer security, so the contribution of this study is novel. However, the suggestion that users lack understanding of a critical element in security relates to the proposal for improving users' mental models by Bravo-Lillo et al. (2010).

The next step. Evidence from this study suggests that users have a difficult time understanding the risks involved in engaging in online activities. As such, the next logical 
step was determined to be testing user understanding of the Internet, zeroing in on specific online activities that carry the most risk (i.e., downloading and installing software, making online purchases, online banking, etc.). 


\section{Study Three: Understanding of Internet Software Abilities}

The third study focused on investigating the mental model participants possess in regards to the threats that exist in relationship to various popular online activities.

In this study, the intention was to follow the work of Morgan et al. (2002) on mental models. They suggested that a person's mental model is unlikely to exactly match new situations, and this may lead to significant difficulties in correctly assessing risk. Morgan et al. suggested a "Mental Model Interview" method. This approach ressembles the method used in study one, which was found to have limitations in the context of online security issues. For example, users who initially said they would trust websites, when asked to articulate were hesitant and often changed their minds without explanation. As an alternative, this study adopted a questionnaire-based approach. This survey was designed in order to investigate user understanding of the critical elements that support online security, as outlined in the Computer Security section of this thesis. The questions in the survey tested user knowledge of a broad range of security issues and technologies. Participant responses were then compared with ground truth, being "the real or underlying facts: information that has been checked or facts that have been collected at source" (Dictionaries, 2016). Determining ground truth in computer security can be very difficult because of the complex issues involved and multiple expert perspectives. For the comparisons made in this study, standard reference works were used, such as Anderson (2008), industry reports such as the Verizon Data Breach Investigations Report (Verizon, 2012), and the expert knowledge of the researchers. Where differences were found between participant responses and this "ground truth", the participant responses were considered evidence of a faulty mental model.

\section{Method}

Experimental design. The Limesurvey online survey tool was used in order to obtain participant data in this study. The survey contained questions in the following four categories: engaging in online banking, making an online purchase, installing software applications downloaded from the Internet, and using a search engine. There were ten 
questions for each category. Using the format of the Likert Scale, answers were rated from -3 to +3 . The participants selected the appropriate rating according to the direction given (i.e., $-3=$ Strongly disagree, $-2=$ Disagree, $-1=$ Slightly disagree, $0=$ Neutral, $+1=$ Slightly agree, $+2=$ Agree, $+3=$ Strongly agree) in the rating scale. Participants were asked to respond, to the best of their abilities, to each of the 10 rating scale questions for each of the four categories. These rating scales reflected subject matter appropriate to each category. The order of the rating scales was randomly generated by the Limesurvey tool so that each participant was shown the questions in a random order. The study was approved by the Carleton University Psychology Research Ethics Board Appendix M.

\section{Participants}

In all, 221 individuals were recruited. Ages ranged from 18 to 72 years. Of the 221 participants, 100 were male and 120 were female, with 1 respondent opting not to provide an answer. Educational backgrounds varied from high school to university graduate degrees. Occupations also varied, from students to medical doctors. The pre-requisites of eligibility for participating in the study were for participants to be at least 18 years of age, not an expert or student in Computer Security, possess a minimum of 2 years of experience with the Internet, and be regular users (i.e., use the Internet at least 2 times each week). The majority of participants stated that they use the Internet multiple times daily. The majority of respondents reported the most used operating system as being Microsoft Windows, with Mozilla Firefox and Google Chrome as the most used Internet browsers. Age distribution for each gender was approximately the same, with an average age of 32 years; the education responses show that the majority of the participant pool has at least some college or university education; the self-reported expertise statement results show that users report being more confident in their knowledge of computer and Internet security than average. 


\section{Hypotheses}

The following hypotheses were created regarding the study. Their alternate versions are also provided. Each of these hypotheses was created based on the results obtained from Study 1 and Study 2, as well as from the results obtained from the Verizon Data Breach Investigations Report (Verizon, 2012). This report concluded that a tremendous amount of incidents were reported by computer users throughout the world regarding Cybersecurity breaches, partly resulting in the theft of personal information and the loss of financial information. Studies 1 and 2 from this thesis work showed that users have a general misunderstanding of the dangers prominent on the Internet while engaging in online activities, and the mechanisms that are in place to help protect them from these threats.

- $\mathbf{H} \mathbf{1}_{o}$ : Participants misunderstand malware in general compared to other areas of computer security.

- H1 $1_{a}$ : Participants do not misunderstand malware in general compared to other areas of computer security.

- H2 ${ }_{o}$ : There are specific aspects of malware where users lack knowledge.

- $\mathbf{H 2} \mathbf{2}_{a}$ : There are no specific aspects of malware where users lack knowledge.

\section{Materials}

The materials used in the study included task instructions Appendix O, a demographics questionnaire Appendix N, the list of activities Appendix P, and the rating scale questions (Appendix Q).

\section{Procedure}

Before the main task, participants answered a short demographics questionnaire, which included questions on age, gender, occupation, education, and frequency of Internet use. This data was collected for exploratory data analysis based on individual differences 
in demographic information. After the main task, participants answered a short post-test questionnaire which asked questions on their Internet activity usage and their self-reported rating on knowledge about computers and Internet security.

The main task questionnaire was created using the Limesurvey tool installed on university secure servers $($ Schmitz, 2010). For each of the four online activities used in the study, an image of the website in question was shown to the participant; this image was embedded directly within the Limesurvey page where the activity name, scenario, and rating scales were located. Participants were told that the screenshots were of the actual websites, and had not been altered in any way. They were told that their task was to observe the website screenshot, taking in the information about the website and the website scenario, and then provide their opinion on the 10 statements about the website in question. The statements themselves dealt with various threats and security measures associated with and specific to each online activity.

Participants were tested on four different online activities: "Doing your banking online", "Making an online purchase", "Installing a software application downloaded from the Internet", and "Using a search engine". Each of these online activities was associated to ten different rating scales. They appear in Tables 7 through 13 . The first column of each table depicts the rating scale in question, and the second column depicts whether the statement is true or false, as determined before the study was conducted. Internet security is inherently complex and phrasing statements that accurately reflect truth or falsehood is difficult. The wording was chosen in consideration of the need to make the statement clear to participants with a range of expertise. Figure 23 depicts one of the websites used during testing, along with a few of the rating scale statements posed to participants.

Participants were informed that the task was designed to investigate how people rate online activities on various different characteristics. Participants were then asked to complete a questionnaire regarding four different online activities with ten statements each, for a total of 40 statements. Each online activity introduced participants to a scenario which was relevant to each online activity. These scenarios are shown below. 


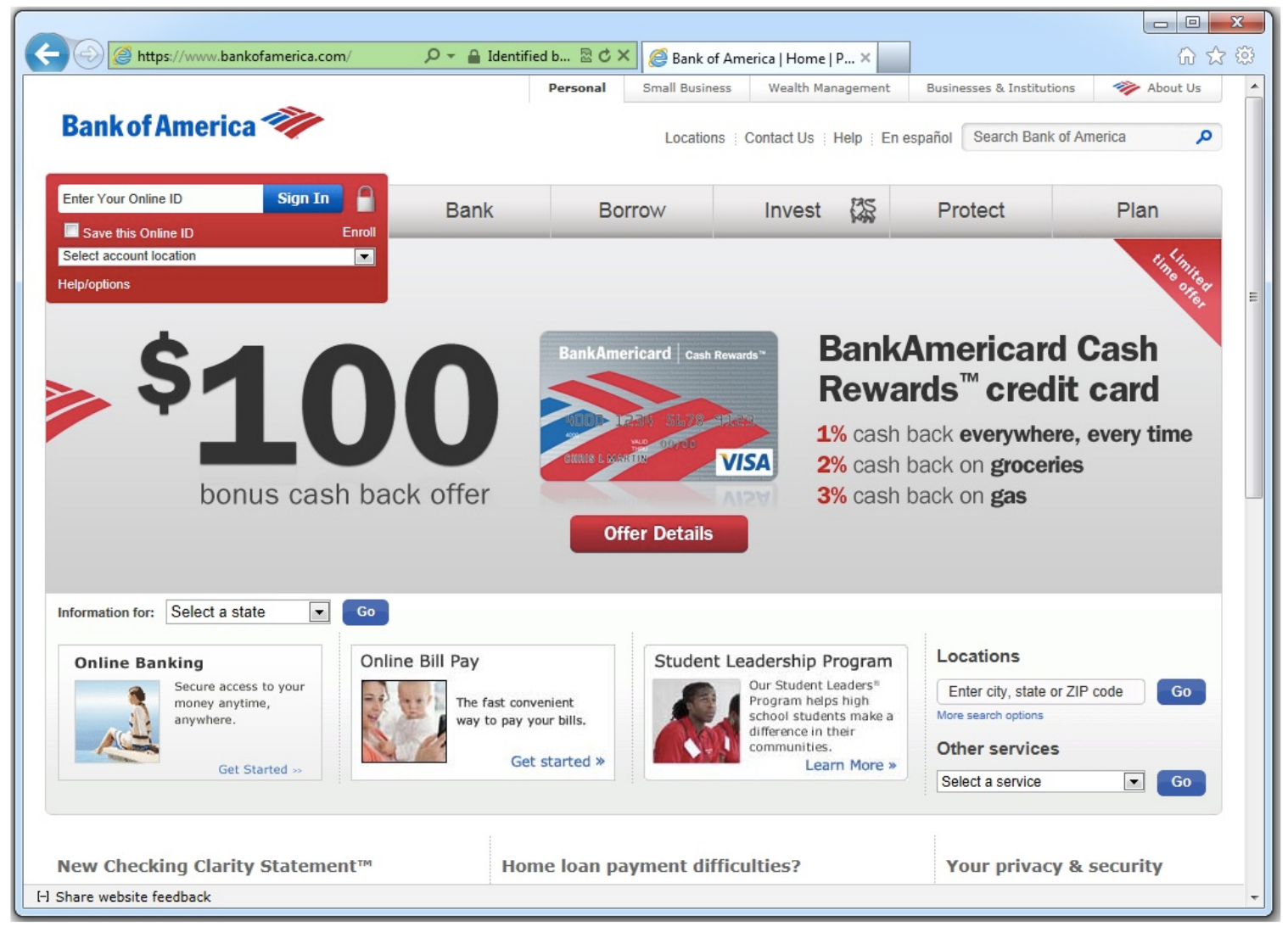

$\rightarrow$ Share website feedback

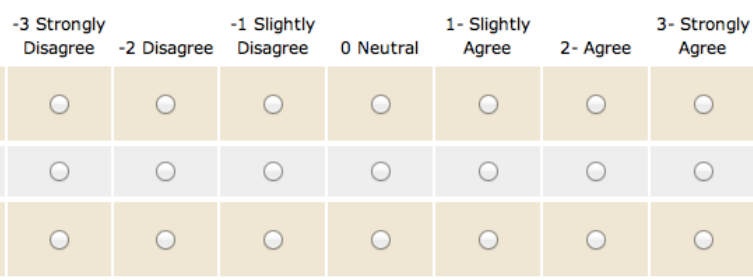

My Bank of America account information (e.g., client card number, password) is at risk from shoulder-surfing (someone looking at my keyboard as I enter the information) if I log in using a computer in a public place.

My computer can be infected by Malware (malicious software program) by using the Bank of America website.

Using the Web address shown in the address bar in the image to reach the Bank of America website, I may be sent to a phishing website (which looks like the correct website but is not genuine).

Figure 23. Questionnaire page demonstrating the activity scenario, website image screenshot, and rating scale statements found within the survey.

Activity scenario: Doing your banking online (Bank of America). You need to access your Bank of America online account, and you click on a link that shows the following webpage. Look closely at the image, and rate each statement below.

Activity scenario: Making an online purchase (Amazon). You need to access your Amazon online account, and you click on a link that shows the following webpage. Look closely at the image, and rate each statement below.

Activity scenario: Installing a software application downloaded from the Internet (CNet Download). You want to download a particular software program for 
your computer. You click on a link in Google that shows the following webpage. Look closely at the image, and rate each statement below.

Activity scenario: Using a search engine (Google). You are looking for something specific on the Internet, and decide to look it up on the Google search engine. Look closely at the image, and rate each statement below.

\section{Results}

Data distributions. Figures 26, 27, 28, and 29, show the distributions of responses to the rating scales as histograms. The left column of each histogram figure depicts the five true statements for the activity in question. For participant responses to be considered as correct, the rating scale responses were 5,6 , or 7 . Incorrect response bars are shown in red, and correct responses are shown in green. The rating scale response 4 represented as being an "I don't know" response and is shown in yellow. For the purposes of statistical analysis, this was also counted as being incorrect. The right column of each histogram figure depicts the five false statements for the activity in question. For participant responses to be considered as correct, the rating scale responses were 1,2, or 3 . However, for ease of comparison, the responses are reversed, and coded as 5, 6, and 7; similarly, the incorrect responses are coded as 1,2 , and 3 . The color coding of the right column is the same as the left column.

Many of the histograms shown here indicate that the responses to the rating scales were widely distributed. For example, Figure 26 shows that there was a lack of consensus between participants when rating the "WebAddressShown" statement, which asked participants whether the Web address shown on the Bank of America website screenshot was associated or not with the Web address www.bankofamerica.secure.com. In this case, participant responses were widely distributed.

Observations of activity 1 responses: Doing your banking online. Out of the 10 rating scales used for the first activity in the study, "Doing your banking online", a select few were found to be problematic in relation to participant responses. Primarily, 


\section{Age and Gender}

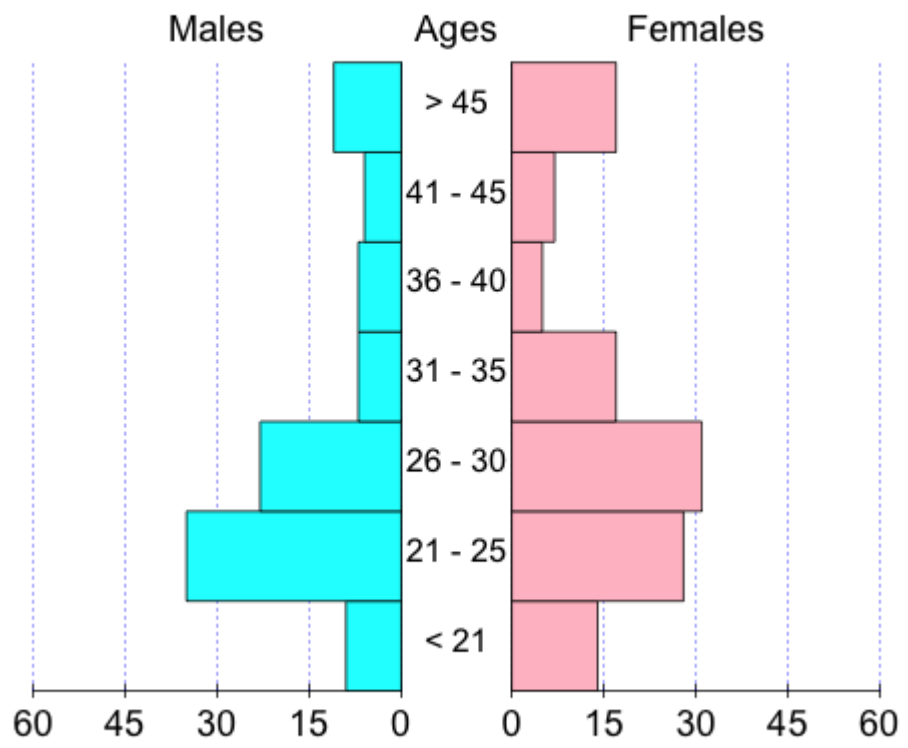

Figure 24. Study participants distribution of age and gender.

Education

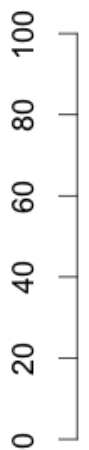

$\circ-$

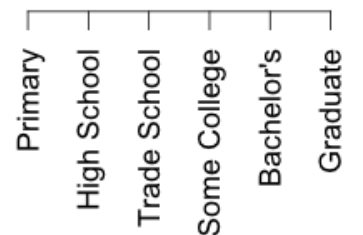

Expertise

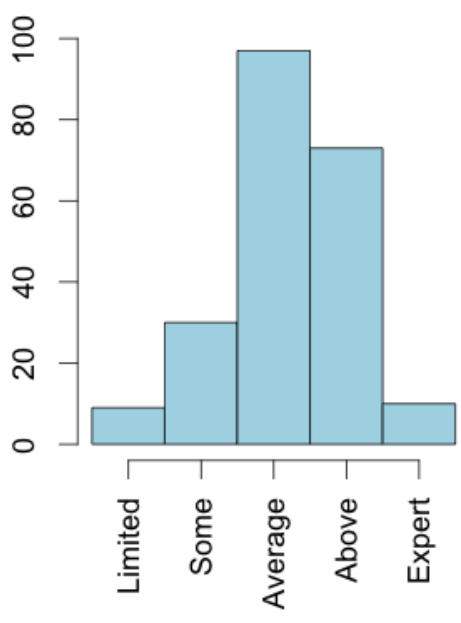

Figure 25. Study participants distribution of education and expertise. 
Table 7

Rating Scales Used in the Main Task Survey for the Activity "Doing Your Banking Online".

Activity: Doing your banking online (Bank of America)

The https indicator in the address bar signifies communication between my computer and the Bank of America website server is encrypted (sent using a secret code)

The Web address shown is www.bankofamerica.com. The Web address www.bankofamerica.secure.com might be associated with a different organization from the Bank of America

If there is malware (malicious software program) installed on the computer I use to gain access to my Bank of America online account, there is a chance that my login information can be copied and sent to a third party

Searching for Bank of America using a search engine (e.g., Google) may take me to a phishing website (which looks like the correct website but is not genuine)

My Bank of America account information (e.g., client card number, password) is at risk from shoulder-surfing (someone looking at my keyboard as I enter the information) if I log in using a computer in a public place

Other Internet users are able to intercept my Bank of America information (e.g., username, password, client card number) when I submit it via the login form found on the homepage

My Internet Service Provider (e.g., Comcast, Time Warner, AT\&T) is able to monitor my personal information (e.g., account balances, etc.) regarding my Bank of America account usage

Using the Web address shown in the address bar in the image to reach the Bank of America website, I may be sent to a phishing website (which looks like the correct website but is not genuine)

Friends from my social networking website (e.g., Facebook, MySpace) can see that I do banking at the Bank of America

Abbreviation

$\mathbf{T} / \mathbf{F}$

HTTPS

$\mathrm{T}$

WebAddressShown

${ }^{*}$ Notes. $\mathrm{T}=$ True. $\mathrm{F}=$ False.

these issues were found to be related to the responses relating to the following rating scale statements: "WebAddressShown", "SearchEnginePhishing", and "ISPCanMonitor". For the rating scale details, see Table 7, for the distribution of responses, see Figure 26, and for the details of participant responses, see Table 8

Many of the responses to the rating scale questions showed a moderately high degree of participant understanding of the various implications of engaging in the activity of online banking. As can be seen in Figure 26, many participant responses are found to be 5,6 , or 7 , represented by the green bars. This suggests a moderate to strong grasp of the many dangers (i.e., attacks) and security mechanisms in place to protect users against these dangers. 


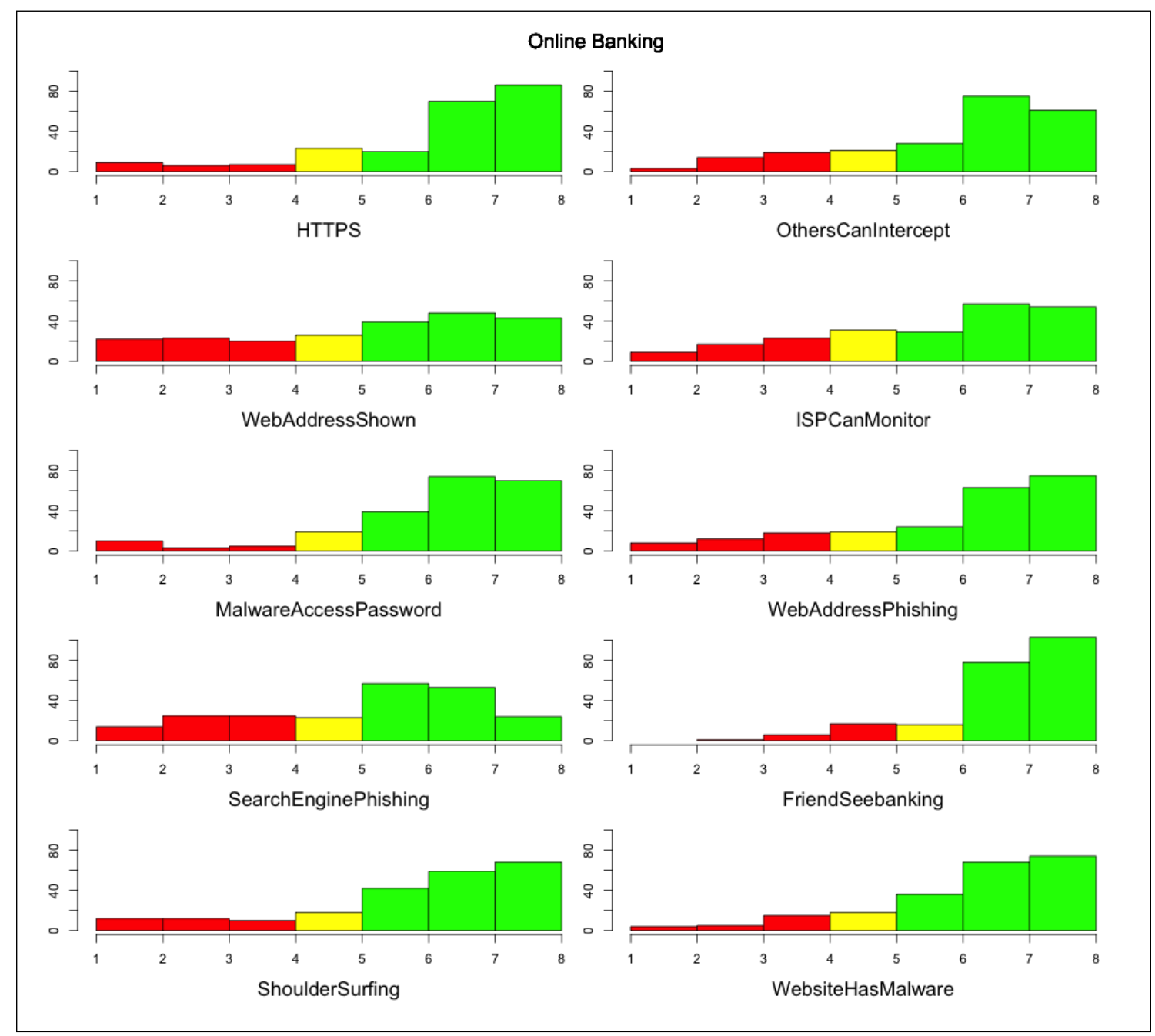

Figure 26. Histograms of the rating scales for the activity "Doing Your Banking Online".

However, some of these rating scale questions showed a high degree of incorrect responses, as well as a high frequency of participants selecting the "Neutral" option, suggesting that they did not know how to correctly answer the question. In some cases, such as the rating scale statement "WebAddressShown", participant responses that were incorrect account for more than $41 \%$ of the total responses. These results suggest problematic issues pertaining to users' understanding of the dangers on the Internet, as well as the security mechanisms of their browser, in conjunction with this online activity.

Observations of activity 2 responses: Making an online purchase. Out of the 10 rating scales used for the second activity in the study, "Making an Online Purchase", 
Table 8

Participant Responses to the Rating Scales Used in the Main Task Survey for the Activity "Doing Your Banking Online".

\begin{tabular}{|c|c|c|c|c|c|c|c|c|c|}
\hline \multirow[t]{2}{*}{ Abbreviation } & \multirow[t]{2}{*}{$\mathbf{T} / \mathbf{F}$} & \multicolumn{8}{|c|}{ Responses (out of 221 participants) } \\
\hline & & -3 & -2 & -1 & 0 & 1 & 2 & 3 & NR \\
\hline \multirow[t]{2}{*}{ HTTPS } & True & 9 & 6 & 7 & 23 & 20 & 70 & 86 & 0 \\
\hline & & $4.07 \%$ & $2.71 \%$ & $3.17 \%$ & $10.41 \%$ & $9.05 \%$ & $31.67 \%$ & $38.91 \%$ & $0.00 \%$ \\
\hline \multirow[t]{2}{*}{ WebAddressShown } & True & 22 & 23 & 20 & 26 & 39 & 48 & 43 & 0 \\
\hline & & $9.95 \%$ & $10.41 \%$ & $9.05 \%$ & $11.76 \%$ & $17.65 \%$ & $21.72 \%$ & $19.46 \%$ & $0.00 \%$ \\
\hline \multirow[t]{2}{*}{ MalwareAccessPassword } & True & 10 & 3 & 5 & 19 & 39 & 74 & 70 & 1 \\
\hline & & $4.52 \%$ & $1.36 \%$ & $2.26 \%$ & $8.60 \%$ & $17.65 \%$ & $33.48 \%$ & $31.67 \%$ & $0.45 \%$ \\
\hline \multirow[t]{2}{*}{ SearchEnginePhishing } & True & 14 & 25 & 25 & 23 & 57 & 53 & 24 & 0 \\
\hline & & $6.33 \%$ & $11.31 \%$ & $11.31 \%$ & $10.41 \%$ & $25.79 \%$ & $23.98 \%$ & $10.86 \%$ & $0.00 \%$ \\
\hline \multirow[t]{2}{*}{ ShouldSurfing } & True & 12 & 12 & 10 & 18 & 42 & 59 & 68 & 0 \\
\hline & & $5.43 \%$ & $5.43 \%$ & $4.52 \%$ & $8.14 \%$ & $19.00 \%$ & $26.70 \%$ & $30.77 \%$ & $0.00 \%$ \\
\hline \multirow[t]{2}{*}{ OthersCanIntercept } & False & 61 & 75 & 28 & 21 & 19 & 14 & 3 & 0 \\
\hline & & $27.60 \%$ & $33.94 \%$ & $12.67 \%$ & $9.50 \%$ & $8.60 \%$ & $6.33 \%$ & $1.36 \%$ & $0.00 \%$ \\
\hline \multirow[t]{2}{*}{ ISPCanMonitor } & False & 54 & 57 & 29 & 31 & 23 & 17 & 9 & 1 \\
\hline & & $24.43 \%$ & $25.79 \%$ & $13.12 \%$ & $14.03 \%$ & $10.41 \%$ & $7.69 \%$ & $4.07 \%$ & $0.45 \%$ \\
\hline \multirow[t]{2}{*}{ WebAddressPhishing } & False & 75 & 63 & 24 & 19 & 18 & 12 & 8 & 2 \\
\hline & & $33.94 \%$ & $28.51 \%$ & $10.86 \%$ & $8.60 \%$ & $8.14 \%$ & $5.43 \%$ & $3.62 \%$ & $0.90 \%$ \\
\hline \multirow[t]{2}{*}{ FriendSeeBanking } & False & 103 & 78 & 16 & 17 & 6 & 1 & 0 & 0 \\
\hline & & $46.61 \%$ & $35.29 \%$ & $7.24 \%$ & $7.69 \%$ & $2.71 \%$ & $0.45 \%$ & $0.00 \%$ & $0.00 \%$ \\
\hline \multirow[t]{2}{*}{ WebsiteHasMalware } & False & 74 & 68 & 36 & 18 & 15 & 5 & 4 & 1 \\
\hline & & $33.48 \%$ & $30.77 \%$ & $16.29 \%$ & $8.14 \%$ & $6.79 \%$ & $2.26 \%$ & $1.81 \%$ & $0.45 \%$ \\
\hline
\end{tabular}

*Notes. Rating scale response choices: $-3=$ Strongly Disagree. $-2=$ Disagree. $-1=$ Slightly Disagree. $0=$ Neutral. $1=$ Slightly Agree. $2=$ Agree. $3=$ Strongly Agree. $\mathrm{NR}=$ No Response. Correct "True" responses are choices 5, 6, and 7, and are coded in green; Correct "False" responses are choices 1, 2, and 3, and are coded in green. "Neutral" responses for all rating scale statements are considered incorrect, and are coded in yellow. "No Response" is coded in gray.

a select few were found to be problematic in relation to participant responses. Primarily, these issues were found to be related to the responses relating to the following rating scales: "WirelessAccessPoint" and "SearchEnginePhishing", "CCHasAccess". For the rating scale details, see Table 9, for the distribution of responses, see Figure 27, and for the details of participant responses, see Table 10.

In contrast to the observations of the first activity participant responses, some of the rating scale questions for the activity of "Making an online purchase" showed more drastic participant responses for some of the rating scale questions. In particular, large proportions of participants answered incorrectly to the "WirelessAccessPoint" and "SearchEnginePhish- 
Table 9

Rating Scales Used in the Main Task Survey for the Activity "Making An Online Purchase".

Activity: Making an online purchase (Amazon)

Amazon can track my browsing history on their website, and recommend specific products, by installing and checking cookies (identifying information stored on my computer)

Searching for Amazon using a search engine (e.g., Google) may take me to a phishing website (which looks like the correct website but is not genuine)

If there is malware (malicious software program) installed on the computer I use to $\log$ in to Amazon, there is a chance that my login information can be copied and sent to a third party

The https indicator in the address bar signifies communication between my computer and the Amazon website server is encrypted (sent using a secret code)

Using a wireless Internet access point (e.g., at a Starbucks coffee shop), when I $\log$ in to Amazon, other users are unable to gain access to my Amazon account

Looking at the Amazon website screenshot image above, I feel it is possible that it is a phishing website (which looks like the correct website but is not genuine)

Other Internet users are able to intercept my personal information (e.g., username, password, credit card number) when I submit it via the Amazon sign in form

My computer can be infected by malware (malicious software program) by using the Amazon website

Using the Web address shown in the address bar in the image to reach the Amazon website, I may be sent to a phishing website (which looks like the correct website but is not genuine)

When I purchase something on Amazon using my credit card, the credit card company (e.g., Visa, MasterCard) always has access to all of the personal information (e.g., username, password, shopping cart contents) found on my Amazon account

Abbreviation

BrowsingHistory

$\mathbf{T} / \mathbf{F}$

$\mathrm{T}$

SearchEnginePhishing

$\mathrm{T}$

MalwareAccessPassword

$\mathrm{T}$

HTTPS

WirelessAccessPoint $\mathrm{T}$

LookingAmazonWebsite

F

OthersCanIntercept

F

InfectedByMalware

F

WebAddressPhishing

F

CCHasAccess

F

${ }^{*}$ Notes. $\mathrm{T}=$ True. $\mathrm{F}=$ False.

ing" rating scale questions. In the former, well over half of the responses - approximately $51 \%$ - were incorrect or answered as "neutral", as can be seen in Figure 27. These findings suggest, in relation to these particular rating scale question, a weak grasp of the many dangers (e.g., attacks) and security mechanisms in place to protect users against these dangers.

\section{Observations of activity 3 responses: Installing a software application}

downloaded from the Internet. Out of the 10 rating scales used for the third activity in the study, "Installing a software application downloaded from the Internet", some rating scale statements were found to be problematic. These specific rating scale statements, "WebAddressPhishing" and "MaliciousCodeGetsInformation" are particularly problematic, as 


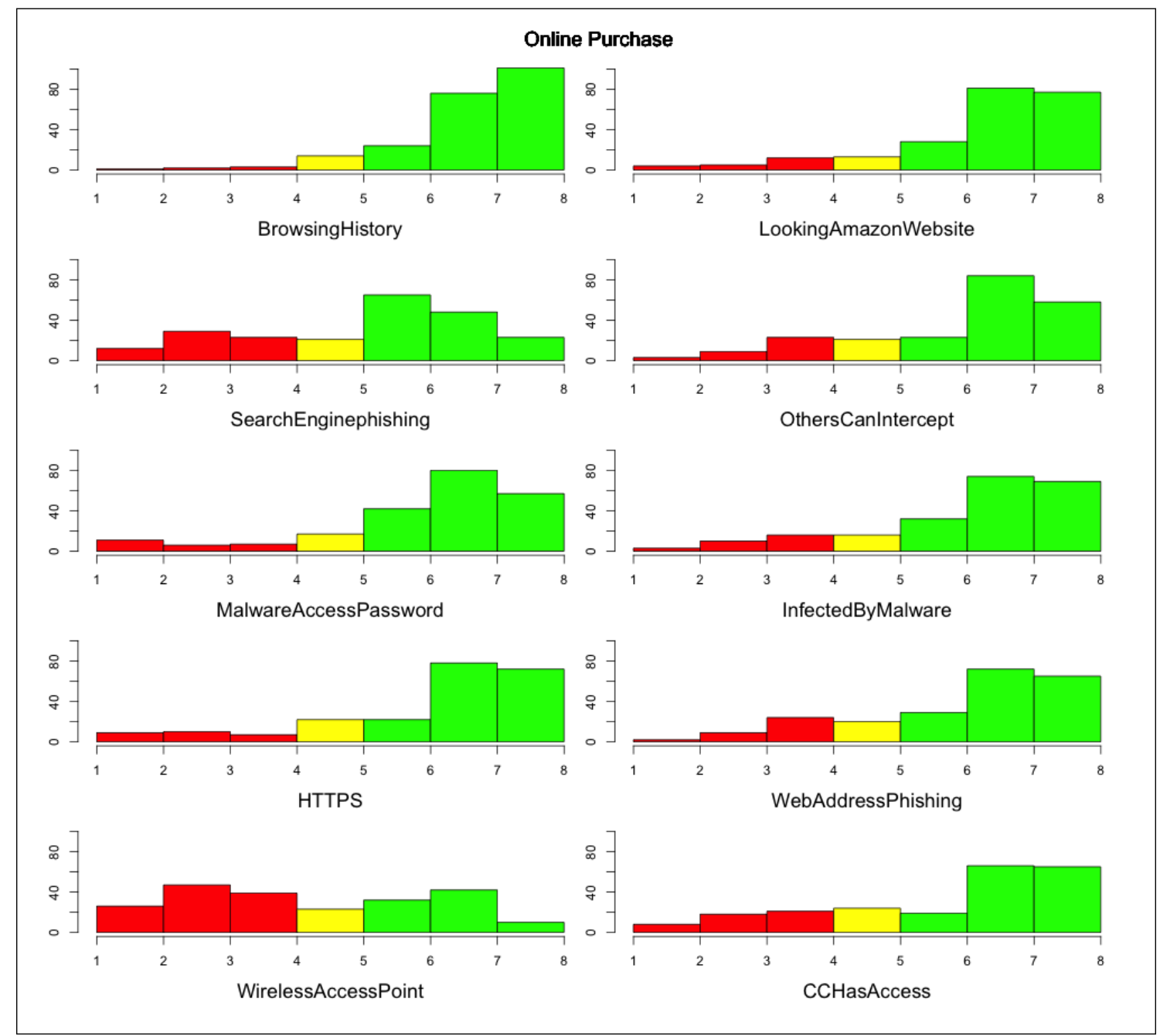

Figure 27. Histograms of the rating scales for the activity "Making an Online Purchase".

the distribution of participant responses is found to be on the side of the incorrect responses. For the rating scale details, see Table 11, for the distribution of responses, see Figure 28, and for the details of participant responses, see Table 12 . The total combined participant responses for either of these rating scale statements total over $60 \%$ of the total responses. This indicates that participants do not understand the implications of these particular situations in an online setting. Participant responses for other rating scale statements, such as "KnownSoftwareDuplicated", and "OtherUsersPrevented", are also problematic, but with slightly less dramatic response distributions, averaging between $30 \%$ and $40 \%$ of incorrect responses. Out of the four online activities in this study, this activity is found to be the most 
Table 10

Participant Responses to the Rating Scales Used in the Main Task Survey for the Activity "Making an Online Purchase".

\begin{tabular}{|c|c|c|c|c|c|c|c|c|c|}
\hline \multirow[t]{2}{*}{ Abbreviation } & \multirow[t]{2}{*}{$\mathbf{T} / \mathbf{F}$} & \multicolumn{8}{|c|}{ Responses (out of 221 participants) } \\
\hline & & -3 & -2 & -1 & 0 & 1 & 2 & 3 & NR \\
\hline \multirow[t]{2}{*}{ Browsing History } & True & 1 & 2 & 3 & 14 & 24 & 76 & 101 & 0 \\
\hline & & $0.45 \%$ & $0.90 \%$ & $1.36 \%$ & $6.33 \%$ & $10.86 \%$ & $34.39 \%$ & $45.70 \%$ & $0.00 \%$ \\
\hline \multirow[t]{2}{*}{ SearchEnginePhishing } & True & 12 & 29 & 23 & 21 & 65 & 48 & 23 & 0 \\
\hline & & $5.43 \%$ & $13.12 \%$ & $10.41 \%$ & $9.50 \%$ & $29.41 \%$ & $21.72 \%$ & $10.41 \%$ & $0.00 \%$ \\
\hline \multirow[t]{2}{*}{ MalwareAccessPassword } & True & 11 & 6 & 7 & 17 & 42 & 80 & 57 & 1 \\
\hline & & $4.98 \%$ & $2.71 \%$ & $3.17 \%$ & $7.69 \%$ & $19.00 \%$ & $36.20 \%$ & $25.79 \%$ & $0.45 \%$ \\
\hline \multirow[t]{2}{*}{ HTTPS } & True & 9 & 10 & 7 & 22 & 22 & 78 & 72 & 1 \\
\hline & & $4.07 \%$ & $4.52 \%$ & $3.17 \%$ & $9.95 \%$ & $9.95 \%$ & $35.29 \%$ & $35.58 \%$ & $0.45 \%$ \\
\hline \multirow[t]{2}{*}{ WirelessAccessPoint } & True & 26 & 47 & 39 & 23 & 32 & 42 & 10 & 2 \\
\hline & & $11.76 \%$ & $21.27 \%$ & $17.65 \%$ & $10.41 \%$ & $14.48 \%$ & $19.00 \%$ & $4.52 \%$ & $0.90 \%$ \\
\hline \multirow[t]{2}{*}{ LookingAmazonWebsite } & False & 77 & 81 & 28 & 13 & 12 & 5 & 4 & 1 \\
\hline & & $34.84 \%$ & $36.65 \%$ & $12.67 \%$ & $5.88 \%$ & $5.43 \%$ & $2.26 \%$ & $1.81 \%$ & $0.45 \%$ \\
\hline \multirow[t]{2}{*}{ OthersCanIntercept } & False & 58 & 84 & 23 & 21 & 23 & 9 & 3 & 0 \\
\hline & & $26.24 \%$ & $38.01 \%$ & $10.41 \%$ & $9.50 \%$ & $10.41 \%$ & $4.07 \%$ & $1.36 \%$ & $0.00 \%$ \\
\hline \multirow[t]{2}{*}{ InfectedByMalware } & False & 69 & 74 & 32 & 16 & 16 & 10 & 3 & 1 \\
\hline & & $31.22 \%$ & $33.48 \%$ & $14.48 \%$ & $7.24 \%$ & $7.24 \%$ & $4.52 \%$ & $1.36 \%$ & $0.45 \%$ \\
\hline \multirow[t]{2}{*}{ WebAddressPhishing } & False & 65 & 72 & 29 & 20 & 24 & 9 & 2 & 0 \\
\hline & & $29.41 \%$ & $32.58 \%$ & $13.12 \%$ & $9.05 \%$ & $10.86 \%$ & $4.07 \%$ & $0.90 \%$ & $0.00 \%$ \\
\hline \multirow[t]{2}{*}{ CCHasAccess } & False & 65 & 66 & 19 & 24 & 21 & 18 & 8 & 0 \\
\hline & & $29.41 \%$ & $29.86 \%$ & $8.60 \%$ & $10.86 \%$ & $9.50 \%$ & $8.14 \%$ & $3.62 \%$ & $0.00 \%$ \\
\hline
\end{tabular}

*Notes. Rating scale response choices: $-3=$ Strongly Disagree. $-2=$ Disagree. $-1=$ Slightly Disagree. $0=$ Neutral. $1=$ Slightly Agree. $2=$ Agree. $3=$ Strongly Agree. $\mathrm{NR}=$ No Response. Correct "True" responses are choices 5, 6, and 7, and are coded in green; Correct "False" responses are choices 1, 2, and 3, and are coded in green. "Neutral" responses for all rating scale statements are considered incorrect, and are coded in yellow. "No Response" is coded in gray.

problematic in relation to participant understanding of the dangers involved in installing software applications downloaded from the Internet.

Observations of activity 4 responses: Using a search engine. Out of the 10 rating scales used for the second activity in the study, "Using a search engine", two of them are found to be the most problematic, "MapsWithoutPermission" and "ISPMonitorsSearches". For the rating scale details, see Table 13, for the distribution of responses, see Figure 29, and for the details of participant responses, see Table 14. Using a search engine is quite different from the other activities used in this study. As such, it is believed that the results from the rating scale statements that were shown to be problematic result from 
Table 11

Rating Scales Used in the Main Task Survey for the Activity "Installing a Software Application Downloaded from the Internet".

\begin{tabular}{|c|c|c|}
\hline $\begin{array}{l}\text { Activity: Installing a software application downloaded from the } \\
\text { Internet (CNet Download) }\end{array}$ & Abbreviation & $T / F$ \\
\hline $\begin{array}{l}\text { A malicious software application (e.g., malware, virus) downloaded from the } \\
\text { Internet, and installed on my computer, can change any of the files contained } \\
\text { on my computer's hard drive }\end{array}$ & MalwareChangeFiles & $\mathrm{T}$ \\
\hline $\begin{array}{l}\text { A malicious software application (e.g., malware, virus) downloaded from the } \\
\text { Internet, and installed on my computer, can access files (such as Microsoft } \\
\text { Word files and PDF files) in order to collect my personal information }\end{array}$ & MalwareAccessFiles & $\mathrm{T}$ \\
\hline $\begin{array}{l}\text { Any software application I download from the Internet can potentially contain } \\
\text { malicious programming code }\end{array}$ & SoftwareMaliciousCode & $\mathrm{T}$ \\
\hline $\begin{array}{l}\text { A commonly known software application (e.g., Adobe Reader, Microsoft In- } \\
\text { ternet Explorer) can be duplicated before it is downloaded, and can contain } \\
\text { malicious programming code in order for someone to "attack" my computer } \\
\text { (e.g., damage or destroy computer files, collect personal information) }\end{array}$ & KnownSoftwareDuplicated & $\mathrm{T}$ \\
\hline $\begin{array}{l}\text { If I download a software application on a downloads website (e.g., } \\
\text { www.download.cnet.com) other than the software company's official website } \\
\text { (e.g., www.microsoft.com), then I run a higher risk of downloading and in- } \\
\text { stalling malware instead of the real, genuine software application }\end{array}$ & InstallingMalwareNotGenuine & $\mathrm{T}$ \\
\hline $\begin{array}{l}\text { If the software application I download from the Internet contains malicious } \\
\text { programming code, then all of my usernames and passwords for each of my } \\
\text { various online accounts (e.g., email, banking, etc.) will automatically be } \\
\text { copied and sent to a third party }\end{array}$ & MaliciousCodeGetsInformation & $\mathrm{F}$ \\
\hline $\begin{array}{l}\text { A virus scan program (e.g., McAfee Antivirus, Norton Antivirus) will de- } \\
\text { tect with } 100 \% \text { accuracy any malicious software applications that attempt to } \\
\text { compromise (i.e., "attack") my computer }\end{array}$ & VirusDetects100 & $\mathrm{F}$ \\
\hline $\begin{array}{l}\text { When I browse the CNet Download.com website as shown above, other In- } \\
\text { ternet users are prevented from knowing which pages I view }\end{array}$ & OtherUsersPrevented & $\mathrm{F}$ \\
\hline $\begin{array}{l}\text { The http indicator in the address bar on the Download.com homepage sig- } \\
\text { nifies that my information is encrypted during communications between my } \\
\text { computer and the Download.com website server }\end{array}$ & HTTP & $\mathrm{F}$ \\
\hline $\begin{array}{l}\text { If I use the Web address as shown for the Download.com website, I may be } \\
\text { open to phishing attacks }\end{array}$ & WebAddressPhishing & $\mathrm{F}$ \\
\hline
\end{tabular}

*Notes. $\mathrm{T}=$ True. $\mathrm{F}=$ False.

a misunderstanding not of the possible dangers associated with engaging in online activities, but the rating statements themselves, and how each was structured within the study's design. It is quite feasible that participants simply do not understand the finer details of how mapping websites and Internet Service Providers obtain information relating to users who opt to use their services. The remaining rating scale statements do not suggest user difficulty in understanding the possible issues in engaging in the activity of using a search engine. As such, it is believed that this activity is generally better understood by users than the previous three activities in this study. 


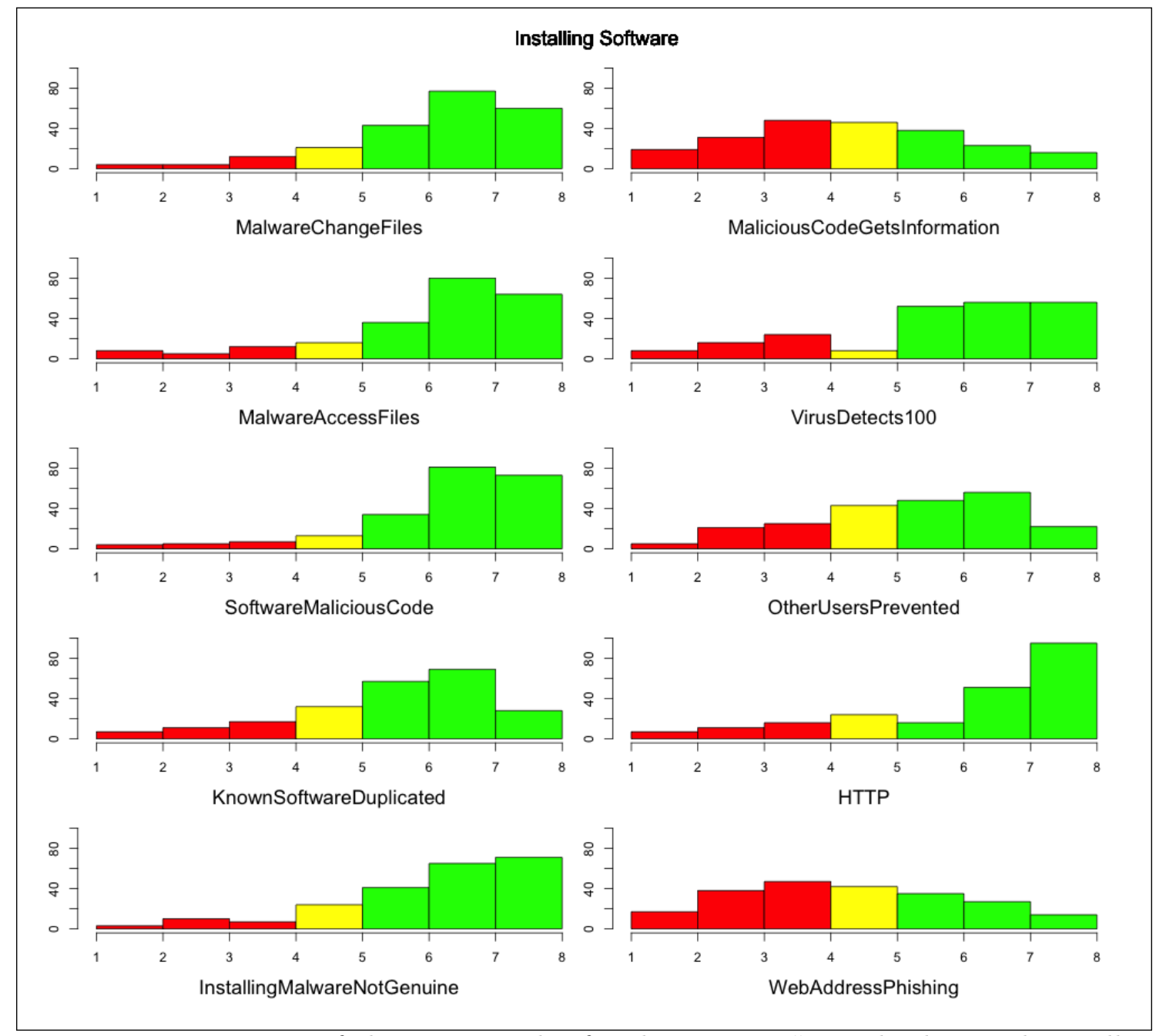

Figure 28. Histograms of the rating scales for the activity "Downloading and Installing Software".

Statistical analysis. Hypothesis 1 was "Participants misunderstand malware in general compared to other areas of computer security." As shown in the distribution figures, participant responses were made consistent so that responses of 5,6 , and 7 indicated correct responses in all cases. Then, normality tests were conducted. The skewness and kurtosis values were within acceptable range, indicating that the data was normality distributed (see Table 16). A between-groups Analysis of Variance was conducted on the four activities used in the study. The results showed a significant difference between activities, with $\mathrm{F}(3)$ $=7.682, \mathrm{p}<0.001$. This supports hypothesis 1 . Following this ANOVA, a Tukey HSD test 
Table 12

Participant Responses to the Rating Scales Used in the Main Task Survey for the Activity "Downloading and Installing Software".

\begin{tabular}{|c|c|c|c|c|c|c|c|c|c|}
\hline \multirow[t]{2}{*}{ Abbreviation } & \multirow[t]{2}{*}{$\mathbf{T} / \mathbf{F}$} & \multicolumn{8}{|c|}{ Responses (out of 221 participants) } \\
\hline & & -3 & -2 & -1 & 0 & 1 & 2 & 3 & NR \\
\hline \multirow[t]{2}{*}{ MalwareChangeFiles } & True & 4 & 4 & 12 & 21 & 43 & 77 & 60 & 0 \\
\hline & & $1.81 \%$ & $1.81 \%$ & $5.43 \%$ & $9.50 \%$ & $19.46 \%$ & $34.84 \%$ & $27.15 \%$ & $0.00 \%$ \\
\hline \multirow[t]{2}{*}{ MalwareAccessFiles } & True & 8 & 5 & 12 & 16 & 36 & 80 & 64 & 0 \\
\hline & & $3.62 \%$ & $2.26 \%$ & $5.43 \%$ & $7.24 \%$ & $16.29 \%$ & $36.20 \%$ & $28.96 \%$ & $0.00 \%$ \\
\hline \multirow[t]{2}{*}{ SoftwareMaliciousCode } & True & 4 & 5 & 7 & 13 & 34 & 81 & 73 & 4 \\
\hline & & $1.81 \%$ & $2.26 \%$ & $3.17 \%$ & $5.88 \%$ & $15.38 \%$ & $36.65 \%$ & $33.03 \%$ & $1.81 \%$ \\
\hline \multirow[t]{2}{*}{ KnownSoftwareDuplicated } & True & 7 & 11 & 17 & 32 & 57 & 69 & 28 & 0 \\
\hline & & $3.17 \%$ & $4.98 \%$ & $7.69 \%$ & $14.48 \%$ & $25.79 \%$ & $31.22 \%$ & $12.67 \%$ & $0.00 \%$ \\
\hline \multirow[t]{2}{*}{ InstallingMalwareNotGenuine } & True & 3 & 10 & 7 & 24 & 41 & 65 & 71 & 0 \\
\hline & & $1.36 \%$ & $4.52 \%$ & $3.17 \%$ & $10.86 \%$ & $18.55 \%$ & $29.41 \%$ & $32.13 \%$ & $0.00 \%$ \\
\hline \multirow[t]{2}{*}{ MaliciousCodeGetsInformation } & False & 16 & 23 & 38 & 46 & 48 & 31 & 19 & 0 \\
\hline & & $7.24 \%$ & $10.41 \%$ & $17.19 \%$ & $20.81 \%$ & $21.72 \%$ & $14.03 \%$ & $8.60 \%$ & $0.00 \%$ \\
\hline \multirow[t]{2}{*}{ VirusDetects100 } & False & 56 & 56 & 52 & 8 & 24 & 16 & 8 & 1 \\
\hline & & $25.34 \%$ & $25.34 \%$ & $23.53 \%$ & $3.62 \%$ & $10.86 \%$ & $7.24 \%$ & $3.62 \%$ & $0.45 \%$ \\
\hline \multirow[t]{2}{*}{ OtherUsersPrevented } & False & 22 & 56 & 48 & 43 & 25 & 21 & 5 & 1 \\
\hline & & $9.95 \%$ & $25.34 \%$ & $21.72 \%$ & $19.46 \%$ & $11.31 \%$ & $9.50 \%$ & $2.26 \%$ & $0.45 \%$ \\
\hline \multirow[t]{2}{*}{ НTTP } & False & 95 & 51 & 16 & 24 & 16 & 11 & 7 & 1 \\
\hline & & $42.99 \%$ & $23.08 \%$ & $7.24 \%$ & $10.86 \%$ & $7.24 \%$ & $4.98 \%$ & $3.17 \%$ & $0.45 \%$ \\
\hline \multirow[t]{2}{*}{ WebAddressPhishing } & False & 14 & 27 & 35 & 42 & 47 & 38 & 17 & 1 \\
\hline & & $6.33 \%$ & $12.22 \%$ & $15.84 \%$ & $19.00 \%$ & $21.27 \%$ & $17.19 \%$ & $7.69 \%$ & $0.45 \%$ \\
\hline
\end{tabular}

*Notes. Rating scale response choices: $-3=$ Strongly Disagree. $-2=$ Disagree. $-1=$ Slightly Disagree. $0=$ Neutral. $1=$ Slightly Agree. $2=$ Agree. $3=$ Strongly Agree. $\mathrm{NR}=$ No Response. Correct "True" responses are choices 5, 6, and 7, and are coded in green; Correct "False" responses are choices 1, 2, and 3, and are coded in green. "Neutral" responses for all rating scale statements are considered incorrect, and are coded in yellow. "No Response" is coded in gray.

was conducted, and showed the largest differences involved the activity "Installing software downloaded from the Internet".

Hypothesis 2 was "There are specific aspects of malware where users lack knowledge." A follow-up Kruskal-Wallis Chi-Squared test was conducted in order to further examine the statistical significance within rating scale statements for this activity. The results of the Chi-Squared test demonstrated that there were significant differences between rating scale statements. This supports hypothesis 2 , and provides direction as to the specific aspects where users lack knowledge. These results are consistent with the observations made in the previous section in regards to the participant responses to the "Installing software" activity. 
Table 13

Rating Scales Used in the Main Task Survey for the Activity "Using a Search Engine".

\begin{tabular}{|c|c|c|}
\hline Activity: Using a Search Engine (Google) & Abbreviation & $\mathbf{T} / \mathbf{F}$ \\
\hline $\begin{array}{l}\text { Clicking on a link found in the Google search engine results can bring me to } \\
\text { a phishing website (which looks like the correct website but is not genuine) }\end{array}$ & ClickingLinkPhishing & $\mathrm{T}$ \\
\hline $\begin{array}{l}\text { My Internet Service Provider (e.g., Comcast, Time Warner, AT\&T) is able } \\
\text { to monitor the things I search for on Google, as well as the results that are } \\
\text { returned to me }\end{array}$ & ISPMonitorsSearches & $\mathrm{T}$ \\
\hline $\begin{array}{l}\text { Using a wireless Internet access point (e.g., at a Starbucks coffee shop) can } \\
\text { allow for my search results on Google to be monitored by other users }\end{array}$ & WirelessAccessAltersResults & $\mathrm{T}$ \\
\hline $\begin{array}{l}\text { Clicking on an advertisement on a Google results page can potentially bring } \\
\text { me to a phishing website (which looks like the correct website but is not } \\
\text { genuine) }\end{array}$ & ClickingAdPhishing & $\mathrm{T}$ \\
\hline $\begin{array}{l}\text { Malware (malicious software program) that is installed on my computer can } \\
\text { purposely alter my Google results in favor of other results }\end{array}$ & MalwareAltersResults & $\mathrm{T}$ \\
\hline $\begin{array}{l}\text { The http indicator in the address bar signifies communication between my } \\
\text { computer and the Google website server is encrypted (sent using a secret } \\
\text { code) }\end{array}$ & HTTP & $\mathrm{F}$ \\
\hline $\begin{array}{l}\text { Using Google allows other Internet users to spy on my computer and obtain } \\
\text { my personal information (e.g., usernames, passwords, banking information) }\end{array}$ & UsersSpyPersonalInformation & $\mathrm{F}$ \\
\hline $\begin{array}{l}\text { Google monitors each and every website listed on their search engine, and } \\
\text { malicious links are always excluded from their search results }\end{array}$ & GoogleMonitorsWebsites & $\mathrm{F}$ \\
\hline $\begin{array}{l}\text { Using Google's map service allows other Internet users to find where I am } \\
\text { presently located, even without my permission }\end{array}$ & MapsWithoutPermission & $\mathrm{F}$ \\
\hline $\begin{array}{l}\text { Using the Google search engine allows other Internet users to intercept my } \\
\text { Google Mail (i.e., Gmail) account information (e.g., username and password) }\end{array}$ & UsersInterceptGoogleMail & $\mathrm{F}$ \\
\hline
\end{tabular}

*Notes. $\mathrm{T}=$ True. $\mathrm{F}=$ False.

Notable responses. Reviewing the histograms for the Internet activity of online banking (Figure 26), strong understanding on most scales was seen. Problematic rating scale statements included users' knowledge about the Internet address shown in the website image, the presence of phishing websites on search engines, and what information can be monitored by their Internet Service Provider.

For the making an online purchase activity (Figure 27), problematic rating scale statements included users' knowledge about information being intercepted by using a wireless access point in a public place, the presence of phishing websites on search engines, and what amount of personal information a credit card provider has about the user's personal information while making an online purchase.

For the downloading and installing software applications activity (Figure 28), understanding seemed weaker than the other activities. Problematic rating scale statements 


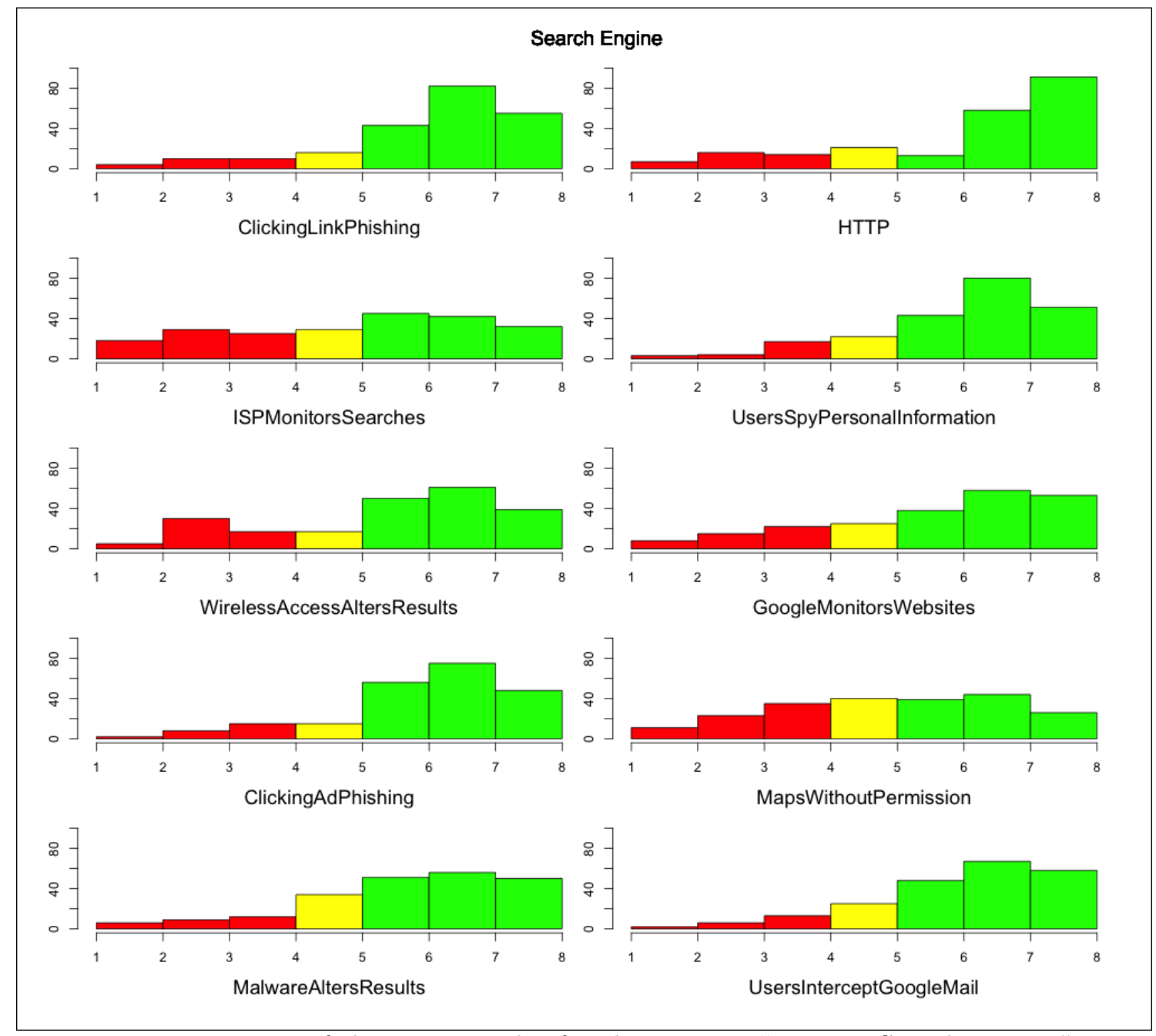

Figure 29. Histograms of the rating scales for the activity "Using a Search Engine".

included users' erroneous conclusions that correct Internet addresses can lead to phishing attacks, their misunderstanding that malware installed on their computer will automatically and always send their personal information to a third party, as well as their misconceptions concerning the communications between their computer and the software downloads website server being secured at all times when downloading software.

For the using a search engine activity (Figure 29), problematic rating scale statements included users' erroneous assumptions that other Internet users are able to locate them geographically, without their permission, when they are using Google Maps; also, some users erroneously reported that they believe that their Internet Service Provider is unable 
Table 14

Participant Responses to the Rating Scales Used in the Main Task Survey for the Activity "Using a Search Engine".

\begin{tabular}{|c|c|c|c|c|c|c|c|c|c|}
\hline \multirow[t]{2}{*}{ Abbreviation } & \multirow[t]{2}{*}{$\mathbf{T} / \mathbf{F}$} & \multicolumn{8}{|c|}{ Responses (out of 221 participants) } \\
\hline & & -3 & -2 & -1 & 0 & 1 & 2 & 3 & NR \\
\hline \multirow[t]{2}{*}{ ClickingLinkPhishing } & True & 4 & 10 & 10 & 16 & 43 & 82 & 55 & 1 \\
\hline & & $1.81 \%$ & $4.52 \%$ & $4.52 \%$ & $7.24 \%$ & $19.46 \%$ & $37.10 \%$ & $24.89 \%$ & $0.45 \%$ \\
\hline \multirow[t]{2}{*}{ ISPMonitorsSearches } & True & 18 & 29 & 25 & 29 & 45 & 42 & 32 & 1 \\
\hline & & $8.14 \%$ & $13.12 \%$ & $11.31 \%$ & $13.12 \%$ & $20.36 \%$ & $19.00 \%$ & $14.48 \%$ & $0.45 \%$ \\
\hline \multirow[t]{2}{*}{ WirelessAccessAltersResults } & True & 5 & 30 & 17 & 17 & 50 & 61 & 39 & 2 \\
\hline & & $2.26 \%$ & $13.57 \%$ & $7.69 \%$ & $7.69 \%$ & $22.62 \%$ & $27.60 \%$ & $17.65 \%$ & $0.90 \%$ \\
\hline \multirow[t]{2}{*}{ ClickingAdPhishing } & True & 2 & 8 & 15 & 15 & 56 & 75 & 48 & 2 \\
\hline & & $0.90 \%$ & $3.62 \%$ & $6.79 \%$ & $6.79 \%$ & $25.34 \%$ & $33.94 \%$ & $21.72 \%$ & $0.90 \%$ \\
\hline \multirow[t]{2}{*}{ MalwareAltersResults } & True & 6 & 9 & 12 & 34 & 51 & 56 & 50 & 3 \\
\hline & & $2.71 \%$ & $4.07 \%$ & $5.43 \%$ & $15.38 \%$ & $23.08 \%$ & $25.34 \%$ & $22.62 \%$ & $1.36 \%$ \\
\hline \multirow[t]{2}{*}{ HTTP } & False & 91 & 58 & 13 & 21 & 14 & 16 & 7 & 1 \\
\hline & & $41.18 \%$ & $26.24 \%$ & $5.88 \%$ & $9.50 \%$ & $6.33 \%$ & $7.24 \%$ & $3.17 \%$ & $0.45 \%$ \\
\hline \multirow[t]{2}{*}{ UsersSpyPersonalInformation } & False & 51 & 80 & 43 & 22 & 17 & 4 & 3 & 1 \\
\hline & & $23.08 \%$ & $36.20 \%$ & $19.46 \%$ & $9.95 \%$ & $7.69 \%$ & $1.81 \%$ & $1.36 \%$ & $0.45 \%$ \\
\hline \multirow[t]{2}{*}{ GoogleMonitorsWebsites } & False & 53 & 58 & 38 & 25 & 22 & 15 & 8 & 2 \\
\hline & & $23.98 \%$ & $26.24 \%$ & $17.19 \%$ & $11.31 \%$ & $9.95 \%$ & $6.79 \%$ & $3.62 \%$ & $0.90 \%$ \\
\hline \multirow[t]{2}{*}{ MapsWithoutPermission } & False & 26 & 44 & 39 & 40 & 35 & 23 & 11 & 3 \\
\hline & & $11.76 \%$ & $19.91 \%$ & $17.65 \%$ & $18.10 \%$ & $15.84 \%$ & $10.41 \%$ & $4.98 \%$ & $1.36 \%$ \\
\hline \multirow[t]{2}{*}{ UsersInterceptGoogleMail } & False & 58 & 77 & 48 & 25 & 13 & 6 & 2 & 2 \\
\hline & & $26.24 \%$ & $30.32 \%$ & $21.72 \%$ & $11.31 \%$ & $5.88 \%$ & $2.71 \%$ & $0.90 \%$ & $0.90 \%$ \\
\hline
\end{tabular}

*Notes. Rating scale response choices: $-3=$ Strongly Disagree. $-2=$ Disagree. $-1=$ Slightly Disagree. $0=$ Neutral. $1=$ Slightly Agree. $2=$ Agree. $3=$ Strongly Agree. $\mathrm{NR}=$ No Response. Correct "True" responses are choices 5, 6, and 7, and are coded in green; Correct "False" responses are choices 1, 2, and 3, and are coded in green. "Neutral" responses for all rating scale statements are considered incorrect, and are coded in yellow. "No Response" is coded in gray.

to monitor the information that they search for using any search engine.

\section{Discussion}

This study was motivated by the desire to find out the ground truth about what people do or do not know about Internet security. The research question asked whether users could correctly identify the threats that exist in online activities, and the security mechanisms that are in place in order to help them protect themselves from these dangers.

It was hypothesized that computer users misunderstand malware in general compared to other areas of computer security. Furthermore, it was believed that there are specific 
Table 15

Participant Responses to the Rating Scales Used in the Post-Test Survey.

\begin{tabular}{|c|c|c|}
\hline Response & Count & Percentage \\
\hline \multicolumn{3}{|c|}{ Question 1: How often do you do online banking? } \\
\hline Never & 24 & $10.86 \%$ \\
\hline Once a month & 35 & $15.84 \%$ \\
\hline Once a week & 57 & $25.79 \%$ \\
\hline Several times a week & 71 & $32.13 \%$ \\
\hline Daily & 28 & $12.67 \%$ \\
\hline Multiple times daily & 5 & $2.26 \%$ \\
\hline No answer & 1 & $0.45 \%$ \\
\hline \multicolumn{3}{|c|}{ Question 2: How often do you do make online purchases? } \\
\hline Never & 7 & $3.17 \%$ \\
\hline Once a month & 136 & $61.54 \%$ \\
\hline Once a week & 57 & $25.79 \%$ \\
\hline Several times a week & 18 & $8.14 \%$ \\
\hline Daily & 1 & $0.45 \%$ \\
\hline Multiple times daily & 1 & $0.45 \%$ \\
\hline No answer & 1 & $0.45 \%$ \\
\hline \multicolumn{3}{|c|}{ Question 3: How often do you do install software downloaded from the Internet? } \\
\hline Never & 17 & $7.69 \%$ \\
\hline Once a month & 139 & $62.90 \%$ \\
\hline Once a week & 42 & $19.00 \%$ \\
\hline Several times a week & 17 & $7.69 \%$ \\
\hline Daily & 4 & $1.81 \%$ \\
\hline Multiple times daily & 1 & $0.45 \%$ \\
\hline No answer & 1 & $0.45 \%$ \\
\hline \multicolumn{3}{|c|}{ Question 4: How often do you use search engines to look for things on the Internet? } \\
\hline Never & 0 & $0.00 \%$ \\
\hline Once a month & 3 & $1.36 \%$ \\
\hline Once a week & 6 & $2.71 \%$ \\
\hline Several times a week & 25 & $11.31 \%$ \\
\hline Daily & 41 & $18.55 \%$ \\
\hline Multiple times daily & 146 & $66.06 \%$ \\
\hline No answer & 0 & $0.00 \%$ \\
\hline \multicolumn{3}{|c|}{ Question 5: How would you rate your knowledge of computer and Internet security? } \\
\hline Limited knowledge & 9 & $4.07 \%$ \\
\hline Some knoweldge & 30 & $13.57 \%$ \\
\hline About average & 97 & $43.89 \%$ \\
\hline More than average knowledge & 73 & $33.03 \%$ \\
\hline Expert knowledge & 10 & $4.52 \%$ \\
\hline No answer & 2 & $0.90 \%$ \\
\hline
\end{tabular}


Table 16

Statistical Tests Conducted on the Study Data

\begin{tabular}{|c|c|c|c|c|c|}
\hline \multicolumn{6}{|c|}{ Skewness and Kurtosis Normality Tests } \\
\hline Activity & Test & Statistic & Standard Error & $t$-Value & $p$-Value \\
\hline $1\left(\mathrm{OB}^{*}\right)$ & Skewness & -0.1132 & 0.1671 & -0.6780 & 0.2489 \\
\hline $1\left(\mathrm{OB}^{*}\right)$ & Kurtosis & -0.3839 & 0.3341 & -1.1489 & 0.1253 \\
\hline $2\left(\mathrm{OP}^{*}\right)$ & Skewness & -0.3486 & 0.1678 & -2.0769 & 0.0189 \\
\hline $2\left(\mathrm{OP}^{*}\right)$ & Kurtosis & -0.1684 & 0.3357 & -0.5018 & 0.3079 \\
\hline 3 (IS*) & Skewness & -0.1407 & 0.1686 & -0.8343 & 0.2021 \\
\hline 3 (IS*) & Kurtosis & -0.1692 & 0.3373 & -0.5017 & 0.3079 \\
\hline $4\left(\mathrm{SE}^{*}\right)$ & Skewness & -0.0467 & 0.1682 & -0.2773 & 0.3908 \\
\hline $4\left(\mathrm{SE}^{*}\right)$ & Kurtosis & -0.2403 & 0.3365 & -0.7141 & 0.2376 \\
\hline \multicolumn{6}{|c|}{ Analysis of Variance (ANOVA) Test } \\
\hline Activities & DF & Sum of Squares & Mean Square & F-Value & $p$-Value \\
\hline $1-4$ & 3 & 11.0 & 3.669 & 7.682 & $<0.001$ \\
\hline \multicolumn{6}{|c|}{ Kruskal-Wallis Chi-Squared Test } \\
\hline Activity & DF & $\mathbf{N}$ & H-value & $p$-value & \\
\hline 3 (IS*) & 9 & 221 & 372.23 & $<0.001$ & \\
\hline
\end{tabular}

*Notes. Activities: $\mathrm{OB}=$ Doing your banking online. $\mathrm{OP}=$ Making an online purchase. IS = Installing software downloaded from the Internet. SE = Using a search engine.

aspects of malware where users lack this knowledge. The statistical tests conducted in order to answer these hypotheses showed that, in fact, users do demonstrate a lack of knowledge in understanding malware in various aspects, from immediacy and/or delay of effects, to non-restricted file access once installed on a user's machine.

Also of interest were group differences obtained via another set of statistics. The study showed that confidence rating scale responses were different between male and female participants. Even though both provided a similar answer, the Likert scale value was usually found in the extreme values for males, and less so for females. This is a well-known gender style difference in psychology literature (Beyer \& Bowden, 1997).

The results suggest that the issues that arose within each of the problematic rating scale responses were topic-based. It is believed that it is a result of user misunderstanding of the system and its subcomponents that comprise the series of events found in an online activity. The intention of this study was to explore the match between people's mental 

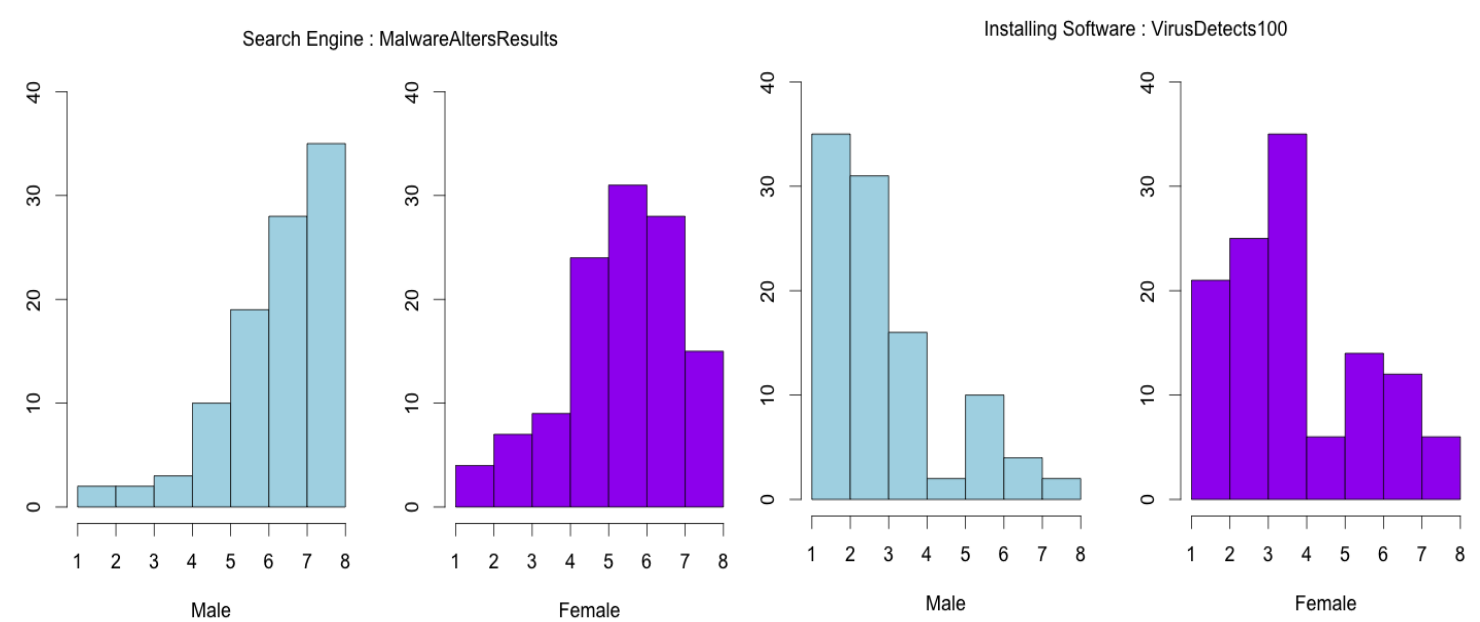

Figure 30. Figure showing gender differences in participant responses to activities. In the left set of histograms, the figure shows the gender differences in responses to the "Using a search engine" activity on the "Using the Google search engine allows other Internet users to intercept my Google Mail account information" rating scale. In the right set of histograms, the figure shows the gender differences in responses to the "Downloading and installing a software application" activity on the "A virus scan program (e.g., McAfee Antivirus, Norton Antivirus) will detect with $100 \%$ accuracy any malicious software applications that attempt to compromise (i.e., "attack") my computer " rating scale.

models and the true nature of online security threats and defences. There are many elements involved in Internet security. For example, a model might begin with the user's physical world, where their computer is located within their workspace; the next component is the user's computer, where they engage in the online activity; the browser gives them access to various websites; the Internet Service Provider allows the user to communicate with other servers found on the Web; the servers enter into communication with the ISP and, ultimately, the end-user; finally, the third-party component relates to any of the ancillary Web services, such as credit card processing servers.

For many of the rating scale statements, the rating scale responses demonstrate a lack of understanding of the connection between these elements. This could be attributed to the notion that some users do not understand the series of events that can lead to their computer being attacked, or their online browsing session to be monitored by a third party, as a few examples. Research conducted on probabilities of events, in accordance with the conjunction rule, has shown that people tend to violate this rule systematically in a variety 
of different situations, such as decisions about risk (Tversky \& Kahneman, 1983). It is believed that this psychological phenomenon has surfaced in the results obtained in this study. For example, users stated that malware software applications always compromise a user's computer by sending their information to a third party, but they reported less certainty about the same malware programs gaining access to and changing various types of files found on their computer. This disconnect between what experts know as a likely chain of events, shows that a large proportion of users do not understand how one threat can, through this sequence of events, lead to the compromising of different parts of the user's system.

This study was motivated by speculation that users had a poor mental model of computer security issues, particularly malware. The study was motivated by the proposed importance of mental models (Bravo-Lillo et al. 2010), and the work of Morgan et al. (2002) on exploring mental models relating to risk. The findings about phishing are in line with earlier studies of phishing (e.g., (Downs et al., 2006)). The findings about malware appear novel, despite industry experts highlighting the critical importance of malware Verizon, 2012).

The next step. Given the evidence from the third study, it was decided that the best step forward would be an exploratory analysis of in-depth user understanding of online interactions surrounding websites, communications between computers and first and third party servers, as well as malicious software applications (malware). 


\section{Study Four: Exploring Understanding and Confidence}

Studies two and three showed that there is a major misunderstanding on the part of users regarding malware (its capabilities) and phishing (particularly concerning assessing the trustworthiness of domain names). In order to build better information systems and security mechanisms to help users avoid the dangers of online attacks, it is important to understand where these misunderstandings originate. The third study was designed to explore user mental models in relation to several common scenarios of Internet usage. The fourth study was to conduct deeper exploratory analysis on participants' understanding of Internet structure and security mechanisms. Moreover, another aspect, confidence, was investigated.

As with study three, participant responses were compared with "ground truth", in order to determine where user mental models were faulty or weak.

\section{Method}

Experimental design. The study targeted various elements of Internet usage, as discussed in the conclusion to study three. It assessed the knowledge participants possess in regards to the threats of each element, as well as the security mechanisms that are in place to protect them from these threats. For example, one person's mental model might differ greatly from another person's mental model in regards to their understanding of the mechanisms associated with the communications between their computer, their Internet Service Provider, and the website server of a given website. For the purposes of the study, three separate areas were included in the experimental design. These areas were selected based on the results from the previous three studies of this thesis research work. The areas of user-engaged online activities that were involved in the testing stages of this study included websites and browsers, communications between computers and servers, and malicious software applications. Each of these areas were tested independently — with their own specific sets of questions - throughout the study. The study was approved by the Carleton University Psychology Research Ethics Board Appendix T. 


\section{Participants}

Ninety-three participants from the Amazon Mechanical Turk online work marketplace (https://www.mturk.com/mturk/) were recruited to participate in the survey. Fifty-one were male, 42 were female. Ages ranged from 18 to 68 years. Invitations were limited to users who resided in the United States, to increase the consistency of knowledge and allowing us to prepare relevant sample websites and example material.

\section{Materials}

The materials used in the study included the following: task instructions Appendix V), a demographics questionnaire created with the Limesurvey Web survey system, running on the HotSoft lab secure Web server Appendix U); and, the Limesurvey questionnaire Appendix W). Participant answers to the demographics survey were kept on the secure server located within the HotSoft lab at Carleton University.

\section{Procedure}

Demographics data. Before the main task, participants answered a short demographics questionnaire, which included questions on age, gender, occupation, education, and frequency of Internet use. This data was collected for exploratory analysis based on individual differences in demographic information.

Task. The task presented to each participant asked him or her to answer different types of questions (multiple choice, rating scales, probability judgments, etc.) based on three different categories of online interactions. The questions can be found in Appendix W. The three categories of online interactions that were tested are as follows: Website/browser, communications, and malicious software applications. A mixture of multiple choice questions, Likert rating scale questions, and follow-up confidence questions were used for each of the three categories found in the main task. One follow-up confidence question was present after each question (i.e., a multiple choice question) or question group (a rating scale statement group of questions), and asked each participant to provide his or her confidence level 
regarding the subject matter of each question posed during testing. The confidence information was collected for analysis purposes in order to compare overall responses to the questions posed and their corresponding confidence ratings.

\section{Results}

Exploratory analysis. This study was based on the results obtained from studies one, two, and three. As such, it was decided that in order to delve deeper into users understanding of Internet security, a study on exploratory analysis of this topic was necessary. The results obtained from this study showed many of the same patterns and trends seen in the previous studies. Each of the following three sections reveals in more detail what observations were made based on the results obtained from the participants. For each section, a selection of the figures and tables were inserted into the main thesis document. For the complete list of all figures and tables, refer to Appendix X.

Website/browser. The results obtained within the website/browser category suggest that users demonstrate difficulty in understanding subdomains. This can be a problem for users when engaging in online activities, as it leaves them open to phishing attacks if they are unable to identify and avoid malicious websites with Web addresses that contain domain names that appear legitimate, such as http://www.servicecanada.userinfo.ca, but are in fact not. In the aforementioned domain name, the userinfo.ca domain is in control of the servicecanada subdomain, and users who navigate to this website could potentially fall victim to a malicious attack on their personal information. This is because userinfo.ca might have been registered by anyone, and not have any connection to the Service Canada government department. Tables 17 and 18 show this user misunderstanding of who can create subdomains, as well as who controls these subdomains.

These tables also reflect a wide range of confidence ratings, regardless of the answer provided by each participant. As can be seen in Table 17, which discusses the creation of subdomains, the overall responses show that approximately 40 participants provided the correct response, while more than 50 provided the incorrect response. However, the table 
also shows that, out of the correct responses, only 20 or so participants stated a "very confident" rating in regards to their response, while the remaining participants provided a "somewhat confident" rating or a "not confident" rating. To a slightly lesser extent, this same pattern can be seen in Table 18, which discusses the control of a domain name. In the figure shown, only half of the responses provided were paired with a high confidence rating. This seems to indicate that users do possess some knowledge about websites and browsers, but are not very confident in regards to this knowledge.

Table 17

Website/Browser Category Question 8: Who is Able to Create the weeklyad in weeklyad.walmart.com/walmart?

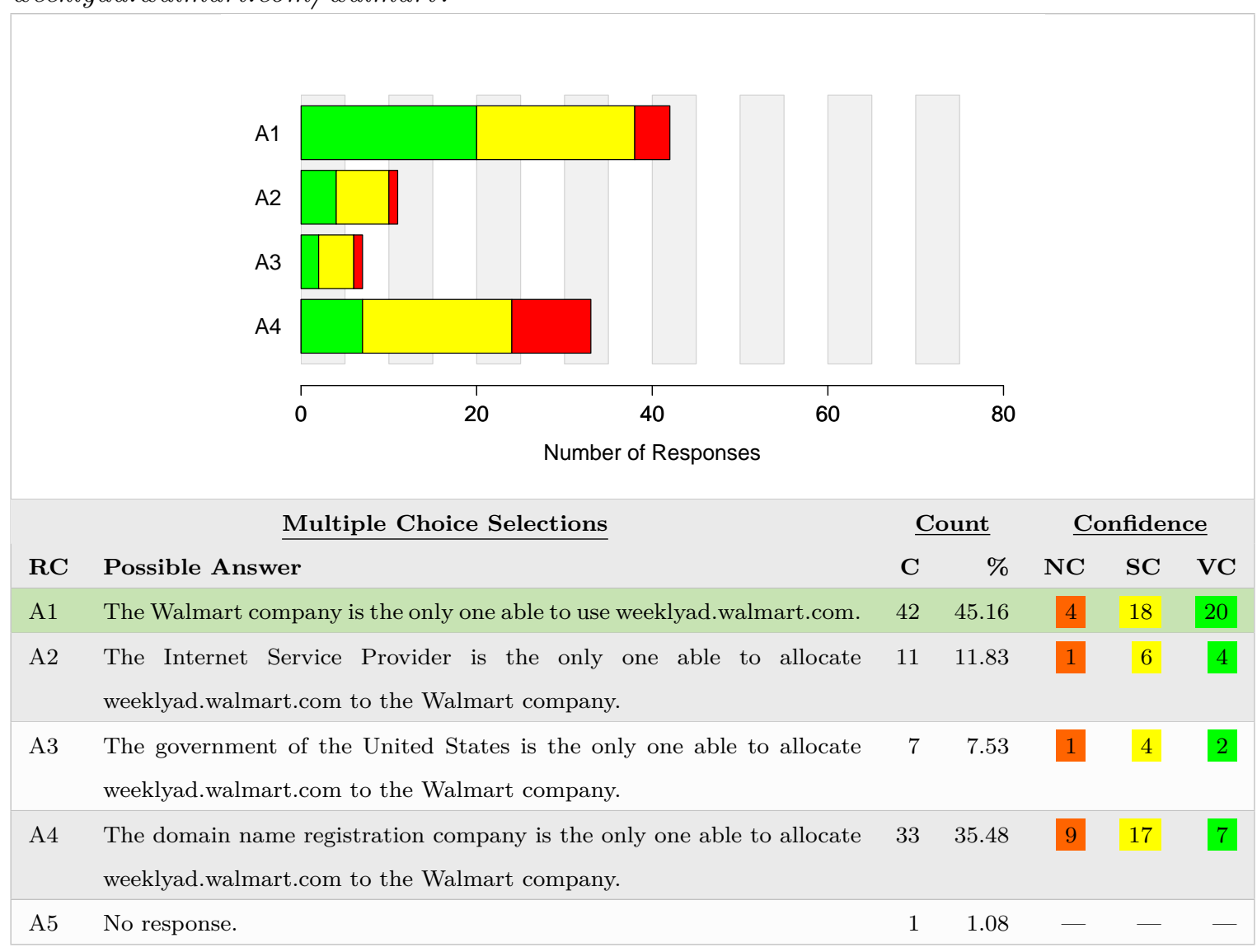

$*$ Notes. $\mathrm{RC}=$ Response Code. $\mathrm{C}=$ Count. $\mathrm{NC}=$ Not Confident; Not Confident response counts are coded in red . $\mathrm{SC}=$ Somewhat Confident; Somewhat Confident responses are coded in yellow $. \mathrm{VC}=$ Very Confident; Very Confident responses are coded in green . The correct multiple choice answer is coded in pastel green . 
Table 18

Website/Browser Category Question 12: Once a Company/Organization Obtains a Domain Name, Who is in Control of the Domain Name (i.e., is Allowed to Make Changes, Associate the Domain Name to a Website, etc.)?

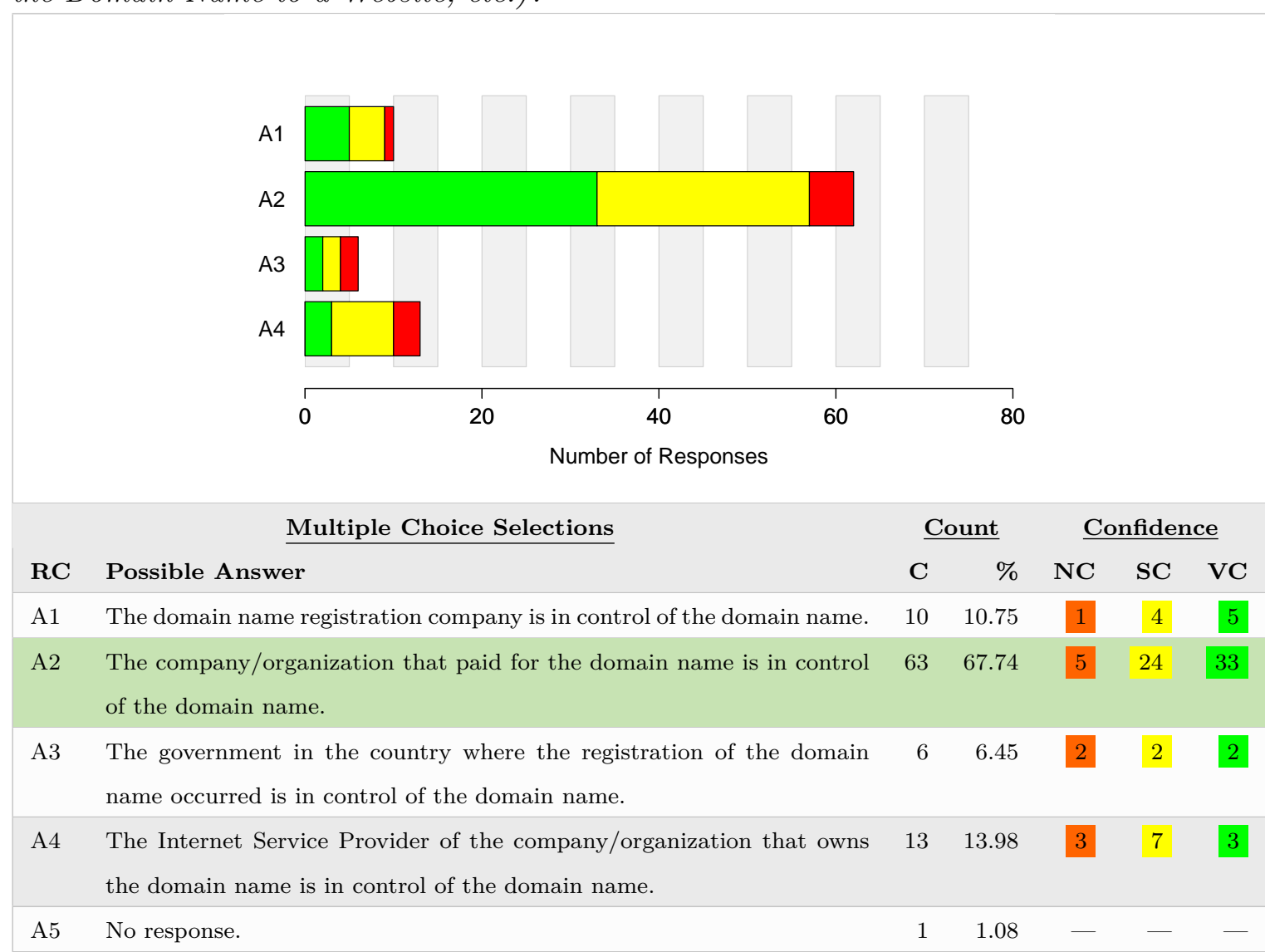

*Notes. $\mathrm{RC}=$ Response Code. $\mathrm{C}=$ Count. $\mathrm{NC}=$ Not Confident; Not Confident response counts are coded in red . $\mathrm{SC}=$ Somewhat Confident; Somewhat Confident responses are coded in yellow $. \mathrm{VC}=$ Very Confident; Very Confident responses are coded in green . The correct multiple choice answer is coded in pastel green .

Communications. The results obtained within the communications category of this study suggest that users are often uncertain about communications between their computer, website servers, Internet Service Provider servers, and any computer interconnected within this system. Furthermore, the study shows that users have difficulty understanding what security certificates are, who can obtain them, and who administers them (i.e., security certificate authorities). Table 19 discusses the access to information granted to Internet Service Providers. Table 20 examines the ways individuals and companies obtain security 
certificates. Table 21 deals with the definition of security certificate authorities. The data reflected in these tables suggest that users show a significant lack of confidence. Table 19 depicts confidence ratings of "somewhat confident" or "not confident" in well over $70 \%$ of all responses, while Tables 20 and 21 depict confidence ratings representing a very high proportion (i.e., approximately $50 \%$ of all responses) of users providing a "not confident" response.

Table 19

Communications Category Question 1: Can Your Internet Service Provider (e.g., Time Warner, Comcast, ATET) See the Personal Information You Submit Through Online Forms, as Well as the Information that is Sent Back to You by the Website Server?

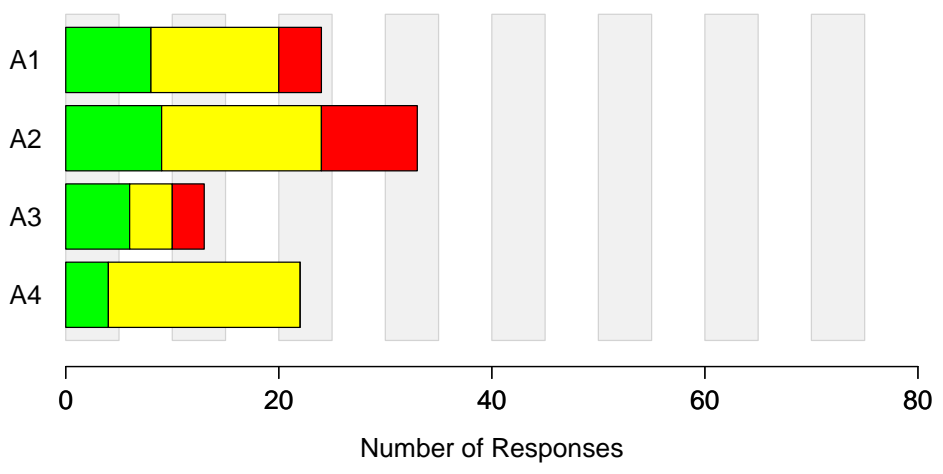

Multiple Choice Selections

\begin{tabular}{|c|c|c|c|c|c|c|}
\hline $\mathbf{R C}$ & Possible Answer & $\mathbf{C}$ & $\%$ & $\mathrm{NC}$ & $\mathrm{SC}$ & VC \\
\hline $\mathrm{A} 1$ & $\begin{array}{l}\text { Yes, but only if the website is using the http protocol to communicate } \\
\text { with your computer. }\end{array}$ & 24 & 25.81 & 4 & 12 & 8 \\
\hline A 2 & $\begin{array}{l}\text { Yes, your ISP can see all of the information sent through all online forms; } \\
\text { their logs record all incoming and outgoing activity. }\end{array}$ & 33 & 35.48 & 9 & 15 & 9 \\
\hline A3 & $\begin{array}{l}\text { No, your ISP does not keep any records of incoming and outgoing infor- } \\
\text { mation between your computer and various website servers. }\end{array}$ & 13 & 13.98 & 3 & 4 & 6 \\
\hline A 4 & $\begin{array}{l}\text { No, your ISP is not allowed, by law, to see the information you submit } \\
\text { through online forms. }\end{array}$ & 23 & 24.73 & 0 & 18 & 4 \\
\hline A5 & No response. & 0 & 0.00 & - & - & - \\
\hline
\end{tabular}

${ }^{*}$ Notes. $\mathrm{RC}=$ Response Code. $\mathrm{C}=$ Count. $\mathrm{NC}=$ Not Confident; Not Confident response counts are coded in red. $\mathrm{SC}=$ Somewhat Confident; Somewhat Confident responses are coded in yellow . VC = Very Confident; Very Confident responses are coded in green. The correct multiple choice answer is coded in pastel green . 
Table 20

Communications Category Question 3: How Does a Company/Organization or Individual Obtain a Security Certificate?

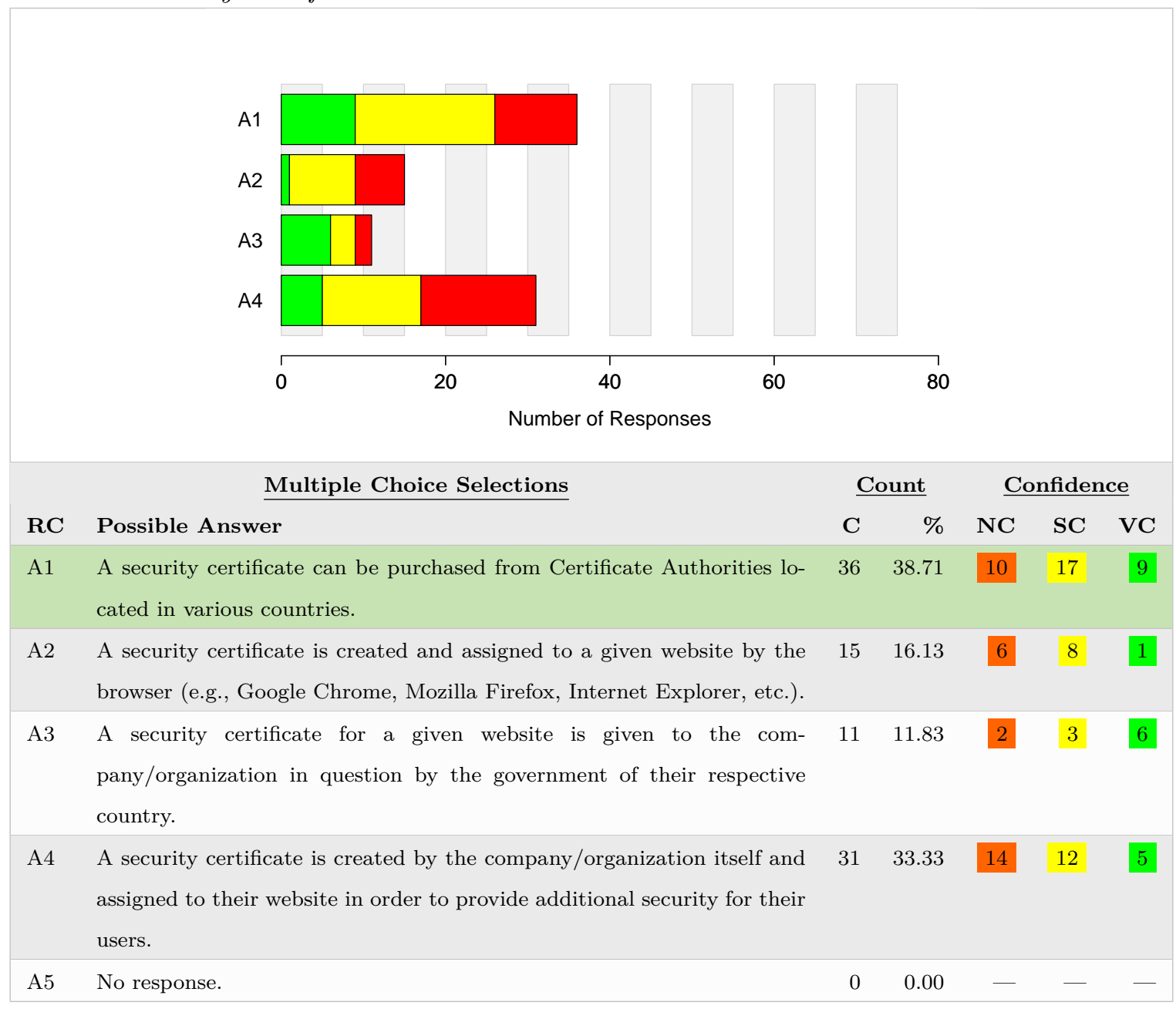

*Notes. $\mathrm{RC}=$ Response Code. $\mathrm{C}=$ Count. $\mathrm{NC}=$ Not Confident; Not Confident response counts are coded in red . $\mathrm{SC}=$ Somewhat Confident; Somewhat Confident responses are coded in yellow $. \mathrm{VC}=$ Very Confident; Very Confident responses are coded in green . The correct multiple choice answer is coded in pastel green . 
Table 21

Communications Category Question 6: What are Security Certificate Authorities?

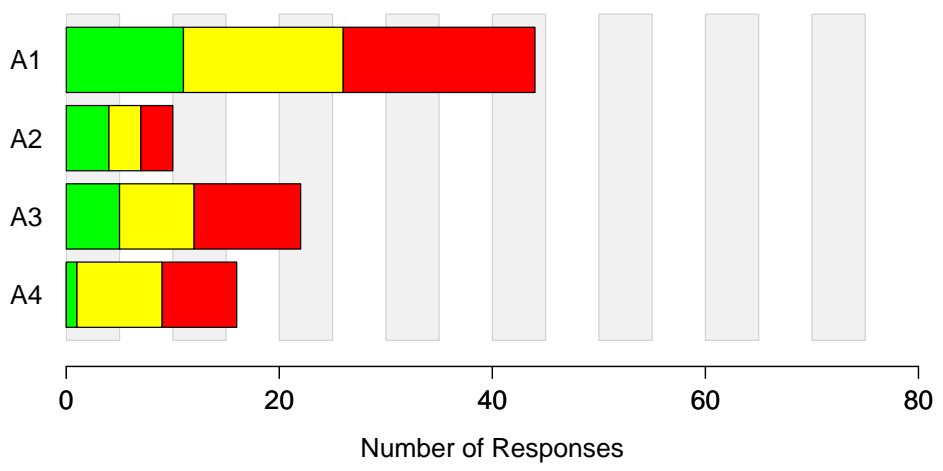

Multiple Choice Selections

\section{Count}

Confidence

RC Possible Answer C $\% \quad$ NC $\quad$ SC $\quad$ VC

A1 A security certificate authority is an entity (company/organization) that $\quad \begin{array}{lllllll}44 & 47.31 & 18 & 15 & 11\end{array}$ issues digital certificates to website owners.

A2 A security certificate authority is a branch of the government that issues $\quad \begin{array}{lllllll}10 & 10.75 & 3 & 3 & 4\end{array}$ digital certificates to website owners.

\begin{tabular}{llllll|l|l|l} 
A3 & A security certificate authority is the website hosting company, which & 22 & 23.66 & 10 & 7 & 5
\end{tabular} has the ability to issue digital certificates to the website owner.

A4 A security certificate authority is the Internet Service Provider, which $\quad \begin{array}{llllll}16 & 17.20 & 7 & 8 & 1\end{array}$ has the ability to issue digital certificates to the website owner.

A5 No response. $\quad \begin{array}{llllll} & 1 & 1.08 & - & -\end{array}$

${ }^{*}$ Notes. $\mathrm{RC}=$ Response Code. $\mathrm{C}=$ Count. $\mathrm{NC}=$ Not Confident; Not Confident response counts are coded in red . $\mathrm{SC}=$ Somewhat Confident; Somewhat Confident responses are coded in yellow . VC = Very Confident; Very Confident responses are coded in green . The correct multiple choice answer is coded in pastel green .

Malware. The results obtained within the malware category suggest that users misunderstand how malware works, and underestimate the hugely negative effects different types of malicious software applications can have on their computer. Users' general misunderstanding of the negative effects of malware comes in the form of what malware software applications can do to their computer once installed and how antivirus programs (e.g., Norton AntiVirus, McAfee AntiVirus, and Microsoft Security Essentials) detect any threats present on their machine. Tables 22, 23, and 24 show the misunderstandings users have in these aforementioned areas of Internet security. Contrary to the two previous testing cat- 
egories, these tables show an increase in confidence paired with responses to the questions being posed. The proportion of participants who stated they were very confident in their responses provided is much higher, with the exception of Table 24, where users showed a tendency to respond to a lower confidence rating or lack of confidence altogether. Overall, however, these tables depict a large proportion of users providing a confidence rating of "somewhat confident" to each of the responses provided. This would suggest that users are able to provide some of the correct responses to the questions being posed, but show a significant lack of confidence in their selection of responses. 
Table 22

Malware Category Question 2: What Can Malicious Software Applications Do Once Installed on Your Computer? (Select all that Apply).

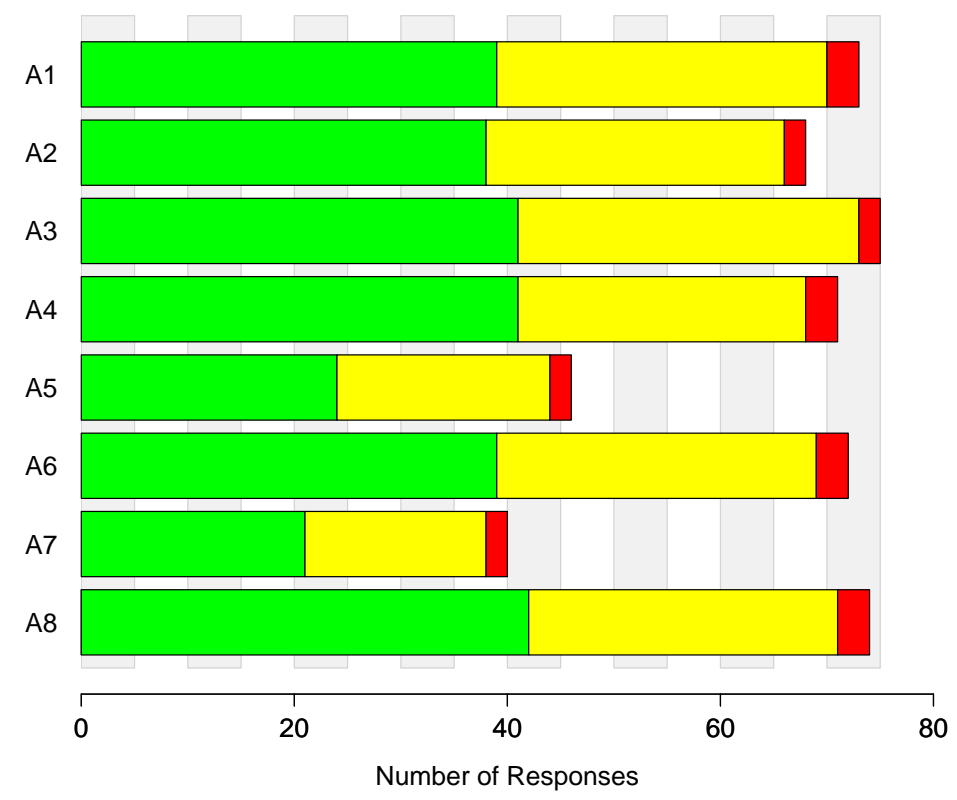

\begin{tabular}{|c|c|c|c|c|c|c|}
\hline & Checklist Selections & & unt & & Ifide & \\
\hline RC & Possible Answer & C & $\%$ & $\mathrm{NC}$ & $\mathrm{SC}$ & VC \\
\hline A1 & $\begin{array}{l}\text { Malicious software applications can collect personal information found } \\
\text { on my computer and send it to a third party. }\end{array}$ & 73 & 78.49 & 3 & 31 & 39 \\
\hline A2 & $\begin{array}{l}\text { Malicious software applications can delete any of the files found on my } \\
\text { computer. }\end{array}$ & 68 & 73.12 & 2 & 28 & 38 \\
\hline A3 & $\begin{array}{l}\text { Malicious software applications can alter the software applications in- } \\
\text { stalled on my computer. }\end{array}$ & 75 & 80.65 & 2 & 32 & 41 \\
\hline A4 & $\begin{array}{l}\text { Malicious software applications can send emails to other users from my } \\
\text { email account. }\end{array}$ & 71 & 76.34 & 3 & 27 & 41 \\
\hline A5 & $\begin{array}{l}\text { Malicious software applications can fry my computer's processor, ren- } \\
\text { dering the computer unusable. }\end{array}$ & 46 & 49.46 & 2 & 20 & 24 \\
\hline A6 & $\begin{array}{l}\text { Malicious software applications can track my usernames and passwords } \\
\text { as I type them when logging in to my various accounts (e.g., email, online } \\
\text { banking, etc.). }\end{array}$ & 72 & 77.42 & 3 & 30 & 39 \\
\hline A7 & $\begin{array}{l}\text { Malicious software applications can shut down my Internet connection } \\
\text { permanently. }\end{array}$ & 40 & 43.01 & 2 & 17 & 21 \\
\hline A 8 & $\begin{array}{l}\text { Malicious software applications can allow other users direct access to my } \\
\text { computer through the Internet. }\end{array}$ & 74 & 79.57 & 3 & 29 & 42 \\
\hline
\end{tabular}

${ }^{*}$ Notes. $\mathrm{RC}=$ Response Code $\mathrm{C}=$ Count. $\mathrm{NC}=$ Not Confident; Not Confident response counts are coded in red. $\mathrm{SC}=$ Somewhat Confident; Somewhat Confident responses are coded in yellow . VC = Very Confident; Very Confident responses are coded in green. The correct checklist answers are coded in pastel green . 
Table 23

Malware Category Question 4: How Does Anti-Virus Software Help to Protect Your Computer From Malicious Software Applications? (Select all that Apply).

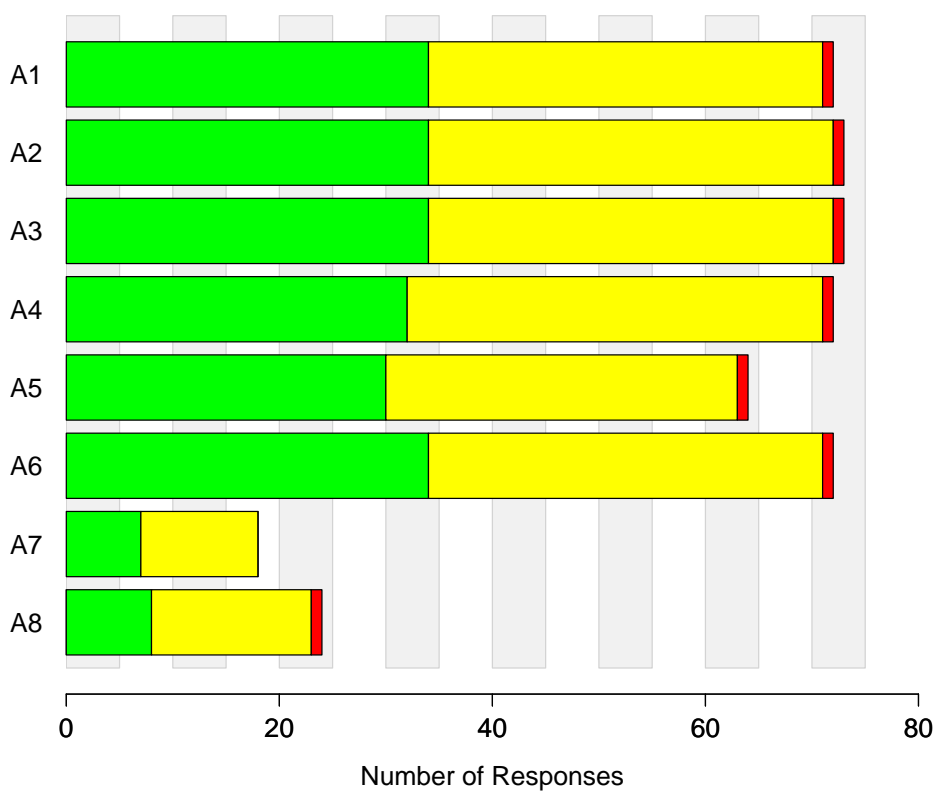

\begin{tabular}{|c|c|c|c|c|c|c|}
\hline \multirow[b]{2}{*}{$\mathbf{R C}$} & \multirow[t]{2}{*}{ Checklist Selections } & \multicolumn{2}{|c|}{$\underline{\text { Count }}$} & \multicolumn{3}{|c|}{ Confidence } \\
\hline & & $\mathbf{C}$ & $\%$ & NC & $\mathrm{SC}$ & VC \\
\hline A1 & By alerting me when a malicious software application is already installed. & 72 & 77.42 & 1 & 37 & 34 \\
\hline A2 & $\begin{array}{l}\text { By alerting me when a malicious software application is attempting to } \\
\text { install. }\end{array}$ & 74 & 79.57 & 1 & 38 & 34 \\
\hline A3 & $\begin{array}{l}\text { By alerting me that certain websites are reputed to distribute malicious } \\
\text { software applications. }\end{array}$ & 73 & 78.49 & 1 & 38 & 34 \\
\hline A4 & $\begin{array}{l}\text { By doing system checks over specific time intervals in order to verify for } \\
\text { the presence of malicious software applications. }\end{array}$ & 72 & 77.42 & 1 & 39 & 32 \\
\hline A5 & $\begin{array}{l}\text { By not allowing me to browse to websites that are known to distribute } \\
\text { malicious software applications. }\end{array}$ & 64 & 68.82 & 1 & 33 & 30 \\
\hline A6 & $\begin{array}{l}\text { By identifying and deleting all malicious software applications that are } \\
\text { or may be installed on my computer. }\end{array}$ & 73 & 78.49 & 1 & 37 & 34 \\
\hline A7 & $\begin{array}{l}\text { By shutting down my computer when a malicious software application } \\
\text { is found on my computer. }\end{array}$ & 18 & 19.35 & 0 & 11 & 7 \\
\hline A 8 & $\begin{array}{l}\text { By shutting down my Internet connection when a malicious software } \\
\text { application is found on my computer. }\end{array}$ & 24 & 25.81 & 1 & 15 & 8 \\
\hline
\end{tabular}

*Notes. $\mathrm{RC}=$ Response Code. $\mathrm{C}=$ Count. $\mathrm{NC}=$ Not Confident; Not Confident response counts are coded in red . $\mathrm{SC}=$ Somewhat Confident; Somewhat Confident responses are coded in yellow . VC = Very Confident; Very Confident responses are coded in green. The correct checklist answers are coded in pastel green . 
Table 24

Malware Category Question 5: How Does Anti-Virus Software Determine Whether There are Malicious Software Applications Installed on My Computer? (Select all that Apply).

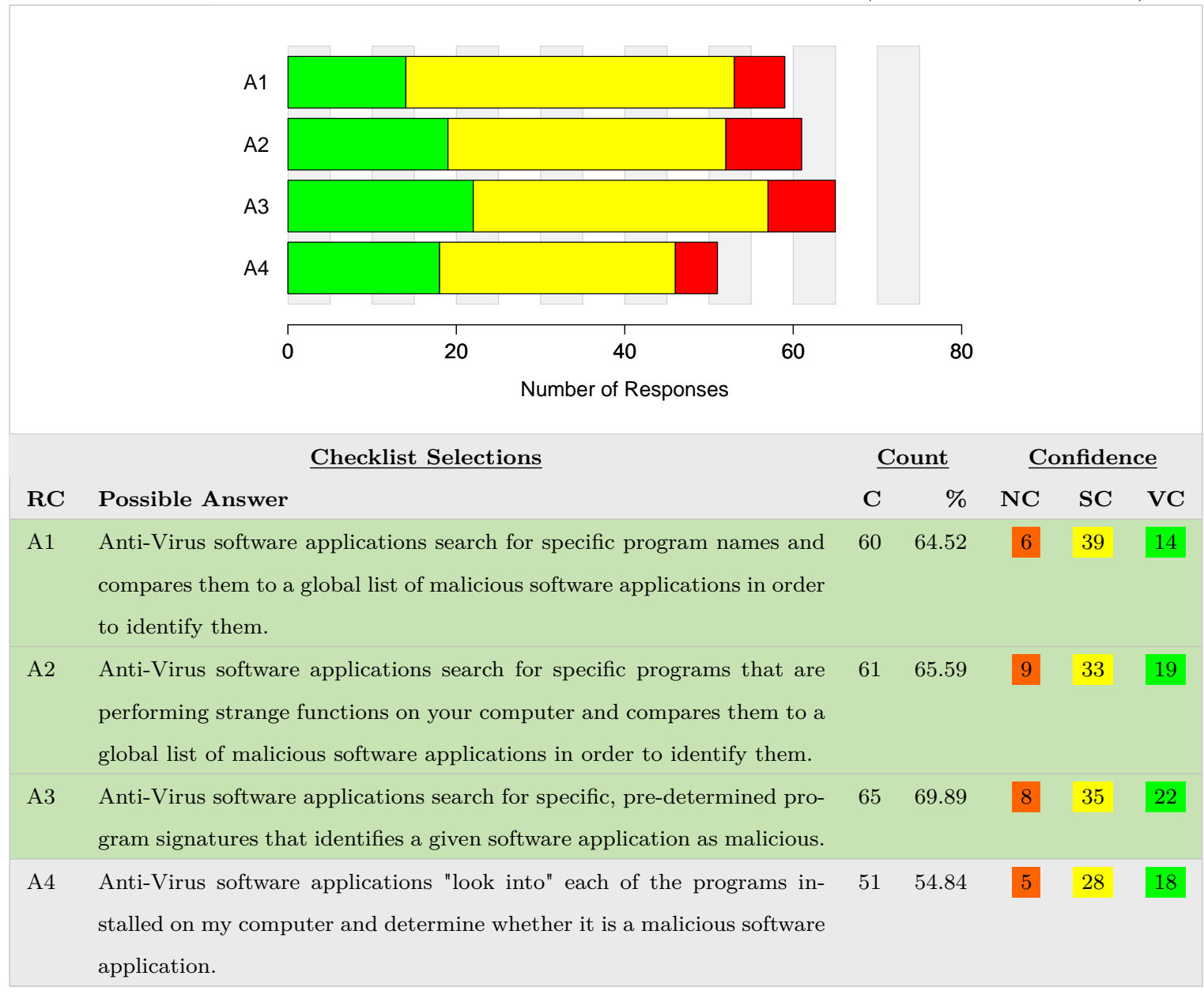

$*$ Notes. $\mathrm{RC}=$ Response Code. $\mathrm{C}=$ Count. $\mathrm{NC}=$ Not Confident; Not Confident response counts are coded in red. $\mathrm{SC}=$ Somewhat Confident; Somewhat Confident responses are coded in yellow . VC = Very Confident; Very Confident responses are coded in green. The correct multiple choice answer is coded in pastel green .

\section{Discussion}

The results from the exploratory analysis for study four led to several insights into user understanding in regards to engaging in various online activities over the Internet. The study consisted of three sections, each addressing an important element in online security. In the website/browser category, it was found that participants had a difficult time understanding how subdomains work, as well as the domain registration capabilities of individuals 
and organizations, potentially leaving users open to phishing attacks because of this. An attacker could easily create a website with a reputable name as a subdomain, and users would most likely accept it as valid. For example, a phishing attack on www.royalbank.com might involve a website named www.royalbank.financials.com.

In the communications category, evidence suggests that the participants were uncertain as to the ways communication works between a user's computer, a website hosting server, ISPs, and any other computer connected within this same network. Moreover, they showed a lack of understanding about the process of obtaining and administrating security certificates. As such, an attacker could use a domain validated certificate for their website, with a name that suggests a reputable company, luring users to provide their information through forms found on the website. These findings are consistent with earlier results obtained in studies of user understanding of browser warnings and SSL certificates (e.g., Shi et al. 2011, Akhawe \& Felt, 2013).

In the malware category, the study results suggest that the participants lack understanding of what malware can do once installed on their computer, particularly in terms of the many different file types and information it can gain access to. Participants also showed general misunderstanding of how antivirus software applications identify threats and alert the user. For example, users might not realize that software to manage photographs could also log keystrokes or open Microsoft Word files. For antivirus software, it is speculated that users may not be aware that these programs are limited in terms of recognizing and eliminating new malware. These results do not appear related to earlier literature.

The most significant of these results is that the participants showed an extensive lack of confidence about important issues, especially those mentioned above, such as those tested during the study. This raises the concern that, in order for users to cope with their lack of knowledge in these areas of Internet security, they may look for anything that helps them make a favourable or unfavourable decision in regards to engaging in a given online activity (e.g., making an online purchase, using an online banking website, installing a software application downloaded from the Internet). These results are consistent with the results 
from study one, where users showed inability to elaborate on their decision making process, suggesting lack of confidence despite using the Internet regularly. As was discussed in study one, this appears to relate to the work on "judgment under uncertainty" by Tversky and Kahneman (1974). These decisions made by users are addressed as part of the next and final study within the thesis research.

The next step. Evidence from the fourth study suggests that users do understand a small portion of the security mechanisms of the Internet, and are able, to some extent, to identify potential threats. There were still many identified weak points, however, where users misunderstand the relationships between websites, server communications, and malware. Most of the time, users are called upon to make decisions in which they are not particularly confident. As such, it was determined that the next logical step in concluding the series of five studies would be to investigate whether users make use of specific strategies when making decisions about certain aspects of online interactions, such as browsing to various websites via their domain name, and downloading and installing software from the Internet. 


\section{Study Five: Heuristics, Knowledge, and Self-Perception}

The previous four studies included in this thesis research demonstrated that there exists much uncertainty on the part of users when it comes to making decisions about engaging in online activities. Also, their limited knowledge when it comes to Internet security demonstrates problems with how users make these decisions. Study one showed that participants made trust decisions regarding a variety of different websites, but were unable to or didn't want to explain the reasoning behind those trust decisions. Study two looked at the risks associated with engaging in online activities, and showed that users made a few significant errors in rating these risks. As a follow-up to study two, study three confirmed that the main problems users are having are located within the areas of domain names and malware. The results from the exploratory study four suggested that the underlying issue for these problematic areas lies in users' uncertainty in dealing with situations in an online setting. The fifth and final study aimed to explore the basis on which users make decisions regarding domain names and malware specifically, as well as other problem areas. In order to delve deeper into user understanding of these issues, study five was divided into three parts: heuristic testing, the relationship between knowledge about Internet security and perception of risk, and self-reporting of attitudes and behaviours. The results obtained from the previous studies led the way for new hypotheses to be constructed in regards to user decision-making practices when engaging in online activities. These hypotheses are principally grounded in heuristics.

Previous research has shown that people make judgments about specific things and situations when they are uncertain about the mechanics involved, or decide not to take the extra time needed in order to better understand the situation or thing at hand (Tversky \& Kahneman, 1974, Tversky, Kahneman, \& Moser, 1990). This research has shown that people have pre-defined notions, models, and ideas about everything that is found within their surroundings. People are more likely to trust others who they know or have had encounters with versus people they have never met, regardless of the setting. When a person is faced with a complex problem, be it a mathematical calculation, a decision to 
purchase a new car, or asked to participate in a new activity, to name a few examples, he or she will have pre-conceived notions about the given situation. These pre-conceived notions are what help people to make decisions regarding situations and problems where they have little to no experience. The research to support this theory suggests that a system is in place in every person's decision-making processes, where the system will substitute an easier - or more satisfactory - response to a question, or a solution to a problem, when the person is unsure about the real answer or solution. Tversky and Kahneman refer to this decision-making process as a heuristic. Kahneman defines the heuristic as "...a simple procedure that helps find adequate, though often imperfect, answers to difficult questions" (Kahneman, 2011, p. 98).

The first two tasks in study five were constructed to test whether computer users make use of heuristics when identifying and making judgments about various aspects of Internet security. These two tasks tested users on heuristics related to the availability heuristic. This heuristic relates to a person's prior exposure to a given situation or thing, making them have strong beliefs or feelings about it, thus making a favourable or unfavourable decision or judgment about the situation or thing (Tversky \& Kahneman, 1973).

For the purposes of this study, it is believed that people making decisions in an online environment will make use of these heuristics when deciding whether to trust or distrust a given website or software application. The notion is that the availability of information in people's memory in regards to recognition will aid them in making these decisions. As an example, should a person be asked to decide which product they would most like to purchase out of a series of products, the availability heuristic stipulates that they will select the product they are most familiar with in order to come to a final decision on which one to select. The heuristics related to availability proposed are: the keyword heuristic and the functionality heuristic, as defined below.

Keyword heuristic. The keyword heuristic is defined as "a domain name that includes a familiar term can be trusted." 
Functionality heuristic. The functionality heuristic is defined as "a software application is only able to access data related to its purpose."

\section{Method}

Experimental design. The Limesurvey online survey tool was used in order to obtain participant data in this study. The survey contained questions in the following five categories: domain names, malware, knowledge, perception of risk, and attitudes, beliefs, and self-reporting. Questions were distributed among each category as follows: ten questions for category one; twelve questions for category two; ten questions for category three; ten questions for category four; and, seven questions for category five. Categories one, two, three, and five made use of the Likert Scale format. The participants selected the appropriate rating according to the direction given for each question (e.g., $0=$ Not risky, 6 = Extremely risky) in the rating scale. The rating scale question order was randomly generated by the Limesurvey tool so that each participant was shown the questions in a random order. The study was approved by the Carleton University Psychology Research Ethics Board Appendix Z).

Section one of study five includes hypothesis testing on the heuristics defined in the previous section. For section two, exploratory analysis was conducted on user knowledge and their perception of risk. Section three examined user attitudes and beliefs related to online security.

\section{Participants}

In all, 384 participants were recruited from the Amazon Mechanical Turk online work marketplace (https://www.mturk.com/mturk/). Two hundred were male, 182 were female, with 2 respondents opting not to answer the question. Ages ranged from 18 to 72 years. Invitations were limited to users who resided in the United States, in order to increase the consistency of knowledge of website activities. Participants were recruited by way of a website posting on the Amazon Mechanical Turk online work marketplace website. Each of 
the participants who completed the main task were experienced Internet users - with at least two years of experience using the Internet - and reported using the Internet at least twice per week. They were required to not be experts or students in either Internet security or computer security.

\section{Hypotheses}

The following hypotheses were created regarding the study. Their alternate versions are also provided.

- $\mathbf{H 1} 1_{o}$ Users do not make use of the keyword heuristic when determining the risk associated with domain names.

- $\mathbf{H} \mathbf{1}_{a}$ Users do use the keyword heuristic when determining the risk associated with domain names.

- H2 $2_{o}$ Users do not make use of the functionality heuristic when determining the risk associated with malware.

- H2 ${ }_{a}$ Users do use the functionality heuristic when determining the risk associated with malware.

\section{Materials}

A demographics questionnaire was used in the study, created using the Limesurvey web survey system, running on the HotSoft Lab secure web server. Participant answers to this demographics form were kept on the secure servers located within the HotSoft Lab at Carleton University. This questionnaire can be found in Appendix AA A Limesurvey online questionnaire was used in the study for the main task, and can be found in Appendix AC. Task instructions can be found in Appendix AB.

Testing interface. This study tested users through the Amazon Mechanical Turk online work marketplace. As such, each individual user completed each of the tasks involved in the study using their own computer. 


\section{Procedure}

The tasks presented to each participant asked him or her to answer multiple-choice questions as well as Likert rating scale questions on various topics regarding the Internet. The multiple-choice questions offered participants a choice of four possible answers to each question, while the Likert rating scale questions were based on a seven-point scale. The main task description can be found in Appendix $\mathrm{AB}$. The list of questions and rating scale statements can be found in Appendix AC. Each participant was shown the tasks in the same order. The questions and rating scale statements were randomly shown to each participant by the Limesurvey questionnaire tool.

\section{Results for Section One: Domain Names}

Analysis. In the section one study of domain names, the participants were shown ten domain names, some of which were high risk and some of which were low risk. The low risk domain names were actual and reputable company domains, while the high risk domain names used domain names or subdomains that merely resembled those of reputable companies. Table 26 lists each of the domain names, their level of risk, and how participants rated the risk for each domain name. Figure 31 shows the distribution of participant responses to each of these domain names. In each domain name, there is a keyword identifying a well known business. For each low-risk domain name, the keyword was placed last, suggesting that the business would be in control of the entire domain. For each high-risk domain name, the keyword was placed elsewhere, either as a subdomain or merely as a component of the entire domain. This would not provide any assurance that the business name is in control of the domain. This design allowed for the determination of whether participants were influenced more by the keyword heuristic, or more by the domain name structure, independent of the placement of the keyword. While in the study all keywords represented well-known businesses, a potential addition would be to test "keywords" that were unlikely to be known, such as "optmart", and investigate whether this produced different results than with well-known keywords. 
The participants rated the risk of each domain name using a 7-point Likert rating scale. For the descriptive statistics, the mean and standard deviation were calculated for the participant responses to each of the domain names. Figure 31 shows the distribution of participant responses for each of the domain names. The graph shows the distribution of participant responses with low assessment of risk shown in blue, and high assessment of risk shown in red.

Of particular interest in this study was finding out whether there exists a significant difference between participant responses to the categories of domain names, high-risk versus low-risk. A paired $t$-test (2-tailed) was conducted on the data. The results showed a significant difference between means, $t(383)=6.89, p<0.001$. The descriptive statistics for the low-risk and high-risk domain name categories, as well as the corresponding boxplot, are found in Table 25. Normality distribution tests were conducted on the data prior to conducting the statistical analysis.

Table 25

Part 1 - Domain Names Statistical Analyses.

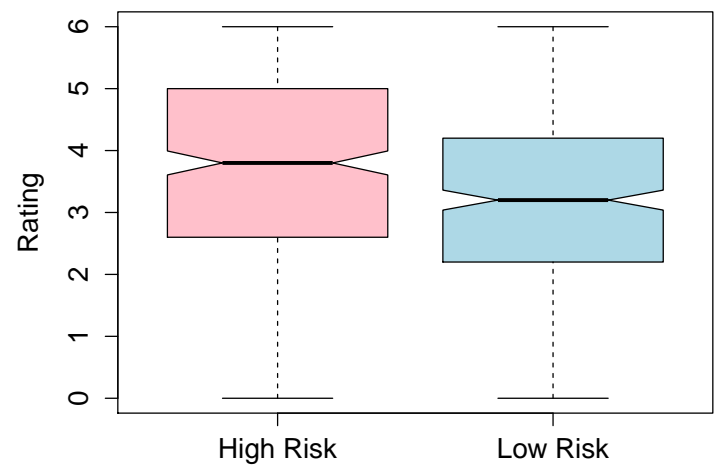

\begin{tabular}{lllllll} 
& \multicolumn{5}{c}{ Descriptive Statistics } \\
Test & df & $t$-Value & $p$-Value & & & \\
Paired $t$-test & 383 & 6.89 & $<0.001$ & & & \\
DN* Category & Minimum & 1st Quartile & Median & Mean & 3rd Quartile & Maximum \\
Low risk & 0.000 & 2.600 & 3.800 & 3.764 & 5.000 & 6.000 \\
High risk & 0.000 & 2.200 & 3.200 & 3.240 & 4.200 & 6.000
\end{tabular}

*Notes. $\mathrm{DN}=$ Domain Name. 
Follow-up, post-hoc paired $t$-tests were then conducted on each of the domain name pairs (e.g., Facebook (low-risk) compared with Facebook (high-risk), and eBay (low risk) compared with eBay (high-risk), etc.). Each of these paired $t$-tests was significant, with the exception of the Bank of America domain name pair. Results were as follows (uncorrected for multiple tests):

- Walmart domain names pair: $t(381)=2.5603, p=0.0108$

- Bank of America domain names pair: $t(379)=-0.1727, p=0.863$

- Facebook domain names pair: $t(380)=2.2694, p=0.024$

- Amazon domain names pair: $t(376)=9.2477, p<0.001$

- eBay domain names pair: $t(378)=11.8577, p<0.001$

The overall paired $t$-test result, as well as each of the paired domain names $t$-test results, seem to suggest that participants were in fact not making use of the keyword heuristic when making judgments about the risk involved in browsing to each of these domains. In order for the interpretation to tilt towards a positive keyword heuristic result, no significant differences would have been expected. 
Table 26

Part 1 - Domain Names.

\begin{tabular}{|c|c|c|c|c|c|c|c|c|c|c|c|}
\hline & & & & Risk & atings & & & & & & \\
\hline Domai & Name & Risk & & $\mathbf{0}$ & 1 & 2 & 3 & 4 & 5 & 6 & NR \\
\hline www.e & lying.walmart.com & Low & \# & 25 & 42 & 35 & 69 & 66 & 71 & 74 & 2 \\
\hline & & & $\%$ & 6.51 & 10.94 & 9.11 & 17.97 & 17.19 & 18.49 & 19.27 & 0.52 \\
\hline www.v & Imart.ebuying.com & High & \# & 15 & 37 & 34 & 67 & 76 & 60 & 95 & 0 \\
\hline & & & $\%$ & 3.91 & 9.64 & 8.85 & 17.45 & 19.79 & 15.62 & 24.74 & 0.00 \\
\hline www.l & bankofamerica.com & Low & \# & 41 & 54 & 42 & 64 & 41 & 61 & 78 & 3 \\
\hline & & & $\%$ & 10.68 & 14.06 & 10.94 & 16.67 & 10.68 & 15.89 & 20.31 & 0.78 \\
\hline www.l & kofamerica.us.com & High & \# & 46 & 58 & 44 & 55 & 40 & 46 & 94 & 1 \\
\hline & & & $\%$ & 11.98 & 15.10 & 11.46 & 14.32 & 10.42 & 11.98 & 24.48 & 0.26 \\
\hline www.t & ps.facebook.com & Low & \# & 19 & 23 & 22 & 59 & 83 & 84 & 92 & 2 \\
\hline & & & $\%$ & 4.95 & 5.99 & 5.73 & 15.36 & 21.61 & 21.88 & 23.96 & 0.52 \\
\hline www.f & ebook.eapps.com & High & \# & 12 & 21 & 18 & 56 & 82 & 85 & 109 & 1 \\
\hline & & & $\%$ & 3.12 & 5.47 & 4.69 & 14.58 & 21.35 & 22.14 & 28.39 & 0.26 \\
\hline www.l & ks.amazon.com & Low & \# & 38 & 53 & 45 & 52 & 47 & 55 & 91 & 3 \\
\hline & & & $\%$ & 9.90 & 13.80 & 11.72 & 13.54 & 12.24 & 14.32 & 23.70 & 0.78 \\
\hline www.l & oks-amazon.com & High & \# & 70 & 85 & 50 & 67 & 39 & 34 & 35 & 4 \\
\hline & & & $\%$ & 18.23 & 22.14 & 13.02 & 17.45 & 10.16 & 8.85 & 9.11 & 1.04 \\
\hline www.s & p.ebay.com & Low & \# & 48 & 64 & 60 & 79 & 50 & 39 & 43 & 1 \\
\hline & & & $\%$ & 12.50 & 16.67 & 15.62 & 20.57 & 13.02 & 10.16 & 11.20 & 0.26 \\
\hline www.s & p-ebay.com & High & \# & 19 & 24 & 32 & 71 & 60 & 77 & 97 & 4 \\
\hline & & & $\%$ & 4.95 & 6.25 & 8.33 & 18.49 & 15.62 & 20.05 & 25.26 & 1.04 \\
\hline & & & & atistic & Analy & & & & & & \\
\hline Code & Level of Risk & $\overline{\mathbf{x}}$ & $s$ & & Code & Leve & l of Risl & & $\overline{\mathbf{x}}$ & $s$ & \\
\hline DN-1 & Low risk (-) & 3.618 & & 46 & $\mathrm{DN}-2$ & High & risk $(+)$ & & 3.854 & 1.77 & \\
\hline DN-3 & Low risk (-) & 3.325 & & 35 & $\mathrm{DN}-4$ & High & risk $(+)$ & & 3.303 & 2.12 & \\
\hline DN-5 & Low risk (-) & 4.052 & & 06 & DN-6 & High & risk $(+)$ & & 4.261 & 1.62 & \\
\hline DN-7 & Low risk (-) & 3.433 & & 61 & DN-8 & High & risk $(+)$ & & 2.426 & 1.91 & \\
\hline DN-9 & Low risk $(-)$ & 2.804 & & 63 & DN-10 & High & risk $(+)$ & & 3.968 & 1.76 & \\
\hline
\end{tabular}

${ }^{*}$ Notes. $\mathrm{DN}=$ Domain Name. $0=$ Not Risky. $6=$ Extremely Risky. NR $=$ No Response 


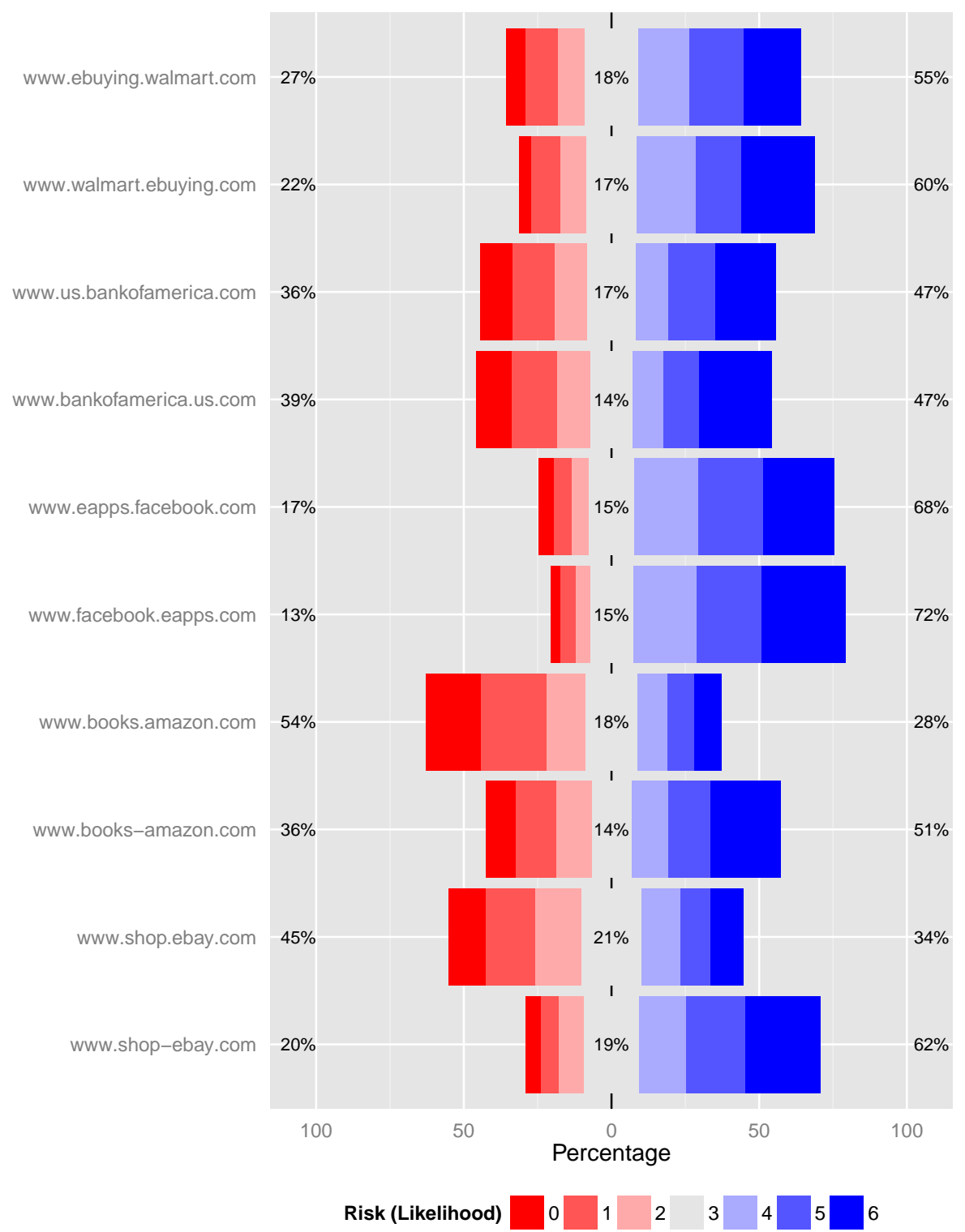

Figure 31. Distribution of participant responses for the high risk and low risk domain names.

Interpretation. The results of most of these tests suggested that participants were not making use of the keyword heuristic. If participants had been making use of the keyword heuristic, they would not have reported the risk involved within each pair of domain names as roughly equal. If they judged the domain name in question based on the keyword alone, 
without any other factors in play, then they would have seen each set of domain names as being approximately the same, without reporting any differences in risk between each pair. As an example, when the keyword Walmart showed up in any of the domain names, in any configuration, it was expected that they would have used the keyword heuristic if they judged the domain name as equal risk, no matter where it was placed in the domain name. However, this is not what the results of the statistical tests show. Since a significant difference between most of the pairs was found, then it was determined that they were not making use of the keyword heuristic.

However, this was not the case for the bankofamerica keyword. There were almost no differences in the ratings of risk attributed to both domain names www . bankofamerica. us.com and www. us.bankof america.com, indicating that participants judged both to be of similar reputability. This represents a danger, because users might fall for a phishing attack if they believe both of these domain names to be legitimate. This suggests that they were applying a keyword heuristic on this domain names pair, associated with the Bank of America name. This is a surprising and important finding, as this is the domain name pair out of the five tested that would be most severe should someone be caught in a phishing attack.

The results showed that the www. eapps.facebook. com is rated almost as risky as the www. facebook. eapps.com domain. This could indicate that people do not trust Facebook applications or lack confidence in unfamiliar aspects of Facebook. They might possibly have heard about the difficulties users are having in relation to privacy and Facebook from the media, or from friends and relatives on their list of contacts. It was also noticed that the Facebook domain names were the highest rated in terms of risk. It was speculated that this might be the result of having integrated the eapps subdomain and main domain into the two domain names. Participants may have interpreted eapps as "electronic applications", and might have rated the risk of these two domain names in terms of possible software associated with the domain name, in addition to the representation of Facebook itself. It is also possible that, in general, the data might be showing people lacking knowledge 
regarding what a domain name is, but instead might recognize patterns of characters such as facebook.com, amazon. com, or ebay.com. This could relate to a person's familiarity with widely known companies, organizations, and brands, which is ingrained into someone's mind through long-term and repetitive visual and/or auditory presentation. As such, it is logical to suggest that a degree of familiarity with a name found within a domain name can be an important criterion people use when making judgments about risk.

\section{Results for Section One: Malware}

Analysis. In the section one study of malware, the participants are presented with two scenarios. In the first scenario, they are asked to consider downloading software to edit photographs, and are asked to rate a series of events describing potential consequences of using this software (see Table 27). In the second scenario, they are asked to consider downloading game software, and are asked to rate a different series of events describing potential consequences of using that software (see Table 29). Some of the events are directly related to the intended functions of each software application, while the others are unrelated to the intended functions of each software application.

In the case of the photos software, related events involved something happening with photo files, while unrelated events focused on other files, software applications, or computer functions. In the case of the games software, related events involved something happening to a user's installed games or game score, while unrelated events focused on other files, games applications, or computer functions. The rating scales for both scenarios can be found in Table 27 and Table 29. The complete breakdown of responses to the rating scales can be found in Tables 28 and 30 , and in Figures 32 and 33 .

The statistical analyses conducted were two 1x6 Within-Subjects Analysis of Variance (one for each malware category), as well as two paired $t$-tests, based on each participant's responses to the functionality-related and functionality-unrelated questions. This was done in order to observe for significant differences between responses to each of the six events within each of the malware scenarios. The results can be found in Table 28 for the first mal- 
ware scenario (photos software application), and Table 30 for the second malware scenario (games software application).

As can be seen from the tables, all the results of the tests were significant. This shows that the participant responses to the questions related to functionality were significantly different to the questions not related to the functionality of the software, for each of the two scenarios. For example, the risks for event 1 in the photos software scenario (unexpectedly sharing your photos without your permission) were rated as more likely by the participants than the risks for event 4 in the photos software (the software changes your Microsoft Word files). Also, the risk-rating responses for event 1 in the games software (the software application downloads unwanted games) were once again rated as more likely by the participants than the risk-rating responses for event 6 in the games software scenario (software application deletes all music files).

\section{Interpretation}

These results suggest that participants were in fact making use of the functionality heuristic when making judgments about risk in conjunction with events related to the downloading and installation of software applications found on the Internet. The results offer insight into the difficulties users demonstrate in understanding the power of malware in general. Users may not understand how unconstrained software is once installed. They appear to feel that when software acts maliciously, it will still only be able to access and change files related to its intended purpose. In many ways, this perspective is reasonable. For example, with motor vehicles, the functionality of headlights are unrelated to the purchase and installation of new tires. Accordingly, anyone would be shocked if the new tires would negatively affect the headlights (e.g., dimming the illumination of the lights). Most domains where users have familiarity (e.g., in the motor vehicle domain), the functions and the limitations are clear, whereas within the area of computer technology users struggle to understand the mechanisms involved, as well as the dangers that are possible.

Overall, these results suggest the distinct possibility that users, lacking a strong 
mental model, do make decisions using heuristics, as suggested by Tversky and Kahneman (1974) and elaborated by Kahneman (2011).

Table 27

Malware: Events relating to the download and installation of photo editing software. Three of these events are functionality-related, and the other three are functionality-unrelated to the software application.

$\begin{array}{llll}\text { Event } & \text { Functionality } & \text { Mean }(\bar{x}) & \text { SD }(s) \\ \begin{array}{l}\text { The software application you obtained posts some of your pho- } \\ \text { tos on the Internet without your permission }\end{array} & \text { Related } & 3.071 & 2.144 \\ \begin{array}{l}\text { The software application you obtained deletes some of your } \\ \text { existing photos from your computer }\end{array} & \text { Related } & 2.882 & 2.117 \\ \begin{array}{l}\text { The software application you obtained overlays advertising } \\ \text { banners on your photos }\end{array} & \text { Related } & 3.076 & 2.828 \\ \begin{array}{l}\text { The software application you obtained changes some of your } \\ \text { Word (.doc, docx) documents }\end{array} & & 2.128 \\ \begin{array}{l}\text { The software application you obtained stops you from using } \\ \text { your Internet connection }\end{array} & \text { Unrelated } & 2.380 & 2.209 \\ \begin{array}{l}\text { The software application you obtained deletes your email con- } \\ \text { tacts list }\end{array} & \text { Unrelated } & 2.151 & 2.269\end{array}$




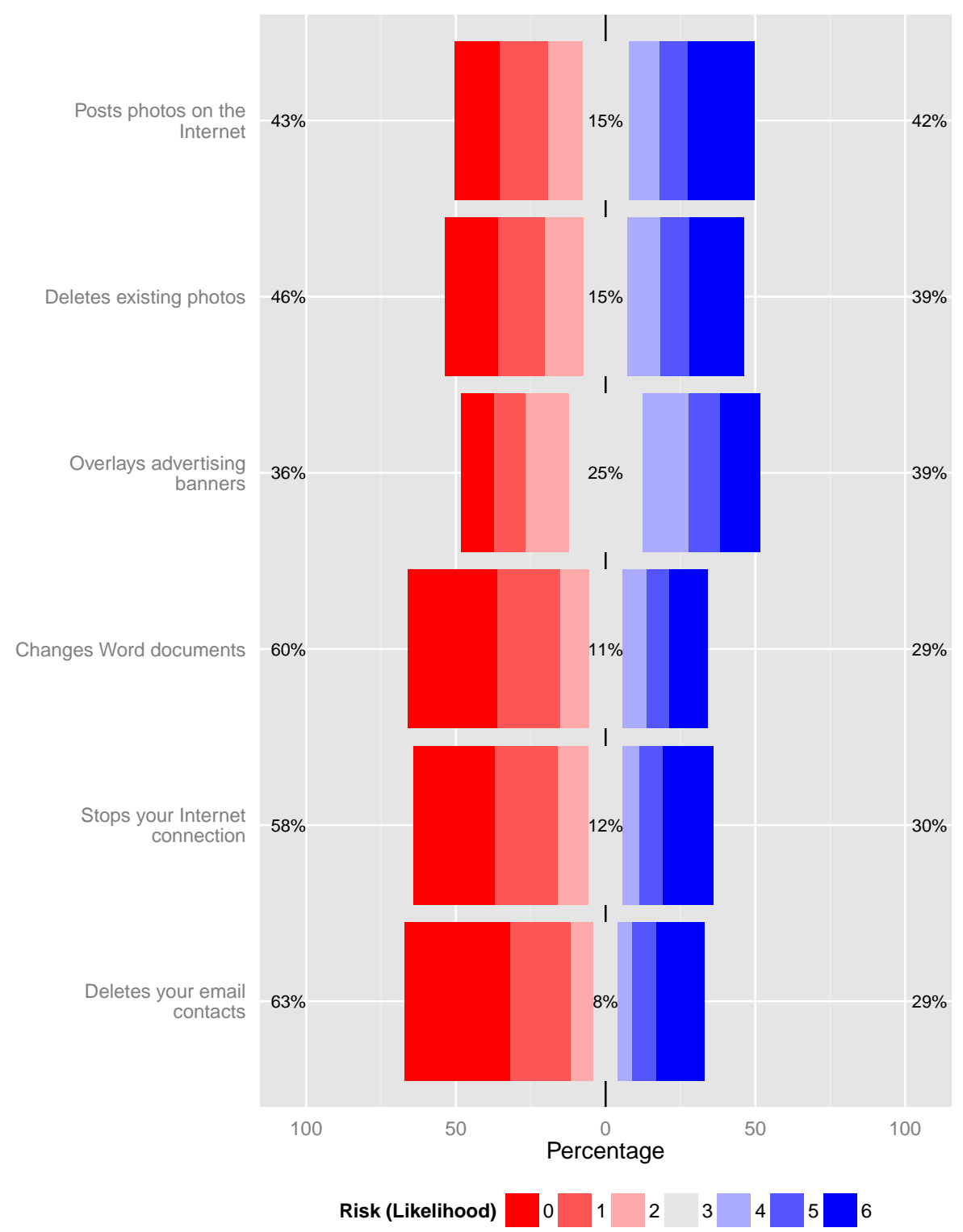

Figure 32. Rating scale responses for each of the six funcionality-related and functionalityunrelated events of the photo software application. A rating of 0 represents the lowest risk, and a rating of 6 represents the highest risk. 
Table 28

Part 1 - Malware Scenario 1 (Photo Editing Software).

\section{Scenario}

You've obtained a software application in order to retouch a set of photographs you've recently transferred to your computer. Please rate the risk, in terms of likelihood, of the following events.

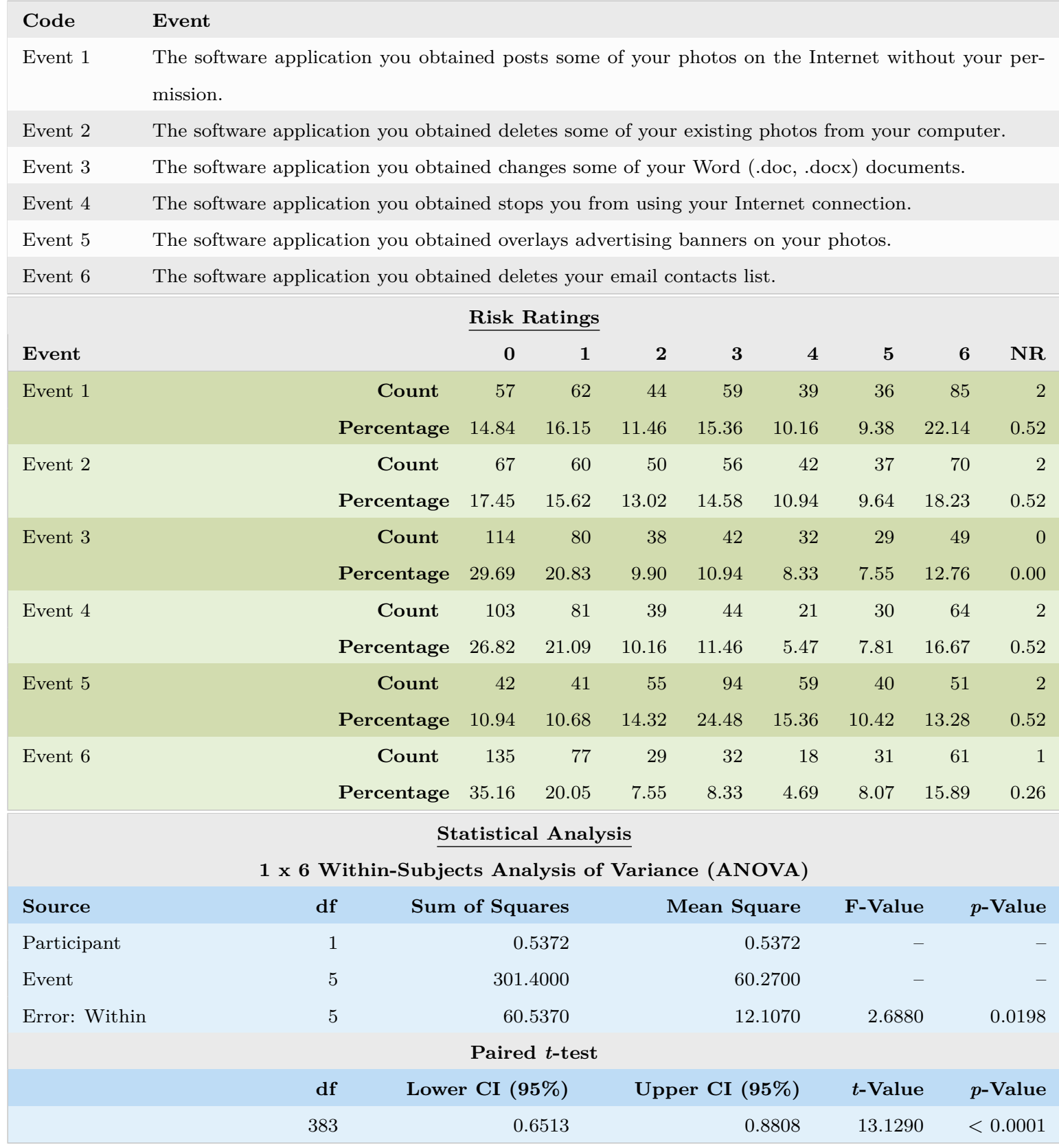

${ }^{*}$ Notes. $0=$ Low Risk. $3=$ Moderate Risk. $6=$ High Risk. NR $=$ No Response 
Table 29

Malware: Events relating to the download and installation of game software. Three of these events are functionality-related, and the other three are functionality-unrelated to the software application.

\begin{tabular}{|c|c|c|c|}
\hline Event & Functionality & Mean $(\bar{x})$ & $\mathrm{SD}(s)$ \\
\hline $\begin{array}{l}\text { The software application you obtained downloads and installs } \\
\text { several other games you don't want }\end{array}$ & Related & 3.815 & 1.774 \\
\hline $\begin{array}{l}\text { The software application you obtained deletes all other games } \\
\text { on your computer }\end{array}$ & Related & 2.281 & 2.165 \\
\hline $\begin{array}{l}\text { The software application you obtained resets your high scores } \\
\text { for all your other games }\end{array}$ & Related & 2.148 & 1.974 \\
\hline $\begin{array}{l}\text { The software application you obtained copies each of the per- } \\
\text { sons on your contacts list, and sends the information to a third } \\
\text { party }\end{array}$ & Unrelated & 3.229 & 2.136 \\
\hline $\begin{array}{l}\text { The software application you obtained installs a special appli- } \\
\text { cation that allows another user to gain control of your com- } \\
\text { puter remotely }\end{array}$ & Unrelated & 3.200 & 2.208 \\
\hline $\begin{array}{l}\text { The software application you obtained deletes all of the music } \\
\text { files on your computer }\end{array}$ & Unrelated & 2.133 & 2.198 \\
\hline
\end{tabular}




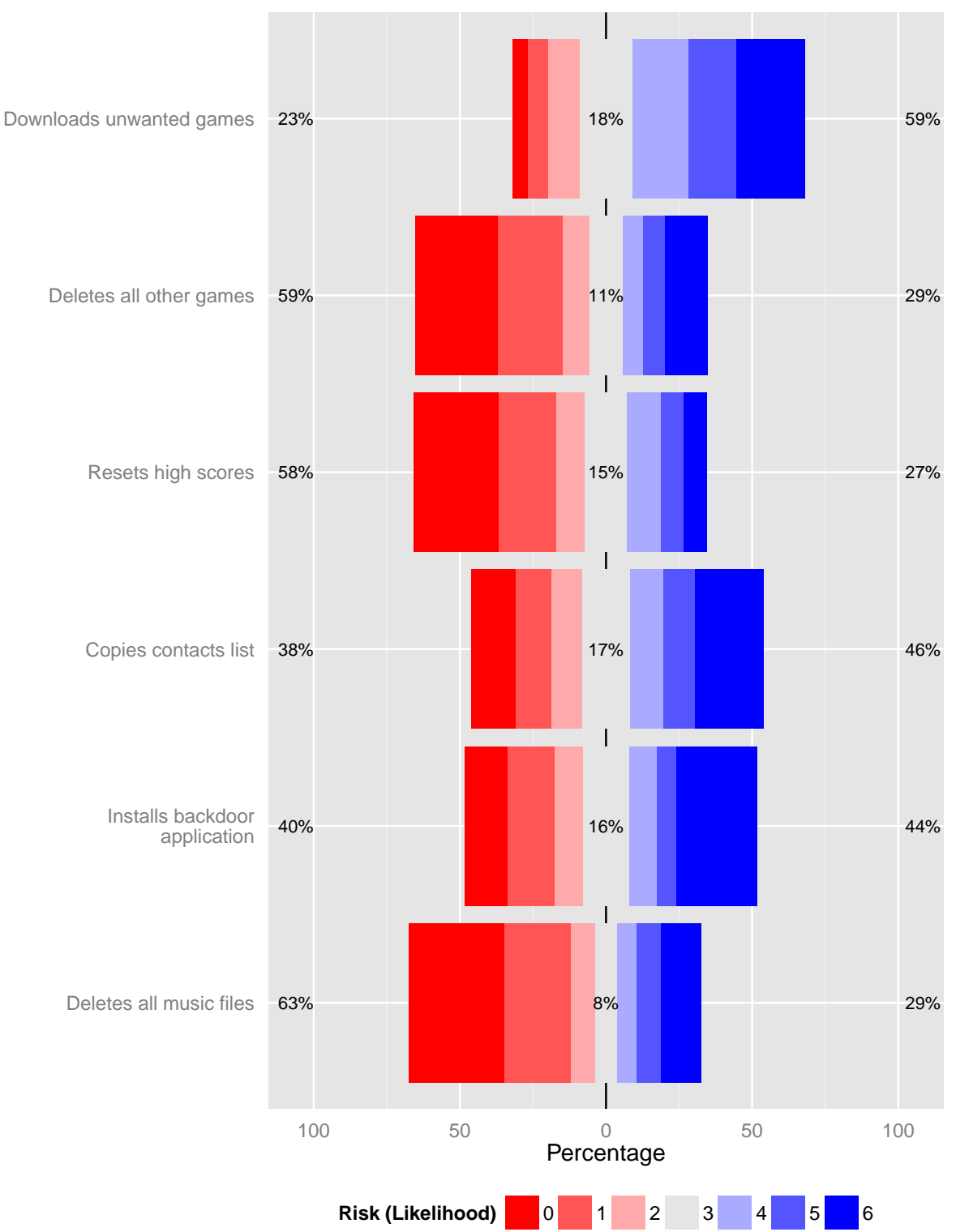

Figure 33. Rating scale responses for each of the six functionality-related and functionalityunrelated events of the game software application. A rating of 0 represents the lowest risk, and a rating of 6 represents the highest risk. 
Table 30

Part 1 - Malware Scenario 2 (Game Software).

\section{Scenario}

Scenario: You've obtained a game software for your computer. Please rate the risk, in terms of likelihood, of the following events.

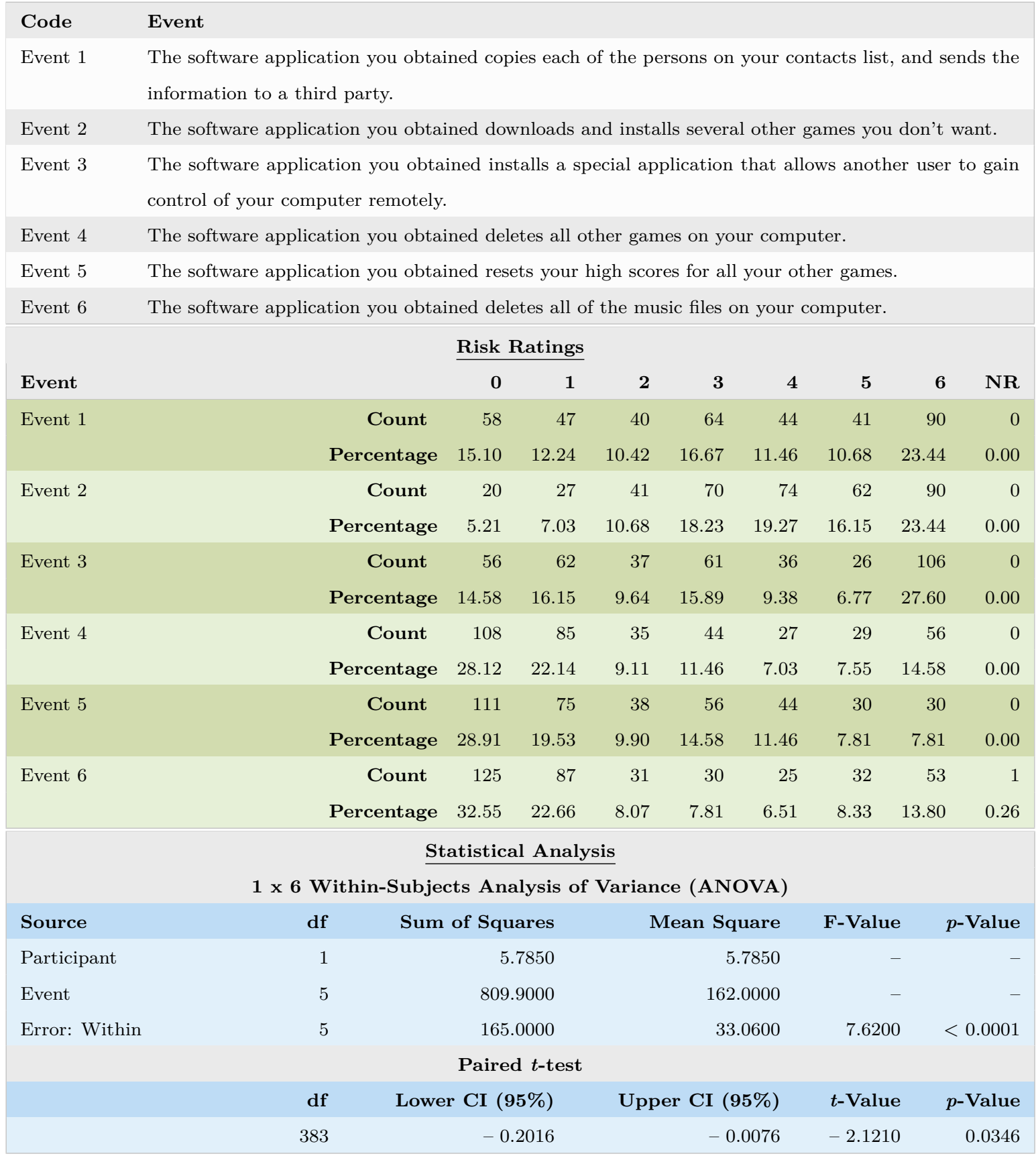

*Notes. 0 = Low Risk. $3=$ Moderate Risk. $6=$ High Risk. NR = No Response. 


\section{Results for Section Two: Knowledge and Risk}

Analysis. In this section, exploratory research was conducted on the relationship between knowledge and risk. Each participant's knowledge score was calculated (i.e., the number of questions they answered correctly), as well as their risk assessment score, for each of ten risk rating scales. Table 31 shows the questions found in the knowledge test section of the main task questionnaire. This table also includes descriptive statistics of how many participants got the correct answer to each question, along with the corresponding percentage. Appendix AD shows the figures, questions, and answers associated with each of the ten questions in the multiple choice knowledge questionnaire. Table 32 shows the perception of risk rating scales used in the main task questionnaire. 
Table 31

Knowledge Test Questions.

Histogram of Knowledge Scores

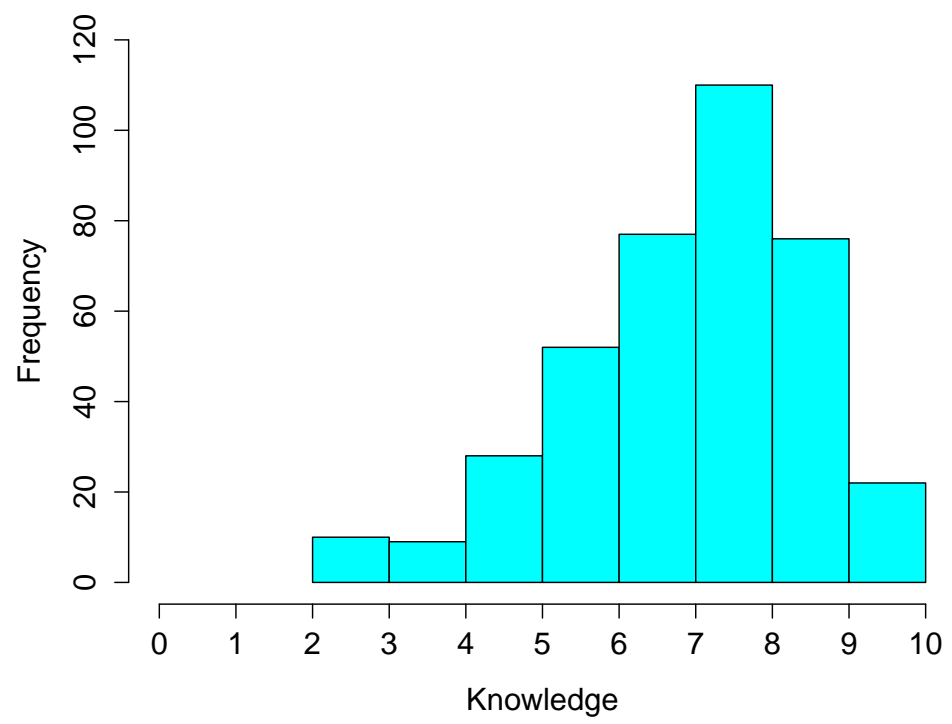

Knowledge Test Overall Response Results

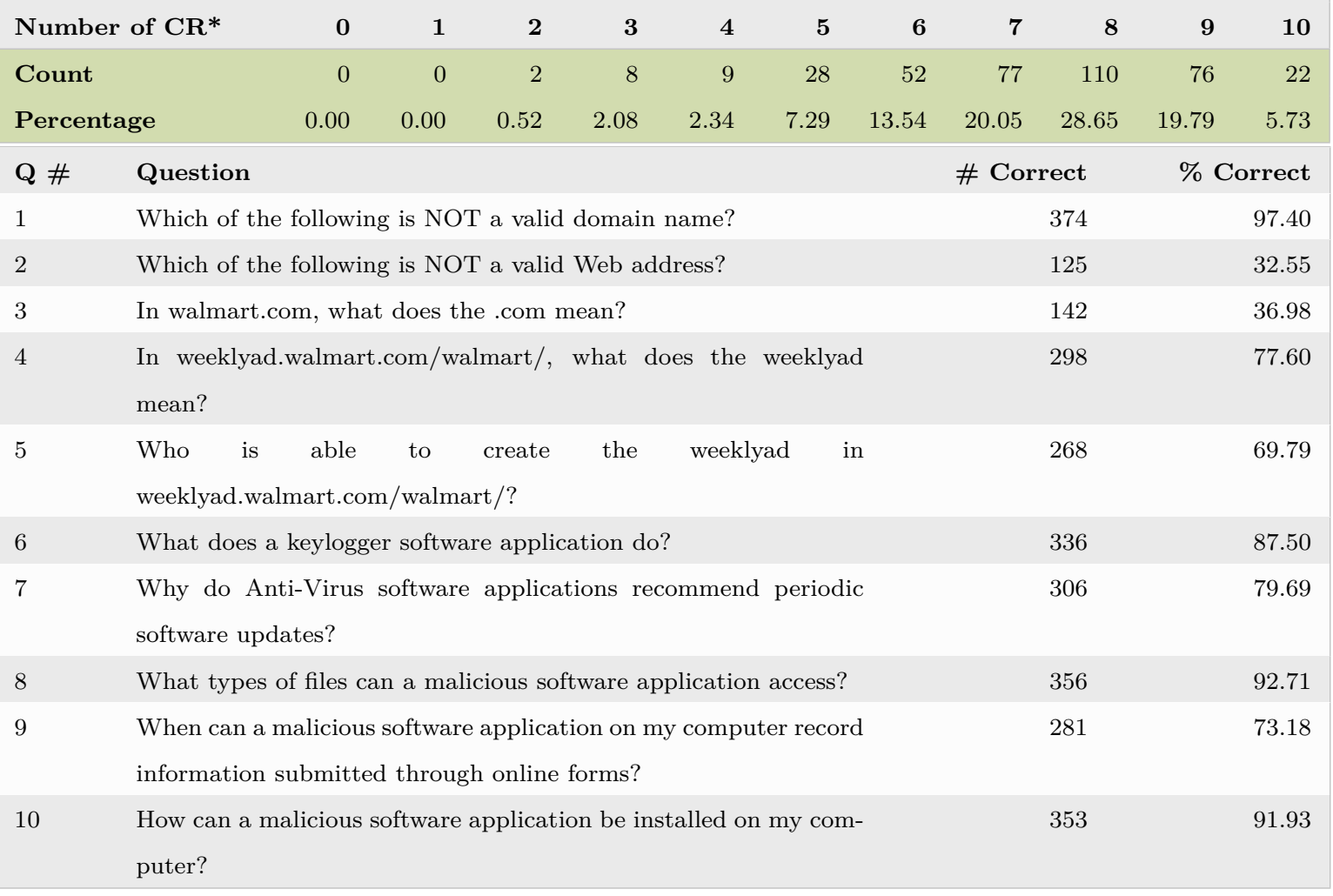




\section{Table 32}

Perception of Risk Rating Scale Statements.

\begin{tabular}{|c|c|c|c|}
\hline Code & Abbreviation & Risk Rating Scale Statement & Category \\
\hline S1 & BOA & $\begin{array}{l}\text { Typing the domain name www.bankofamerica.com into your } \\
\text { browser's address bar could lead to the loss of personal infor- } \\
\text { mation. }\end{array}$ & Browser \\
\hline $\mathrm{S} 2$ & EmailBOA & $\begin{array}{l}\text { Clicking on a link found in an email, where the link displays the } \\
\text { domain name www.bankofamerica.com }\end{array}$ & Browser \\
\hline S3 & PPUS & $\begin{array}{l}\text { Typing the domain name www.paypal.us.com into your browser's } \\
\text { address bar. }\end{array}$ & Browser \\
\hline $\mathrm{S} 4$ & Email PP & $\begin{array}{l}\text { Clicking on a link found in an email, where the link displays the } \\
\text { domain name www.paypal.com }\end{array}$ & Browser \\
\hline S5 & Google BOA & $\begin{array}{l}\text { Clicking on a link in a Google search result which brings } \\
\text { you to the Web address https://www.bankofamerica.com/credit- } \\
\text { cards/overview.go }\end{array}$ & Browser \\
\hline S6 & Tickets & $\begin{array}{l}\text { Installing software downloaded from an unknown source on the } \\
\text { Internet. }\end{array}$ & Download \\
\hline $\mathrm{S} 7$ & PDF & $\begin{array}{l}\text { Downloading a PDF file containing a sales brochure for a new } \\
\text { car, and viewing it in Adobe Reader. }\end{array}$ & Download \\
\hline S8 & Attach & $\begin{array}{l}\text { Reading an email from an unknown source and opening the at- } \\
\text { tachment present with the email message. }\end{array}$ & Download \\
\hline S9 & Github & $\begin{array}{l}\text { Downloading and installing a new toolbar for your browser via } \\
\text { the website http://www.github.com }\end{array}$ & Download \\
\hline $\mathrm{S} 10$ & Tickets & $\begin{array}{l}\text { Browse to a new concert ticket buying website by typing the Web } \\
\text { address www.ticketsforyou.com }\end{array}$ & Browser \\
\hline
\end{tabular}


Table 33

Perception of Risk Rating Scales: Rating the Risk Involved in Terms of the Severity of Consequences.

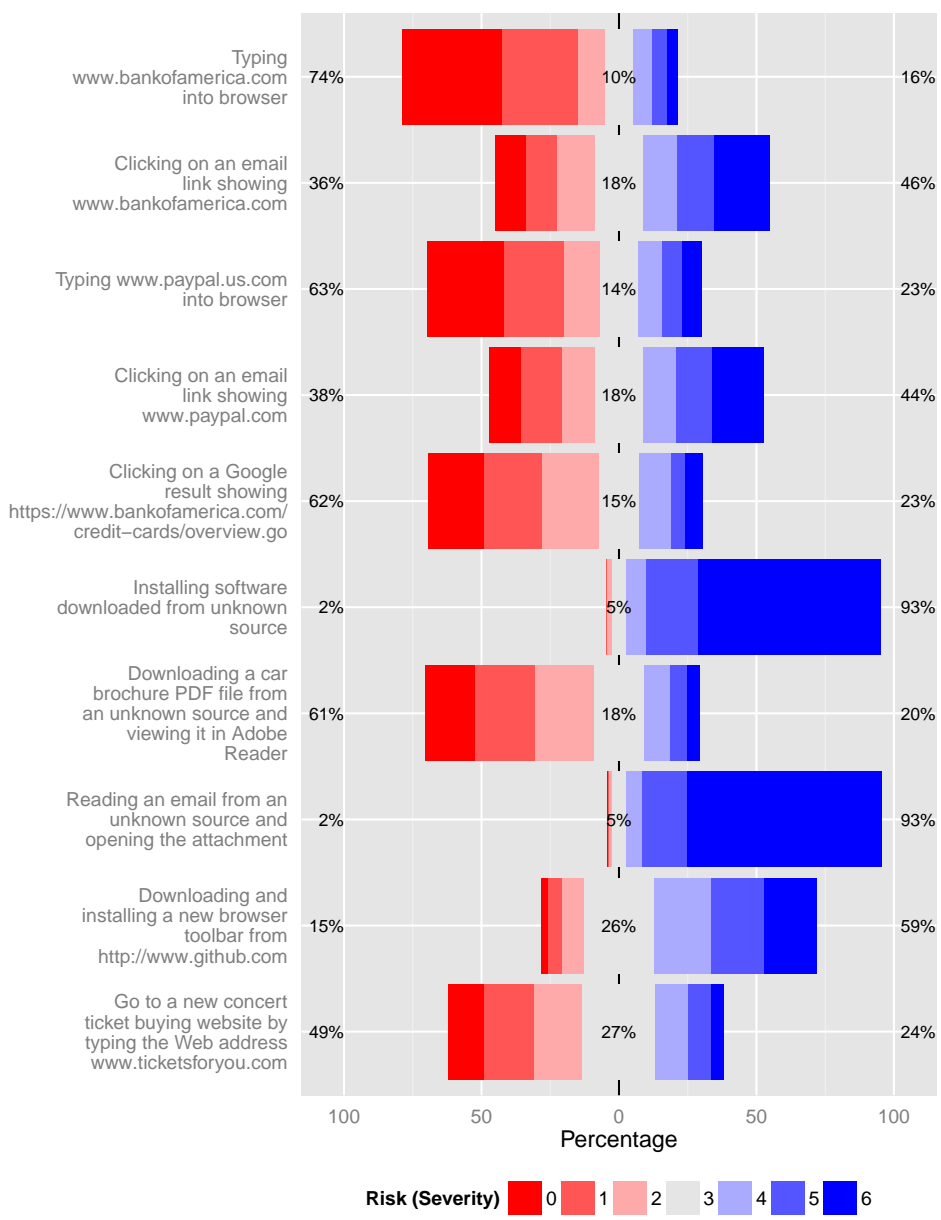

\begin{tabular}{|c|c|c|c|c|c|c|c|c|c|c|c|c|c|c|c|c|}
\hline \multicolumn{17}{|c|}{ Count / Percentage } \\
\hline $\mathbf{S}$ & $\mathbf{0}$ & $\%$ & 1 & $\%$ & 2 & $\%$ & 3 & $\%$ & 4 & $\%$ & 5 & $\%$ & 6 & $\%$ & NR & $\%$ \\
\hline S1 & 137 & 35.68 & 106 & 27.60 & 37 & 9.64 & 39 & 10.16 & 27 & 7.03 & 20 & 5.21 & 14 & 3.65 & 4 & 1.04 \\
\hline $\mathrm{S} 2$ & 42 & 10.94 & 44 & 11.46 & 53 & 13.80 & 68 & 17.71 & 48 & 12.50 & 51 & 13.28 & 78 & 20.31 & 0 & 0.00 \\
\hline S3 & 106 & 27.60 & 84 & 21.88 & 49 & 12.76 & 54 & 14.06 & 32 & 8.33 & 29 & 7.55 & 27 & 7.03 & 3 & 0.78 \\
\hline S4 & 44 & 11.46 & 57 & 14.84 & 46 & 11.98 & 68 & 17.71 & 46 & 11.98 & 50 & 13.02 & 72 & 18.75 & 1 & 0.26 \\
\hline S5 & 77 & 20.05 & 81 & 21.09 & 79 & 20.57 & 57 & 14.84 & 44 & 11.46 & 20 & 5.21 & 25 & 6.51 & 1 & 0.26 \\
\hline S6 & 0 & 0.00 & 1 & 0.26 & 7 & 1.82 & 20 & 5.21 & 28 & 7.29 & 73 & 19.01 & 253 & 65.89 & 2 & 0.52 \\
\hline S7 & 69 & 17.97 & 85 & 22.14 & 82 & 21.35 & 70 & 18.23 & 37 & 9.64 & 24 & 6.25 & 17 & 4.43 & 0 & 0.00 \\
\hline $\mathrm{S} 8$ & 1 & 0.26 & 1 & 0.26 & 5 & 1.30 & 19 & 4.95 & 23 & 5.99 & 63 & 16.41 & 271 & 70.57 & 1 & 0.26 \\
\hline S9 & 9 & 2.34 & 20 & 5.21 & 30 & 7.81 & 99 & 25.78 & 79 & 20.57 & 74 & 19.27 & 73 & 19.01 & 0 & 0.00 \\
\hline S10 & 50 & 13.02 & 69 & 17.97 & 68 & 17.71 & 103 & 26.82 & 45 & 11.72 & 32 & 8.33 & 17 & 4.43 & 0 & 0.00 \\
\hline
\end{tabular}

${ }^{*}$ Notes. $\mathrm{S}=$ Scale. $0=$ Low Severity. $3=$ Moderate Severity. $6=$ High Severity. $\mathrm{NR}=$ No Response. 
The relationships of interest in the study were the knowledge by risk assessment correlations. For the analysis, the correlations were divided into two themed groups: the browser category group and the download category group. Each group of correlations depicts the correlations between knowledge score and risk assessment. Table 34 shows the correlation statistics broken down by category. Figures 35 and 34 show the group correlations between knowledge score and risk assessment. In each figure, the knowledge score is related to each of the scales in the categories. Also, each rating scale is compared with every other rating scale found in the same category. In each figure, the diagonal shows histograms representing the distributions of each individual scale. Above the diagonal, correlation values are shown for every pair of scales. Below the diagonal, scatterplots are shown for every pair of scales. The red line in each scatterplot is the Loess curve, indicating the relationship between the two scales.

Table 34

Knowledge and Risk Statistics.

\begin{tabular}{|llr|} 
& \multicolumn{1}{c}{ Statistical Analysis } & $\rho^{*}$ \\
\hline Score & Rownload Category & 0.13 \\
\hline Knowledge Score & Install* Rating Scale & -0.06 \\
Knowledge Score & PDF* Rating Scale $^{*}$ & 0.14 \\
Knowledge Score & Attach* Rating Scale & -0.08 \\
Knowledge Score & Github* Rating Scale & $\rho^{*}$ \\
\hline Score & \multicolumn{1}{c}{ Browser Category } & -0.09 \\
\hline Knowledge Score & Rating Scale & 0.07 \\
Knowledge Score & BOA* Rating Scale & 0.09 \\
Knowledge Score & Email BOA* Rating Scale & 0.04 \\
Knowledge Score & PPUS* Rating Scale & -0.13 \\
Knowledge Score & Email PPUS* Rating Scale \\
Knowledge Score & Google BOA* Rating Scale & -0.02 \\
\hline Notes. CR $=$ Correct Responses. $\rho:$ Spearman's correlation coefficient was used between ranked (i.e., rating scale) \\
\hline variables. For the list of full abbreviated rating scales, refer to Table 32
\end{tabular}




\section{Interpretation}

The correlations between knowledge and risk assessment suggest that these two dimensions don't correlate very highly at all, whether positively or negatively. First, the highest observed correlation within the download category was between knowledge and the "Attach" (opening unknown email attachments) risk assessment rating scale, where the Spearman correlation statistic $(\rho)$ showed a low positive correlation of 0.14 . The highest observed correlation within the category was between knowledge and the "Google BOA" (clicking a search link for Bank of America) risk assessment rating scale, where the Spearman correlation statistic $(\rho)$ showed a low negative correlation of -0.13 . These resulting correlations are puzzling, as stronger correlations between knowledge and risk were expected, whether they would have been stronger negative correlations or stronger positive correlations.

As such, based on these results, it appears that participants with higher knowledge scores are rating some of the rating scales as low-risk — even though they're high-risk — and participants with lower knowledge scores are rating these same rating scales as high-risk. This has the effect of counterbalancing the results, resulting in these almost non-existant correlations.

In earlier work, Rafalak et al. (2014) conducted a large study relating personality characteristics to risk taking within the context of browsing the Internet. The results showed a wide range of behaviours on the part of the users, and specifically noted that propensity for taking risk distinguishes different kinds of behaviour. Personality characteristics were not investigated in the present study. If personality characteristics do strongly influence risk taking, this may explain the lack of correlations seen in the results. 


\section{Download category rating scales}

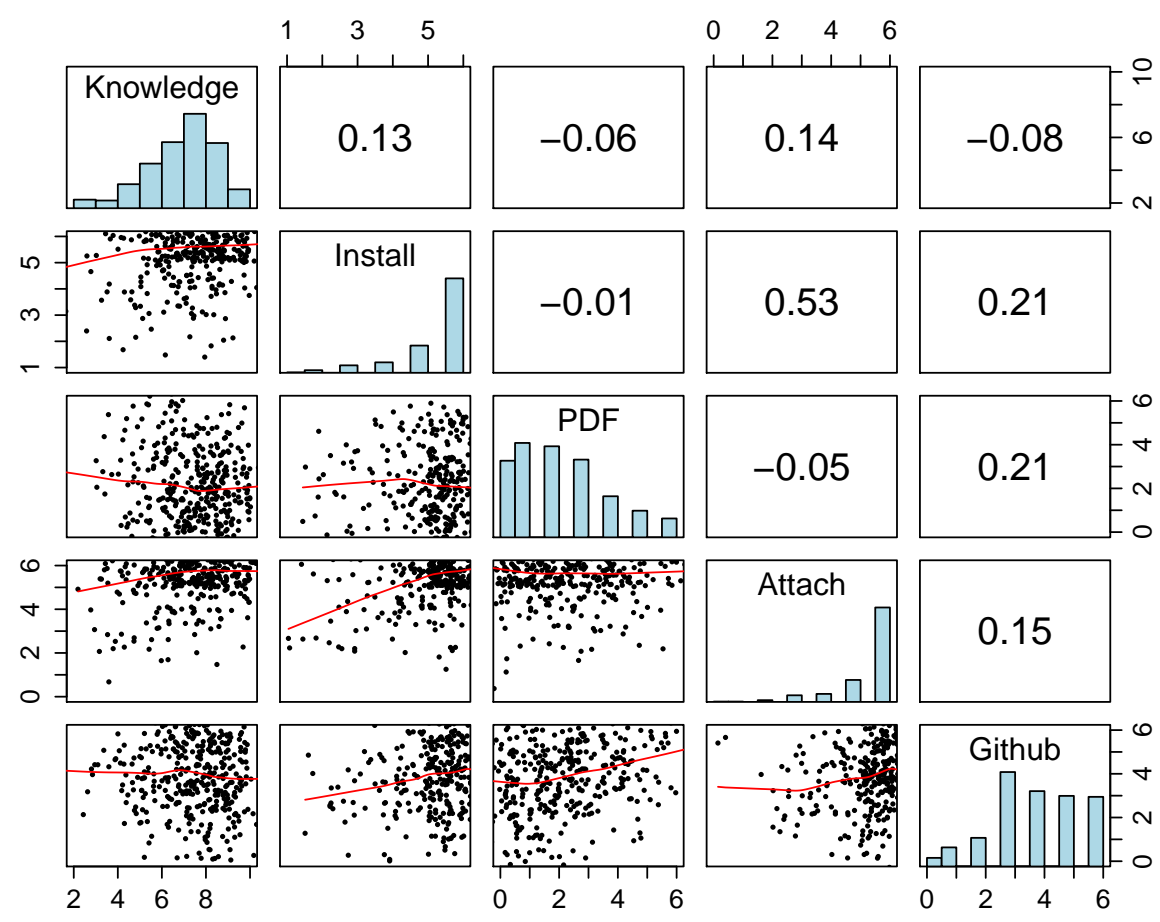

Figure 34. Correlation matrix of knowledge and risk assessment for the "download" theme. 


\section{Browser category rating scales}

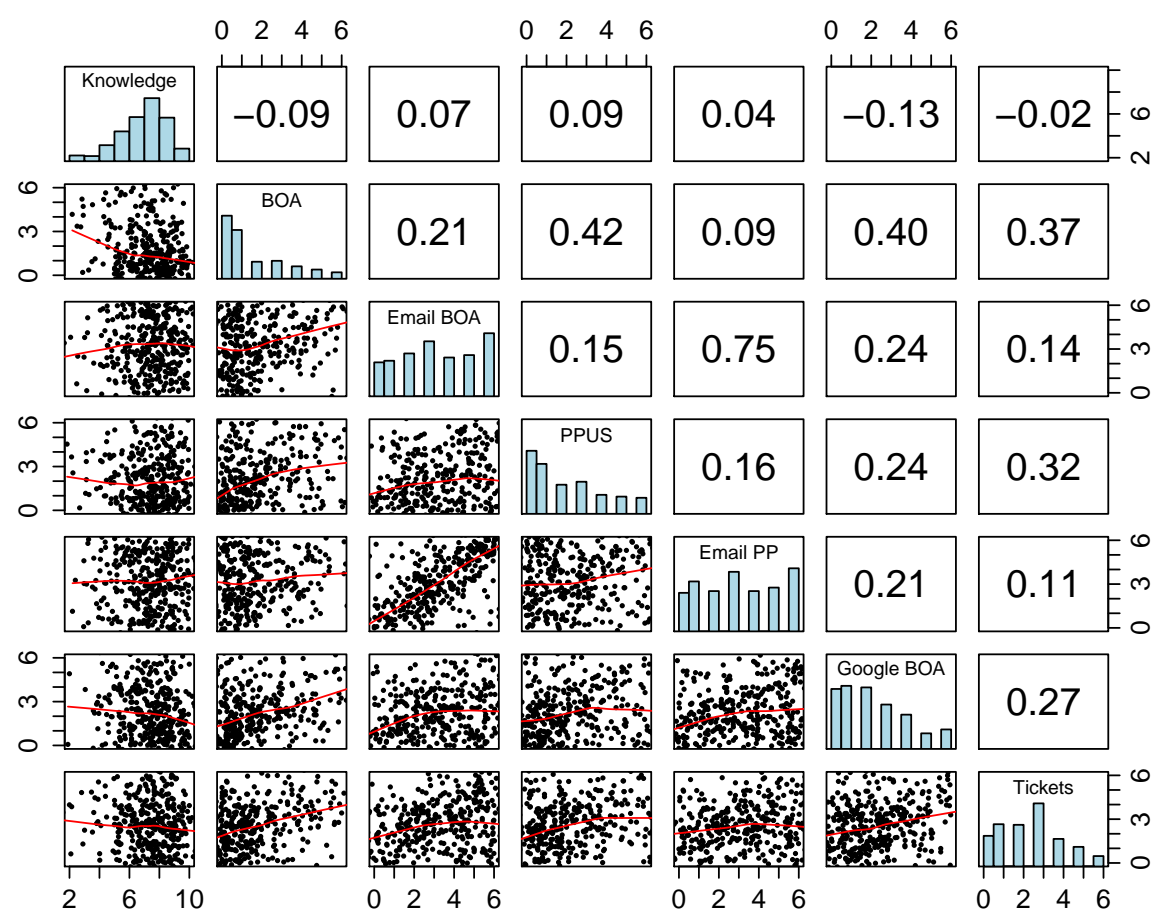

Figure 35. Correlation matrix of knowledge and risk assessment for the "browser" theme. 


\section{Results for Section Three: Attitudes and Beliefs}

Analysis. For this section, it was speculated that there might be relationships between assessed knowledge of Internet security, self-reported knowledge of Internet security, and several dimensions of self-reported perceptions and attitudes related to learning about Internet security. These dimensions were: learning capability, difficulty in learning, time available to learn, and motivation to learn. It is important to note that, for these five dimensions in this section, the four rating scale statements are the same. Table 35 shows these scales. Also examined were attitudes about responsibility for Internet security, and whether participants had experienced Internet security problems.

Table 35

Rating scales used in each of the five dimensions (Knowledgeable, Capable, Difficult, Time, Motivated), along with each corresponding rating scale abbreviation.

\begin{tabular}{ll} 
Abbreviation & Rating Scale \\
\hline Works & How the Internet works? \\
Protect & How to protect your security and privacy on the Internet? \\
Avoid & How to avoid malicious software applications being installed on your computer? \\
Identify & How to identify potentially harmful websites? \\
\hline
\end{tabular}

In this section, the analyses conducted were similar to the previous section. Each participant's knowledge score was correlated with their rating scale score for each of the five learning dimensions. These are abbreviated as knowledgeable, capable, difficulty, time, and motivated. Figures showing these correlations were created, one for each dimension. It was found that, for each of the dimensions, all five of the rating scales were strongly correlated with each other, but were very weakly correlated with assessed knowledge. Appendix AE includes these correlations in Tables 71 to 75 and Figures 48 to 52 .

Also, responsibility and participant experience were examined and compared with assessed knowledge. For responsibility, participants were asked for their assessment about several different entities and their responsibility related to Internet security (e.g., themselves, the police, their Internet Service Provider, etc.). For participants' experience, they were asked for their agreement or disagreement that several events occurred to them or their family in the past. These results are shown in Tables 36 (responsibility) and 37 (experience). 
Table 36

Part 3 - Post Test Question 6: How Responsible do You Think the Following Entities are for Protecting You from Malicious Software Applications?

\begin{tabular}{|ll|}
\hline Code & Rating Scale Statement \\
S1 & My Internet Service Provider (e.g., Comcast, Time Warner, etc.). \\
S2 & My Anti-Virus software application provider (e.g., Norton, McAfee, etc.). \\
S3 & My operating system provider (e.g., Microsoft, Apple, etc.). \\
S4 & The police. \\
S5 & The government of the United States. \\
S6 & Myself. \\
\hline
\end{tabular}

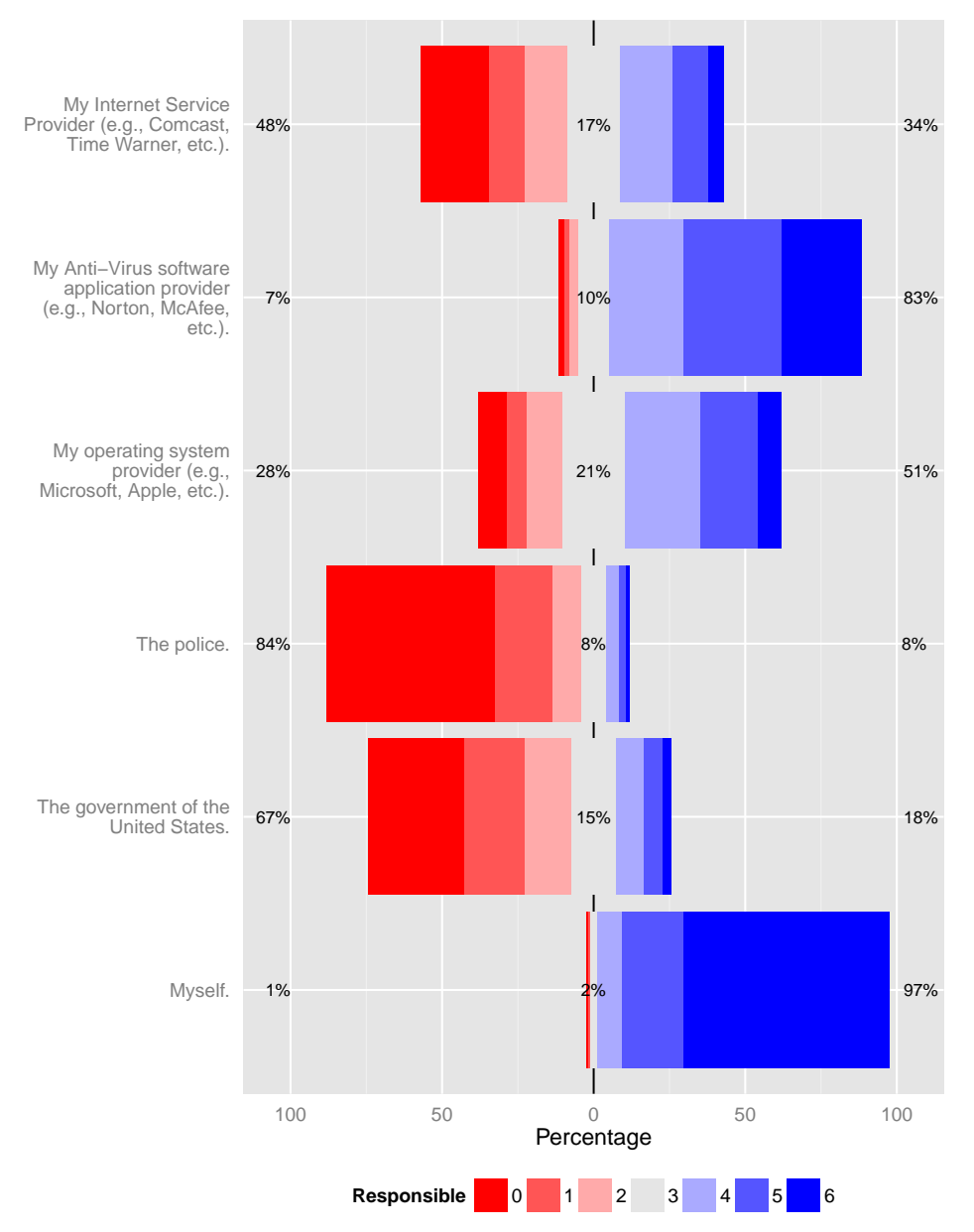

\section{Count / Percentage}

\begin{tabular}{|lrrrrrrrrrrrrrrrr} 
S & $\mathbf{0}$ & $\mathbf{\%}$ & $\mathbf{1}$ & $\mathbf{\%}$ & $\mathbf{2}$ & $\mathbf{\%}$ & $\mathbf{3}$ & $\mathbf{\%}$ & $\mathbf{4}$ & $\mathbf{\%}$ & $\mathbf{5}$ & $\mathbf{\%}$ & $\mathbf{6}$ & $\mathbf{\%}$ & NR & \% \\
S1 & 86 & 22.40 & 45 & 11.72 & 54 & 14.06 & 67 & 17.45 & 67 & 17.45 & 44 & 11.46 & 20 & 5.21 & 1 & 0.26 \\
S2 & 7 & 1.82 & 7 & 1.82 & 11 & 2.86 & 39 & 10.16 & 94 & 24.48 & 123 & 32.03 & 101 & 26.30 & 2 & 0.52 \\
S3 & 36 & 9.38 & 26 & 6.77 & 44 & 11.46 & 81 & 21.09 & 94 & 24.48 & 73 & 19.01 & 29 & 7.55 & 1 & 0.26 \\
S4 & 212 & 55.21 & 73 & 19.01 & 36 & 9.38 & 32 & 8.33 & 16 & 4.17 & 9 & 2.34 & 5 & 1.30 & 1 & 0.26 \\
S5 & 121 & 31.51 & 75 & 19.53 & 59 & 15.36 & 58 & 15.10 & 35 & 9.11 & 23 & 5.99 & 11 & 2.86 & 2 & 0.52 \\
S6 & 2 & 0.52 & 2 & 0.52 & 1 & 0.26 & 8 & 2.08 & 32 & 8.33 & 78 & 20.31 & 260 & 67.71 & 1 & 0.26 \\
\hline
\end{tabular}

*Notes. $\mathrm{S}=$ Scale. $0=$ Not at all Responsible. $6=$ Completely Responsible. $\mathrm{NR}=$ No Response. 
Table 37

Part 3 - Post Test Question 7: Please State Your Level of Agreement or Disagreement With the Following Statements.

$\begin{array}{ll}\text { Code } & \text { Rating Scale Statement } \\ \text { S1 } & \text { I have been the victim of a phishing website (which looks like the correct website but is not genuine). } \\ \text { S2 } & \text { I have had malicious software applications installed on my computer. } \\ \text { S3 } & \text { One or more of my family members has been the victim of a phishing website (which looks like the } \\ & \text { correct website but is not genuine). } \\ \text { S4 } & \text { One or more of my family members has had malicious software applications installed on their computer. } \\ \text { S5 } & \text { When I browse the Internet, I am confident that I know how to avoid phishing websites (which look } \\ & \text { like the correct websites but are not genuine) and malicious software applications. }\end{array}$

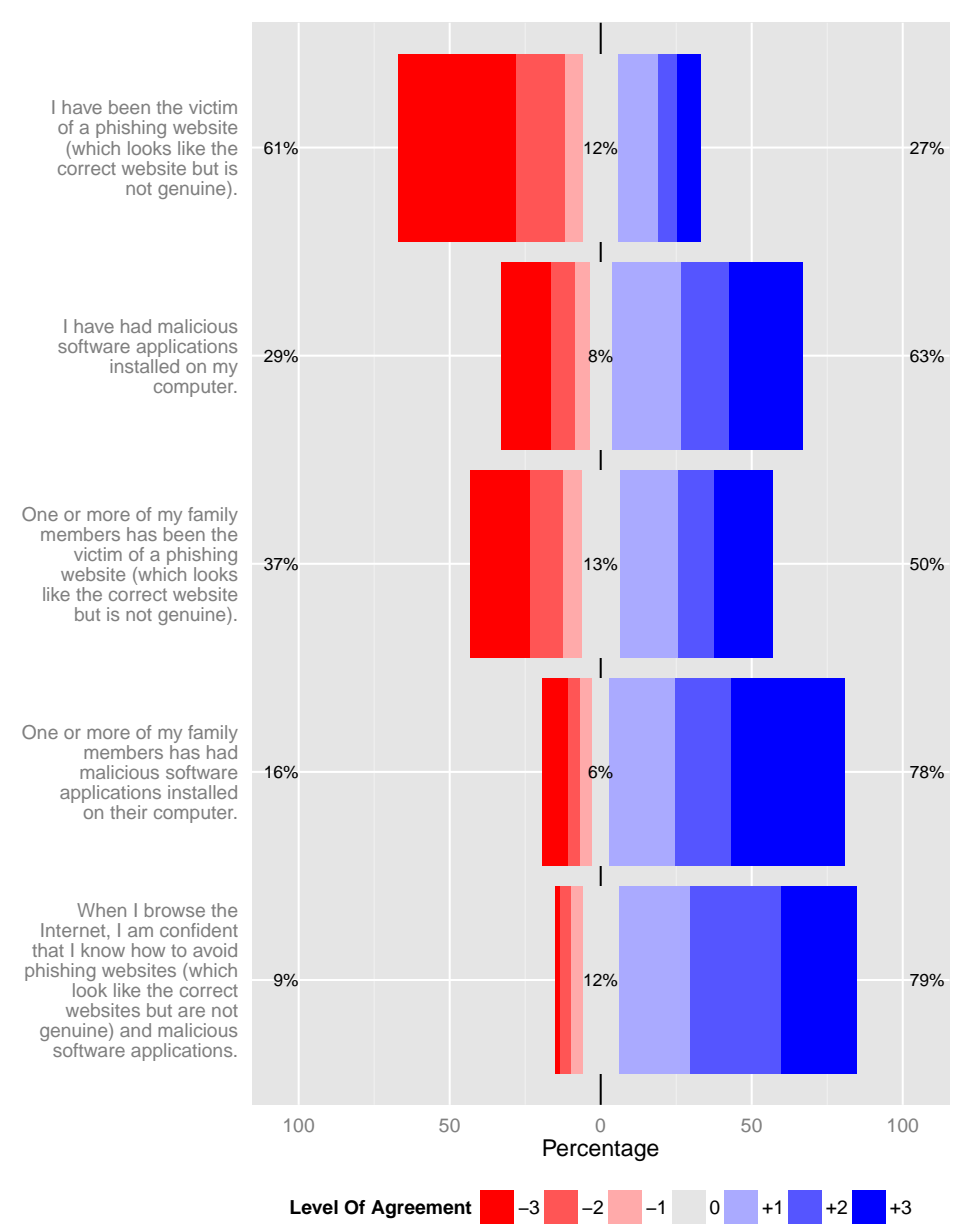

\begin{tabular}{|c|c|c|c|c|c|c|c|c|c|c|c|c|c|c|c|c|}
\hline \multicolumn{17}{|c|}{ Count / Percentage } \\
\hline $\mathbf{S}$ & -3 & $\%$ & -2 & $\%$ & -1 & $\%$ & $\mathbf{0}$ & $\%$ & +1 & $\%$ & +2 & $\%$ & +3 & $\%$ & NR & $\%$ \\
\hline S1 & 149 & 38.80 & 62 & 16.15 & 23 & 5.99 & 45 & 11.72 & 50 & 13.02 & 24 & 6.25 & 30 & 7.81 & 1 & 0.26 \\
\hline S2 & 62 & 16.15 & 31 & 8.07 & 18 & 4.69 & 29 & 7.55 & 87 & 22.66 & 61 & 15.89 & 92 & 23.96 & 4 & 1.04 \\
\hline S3 & 76 & 19.79 & 42 & 10.94 & 23 & 5.99 & 50 & 13.02 & 74 & 19.27 & 45 & 11.72 & 74 & 19.27 & 0 & 0.00 \\
\hline S4 & 32 & 8.33 & 15 & 3.91 & 16 & 4.17 & 22 & 5.73 & 84 & 21.88 & 71 & 18.49 & 144 & 37.50 & 0 & 0.00 \\
\hline S5 & 6 & 1.56 & 14 & 3.65 & 15 & 3.91 & 46 & 11.98 & 91 & 23.70 & 116 & 30.21 & 96 & 25.00 & 0 & 0.00 \\
\hline
\end{tabular}

*Notes. $\mathrm{S}=$ Scale. $-3=$ Strongly Disagree. $+3=$ Strongly Agree. NR $=$ No Response. 
Responsibility. The correlation between knowledge score and responsibility is shown in Figure 36. The highest observed positive correlation was between the knowledge score and any of the rating scales was 0.16 . This relationship was between the knowledge score and the "Myself" rating scale (indicating that the users themselves are responsible). In contrast, the highest observed negative correlation between the knowledge score and any of the rating scales was -0.18 . This relationship was between the knowledge score and the "Internet Service Provider (ISP)" rating scale (indicating that the ISP is responsible). In fact, the highest observed positive correlation was the only positive correlation between the knowledge score and any of the rating scales.

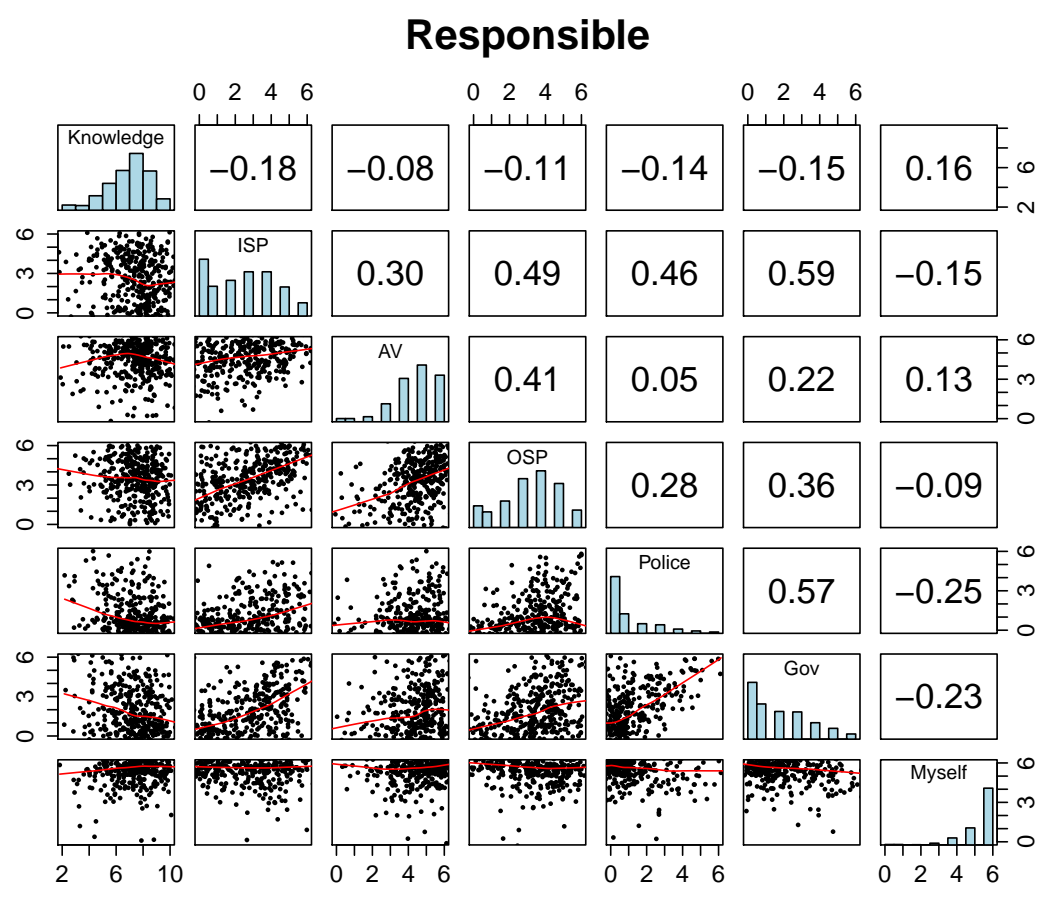

Figure 36. Correlation matrix of assessed knowledge by responsible dimension rating scales.

Experience. The correlation between knowledge score and experience is shown in Figure 37. The highest observed positive correlation was between the knowledge score and any of the rating scales was 0.16 . This relationship was between the knowledge score and the "CanAvoid" rating scale (indicating that the user is confident in avoiding phishing 
websites and malware). In contrast the highest observed negative correlation between the knowledge score and any of the rating scales was -0.23 . This relationship was between the knowledge score and the "MePhishing" rating scale (indicating that the user has fallen to a phishing attack). These correlations with the participant knowledge scores are low to moderate at best. However, it is important to note that participants' responses to their personal experiences with phishing and malware are much more highly correlated with their confidence in avoiding these threats than are their responses to their beliefs about others' experience with phishing and malware. To highlight this, the correlation between participants' personal experience with phishing and their confidence in avoiding phishing is -0.28 , indicating that they have low confidence in being able to avoid phishing if they've ever been affected by it. In contrast, the correlation between participants' responses to others' experience with phishing and their confidence in avoiding phishing is -0.02 , indicating that others' experience with phishing has no bearing on their personal experience in avoiding phishing and malware. Also, the correlation between participants' responses to their experience with malware and their confidence in avoiding malware is -0.06 , indicating that there's no relationship between their personal experience with malware and their confidence in avoiding malware. In contrast, the correlation between participants' responses to others' experience with malware and their confidence in avoiding phishing is 0.20 , indicating that others' experience with malware is low to moderately correlated with their confidence in avoiding phishing and malware. 


\section{Agree/Disagree rating scales}

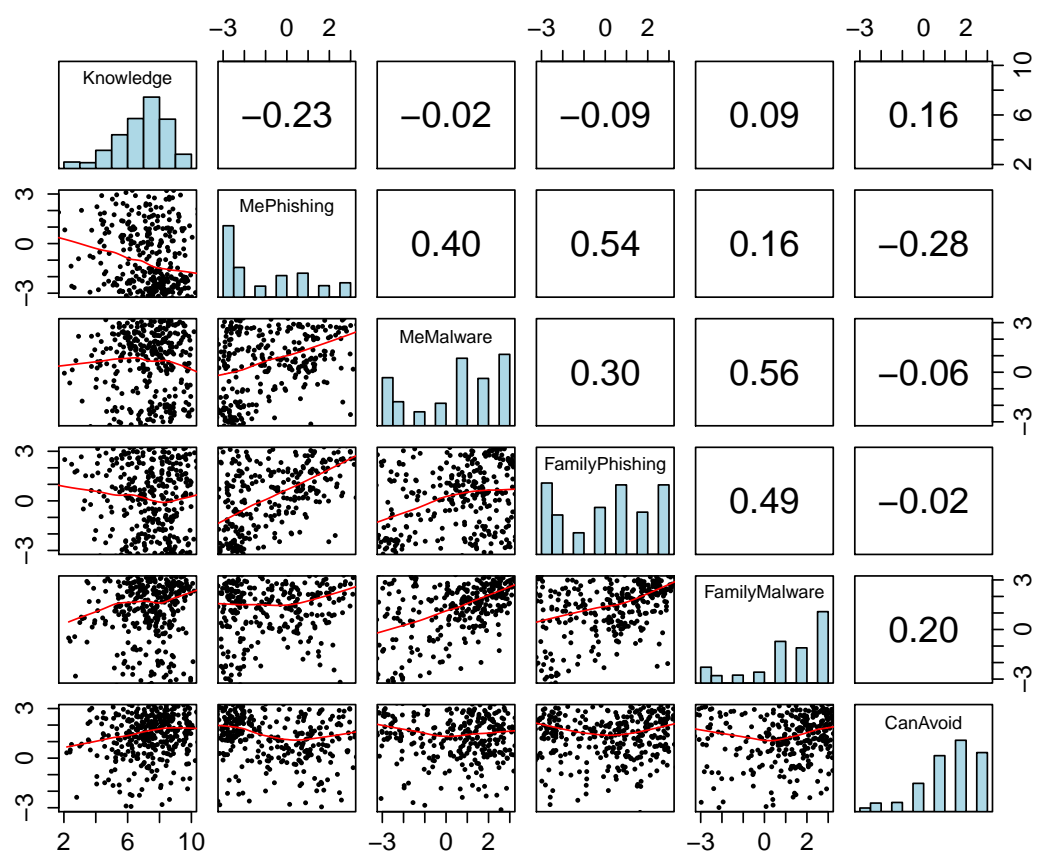

Figure 37. Correlation matrix of assessed knowledge by the agree/disagree rating scales.

\section{Interpretation}

In this section, the goal was to explore relationships between knowledge and selfratings of attitudes, beliefs, and experience. There was little correlation between assessed knowledge and self-rated knowledge. The learning dimensions were all highly correlated with one another, but had little to no relationship with assessed knowledge. Perhaps the most interesting finding involved responsibility and assessed knowledge. Correlations were not strong, but suggested that higher knowledge about Internet security was associated with greater acceptance of personal responsibility in terms of protection against threats. Conversely, people with lower knowledge were more likely to feel some other organization was responsible. This indicates a danger for those with less knowledge because they may be assuming that some organization is taking responsibility for their security, but in many cases this is in fact not true. It has been suggested that promoting greater personal responsibility for Internet safety is the best strategy (LaRose, Rifon, \& Enbody, 2008). Some research 
has shown that many users do exhibit feelings of responsibility for their computer security, but this may still leave many users attributing responsibility elsewhere (Furnell, Bryant, \& Phippen, 2007). 


\section{General Discussion}

The study sections of this thesis document outlined the research methods and obtained results. This section provides a more in-depth interpretation and discussion of the results that were obtained within the five studies. The studies examined various aspects of engaging in online activities and, as such, provide various themes to discuss in this section. Each of these themes can be examined over the thesis work as a whole, in such a way as to provide a consistent and cohesive story with the goal of explaining people's knowledge, beliefs, perceptions, and misunderstandings regarding security when engaging in online activities.

\section{Study One: Determining Website Legitimacy}

The first study was to explore participants' abilities to make appropriate decisions regarding trustworthiness of websites.

The findings of the first experiment indicated that users are not able to determine, with any confidence, website legitimacy by locating and identifying security mechanisms embedded within the browser chrome. It is believed that the users partaking in the study were unable to make proper decisions regarding which websites to trust, due to a lack of understanding of the information presented to them during testing. When provided with various web browser screenshots, they would often claim recognition of the website in question, but when questioned about their willingness to use and to trust the same website,

they would, more often than not, declare an unwillingness to trust and subsequently browse to the same website. They initially appeared inclined to trust, but not confident when questioned about their reasoning.

This has important implications for user choice in online interactions, as it suggests that users make important decisions about browsing to and interacting with a given website with little to no information about how they themselves are protected or not from any potentially malicious intent on behalf of the website administrators (i.e., phishing websites).

This thesis discussed in detail phishing websites in the Computer Security Background section. It is widely known by researchers in the field of computer security that these types 
of websites attempt to lure users into a false sense of security — by appearing like the real website - and then obtain usernames and passwords entered by the user in order for the attackers to access the user's accounts on the legitimate websites (Dhamija et al., 2006).

Results from the first experiment suggest that users may be easily fooled by the deceptive methods used in phishing and malicious websites. Users are easily drawn to cues located within the website itself, meant to indicate a trustworthy and legitimate website. These cues can come in the form of lock icons, testimonials, reviews by recognized websites and magazines (e.g., CNet Reviews, PC Magazine), among others.

These methods of deception can easily be integrated into any website. The problem comes from the fact that users don't quite understand that the security mechanisms that help protect them are only found within the browser chrome, and not within the webpage itself. Most of the users partaking in the first experiment reported deciding in favor of trusting a small proportion of the websites shown, even though they depicted fake security mechanisms, stating that they saw a lock icon on the page, or an award given to the website by a trusted outside source, which led them to believe that the website in question was trustworthy. This has serious implications for user vulnerability while browsing the Internet, as there are a plethora of websites which exist online that use these exact security mechanism cues in order to fool users into trusting them, leading them to giving up personal information, or downloading malicious software applications that will compromise their computer's files or copy personal and/or financial information.

These vulnerabilities give rise to questions involved in engaging in online interactions, the user understanding of these risks, and the potential outcomes should something go wrong while engaging in said activities.

Themes. Overall, participants responded to the questions posed during testing with a reliance on superficial characteristics within the websites shown. However, they were reticent to discuss their thoughts and feelings about a given website, they also put up blocks when questioned about trusting or distrusting a given website. This was surprising, as initially participants appeared willing to trust a given website when questioned about it, 
but as the study session progressed, their responses became less and less positive towards each of the websites shown. This was interpreted as uncertainty about the deeper issues and, as such, the overarching theme that emerged in this study was a lack of confidence.

\section{Study Two: Risk Perception of Internet-Related Activities}

The second study was to explore participants' perception of risk related to online activities.

Study 2 concerned perception of risk involved in online interactions and revealed some interesting results. The materials used in the study addressed user understanding of the various risks involved in many online activities, and a few offline activities. These activities were distributed between three categories: financially-linked activities, potentially embarrassing activities, and a third category which encompassed the remaining activities, classified as "other". This last category encompassed activities which were not related to personal information, nor financial information directly. Online activities classified as "other" included, but were not limited to, "using a search engine (e.g., Google, Yahoo, Bing)", "downloading pirated movies or music", and "sending and receiving email".

The results obtained showed some very interesting patterns. First, financially-linked activities, such as banking online, were attributed to carry a high level of severity of consequences should something go wrong while engaging in this activity. While this category of activities was determined to carry a high level of severe consequences, potentially embarrassing activities were attributed to carry a high level of likelihood of something going wrong, such as someone leaking personal information about a friend or relative on a social networking website.

Participants rated some offline activities, such as giving a credit card over the phone, as being more severe than using a credit card to make an online purchase through a secure website. A plausible explanation for these results would be that users believe the action of giving their credit card number over the phone to a customer service representative or salesperson would make them vulnerable to having their credit card information copied and 
used by these people. However, users do not completely understand that the severity of consequences in giving their credit card over an online form, even through a secured online form, would represent a higher risk of having their account information copied should their computer be infected by a malicious software application. A program such as this could copy the account information and send it to a third party without the user's knowledge. This latter scenario would represent a much higher severity of consequences than the former scenario, should something go wrong.

A more serious finding concerned malware explicitly. Participants attributed more likely, but less severe, consequences of installing a software application when compared to divulging financial information over the Internet. This suggests that users do not understand the link between malicious software applications and the permissions obtained by the software once installed on a user's machine. Should a user download and install a malicious software application from the Internet, whether knowingly or by mistake, this software application can then obtain access to any of the files located on the computer. Also, it can copy any information the user types into an Internet browser on any website. This has serious implications for the severity of consequences of the loss of any kind of information, be it financial, personal, or potentially embarrassing. The critical observation here is that users do not appear to understand that the downloading and installation of malicious software applications trump any other potentially dangerous online activity, as it can gain access to any information once it has been installed on the user's machine. This shows a drastic lack of understanding of the risks of engaging in online interactions, when dealing specifically with malicious software applications. A related misunderstanding concerns the immediacy of effect of downloading and installing a malicious software application from the Internet. Users demonstrate difficulty in understanding that malicious software applications can gain access to their computer files both immediately after having been installed on their machine, as well as after some time has passed (e.g., a few hours, days, or even weeks).

These points suggest a lack of user understanding concerning malware and phishing attacks. Overall, the distribution of participant responses shows that users do, to some 
extent, understand some of the risks in online interactions, but are often unable to measure both the appropriate likelihood of something going wrong, and the corresponding severity of consequences associated with the online activity in question.

Themes. Overall, study two showed that users rated the risks associated with financially linked activities as severe, at the same time rating the risks associated with activities linked to embarrassing situations as more likely to occur. However, there were some important mismatches between people's ratings of related activities, such as the downloading and installation of software applications (which could contain malware) in relation to any other online activity that could be affected by this. As such, the overarching theme that emerged in this study was an inconsistency in assessing risk linked to online activities. This perhaps arises because of a poor mental model supporting the basis for decision making.

\section{Study Three: Understanding of Internet Software Abilities}

The third study was to explore participants' understanding of Internet structure in general, particularly in relation to malware, communications between computers and servers, and website security mechanisms.

The findings of study three demonstrated that some users do not understand the series of events that can lead to their computer being attacked, or their online browsing session to be monitored by a third party. This evidence points to a general misunderstanding that relates to the dangers of what malware can do once it has been installed on a user's computer. They also don't completely understand the mechanisms related to domain names and Web addresses. It can be argued that this could be attributed to users not understanding the series of events that can lead to their computer being attacked, such as downloading a software application from the Internet could potentially lead to a user's online bank account username and password being copied.

Furthermore, this study has shown evidence about a user's perception of risk regarding online activities, in relation to the conjunction rule. The conjunction rule stipulates that the probability that $\mathrm{X}$ and $\mathrm{Y}$ are simultaneously true, is always less than or equal to the 
probability that $\mathrm{X}$ is true. A violation of this rule is known as a conjunction fallacy (Tversky \& Kahneman, 1983). Research conducted on probabilities of events, in accordance with this rule, has shown that people tend to violate it systematically in a variety of different situations, such as decisions about risk. This fallacy has surfaced in the results obtained in the study. For example, users stated that malware programs always compromise a user's computer by sending their information to a third party, but they reported less certainty about the same malware programs gaining access to and changing various types of files found on their computer. This disconnect between what experts know as a likely chain of events, shows that a large proportion of users do not understand how one threat can, through this sequence of events, lead to the compromising of different parts of the user's system.

Themes. Study three showed that users have difficulty in grasping the threats and dangers associated with malware. In particular, people don't understand that malware can negatively affect the information found on — or communicated by - their computer, once a malicious software application has been installed on their computer. The mismatch between their level of understanding and the actual risks involved with malware extends to all activities conducted online as well as offline, and how this relates to general areas of Internet security, such as browsing (e.g., domain names and Web addresses), downloading software, and engaging in social media (e.g., Facebook). As such, the overarching theme that emerged in this study was a misunderstanding of the power of malware.

\section{Study Four: Exploring Understanding and Confidence}

The fourth study was to conduct exploratory analysis on participant understanding of Internet structure in general, based on findings from the previous studies.

Participants showed a general misunderstanding of domain name structure. Specifically, they misunderstand how the various parts of the domain name are structured and what differentiates them from Web addresses. Structurally, domain names can begin with a sequence of specific subdomains (e.g., www, www1, remote, login, webserver, etc.), a dot 
delimiter, the main domain name itself (e.g., walmart), and finally the top level domain (e.g., .com, .net, .org).

During testing, participants demonstrated difficulty recognizing and separating these domain name parts, and deciding which domain names could be seen as potentially illegitimate (e.g., www.walmart.ebuying.com versus www.ebuying.walmart.com). These issues will only become worse with the newly created top-level domains (e.g., .info, .tv, .biz, etc.). As an example, Carleton University's Sprott Business School once used the domain name sprott.carleton.co instead of sprott.carleton.ca. People who are aware of Carleton University and its website (www.carleton.ca) would most likely be confused with this top-level domain, which is actually associated with websites in the country of Columbia.

Another issue in understanding domain names was seen regarding sub-domains. For example, in www.weeklyad.walmart.com, the weeklyad portion of the domain is the subdomain. Participants had a difficult time in understanding what a subdomain such as this one was, who was responsible for its creation on the website server, and who administered it once it was created. This provides further evidence to support the claim of this thesis work that a large proportion of people simply don't understand the makeup of the domain name, its functions, and how it is administered once it is created and assigned to a given website. For example, a subdomain that ressembles a known top-level domain may have no connection whatsoever with the top-level domain, but users might assume it does. This misunderstanding would leave users vulnerable to phishing.

Similar problematic areas were seen regarding Web addresses. A Web address is defined as the complete address of a page, document, or file on a web server (e.g., http://www . walmart.com/cp/toys/4171/). Participants had a difficult time understanding the difference between a domain name and a Web address, and what constitutes a Web address. Some participants were unable to identify which components could be contained within a Web address, such as dots, hyphens, special characters, and so forth. They were even more confused when asked which special characters could be used within a Web address, and where these characters could be placed within the address itself. This offers some 
insight into the complexity of the makeup of a domain name and Web address.

Testing showed that communications between a user's computer and a website server were poorly understood. Participants were surveyed on the differences between the secured https protocol and the unsecured http protocol. Results showed that users do, to some extent, understand that the https protocol is more secure than the http protocol, but they don't seem to understand the mechanisms controlling how these protocols work. Some participants were aware of how the https protocol protects their information submitted via an online form, while others thought that the http protocol did the same. There was a general misunderstanding of how both the security behind the https protocol is rendered useless if a malware application has been installed on the user's machine, as it can copy the information submitted via the online form while the user is typing it into the browser's text input fields. This general misunderstanding of the http and https communications protocols provides strong evidence to support that people don't grasp the importance of protecting their information via different methods, such as only using https protocols when engaging in online activities that require the exchange of financial information via online forms.

A somewhat startling result was observed within participant responses to certain questions about malware protection. It was ascertained that users believe that the abilities of antivirus software applications far surpass their actual abilities. In the study surveys, participants stated that they believe antivirus applications are able to look for, identify, and remove any and all potentially malicious software applications from their computer, regardless of type and recency. However, in fact, antivirus software applications are only able to locate and delete malware that the creators of the software know exist. In particular, the software tests for signatures (patterns that exist in known malware), and simple heuristics, and these are not always sufficient (Heyman, 2007). This is why malware lists are updated frequently with newer signatures and heuristics, and frequent updates to the antivirus software application are needed in order to better protect the user from these dangers of attack. 
In the studies, it was shown that people have some knowledge about Internet security. However, where that knowledge is not strong, users may still not make the right decisions. Evidence has shown that there is a substantial proportion of people who aren't confident about their knowledge regarding Internet technology and security mechanisms. When tested on various questions regarding domain names, communications, malicious software applications, and other themes, people often reported being not confident at all - or only somewhat confident - regarding their answers to the questions in the various sections of the survey. As described above, user understanding of critical security mechanisms in some cases is generally poor. In other cases, however, most users have good understanding but with only limited confidence. This lack of confidence about how the Internet works, and the dangers that exist when engaging in online activities, can also pose a problem because users will not be able to reason appropriately when new threats arise.

By examining user responses to the questions found in the survey, and comparing it to their subsequent confidence ratings for each of these questions, this puts the provided responses more in context with their true beliefs, attitudes, and motivations towards the Internet and its security mechanisms. Thus, this allows for a deeper understanding of what users do know, what they believe they know and, most importantly, what they do not know regarding the potential dangers of the Internet, as well as the security mechanisms that are in place to help protect them from potential attacks on their personal information.

Themes. Following similar testing methods as the previous study, study four once again showed that users have difficulty in understanding malware. In this study, however, a clear lack of uncertainty was seen on the part of the participants when questioned about various aspects of malicious software applications. In particular, even though some participants showed good understanding of the key aspects of software applications, some reported very little and many reported no confidence at all in their answers. The theme that therefore emerged was a lack of confidence. It was also found that users place a large amount of trust in their antivirus software applications to protect them from any and all dangers on the Internet, even though these programs aren't one hundred percent effective at this task, 
and are there in order to help protect people but not to shield them from threats completely. Users understand some aspects of the elements involved in assuring security, but they do not known their limitations and the implications. This brings forward the theme of a poor mental model of the mechanisms of antivirus software.

\section{Study Five: Heuristics, Knowledge, and Self-Perception}

The fifth study was to explore the basis on which users make decisions regarding domain names and malware. The study also explored relationships between knowledge, perception of risk, attitudes, and beliefs about various aspects of the Internet.

This study looked at a novel way of delving into user understanding of various facets of the Internet. The research specifically looked at decisions made regarding the legitimacy of domain names and software applications, and how users make these judgments. The research targeted user understanding of domain names and software applications via heuristics. Specifically, it was investigated whether a "keyword" heuristic was used in assessing domain names, and a "functionality" heuristic was used in assessing capability of malware.

The results mostly suggest that users do not make use of the keyword heuristic when determining risks associated with domain names. Should they have been making use of the keyword heuristic, there would have been no significant differences between legitimate domain names and potentially illegitimate domain names. These findings led to the conclusion that users are able to recognize, to some degree, how a domain name is structured, and how to identify potentially malicious websites. However, a disturbing finding was that the "Bank of America" domain names were rated as having no differences in terms of level of risk by users. This is problematic as, out of the five pairs of domain names, this pair would likely be the most damaging in terms of financial loss should someone become victim to a phishing attack.

Further results show, however, that users do make use of the functionality heuristic when determining risks associated with downloading and installing certain types of software 
applications. Specifically, users appear to believe that software applications can only access the files and perform operations on a user's computer for which the software was supposedly intended to function. Users do not understand that any software application, if programmed as such, can have unfettered access to any of the user's files located on the computer. This has serious implications for user understanding of malware. There appears to be a pattern between the studies in this thesis of user misunderstanding of malicious software applications. Whether it's risk-based, trust-based, or heuristic-based, users simply do not understand that any software application can potentially access any files on a computer, and thus copy any files and information and send it to a third party via the Internet. Moreover, malware can even monitor communications within the user's computer, allowing keystroke logging and access to online forms. Accordingly, it appears that users faced with having to make a decision, they make it on the basis of the intended functionality of the software, thereby allowing malicious software that promises benign functionality.

Results from the final study also provide evidence about users' attitudes, beliefs, and self-reported expertise. These results suggest that correlations exist between some of the self-rated attitude dimensions (e.g., knowledgeability, motivation, etc.). Some of these correlations offer additional insight into people's beliefs about their understanding of the Internet, as well as who they believe is responsible in protecting them when engaging in online activities. In particular, the responsibility dimension was shown to be an issue, with users who possess less knowledge about Internet structure and security mechanisms attributing more responsibility to other people. The results suggest that the less someone knows, the more they think it's someone else's responsibility to keep them secure while engaging in online activities, such as the government, or the police. These users also believe that responsibility should be shouldered by the entities in charge of creating software (e.g., software companies such as Adobe, Microsoft, etc.), entities providing Internet access (e.g., Internet Service Providers), and entities claiming to protect users against malicious software applications (i.e., antivirus software companies, such as McAfee, Norton, etc.). Contrarily, the more someone knows about the Internet, the more they believe it is their 
own responsibility to ensure they are kept safe while engaging in online activities.

It is also worth mentioning that users' knowledge scores did not correlate very highly with their perception of risk associated with engaging in various online activities. It was expected that users with a higher knowledge of the various functions of the Internet, would assess risk more correctly than users with less knowledge of the Internet. It came as a surprise that this was not the case, which leads to the suspicion that these two dimensions, knowledge and risk perception, simply do not correlate, and that there are potentially deeper explanations to explore, which influence the outcome of these results.

Themes. Study five showed evidence that heuristics may be used when people make decisions about trusting low risk versus high risk domain names, as well as make judgments

about the functionality of a particular piece of software. The study also showed that there is little relationship between people's knowledge and perception of risk when questioned Internet security. However, a relationship was found between knowledge and recognition of responsibility for one's own actions when engaging in online activities. Overall, it appears that a theme emerges of willingness to make decisions despite weak understanding. This is reflected both in the use of heuristics and by people with lower knowledge feeling that they had little responsibility for protecting themselves online.

\section{Mental Models}

This last section of the general discussion examines the influence of mental models in user interactions in online activities. It is believed that, due to the strong evidence resulting from the five studies conducted within this thesis work, mental models play an important role in determining how a person will interact with the Internet and online activities in general. The importance of mental models for computer security was proposed by BravoLillo et al. (2010), and the findings in this thesis confirm that importance.

In psychology, mental models play a very important part in understanding how people make decisions that lead to a specific outcome (Morgan et al., 2002). Examples of this could include circumstances where a person chooses to drive his or her car during a snowstorm, 
which relies on the person's understanding of a car's mechanics while being driven in snow, or whether a person decides to trust a given website based on the visual information provided to them by their website browser (e.g., Mozilla Firefox, Google Chrome). Relevant to this thesis work, knowledge about people's mental models could go a long way to understanding why users behave in various ways when engaging in online activities, and what influences their decision-making process when deciding to trust and use a given website, as well as provide personal information to this website in question via online forms. Evidence from this thesis work suggests that users, in general, do not possess accurate and complete mental models that represent the model of location of understanding of Internet usage (i.e., Internet communications), as well as the various security mechanisms in place to keep them safe from online attacks (e.g., phishing websites, malicious software applications, etc.).

A potential model of location of understanding of Internet usage might follow the steps taken by the system, from beginning to end, when a user engages in an online activity. The model could begin with the user's physical world, where their computer is located within their workspace; the next component is the user's computer, where they engage in the online activity; the browser gives them access to various websites; the Internet Service Provider allows the user to communicate with other servers found on the Web; the server section of the model could refer to all other servers that enter into communication with the ISP and, ultimately, the end-user; finally, the third-party component of the model relates to any of the ancillary Web services, such as credit card processing servers. The results from studies three and four about user misunderstanding of the capabilities of software (and hence malicious software applications) suggest that the proposed model might be expanded to cover the levels of protection that govern software and its access to files and resources on the user's computer.

The issue then arises where a user's mental model of the workings of the Internet is either missing information (i.e., incomplete), contains inaccurate information, or is poorly constructed from information obtained by the user and put together over time in a scheme that goes against this flow model. It is believed that these issues surrounding erroneous 
mental models of the Internet are often the cause of the many issues that arise from situations where a user' personal information has been copied, or a username and password have been stolen for an account belonging to this same user. By this same reasoning, it follows that a user will be unable to fully protect himself or herself from the various dangers on the Internet if his or her mental model of the flow of Internet usage is lacking necessary information in order to avoid these dangers, and protect themselves from attacks on their personal information. Research by Wash (2010) found that users had developed "folk models" that lead to problems in the absence of better information. Conversely, other research shows that improving users' mental models enables them to better assess risks (Byrne et al., 2012).

This same issue arises concerning the security mechanisms in place to help users protect themselves from malicious attacks online. Evidence from the experiments comprising this thesis demonstrates that a large proportion of users do not fully understand how these security mechanisms can protect them from having their information copied or stolen by an attacker. The same issue of incomplete or incorrect mental models can also be applied in these cases. In order to better protect themselves from online attacks, users must have a full working mental model of the security mechanisms integrated into the browser chrome. One of the issues regarding problems in understanding these security mechanisms is that there exists a myriad of Internet browsers. These browsers function mostly in the same way, in allowing users to browse the various websites on the Internet, interact with e-commerce websites, participate in social networking, and so forth. The issues stem from the fact that most browsers do not depict the security mechanisms in the same fashion. Some browsers show the lock icon - which represents secure communication between the user's computer and the website server (i.e., https secured communications) - on the left side of the address bar, while other browsers show this same lock icon within the website address itself. Some website browsers hide the lock icon entirely unless the user selects the website address directly, thereby showing the lock icon within the selected website address, and providing security certificate information to the user once he or she clicks directly on the lock icon. 
This lack of chrome consistency between website browsers can greatly affect the creation and sustainability of a full and complete mental model of the security mechanisms of the Internet. Moreover, the design of browser chrome changes continually, increasing the difficulty users would have in understanding the important elements. The importance of the browser chrome might even have been undermined by Google naming their browser "Chrome".

Even if a user has a correct mental model, using this model to determine whether a website is trustworthy or not might represent a considerable investment of time. For example, the user might have to carefully inspect the domain name, along with elements of the browser chrome, and then interact with the browser to inspect the certificate, and assess its contents. Moreover, making a decision about downloading a given piece of software may entail even more effort in attempting to determine its legitimacy. Herley (2009) discusses the cost of learning and applying security knowledge in relationship to risks and benefits, and suggests that some users not following advice might be "rational". Of course, any risk decision will involve a cost/benefit trade-off. Moreover, even the best mental model will not suffice to absolutely protect someone from all dangers. It may well be, however, that critical elements of a correct mental model may present little burden to users and may help avoid many dangers. The human-in-the-loop framework proposed by Cranor (2008) highlights that both automated and human driven processes should be involved in security, and also that human driven processes have limits and capacities. Careful research is needed to determine how much time and energy users can devote to security in order to protect themselves without relying on automated protection. 


\section{Conclusion}

The research question for this thesis work was "How do people perceive, understand, and make decisions regarding the dangers associated with malicious websites, and are they able to identify and understand the various levels of risks associated with engaging in online activities?"

Five studies were conducted in order to address this research question. Study one examined how users determined website legitimacy using a qualitative approach, and showed users were willing to make decisions but, when questioned, showed a strong lack of confidence. Study two investigated users' perception of risk in relation to various online activities, and showed that they do make judgments about risk, but these demonstrate some serious inconsistencies. Study three evaluated user understanding of various aspects of online activities, and found evidence that users have the most trouble in understanding the mechanisms involved in malicious software applications. Study four used exploratory analysis methods to examine users' knowledge of daily interactions on the Internet, and showed that their confidence in relationship to these interactions on the Internet was poor. The final study examined whether people use heuristics in decision-making, and explored several other relationships, finding some support for the role of heuristics, and a relationship between knowledge and the attribution of responsibility to oneself.

Within these five studies were found several themes. Study one illustrated that people made decisions but when questioned showed lack of confidence. Study two showed that participants were inconsistent in assessing risk linked to online activities perhaps because of a poor mental model. Study three showed that participants demonstrated an important misunderstanding of the power of malware. Study four further demonstrated the lack of confidence demonstrated by the participants, as well as their poor mental models in understanding the mechanisms of Internet security, especially antivirus software. Study five showed that people are still willing to make decisions with weak understanding of these mechanisms, reflected both in the use of heuristics and the attribution of responsibility.

In response to the research question, users do show understanding of some of the 
key concepts in Internet security, and often make reasonable decisions. However, there are important anomalies. For example, many users had important misunderstandings about malware, suggesting they had poor mental models about the capabilities of malware and the capabilities of antivirus software applications in protecting them from threats online. Moreover, participants showed lack of confidence across a range of issues, but in practice they were still willing to make decisions even with this uncertainty. Some evidence was found which suggests that users employ heuristics in making such decisions and judgments under uncertainty, such as the proposed keyword and functionality heuristics.

The contributions of this thesis are the five empirical studies exploring the basis of user decision-making in regards to malicious websites and the download and installation of software applications. The key findings are poor mental models, especially with respect to malware, the willingness to make decisions despite significant uncertainty, and the potential role of heuristics in that process.

\section{Limitations}

Some limitations were identified within the studies comprising this thesis research. The topic of Internet security covers a wide range of areas and mechanisms. Within these mechanisms, there exist many exceptions to the rule. The questions that were created for testing purposes within each study were not created to account for each of these exceptions, potentially leading to some confusion on the part of the participants, should their knowledge be advanced enough to spot these omissions. Beyond this, there are also issues of ecological validity outlined by Motiee et al. (2010), involving user attention, responsibility, and genuine risk. Furthermore, participants were recruited via the Amazon Mechanical Turk online marketplace. Previous research has shown that, overall, data collected using Mechanical Turk is reliable (e.g., (Buhrmester et al., 2011, Kittur et al., 2008)). However, there is still some need for caution in interpreting data collected from this participant pool. 


\section{Future Work}

The thesis research opened the door to a few different interesting possibilities for future work. First, as mental models play a very important role in a person's decision-making regarding online activities, it would be a big step forward to develop educational materials that teach security mechanisms to users in general, so they are better able to make informed decisions about browsing, downloading, and engaging in any other online activity. This would help those with poor mental models develop more knowledge of the key areas in Internet security. Second, since people in general have a difficult time in understanding browser chrome security cues (e.g., (Akhawe \& Felt, 2013)), studying the effectiveness of various different browser security indicators would allow software developers to learn more about which cues are effective and which ones are not. This would lead them to design and retool the chrome in order to make it easier for users to understand what the browser is telling them about a particular website. Ultimately, the advice offered via the browser is based on knowledge from certificate authorities or other reputable sources, and so supports the concept of institutional embeddedness, described as one of the core elements for forming trust by Riegelsberger et al. (2005). Third, further research could be conducted in order to pinpoint which heuristics people are actually using when making decisions about online activities. Then, browser chrome security cues should be redesigned with these heuristics in mind. Finally, for those users who have greater difficulty in understanding security mechanisms, and are less willing to learn more about them, operating system and software developers could implement a closed-market type of approach to software application distribution. Closed markets are operated and managed by reputable authorities, suggesting they also support Riegelsberger's concept of institutional embeddedness. Closed markets would make users aware of the permissions each application requires in order to be installed, thus making them aware of which types of files the program in question has access to. The user could then decide whether he or she wants to proceed with the installation, or deny access to the software application. This approach has been applied in the Android operating system for mobile devices, but with questionnable results (Kelley et al., 2012). 


\section{References}

Adams, A., \& Sasse, M. A. (1999). Users are not the enemy. Communications of the ACM, 42(12), 40-46.

Akhawe, D., \& Felt, A. P. (2013). Alice in warningland: A large-scale field study of browser security warning effectiveness. In Presented as part of the 22nd usenix security symposium (usenix security 13) (pp. 257-272). USENIX.

Almuhimedi, H., Felt, A. P., Reeder, R. W., \& Consolvo, S. (2014). Your reputation precedes you: History, reputation, and the chrome malware warning. In Symposium on usable privacy and security (soups).

Anderson, R. (2008). Security engineering. John Wiley \& Sons.

Bacon, M. (2014, November). Social engineering. Retrieved from http://searchsecurity .techtarget.com/definition/social-engineering.

Baxter, K., Malahy, L. W., \& Lubin, J. (2013). Pirates of the search results page. In Proceedings of the sigchi conference on human factors in computing systems (pp. 30233026). ACM.

Beyer, S., \& Bowden, E. M. (1997). Gender differences in self-perceptions: Convergent evidence from three measures of accuracy and bias. Personality and Social Psychology Bulletin, 23(2), 157-172.

Biddle, R., van Oorschot, P. C., Patrick, A. S., Sobey, J., \& Whalen, T. (2009). Browser interfaces and extended validation ssl certificates: an empirical study. In Proceedings of the 2009 acm workshop on cloud computing security (pp. 19-30).

Bontchev, V. (2005). Current status of the caro malware naming scheme. Virus Bulletin (VB2005), Dublin, Ireland.

Bravo-Lillo, C., Cranor, L. F., Downs, J., \& Komanduri, S. (2010). Bridging the gap in computer security warnings: A mental model approach. IEEE Security $\&$ Privacy(2), $18-26$.

Brayton, J., Finneman, A., Turajski, N., \& Wiltsey, S. (2006, October). PKI - public key infrastructure. http://searchsecurity.techtarget.com/definition/PKI. 
Buhrmester, M., Kwang, T., \& Gosling, S. D. (2011). Amazon's mechanical turk a new source of inexpensive, yet high-quality, data? Perspectives on Psychological Science, $6(1), 3-5$.

Burgoon, J. K., Blair, J. P., \& Strom, R. E. (2005). Heuristics and modalities in determining truth versus deception. In HICSS'05. proceedings of the 38th annual hawaii international conference on system sciences (p. 19a).

Burton, I., Kates, R. W., \& White, G. F. (1978). The environment as hazard. New York: Oxford University Press.

Byrne, Z., Weidert, J., Liff, J., Horvath, M., Smith, C., Howe, A., \& Ray, I. (2012). Perceptions of internet threats: Behavioral intent to click again. In Proc. of the society for industrial and organizational psychology conference.

Camp, L. J. (2009, Fall). Mental models of privacy and security. Technology and Society Magazine, IEEE, 28(3), 37-46.

Cialdini, R. B. (2009). Influence: Science and practice. Pearson Education.

Coron, J. S. (2006). What is cryptography? IEEE Security and Privacy, 4(1), 70-73.

Cranor, L. F. (2008). A framework for reasoning about the human in the loop. UPSEC, $8,1-15$.

Cranor, L. F., \& Garfinkel, S. (2005). Security and usability: designing secure systems that people can use. "O’Reilly Media, Inc.".

Crump, M. J., McDonnell, J. V., \& Gureckis, T. M. (2013). Evaluating Amazon's Mechanical Turk as a tool for experimental behavioral research. PLoS ONE, 8(3), e57410.

Dhamija, R., Tygar, J. D., \& Hearst, M. (2006). Why phishing works. In CHI 'O6 proceedings of the conference on human factors in computing systems (pp. 581-590).

Dictionaries, O. (2016). Ground truth. http://wWw.oxforddictionaries.com/ definition/english/ground-truth.

Dodge, R. C., Carver, C., \& Ferguson, A. J. (2007). Phishing for user security awareness. Computer \& Security, 26(1), 73-80.

Douglas, M., \& Wildavsky, A. (1982). Risk and culture: An essay on the selection of 
technological and environmental dangers. Berkeley: University of California Press.

Downs, J. S., Holbrook, M., \& Cranor, L. F. (2007). Behavioral response to phishing risk. In Proceedings of the anti-phishing working groups 2 nd annual ecrime researchers summit (pp. 37-44). ACM.

Downs, J. S., Holbrook, M. B., \& Cranor, L. F. (2006). Decision strategies and susceptibility to phishing. In Proceedings of the second symposium on usable privacy and security (pp. 79-90).

Egelman, S., Cranor, L. F., \& Hong, J. (2008). You've been warned: An empirical study of the effectiveness of web browser phishing warnings. In Proceedings of the sigchi conference on human factors in computing systems (pp. 1065-1074). ACM.

Egger, F. N. (2000). Trust me, I'm an online vendor. Conference on Human Factors in Computing Systems: CHI '00 Extended Abstracts, 1(6), 101-102.

Felt, A. P., Reeder, R. W., Almuhimedi, H., \& Consolvo, S. (2014). Experimenting at scale with google chrome's ssl warning. In Proceedings of the sigchi conference on human factors in computing systems (pp. 2667-2670). ACM.

Fischhoff, B., Slovic, P., Lichtenstein, S., Read, S., \& Combs, B. (1978). How safe is safe enough? A psychometric study of attitudes towards toward technological risks and benefits. Policy Sciences, 9, 127-152.

Fischhoff, B., Slovic, P., Lichtenstein, S., Read, S., \& Combs, B. (2000). How safe is safe enough? A psychometric study of attitudes towards toward technological risks and benefits. In P. Slovic (Ed.), The perception of risk (pp. 80-103). Earthscan Publications Ltd.

Fogg, B. J., Soohoo, C., Danielson, D. R., Marable, L., Stanford, J., \& Tauber, E. R. (2003). How do users evaluate the credibility of web sites?: A study with over 2,500 participants. In Proceedings of the 2003 conference on designing for user experiences (pp. 1-15). New York, NY, USA: ACM. Retrieved from http://doi.acm.org/10 $.1145 / 997078.997097$ doi: 10.1145/997078.997097

Fonteyn, M. E., Kuipers, B., \& Grobe, S. J. (1993). A description of think aloud method 
and protocol analysis. Qualitative Health Research, 3(4), 430-441.

Furnell, S., Bryant, P., \& Phippen, A. D. (2007). Assessing the security perceptions of personal internet users. Computers \& Security, 26(5), 410-417.

Garfinkel, S., \& Lipford, H. R. (2014). Usable security: History, themes, and challenges. Synthesis Lectures on Information Security, Privacy, and Trust, 5(2), 1-124.

Herley, C. (2009). So long, and no thanks for the externalities: The rational rejection of security advice by users. In Proceedings of the 2009 workshop on new security paradigms workshop (pp. 133-144).

Herzberg, A. (2009). Why johnny can’t surf (safely)? attacks and defenses for web users. Computers 6 Security, 28(1âĂŞ2), 63-71.

Heyman, K. (2007). New attack tricks antivirus software. Computer, 40 (5), 18-20.

Huber, M., Kowalski, S., Nohlberg, M., \& Tjoa, S. (2009). Towards automating social engineering using social networking sites. In 2009 international conference on computational science and engineering (pp. 117-124).

Jagatic, T. N., Johnson, N. A., Jakobsson, M., \& Menczer, F. (2007). Social phishing. In Communications of the ACM (Vol. 50, pp. 94-100). ACM: New York.

Kahneman, D. (2011). Thinking, fast and slow. Macmillan.

Kelley, P. G. (2010). Conducting usable privacy \& security studies with Amazon's Mechanical Turk. In Symposium on usable privacy and security (soups). ACM.

Kelley, P. G., Consolvo, S., Cranor, L. F., Jung, J., Sadeh, N., \& Wetherall, D. (2012). A conundrum of permissions: installing applications on an android smartphone. In Financial cryptography and data security (pp. 68-79). Springer.

Kincaid, J. (2011, February). Google rolls out two-factor authentication for everyone. you should use it. http://techcrunch.com/2011/02/10/google-rolls-out-two -factor-authentication-for-everyone-you-should-use-it/.

Kirillov, I., Beck, D., Chase, P., \& Martin, R. (2011). Malware attribute enumeration and characterization. Mitre.

Kirlappos, I., Sasse, M., \& Harvey, N. (2012). Why trust seals donâĂŹt work: A study of 
user perceptions and behavior. Trust and Trustworthy Computing, 308-324.

Kittur, A., Chi, E. H., \& Suh, B. (2008). Crowdsourcing user studies with mechanical turk. In Proceedings of the sigchi conference on human factors in computing systems (pp. $453-456)$.

Kumaraguru, P., Cranshaw, J., Acquisti, A., Cranor, L., Hong, J., Blair, M. A., \& Pham, T. (2009). School of phish: A real-world evaluation of anti-phishing training. In Proceedings of the 5th symposium on usable privacy and security (pp. 3:1-3:12). ACM.

Lacohée, H., Phippen, A. D., \& Furnell, S. M. (2006). Risk and restitution: Assessing how users establish online trust. Computers \& Security, 25 (7), 486-493.

LaRose, R., Rifon, N. J., \& Enbody, R. (2008). Promoting personal responsibility for Internet safety. Communications of the ACM, 51(3), 71-76.

Liang, H., \& Xue, Y. (2009). Avoidance of information technology threats: A theoretical perspective. MIS quarterly, 71-90.

Liang, H., \& Xue, Y. (2010). Understanding security behaviors in personal computer usage: A threat avoidance perspective. Journal of the Association for Information Systems, $11(7), 394-413$.

Lin, E., Greenberg, S., Trotter, E., Ma, D., \& Aycock, J. (2011). Does domain highlighting help people identify phishing sites? In Proceedings of the sigchi conference on human factors in computing systems (pp. 2075-2084). ACM.

Lindgaard, G., Fernandes, G., Dudek, C., \& Brown, J. (2006). Attention Web designers: You have 50 milliseconds to make a good first impression! Behaviour $\&$ Information Technology, 25(2), 115-126.

Mellor, C. (2005, September). TPM - trusted platform module. http://features .techworld.com/storage/1777/tpm-trusted-platform-module/\#23.

Menezes, A. J., Vanstone, S. A., \& Oorschot, P. C. v. (1996). Handbook of applied cryptography. CRC Press.

Metzger, M. J., Flanagin, A. J., \& Medders, R. B. (2010). Social and heuristic approaches to credibility evaluation online. Journal of Communication, 60(3), 413-439. 
Microsoft. (2005, December). Data confidentiality. http://msdn.microsoft.com/en-us/ library/ff650720.aspx.

Millar, M. G., \& Millar, K. U. (1997). The effects of cognitive capacity and suspicion on truth bias. Communication Research, 24(5), 556-570.

Morgan, M. G., Fischhoff, B., Bostrom, A., \& Atman, C. J. (2002). Risk communication: A mental models approach. Cambridge University Press.

Motiee, S., Hawkey, K., \& Beznosov, K. (2010). The challenges of understanding users' security-related knowledge, behaviour, and motivations. In Symposium on usable privacy and security.

Neupane, A., Rahman, M. L., Saxena, N., \& Hirshfield, L. (2015). A multi-modal neurophysiological study of phishing detection and malware warnings. In Proceedings of the 22nd acm sigsac conference on computer and communications security (pp. 479-491). ACM.

Operations, C. S. I. (2015). What is the difference: Viruses, Worms, Trojans, and Bots? http://www.cisco.com/web/about/security/intelligence/virus-worm -diffs.html

Patrick, A. (2007, March). Commentary on research on new security indicators. Retrieved from http://www. andrewpatrick.ca/essays/commentary-on-research-on -new-security-indicators/

Patrick, A. S., Briggs, P., \& Marsh, S. (2005). Designing systems that people will trust. In L. F. Cranor \& S. Garfinkel (Eds.), Security and usability: Designing secure systems that people can use (pp. 75-99). Sebastopol, CA: O'Reilly Media, Inc.

Prabha, C., Connaway, L. S., Olszewski, L., \& Jenkins, L. R. (2007). What is enough? satisficing information needs. Journal of Documentation, 63(1), 74-89.

Qin, T. (2007). An investigation of heuristics of human judgment in detecting deception and potential implications in countering social engineering. In Intelligence and security informatics (pp. 152-159). IEEE.

Rafalak, M., Abramczuk, K., \& Wierzbicki, A. (2014). Incredible: Is (almost) all web 
content trustworthy? analysis of psychological factors related to website credibility evaluation. In Proceedings of the 23rd international conference on world wide web (pp. 1117-1122). International World Wide Web Conferences Steering Committee.

Rasmussen, R., Aaron, G., \& Routt, A. (2011). Global phishing survey: trends and domain name use in 1h2011. Anti-Phishing Working Group.

Riegelsberger, J., Sasse, M. A., \& McCarthy, J. D. (2005). The mechanics of trust: A framework for research and design. International Journal of Human-Computer Studies, 62(3), 381-422.

Rieh, S. Y., \& Belkin, N. J. (1998). Understanding judgment of information quality and cognitive authority in the www. In Proceedings of the 61st annual meeting of the american society for information science (Vol. 35, pp. 279-289).

Sagiroglu, S., \& Canbek, G. (2009, Fall). Keyloggers. Technology and Society Magazine, IEEE, 28(3), 10-17.

Schechter, S. E., Dhamija, R., Ozment, A., \& Fischer, I. (2007). The emperor's new security indicators: An evaluation of website authentication and the effect of role playing on usability studies. In Security and privacy, 200\% proceedings of the 2007 ieee symposium on.

Schmitz, C. (2010). Limesurvey [computer software].

Sheng, S., Magnien, B., Kumaraguru, P., Acquisti, A., Cranor, L. F., Hong, J., \& Nunge, E. (2007). Anti-phishing phil: The design and evaluation of a game that teaches people not to fall for phish. In Proceedings of the 3rd symposium on usable privacy and security (pp. 88-99).

Shi, P., Xu, H., \& Zhang, X. L. (2011). Informing security indicator design in web browsers. In Proceedings of the 2011 iconference (pp. 569-575). ACM.

Shinder, D. (2005, June). Code signing: Is it a security feature? Retrieved from http:// WwW.windowsecurity.com/articles/Code-Signing.html.

Short, J. F. (1984). The social fabric at risk: Toward social transformation of risk analysis. American Sociological Review, 49, 711-725. 
Simon, H. (1956). Rational choice and the structure of the environment. Psychological Review, 63, 129-138.

Slovic, P. (1975). Choice between equally valued alternatives. Journal of Experimental Psychology, 1(3), 280-287.

Slovic, P. (1987). Perception of risk. Science, 236, 280-285.

Slovic, P., \& Lichtenstein, S. (1968). Relative importance of probabilities and payoffs in risk taking. Journal of Experimental Psychology Monograph, 78(3, Part 2), 1-18.

Sobey, J., Biddle, R., Oorschot, P. C., \& Patrick, A. S. (2008). Exploring user reactions to new browser cues for extended validation certificates. In Proceedings of the 13th european symposium on research in computer security: Computer security (pp. 411427). Berlin, Heidelberg: Springer-Verlag.

Song, J., \& Zahedi, F. M. (2005). A theoretical approach to Web design in e-commerce: A belief reinforcement model. Management Science, 51(8), 1219-1235.

Sotirakopoulos, A., Hawkey, K., \& Beznosov, K. (2011). On the challenges in usable security lab studies: Lessons learned from replicating a study on ssl warnings. In Proceedings of the seventh symposium on usable privacy and security (pp. 3:1-3:18). New York, NY, USA: ACM. Retrieved from http://doi.acm.org/10.1145/2078827.2078831 doi: $10.1145 / 2078827.2078831$

Stajano, F., \& Wilson, P. (2011, March). Understanding scam victims: seven principles for systems security. Communications of the ACM, 54(3), 70-75.

Starr, C. (1969). Social benefit versus technological risk. Science, 165, 1232-1238.

Sunshine, J., Egelman, S., Almuhimedi, H., Atri, N., \& Cranor, L. F. (2009). Crying wolf: An empirical study of ssl warning effectiveness. In Proceedings of the 18th conference on usenix security symposium (pp. 399-416). USENIX Association.

Tekedia. (2011, May). Open vs. close - google android market and apple app store. http:// tekedia. $\operatorname{com} / ? \mathrm{p}=7216$

Tversky, A., \& Kahneman, D. (1973). Availability: A heuristic for judging frequency and probability. Cognitive Psychology, 5(2), 207-232. 
Tversky, A., \& Kahneman, D. (1974). Judgment under uncertainty: Heuristics and biases. Science, 185, 1124-1131.

Tversky, A., \& Kahneman, D. (1983). Extensional versus intuitive reasoning: The conjunction fallacy in probability judgment. Psychological Review, 90(4), 293-315.

Tversky, A., Kahneman, D., \& Moser, P. (1990). Judgment under uncertainty: Heuristics and biases. Rationality in Action: Contemporary Approaches, 171-188.

Tversky, A., Slovic, P., \& Sattath, S. (1988). Contingent weighting in judgment and choice. Psychological Review, 95(3), 371-384.

Verizon. (2012). Data breach investigations report. Verizon, Inc.

Wash, R. (2010). Folk models of home computer security. In Proceedings of the sixth symposium on usable privacy and security (p. 11).

Whalen, T., \& Inkpen, K. M. (2005). Gathering evidence: Use of visual security cues in web browsers. In Proceedings of graphics interface 2005 (pp. 137-145).

Whitten, A., \& Tygar, J. D. (1999). Why johnny can’t encrypt: A usability evaluation of pgp 5.0. In Usenix security (Vol. 1999).

Workman, M. (2008). Wisecrackers: A theory-grounded investigation of phishing and pretext social engineering threats to information security. Journal of the American Society for Information Science and Technology, 59(4), 662-674.

Wu, M., Miller, R. C., \& Garfinkel, S. L. (2006). Do security toolbars actually prevent phishing attacks? In Proceedings of the sigchi conference on human factors in computing systems (pp. 601-610). ACM.

Zurko, M. E., \& Simon, R. T. (1996). User-centered security. In Proceedings of the 1996 workshop on new security paradigms (pp. 27-33). ACM. 


\section{Appendix A}

\section{Study One: Ethics Application (Determining Website Legitimacy)}

\section{Purpose of Study}

Previous research shows that Internet users are likely to trust the majority of websites, whether the website's intent is legitimate or malicious. Earlier work has explored how users determine the trustworthiness of websites; for example, see the trust models of Riegelsberger (Riegelsberger, 2005) and Egger (Egger, 2000). More recent work has shown that users pay little attention to operating system and browser security indicators (Schecter et al., 2007).

Our research will examine the steps Internet users follow in order to determine the legitimacy of websites and/or online content. For example, some malicious websites may try to lure users to download malicious software, or to fraudulently obtain users' credentials. By better understanding how users determine which websites they trust, we can develop software to help them identify illegitimate content, and avoid the danger it represents.

\section{Method}

\section{Materials:}

- One standard Dell desktop computer running Windows 7 operating system with standard web browsers (e.g., Microsoft Internet Explorer, Mozilla Firefox).

- A series (approximately 5 to 10) of websites (including webpages, pop-up windows, and so forth), selected to exhibit a range of features that may or may not lead to concerns about the legitimacy of the website or content. The websites will be

carefully selected so as not to include any offensive or emotionally disturbing content. These websites will be based on the PSYC 6901 Independent Research conducted by Daniel LeBlanc in the Summer term 2010. Please see Appendix C for an example. 
- 2 questionnaires (demographics, multiple-choice post-test) created with the Limesurvey web survey system, running on our own secure web server. These questionnaires can be found in Appendices A and B.

- A role-play scenario script will be used in order to give participants a story in which to follow, so that they may assess the websites presented to them during the main research task (see Appendix D for our script including example websites and scenarios). Participants:

- 20 to 30 participants, aged 18 or older.

- Participants must be experienced Internet users (at least 2 years), and must use the Internet at least twice weekly. They must not be either experts or students in computer security.

- Participant pool will be gender balanced.

\section{$\underline{\text { References }}$}

Egger, F. N. (2000). Trust me, I'm an online vendor. Conference on Human Factors in Computing Systems: CHI 'O0 Extended abstracts on Human Factors in Computing Systems, 1(6), 101-102.

Riegelsberger, J. (2005). The mechanics of trust: A framework for research and design. International Journal of Human-Computer Studies, 62(3), 381-422.

Schecter, S. E., Dhamija, R., Ozment, A., and Fischer, I. (2007). Emperor's new security indicators: An evaluation of website authentication and the effect of role playing on usability studies. In Proceedings of the 2007 IEEE Symposium on Security and Privacy. 


\section{Informed Consent}

The purpose of an informed consent is to ensure that you understand the purpose of the study and the nature of your involvement. The informed consent must provide sufficient information such that you have the opportunity to determine whether you wish to participate in the study.

\section{Research Study: Determining Website Legitimacy}

Research personnel: The following people are involved in this research project, and may be contacted at anytime if you have questions or concerns: Daniel LeBlanc (dblanc@connect.carleton.ca, phone: 613-520-2600 ext. 1987), Dr. Robert Biddle (Faculty Sponsor, email: robert_biddle@carleton.ca, 613-520-2600 ext. 6317), Dr. Andrew Patrick (Adjunct Research Professor, email: andrew@andrewpatrick.ca, phone: 613-2779211). Should you have any ethical concerns about this research, please contact Dr. Monique Sénéchal, at monique_senechal@carleton.ca (613-520-2600 ext. 1155). For any other concerns, please contact Dr. Janet Mantler (Chair, Department of Psychology, 613520-2600 ext. 4171, psychchair@carleton.ca).

Purpose: The purpose of this study is to investigate the ways in which people are able to identify potentially dangerous websites and/or online content.

Task requirements: We are asking you to fill out a demographics questionnaire regarding your background. We will also ask you to answer a few brief multiple-choice and openended questions (approximately 10 to 15 questions) regarding your experience with online environments. The latter questionnaire will take approximately 5 minutes to complete and will be completed after the main research task. For the main task, we will ask you to inspect and assess several websites and explain to us your impression of the legitimacy of the content, and how you determined it. You will receive either a $1 \%$ increase in your final 
grade of PSYC 1001, PSYC 1002, PSYC 2001, or PSYC 2002 for participating in this study, or a $\$ 10.00$ honorarium. Testing will take place at the HCI lab (room 2110) on the Carleton University campus.

Potential risk/discomfort: There are no physical risks to participating in this study, beyond those involved in normal computer usage. If you feel any discomfort or distress, you may choose not to answer specific questions, and you will not be penalized in any way if you choose to do this. The debriefing form given to you at the end of the study provides contact details that you may use if you wish to obtain additional information.

Anonymity/Confidentiality: The data collected in this experiment are strictly confidential. All data are coded such that your name is not associated with the responses you provide. Any identifying information associated with your code will be confined to a single page that will be separated from your questionnaire, and kept in a separate, secured file by the research investigators, who will keep this information confidential.

Right to withdraw: Your participation in this study is entirely voluntary. At any point during the study, you have the right not to complete certain questions, or to withdraw from the study without penalty.

This study has received clearance by the Carleton University Psychology Ethics Board (Reference \#10-200). 


\section{Signatures}

I have read the above form and understand the conditions of my participation. My participation in this study is voluntary, and I understand that if at anytime I wish to leave the experiment, I may do so without having to give an explanation and with no penalty whatsoever. Furthermore, I am also aware that the data gathered in this study are confidential and anonymous with respect to my personal identity. My signature indicates that I agree to participate in this study.

Participant's Name:

Participant's Signature:

Date:

Researcher's Name:

Researcher's Signature

Date: 


\section{$\underline{\text { Debriefing }}$}

\section{Research Study: Determining Website Legitimacy}

\section{What are we trying to learn in this research?}

This research examines the steps Internet users follow in order to determine the legitimacy of websites and/or online content. For example, some malicious websites may try to lure users to download malicious software, or to fraudulently obtain users' credentials.

\section{Why is this important to scientists or the general public?}

Previous research has shown that Internet users are likely to trust the majority of websites, whether their intent is legitimate or malicious (see

http://hotsoft.carleton.ca/hotsoft/resources/website-trust/ for more information). By better understanding how users determine which websites they trust, we can develop software to help them identify illegitimate content, and avoid the danger it represents.

\section{What are our hypotheses and predictions?}

We expect that users will use a variety of methods, including the appearance of content, the reputation of companies, and some information provided by the operating system, in order to determine website legitimacy.

\section{Where can I learn more?}

In order to learn more about our research on security and trust in an online environment, please see our website at http://hotsoft.carleton.ca/security/ 


\section{What if I have questions later?}

If you have any remaining concerns, questions, or comments about the experiment, please feel free to contact Daniel LeBlanc (Principal Investigator) at: dblanc@connect.carleton.ca (613-520-2600 ext. 1987), Dr. Robert Biddle (Faculty Sponsor) at: robert_biddle@carleton.ca, 613-520-2600 ext. 6317, Dr. Andrew Patrick (Adjunct Research Professor) at: andrew@andrewpatrick.ca, 613-277-9211. Should you have any ethical concerns about this research, please contact Dr. Monique Sénéchal, at monique_senechal@carleton.ca (613-520-2600 ext. 1155). For any other concerns, please contact Dr. Janet Mantler (Chair, Department of Psychology, 613-520-2600 ext. 4171, psychchair@carleton.ca).

Thank you for participating in this research! 


\section{Announcement for recruiting participants (SONA system)}

Study Name: Browsing Websites

Description: This research examines the steps Internet users follow in order to determine the legitimacy of websites and/or online content. For example, some malicious websites may try to lure users to download malicious software, or to fraudulently obtain users' credentials.

Eligibility Requirements: We are looking for adults 18 years of age or older, who possess a minimum of 2 years of experience with the Internet, and be regular users (i.e., use the Internet at least 2 times each week). We wish to exclude anyone who is an expert or student in computer security.

Duration and Locale: 1 hour at he HCI lab (2110 HCI building). Instructions to the lab will be provided via an email once we receive your notice of participation.

Compensation: You will receive either a 1\% credit towards a psychology course (PSYC 1001, PSYC 1002, PSYC 2001, PSYC 2002), or a $\$ 10.00$ honorarium for your time.

\section{Researchers:}

Daniel LeBlanc (Principal Investigator), dblanc@connect.carleton.ca, 613-355-6533

Dr. Robert Biddle (Faculty Sponsor), robert_biddle@carleton.ca, 613-520-2600 ext. 6317

Dr. Andrew Patrick (Adjunct Research Professor), andrew@andrewpatrick.ca, 613-2779211

This study has received clearance by the Carleton University Psychology Ethics Board (Reference \#10-200). 


\section{Appendix B}

\section{Study One: Participant Information}

This information will be held completely confidential.

(Please, do not put your name on this form!)

Age: _— years

Sex: $\bigcirc$ Male $\bigcirc$ Female

In what academic program are you enrolled?

At what level are you studying?

$\bigcirc$ Undergraduate $\bigcirc$ Masters $\bigcirc$ Ph.D $\bigcirc$ Other

What year of study are you in?

One a scale of 1 to 10, how would you rate yourself with respect to your computer skills?

$\bigcirc 1$ Novice $\bigcirc 2 \bigcirc 3 \bigcirc 4 \bigcirc 5 \bigcirc 6 \quad \bigcirc 7 \bigcirc 8 \bigcirc 9$ Expert

How often do you browse the Web?

$\bigcirc$ Daily $\bigcirc$ Several times a week $\bigcirc$ Once a week $\bigcirc$ Less than once a week 


\section{Appendix C}

\section{Study One: Main Task Script}

Introduction: Today, you'll be asked to browse six different websites. We're going to give you a role-play scenario for each one of the websites, and ask you to talk aloud while you're viewing the website in question. I'll give you the role-play scenario before each of the six websites you're about to see, one at a time.

\section{Website \#1: CNet RTF to PDF Converter}

Context : Imagine you are a student, and your professor has sent you a document in .rtf format. You are looking on the Internet for a method to read this file, and you come upon this website. How do you decide whether or not to download and install this software application?

\section{Website \#2: 1 Click SpyClean}

Context : Based on the previous scenario, you've downloaded the document your professor sent you via email, but are now concerned about the possibility that a virus has been integrated into this file. You decide to browse Google for an antivirus software program, and come upon this screen. How do you decide whether or not to download and install this software application?

\section{Website \#3: XP/Vista Antivirus Software}

Context : You're not completely satisfied that the 1 Click Spy Clean program will fix your computer properly. You decide to browse Google once more and come upon this software application. How do you decide whether or not to download and install this program? 


\section{Website \#4: Ashisoft Duplicate File Finder 2009}

Context : After having downloaded the .rtf file from your professor, you've discovered that the file has been duplicated on your hard drive. You find the application "Duplicate Finder 2009", which will help you find and remove duplicate files on your computer. How do you decide whether or not to download and install this program?

\section{Website \#5: Tucows SpeedUpMyPC Software}

Context : After having cleaned your computer of duplicate files, you find that the computer speed is slower than usual. You decide to browse Google once more and you come upon this website that is offering a downloadable version of the SpeedUpMyPC software. How do you decide whether or not to download and install this program?

\section{Website \#6: CNet Photoshop}

Context : After having spent time looking for various computer fix-it programs, you decide to browse Google in search of the Adobe Photoshop application, in order to improve your photo enhancing skills. You come upon the CNet website once more, where this program is advertised for download. How do you decide whether or not to download and install this program? 


\section{Questions that will be asked during testing:}

Please Note: This is a semi-structured interview. These questions will likely change as a result of the answers provided by the participants. This reformulating of the questions follows the constant comparative technique for the better formulating of questions during successive trials.

1. Would you recommend this webpage to anyone you know?

2. How does this webpage compare to another webpage you trust and use consistently?

3. Is there a particular aspect of the webpage that makes you trusting or mistrusting of it?

4. Do you think the page looks professional? If yes, how? If not, why?

5. With the information provided, can you tell who is responsible for the website?

6. Did you look at the Internet address? If so, does it tell you anything?

7. Was there a lock icon present on the webpage? If so, what does this tell you?

8. Now that you've had the time to assess this webpage, would you decide in favor of trusting or distrusting this website? 


\section{Appendix D}

\section{Study One: Post-Test Questionnaire}

1. What is a Web browser?

(A) An operating system, such as Microsoft Windows.

(B) A computer program that shows websites and allows the user to interact with them.

(C) A search engine that allows users to search for content on the Internet.

(D) I don't really know the answer.

2. What does a picture of a lock icon in a Web browser indicate?

(A) The website is free of viruses.

(B) The website content has been approved by Microsoft.

(C) It indicates secure communication with the website server.

(D) I don't really know the answer.

3. The information you provide on a website can sometimes be observed by someone else over the Internet. Can you tell us how this might happen? 
4. What is a website security certificate?

(A) A mathematical key used to prove the website's identity.

(B) A government license legally required for all websites.

(C) The numeric Internet address of the website.

(D) I don't really know the answer.

5. What does https:// mean at the beginning of the website address?

(A) It specifies that the connection with the website must be encrypted.

(B) It means that the website has been certified secure by Google.

(C) It means that the website should only be used for administrative purposes.

(D) I don't really know the answer.

6. What does this seal by Better Business Bureau mean?

(A) The Better Business Bureau continually monitors communication with this website.

(B) The Better Business Bureau operates this website on behalf of the company.

(C) The Better Business Bureau recognizes the website as meeting their standards.

(D) I don't really know the answer.

7. How do you know the name of a person or company who is responsible for a website? 
8. What is a phishing website?

9. What is a domain name?

10. Approximately how many web sites do you visit that require a username and password?

11. How often do you re-use the same password on different web sites?

12. Are you concerned about the security of your passwords? 
13. Are you concerned about websites that attempt to access your private information or download dangerous software?

14. Do you use any antivirus or other Internet security software (e.g., McAfee, Norton, Microsoft Security Essentials, etc.)?

15. Please respond to the following statements:

I am concerned that, in the past, I have provided personal information to a fake website.

$\bigcirc$ Yes $\bigcirc$ No $\bigcirc$ Unsure

I feel confident that I can identify fake or malicious websites.

$\bigcirc$ Not at all confident $\bigcirc$ Somewhat confident $\bigcirc$ Extremely confident

I believe that, if people get tricked into providing personal information to a fake/malicious website, it is their own fault.

$\bigcirc$ Yes $\quad \bigcirc$ No $\quad \bigcirc$ It depends on the circumstances 


\section{Appendix E}

\section{Study One: Website Images}

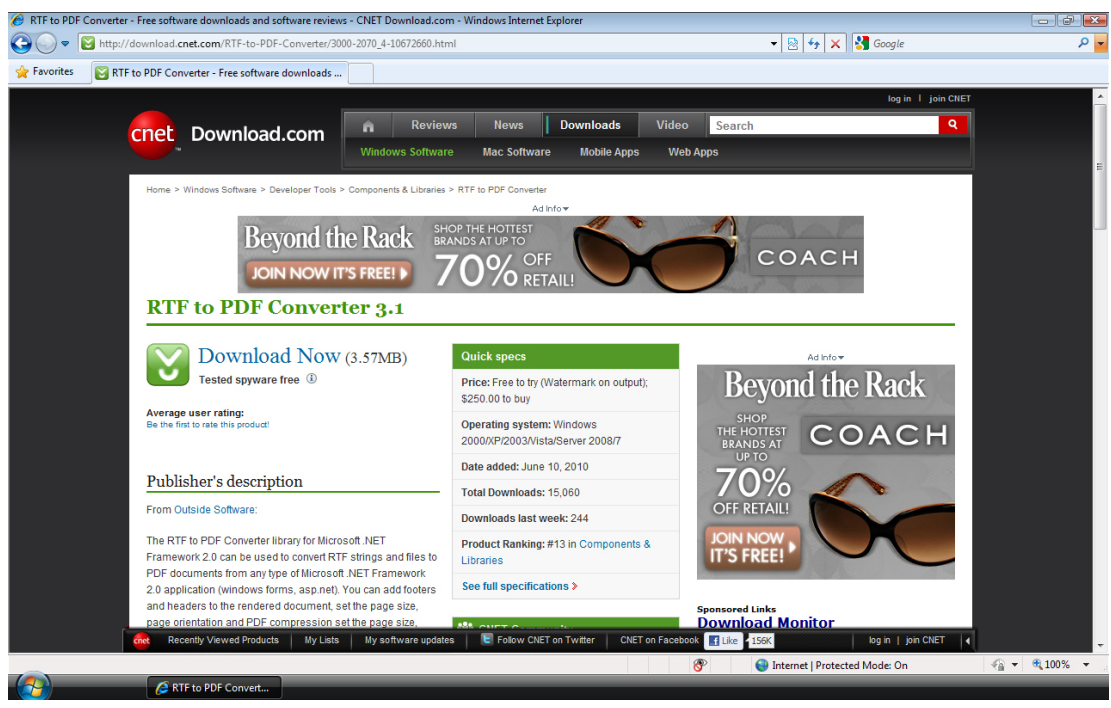

Figure 38. CNet RTF to PDF Converter website screenshot.

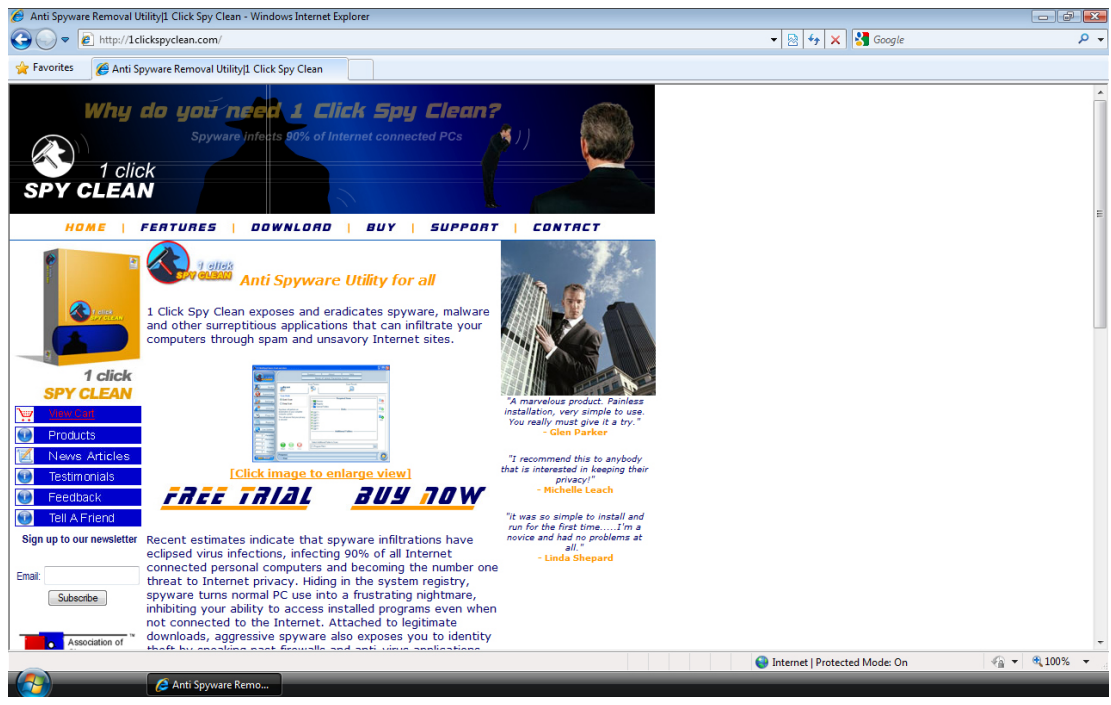

Figure 39. 1ClickSpyClean website screenshot. 


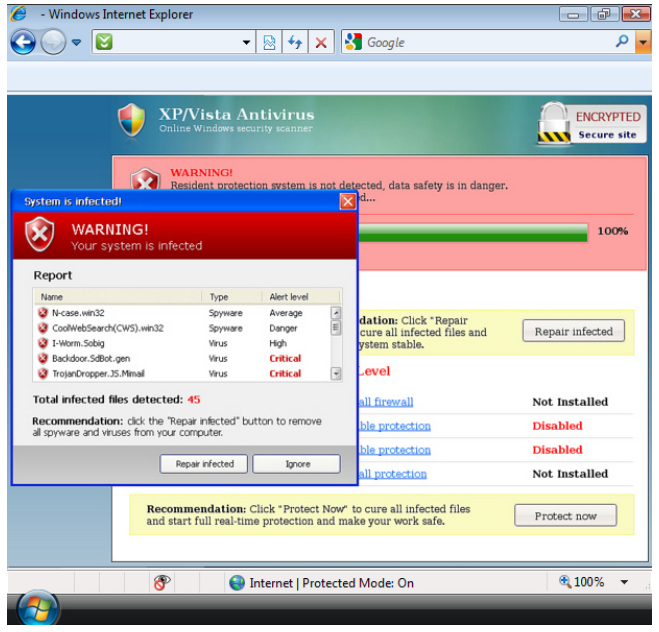

Figure 40. XP/Vista Antivirus website screenshot.

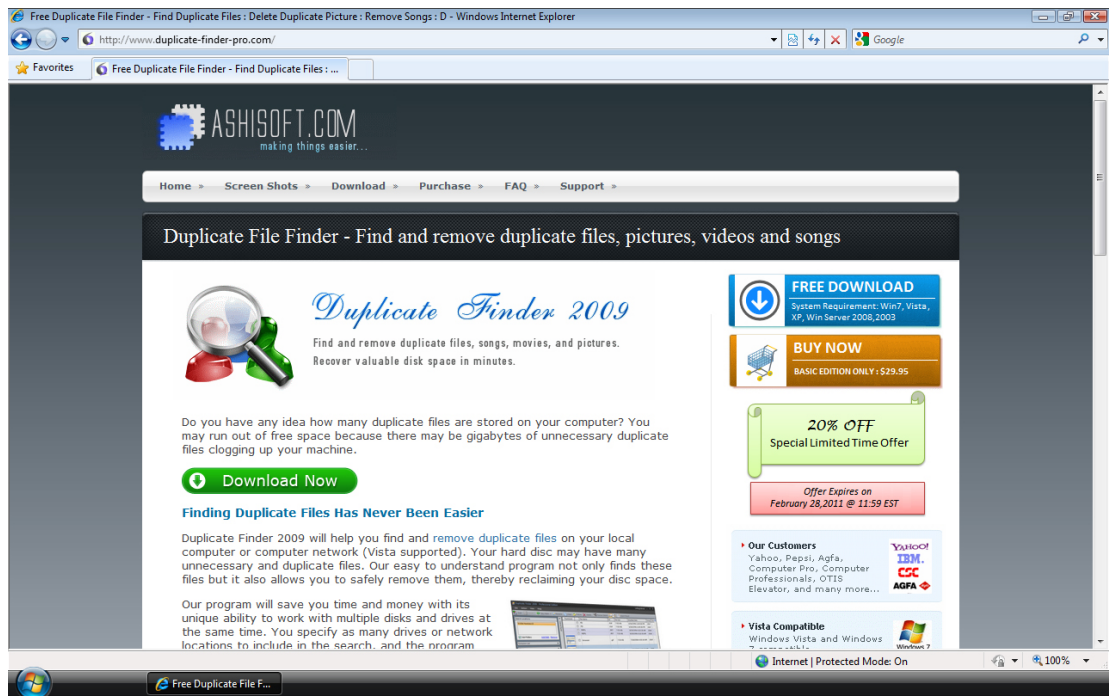

Figure 41. Ashisoft Duplicate File Finder 2009 website screenshot. 


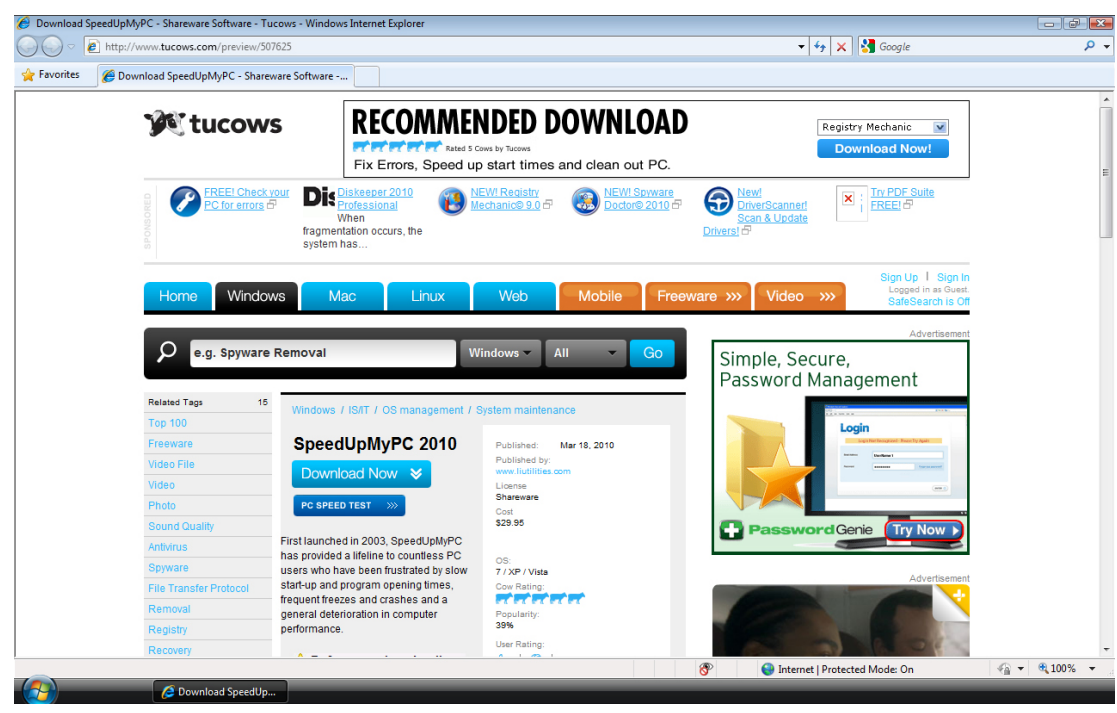

Figure 42. Tucows SpeedUpMyPC website screenshot.

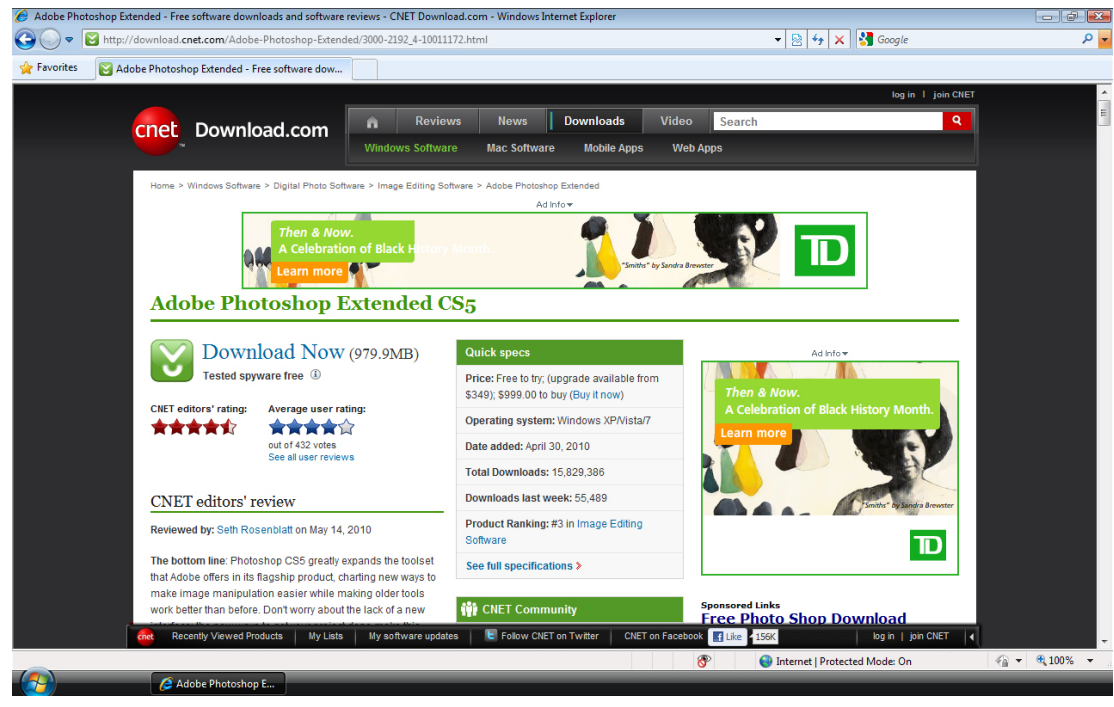

Figure 43. CNet Photoshop website screenshot. 


\section{Appendix F}

\section{Study One: Qualitative Interview Transcripts}

This appendix contains the qualitative interview transcripts from the 12 participants in the study. P1 through $\mathbf{P 1 2}$ indicate participants 1 through 12.

\section{Website 1: CNet RTF to PDF Converter}

Question 1: How does this webpage compare to another webpage you trust and use consistently?

P1 I trust my antivirus because it blocks sites. It tells me what sites are good or bad.

P2 It is quite similar. States operating system. All the information is included about the program.

P3 It's quite similar. It looks like a site I've used before. I've used CNet before as well. The ads here I've seen on other sites before. The program has been tested spyware free, and I've seen that on other sites as well.

P4 No user ratings. Ads everywhere. No Mac support.

P5 The ads on it are consistent with other websites. There are user ratings, and trusted sites have higher user ratings. The tabs are the same as other websites. A lot of websites use Facebook and Twitter.

P6 It seems legitimate to me. I don't know this website. I noticed the URL (downloads.com). Legitimate websites have good URLs. There is a lot of information on this page (quick specifications).

P7 It's supported by ads. It says it has a lot of downloads, but it's only been a few months. The fact that no one rated this program is disconcerting. There are a lot of downloads, but no ratings. I'm not familiar with CNet.

P8 There are more, larger ads, which is a bad thing. Websites with a lot of large ads make them less legitimate.

P9 The website doesn't have a name I recognize. It talks about spyware, which makes 
me feel better. The price is indicated. The total number of downloads is indicated.

P10 I don't trust this one. The ads aren't trustable. I'm not interested in it, I don't trust downloading. I don't download from online.

P11 The better the design, the more I am to trust it. I would look on Google for reviews about it. I would ask my dad, who is an engineer, what he thinks.

P12 I use reliable sources, not downloads.com.

Question 2: Is there a particular aspect of the webpage that makes you trusting or mistrusting of it?

P1 Trust: they offer a publisher's description. They make note of the number of downloads overall.

P2 Trusting: because it says "Tested spyware free". Lots of information provided on the website. More downloads is better than less downloads.

P3 Trusting: I've used it before. There is a lot of information on it, including full specifications. The ads showing expensive glasses make me trust it.

P4 The fact that there are ads and no user ratings makes me distrust the website.

P5 Mistrust: no user ratings (no feedback). Not sure if I trust the title of the website (downloads.com). Trusting: there are different opportunities to find out information on the website.

P6 The ads make me trust it, as they are reputable.

P7 Trust: the way the publisher describes it. Windows and Mac support gives the impression of legitimacy. The bar at the bottom makes it legitimate.

P8 Mistrust: ads don't match. Trust: bottom bar makes me think that a lot of people use it. There is a lot of information for what you'll download.

P9 It talks about spyware, which is good.

P10 Nothing in particular.

P11 It's nice to see the information, but it's easy to fake. I'd be more likely to trust the site, but not without reserve. The more well-known it is, the more I trust it. I don't 
know CNet.

$\mathbf{P 1 2}$ "Be the first to rate product" - not good. Distrust: 15000 downloads is not a good amount of downloads. No one gave any feedback.

Question 3: Do you think the page looks professional? If yes, how? If not, why?

P1 In between yes and no. There are a lot of ads, which is good. The layout is weird though.

P2 Ads are not usually professional. Facebook isn't trustworthy. The web hosting forces them to put ads on their website.

P3 Yes, the specifications tabs are good. There is a search bar. You can login to an account. Facebook and Twitter are good. I would trust it.

P4 Not entirely professional. It has stuff for Facebook and Twitter, which is bad. If it's to be professionally made, there can’t be social networking sites on it.

P5 It does, but the ads make it look unprofessional. It has both Windows and Mac, which makes it diverse. Not really sure what else.

P6 You can tell the layout was created professionally.

P7 Yeah, kind of. Mostly yes, a bit no. The ads make it look bad sort of. It's clean, with lots of info. It's organized a lot.

P8 Yes, nicely laid out. Nice navigation bar. Simple layout.

P9 Yes, it's organized, easy to navigate. The placement of ads is organized. The ads are trying to promote products, which makes me trust it less. The ads aren't concerned with their own product.

P10 Yeah, there's a lot of ads, which is unprofessional. Yes, it's professional because of the content.

P11 It looks fairly professional. There is font similarity. It's well organized, and not simply thrown together. The links to Facebook and Twitter make me trust it (this is hard to fake).

P12 It looks professional. The layout is good. It describes a lot of stuff (Operating System 
used, etc.).

Question 4: With the information provided, can you tell who is responsible for the website?

P1 I don't think so, no. ASP.net maybe? I'm not sure.

P2 I can't tell from the information provided.

P3 No, it applies to a lot of websites. I would have to research them, probably using Wikipedia.

P4 Not really, no.

P5 I don't think so, no. No contact information to find out more.

P6 No, I can’t tell.

P7 CNet is responsible for the whole website.

P8 The CNet company.

P9 CNet, which is a trademark. It doesn't say who CNet is though.

P10 No, I can't tell.

P11 I guess not. Is CNet its own company?

$\mathbf{P 1 2}$ CNet is responsible.

Question 5: Did you look at the Internet address? If so, does it tell you anything?

P1 Yes, but it doesn't say anything of importance to me.

P2 They have other things you can download, like software.

P3 No, I didn't look. It has "http". There's nothing wrong with the address. Isn't ".html" an image though?

P4 It tells me it's an RTF to PDF converter.

P5 It gives the title, but it doesn't give me the background information.

P6 It tells me the URL is professional (there are no mistakes present). They paid to get it.

P7 I did, yes. CNet in main part of it. It doesn't match up. The missing hints in the URL puts me off. 
P8 In the download portion of the CNet.com website. The favicon in the URL bar is a good thing.

P9 It's a dot.com website. The URL is a download link, which is linked from the homepage.

P10 Uh, no, I didn't look. It's Downloads.com.

P11 I didn't look. I don't really know what it says.

P12 download.cnet.com. Nothing specific for me.

Question 6: Would you recommend this webpage to anyone you know?

P1 Yes, I use downloads.com all the time. It's spyware free. They offer specs.

P2 Only if they were in desperate need of the program listed.

P3 Yes, I've used it before. I'de use Google to find it. I've never used this program before.

P4 No, I wouldn't use it, so I wouldn't recommend it to anyone. I don't trust the website. I don't download software from the Internet, because I've had bad experiences in the past.

P5 I'd have to look into it more, and then it would depend on what I find out.

P6 If they were looking for this program, then yes. I don't download from the Internet myself, since it's risky (viruses and stuff like that).

P7 In general, probably not. I'm hesitant about it. I wouldn't use the program. I would email the professor in the scenario and ask for a different file type.

P8 Yes, if they needed it. I would check it first to see if it works.

P9 Probably not. I don't know anything about it. I can find something more popular to use. I'd find out what my friends are using.

P10 No, I wouldn't recommend it because I don't download things. I wouldn't recommend the product itself.

P11 Not unless I could find out more about the website first. I would search Google for CNet scams or viruses. I would check Twitter. The fact that it says it's been tested 
spyware free can easily be faked.

P12 If I didn't use it, no. No other reasons why, I need to know about the product first.

Question 7: Was there a lock icon present on the webpage? If so, what does this tell you?

P1 It tells me that it's secure. They can't take the information from the site? The browser is protecting you from phishing scams and information theft.

P2 I didn't notice one.

P3 I don't know what it does. I use Chrome, and I've never looked for it.

P4 No idea what it means (lock icon in corner).

P5 I don't see one. Does logging in give you this?

P6 I didn't notice one, no.

P7 I don't see a lock icon. Hmm, now I see it, but I don't understand it. It might mean Internet Protected mode is on?

P8 I don't see one.

P9 I don’t really see one.

P10 I don't see one.

P11 I don't see a lock icon. Oh, in the corner? I don't know what it does.

P12 There is one, but it's.....I don't know what you call it. It doesn't tell me anything.

Question 8: Now that you've had the time to assess this webpage, would you decide in favor of trusting or distrusting this website?

P1 Trust it. I use it a lot.

P2 I would not trust it. I can't directly comment on the website. I would have to go on another website (blog or something) to find out more information about this website first.

P3 I would trust it. I covered why in what I said before.

P4 I would distrust it. Same reasons as before. I have nothing else to add.

P5 I would probably distrust it. A lot of download websites I've been on in the past were bad. I'd have to find out more about it first. 
P6 I would trust it.

P7 I would probably distrust it. I'm paranoid about some aspects of it.

P8 I would trust it. You can see that a lot of people have downloaded it. It's free to try, then you can buy it after a while.

P9 I would distrust it. Just mostly because of the ads. Large companies don't need ads to sell products. I can't be too sure of the site's legitimacy.

P10 I would distrust it. Same reasons as before.

P11 Based on this information, I would not trust it. I would have to find out more about it first (as I previously mentioned).

P12 Distrusting, for the same reasons. 


\section{Website 2: 1 Click Spy Clean}

Question 1: How does this webpage compare to another webpage you trust and use consistently?

P1 The format looks like someone was in a hurry to create the site. It looks less professional.

P2 It has a lot of aspects I look for in websites (product information, articles, user feedback, testimonials, "Buy Now" option. It has everything you need to know. The color scheme is off, however. Also, I never download from the Internet; there are too many add-ons.

P3 It has empty space. They usually fill in all the space on a website. It's not a very professional polish (fonts, colours, scheme). The right side testimonials I would distrust.

P4 It doesn't look professional. It looks outdated, not as clean as other websites. The fonts are generic. The links aren't detailed enough.

P5 It has contact area/support. There is advertising of products with information. There are email links.

P6 It's not as legitimate. It's not professional. There is no information on how it works (specs). They have a more direct approach to buying.

P7 This looks like a cheap ad on television. The motto is bad. I can't believe the name of the program. Antivirus programs don't act like this.

P8 It doesn't as legitimate as other programs (Norton, etc.). It looks like a pop-up. The fonts don't look right. The name is weird. The testimonials on the pageare bad, and they make me think it's weird.

P9 The site seems less professional.

P10 The site looks fine. I'm not sure of the product though. The site looks clean (there are no ads everywhere).

P11 It looks like a lot of shopping sites. The testimonials seem fake.

P12 Same thing as the first one. I wouldn't trust it. 
Question 2: Is there a particular aspect of the webpage that makes you trusting or mistrusting of it?

P1 "Buy Now" makes it trustable. I'm worried that if I download it, it won't work properly.

P2 The website gives you all the cliches. Fake quotes make the website distrustful.

P3 The testimonials make me distrust it. The tab at the top would make me trust them more, since I could call or contact them. The news articles could be forged, which makes me distrust the website. The overall scheme makes me more distrustful.

P4 The fact that it doesn't look professional makes me mistrust it. There is no real company name on it.

P5 Trusting: having the ability to contact people. The testimonials are good.

P6 Mistrust: The top banner looks thrown together. The reviews (testimonials) are not trustworthy.

P7 Trusting: newsletter, testimonials. They can make these up, though. OK, nothing makes me trust it.

P8 No comment provided.

P9 Mistrust: the option to view your cart? Already? Also, the testimonials are fake. I've never heard of this program.

P10 Trusting: free trial, testimonials. Distrusting: I've never heard of the product.

P11 I would buy my spyware at a store. If you download anything online without a protection program, you open yourself up to possible problems. I like the contact button, but it could be a scam.

P12 It's a known spyware software. I would use one I know first. I would research it first.

Question 3: Do you think the page looks professional? If yes, how? If not, why?

P1 No answer provided.

P2 The colour scheme is off. That's my main complaint.

P3 Unprofessional, as I said in my previous answer. 
P4 Same as the previous question.

P5 Yes, more than the last one (CNet). Less ads is better. The website just seems more professional.

P6 It's not professional. The colour scheme is bad. It could fool the average internet user.

P7 Not particularly, no. High school students could make this.

P8 The fonts don't match up everywhere. Maybe they had a bad website designer? The pictures are weird (a guy looking down over a building?)

P9 It seems homemade. Anyone could make this. The colour contrast is bad. The general layout isn't good.

$\mathbf{P 1 0}$ Yes, because it has no ads. There's a picture of the program, and a picture of a guy in a suit, which is good.

P11 It looks fairly professional. The left menu isn't right (red over blue). The layout isn't good. The logo is ok. The site is well organized.

P12 It's not professional. It needs more options (the first one had more options).

Question 4: With the information provided, can you tell who is responsible for the website?

P1 "Association of...." on the left side bottom.

P2 Not in particular. There is a link provided in order to contact someone (call, email).

P3 Not on this page. The contact/support would offer more information if I could click on them. "Association....." on the bottom left side of the page, I can't see it all.

P4 I can't tell who is responsible.

P5 Not immediately, but you can contact them to find out.

P6 No, I can't tell.

P7 No. "Association of....." Is this a sponsor?

P8 Something at the bottom. "Association of....." might be responsible for the website.

P9 No. SpyClean I guess?

P10 The product. Not sure specifically who. 
P11 I don't think so, no.

P12 clickspyclean.com.

Question 5: Did you look at the Internet address? If so, does it tell you anything?

P1 The site doesn't look professional. There are pop-ups with numbers.

P2 I know what a URL is, but the address here doesn't tell me anything.

P3 Yes, I did for this website. It looks complicated. I don't get any information from the address.

P4 I didn't look, but it just tells you the name of the website/product.

P5 It seems more direct than the last one (CNet). There is less confusing information in the URL.

P6 The URL doesn't look professional. It looks like a pop-up.

P7 Yep, it tells me the company name. Anyone can buy a domain name. They're not validated in any way.

P8 The exact same as the product name. They registered the domain name for that particular product.

P9 It says "Click SpyClean". It indicates that it's sort of like a pop-up window.

P10 I didn't look.

P11 Same name as the title of the website.

P12 No, it just says "click spy clean".

Question 6: Would you recommend this webpage to anyone you know?

P1 The links don't look like all the other sites I've seen before. I would Google it with a search for scams. I wouldn't recommend this website.

P2 I don't trust it myself, so I wouldn't recommend it.

P3 I don't like it, so no, I wouldn't. I'd look up the site to find out more about it. I'd check other spyware first (ABG Spyware). I'd buy it in store before downloading it online. 
P4 No, same reasons as before.

P5 I probably would. It has a general feeling of professionalism.

P6 No, I wouldn't recommend it. For the same reasons as before.

P7 No, for the same reasons. It looks untrustworthy in general.

P8 No, I wouldn't.

P9 No, just because I don't know anything about it. There are better spyware programs out there.

P10 No, because I haven't ever heard of the product before. I would recommend what I already know.

P11 No, I've never heard of it, and there isn't enough information available.

P12 No, same reasons as before.

Question \%: Was there a lock icon present on the webpage? If so, what does this tell you?

P1 The lock doesn't guarantee trustability.

P2 I didn't notice one.

P3 Yes, but it doesn't tell me anything.

P4 There's one at the bottom corner like before, I don't know what it means.

P5 No, I don't see one.

P6 Yes, bottom right. It doesn't tell me anything.

P7 Same one as before. I don't understand it.

P8 I can't see one here.

P9 There is no lock icon.

P10 No, I didn't notice one.

P11 I don't know what that lock icon means.

P12 It's grey, and open. It doesn't tell me anything.

Question 8: Now that you've had the time to assess this webpage, would you decide in favor of trusting or distrusting this website? 
P1 I wouldn't trust it for all the reasons I mentioned before.

P2 I would definitely distrust the site.

P3 I would distrust it.

P4 I would distrust this website.

P5 I would probably trust it. I'm paranoid for my computer. I'd have to look at the website more and find out more about it.

P6 I would distrust this website. For the same reasons as before.

P7 I would distrust this website. Same reason as before.

P8 I would distrust this website. For the same reasons as before.

P9 I would distrust this website. For the same reasons as before.

P10 I would trust it. For the same reasons as before.

P11 I would distrust this website. For the same reasons as before.

P12 Distrusting this site. 


\section{Website 3: XP/Vista Antivirus}

Question 1: How does this webpage compare to another webpage you trust and use consistently?

P1 Pop-ups with danger warnings. There are no options for customization.

P2 I'm not sure. The website is really different. I've never seen it before. "Social Spy Network" was installed on my computer.

P3 There is no URL for some reason. I've never seen that before. He lock icon looks fake, too large. I've never seen one like that before. There is no lock icon shown on the Chrome browser. It has the same logo as XP/Vista, I'm not sure if that's good or bad.

P4 It's crowded, there's a pop-up on top. You can't tell what you're doing on it.

P5 It's along the lines of a virus. It stands out more as being unsafe. I see it as a threat (warning).

P6 It looks like it's by Windows. It's a Windows site, directed to people who use Windows.

P7 I would trust this automatically. It's run by XP/Vista. It doesn't say who runs it though.

P8 It looks similar. It looks like Windows antivirus. The icons and colours are the same.

P9 Is that a pop-up?

P10 It looks trustworthy. It's telling you what's wrong with your PC (they're doing the work).

P11 I've never used one like this before. I use Windows 7 now, not Vista.

$\mathbf{P 1 2}$ This is a spyware/virus.

Question 2: Is there a particular aspect of the webpage that makes you trusting or mistrusting of it?

P1 Nothing at all makes me trust it.

P2 I would mistrust the warnings. The program itself put them there. 
P3 Trusting: I know the virus types listed by the website. Mistrusting: I've seen these things before, and I've never seen so many problems at once. Also, one-click fix is good for me. I might stop it if it causes problems.

P4 Mistrusting: there is no URL. A lot of stuff looks copy/pasted from another website.

P5 I would probably distrust this one for those same reasons.

P6 At first, I was skeptical (pop-up window). Now, I recognize the logos and icons.

P7 Distrust: pop-up with infection warnings. This could be a random program with popups. The option to repair right away is bad. They're selling you a product. There's a pop-up every time.

P8 Mistrust: the amount of red warnings. I've seen pop-ups like these before, and they were bad.

P9 Distrust: Windows Vista doesn't look like that. I probably would trust it if it's not a pop-up. I wouldn't trust it, since the notification would not pop-up. The messages would be integrated directly into the browser, and not a pop-up window.

P10 The information about the computer's status makes me trust it (Status On/Off).

P11 I'd be really suspicious of this. It looks like Windows 7. I'd be really suspicious of anything that gives me huge warnings like this.

P12 Distrust: It says infected, when did it check my system? It takes longer than that to check a computer.

Question 3: Do you think the page looks professional? If yes, how? If not, why?

P1 Not a lot, no. The background looks like Windows, which is made by Microsoft.

P2 It looks professional. I trust Vista's logos. This is an encrypted and secure site.

P3 Yes, it looks more like sites I trust. The same window scheme as Windows, so I can trust it. There is more info, which helps, so it looks professional.

P4 No, same as previous question.

P5 No, I don't think so. It looks like there's a pop-up. Pop-ups are normally viruses for me. This website is unprofessional. There isn't a lot of information, but the 
information there is is too forceful.

P6 It looks professional (the logos and the background).

P7 At first glance, yes. But after, no. The colours are good. I lose faith after a while.

P8 Somewhat. I have a Mac, so I'm not sure on that aspect. It would raise red flags on the Mac.

P9 It looks fairly professional. The colour scheme is good. It looks like XP/Vista. The font doesn't look the same as Windows fonts.

P10 Yes, because they have proper names for everything: virus names, urgency, etc. They show details rather than being vague.

P11 Yeah, the logos are good. It presents information like XP/Vista. The font is different than Microsoft Windows' site though.

P12 It's not professional. Not a site, 2 options only, it doesn't tell you any information at all.

Question 4: With the information provided, can you tell who is responsible for the website?

P1 There is no indication.

P2 XP/Vista is responsible for the website.

P3 Yes, I would guess that it's Microsoft corporation that set it up (the colour scheme is similar).

P4 Not at all, no idea.

P5 No, I don't see a place where it says.

P6 Windows.

P7 No, can't tell.

P8 Windows. Microsoft Windows is responsible.

P9 It says XP/Vista, but it doesn't show as a trademark, so I'm unsure where it's from.

$\mathbf{P 1 0} \mathrm{XP} /$ Vista Antivirus is responsible.

P11 We're supposed to think it's Windows, but hmm.... I can't tell.

P12 No, I can't tell. It says Windows, but I don't think so. 
Question 5: Did you look at the Internet address? If so, does it tell you anything?

P1 There is no Internet address, so it's untrustworthy.

P2 There is no lock icon.

P3 Yes, it's absent, which is very odd.

P4 There is no URL. Since it's absent, I don't know what that tells me.

P5 No, there isn't one. Not sure what that would tell me. I've never thought about it.

P6 I looked there, but there is nothing there. There should be something there. Hmm.....

P7 There is no URL. The icon image for the lock is the same as the first website (RTF to PDF Converter).

P8 It says it's encrypted. This could be just a graphic. It would be more trusted if it was in the URL bar (certificate).

P9 It doesn't have one. I see one now, but I'm not sure where it came from. If it came from my PC, it would give me a URL.

P10 There's nothing (no address). That tells me it doesn't seem like a website.

P11 There's no URL present, and the window is too small. There's something wrong, sites always have URLs. I'd refresh the page to see if it helps bring it up.

P12 No URL or Internet address. That tells me it's not a webpage.

Question 6: Would you recommend this webpage to anyone you know?

P1 I would not recommend this website to anyone. There are no links present on the page. I wouldn't trust something that shows me errors on my PC.

P2 No, there is no URL address. It's fake/non-existant.

P3 If I ran it and was happy with it, then yes, I would. If not, then no, I wouldn't.

P4 No, it just doesn't look legitimate.

P5 Probably not, I wouldn’t. I wouldn't trust it, nor recommend it.

P6 Yes, I would recommend.

P7 No, for the same reasons as before.

P8 No. 
P9 No, because I don't know what it's called or where it's from. How can I recommend it?

P10 Yeah, because it looks really real. I would recommend it to XP and Vista users.

P11 No, I would not. I don't like warnings.

P12 No, I wouldn't.

Question 7: Was there a lock icon present on the webpage? If so, what does this tell you?

P1 The big lock icon put there might be a trick.

P2 Yes, it means the website is encrypted, it's a secure site. It's approved by XP/Vista.

P3 No lock icon on the bottom. Mixed with no URL, this isn't good. The large encrypted lock icon is replicated. It's too big and begging for attention.

P4 Top-right, it looks copy/pasted. The lock tells me it's encrypted. But, it's not on the bar at the bottom, so it's probably fake.

P5 Yes, in the upper corner. Not sure what encrypted means. It's not a good thing (that's just a hunch). There is no information provided, and I cant find out what it is.

P6 The lock icon tells me it's secure. The information isn't being shared with anyone else. It is operated by Windows.

P7 Encrypted, secure site lock icon directly on the site. It could be pasted on there. It's probably not secure, it's probably fake.

P8 There wasn't a URL. The pop-ups are suspicious.

P9 There is a picture of a lock. I don't recognize it, and am unsure what it means. It doesn't seem very promising.

P10 Yes, encrypted, secure site. It means it's secure, and not filled with viruses.

P11 The lock icon looks like clip art. It makes me suspicious. This should be on the browser, not in the page itself. It's too easy to post it on the website.

P12 There is no lock present. I'm not sure, maybe that's something to consider as being bad. 
Question 8: Now that you've had the time to assess this webpage, would you decide in favor of trusting or distrusting this website?

P1 I would distrust the website.

P2 I would distrust this website, for all the reasons I mentioned.

P3 It's a toss up. I'm not sure now. The lock icon isn't right. I'd distrust it now.

P4 I would mistrust this website.

P5 I would distrust this website, I summed up why already.

P6 I would trust this website. For the same reasons as before.

P7 I would distrust it. Same reasons as before. The lock icon is gone, I'm not sure why.

P8 I would distrust this website, for all the reasons I mentioned.

P9 I would probably distrust it, because I'm unsure where it came from.

P10 I would trust it. For the same reasons as before.

P11 I would definitely distrust the site.

P12 Distrust, same reasons as before. 


\section{Website 4: Ashisoft Duplicate File Finder 2009}

Question 1: How does this webpage compare to another webpage you trust and use consistently?

P1 I don't see specs. "Buy Now" option is good. I like downloads.com.

P2 You get a pretty good description of its uses. It's Vista compatible. It lists their customers. It gives you a slew of information available for the product.

P3 It's very similar. The tabs, FAQs, support options. There are similar links (download tab, free downloads icon). It has logos, slogans, these make it more professional.

P4 It looks professional, more complex than the 1 Click SpyClean website. It's not crowded with ads.

P5 It looks similar to a website I would use. The information is accessible.

P6 It's hard to say whether there are things here that are trustable or not.

P7 This is a stupid program. Why not just find the files yourself?

P8 Things are around the same as a site I've used before. It's a bit less professional looking. The fonts are all different from each other. Some of the graphics are the same.

P9 It seems more trustworthy. It has a good layout. It has a "Download Now" button.

P10 It looks trustable, but I wouldn't use it. You can find duplicate files on your own without a program.

P11 Duplicate files exist? I have a lot of RAM, I'm not worried about duplicate files.

P12 I might use this.

Question 2: Is there a particular aspect of the webpage that makes you trusting or mistrusting of it?

P1 The website looks legitimate because of the date of expiry of the product offer. Why is Pepsi a customer?

P2 I think the special limited time offer makes this a cheap product, they're just trying to get rid of it. 
P3 The lock icon will make me trust it. The Internet address doesn't look odd to me. The URL doesn't match the logo name.

P4 Trusting: it lists their customers on the right side. It sows that more people have used it before.

P5 Nothing in particular I can think of. It comes across as trustable.

P6 I wouldn't use it. The fonts make me not trust it. The logo looks good though. The links on the right side are good.

P7 There are a list of customers (Pepsi, Yahoo). This makes me trust it. I'd verify first through other sources. The downloads tabs make me trust it. This could be a legitimate program. It seems invasive though. The program might be bad for file deletion. It might not correctly delete the files. Does it gain access to the entire computer?

P8 Trust: they have their own favicon. They have a "our customers" banner. Distrust: the fonts don't match.

P9 Trusting: it seems I can trust it. The layout, options, feedback, support buttons are good.

P10 There are no ads, which is good. The banners, text, and so forth are very organized.

P11 I would ask someone about this. Right side clip art looks "iffy". The customers list can be faked. It's nice to have a FAQ section (it helps).

P12 It looks sketchy, I've never heard of this type of program.

Question 3: Do you think the page looks professional? If yes, how? If not, why?

P1 It looks ok. It looks trustable because of logos, colours, and background.

P2 No, things look like they were made with "Publisher". It looks like it was made by someone in a basement.

P3 Yes, all the tabs at the top. Not very different from CNet. They list their customer, but I'm not sure if that's legitimate. The cost of it makes me trust it, as opposed to free downloads. 
P4 The writing, the links, and the layout are more professional.

P5 It looks more professional than the last one. No particular reason why. The FAQ section means people have provided feedback to them. The ads are for the product itself, which is professional.

P6 The layout is professional. Everything is organized professionally.

P7 No comment provided.

P8 Yes, it has a clean layout (buttons).

P9 The layout is professional. The options bar on top is good. The name, slogan, summary, and the variety of information is good.

P10 The headings, text, buttons, and nice fonts are professional.

P11 It looks like sites that are reliable. It gives you information. It's fairly professional. The download is free.

P12 It looks professional, the layout, options, everything.

Question 4: With the information provided, can you tell who is responsible for the website?

P1 I can't tell from the information provided.

P2 No, I can't tell.

P3 Ashisoft.com, logo plus slogan, so yes.

P4 Not entirely. Maybe Ashisoft.com?

P5 I don't see who. But a support section tells you who is responsible for it.

P6 No, I can't tell.

P7 Ashisoft, can’t see any other company.

P8 Ashisoft.com is responsible.

P9 On the main page, it says Ashisoft.com. They list customers that I recognize, which makes me trust it. They can't just use their names without permission.

P10 Duplicate File Finder.

P11 No, I can't.

P12 Ashisoft.com. But on the URL is says Duplicate Finder Pro, so I'm not sure who 
made it.

Question 5: Did you look at the Internet address? If so, does it tell you anything?

P1 Doesn't look familiar. I doesn't tell me anything.

P2 Nothing in particular other than the name of the product.

P3 There is nothing wrong with it. They don't match up with the logo, which is odd. The page should be under Ashisoft's link.

P4 It's for the Duplicate Finder Pro.

P5 Much like the 1 Click SpyClean URL. It's direct, and not confusing.

P6 The URL looks legitimate. It's short, there is no extra stuff in there.

P7 Yes, it suggests that it's the homepage. Anyone can buy a domain name though.

P8 It clashes with the rest of the titles for the program on the page.

P9 The Url is straight to the point, there are no add-ons on it.

P10 It tells you the name of it (Duplicate File Finder).

P11 Pretty much the same (name of product).

$\mathbf{P 1 2}$ It says what the site is all about. But the site is another name, so it's not right.

Question 6: Would you recommend this webpage to anyone you know?

P1 I would recommend this website, I think. I don't see anything wrong with it. I would show it to someone who needs the product.

P2 No, I wouldn't trust it.

P3 I wouldn't, since I don't use this type of software. There is nothing wrong with the site. The URL/name don't match. I might recommend it with a note of caution.

P4 Probably not, not really, no particular reason. I don't download these programs.

P5 I would, yes, if they were looking for such a product. I would caution them to find out more information first.

P6 No, special ads make it look cheap. Particularly the clip art on the right side.

P7 Probably not, because there are too many negative aspects to it. 
P8 No, not unless I checked it out first. I would Google it and see if others have used it.

P9 Yes, I think I might, because it seems trustworthy. 2009 year throws me off though, since programs are usually updated and rereleased every year.

P10 I would tell someone about it, but I wouldn't recommend they download it. They can find duplicate files themselves.

P11 No, same reasons. I'd need to search first. Need to find out more from someone else.

P12 No, because I don't know if there is something called Duplicate File Finder Pro.

Question 7: Was there a lock icon present on the webpage? If so, what does this tell you?

$\mathbf{P 1}$ There is no lock icon present.

P2 There is no lock icon.

P3 Yes, I think it's secure. It's legitimate, and not a virus, and it's selling what they're advertising.

P4 One on the bottom-right, not on the webpage.

P5 I don't see one, no.

P6 There is none on the actual page. I'm confused about that.

P7 The same as before.

P8 No lock icon noticed.

P9 Yes, one lock icon. I'm unsure what it means. If it's locked, then it's secure? I'm just guessing.

P10 No lock icon.

P11 The lock icon has been the same every time. I'm not sure what it means.

$\mathbf{P 1 2}$ There is a lock icon, but I don't know what it means.

Question 8: Now that you've had the time to assess this webpage, would you decide in favor of trusting or distrusting this website?

P1 The identity is unknown, so I am not sure if I should trust it.

P2 I wouldn't trust it. The product looks cheap, and the limited time offer makes it cheap. 
P3 I would trust it, for all the reasons I mentioned. There is an Internet protected mode? Hmm, I have no idea what this means. The Internet browser's protecting me from cookies and pop-ups.

P4 I trust it, but I still wouldn't download the program.

P5 I would probably trust it. But, I would have to look into it more first.

P6 I would distrust this website. Same reasons as before.

P7 Distrusting again. Nothing to add about that. I'm at a toss-up about it, but I probably wouldn't trust it or use it.

P8 I would trust the website. OK, no, I wouldn't. The little discrepancies bother me. I would Google it first.

P9 I would trust it. It points out the 2011 year expiry date. It would work as best as it did in 2009 .

P10 I would distrust it because of the product itself. I wouldn't buy it. I would wonder why they'd make such a program.

P11 I'd distrust the site, for the same reasons as before. I'd need to find out more first.

P12 Distrust, same reasons. 


\section{Website 5: Tucows SpeedUpMyPC 2010}

Question 1: How does this webpage compare to another webpage you trust and use consistently?

P1 There is a cost to the product, it isn't free. The user ratings and date of publication are good.

P2 The website ratings. The license, cost, publisher, when it was published, are all listed on the website. There are outside links provided. Here is a search bar. It's a multi-platform software product.

P3 It has tabs, but they're for different Operating Systems, not support or contact pages, which are useful. Same tabs as websites I use. Advertisements for more software, not actual product.

P4 It's a bit more crowded. The links go to another website.

P5 It's different than sites I use. It jumps around (caters to a lot of different things). Tucows? What's that? There are a lot of ads. When I do research, I try to find sites with less ads.

P6 It looks really professional. They have a bunch of ratings on the site.

P7 It looks professional.

P8 The website is more cluttered with ads and banners, and the tags on the side.

P9 The layout is chaotic. There's too much going on. They have cow ratings? That's strange. I'm not sure what they mean.

P10 I don't trust this. The ads make me distrust it. Also, something professional shouldn't be called Tucows.

P11 You can buy this in a store. I wouldn't use this. I can think of other options that are less risky.

P12 First time I hear of this too.

Question 2: Is there a particular aspect of the webpage that makes you trusting or mistrusting of it? 
P1 The name matches the year advertised. "Published by" is shown on the website.

P2 It's mistrustful. There are too many ads. The cows used for rating are unprofessional. It's not up-to-date (2010). The program isn't credible (SpeedUpMyPC?).

P3 Distrust: no support/contact pages, which are useful when present. There's no background, just a white page. The sign-up with account option is good. Mistrust: the banner that says "Recommended Downloads" is pushy.

P4 Mistrust: it says "safe is off" on the right side. 39\% popularity.

P5 The fact that I don't know what Tucows is or does, I would distrust it until I find out more about it.

P6 It looks professional. It looks like an actual professional product. Someone with experience must have built it.

P7 The login option makes me trust it. I don't like the bar with the tabs. There is too much variety. These things make me hesitant to trust it.

P8 Mistrust: large amounts of advertisements. The ads mean they're not self-sufficient (not doing well).

P9 Distrust: the ads, layout, cow ratings. It might be a low budget site. It looks low budget.

P10 Mistrusting: there are a lot of ads on the whole top and right side of the page. It doesn't look very professional. The cow ratings aren't professional.

P11 Distrust: Tucows name? Hmm, nothing else sticks out.

P12 I wouldn't trust the site. It's not usually a problem with my PC's speed. This is probably a virus.

Question 3: Do you think the page looks professional? If yes, how? If not, why?

P1 It looks semi-professional. They need a better layout for the ads for the software.

P2 No, recommended downloads in ad form. Broken link in banner.

P3 The page is centered and cut/pasted. There's no background. It's not very appealing, there's a square look to it. It's not good design, too square. 
P4 Not entirely, the page is not specifically for the product. It's a generic page for a bunch of other products.

P5 I don't think so. They're throwing ads in your face. There is no focus on the product itself, there are too many ads.

P6 The logo is good. Everything is organized. Having a search bar is good. Sign-in is for members only. The toolbar looks good.

P7 It looks professional. It seems organized. The tabs for all the different formats isn't good.

P8 Some parts, yes: toolbars, tags on left menu, and ads.

P9 I'm in the middle, it's not on the higher end of the spectrum. I would make the ads smaller on the side. I'd change the Tucows name, since I can't take it seriously. This cow rating system isn't legitimate. The popularity of the program is odd, sitting at $39 \%$.

P10 It looks unprofessionally made, because of the ads, and the cows, etc.

P11 Yeah, it looks like other sites like torrents and stuff. These look the same. There are third-party sites on here. I'd download music or movies before I'd use this (those give less problems).

P12 No comment given.

Question 4: With the information provided, can you tell who is responsible for the website?

P1 The website itself, no. For the program, yes.

P2 The publisher is the Liutilities company.

P3 I assume Tucows, but past that I'm not sure.

P4 No, it just says "Tucows".

P5 No, I can't tell.

P6 "Liutilities.com" is responsible.

P7 Tucows is responsible. Tucows is the host. The program was developed by "Liutilities". 
P8 Tucows is responsible.

P9 Tucows, it looks like.

P10 Tucows is responsible.

P11 It's a mix between Tucows and "Liutilities.com". Tucows hosts the software.

P12 Tucows, "Liutilities".

Question 5: Did you look at the Internet address? If so, does it tell you anything?

P1 The link from the actual website. Seems to come from Tucows.

P2 Tucows name is extremely unprofessional.

P3 It seems normal to me, nothing seems wrong. It seems like it's pointing to a trial offer.

P4 It tells me it's for Tucows, and not for "SpeedUpMyPc 2010".

P5 The URL looks professional. It's short, to the point, and doesn't look made-up.

P6 I don't like it (preview/number). It's not trustable.

P7 This is part of the Tucows overall website. It's a subsection of the main website.

P8 The URL isn't too bad. They offer a "preview" for the program. The URL doesn't throw me off.

P9 Yeah, it's called "Tucows", but it doesn't talk about the product itself. The product itself has nothing to do with cows.

P10 No answer provided.

P11 Nope, I keep feeling like it should, but no.

P12 It just shows you the Tucows page. It previews the "Windows" tab.

Question 6: Would you recommend this webpage to anyone you know?

P1 I think I would. It looks semi-professional though. The costs are shown, who published it is known, there are ratings, as well as the number of downloads so far.

P2 No, there is more to getting a PC to speed up than simply using a program to do it. You need to upgrade the PC's components. 
P3 I don't like the look of it, so no, I would not. It costs money, I wouldn't want to spend it.

P4 No, same reasons as before.

P5 Probably not. It wouldn't be my top choice for such a program. "Safe searches off" makes me trust it less. The searches aren't safe?

P6 Yes, I would recommend.

P7 No, I wouldn't. Same reasons I listed.

P8 No, it doesn't look like the best option. The user ratings for the product aren't good (39\% rating).

P9 Probably not. I'm unsure of it myself, so I wouldn't recommend to anyone else.

P10 No, same reasons.

P11 Nope, same reasons. I've never heard of Tucows before.

P12 No, same reasons.

Question 7: Was there a lock icon present on the webpage? If so, what does this tell you?

P1 I'm ignoring the lock icon on the bottom. This icon means that the browser is semi protecting me.

P2 None.

P3 Yes, the webpage is secure or the Internet "Protected mode" is on.

P4 The one on the bottom-right of the page. I still have no idea what it means.

P5 No lock, but the sign-in page might provide more security to you once you are logged in.

P6 No, I can't see one.

P7 The same lock as before.

P8 No lock icon, no.

P9 Well, yes, bottom right. Aren't they normally a gold colour? This one doesn't reassure me. It should be golden, and tell me if the site is secure or not.

P10 No lock icon. 
P11 Same one as all the other ones.

P12 Yeah, same as the other ones, I have no idea.

Question 8: Now that you've had the time to assess this webpage, would you decide in favor of trusting or distrusting this website?

P1 I would trust it, yes, for the same reasons I mentioned.

P2 I would distrust the website, for the same reasons.

P3 I would distrust the website. I've covered the reasons in the other questions.

P4 I wouldn't trust it even if I did download programs from the Internet.

P5 I would distrust this website. For the same reasons as before.

P6 I would trust this website.

P7 I would distrust this website. Same reasons as before.

P8 I would distrust the website, for the same reasons.

P9 I would distrust it. There are not enough reasons for me to trust it.

P10 I would distrust the website, for the same reasons.

P11 Distrust, same reasons.

P12 Distrusting, same reasons. 


\section{Website 6: CNet Adobe Photoshop}

Question 1: How does this webpage compare to another webpage you trust and use consistently?

P1 The website has more stuff, including CNet editor ratings.

P2 There are rankings provided, the space required for the program, the date it was added to CNet, reviews, the total number of downloads.

P3 It's very similar, the tabs, company name, sponsors, reputable company (TD Bank), login for account, Facebook, good colour scheme, background.

P4 It has more ads. It looks like a normal website, like the ones I use.

P5 There is feedback on the front. It leads me to trust it more. It tells you what the website is. It looks the same as the first one (CNet).

P6 It looks similar to the 1st one (RTF to PDF Converter). There are ratings (votes), lots of people voted. There are reviews of the actual program, there are quick specs and lots of information to be found.

P7 Same as the first one (RTF to PDF Converter). Seeing it twice gives more credibility to it.

P8 The features are around the same. The specifications, download now button, the toolbars and navigation bars.

P9 It seems alright. It's similar to the first one (RTF to PDF Converter). Seeing it again reassures me (they have many products). 15 million downloads is good. The rating of the program doesn't say it's 5 stars, though. I'd be more inclined to download it if it had a 5-star rating.

P10 It's the same as the first one (RTF to PDF Converter). I don't like the ads. The product costs $\$ 1000$. I wouldn’t download something that costs $\$ 1000$.

P11 $\$ 999$ price? What costs this? I might want to buy this from a store.

P12 This is the same website as the first one (RTF to PDF Converter).

Question 2: Is there a particular aspect of the webpage that makes you trusting or mistrusting 
of it?

P1 Everything looks trusting. The product is $\$ 999$. Isn’t that too high of a cost to buy this product? I would hesitate to buy because of this price.

P2 Trusting: it's got a lot of good information, including in-depth reviews. It's mistrustful because there is no contact information for the product.

P3 It has tabs at the top, reviews of actual product to read, ratings are reassuring, which is nice, the list of specs is nice, there is a lot of info to find.

P4 Same reasons as the first CNet website (RTF to PDF Converter).

P5 They have specific sites for each program. I would trust it or mistrust it based on the 1st program (RTF to PDF Converter).

P6 I have no trust issues with it.

P7 I would trust it more, the same as with the RTF to PDF Converter site. The TD Bank ads make it more trustable. Banks wouldn't advertise on bad websites.

P8 Trusting: TD ads make me trust it more. Links to Facebook and Twitter are good. The number of user downloads is good.

P9 No comment provided.

P10 It looks like the ads aren't trustworthy. The table looks nice though. But a $\$ 1000$ product isn't trustable. I would buy it in a store if it was good, and not online.

P11 I would do research first to see about the program. The user reviews are a good thing. I'd be swayed by bad reviews though, since I give credence to lower reviews. This website has its own host (downloads.com).

P12 I would trust it more. This is the second time it pops up, which is good. The ratings are shown, there are 15 million downloads.

Question 3: Do you think the page looks professional? If yes, how? If not, why?

P1 The website looks semi-professional, like the first CNet website. The colours and contrast aren't right.

P2 A little, the ads are unrelated to the program for download. 
P3 Yes, same reasons as the first one we were at (CNet RTF to PDF Converter).

P4 Somewhat. The social networking sites, ads, these aren't things to add to a website to make it look professional.

P5 It looks professional in a way (the reviews), but the ads make it less professional.

P6 It looks professional. The ads are by legitimate people. The logo and colour scheme are good.

P7 Yes, it's very clean. There is a lot of information. There are related tags and tabs. The ads are understandable. There is a trial version available.

P8 Yes, it does. It's really clean, and well laid out.

P9 For the most part, yes. It's organized. You can follow it on Twitter and Facebook.

P10 The left side of the page looks professional, but there are too many ads.

P11 Yeah. CNet looks the most professional of all the 6 sites. It's organized, the menu gives you the path followed to get to the page. The themes are similar from product to product.

P12 Yeah, it shows all the softwares available, for every Operating System available. The way it looks is professional.

Question 4: With the information provided, can you tell who is responsible for the website?

P1 CNet is the one responsible for the website.

P2 Not a particular individual. The CNet company.

P3 Yes, downloads.com is responsible. I would imagine CNet owns downloads.com.

P4 No, I can't tell.

P5 No, not right off the bat.

P6 No, can't tell.

P7 CNet is responsible.

P8 CNet is responsible for the website.

P9 It looks like CNet again.

P10 Downloads.com. 
P11 Not really, not more than CNet itself.

P12 CNet.

Question 5: Did you look at the Internet address? If so, does it tell you anything?

P1 It comes from CNet.

P2 It tells me about the product for download on the website.

P3 No, the address doesn't tell me anything other than the CNet name, which is consistent.

P4 It's CNet and it's for Photoshop.

P5 It looks again more confusing, not a direct link to a program.

P6 Yeah, the same as the 1st webpage (RTF to PDF Converter). It's an actual website. The address is short and tells you where you are and stuff. It doesn't look made up.

P7 Yep, it tells me that it's not legitimate. CNet to Adobe website? There is missing information in the URL. Bookmarks would make it easier to find. The numbers in the URL make me distrust it.

P8 You're in the downloads portion of CNet.com.

P9 Yes, it shows me where CNet/Photoshop is. It doesn't shock me. It says how I got here.

P10 It tells you the name of the product, which is a good thing. It also shows the name of the website itself (downloads.com).

P11 Nope, nothing.

P12 It just tells you you're downloading Photoshop Extended from CNet.

Question 6: Would you recommend this webpage to anyone you know?

P1 I would trust it, as I use it a lot. It is tested spyware free. Reviews are present. Quick specs are listed. Full specs are listed.

P2 Yes, if they need the product for anything. 
P3 Yes, it looks similar to sites I use. It has links to the company, there is overall a lot of information, it has reputable and reassuring ads from top companies. Facebook and Twitter are reliable.

P4 Probably not, no other reasons why.

P5 Yes, but first I would do some research and background checking to make sure of its legitimacy.

P6 Yes, I would. Same reasons as before. It looks legitimate.

P7 Probably not, because I wouldn't want to be responsible if something went wrong. I'm careful when downloading. The price is too high, it might be fake.

P8 Yes, it looks legitimate. There are lots of user ratings, the total number of downloads is shown.

P9 Maybe, as I look at it more. The login option is good.

P10 No, because of the ads. And, the fact that the product costs $\$ 1000$.

P11 No, I would research it first. I'd ask someone knowledgeable in this area.

P12 No, I wouldn't. I don't recommend stuff I've never used when I don't know what it is.

Question 7: Was there a lock icon present on the webpage? If so, what does this tell you?

P1 Just the browser lock icon, none other on the website.

P2 No lock icon.

P3 Yes, same as other sites.

P4 Just on the bottom toolbar, same as the others.

P5 No lock, but a login option. Logging in would help protect you.

P6 No, I did not.

P7 Same as before.

P8 No, no lock icon.

P9 Same as I saw before. It looks like it's locked. I assume it's secure, and not harmful to my computer. 
P10 There is no lock icon.

P11 Same as all the other ones.

P12 Yes, it doesn't tell me anything.

Question 8: Now that you've had the time to assess this webpage, would you decide in favor of trusting or distrusting this website?

P1 I would trust it because of how it looks, the specs listed. I would also trust it based on personal experience.

P2 I would trust it. It's the same as the 1st website. This program has much more downloads than the first one (RTF to PDF Converter). It also has a higher raking than the first one.

P3 I would trust it, for the same reasons I listed. Also for the sheer amount of information and everything is good.

P4 I wouldn't trust it because I don't download programs from the Internet.

P5 I would trust it probably. If it worked for the RTF program, then yes I would. If not, then I wouldn't.

P6 I would trust this website. I like the big review information, how someone spent time reviewing it.

P7 I would distrust the site. Same reasons as before.

P8 I would trust it. For the same reasons I mentioned before.

P9 I would trust it, but I would download it and cringe and hope for the best.

P10 I would distrust the site. Same reasons as before.

P11 Distrusting, same reasons as before.

P12 Distrusting it. I could go to the Adobe website instead and get it for free. 


\section{Appendix G}

\section{Study One: Post-Test Questionnaire Responses}

This appendix contains the post-test questionnaire responses from the 12 participants in the study. P1 through $\mathbf{P 1 2}$ indicate participants 1 through 12.

\section{Question 1: What is a Web browser?}

(A) An operating system, such as Microsoft Windows.

(B) A computer program that shows websites and allows the user to interact with them.

(C) A search engine that allows users to search for content on the Internet.

(D) I don't really know the answer.

$\begin{array}{ll}\text { P1 } & \text { B (Correct) } \\ \text { P2 } & \text { C } \\ \text { P3 } & \text { B (Correct) } \\ \text { P4 } & \text { C } \\ \text { P5 } & \text { C } \\ \text { P6 } & \text { C } \\ \text { P7 } & \text { B (Correct) } \\ \text { P8 } & \text { B (Correct) } \\ \text { P9 } & \text { C } \\ \text { P10 } & \text { A } \\ \text { P11 } & \text { B (Correct) } \\ \text { P12 } & \text { B (Correct) }\end{array}$

Question 2: What does a picture of a lock icon in a Web browser indicate?

P1 C (Correct)

P2 C (Correct) 
P3 D

P4 D

P5 C (Correct)

P6 D

P7 C (Correct)

P8 C (Correct)

P9 B

P10 A

P11 D

P12 D

Question 3: The information you provide on a website can sometimes be observed by someone else over the Internet. Can you tell us how this might happen?

P1 No answer provided by participant

P2 A virus implanted on your computer which allows someone else to possibly over the Internet to record your key strokes.

P3 No I am unsure.

P4 I'm not sure.

P5 Someone searching content that may be the same as the content you provided is likely to come across it.

P6 The term "world wide web" tells a person that anything put on the Internet could potentially be shared with anyone else. Most people even assume so and know not to share anything they wouldn't want known even if the website was secure.

P7 When you post on a website like Facebook, what you posted is immediately on the website and it is there for all to see (who have access). If you are registering for an email, for example, the information you provided should not be accessible to others because it should be secure. 
P8 If the website is not secure there is a chance that the information you provide can be seen by anyone who is trying to get at it.

P9 When you do not have a secure internet connection, it will make what you do potentially visible to other users.

P10 People can hack into website. The cops can look at things written on the internet.

P11 The internet itself is letting me view information others have provided, so it makes sense that someone could see the information I provide using the same method.

P12 i donot know

Question 4: What is a website security certificate?

$\begin{array}{ll}\text { P1 } & \text { A (Correct) } \\ \text { P2 } & \text { B } \\ \text { P3 } & \text { A (Correct) } \\ \text { P4 } & \text { D } \\ \text { P5 } & \text { B } \\ \text { P6 } & \text { D } \\ \text { P7 } & \text { B } \\ \text { P8 } & \text { D } \\ \text { P9 } & \text { D }\end{array}$

P10 D

P11 B

P12 D

Question 5: What does https:// mean at the beginning of the website address?

P1 D

P2 D

P3 A (Correct)

P4 A (Correct) 


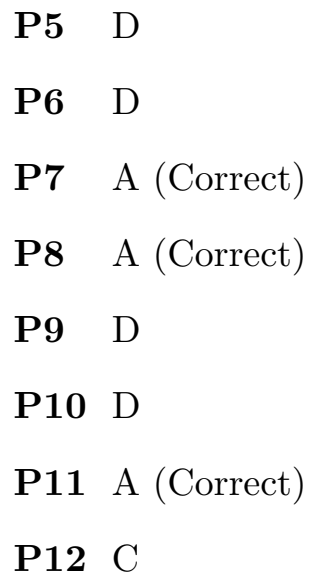

Question 7: How do you know the name of a person or company who is responsible for a website?

P1 at the end of the page, where it says "all rights reserved", or, if it is a company it might be stated at the top of the page. example: "cnet tm".

P2 It should be listed in the copyright details or a specific link should be provided with the info. 
P3 By the logo or company name present on the site. Perhaps in the URL. Sponsored by.

P4 The domain should tell you or somewhere on the website it should say

P5 The name and/or company is eaither listed under the "contact us" tab or located at the bottem of the page where copy right information might be

P6 You would only know this if it were mentioned or advertised.

P7 There is information in the section where you would find copyright information.

P8 Generally the person or company that is responsible has a header on the page, or the site falls under their overall domain name, or they are recognized somwhere on the page.

P9 Should be on the web page.

P10 By looking at either the web address or a logo on the top of the webpage.

P11 No idea. Generally, on the bottom of the page, it will say the person or company, and/or a copyright date, but I didn't see any of this on the pictures provided.

$\mathbf{P 1 2}$ usually its on the url or it is shown on the website

\section{Question 8: What is a phishing website?}

P1 an unsafe website

P2 I don’t know.

P3 A phishing website is a website that attempts to replicate legitimate sites in order to obtain confidential information from humans, such as credit card numbers.

P4 A fake website that tries to steal your information.

P5 i dont know

P6 I don't really know the answer

P7 A website that is looking for your information in order to use it for fraudulent means.

P8 A website that attempts to get you give them personal information m(usually by deception) which will then be used against you - either in a money scam or to compromise your identity online (identity theft) 
P9 N/A

P10 I am not sure.

P11 I website that phishes for your information. I don't know why the word "phish" is used, but I think it MEANS that others can get information off your computer through the internet.

P12 i dont know

\section{Question 9: What is a domain name?}

$\mathbf{P 1}$ is a company that hosts websites. or the regular website name.

P2 I don’t know.

P3 I am unausre.

P4 The name in the website address.

P5 i dont know

P6 I don't really know the answer

P7 The address. www.domainname.com. Anyone can purchase a domain name.

P8 The unique name registered to a particular website? Not completely sure what the formal definition is.

P9 N/A

P10 I'm not sure.

P11 The website address?

P12 i dont know

Question 10: Approximately how many websites do you visit that require a username and password?

P1 8

P2 5

P3 7

P4 5 
P5 4

P6 5

P7 5-7

P8 6

P9 5-7

P10 2

P11 15

P12 5-7

Question 11: How often do you re-use the same password on different websites?

P1 7

P2 very often

P3 Fairly often.

P4 Most of the time

P5 never

P6 Almost always

P7 never

P8 often

P9 sometimes

$\mathbf{P 1 0}$ almost all the time

P11 very often

P12 2

Question 12: Are you concerned about the security of your passwords?

P1 I am sometimes.

P2 nope.

P3 No Not right now.

P4 Not really 
P5 i haven't been in the past because i havent been given reason to. if a website looks like the one i distrust $\mathrm{i}$ wont put personal information on it and $\mathrm{i}$ never save my passwords.

P6 I am only when websites I don't trust ask for them. Otherwise, I'm not too concerned.

P7 I occasionally change them up, but I try my best in the first place to use as many different characters as possible.

P8 Yes

P9 no

P10 I make a password that is secure with different kinds of characters like letters and numbers and symbols so I feel comfotable using it a lot.

P11 Minorly, but I've never had a problem, and I think my password(s) are a good mix of easy for me to remember and hard for others to guess. When I don't trust websites, I use a different password.

$\mathbf{P 1 2}$ yes

Question 13: Are you concerned about websites that attempt to access your private information or download dangerous software?

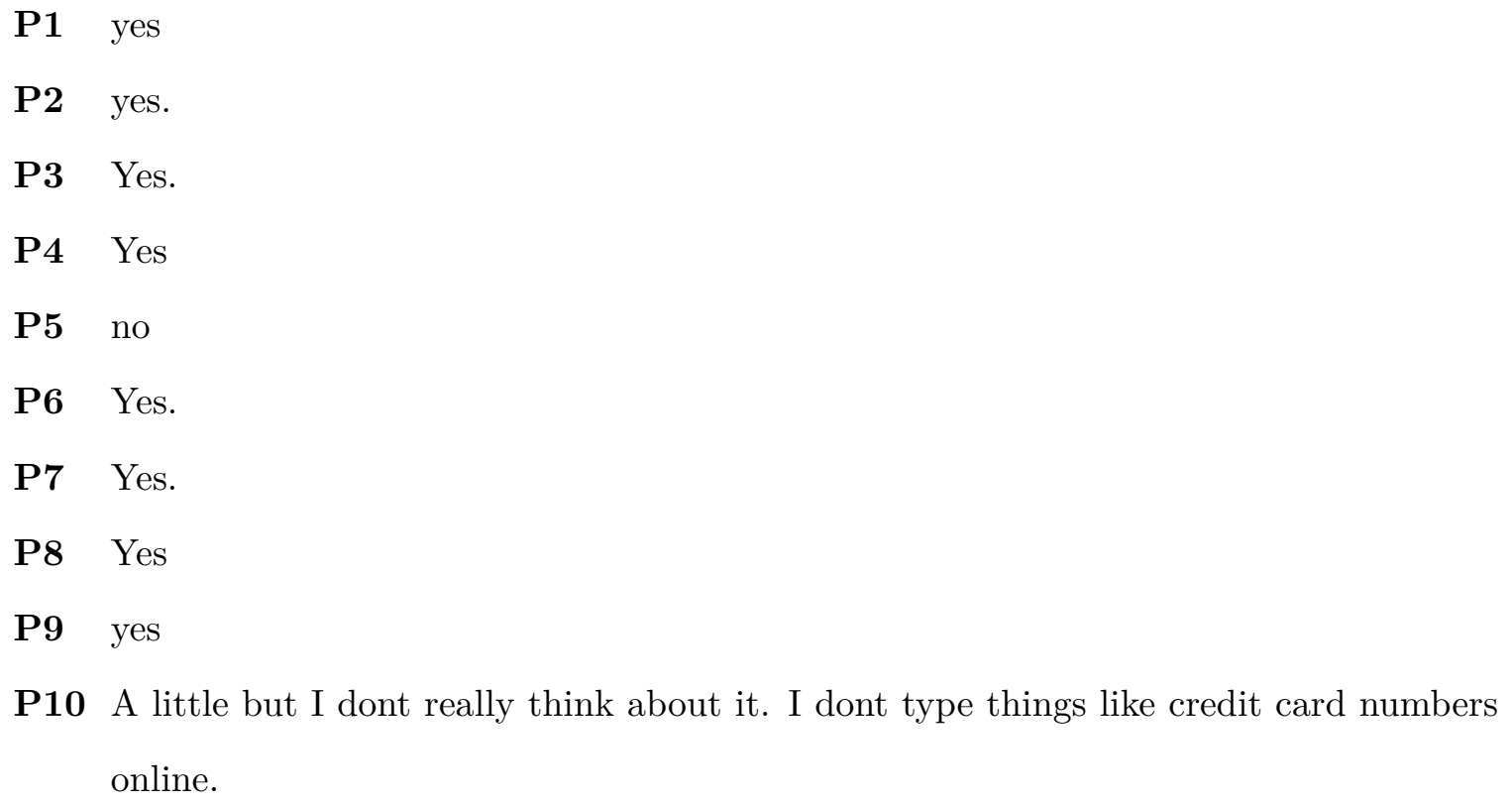

P10 A little but I dont really think about it. I dont type things like credit card numbers online. 
P11 A bit.

P12 yes

Question 14: Do you use any anti-virus or other Internet security software (e.g., McAfee, Norton, Microsoft Security Essentials, etc.)?

P1 McAfee

P2 Bit Defender.

P3 Yes. AVG.

P4 No

P5 yes i use bell sypatico

P6 Yes.

P7 No, I have a Mac.

P8 No, I am a Mac user, so in general anti-virus is unecessary.

P9 Norton

P10 McAfee

P11 Yes, I use Norton.

P12 yes norton

Question 15 (Part 1): I am concerned that, in the past, I have provided personal information to a fake website.
P1 yes
P2 no
P3 no
$\mathbf{P 4}$ no
P5 no
P6 yes
P7 no
P8 no 
P9 no

$\mathbf{P 1 0}$ no

P11 unsure

$\mathbf{P 1 2}$ no

Question 15 (Part 2): I feel confident that I can identify fake or malicious websites.

P1 Somewhat confident

P2 Not confident

P3 Not confident

P4 Somewhat confident

P5 Somewhat confident

P6 Somewhat confident

P7 Extremely confident

P8 Somewhat confident

P9 Somewhat confident

P10 Somewhat confident

P11 Somewhat confident

P12 Somewhat confident

Question 15 (Part 3): I believe that, if people get tricked into providing personal information to a fake/malicious website, it is their own fault.

P1 depends

P2 no

P3 no

P4 depends

P5 depends

P6 depends 


P7 yes
P8 no
P9 depends
P10 depends
P11 no
P12 depends




\section{Appendix $\mathbf{H}$}

\section{Study Two: Ethics Application (Activities And Risk)}

\section{Purpose of Study}

Previous research has shown that people perceive the risks associated with specific activities and technologies differently than others, depending on their general knowledge and expertise regarding the activity or technology in question (Fischhoff et al, 1978; Starr, 1969). Our research will take the earlier work conducted by Fischfoff et al. and restructure it to test participants on the perceived risks relating to activities over the Internet and in everyday life. We are interested in finding out their perception of the risks involved with each activity in terms of having their personal, confidential, and financial information lost as a result of engaging in each activity. For example, a person might report that downloading software from the Internet is a very risky activity for the potential loss of personal information because of embedded "Trojan horse" malware, while this same person might attribute a low risk to using an automatic teller machine at a local bank.

By better understanding how people perceive the risks in conjunction with the many activities involved while using the Internet, we can develop tools to help educate users on the many dangers and threats prominent over the Internet. In this research experiment, our methods would generally be the same as Fischhoff et al.'s study, except for the list of activities used and the rating scales given to each participant. Our study would ask participants to rate up to 30 activities on up to ten different 7 -point rating scales.

\section{Method}

Materials: We will use one demographics questionnaire created with the Limesurvey Web survey system, running on our Hotsoft Lab secure Web server. This questionnaire can be found in Appendix Appendix 1.

We will make use of one task to ask participants to rate up to 30 activities on various 7-point Likert scales. This task will be in survey form. The survey will be created using the 
Limesurvey web survey system. The task description can be found in Appendix Appendix J.

Participants: We will recruit 300 participants from the Amazon Mechanical Turk (AMT) online work marketplace. AMT is an online service where participants sign up to do tasks in exchange for modest payment. See https://www.mturk.com/mturk/welcome for additional details. We will use AMT for recruitment and payment purposes and consent forms and research questionnaires will be administered from our own secure servers (http://mvp.soft.carleton.ca/). Each participant will receive a $\$ 1$ USD honorarium for his or her time. Note that this is lower than the in-lab participants, but reflects a good AMT rate and higher rates are thought to attract inappropriate participants. The participants must be experienced Internet users (at least 2 years), and must use the Internet at least twice weekly. They must not be either experts or students in computer security.

Procedure: One task will be presented to each participant. This task will ask participants to rate up to 30 activities on various 7-point Likert scales. The task description can be found in Appendix Appendix J. This same list of activities will be used for each of the Likert rating scales. The list of activities is found in Appendix Appendix K. The list of rating scales is found in Appendix Appendix L As there are many activities and rating scales, each participant will be given a random selection of 20 of the 30 activities and 5 of the 10 rating scales. For example, for a particular participant, we might show activities 4, $7,10,12 \ldots 29$, with rating scales $3,4,7,8$, and 10 shown for each one.

\section{$\underline{\text { References }}$}

Fischhoff, B., Slovic, P., Lichtenstein, S., Read, S. \& Combs, B. (1978). How safe is safe enough? A psychometric study of attitudes towards technological risks and benefits. Policy Sciences, 9 (2), 127-152.

Starr, C. (1969). Social benefits vs. technological risk. Science, 165, 1232-1238. 


\section{Informed Consent}

The purpose of an informed consent is to ensure that you understand the purpose of the study and the nature of your involvement. The informed consent must provide sufficient information such that you have the opportunity to determine whether you wish to participate in the study.

\section{Present Study: Activities and Risk}

Research personnel: The following people are involved in this research project, and may be contacted at anytime if you have questions or concerns: Daniel LeBlanc (dblanc@connect.carleton.ca, phone: 613-520-2600 ext. 1987), Dr. Robert Biddle (Faculty Sponsor, email: robert_biddle@carleton.ca, 613-520-2600 ext. 6317), and Dr. Andrew Patrick (Adjunct Research Professor, email: andrew@andrewpatrick.ca, phone: 613-2779211).

Ethical concerns: Should you have any ethical concerns about this research, please contact Dr. Monique Sénéchal, at monique_senechal@carleton.ca (613-520-2600 ext. 1155).

Any other concerns: For any other concerns, please contact Dr. Anne Bowker (Chair, Department of Psychology, 613-520-2600 ext. 4171, psychchair@carleton.ca).

Purpose: The purpose of this study is to investigate how people identify risk in everyday activities.

Description: We are asking you to fill out a demographics questionnaire regarding your background. For the main task, we will ask you to rate a list of activities on a few different rating scales.

Duration: The session will last approximately 30 minutes.

Remuneration: You will receive a $\$ 1$ honorarium for your participation in the research. 
Potential Risk/Discomfort: There are no physical risks to participating in this study, beyond those involved in normal computer usage. If you feel any discomfort or distress, you may choose not to answer specific questions, and you will not be penalized in any way if you choose to do this. The debriefing form given to you at the end of the study provides contact details that you may use if you wish to obtain additional information.

Anonymity/Confidentiality: The data collected in this experiment are strictly confidential. All data are coded such that your name is not associated with the responses you provide. Any identifying information associated with your code will be confined to a single page that will be separated from your questionnaire, and kept in a separate, secured file by the research investigators, who will keep this information confidential. To respect Ontario Government FIPPA requirements, we will keep your data in our secure records for no longer than 5 years.

Right to withdraw: Your participation in this study is entirely voluntary. At any point during the study, you have the right not to complete certain questions, or to withdraw from the study. 


\section{Consent}

I have read and understand the above terms of testing and I understand the conditions of my participation. My choice of pressing the agree button indicates that I agree to participate in this experiment. (If you agree to participate, the instructions will be sent to your email address.)

Name:

Email Address:

[Button] Yes, I agree to participate in this study (Provide name and email address above). [Button] No, I will not participate in this study (Leave name and email address empty).

This study has received clearance by the Carleton University Psychology Research Ethics Board (Reference \#12-203). 


\section{$\underline{\text { Debriefing }}$}

\section{What are we trying to learn in this research?}

This research examines the perceived risks that people attribute to everyday activities on the Internet and in everyday life. For example, some people might perceive engaging in social websites (e.g., Facebook) as particular risky, while others may think this activity as being not so risky.

\section{Why is this important to scientists or the general public?}

Previous research has shown that people perceive the risks associated with specific activities and technologies differently than others, depending on their general knowledge and expertise regarding the activity or technology in question. Our research takes this work and restructures it to find out more information about the perceived risks relating to activities over the Internet. By better understanding how people perceive these risks, we can develop educational tools to help them identify the more dangerous elements involved with interactions over the Internet.

\section{What are our hypotheses and predictions?}

We expect that users will perceive the risks of Internet activities very differently than they perceive everyday activities.

\section{Where can I learn more?}

In order to learn more about our research on security and trust in an online environment, please see our website at http://hotsoft.carleton.ca/security/ 


\section{What if I have questions later?}

If you have any remaining concerns, questions, or comments about the experiment, please feel free to contact Daniel LeBlanc (Principal Investigator) at: dblanc@connect.carleton.ca (613-520-2600 ext. 1987), Dr. Robert Biddle (Faculty Sponsor) at: robert_biddle@carleton.ca, 613-520-2600 ext. 6317, and Dr. Andrew Patrick (Adjunct Research Professor) at: andrew@andrewpatrick.ca, 613-277-9211. Should you have any ethical concerns about this research, please contact Dr. Monique Sénéchal, at monique_senechal@carleton.ca (613-520-2600 ext. 1155). For any other concerns, please contact Dr. Anne Bowker (Chair, Department of Psychology, 613-520-2600 ext. 4171, psychchair@carleton.ca).

Thank you for participating in this research! 


\section{Doing Research Online}

Recruiting participants online: For the purposes of the research study, we will recruit participants using the Amazon Mechanical Turk online work marketplace. An ad will be placed on their website (https://www.mturk.com/mturk/). Once participants have agreed to participate in the research study, they will be redirected from the Mechanical Turk website to our HotSoft website (http://mvp.soft.carleton.ca/), where they will be able to complete the online survey. The online survey will be created using the LimeSurvey freeware application (http://www.limesurvey.org/), which is hosted on our HotSoft server.

\section{Announcement for recruiting participants via Amazon Mechanical Turk}

\section{Study Name: Activities and Risk}

Description: This research examines the perceived risks that people attribute to everyday activities on the Internet and in everyday life. For example, some people might perceive engaging in social websites (e.g., Facebook) as particular risky, while others may think this activity as being not so risky.

Eligibility Requirements: We are looking for adults 18 years of age or older, who possess a minimum of 2 years of experience with the Internet, and be regular users (i.e., use the Internet at least 2 times each week). We wish to exclude anyone who is an expert or student in computer security.

Duration: The session will last approximately 30 minutes. Instructions will be provided via email once we receive your notice of participation. 
Compensation: You will receive a $\$ 1$ USD honorarium for your time.

\author{
Researchers: \\ Daniel LeBlanc (Principal Investigator) \\ Dr. Robert Biddle (Faculty Sponsor) \\ Dr. Andrew Patrick (Adjunct Research Professor)
}

This study has received clearance by the Carleton University Psychology Research Ethics Board (Reference \#12-203). 


\section{Appendix I}

\section{Study Two: Pre-Test Demographics Questionnaire}

This questionnaire asks a few questions about your demographic information. All data is confidential and is coded for anonymity. You are not obliged to answer any of the questions.

In this questionnaire, we'll be asking some brief questions about your demographic information, as well as your level of Internet knowledge. All data is confidential and is coded for anonymity. You are not obliged to answer any of the questions.

There are 5 questions in this survey.

Question 1: What is your age?

Question 2: What is your gender?

Male

$\bigcirc$ Female

Question 3: What is your occupation?

Question 4: What is your highest level of education?

$\bigcirc$ Primary

$\bigcirc$ Secondary or high school

$\bigcirc$ Trade or professional school

Some college or university

$\bigcirc$ Bachelor's degree

Graduate degree

Other: 
Question 5: How often do you browse the Web?

Once a month

Once a week

Several times a week

$\bigcirc$ Daily

Multiple times daily

Thank you for completing the demographics questionnaire. Please see the experimenter for further instructions. 


\section{Appendix $\mathbf{J}$}

\section{Study Two: Main Task Description}

We are asking you to rate a list of activities in relation to the risk of the loss of personal information. Personal information loss can be seen as the following:

- The loss of personal information that is harmful to you (for example: your personal information is stolen from your medical records at your doctor's office; your personal information is stolen from your office computer).

- The loss of confidential information (for example: your co-worker tells your boss about your actions during this year's office Christmas party; your child tells your cousin about your embarrassing habits).

- The loss of financial information (for example: your credit card number is copied by a restaurant waiter; your bank card personal identification number (PIN) is seen by someone behind you at an automated teller machine (ATM)).

For each rating scale, provide the number that fits best with your understanding of the risks involved with the activity. 


\section{Appendix K}

\section{Study Two: List Of Activities}

- Using an automated teller machine (ATM)

- Having your computer fixed at a store

- Giving your credit card number over the phone to buy something

- Belonging to a loyalty program (e.g., Frequent Flyer Points, Supermarket Club Card)

- Paying bills by postal mail (e.g., U.S. Postal Service)

- Installing software downloaded from the Internet

- Sending and receiving email

- Making an online purchase (e.g., Amazon, Dell Computers)

- Doing your banking online (e.g., Bank of America Online Banking, Citibank Online Banking)

- Using a search engine (e.g., Google, Yahoo, Bing)

- Searching for adult content online

- Downloading pirated movies or music

- Using social networking websites (e.g., Facebook, Twitter)

- Using a webcam through a chat program (e.g., MSN Live, Skype)

- Using an online dating service (e.g., Match.com, eHarmony)

- Making a purchase through an auction website (e.g., eBay, eBid)

- Sending money through a payment website (e.g., PayPal, Google Checkout)

- Selling something through a local online classified ad website (e.g., Craigslist, Oodle) 
- Responding to an ad on a local online classified ad website (e.g., Craigslist, Oodle)

- Playing poker or other gambling activity over the Internet 


\section{Appendix L}

\section{Study Two: Rating Scales}

1 - Consider each of the activities below. For each activity, how beneficial is this activity to the general population?

$\bigcirc 1$ No benefits whatsoever $\bigcirc 2 \quad \bigcirc 3 \quad \bigcirc 4 \quad \bigcirc 5 \quad \bigcirc 6 \quad \bigcirc 7$ Extremely beneficial

2 - Consider each of the activities below. For each activity, how likely is it that the loss of personal information would occur as a result of this activity?

$\bigcirc 1$ Not at all likely $\bigcirc 2 \quad \bigcirc 3 \quad \bigcirc 4 \quad \bigcirc 5 \quad \bigcirc 6 \bigcirc 7$ Extremely likely

3 - Consider each of the activities below. For each activity, to what extent is the risk of losing personal information this way immediate?

$\bigcirc 1$ Occurs after long delay $\bigcirc 2 \quad \bigcirc 3 \quad \bigcirc 4 \quad \bigcirc 5 \quad \bigcirc 6 \quad \bigcirc 7$ Occurs immediately

4 - Consider each of the activities below. For each activity, when information loss occurs, after how much time would you be affected by the consequences?

$\bigcirc 1$ Affected after long delay $\bigcirc 2 \quad \bigcirc 3 \quad \bigcirc 4 \quad \bigcirc 5 \quad \bigcirc 6 \quad \bigcirc 7$ Affected immediately

5 - Consider each of the activities below. For each activity, when personal information is lost, how severe are the consequences?

$\bigcirc 1$ Mildly inconvenient $\bigcirc 2 \quad \bigcirc 3 \quad \bigcirc 4 \quad \bigcirc 5 \quad \bigcirc 6$ Extremely severe

6 - Consider each of the activities below. How often do you engage in each of the activities?
1 Never
$\bigcirc 2$
$\bigcirc$
$\bigcirc$
$\bigcirc$
6
7 Very often 


\title{
Appendix M
}

\section{Study Three: Ethics Application (Understanding of Internet Software}

\begin{abstract}
Abilities)
Purpose of Study

Previous research has shown that people perceive the risks associated with specific activities and technologies differently than others, depending on their general knowledge and expertise regarding the activity or technology in question (Fischhoff et al, 1978; Starr, 1969). Our current research will examine users' knowledge about the many facets that co-exist during their daily interactions on the Internet, especially those facets having to do with malicious websites. This experiment will build upon the knowledge we accumulated from our latest work, where we were able to determine that participants associate a high amount of risk to specific online activities (e.g., using a search engine, making an online purchase) that experts would normally perceive as being less risky (LeBlanc \& Biddle, 2012). The study will target various online activities, and assess the knowledge participants possess in regards to the threats that exist in relationship to each activity. For example, a person might report that downloading software from the Internet is a very risky activity for the potential installation of a Malware software application, while this same person might report that the activity of online banking is completely safe from any dangers.

By better understanding how people assess threats in conjunction with online activities, we can develop tools to help educate users on the many dangers prominent over the Internet. Our study would ask participants to rate 4 online activities on 40 (10 for each activity) 7-point rating scales.
\end{abstract}

\section{Method}

Materials: We will use one standard Dell desktop computer running the Windows 7 operating system with standard web browsers (e.g., Microsoft Internet Explorer, Mozilla Firefox). 
We will use one demographics questionnaire created with the Limesurvey web survey system, running on our Hotsoft Lab secure web server. This questionnaire can be found in Appendix Appendix N.

We will ask participants to rate 4 different online activities on a total of 40 (10 for each activity) 7-point Likert scales. This task will be in survey form. The survey will be created using the Limesurvey web survey system. The task description can be found in Appendix Appendix O. For each online activity, images of the website will be shown to the participant; these images will be embedded directly within the Limesurvey page where the activity name and rating scales are located. In terms of the website image screenshots, we understand that our use of these companies' website homepages is acceptable under the "Fair Dealing" provision of the Copyright Act, section 29.

Participant answers on the demographics questionnaire and the main task questionnaire will be kept on our secure server located within our HotSoft lab at Carleton University.

Participants: 200 online participants will be recruited through the Amazon Mechanical Turk online marketplace. Participants will be recruited by way of a website posting on the Amazon Mechanical Turk online marketplace website. Each participant will receive a $\$ 1$ honorarium for his or her time. If, at any point during testing, should the Amazon Mechanical Turk online marketplace participant choose to quit the study before its completion, the participant will be redirected to a debriefing page.

The participants must be experienced Internet users (at least 2 years), and must use the Internet at least twice weekly. They must not be either experts or students in computer security.

The participants will be limited to the United States. As such, the rating scale material has been created with American companies in mind.

Procedure: One task will be presented to each participant. This task will ask participants to rate 4 different online activities on a total of 40 (10 for each activity) 7-point Likert scales. The task description can be found in Appendix Appendix O. This same list of online activities will be used for each of the Likert rating scales. The list of activities is found in 
Appendix Appendix P. The list of rating scales is found in Appendix Appendix Q, The list of website images is found in Appendix Appendix R. Each participant will be shown the 4 online activities in a specified order, and the Likert rating scales will be randomly shown to the participant by the Limesurvey program.

Confidentiality: In our study, we ask participants for their name and email address in order to obtain consent for their participation in the research. In order to ensure complete separation between participants' identity and their study data, we will store their name and email address in a separate database from the one storing the research data. This method of securing the data will be used for both the local sample of participants and the online sample of participants.

\section{References}

Fischhoff, B., Slovic, P., Lichtenstein, S., Read, S. \& Combs, B. (1978). How safe is safe enough? A psychometric study of attitudes towards technological risks and benefits. Policy Sciences, 9 (2), 127-152.

LeBlanc, D., \& Biddle, R. (2012). Risk perception of Internet-related activities. Twelfth Annual International Conference on Privacy, Security, and Trust (PST 2012), 8895.

Starr, C. (1969). Social benefits vs. technological risk. Science, 165, 1232-1238. 


\section{Informed Consent}

The purpose of an informed consent is to ensure that you understand the purpose of the study and the nature of your involvement. The informed consent must provide sufficient information such that you have the opportunity to determine whether you wish to participate in the study.

\section{Present Study: Understanding of Internet Software Abilities}

Research personnel: The following people are involved in this research project, and may be contacted at anytime if you have questions or concerns: Daniel LeBlanc (dblanc@connect.carleton.ca, phone: 613-520-2600 ext. 1987), Dr. Robert Biddle (Faculty Sponsor, email: robert_biddle@carleton.ca, 613-520-2600 ext. 6317), and Dr. Andrew Patrick (Adjunct Research Professor, email: andrew@andrewpatrick.ca, phone: 613-2779211).

Ethical concerns: Should you have any ethical concerns about this research, please contact Dr. Avi Parush (Chair, Psychology Ethics Board, avi_parush@carleton.ca, 613-520-2600, ext. 6026 .

Any other concerns: For any other concerns, please contact Dr. Anne Bowker (Chair, Department of Psychology, 613-520-2600 ext. 8218, psychchair@carleton.ca).

Purpose: The purpose of this study is to investigate how people rate online activities on various different characteristics.

Description: We are asking you to fill out a demographics questionnaire regarding your background. For the main task, we will ask you to rate a short list of online activities on a few different rating scales.

Duration: The session will last approximately 45 minutes.

Remuneration: You will receive a $\$ 1$ honorarium for your participation in the research. 
Potential Risk/Discomfort: There are no physical risks to participating in this study, beyond those involved in normal computer usage. If you feel any discomfort or distress, you may choose not to answer specific questions, and you will not be penalized in any way if you choose to do this. The debriefing form given to you at the end of the study provides contact details that you may use if you wish to obtain additional information.

Anonymity/Confidentiality: The data collected in this experiment are strictly confidential. All data are coded such that your name is not associated with the responses you provide. Any identifying information associated with your code will be confined to a single page that will be separated from your questionnaire, and kept in a separate, secured file by the research investigators, who will keep this information confidential. To respect FIPPA requirements, we will keep your data in our secure records for no longer than 5 years.

Right to withdraw: Your participation in this study is entirely voluntary. At any point during the study, you have the right not to complete certain questions, or to withdraw from the study without penalty. Should you decide to withdraw during the testing session, you will still receive remuneration for your time. 


\section{Consent}

I have read the above form and understand the conditions of my participation. My participation in this study is voluntary, and I understand that if at anytime I wish to leave the experiment, I may do so without having to give an explanation and with no penalty whatsoever. Furthermore, I am also aware that the data gathered in this study are confidential and anonymous with respect to my personal identity. Entering my name and my email address and pressing the "Submit" button indicates that I agree to participate in this study.

Name:

Email Address:

[Button] Yes, I agree to participate in this study (Provide name and email address above). [Button] No, I will not participate in this study (Leave name and email address empty).

This study has received clearance by the Carleton University Psychology Research Ethics Board (Reference \#12-147). 


\section{$\underline{\text { Debriefing }}$}

\section{What are we trying to learn in this research?}

This research examines the threats that people perceive in everyday activities on the

Internet. For example, some people might perceive the threat of downloading and installing a software application while engaging in social websites (e.g., Facebook) as particular risky, while others may think this particular threat as being not so risky in conjunction with the same online activity.

\section{Why is this important to scientists or the general public?}

Previous research has shown that people perceive the risks associated with specific activities and technologies differently than others, depending on their general knowledge and expertise regarding the activity or technology in question. Our current research examines in greater detail people's perceptions of the threats that exist in conjunction with online activities, such as online banking and online purchasing. By better understanding how people perceive the threats associated with various online activities, we can develop educational tools to help them identify the more dangerous elements involved with interactions over the Internet.

\section{What are our hypotheses and predictions?}

We expect that users will correctly identify and assess some of the threats associated with Internet activities, but will also incorrectly assess other threats that they perceive as less likely or less severe if something were to occur.

\section{Where can I learn more?}

In order to learn more about our research on security and trust in an online environment, please see our website at http://hotsoft.carleton.ca/security/ 


\section{What if I have questions later?}

If you have any remaining concerns, questions, or comments about the experiment, please feel free to contact Daniel LeBlanc (Principal Investigator) at: dblanc@connect.carleton.ca (613-520-2600 ext. 1987), Dr. Robert Biddle (Faculty Sponsor) at: robert_biddle@carleton.ca, 613-520-2600 ext. 6317, and Dr. Andrew Patrick (Adjunct Research Professor) at: andrew@andrewpatrick.ca, 613-277-9211. Should you have any ethical concerns about this research, please contact Dr. Avi Parush (Chair, Psychology Ethics Board, avi_parush@carleton.ca, 613-520-2600, ext. 6026). For any other concerns, please contact Dr. Anne Bowker (Chair, Department of Psychology, 613-520-2600 ext. 8218, psychchair@carleton.ca).

Thank you for participating in this research! 


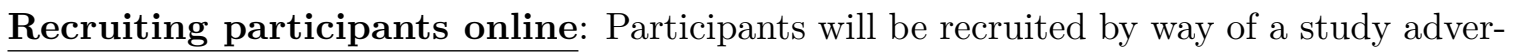
tisement on the Amazon Mechanical Turk online marketplace website.

\section{Announcement for recruiting participants via Amazon Mechanical Turk}

\section{Study Name: Understanding of Internet Software Abilities}

Description: This research examines issues that arise in online activities. For example, some people might perceive certain issues associated with social networking websites (e.g., Facebook), while others may think this activity as being unrelated to those issues.

Eligibility Requirements: We are looking for adults 18 years of age or older, who possess a minimum of 2 years of experience with the Internet, and be regular users (i.e., use the Internet at least 2 times each week). We wish to exclude anyone who is an expert or student in computer security.

Duration: The session will last approximately 45 minutes.

Compensation: You will receive a $\$ 1$ USD honorarium for your time.

Contact: For more details, please contact Daniel LeBlanc at riskstudy@mvp.soft.carleton.ca

This study has received clearance by the Carleton University Psychology Research Ethics Board (Reference \#12-147). 


\section{Appendix N}

\section{Study Three: Pre-Test Demographics Questionnaire}

This questionnaire asks a few questions about your demographic information. All data is confidential and is coded for anonymity. You are not obliged to answer any of the questions.

In this questionnaire, we'll be asking some brief questions about your demographic information, as well as your level of Internet knowledge. All data is confidential and is coded for anonymity. You are not obliged to answer any of the questions.

There are 5 questions in this survey.

Question 1: What is your age?

Question 2: What is your gender?

Male

$\bigcirc$ Female

Question 3: What is your occupation?

Question 4: What is your highest level of education?

$\bigcirc$ Primary

$\bigcirc$ Secondary or high school

$\bigcirc$ Trade or professional school

Some college or university

Bachelor's degree

Graduate degree

Other: 
Question 5: How often do you browse the Web?

Once a month

Once a week

Several times a week

$\bigcirc$ Daily

Multiple times daily

Thank you for completing the demographics questionnaire. 


\section{Appendix $\mathrm{O}$}

\section{Study Three: Task Description}

You are now at the main task.

Over the next few pages, you'll be presented with different website screenshots. These screenshots are of the actual websites, and have not been altered in any way.

Your task is to observe the website screenshot, taking in the information about the website and the website scenario, and then provide your opinion on a few statements about the website in question. 


\section{Appendix $\mathbf{P}$}

\section{Study Three: List of Activities}

1. Doing your banking online

2. Making an online purchase

3. Installing software downloaded from the Internet

4. Using a search engine 


\section{Appendix Q \\ Study Three: Rating Scales \\ Activity: Online Banking (Bank of America)}

\section{True Statements}

1. The https indicator in the address bar signifies that my information is encrypted during communications between my computer and the Bank of America website server.

2. The Web address www.bankofamerica.versipass.com is not associated to the Bank of America's website.

3. If there is Malware installed on the computer I use to gain access to my Bank of America online account, there is a chance that my login information can be copied and sent to a third party.

4. Following a given link found on a search engine (e.g., Google) that takes me to the Bank of America website can leave me open to a phishing attack.

5. My Bank of America account information (e.g., client card number, password) can be stolen by "shoulder-surfing" attacks. 


\section{Activity: Online Banking (Bank of America)}

\section{False Statements}

1. Other Internet users are able to intercept my Bank of America information (e.g., username, password, client card number) when I submit it via the login form found on the homepage.

2. My Internet Service Provider (e.g., Comcast, Time Warner, AT\&T) is able to monitor my personal information regarding my bank accounts, email accounts, usernames, and passwords.

3. If I use the actual and current Web address to reach the Bank of America website, I may be open to phishing attacks.

4. Friends from my social networking website (e.g., Facebook, MySpace) can see that I do banking at the Bank of America financial institution.

5. My computer can be infected by malware (e.g., a virus, a spyware program) by engaging in this activity. 


\section{Activity: Making a purchase online (Amazon.com)}

\section{True Statements}

1. Cookies used by Amazon can track my browsing history on any their website, and recommend specific products that target my browsing preferences.

2. Following a given link found on a search engine (e.g., Google) that takes me to the Amazon website can leave me open to a phishing attack.

3. If there is Malware installed on the computer I use to make my purchase online, there is a chance that my login information can be copied and sent to a third party.

4. The https indicator in the address bar signifies that my information is encrypted during communications between my computer and Amazon's website server.

5. Using a free wireless access point (e.g., connecting to the Internet at a Starbucks coffee shop) can allow for my search results on Amazon to be altered. 


\section{Activity: Making a purchase online (Amazon.com)}

\section{False Statements}

1. The http indicator in the address bar on the Amazon.com homepage signifies that my information is encrypted during communications between my computer and the Amazon website server.

2. Other Internet users are able to intercept my personal information (e.g., username, password, credit card number) when I submit it via the Amazon.com checkout form.

3. My computer can be infected by malware (e.g., a virus, a spyware program) by engaging in this activity.

4. If I use the actual and current Web address to reach the Amazon website, I may be open to phishing attacks.

5. The payment-processing server that Amazon uses in order to process my credit card payment has access to all of the personal information (e.g., username, password, wish lists, shopping cart contents) found on my Amazon account. 
Activity: Installing a software application downloaded from the Internet (CNet

\section{Download)}

\section{True Statements}

1. A malicious software application (e.g., Malware, virus) downloaded and installed from the Internet can manipulate any of the files contained on my computer's hard drive.

2. A malicious software application (e.g., Malware, virus) downloaded and installed from the Internet can manipulate Microsoft Word files, executable files (i.e., .exe files), and PDF files in order to collect my personal information.

3. Any software application I download from the Internet can potentially contain malicious programming code.

4. A commonly known software application (e.g., Adobe Reader, Microsoft Internet Explorer) can be duplicated before it is downloaded, and can contain malicious programming code in order for someone to "attack" my computer (e.g., damage or destroy computer files, collect personal information).

5. If I download a software application on a downloads website (e.g., www.download.cnet.com) other than the official company's website (e.g., www.microsoft.com), then I run a higher risk of downloading and installing malware instead of the real, genuine software application. 
Activity: Installing a software application downloaded from the Internet (CNet

\section{Download)}

\section{False Statements}

1. If the software application I download from the Internet contains malicious programming code, then all of my usernames and passwords for each of my various online accounts (e.g., email, banking, etc.) will automatically be copied and sent to a third party.

2. A virus scan program (e.g., McAfee Antivirus, Norton Antivirus) will detect with 100\% accuracy any malicious software applications that attempt to compromise (i.e., "attack") my computer.

3. Other Internet users are able to intercept my personal information (e.g., username, password) when I download a software application from the Internet.

4. The http indicator in the address bar on the Downloads.com homepage signifies that my information is encrypted during communications between my computer and the Downloads.com website server.

5. If I use the actual and current Web address of the Downloads.com website, I may be open to phishing attacks. 


\section{Activity: Using a search engine (Google)}

\section{True Statements}

1. Some Google search engine results can leave me open to phishing attacks when I click on the given link.

2. My Internet Service Provider (e.g., Comcast, Time Warner, AT\&T) is able to monitor the things I search for, as well as the results that are returned to me, using Google.

3. Using a free wireless access point (e.g., connecting to the Internet at a Starbucks coffee shop) can allow for my search results on Google to be altered.

4. Clicking on a Google ad can potentially bring me directly to a phishing website.

5. Malicious software (e.g., Malware) that is installed on my computer can purposely alter my Google results in favour of other results. 


\section{Activity: Using a search engine (Google)}

\section{False Statements}

1. The http indicator in the address bar on the Google.com homepage signifies that my search criteria is encrypted during communications between my computer and Google's servers.

2. Using Google can allow other Internet users to spy on my computer and obtain my personal information (e.g., usernames, passwords, bank information).

3. Google monitors each and every website listed on their search engine, and purposefully leaves out all potentially malicious links from its search results.

4. Using Google's map service allows other Internet users to find where I am presently located.

5. Using the Google search engine allows other Internet users to intercept my Google Mail (i.e., GMail) account information (e.g., username and password). 


\section{Appendix R}

\section{Study Three: Website Images}

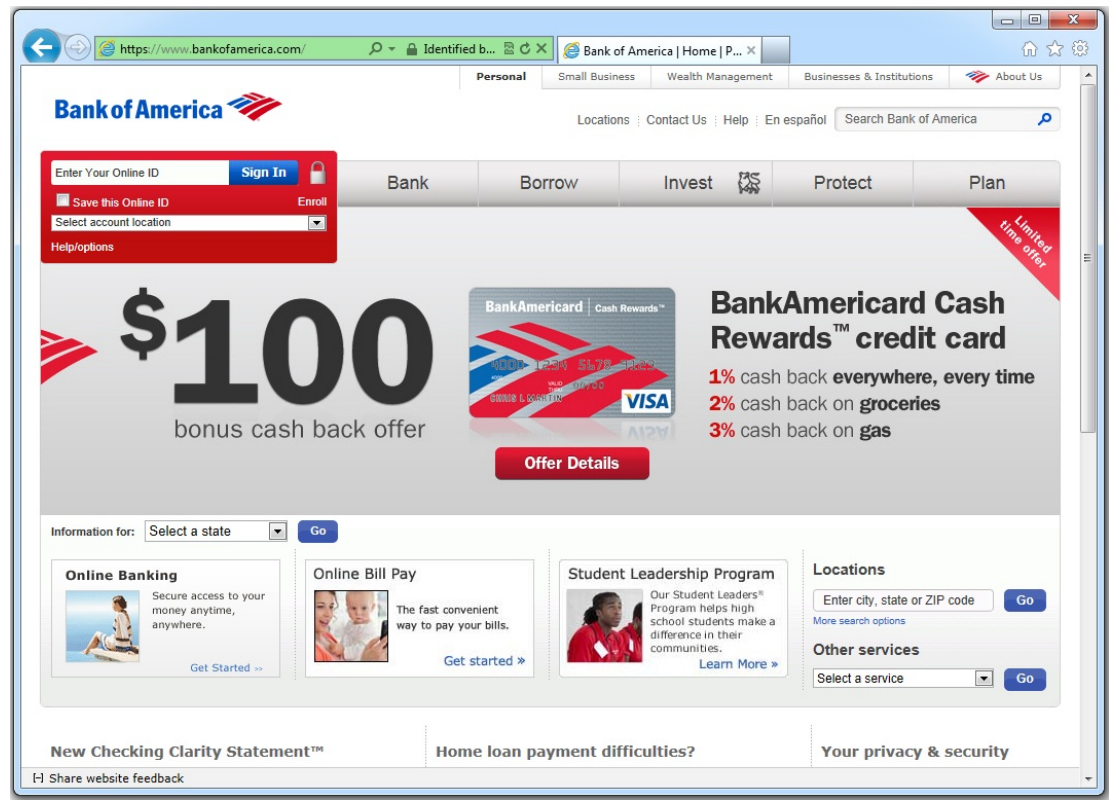

Figure 44. Bank of America website screenshot.

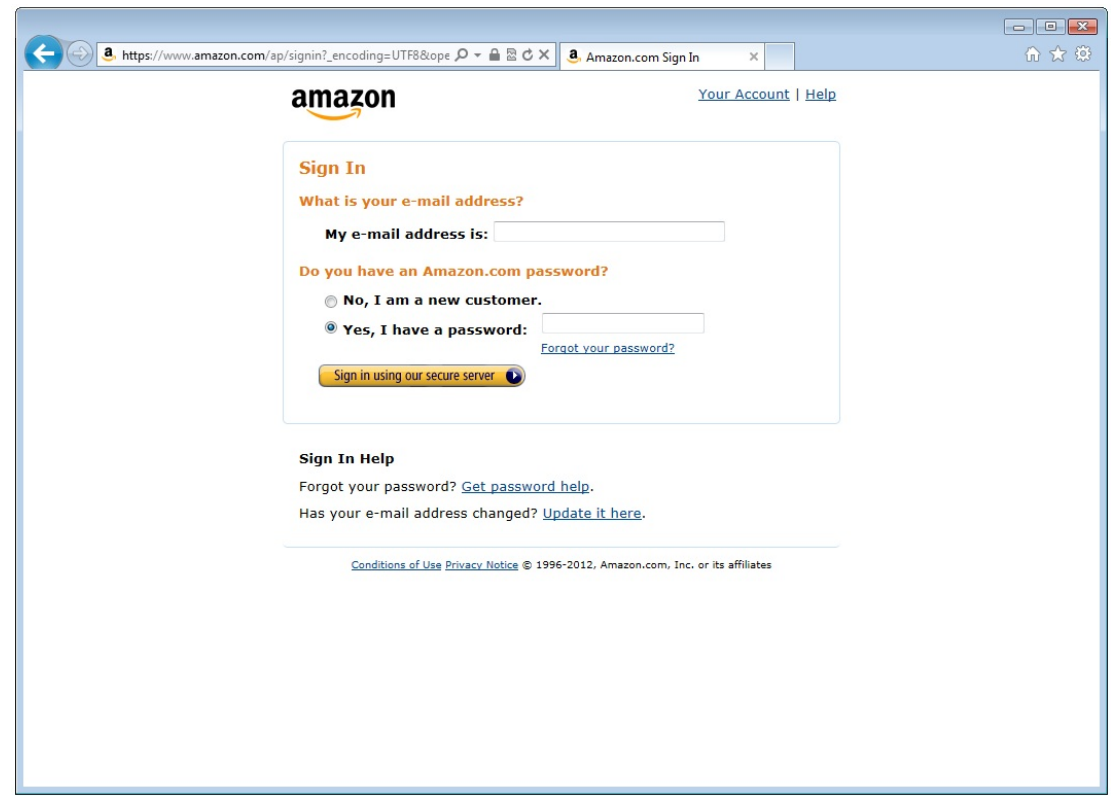

Figure 45. Amazon website screenshot. 


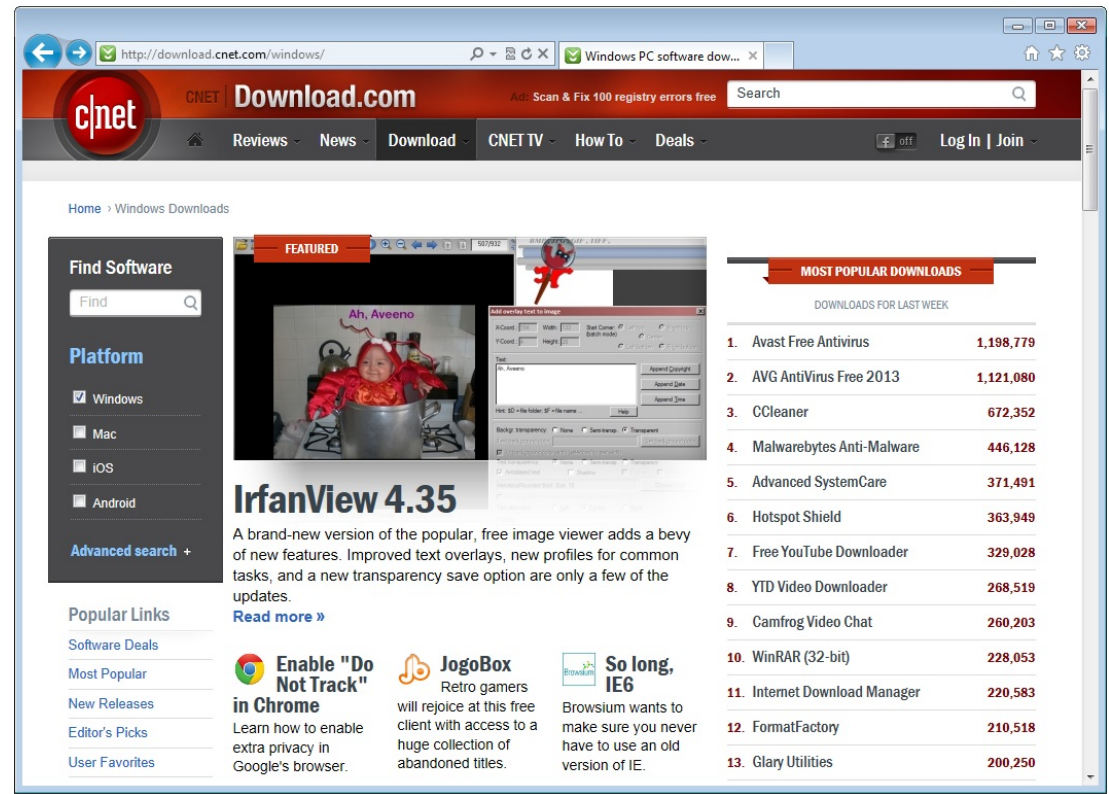

Figure 46. CNet website screenshot.

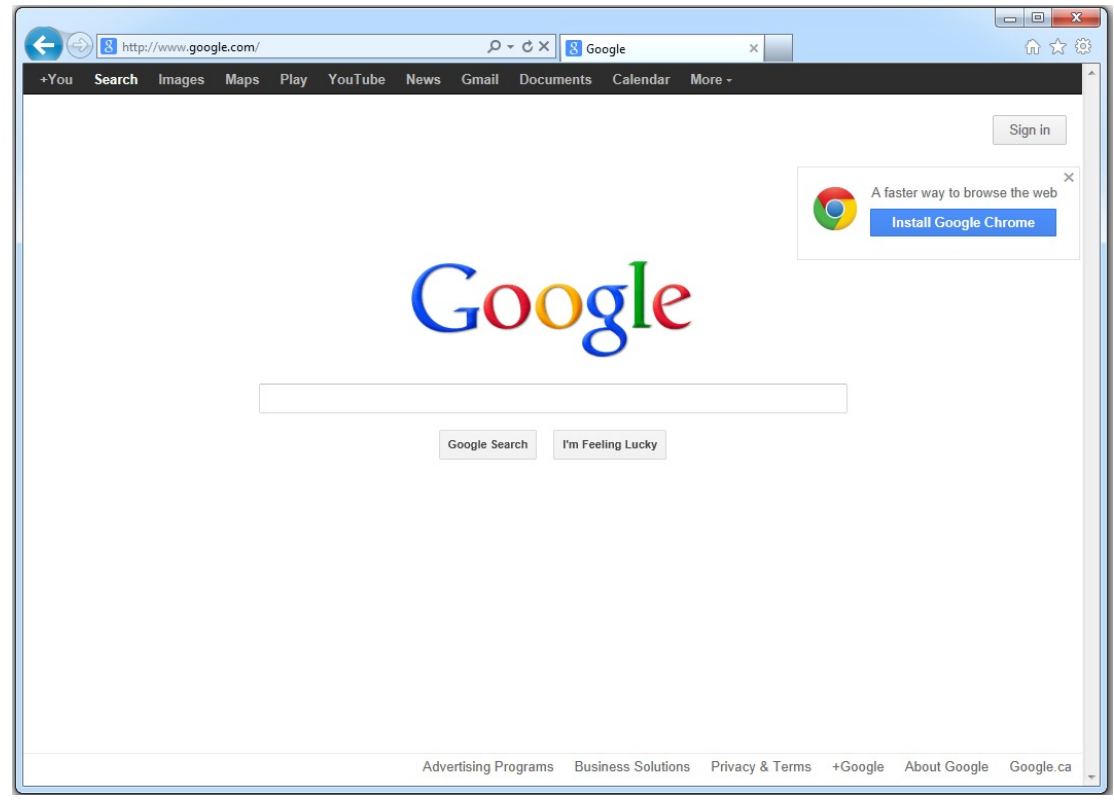

Figure 4\%. Google website screenshot. 


\section{Appendix $\mathrm{S}$}

\section{Study Three: Post-Test Questionnaire}

The survey is almost done. Please take a moment to answer the following post-test questions.

1 - How often do you do online banking?

$\bigcirc$ Never

Once a month

Once a week

Several times a week

$\bigcirc$ Daily

Multiple times daily

2 - How often do you make online purchases?

$\bigcirc$ Never

Once a month

$\bigcirc$ Once a week

Several times a week

$\bigcirc$ Daily

Multiple times daily 
3 - How often do you install software applications downloaded from the Internet?

$\bigcirc$ Never

Once a month

Once a week

Several times a week

Daily

Multiple times daily

4 - How often do you use search engines to look for things on the Internet?

$\bigcirc$ Never

Once a month

Once a week

Several times a week

$\bigcirc$ Daily

Multiple times daily

5 - How would you rate your knowledge of computer and Internet security?

$\bigcirc$ Limited knowledge

Some knowledge

About average

More than average knowledge

$\bigcirc$ Expert knowledge 


\section{Appendix T}

\section{Study Four: Ethics Application (Interpreting Internet Structure)}

\section{Purpose of Study}

Previous research has shown that people perceive the risks associated with specific activities and technologies differently than others, depending on their general knowledge and expertise regarding the activity or technology in question (Fischhoff et al, 1978; Starr, 1969). Research in risk communication stipulates that a better understanding of the population's beliefs about risks associated with a specific event is necessary in order to develop teaching tools to help them better understand these risks (Morgan et al, 2002).

Our current research will examine users' knowledge about the many facets that coexist during their daily interactions on the Internet. The research will also attempt to determine whether users have complete and correct mental models of how the flow model of Internet usage is constructed, based on expert input. This experiment will build upon the knowledge we accumulated from our latest studies, where we were able to determine that participants associate a high amount of risk to specific online activities (e.g., using a search engine, making an online purchase) that experts would normally perceive as being less risky (LeBlanc \& Biddle, 2012); we also determined that there are problematic areas in user understanding of the various threats and security mechanisms associated with various Internet activities (e.g., using a wireless access point, downloading and installing malicious software).

The study will target various online activities associated with our flow model of Internet usage, and assess the knowledge participants possess in regards to the threats that exist in relationship to each activity, as well as the security mechanisms that are in place to protect them from these threats. For example, one person's mental model of the flow of Internet usage might differ greatly from another person's mental model in regards to their understanding of the mechanisms associated with the communications between their computer, their Internet Service Provider, and the website server of a given website. 
By better understanding people's mental model of the flow model of Internet usage, we would be in a better position to propose the development of specific tools to help educate users on the many dangers prominent over the Internet. Our study will utilize the semi-structured interview method, where we will ask questions to participants and assess their mental models of the flow of Internet usage.

\section{$\underline{\text { Method }}$}

Materials: We will use one standard Dell desktop computer running the Windows 7 operating system with standard web browsers (e.g., Microsoft Internet Explorer, Mozilla Firefox, Google Chrome).

We will use one demographics questionnaire created with the Limesurvey web survey system, running on our Hotsoft Lab secure web server. Participant answers on the demographics questionnaire will be kept on our secure server located within our HotSoft lab at Carleton University. This questionnaire can be found in Appendix Appendix U.

The task presented to each participant will ask him or her to answer different types of questions (multiple choice, rating scales, probability judgments, etc.) based on three different categories of online interactions. The questions can be found in Appendix Appendix W] The three categories of online interactions to be tested are as follows: Website/Browser, Communications, and Malicious Software Applications. A mixture of 5-point ordinal level scales, ratio level scales, true/false statements, and probability judgments will be used for each of the three categories found in the main task.

Participants: 300 online participants will be recruited through the Amazon Mechanical Turk online marketplace (https://www.mturk.com/mturk/welcome). Participants will be recruited by way of a website posting on the Amazon Mechanical Turk online marketplace website. Each participant will receive a $\$ 2.50$ honorarium for his or her time. If, at any point during testing, should the Amazon Mechanical Turk online marketplace participant choose to quit the study before its completion, the participant will be redirected to a debriefing page. 
Participants must be experienced Internet users (at least 2 years), and must use the Internet at least twice weekly. They must not be either experts or students in Internet security or computer security.

The Amazon mechanical Turk online marketplace participants will be limited to the United States. This is done in an effort to control for reliability within the test results. As such, the rating scale material has been created with American companies/organizations in mind.

Recruitment: We plan to post a recruitment notice on the Amazon Mechanical Turk online marketplace website in order to recruit participants.

Procedure: The task presented to each participant will ask him or her to rate different facets of online interactions using a variety of rating scales. The three categories of online interactions to be tested are as follows: Website/Browser, Communications, and Malicious Software Applications. A mixture of 5-point ordinal level scales, ratio level scales, and probability judgments will be used for each of the three categories found in the main task. The task description can be found in Appendix Appendix V. The list of questions and statements can be found in Appendix Appendix W. Each participant will be shown the three categories of online interactions in a specified order; the questions and rating scale statements will be randomly shown to the participant by the Limesurvey program.

Confidentiality: In our study, we ask participants for their name and email address in order to obtain consent for their participation in the research. In order to ensure complete separation between participants' identity and their study data, we will store their name and email address in a separate database from the one storing the research data.

Amazon Mechanical Turk online marketplace participants will be redirected to the debriefing form following the main task. The online marketplace allows us to keep records of the participants who were paid the honorarium for participating in the study. 


\section{References}

Fischhoff, B., Slovic, P., Lichtenstein, S., Read, S. \& Combs, B. (1978). How safe is safe enough? A psychometric study of attitudes towards technological risks and benefits. Policy Sciences, 9 (2), 127-152.

LeBlanc, D., \& Biddle, R. (2012). Risk perception of Internet-related activities. Twelfth Annual International Conference on Privacy, Security, and Trust (PST 2012), 8895.

Morgan, M. G, Fischhoff, B., Bostrom, A., \& Atman, C. J. (Eds.). (2002). Risk Communication: A Mental Models Approach, $(63-79)$. Cambridge, UK: Cambridge University Press.

Starr, C. (1969). Social benefits vs. technological risk. Science, 165, 1232-1238. 


\section{Informed Consent}

The purpose of an informed consent is to ensure that you understand the purpose of the study and the nature of your involvement. The informed consent must provide sufficient information such that you have the opportunity to determine whether you wish to participate in the study.

\section{Present Study: Interpreting Internet Structure}

Research personnel: The following people are involved in this research project, and may be contacted at anytime if you have questions or concerns: Daniel LeBlanc (danielleblanc3@cmail.carleton.ca, phone: 613-520-2600 ext. 1987), Dr. Robert Biddle (Faculty Sponsor, email: robert_biddle@carleton.ca, 613-520-2600 ext. 6317).

Ethical concerns: Should you have any ethical concerns about this research, please contact Dr. Shelley Brown (Chair, Psychology Ethics Board, shelley.brown@carleton.ca, 613-5202600, ext. 1505).

Any other concerns: For any other concerns, please contact Dr. Anne Bowker (Chair, Department of Psychology, 613-520-2600 ext. 8218, psychchair@carleton.ca).

Purpose: The purpose of this study is to investigate people's understanding of different characteristics involved with various online activities.

Description: We are asking you to fill out a demographics questionnaire regarding your background. For the main task, we will ask you various questions in relation to online activities. These questions will be divided into three parts.

Duration: The session will last approximately 60 minutes.

Remuneration: You will receive a $\$ 2.50$ honorarium for your participation in the research. 
Potential Risk/Discomfort: There are no physical risks to participating in this study, beyond those involved in normal computer usage. If you feel any discomfort or distress, you may choose not to answer specific questions, and you will not be penalized in any way if you choose to do this. Please keep in mind that we are testing systems associated with the Internet, and not your personal abilities. The debriefing form given to you at the end of the study provides contact details that you may use if you wish to obtain additional information.

Anonymity/Confidentiality: The data collected in this experiment are strictly confidential. All data are coded such that your name is not associated with the responses you provide. Any identifying information associated with your code will be confined to a single page that will be separated from your questionnaire, and kept in a separate, secured file by the research investigators, who will keep this information confidential. To respect FIPPA (Freedom of Information and Protection of Privacy Act) requirements, we will keep your data in our secure records for no longer than 5 years, after which they will be deleted. Your online consent form and honorarium payment records will be kept in our Amazon Mechanical Turk online marketplace secured account for no longer than 5 years, after which they will be deleted. The data portion of the study will be kept on our secure servers during the aforementioned period of time.

Right to withdraw: Your participation in this study is entirely voluntary. At any point during the study, you have the right not to complete certain questions, or to withdraw from the study without penalty. Should you decide to withdraw during the testing session, you will still receive remuneration for your time. 


\section{Consent}

I have read the above form and understand the conditions of my participation. My participation in this study is voluntary, and I understand that if at anytime I wish to leave the experiment, I may do so without having to give an explanation and with no penalty whatsoever. Furthermore, I am also aware that the data gathered in this study are confidential and anonymous with respect to my personal identity. By clicking on the Agree button below, I agree to participate in this study.

[Button] I agree to participate in this study.

[Button] I do not wish to participate.

This study has received clearance by the Carleton University Psychology Research Ethics Board (Reference \#13-159). 


\section{$\underline{\text { Debriefing }}$}

\section{What are we trying to learn in this research?}

This research examines users' knowledge about the many facets that co-exist during their

daily interactions on the Internet. We're hoping to determine whether users have complete and correct mental models of the flow model of Internet usage, based on expert input. This experiment builds upon previously conducted studies, where we were able to determine that participants associate a high amount of risk to specific online activities (e.g., using a search engine, making an online purchase) that experts would normally perceive as being less risky.

\section{Why is this important to scientists or the general public?}

Previous research has shown that people perceive the risks associated with specific activities and technologies differently than others, depending on their general knowledge and expertise regarding the activity or technology in question. Our current research examines in greater detail people's perceptions of the threats that exist in conjunction with online activities, such as online banking and online purchasing. By better understanding how people perceive the threats associated with various online activities, we can develop educational tools to help them identify the more dangerous elements involved with interactions over the Internet.

\section{What are our hypotheses and predictions?}

We expect that users will have widely varying mental models of the expert flow model of Internet usage. Some of these mental models will be more complete, while some will be less complete. We are also expecting that some mental models will be incorrect when compared to the flow model of Internet usage. 


\section{Where can I learn more?}

In order to learn more about our research on security and trust in an online environment, please see our website at http://hotsoft.carleton.ca/security/

\section{What if I have questions later?}

If you have any remaining concerns, questions, or comments about the experiment, please feel free to contact Daniel LeBlanc (Principal Investigator) at: danielleblanc3@cmail.carleton.ca (613-520-2600 ext. 1987), Dr. Robert Biddle (Faculty Sponsor) at: robert_biddle@carleton.ca, 613-520-2600 ext. 6317. Should you have any ethical concerns about this research, please contact Dr. Shelley Brown (Chair, Psychology Ethics Board, shelley.brown@carleton.ca, 613-520-2600, ext. 1505). For any other concerns, please contact Dr. Anne Bowker (Chair, Department of Psychology, 613-520-2600 ext. 8218, psychchair@carleton.ca).

Thank you for participating in this research! 


\section{$\underline{\text { Recruiting participants }}$}

Participants will be recruited by way of a website posting on the Amazon Mechanical Turk online marketplace (https://www.mturk.com/mturk/welcome).

\section{Announcement for recruiting participants (Amazon Mechanical Turk online marketplace)}

\section{Study Name: Interpreting Internet Structure}

Description: This research examines users' knowledge about the many facets that co-exist during their daily interactions on the Internet. We're hoping to determine whether users have complete and correct mental models of the flow model of Internet usage, based on expert input. This experiment builds upon previously conducted studies, where we were able to determine that participants associate a high amount of risk to specific online activities (e.g., using a search engine, making an online purchase) that experts would normally perceive as being less risky.

Eligibility Requirements: We are looking for adults 18 years of age or older, who possess a minimum of 2 years of experience with the Internet, and be regular users (i.e., use the Internet at least 2 times each week). We wish to exclude anyone who is an expert or student in computer or Internet security.

Duration: The session will last approximately 60 minutes.

Compensation: You will receive a $\$ 2.50$ USD honorarium for your time. 
Contact: For more details, please contact Daniel LeBlanc at mmstudy@mvp.soft.carleton.ca

This study has received clearance by the Carleton University Psychology Research Ethics Board (Reference \#13-159). 


\section{Appendix U}

\section{Study Four: Pre-Test Demographics Questionnaire}

This questionnaire asks a few questions about your demographic information. All data is confidential and is coded for anonymity. You are not obliged to answer any of the questions. In this questionnaire, we'll be asking some brief questions about your demographic information, as well as your level of Internet knowledge. All data is confidential and is coded for anonymity. You are not obliged to answer any of the questions.

There are 5 questions in this survey.

Question 1: What is your age?

Question 2: What is your gender?

Male

$\bigcirc$ Female

Question 3: What is your occupation?

Question 4: What is your highest level of education?

$\bigcirc$ Primary

Secondary or high school

Trade or professional school

Some college or university

Bachelor's degree

Graduate degree

Other: 
Question 5: How often do you browse the Web?

Once a month

Once a week

Several times a week

$\bigcirc$ Daily

$\bigcirc$ Multiple times daily

Thank you for completing the demographics questionnaire. 


\section{Appendix V}

\section{Study Four: Main Task Script}

You are now at the main task.

Here, you'll be asked to answer various questions and provide your opinion on different rating scale statements related to the structure of the Internet.

There are three sections to the main task. For each of these sections, you'll be shown various website screenshots, as well as various images depicting file types, software applications, and so on. The questions and rating scale statements can be related to any of these images. Don't worry if you don't know the answer to any of the questions being asked. You can choose not to answer any given question.

Main task begins. 


\section{Appendix W}

\section{Study Four: List of Questions - Website/Browser Category}

1 - What is a domain name?

Choose one of the following answers

$\bigcirc$ It is the component of a Web address that identifies and locates the entity (e.g., an individual, a company, etc.) present on the Internet.

$\bigcirc$ It is the complete Web address of a given website (e.g., http://www.walmart.com/index.gsp).

It is the extension of a Web address found at the end of the address (e.g., .com, .net, .org, etc.).

It is the name of the company/organization that owns the website in question (e.g., Amazon, Microsoft, etc.)

How confident are you of your answer? (Please provide further explanation, if applicable).

Choose one of the following answers

$\bigcirc$ Very confident $\bigcirc$ Somewhat confident $\bigcirc$ Not confident

Please enter your comment here

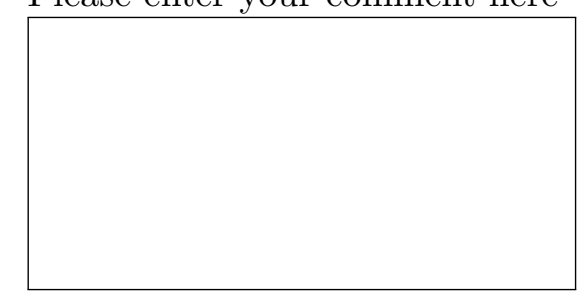


2 - Which of the following is a valid domain name? (Select all that apply)

$\square$ walmart.com

$\square$ harvard.edu

$\square$ walmart.com/browse/computers/

$\square$ ebay.com

$\square$ motors.ebay.com

$\square$ amazon.com.us

$\square$ amazon.com

$\square$ http://www.amazon.com/dp/B007HCCNJU/ref=sa_menu_kdptq

How confident are you of your answer? (Please provide further explanation, if applicable).

Choose one of the following answers

$\bigcirc$ Very confident $\bigcirc$ Somewhat confident $\bigcirc$ Not confident

Please enter your comment here 
3 - What is a Web address?

Choose one of the following answers

$\bigcirc$ A Web address identifies the location of a specific Web page or website on the Internet, also known as the Uniform Resource Locator.

A Web address identifies the domain name of the website.

$\bigcirc$ A Web address is a series of numbers that identify the website (e.g., 255.255.108.1).

A Web address is the path that leads to a specific document found on a website.

\section{How confident are you of your answer? (Please provide further explanation, if applicable).}

Choose one of the following answers

$\bigcirc$ Very confident $\bigcirc$ Somewhat confident $\bigcirc$ Not confident

Please enter your comment here 
4 - Which of the following is a valid Web address? (Select all that apply)

$\square$ http://www.walmart.com

$\square$ localad.walmart.com

$\square$ http://www.amazon.com/mobile-apps/b/ref=sa_menu_adr_app?ie=UTF8\&node=2350149011

$\square$ https://www.chase.com

$\square$ http://www.walmart.com.us

$\square$ http://www.starbucks

$\square$ http://localad.walmart/Walmart/Entry/Flash?

$\square$ http://www.amazon.ca/books-used-books-textbooks/b/ref=sa_menu_bo?ie=UTF8\&node=916520

\section{How confident are you of your answer? (Please provide further explanation, if} applicable).

Choose one of the following answers

$\bigcirc$ Very confident $\bigcirc$ Somewhat confident $\bigcirc$ Not confident

Please enter your comment here 
5 - In walmart.com, what does the .com mean?

Choose one of the following answers

.com is the top-level domain at the end of the domain name.

.com is the Web address of the domain name.

.com is the Uniform Resource Locator of the domain name.

.com is the domain name extension at the end of the domain name, which is exclusive to the United States.

How confident are you of your answer? (Please provide further explanation, if applicable).

Choose one of the following answers

$\bigcirc$ Very confident $\bigcirc$ Somewhat confident $\bigcirc$ Not confident

Please enter your comment here 
6 - In walmart.com, what does the .com mean?

Choose one of the following answers

.com is the top-level domain at the end of the domain name.

.com is the Web address of the domain name.

.com is the Uniform Resource Locator of the domain name.

.com is the domain name extension at the end of the domain name, which is exclusive to the United States.

How confident are you of your answer? (Please provide further explanation, if applicable).

Choose one of the following answers

$\bigcirc$ Very confident $\bigcirc$ Somewhat confident $\bigcirc$ Not confident

Please enter your comment here 
7 - In weeklyad.walmart.com/walmart, what does the weeklyad mean?

Choose one of the following answers

$\bigcirc$ weeklyad represents the subdomain of the walmart website.

weeklyad represents a folder found on the walmart website.

weeklyad represents the Web address of the walmart website.

weeklyad represents the Uniform Resource Locator of the walmart website.

How confident are you of your answer? (Please provide further explanation, if applicable).

Choose one of the following answers

$\bigcirc$ Very confident

Somewhat confident

Not confident

Please enter your comment here

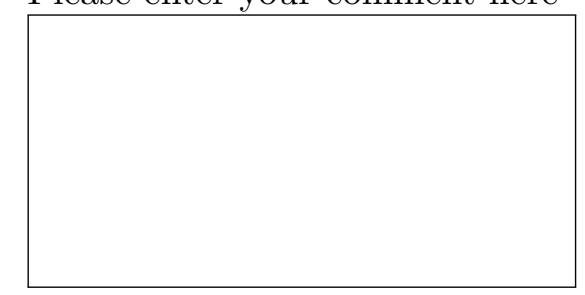


8 - In weeklyad.walmart.com/walmart, what does the weeklyad mean?

Choose one of the following answers

weeklyad represents the subdomain of the walmart website.

$\bigcirc$ weeklyad represents a folder found on the walmart website.

weeklyad represents the Web address of the walmart website.

$\bigcirc$ weeklyad represents the Uniform Resource Locator of the walmart website.

How confident are you of your answer? (Please provide further explanation, if applicable).

Choose one of the following answers

$\bigcirc$ Very confident $\bigcirc$ Somewhat confident $\bigcirc$ Not confident

Please enter your comment here

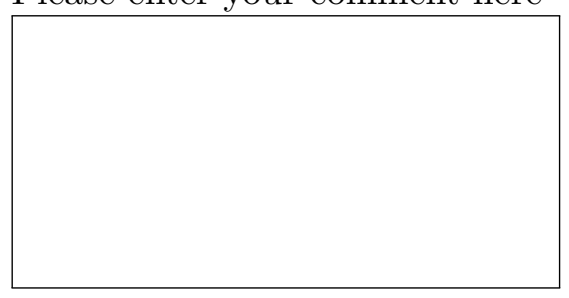


9 - In http://www.apple.com, what does the hyper-text transfer protocol (http) mean?

Choose one of the following answers

http means that the communication between your computer and the website server is sent unsecured (i.e., unencrypted).

$\bigcirc$ http means that the communication between your computer and the website server is sent securely (i.e., encrypted).

http is the prefix of the domain name.

$\bigcirc$ http is the Uniform Resource Locator of the domain name.

\section{How confident are you of your answer? (Please provide further explanation, if applicable).}

Choose one of the following answers

$\bigcirc$ Very confident $\bigcirc$ Somewhat confident $\bigcirc$ Not confident

Please enter your comment here 
10 - In https://www.chase.com, what does the hyper-text transfer protocol secure (https) mean?

Choose one of the following answers

https means that the communication between your computer and the website server is sent unsecured (i.e., unencrypted).

https means that the communication between your computer and the website server is sent secured (i.e., encrypted).

https is the prefix of the domain name.

https is the Uniform Resource Locator of the domain name.

\section{How confident are you of your answer? (Please provide further explanation, if applicable).}

Choose one of the following answers

$\bigcirc$ Very confident $\bigcirc$ Somewhat confident $\bigcirc$ Not confident

Please enter your comment here 
11 - A company/organization may obtain a domain name via the following: (select all that apply).

Choose one of the following answers

They can register a domain name through a domain name registration company.

They can register a domain name through their Internet Service Provider.

They can register a domain name through certain government departments.

They can register a domain name by themselves using their own servers.

How confident are you of your answer? (Please provide further explanation, if applicable).

Choose one of the following answers

$\bigcirc$ Very confident $\bigcirc$ Somewhat confident $\bigcirc$ Not confident

Please enter your comment here 
12 - Once a company/organization obtains a domain name, who is in control of the domain name (i.e., is allowed to make changes, associate the domain name to a website, etc.).

Choose one of the following answers

The domain name registration company is in control of the domain name.

The company/organization that paid for the domain name is in control of the domain name.

The government in the country where the registration of the domain name occurred is in control of the domain name.

The Internet Service Provider of the company/organization that owns the domain name is in control of the domain name.

How confident are you of your answer? (Please provide further explanation, if applicable).

Choose one of the following answers

$\bigcirc$ Very confident $\bigcirc$ Somewhat confident $\bigcirc$ Not confident

Please enter your comment here 


\section{3 - Please provide your opinion on the following statements:}

Individuals can obtain a domain name as well as a company/organization.

$\bigcirc$ False $\bigcirc$ Probably False $\bigcirc$ Don't Know $\bigcirc$ Probably True $\bigcirc$ True

There exist various top-level domains, such as .com, .ca, .net, .org, .edu, .info, and so on.

$\bigcirc$ False $\bigcirc$ Probably False $\bigcirc$ Don't Know $\bigcirc$ Probably True $\bigcirc$ True

Any company/organization or individual may obtain any of these extensions to use in conjunction with their website(s).

$\bigcirc$ False $\bigcirc$ Probably False $\bigcirc$ Don't Know $\bigcirc$ Probably True $\bigcirc$ True

A website can have more than 1 domain name associated with it (e.g., harvard.com and harvard.edu can direct users to the same website).

$\bigcirc$ False $\bigcirc$ Probably False $\bigcirc$ Don’t Know $\bigcirc$ Probably True $\bigcirc$ True

Certain domain name extensions (e.g., .us, .ca) can only be purchased by companies/organizations or individuals related in some way to the region in question.

$\bigcirc$ False $\bigcirc$ Probably False $\bigcirc$ Don't Know $\bigcirc$ Probably True $\bigcirc$ True 
How confident are you of your answer? (Please provide further explanation, if applicable).

Choose one of the following answers

$\bigcirc$ Very confident $\bigcirc$ Somewhat confident $\bigcirc$ Not confident

Please enter your comment here

(2)


14 - What is an IP address?

Choose one of the following answers

$\bigcirc$ An IP address is a numerical label assigned to a device (e.g., a computer server) participating in a network that uses the Internet Protocol for communication.

An IP address is a numerical label assigned to the Web address of a website.

An IP address is a numerical label assigned to the domain name server (DNS) hosting the website.

An IP address is a numerical label assigned to the Uniform Resource Locator of a website.

How confident are you of your answer? (Please provide further explanation, if applicable).

Choose one of the following answers

$\bigcirc$ Very confident $\bigcirc$ Somewhat confident $\bigcirc$ Not confident

Please enter your comment here

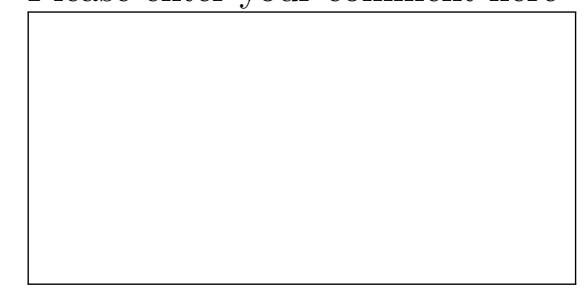


15 - Can a domain name (for example: walmart.com) point to more than one IP address?

Choose one of the following answers

$\bigcirc$ Yes, it can point to individual IP addresses assigned to all servers chosen by the owners of the domain.

No, it can only point to the main server responsible for incoming traffic to the domain name walmart.com.

$\bigcirc$ Yes, it can point to one additional IP address assigned to a backup server, should the main walmart.com server fail.

No, all domain names, regardless of type, can only be assigned one IP address.

How confident are you of your answer? (Please provide further explanation, if applicable).

Choose one of the following answers

$\bigcirc$ Very confident $\bigcirc$ Somewhat confident $\bigcirc$ Not confident

Please enter your comment here 
16 - Can a fake website (i.e., a phishing website [which looks like the correct website but is not genuine]) have the same domain name (e.g., walmart.com) as the real website?

Choose one of the following answers

$\bigcirc$ Yes, a fake website can have the same domain name as the real one; it directs users to a different server.

$\bigcirc$ No, once the domain name is registered to a given company/organization, it can only be used by them.

$\bigcirc$ Yes, the fake website can obtain the same domain name by registering it with a domain name registration company different than the one used to register the legitimate walmart.com domain name.

How confident are you of your answer? (Please provide further explanation, if applicable).

Choose one of the following answers

$\bigcirc$ Very confident $\bigcirc$ Somewhat confident $\bigcirc$ Not confident

Please enter your comment here 


\section{List of Questions - Communications Category}

1 - Can your Internet Service Provider (e.g., Time Warner, Comcast, AT\&T) see the personal information you submit through online forms, as well as the information that is sent back to you by the website server?

Choose one of the following answers

Yes, but only if the website is using the http protocol to communicate with your computer.

$\bigcirc$ Yes, your ISP can see all of the information sent through all online forms; their logs record all incoming and outgoing activity.

$\bigcirc$ No, your ISP does not keep any records of incoming and outgoing information between your computer and various website servers.

No, your ISP is not allowed, by law, to see the information you submit through online forms.

How confident are you of your answer? (Please provide further explanation, if applicable).

Choose one of the following answers

$\bigcirc$ Very confident $\bigcirc$ Somewhat confident $\bigcirc$ Not confident

Please enter your comment here 


\section{2 - What are security certificates?}

Choose one of the following answers

$\bigcirc$ Security certificates are additional security questions asked by various websites in order to better secure your account (e.g., What is the name of your oldest cousin?).

$\bigcirc$ Security certificates are electronic documents that use a digital signature to bind a public key with an identity (e.g., the name of a company/organization).

Security certificates are the lock icons depicted on various parts of the website when you log in to a given account (e.g., an email account, a bank account).

$\bigcirc$ Security certificates are the pop-up window warnings seen on various websites that alert you to potential issues regarding the website in question.

\section{How confident are you of your answer? (Please provide further explanation, if applicable).}

Choose one of the following answers

$\bigcirc$ Very confident $\bigcirc$ Somewhat confident $\bigcirc$ Not confident

Please enter your comment here

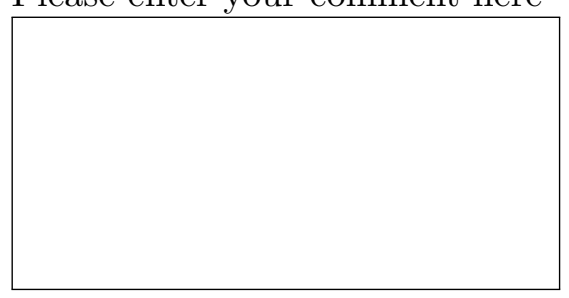


3 - How does a company/organization or individual obtain a security certificate? Choose one of the following answers

$\bigcirc$ A security certificate can be purchased from Certificate Authorities located in various countries.

$\bigcirc$ A security certificate is created and assigned to a given website by the browser (e.g., Google Chrome, Mozilla Firefox, Internet Explorer, etc.).

$\bigcirc$ A security certificate for a given website is given to the company/organization in question by the government of their respective country.

$\bigcirc$ A security certificate is created by the company/organization itself and assigned to their website in order to provide additional security for their users.

How confident are you of your answer? (Please provide further explanation, if applicable).

Choose one of the following answers

$\bigcirc$ Very confident $\bigcirc$ Somewhat confident $\bigcirc$ Not confident

Please enter your comment here

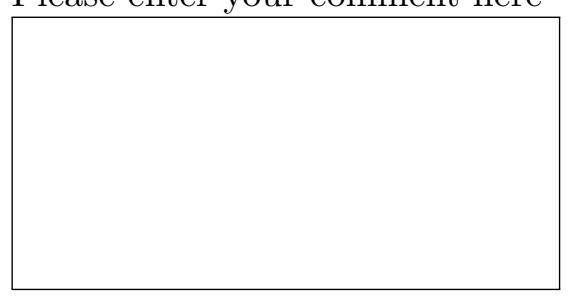


4 - What happens when you visit a website that has an expired security certificate?

Choose one of the following answers

$\bigcirc$ Your browser will warn you that the security certificate has expired, and will ask whether you still want to proceed with viewing the website in question.

$\bigcirc$ Your browser will block your access to the website, explaining that the website is no longer secure.

Nothing will happen; it is the user's responsibility to verify whether the security certificate is still valid when browsing the website in question.

$\bigcirc$ Your browser will remove the lock icons that were present on the website, alerting you to the website no longer being secure.

\section{How confident are you of your answer? (Please provide further explanation, if applicable).}

Choose one of the following answers

$\bigcirc$ Very confident $\bigcirc$ Somewhat confident $\bigcirc$ Not confident

Please enter your comment here 
5 - Do expired security certificates indicate malicious intent in regards to the website?

Choose one of the following answers

$\bigcirc$ No, expired security certificates usually indicate that the administrator of the website in question forgot to renew the certificate.

$\bigcirc$ Yes, expired security certificates are always a sign of malicious websites (e.g., phishing websites).

$\bigcirc$ Yes, expired security certificates indicate that malicious websites are attempting to trick your browser into thinking your information is sent securely, when it is not.

\section{How confident are you of your answer? (Please provide further explanation, if applicable).}

Choose one of the following answers

$\bigcirc$ Very confident $\bigcirc$ Somewhat confident $\bigcirc$ Not confident

Please enter your comment here 
6 - What are security certificate authorities?

Choose one of the following answers

$\bigcirc$ A security certificate authority is an entity (company/organization) that issues digital certificates to website owners.

A security certificate authority is a branch of the government that issues digital certificates to website owners.

A security certificate authority is the website hosting company, which has the ability to issue digital certificates to the website owner.

$\bigcirc$ A security certificate authority is the Internet Service Provider, which has the ability to issue digital certificates to the website owner.

How confident are you of your answer? (Please provide further explanation, if applicable).

Choose one of the following answers

$\bigcirc$ Very confident $\bigcirc$ Somewhat confident $\bigcirc$ Not confident

Please enter your comment here

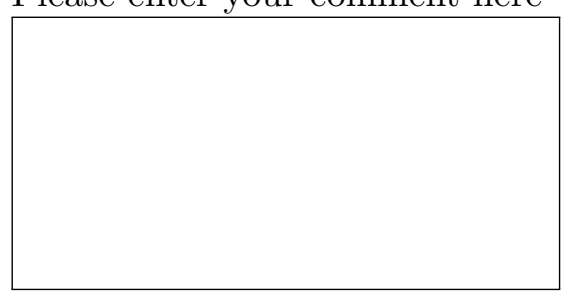


7 - Who is responsible for the security mechanisms embedded into an Internet browser (e.g., Google Chrome, Mozilla Firefox, Microsoft Internet Explorer)? Choose one of the following answers

The company that created the browser (e.g., Microsoft, Mozilla, Google, etc.) is responsible for the security mechanisms embedded into an Internet browser.

The operating system (e.g., Windows, OS X, Linux, etc.) in which the browser is being used is responsible for the security mechanisms embedded into an Internet browser.

The Internet Service Provider (e.g., Time Warner, Comcast, etc.) is responsible for the security mechanisms embedded into an Internet browser.

The computer user is responsible for installing and keeping up to date the security mechanisms embedded into an Internet browser.

\section{How confident are you of your answer? (Please provide further explanation, if applicable).}

Choose one of the following answers

$\bigcirc$ Very confident $\bigcirc$ Somewhat confident $\bigcirc$ Not confident

Please enter your comment here 


\section{8 - Please provide your opinion on the following statements:}

Your Internet Service Provider (e.g., AT\&T, Comcast, Time Warner Cable) can see (i.e., keep records of) the websites you visit.

$\bigcirc$ False $\bigcirc$ Probably False $\bigcirc$ Don’t Know $\bigcirc$ Probably True $\bigcirc$ True

Your Internet Service Provider (e.g., AT\&T, Comcast, Time Warner Cable) can modify the websites you visit.

$\bigcirc$ False $\bigcirc$ Probably False $\bigcirc$ Don’t Know $\bigcirc$ Probably True $\bigcirc$ True

A security certificate expires after a pre-determined amount of time.

$\bigcirc$ False $\bigcirc$ Probably False $\bigcirc$ Don’t Know $\bigcirc$ Probably True $\bigcirc$ True

The expiration date of a security certificate is determined by the owner of the website in question.

$\bigcirc$ False $\bigcirc$ Probably False $\bigcirc$ Don’t Know $\bigcirc$ Probably True $\bigcirc$ True

There are strict rules and regulations that enforce certificate authorities to conduct business in legitimate ways.

$\bigcirc$ False $\bigcirc$ Probably False $\bigcirc$ Don’t Know $\bigcirc$ Probably True $\bigcirc$ True

All security certificate authorities are located in North America (i.e., within the United States and Canada).

$\bigcirc$ False $\bigcirc$ Probably False $\bigcirc$ Don't Know $\bigcirc$ Probably True $\bigcirc$ True

All security certificate authorities are reputable and can be trusted.

$\bigcirc$ False $\bigcirc$ Probably False $\bigcirc$ Don’t Know $\bigcirc$ Probably True $\bigcirc$ True 
Browsing to a website that has a security certificate ensures that your transmitted information is completely safe from being intercepted by another user.

$\bigcirc$ False $\bigcirc$ Probably False $\bigcirc$ Don’t Know $\bigcirc$ Probably True $\bigcirc$ True

All Internet browsers show security certificate information the same way. As an example, they all make use of lock icons and address bar indicators.

$\bigcirc$ False $\bigcirc$ Probably False $\bigcirc$ Don’t Know $\bigcirc$ Probably True $\bigcirc$ True

Other users are able to spy on your Web usage when you login to a wireless (i.e., Wifi) network in a public place (e.g., at your local Starbucks).

$\bigcirc$ False $\bigcirc$ Probably False $\bigcirc$ Don't Know $\bigcirc$ Probably True $\bigcirc$ True

Information sent securely through an https connection can be intercepted when you're logged on to a wireless (i.e., Wifi) network (e.g., at your local Starbucks).

$\bigcirc$ False $\bigcirc$ Probably False $\bigcirc$ Don't Know $\bigcirc$ Probably True $\bigcirc$ True

Secured (i.e., password-protected) Wifi wireless network connections protect you completely from other users intercepting your information while browsing the Web.

$\bigcirc$ False $\bigcirc$ Probably False $\bigcirc$ Don't Know $\bigcirc$ Probably True $\bigcirc$ True

There are software applications that exist which are able to intercept your personal information when you are logged on to a Wifi wireless network.

$\bigcirc$ False $\bigcirc$ Probably False $\bigcirc$ Don’t Know $\bigcirc$ Probably True $\bigcirc$ True 
How confident are you of your answer? (Please provide further explanation, if applicable).

Choose one of the following answers

$\bigcirc$ Very confident $\bigcirc$ Somewhat confident $\bigcirc$ Not confident

Please enter your comment here

(2)




\section{List of Questions - Malware Category}

1 - How do malicious software applications get installed on your computer? (Select all that apply)

$\square$ By downloading pirated computer files (e.g., music, videos, software applications).

$\square$ By downloading and installing a piece of software from an untrusted source.

$\square$ By opening an attachment contained in an email from an untrusted source.

$\square$ By reading an email from an untrusted source.

By purchasing a software application from a physical store and installing it on your computer.

$\square$ By downloading and installing a reputable software application that contains optional program extensions.

$\square$ By sharing computer files with a friend or family member who is already infected by a malicious software application.

$\square$ By dismissing pop-up windows on websites by clicking on the OK button.

How confident are you of your answer? (Please provide further explanation, if applicable).

Choose one of the following answers

$\bigcirc$ Very confident $\bigcirc$ Somewhat confident $\bigcirc$ Not confident

Please enter your comment here 


\section{2 - What can malicious software applications do once installed on your com- puter? (Select all that apply)}

Malicious software applications can collect personal information found on my computer and send it to a third party.

Malicious software applications can delete any of the files found on my computer.

Malicious software applications can alter the software applications installed on my computer.

$\square$ Malicious software applications can send emails to other users from my email account.

Malicious software applications can fry my computer's processor, rendering the computer unusable.

Malicious software applications can track my usernames and passwords as I type them when logging in to my various accounts (e.g., email, online banking, etc.).

Malicious software applications can shut down my Internet connection permanently.

Malicious software applications can allow other users direct access to my computer through the Internet.

How confident are you of your answer? (Please provide further explanation, if applicable).

Choose one of the following answers

$\bigcirc$ Very confident $\bigcirc$ Somewhat confident $\bigcirc$ Not confident

Please enter your comment here

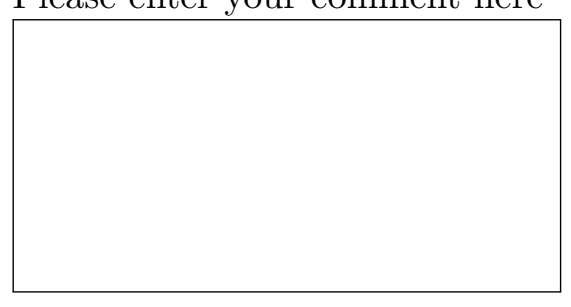


3 - Once a malicious software application is installed on your computer, is it able to do damage to your computer only after some time has passed, are the effects immediate, or are both scenarios possible?

Choose one of the following answers

It is able to do damage to my computer only after some time has passed.

The effects of the damage to my computer are immediate.

$\bigcirc$ It is able to do damage to my computer immediately as well as after some time has passed.

How confident are you of your answer? (Please provide further explanation, if applicable).

Choose one of the following answers

$\bigcirc$ Very confident $\bigcirc$ Somewhat confident $\bigcirc$ Not confident

Please enter your comment here 


\section{4 - How does Anti-Virus software help to protect your computer from malicious software applications? (Select all that apply)}

By alerting me when a malicious software application is already installed.

By alerting me when a malicious software application is attempting to install.

By alerting me that certain websites are reputed to distribute malicious software applications.

By doing system checks over specific time intervals in order to verify for the presence of malicious software applications.

By not allowing me to browse to websites that are known to distribute malicious software applications.

By identifying and deleting all malicious software applications that are or may be installed on my computer.

By shutting down my computer when a malicious software application is found on my computer.

By shutting down my Internet connection when a malicious software application is found on my computer.

\section{How confident are you of your answer? (Please provide further explanation, if applicable).}

Choose one of the following answers

$\bigcirc$ Very confident $\bigcirc$ Somewhat confident $\bigcirc$ Not confident

Please enter your comment here 
5 - How does Anti-Virus software determine whether there are malicious software applications installed on my computer?

Choose one of the following answers

Anti-Virus software applications search for specific program names and compares them to a global list of malicious software applications in order to identify them.

Anti-Virus software applications search for specific programs that are performing strange functions on your computer and compares them to a global list of malicious software applications in order to identify them.

Anti-Virus software applications search for specific, pre-determined program signatures that identifies a given software application as malicious.

$\bigcirc$ Anti-Virus software applications "look into" each of the programs installed on my computer and determine whether it is a malicious software application.

How confident are you of your answer? (Please provide further explanation, if applicable).

Choose one of the following answers

$\bigcirc$ Very confident $\bigcirc$ Somewhat confident $\bigcirc$ Not confident

Please enter your comment here

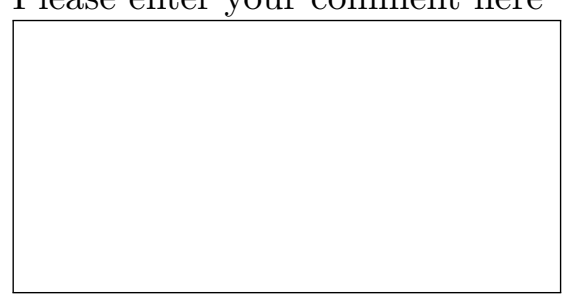




\section{6 - Please provide your opinion on the following statements:}

Once installed, malicious software applications can access any file on your computer.

$\bigcirc$ False $\bigcirc$ Probably False $\bigcirc$ Don't Know $\bigcirc$ Probably True $\bigcirc$ True

Once installed, malicious software applications can access any software application (e.g., Microsoft Word, Microsoft Excel, Adobe Reader) on your computer.

False $\bigcirc$ Probably False $\bigcirc$ Don’t Know $\bigcirc$ Probably True $\bigcirc$ True

Once installed, malicious software applications can obtain personal information found on your computer (e.g., various types of account usernames, passwords, etc.).

$\bigcirc$ False $\bigcirc$ Probably False $\bigcirc$ Don't Know $\bigcirc$ Probably True $\bigcirc$ True

Once installed, malicious software applications can see the information you submit through online forms (e.g., while making an online purchase, while logging on to your bank account, etc.).

$\bigcirc$ False $\bigcirc$ Probably False $\bigcirc$ Don't Know $\bigcirc$ Probably True $\bigcirc$ True

Anti-Virus software protects you from all malicious software applications.

False $\bigcirc$ Probably False $\bigcirc$ Don’t Know $\bigcirc$ Probably True $\bigcirc$ True

The companies that create Anti-Virus software are responsible for your loss of personal information, should their product fail to protect you from malicious programs.

$\bigcirc$ False $\bigcirc$ Probably False $\bigcirc$ Don't Know $\bigcirc$ Probably True $\bigcirc$ True

All Anti-Virus software, created by different companies (e.g., Norton, McAfee), protect you from all the same malicious software applications.

False $\bigcirc$ Probably False $\bigcirc$ Don’t Know $\bigcirc$ Probably True $\bigcirc$ True 
How confident are you of your answer? (Please provide further explanation, if applicable).

Choose one of the following answers

$\bigcirc$ Very confident $\bigcirc$ Somewhat confident $\bigcirc$ Not confident

Please enter your comment here 


\section{Appendix X}

\section{Study Four: Results}

Table 38

Website/Browser Category Question 1: What is a Domain Name?

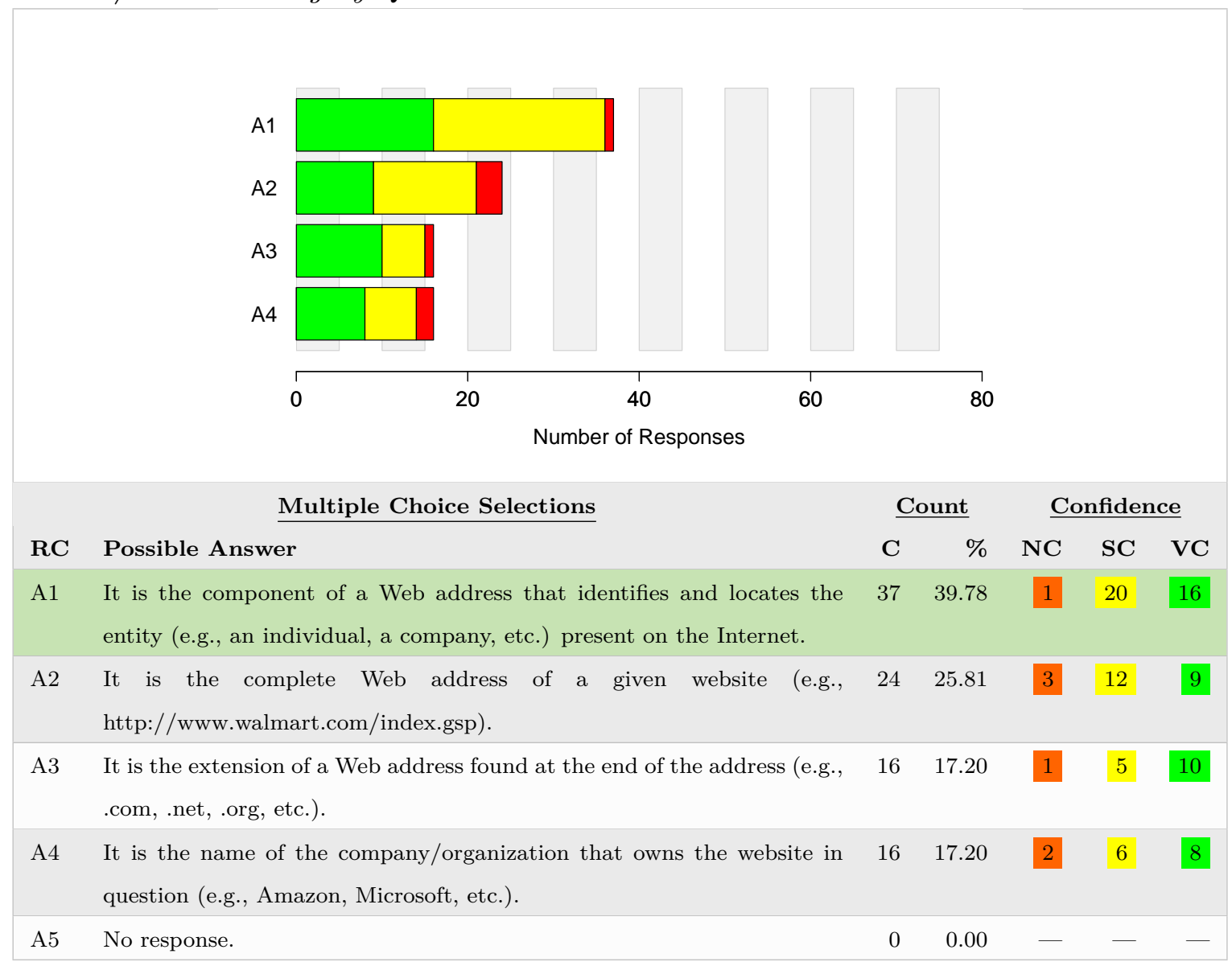

*Notes. $\mathrm{RC}=$ Response Code. $\mathrm{C}=$ Count. $\mathrm{NC}=$ Not Confident; Not Confident response counts are coded in red . $\mathrm{SC}=$ Somewhat Confident; Somewhat Confident responses are coded in yellow . VC = Very Confident; Very Confident responses are coded in green . The correct multiple choice answer is coded in pastel green . 
Table 39

Website/Browser Category Question 2: Which of the Following is a Valid Domain Name? (Select all that Apply).

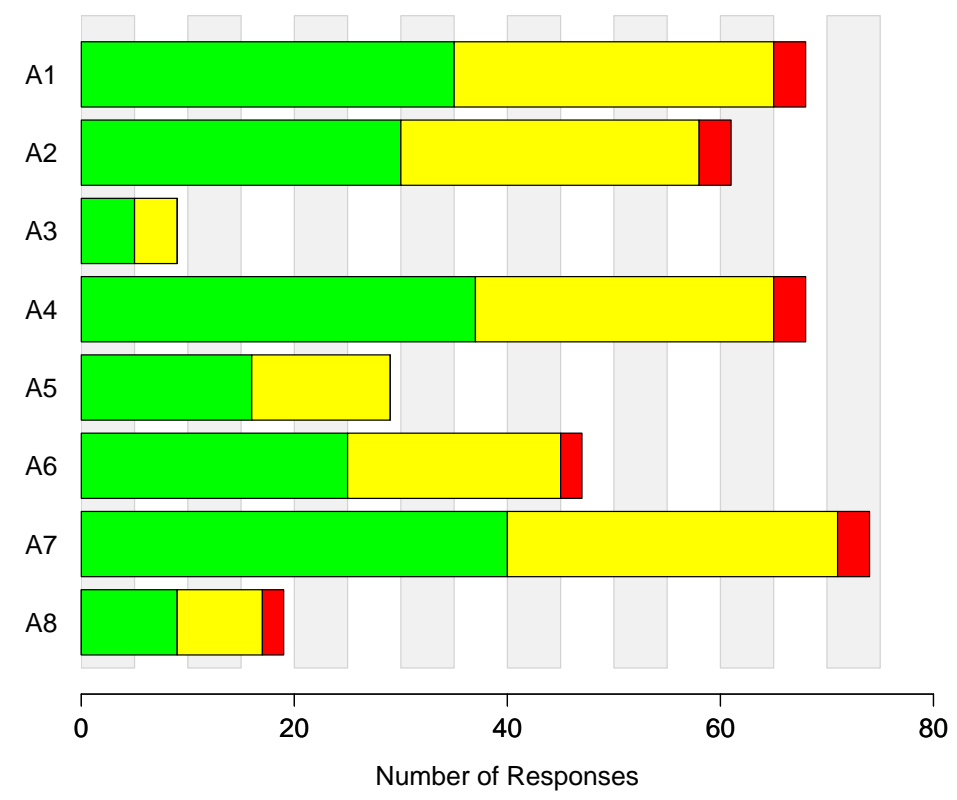

\section{Checklist Selections}

\begin{tabular}{rrrrrr}
\multicolumn{2}{c}{ Count } & \multicolumn{3}{c}{ Confidence } \\
C & $\%$ & NC & SC & VC \\
\hline 68 & 73.12 & 3 & 30 & 35 \\
\hline 61 & 65.59 & 3 & 28 & 30 \\
\hline 9 & 9.68 & 0 & 4 & 5 \\
\hline 68 & 73.12 & 3 & 28 & 37 \\
\hline 29 & 31.18 & 0 & 13 & 16 \\
\hline 47 & 50.54 & 2 & 20 & 25 \\
\hline 74 & 79.57 & 3 & 31 & 40 \\
\hline 19 & 20.43 & 2 & 8 & 9 \\
\hline
\end{tabular}

${ }^{*}$ Notes. $\mathrm{RC}=$ Response Code. $\mathrm{C}=$ Count. $\mathrm{NC}=$ Not Confident; Not Confident response counts are coded in red $. \mathrm{SC}=$ Somewhat Confident; Somewhat Confident responses are coded in yellow . VC = Very Confident; Very Confident responses are coded in green . NR = No Response; No response counts are coded in gray . The correct checklist answers are coded in pastel green . 
Table 40

Website/Browser Category Question 3: What is a Web Address?

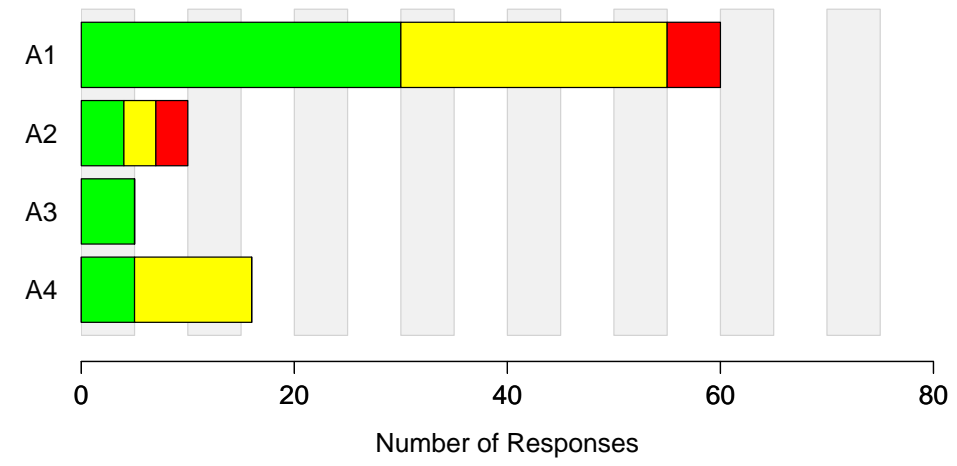

Multiple Choice Selections

Count

Confidence

RC Possible Answer

C $\%$ NC SC VC

A1 A Web address identifies the location of a specific Web page or website $\quad 61 \quad 65.59 \quad 5$ on the Internet, also known as the Uniform Resource Locator.

A2 A Web address identifies the domain name of the website. $\quad 11 \quad 11.83 \quad 3$
255.255.108.1).

A4 A Web address is the path that leads to a specific document found on a $\quad \begin{array}{llll}16 & 17.20 & 0\end{array}$ website.

0.00

${ }^{*}$ Notes. $\mathrm{RC}=$ Response Code. $\mathrm{C}=$ Count. $\mathrm{NC}=$ Not Confident; Not Confident response counts are coded in red. $\mathrm{SC}=$ Somewhat Confident; Somewhat Confident responses are coded in yellow . VC = Very Confident; Very Confident responses are coded in green. The correct multiple choice answer is coded in pastel green . 
Table 41

Website/Browser Category Question 4: Which of the Following is a Valid Web Address? (Select all that Apply).

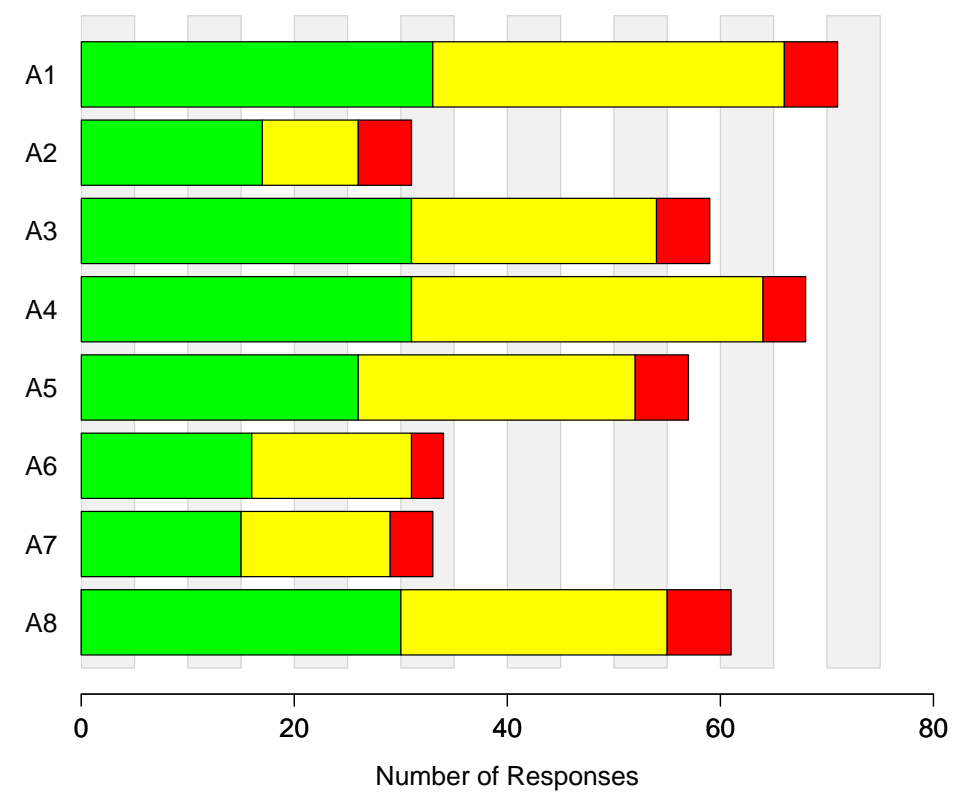

\begin{tabular}{|llrrrr|r|}
\hline & & \multicolumn{1}{c}{ Checklist Selections } & \multicolumn{3}{c|}{ Count } & \multicolumn{3}{c|}{ Confidence } \\
RC & Possible Answer & C & $\%$ & NC & SC & VC \\
\hline A1 & http://www.walmart.com & 72 & 77.42 & 5 & 33 & 33 \\
\hline A2 & localad.walmart.com & 31 & 33.33 & 5 & 9 & 17 \\
\hline A3 & http://www.amazon.com/mobile-apps/b/ref=sa_menu_adr_app?ie=UT & 60 & 64.52 & 5 & 23 & 31 \\
\hline A4 & https://www.chase.com & 71 & 76.34 & 4 & 33 & 31 \\
\hline A5 & http://www.walmart.com.us & 58 & 62.37 & 5 & 26 & 26 \\
\hline A6 & http://www.starbucks & 36 & 38.71 & 3 & 15 & 16 \\
\hline A7 & http://localad.walmart/Walmart/Entry/Flash? & 34 & 36.56 & 4 & 14 & 15 \\
\hline A8 & http://www.amazon.ca/books-used-books- & 63 & 67.74 & 6 & 25 & 30 \\
& textbooks/b/ref=sa_menu_bo?ie=UTF8\&node=916520 & & & & \\
\hline
\end{tabular}

*Notes. $\mathrm{RC}=$ Response Code. $\mathrm{C}=$ Count. $\mathrm{NC}=$ Not Confident; Not Confident response counts are coded in red $. \mathrm{SC}=$ Somewhat Confident; Somewhat Confident responses are coded in yellow. VC $=$ Very Confident; Very Confident responses are coded in green . NR = No Response; No response counts are coded in gray . The correct checklist answers are coded in pastel green . 
Table 42

Website/Browser Category Question 5: In walmart.com, What Does the .com Mean?

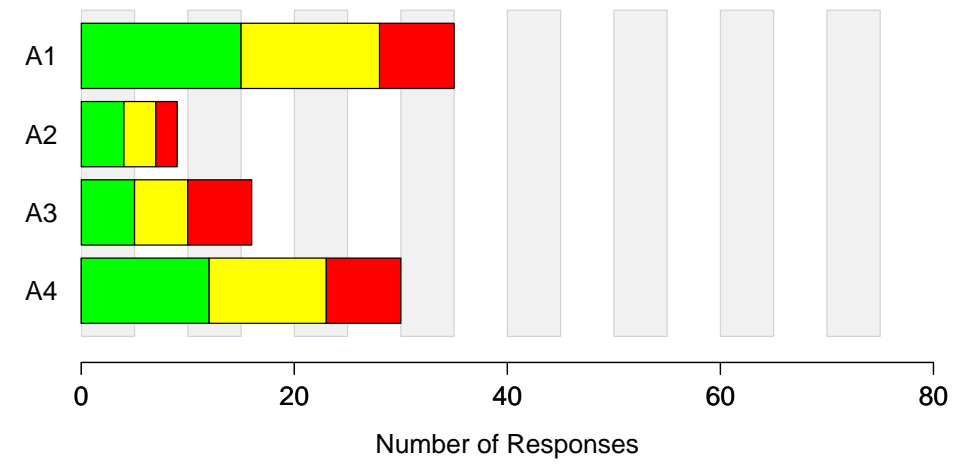

Multiple Choice Selections

\section{Count}

Confidence

RC Possible Answer

C $\quad \% \quad \mathrm{NC} \quad \mathrm{SC} \quad \mathrm{VC}$

A1 .com is the top-level domain at the end of the domain name

\begin{tabular}{ll|l|l|l}
36 & 38.71 & 7 & 13 & 15
\end{tabular}

A2 .com is the Web address of the domain name.

$\begin{array}{llllll}9 & 9.68 & 2 & 3 & 4\end{array}$

A3 .com is the Uniform Resource Locator of the domain name.

$\begin{array}{lllll}16 & 17.20 & 6 & 5 & 5\end{array}$

A4 .com is the domain name extension at the end of the domain name, which

$\begin{array}{lllll}30 & 32.26 & 7 & 11 & 12\end{array}$
is exclusive to the United States.

A5 No response. $\quad 0 \quad 0.00 \quad-\quad-\quad-$

*Notes. $\mathrm{RC}=$ Response Code. $\mathrm{C}=$ Count. $\mathrm{NC}=$ Not Confident; Not Confident response counts are coded in red . $\mathrm{SC}=$ Somewhat Confident; Somewhat Confident responses are coded in yellow $. \mathrm{VC}=$ Very Confident; Very Confident responses are coded in green. The correct multiple choice answer is coded in pastel green . 
Table 43

Website/Browser Category Question 6: In walmart.com, What Does walmart Mean?

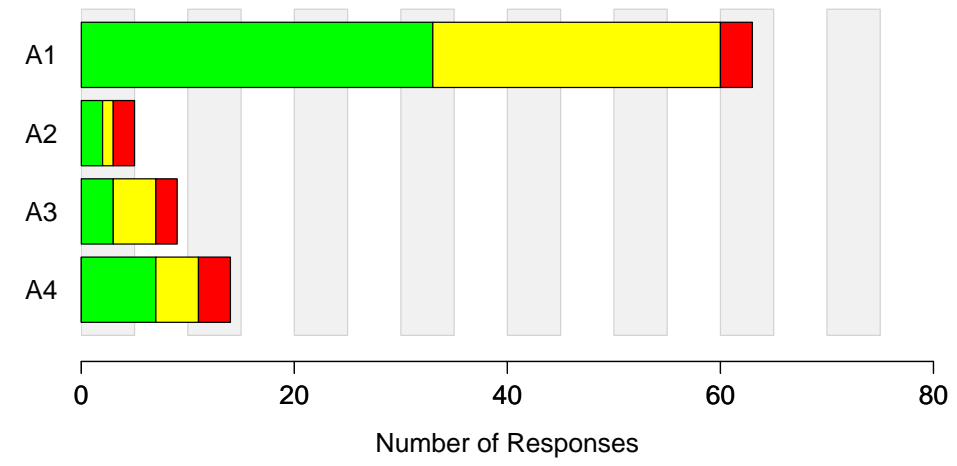

Multiple Choice Selections

Count

Confidence

RC Possible Answer

C $\% \quad \mathrm{NC} \quad \mathrm{SC} \quad \mathrm{VC}$

A1 walmart represents the name registered by Wal-Mart Stores, Inc. for the $\quad$\begin{tabular}{llllll|l}
64 & 68.82 & 3 & 27 & 33
\end{tabular} domain.

$\begin{array}{llllllll}\text { A2 } & \text { walmart represents the name registered by the government for Wal-Mart } & 6 & 6.45 & 2 & 1 & 2\end{array}$ Stores, Inc.

\begin{tabular}{llrrrr|r|} 
A3 & walmart represents the Uniform Resource Locator of the website. & 9 & 9.68 & 2 & 4 & 3 \\
\hline A4 & walmart represents the domain extension of the website. & 14 & 15.05 & 3 & 4 & 7 \\
\hline A5 & No response. & 0 & 0.00 & - & - & -
\end{tabular}

${ }^{*}$ Notes. $\mathrm{RC}=$ Response Code. $\mathrm{C}=$ Count. $\mathrm{NC}=$ Not Confident; Not Confident response counts are coded in red. $\mathrm{SC}=$ Somewhat Confident; Somewhat Confident responses are coded in yellow . VC = Very Confident; Very Confident responses are coded in green. The correct multiple choice answer is coded in pastel green . 
Table 44

Website/Browser Category Question 7: In weeklyad.walmart.com/walmart, What Does the weeklyad Mean?

\begin{tabular}{|l|l|l|l|l|}
\hline & & \\
\end{tabular}

${ }^{*}$ Notes. $\mathrm{RC}=$ Response Code. $\mathrm{C}=$ Count. $\mathrm{NC}=$ Not Confident; Not Confident response counts are coded in red . $\mathrm{SC}=$ Somewhat Confident; Somewhat Confident responses are coded in yellow . VC = Very Confident; Very Confident responses are coded in green. The correct multiple choice answer is coded in pastel green . 
Table 45

Website/Browser Category Question 9: In http://www.apple.com, What Does the HyperText Transfer Protocol (http) Mean?

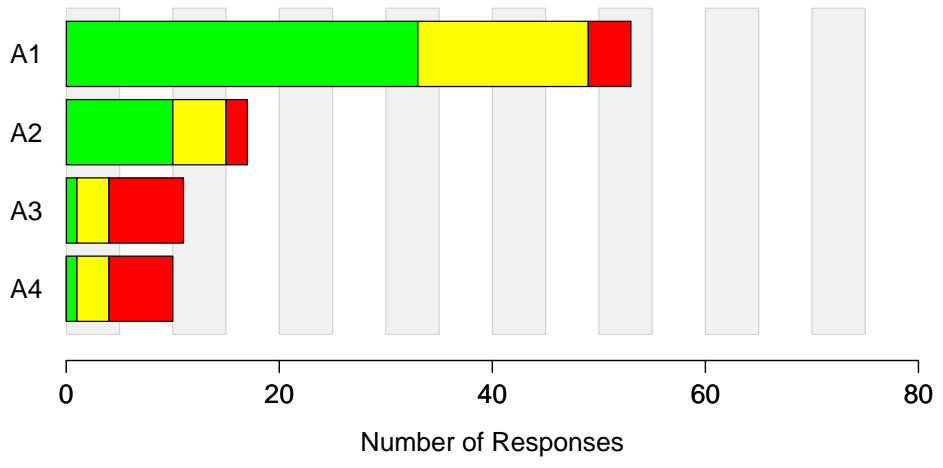

$\underline{\text { Multiple Choice Selections }}$

Count

Confidence

RC Possible Answer

C $\% \quad \mathrm{NC} \quad \mathrm{SC} \quad \mathrm{VC}$

A1 http means that the communication between your computer and the $\quad \begin{array}{llllll}54 & 58.06 & 4 & 16 & 33\end{array}$ website server is sent unsecured (i.e., unencrypted).

A2 http means that the communication between your computer and the $\begin{array}{lllllll}18 & 19.35 & 2 & 5 & 10\end{array}$ website server is sent securely (i.e., encrypted).

\begin{tabular}{|llrrr|r|r|} 
A3 & http is the prefix of the domain name. & 11 & 11.83 & 7 & 3 & 1 \\
\hline A4 & http is the Uniform Resource Locator of the domain name. & 10 & 10.75 & 6 & 3 & 1 \\
\hline A5 & No response. & 0 & 0.00 & - & - & -
\end{tabular}

*Notes. $\mathrm{RC}=$ Response Code. $\mathrm{C}=$ Count. $\mathrm{NC}=$ Not Confident; Not Confident response counts are coded in red . $\mathrm{SC}=$ Somewhat Confident; Somewhat Confident responses are coded in yellow. VC = Very Confident; Very Confident responses are coded in green. The correct multiple choice answer is coded in pastel green . 
Table 46

Website/Browser Category Question 10: In https://www.chase.com, What Does the HyperText Transfer Protocol Secure (https) Mean?

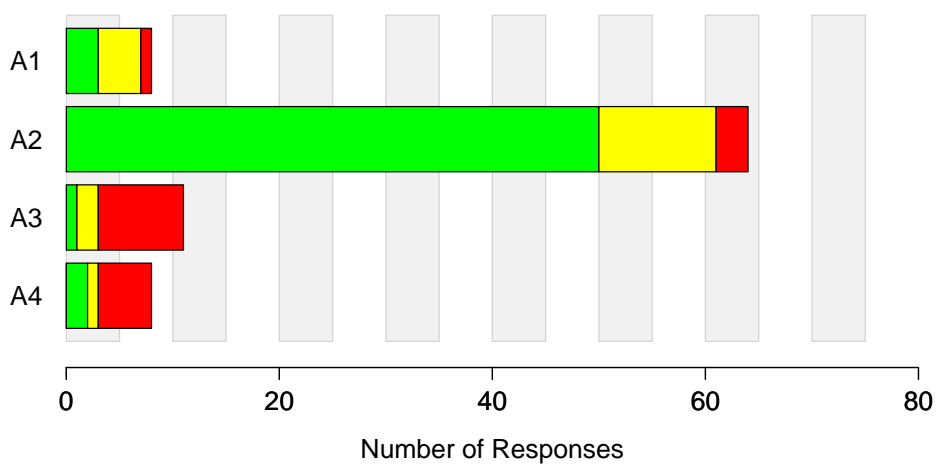

\section{Multiple Choice Selections}

Count

Confidence

RC Possible Answer

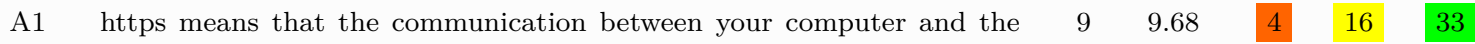
website server is sent unsecured (i.e., unencrypted).

A2 https means that the communication between your computer and the $\quad \begin{array}{llllll}64 & 68.82 & 2 & 5 & 10\end{array}$ website server is sent secured (i.e., encrypted).

\begin{tabular}{|llrrr|r|r|} 
A3 & https is the prefix of the domain name. & 11 & 11.83 & 7 & 3 & 1 \\
\hline A4 & https is the Uniform Resource Locator of the domain name. & 8 & 8.60 & 6 & 3 & 1 \\
\hline A5 & No response. & 1 & 1.08 & - & - & -
\end{tabular}

*Notes. $\mathrm{RC}=$ Response Code. $\mathrm{C}=$ Count. $\mathrm{NC}=$ Not Confident; Not Confident response counts are coded in red . $\mathrm{SC}=$ Somewhat Confident; Somewhat Confident responses are coded in yellow $. \mathrm{VC}=$ Very Confident; Very Confident responses are coded in green. The correct multiple choice answer is coded in pastel green . 
Table 47

Website/Browser Category Question 11: A Company/Organization May Obtain a Domain Name Via the Following: (Select all that Apply).

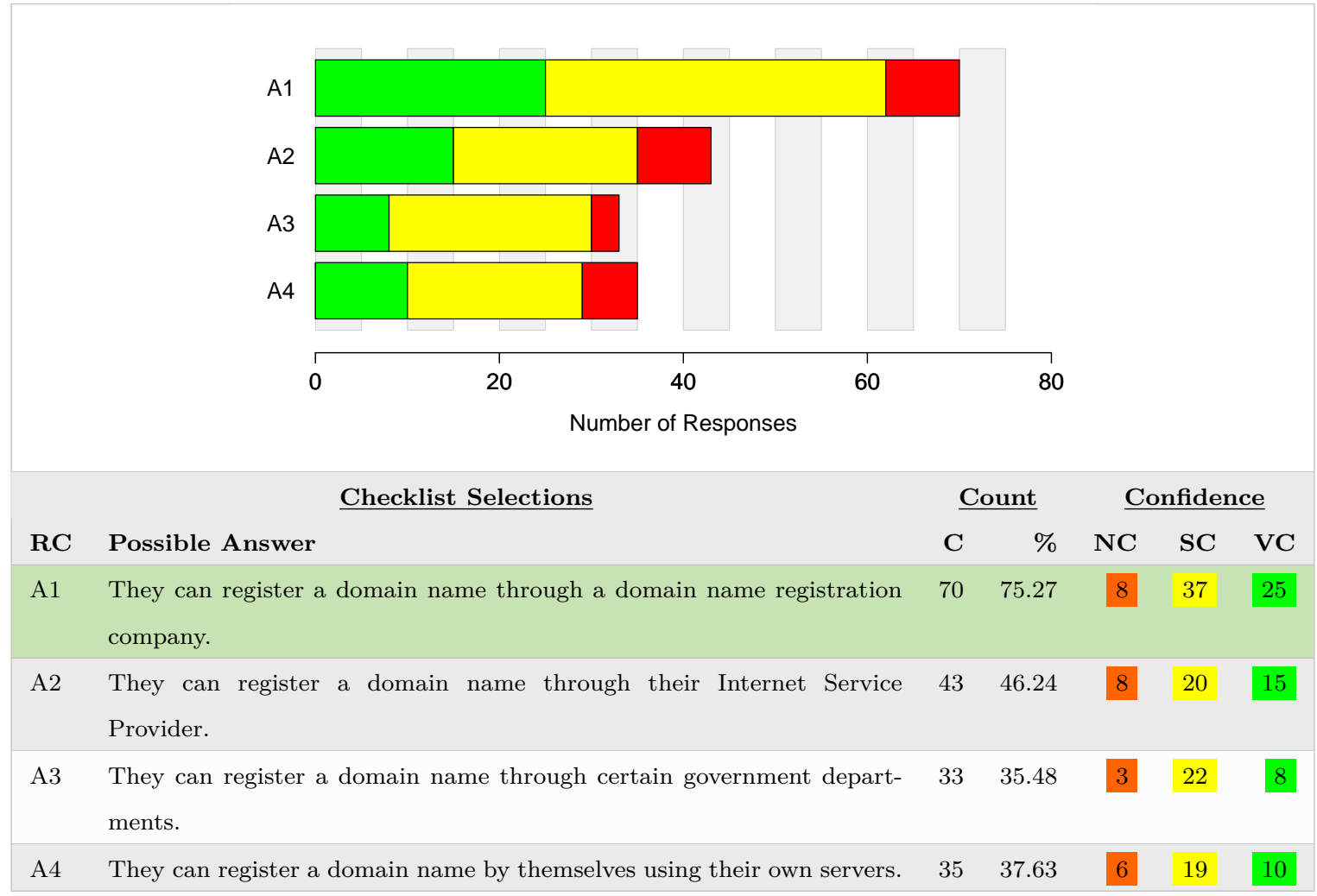

${ }^{*}$ Notes. $\mathrm{RC}=$ Response Code $\mathrm{C}=$ Count. $\mathrm{NC}=$ Not Confident; Not Confident response counts are coded in red. $\mathrm{SC}=$ Somewhat Confident; Somewhat Confident responses are coded in yellow $. \mathrm{VC}=$ Very Confident; Very Confident responses are coded in green. The correct multiple choice answer is coded in pastel green . 
Table 48

Website/Browser Category Question 13: Please Provide Your Opinion on the Following Statements.

$\begin{array}{ll}\text { Code } & \text { Rating Scale Statement } \\ \text { S1 } & \text { Individuals can obtain a domain name as well as a company/organization } \\ \text { S2 } & \text { There exist various top-level domains, such as .com, .ca, .net, .org, .edu, .info, and so on. } \\ \text { S3 } & \text { Any company/organization or individual may obtain any of these extensions to use in conjunction with } \\ & \text { their website(s). } \\ \text { S4 } & \text { A website can have more than } 1 \text { domain name associated with it (e.g., harvard.com and harvard.edu } \\ & \text { can direct users to the same website). } \\ \text { S5 } & \text { Certain domain name extensions (e.g., .us, .ca) can only be purchased by companies/organizations or } \\ & \text { individuals related in some way to the region in question. }\end{array}$

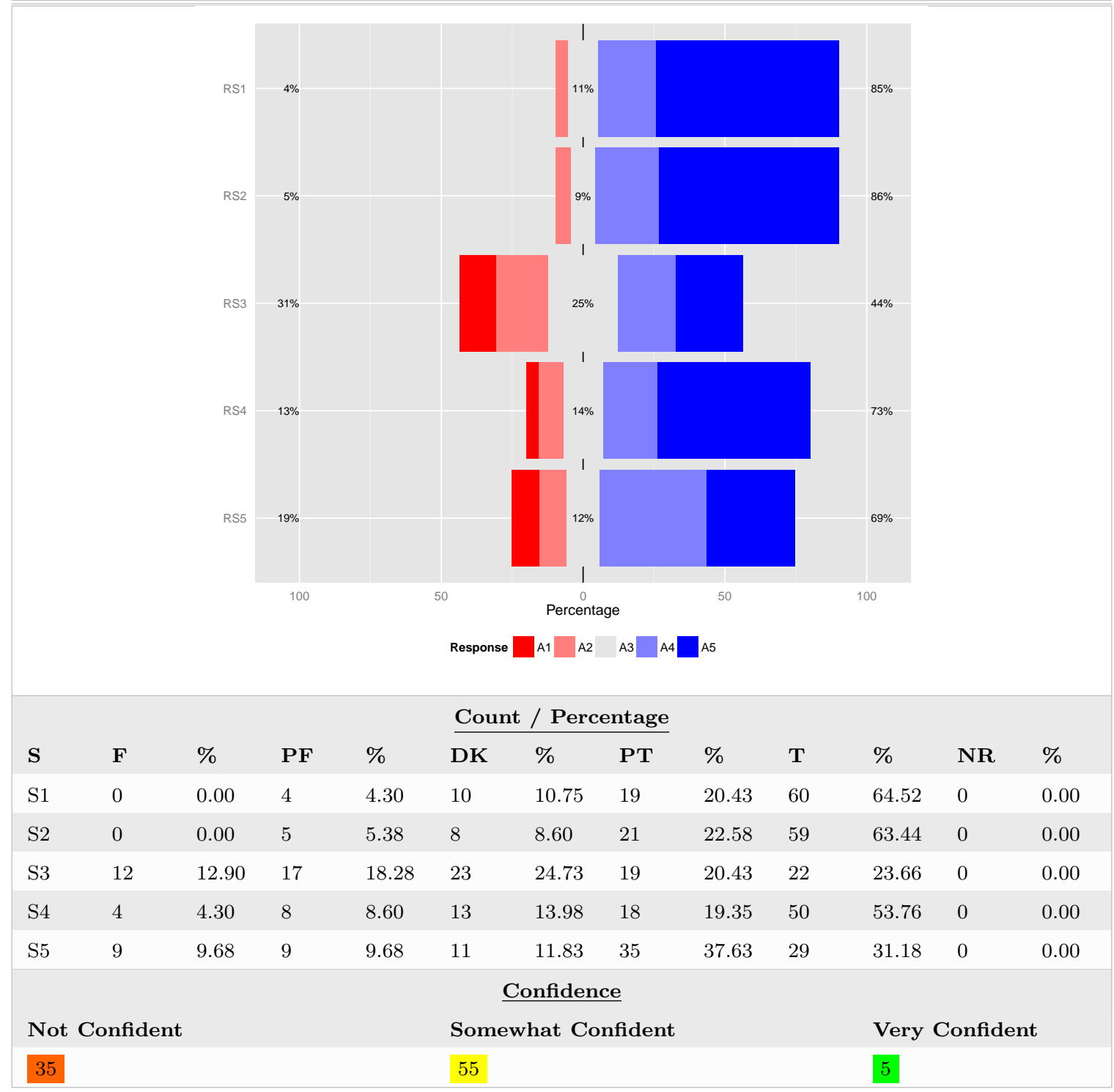

${ }^{*}$ Notes. $\mathrm{S}=$ Scale. $\mathrm{F}=$ False. $\mathrm{PF}=$ Probably False. $\mathrm{DK}=$ Don't Know. $\mathrm{PT}=$ Probably True. $\mathrm{T}=\mathrm{True} . \mathrm{NC}=$ Not Confident; Not Confident response counts are coded in red . SC = Somewhat Confident; Somewhat Confident responses are coded in yellow $. \mathrm{VC}=$ Very Confident; Very Confident responses are coded in green . 
Table 49

Website/Browser Category Question 14: What is an IP Address?

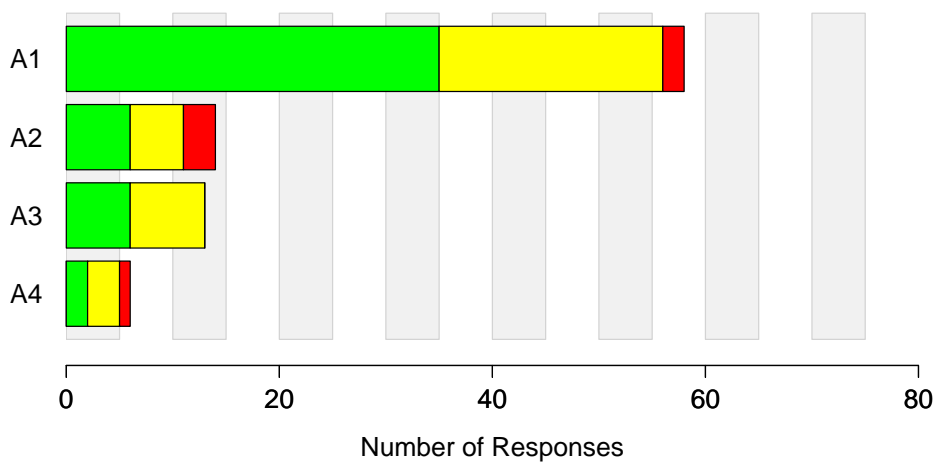

Multiple Choice Selections

\begin{tabular}{rrrrr}
\multicolumn{2}{c}{ Count } & \multicolumn{3}{c}{ Confidence } \\
C & $\%$ & NC & SC & VC \\
58 & 62.37 & 2 & 21 & 35
\end{tabular}

A1 An IP address is a numerical label assigned to a device (e.g., a computer server) participating in a network that uses the Internet Protocol for communication.

A2 An IP address is a numerical label assigned to the Web address of a $\quad \begin{array}{llll}14 & 15.05 & 3\end{array}$ website.

A3 An IP address is a numerical label assigned to the domain name server $\quad 13 \quad 13.98 \quad 13 \quad 6$ (DNS) hosting the website.

A4 An IP address is a numerical label assigned to the Uniform Resource $\quad 6 \quad 6.45 \quad 1$ Locator of a website.

A5 No response. $\quad 1 \quad 1.08 \quad-$

$*$ Notes. $\mathrm{RC}=$ Response Code. $\mathrm{C}=$ Count. $\mathrm{NC}=$ Not Confident; Not Confident response counts are coded in red. $\mathrm{SC}=$ Somewhat Confident; Somewhat Confident responses are coded in yellow . VC = Very Confident; Very Confident responses are coded in green. The correct multiple choice answer is coded in pastel green . 
Table 50

Website/Browser Category Question 15: Can a Domain Name (for Example: walmart.com) Point to More Than One IP Address?

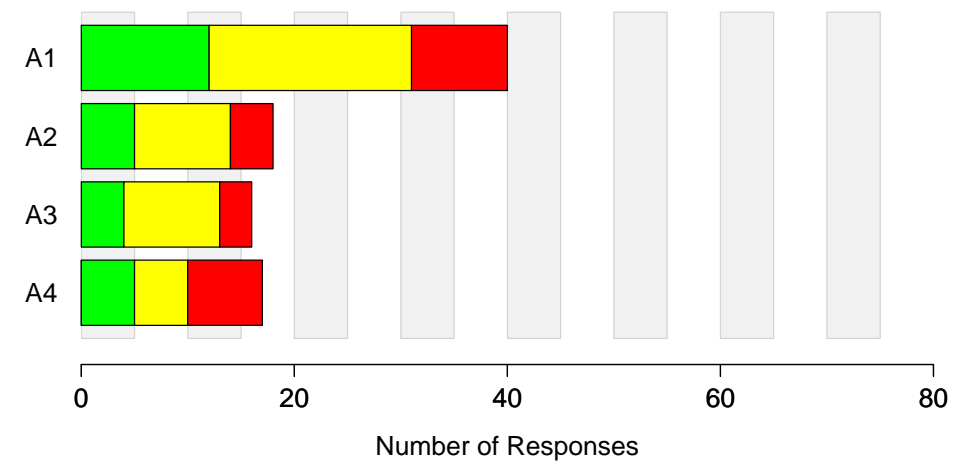

$\underline{\text { Multiple Choice Selections }}$

Count Confidence

RC Possible Answer

A1 Yes, it can point to individual IP addresses assigned to all servers chosen by the owners of the domain.

A2 No, it can only point to the main server responsible for incoming traffic $\quad \begin{array}{llllll}18 & 19.35 & 4 & 9 & 5\end{array}$ to the domain name walmart.com.

$\begin{array}{lllllll}\text { A3 } & \text { Yes, it can point to one additional IP address assigned to a backup server, } & 16 & 17.20 & 3 & 9 & 4\end{array}$ should the main walmart.com server fail.

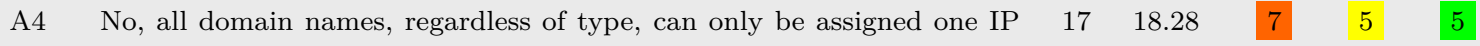
address.

A5 No response.

$*$ Notes. $\mathrm{RC}=$ Response Code. $\mathrm{C}=$ Count. $\mathrm{NC}=$ Not Confident; Not Confident response counts are coded in red . $\mathrm{SC}=$ Somewhat Confident; Somewhat Confident responses are coded in yellow . VC = Very Confident; Very Confident responses are coded in green. The correct multiple choice answer is coded in pastel green . 
Table 51

Website/Browser Category Question 16: Can a Fake Website (i.e., a Phishing Website [Which Looks Like the Correct Website but is not Genuine]) Have the Same Domain Name (e.g., walmart.com) as the Real Website?

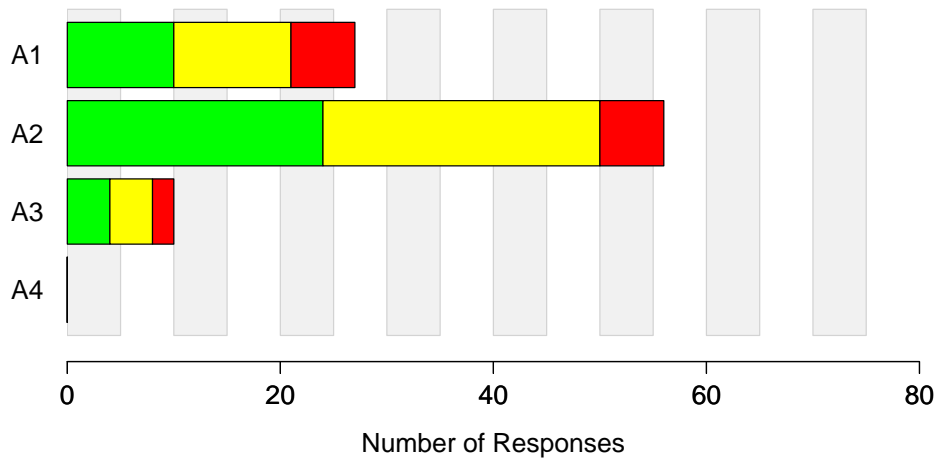

\section{Multiple Choice Selections}

\section{Count Confidence}

\begin{tabular}{lllll|l|l|l} 
A1 Yes, a fake website can have the same domain name as the real one; it & 27 & 29.03 & 6 & 11 & 10
\end{tabular} directs users to a different server.

A2 No, once the domain name is registered to a given company/organization, $\quad \begin{array}{llllll}56 & 60.22 & 6 & 26 & 24\end{array}$ it can only be used by them.

A3 Yes, the fake website can obtain the same domain name by registering $\quad$\begin{tabular}{llll|l|l|}
10 & 10.75 & 2 & 4 & 4
\end{tabular} it with a domain name registration company different than the one used to register the legitimate walmart.com domain name.

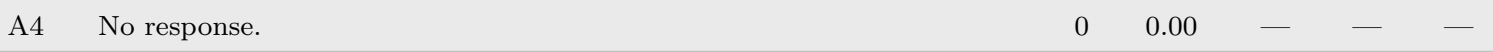

*Notes. $\mathrm{RC}=$ Response Code. $\mathrm{C}=$ Count. $\mathrm{NC}=$ Not Confident; Not Confident response counts are coded in red . $\mathrm{SC}=$ Somewhat Confident; Somewhat Confident responses are coded in yellow . VC = Very Confident; Very Confident responses are coded in green. The correct multiple choice answer is coded in pastel green . 


\section{Table 52}

Communications Category Question 2: What are Security Certificates?

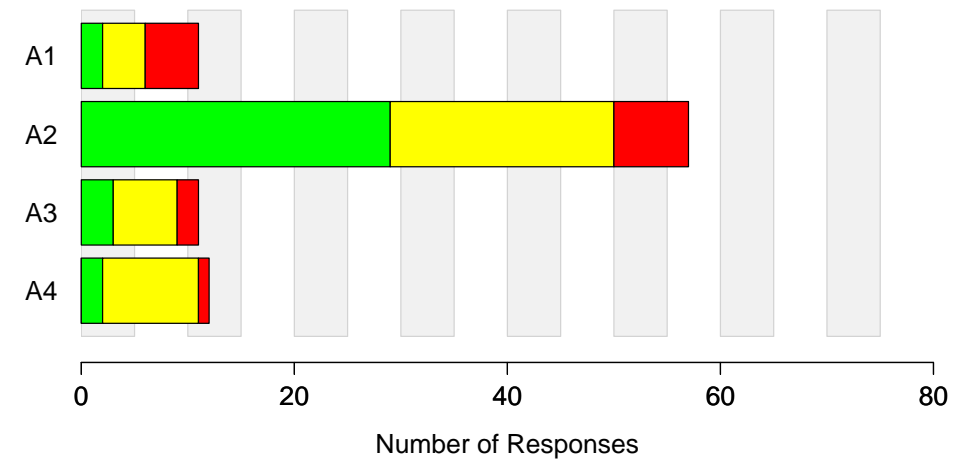

\section{Multiple Choice Selections}

RC Possible Answer

A1 Security certificates are additional security questions asked by various websites in order to better secure your account (e.g., What is the name of your oldest cousin?).

\begin{tabular}{|c|c|c|c|c|c|c|}
\hline A2 & $\begin{array}{l}\text { Security certificates are electronic documents that use a digital signa- } \\
\text { ture to bind a public key with an identity (e.g., the name of a com- } \\
\text { pany/organization). }\end{array}$ & 57 & 61.29 & 7 & 21 & 29 \\
\hline A3 & $\begin{array}{l}\text { Security certificates are the lock icons depicted on various parts of the } \\
\text { website when you log in to a given account (e.g., an email account, a } \\
\text { bank account). }\end{array}$ & 11 & 11.83 & 2 & 6 & 3 \\
\hline A4 & $\begin{array}{l}\text { Security certificates are the pop-up window warnings seen on various } \\
\text { websites that alert you to potential issues regarding the website in ques- } \\
\text { tion. }\end{array}$ & 12 & 12.90 & 1 & 9 & 2 \\
\hline A5 & No response. & 1 & 1.08 & - & & - \\
\hline
\end{tabular}

${ }^{*}$ Notes. $\mathrm{RC}=$ Response Code. $\mathrm{C}=$ Count. $\mathrm{NC}=$ Not Confident; Not Confident response counts are coded in red . $\mathrm{SC}=$ Somewhat Confident; Somewhat Confident responses are coded in yellow . VC = Very Confident; Very Confident responses are coded in green. The correct multiple choice answer is coded in pastel green . 


\section{Table 53}

Communications Category Question 4: What Happens When You Visit a Website that Has an Expired Security Certificate?

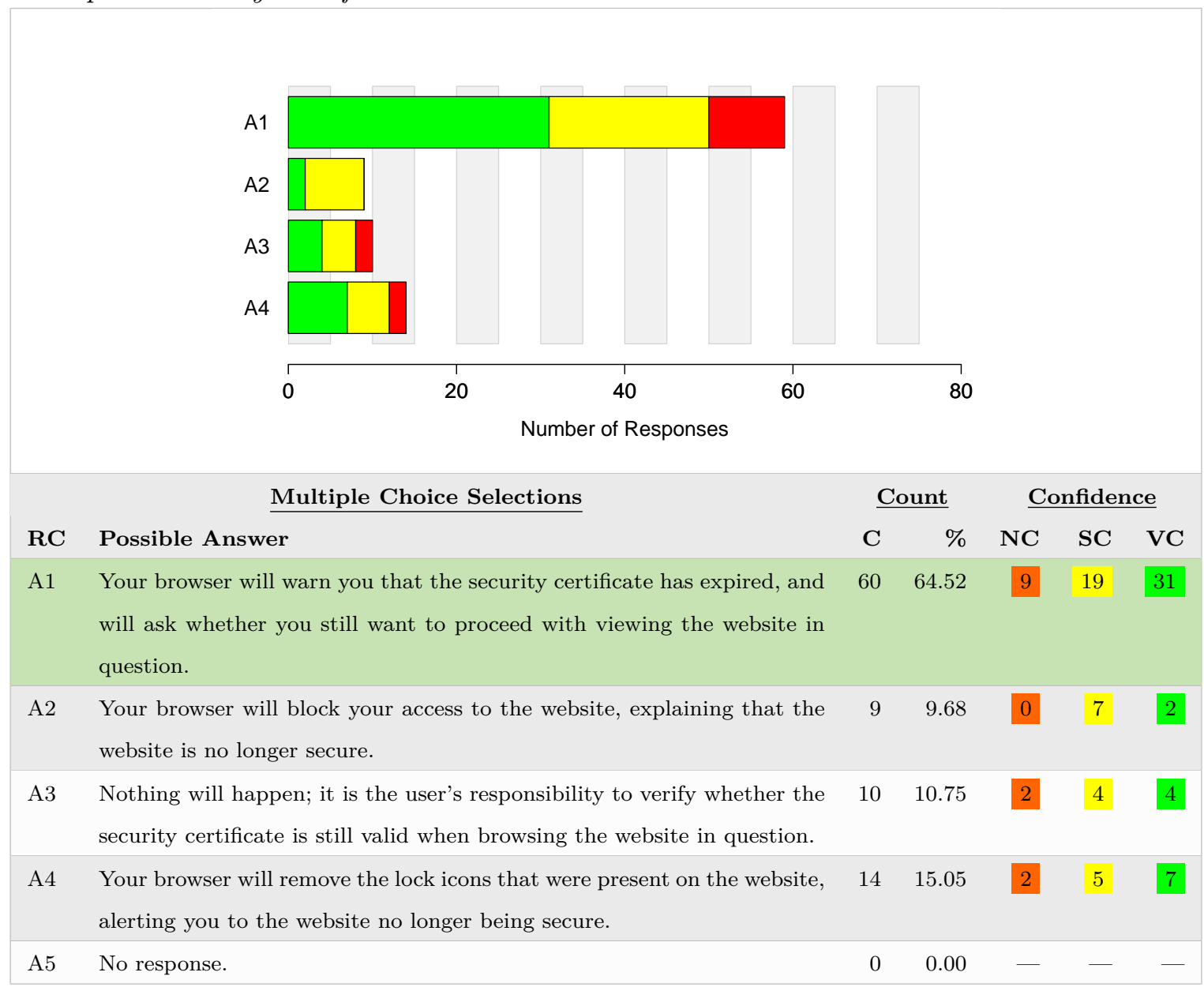

$*$ Notes. $\mathrm{RC}=$ Response Code. $\mathrm{C}=$ Count. $\mathrm{NC}=$ Not Confident; Not Confident response counts are coded in red. $\mathrm{SC}=$ Somewhat Confident; Somewhat Confident responses are coded in yellow. VC = Very Confident; Very Confident responses are coded in green. The correct multiple choice answer is coded in pastel green . 
Table 54

Communications Category Question 5: Do Expired Security Certificates Indicate Malicious Intent in Regards to the Website?

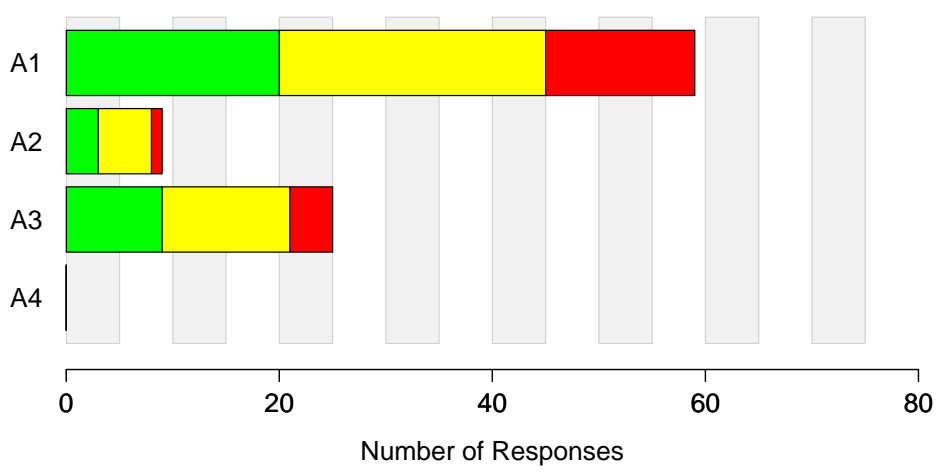

$\underline{\text { Multiple Choice Selections }}$

Count $\quad$ Confidence

RC Possible Answer

A1 No, expired security certificates usually indicate that the administrator of the website in question forgot to renew the certificate.

A2 Yes, expired security certificates are always a sign of malicious websites (e.g., phishing websites).

A3 Yes, expired security certificates indicate that malicious websites are attempting to trick your browser into thinking your information is sent securely, when it is not.

A4 No response. $\quad 0 \quad 0.00$

*Notes. $\mathrm{RC}=$ Response Code. $\mathrm{C}=$ Count. $\mathrm{NC}=$ Not Confident; Not Confident response counts are coded in red $\mathrm{SC}=$ Somewhat Confident; Somewhat Confident responses are coded in yellow $\cdot$ VC = Very Confident; Very Confident responses are coded in green . The correct multiple choice answer is coded in pastel green . 


\section{Table 55}

Communications Category Question 7: Who is Responsible for the Security Mechanisms Embedded into an Internet Browser (e.g., Google Chrome, Mozilla Firefox, Microsoft Internet Explorer)?

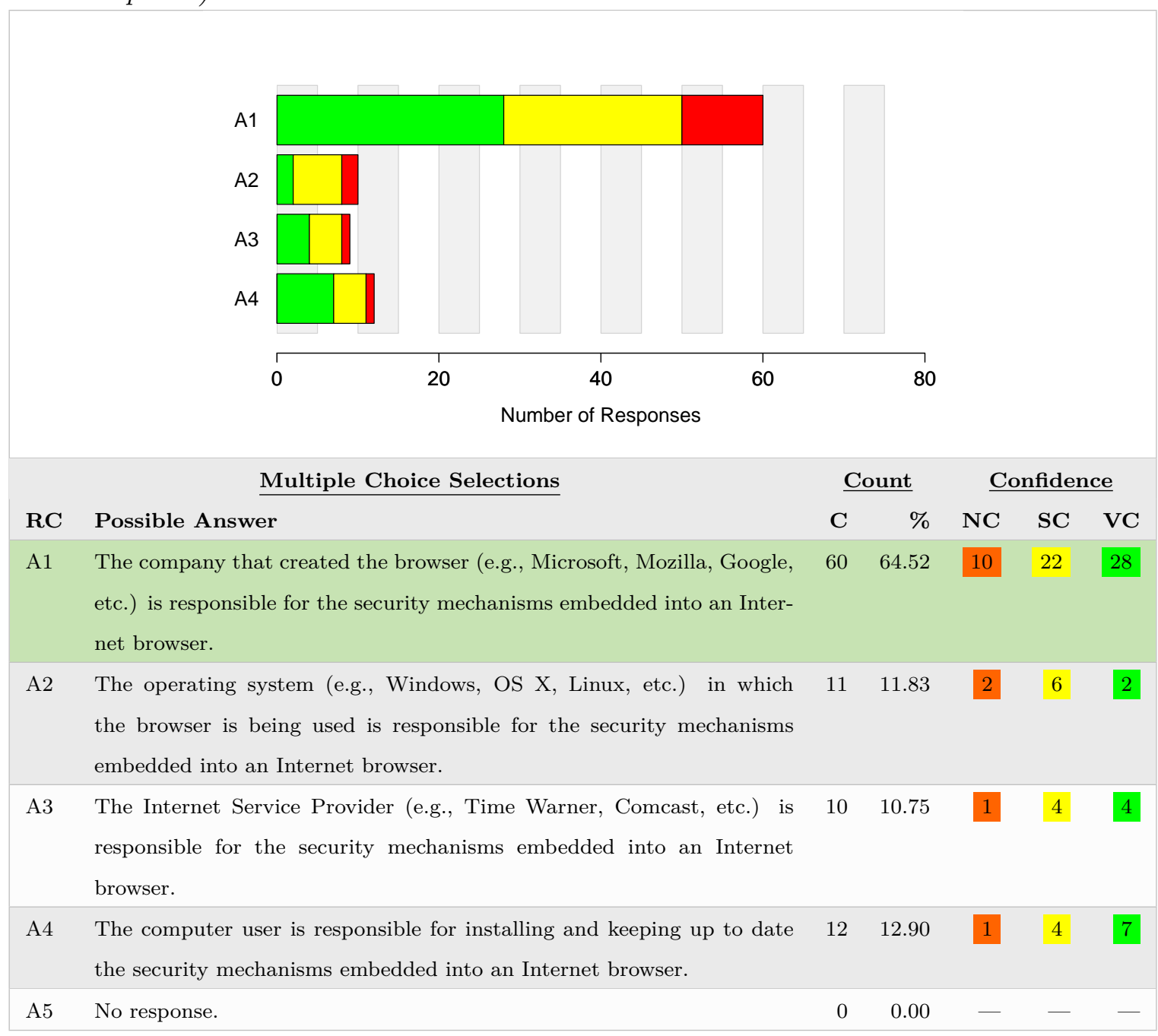

*Notes. $\mathrm{RC}=$ Response Code. $\mathrm{C}=$ Count. $\mathrm{NC}=$ Not Confident; Not Confident response counts are coded in red. $\mathrm{SC}=$ Somewhat Confident; Somewhat Confident responses are coded in yellow $. \mathrm{VC}=$ Very Confident; Very Confident responses are coded in green. The correct multiple choice answer is coded in pastel green . 


\section{Table 56}

Communications Category Question 8: Please Provide Your Opinion on the Following Statements.

\begin{tabular}{|c|c|}
\hline Code & Rating Scale Statement \\
\hline S1 & $\begin{array}{l}\text { Your Internet Service Provider (e.g., AT\&T, Comcast, Time Warner Cable) can see (i.e., keep records } \\
\text { of) the websites you visit. }\end{array}$ \\
\hline $\mathrm{S} 2$ & $\begin{array}{l}\text { Your Internet Service Provider (e.g., AT\&T, Comcast, Time Warner Cable) can modify the websites } \\
\text { you visit. }\end{array}$ \\
\hline S3 & A security certificate expires after a pre-determined amount of time. \\
\hline S4 & The expiration date of a security certificate is determined by the owner of the website in question. \\
\hline S5 & $\begin{array}{l}\text { There are strict rules and regulations that enforce certificate authorities to conduct business in legitimate } \\
\text { ways. }\end{array}$ \\
\hline S6 & $\begin{array}{l}\text { All security certificate authorities are located in North America (i.e., within the United States and } \\
\text { Canada). }\end{array}$ \\
\hline S7 & All security certificate authorities are reputable and can be trusted. \\
\hline S8 & $\begin{array}{l}\text { Browsing to a website that has a security certificate ensures that your transmitted information is } \\
\text { completely safe from being intercepted by another user. }\end{array}$ \\
\hline S9 & $\begin{array}{l}\text { All Internet browsers show security certificate information the same way. As an example, they all make } \\
\text { use of lock icons and address bar indicators. }\end{array}$ \\
\hline S10 & $\begin{array}{l}\text { Other users are able to spy on your Web usage when you login to a wireless (i.e., Wifi) network in a } \\
\text { public place (e.g., at your local Starbucks). }\end{array}$ \\
\hline S11 & $\begin{array}{l}\text { Information sent securely through an https connection can be intercepted when you're logged on to a } \\
\text { wireless (i.e., Wifi) network (e.g., at your local Starbucks). }\end{array}$ \\
\hline $\mathrm{S} 12$ & $\begin{array}{l}\text { Secured (i.e., password-protected) Wifi wireless network connections protect you completely from other } \\
\text { users intercepting your information while browsing the Web. }\end{array}$ \\
\hline S13 & $\begin{array}{l}\text { There are software applications that exist which are able to intercept your personal information when } \\
\text { you are logged on to a Wifi wireless network. }\end{array}$ \\
\hline
\end{tabular}


Table 57

Communications Category Question 8: Please Provide Your Opinion on the Following Statements (continued...).

\begin{tabular}{|c|c|c|c|c|c|c|c|c|c|c|c|c|}
\hline & & RS1 & $12 \%$ & & & 2 & & & & $67 \%$ & & \\
\hline & & RS2 & $42 \%$ & & & 15 & & & & $40 \%$ & & \\
\hline & & RS3 & $4 \%$ & & & 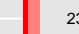 & & & & $73 \%$ & & \\
\hline & & RS4 & $37 \%$ & & & 25 & & & & $34 \%$ & & \\
\hline & & RS5 & $10 \%$ & & & & & & & $71 \%$ & & \\
\hline & & RS6 & $43 \%$ & & & 3 & & & & $22 \%$ & & \\
\hline & & RS7 & $50 \%$ & & & 2 & & & & $26 \%$ & & \\
\hline & & RS8 & $32 \%$ & & & 2 & & & & $47 \%$ & & \\
\hline & & RS9 & $26 \%$ & & & 28 & & & & $46 \%$ & & \\
\hline & & RS10 & $13 \%$ & & & 22 & & & 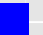 & $65 \%$ & & \\
\hline & & RS11 & $25 \%$ & & & 22 & & & & $53 \%$ & & \\
\hline & & RS12 & $47 \%$ & & & 16 & & & & $37 \%$ & & \\
\hline & & RS13 & $3 \%$ & & & 16 & & & & $80 \%$ & & \\
\hline & & & 100 & & 50 & Perce & & 50 & & 100 & & \\
\hline & & & & & Cou & / Per & ntage & & & & & \\
\hline $\mathbf{S}$ & F & $\%$ & PF & $\%$ & DK & $\%$ & PT & $\%$ & $\mathbf{T}$ & $\%$ & NR & $\%$ \\
\hline S1 & 2 & 2.15 & 9 & 9.68 & 19 & 20.43 & 28 & 30.11 & 34 & 36.56 & 1 & 1.08 \\
\hline S2 & 20 & 21.52 & 18 & 19.35 & 17 & 18.28 & 20 & 21.51 & 16 & 17.20 & 2 & 2.15 \\
\hline S3 & 1 & 1.08 & 3 & 3.23 & 21 & 22.58 & 32 & 34.41 & 35 & 37.63 & 1 & 1.08 \\
\hline $\mathrm{S} 4$ & 12 & 12.90 & 22 & 23.66 & 26 & 27.96 & 21 & 22.58 & 10 & 10.75 & 2 & 2.15 \\
\hline S5 & 2 & 2.15 & 7 & 7.53 & 17 & 18.28 & 47 & 50.54 & 18 & 19.35 & 2 & 2.15 \\
\hline S6 & 26 & 27.96 & 14 & 15.05 & 32 & 34.41 & 12 & 12.90 & 8 & 8.60 & 1 & 1.08 \\
\hline S7 & 15 & 16.13 & 31 & 33.33 & 22 & 23.66 & 16 & 17.20 & 8 & 8.60 & 1 & 1.08 \\
\hline S8 & 14 & 15.05 & 15 & 16.13 & 19 & 20.43 & 26 & 27.96 & 16 & 17.20 & 3 & 3.23 \\
\hline S9 & 11 & 11.83 & 13 & 13.98 & 26 & 27.96 & 31 & 33.33 & 11 & 11.83 & 1 & 1.08 \\
\hline S10 & 4 & 4.30 & 8 & 8.60 & 20 & 21.51 & 26 & 27.96 & 33 & 35.48 & 2 & 2.15 \\
\hline S11 & 6 & 6.45 & 17 & 18.28 & 20 & 21.51 & 34 & 36.56 & 15 & 16.13 & 1 & 1.08 \\
\hline S12 & 23 & 24.73 & 21 & 22.58 & 15 & 16.13 & 19 & 20.43 & 15 & 16.13 & 0 & 0.00 \\
\hline $\mathrm{S} 13$ & 2 & 2.15 & 1 & 1.08 & 15 & 16.13 & 36 & 38.71 & 38 & 40.86 & 1 & 1.08 \\
\hline & & & & & & onfide & & & & & & \\
\hline Not & nfi & & & & Som & hat $\mathrm{C}$ & afident & & & Very & Confid & \\
\hline 18 & & & & & 48 & & & & & 27 & & \\
\hline
\end{tabular}

*Notes. $\mathrm{S}=$ Scale. $\mathrm{F}=$ False. $\mathrm{PF}=$ Probably False. $\mathrm{DK}=$ Don't Know. $\mathrm{PT}=$ Probably True. $\mathrm{T}=\mathrm{True} . \mathrm{NC}=$ Not Confident; Not Confident response counts are coded in red . SC = Somewhat Confident; Somewhat Confident responses are coded in yellow . VC $=$ Very Confident; Very Confident responses are coded in green . 
Table 58

Malware Category Question 1: How do Malicious Software Applications get Installed on Your Computer? (Select all that Apply).

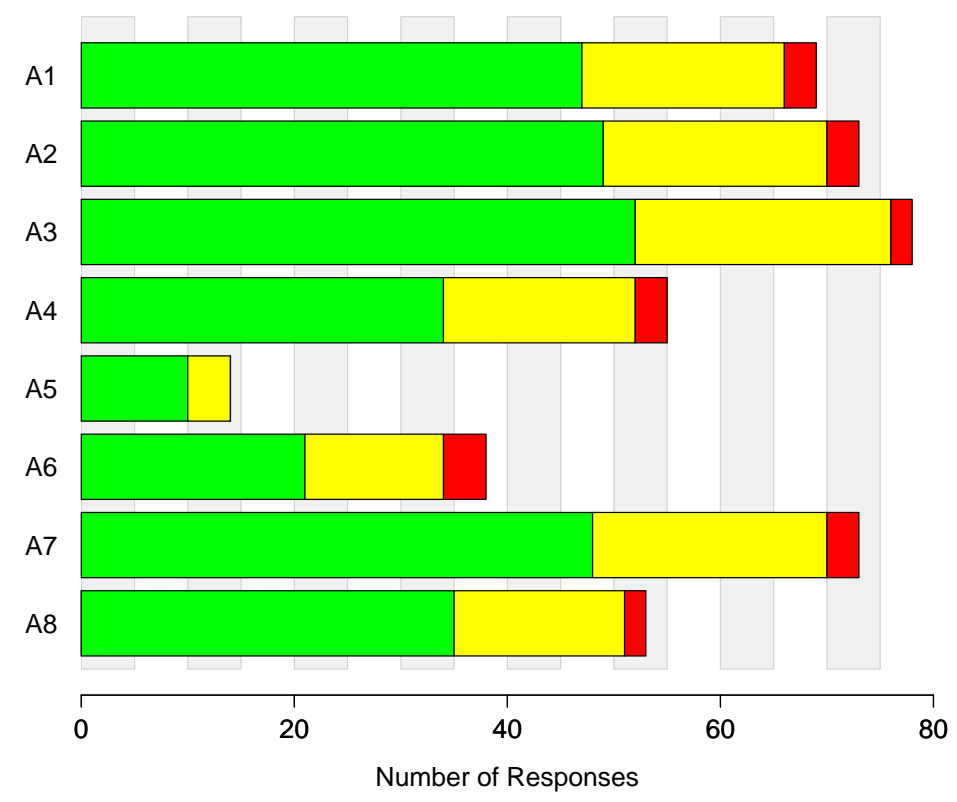

\section{Multiple Choice Selections}

\section{RC Possible Answer}

A1 By downloading pirated computer files (e.g., music, videos, software applications).

$\begin{array}{llllllll}\text { A2 } & \text { By downloading and installing a piece of software from an untrusted } & 73 & 78.49 & 3 & 21 & 49\end{array}$ source.

A3 By opening an attachment contained in an email from an untrusted $\quad \begin{array}{lllllll}78 & 83.87 & 2 & 24 & 52\end{array}$ source.

$\begin{array}{llllllll}\text { A4 } & \text { By reading an email from an untrusted source. } & 55 & 59.14 & 3 & 18 & 34\end{array}$

A5 By purchasing a software application from a physical store and installing $\quad \begin{array}{lllllll}14 & 15.05 & 0 & 4 & 10\end{array}$ it on your computer.

A6 By downloading and installing a reputable software application that con- $\quad$\begin{tabular}{lllll|l}
39 & 41.94 & 4 & 13 & 21
\end{tabular} tains optional program extensions.

$\begin{array}{llllllll}\text { A7 } & \text { By sharing computer files with a friend or family member who is already } & 73 & 78.49 & 3 & 22 & 48\end{array}$ infected by a malicious software application.

$\begin{array}{llllllll}\text { A8 } 8 & \text { By dismissing pop-up windows on websites by clicking on the OK button. } & 53 & 56.99 & 2 & 16 & 35\end{array}$

$*$ Notes. $\mathrm{RC}=$ Response Code. $\mathrm{C}=$ Count. $\mathrm{NC}=$ Not Confident; Not Confident response counts are coded in red . $\mathrm{SC}=$ Somewhat Confident; Somewhat Confident responses are coded in yellow $\cdot \mathrm{VC}=$ Very Confident; Very Confident responses are coded in green. The correct checklist answers are coded in pastel green . 
Table 59

Malware Category Question 3: Once a Malicious Software Application is Installed on Your Computer, is it Able to do Damage to Your Computer Only After Some Time has Passed, are the Effects Immediate, or are Both Scenarios Possible?

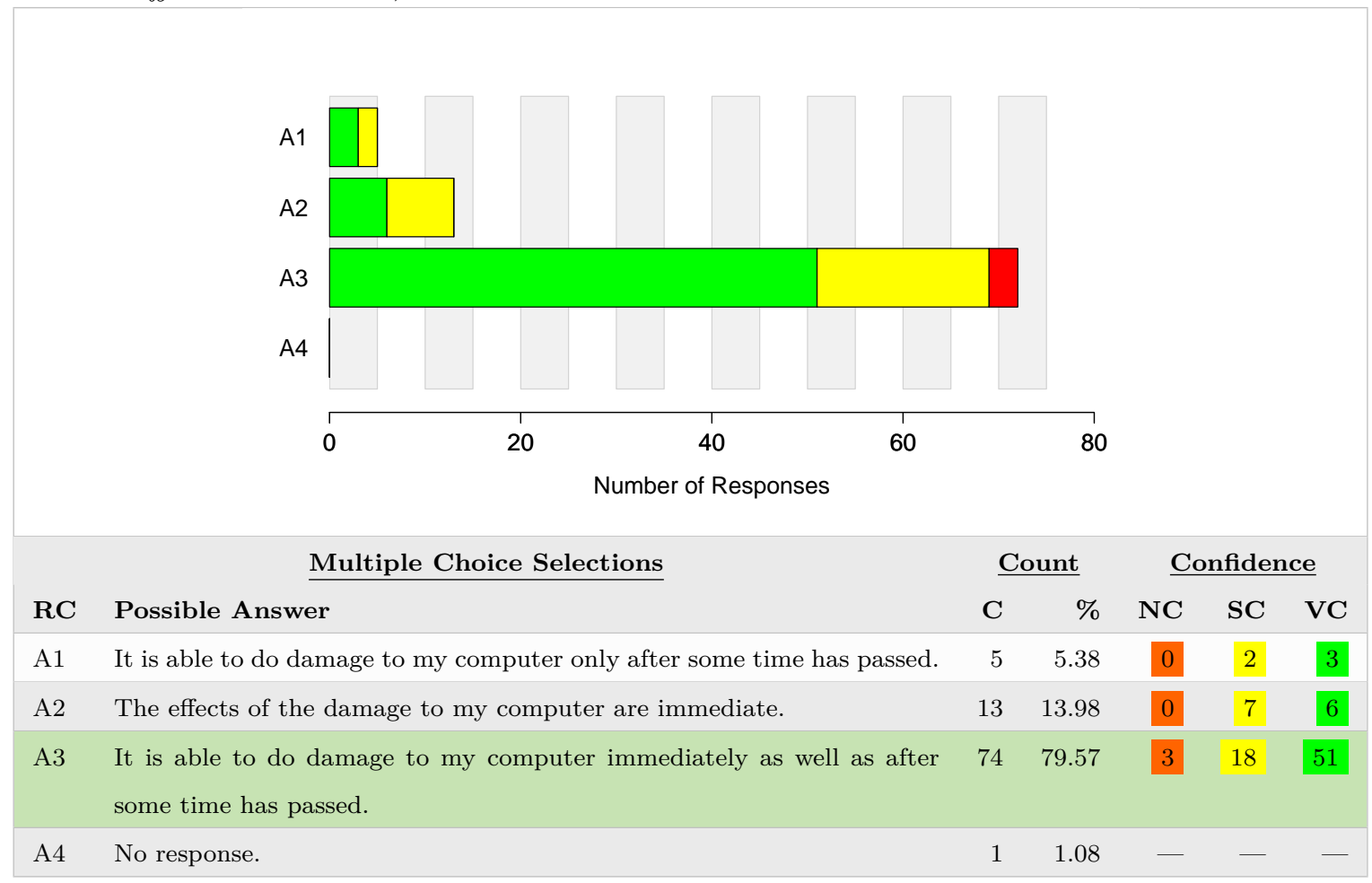

$*$ Notes. $\mathrm{RC}=$ Response Code. $\mathrm{C}=$ Count. $\mathrm{NC}=$ Not Confident; Not Confident response counts are coded in red . $\mathrm{SC}=$ Somewhat Confident; Somewhat Confident responses are coded in yellow . VC = Very Confident; Very Confident responses are coded in green . The correct multiple choice answer is coded in pastel green . 
Table 60

Malware Category Question 6: Please Provide Your Opinion on the Following Statements.

$\begin{array}{ll}\text { Code } & \text { Rating Scale Statement } \\ \text { S1 } & \text { Once installed, malicious software applications can access any file on your computer. } \\ \text { S2 } & \text { Once installed, malicious software applications can access any software application (e.g., Microsoft } \\ & \text { Word, Microsoft Excel, Adobe Reader) on your computer. } \\ \text { S3 } & \text { Once installed, malicious software applications can obtain personal information found on your computer } \\ & \text { (e.g., various types of account usernames, passwords, etc.). } \\ \text { S4 } & \text { Once installed, malicious software applications can see the information you submit through online forms } \\ & \text { (e.g., while making an online purchase, while logging on to your bank account, etc.). } \\ \text { S5 } & \text { Anti-Virus software protects you from all malicious software applications. } \\ \text { S6 } & \text { The companies that create Anti-Virus software are responsible for your loss of personal information, } \\ & \text { should their product fail to protect you from malicious programs. } \\ \text { S7 } & \text { the same malicious software applications. }\end{array}$

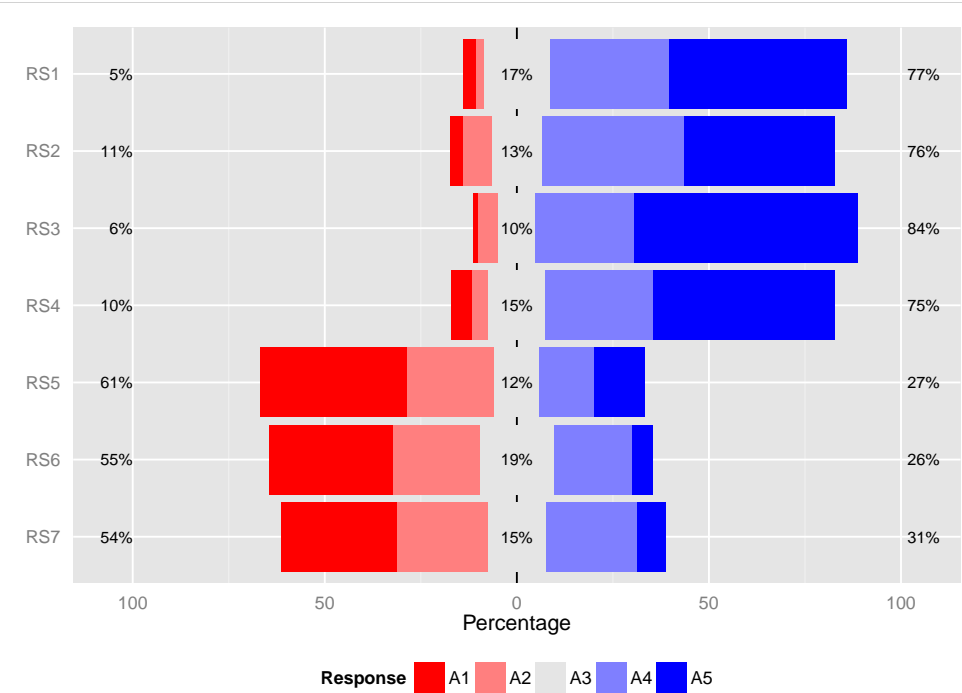

\begin{tabular}{|c|c|c|c|c|c|c|c|c|c|c|c|c|}
\hline \multirow[b]{2}{*}{$\mathrm{S}$} & \multirow[b]{2}{*}{$\mathbf{F}$} & \multirow[b]{2}{*}{$\%$} & \multirow[b]{2}{*}{ PF } & \multirow[b]{2}{*}{$\%$} & \multicolumn{3}{|c|}{ Count / Percentage } & \multirow[b]{2}{*}{$\%$} & \multirow[b]{2}{*}{$\mathbf{T}$} & \multirow[b]{2}{*}{$\%$} & \multirow[b]{2}{*}{ NR } & \multirow[b]{2}{*}{$\%$} \\
\hline & & & & & DK & $\%$ & $\mathbf{P T}$ & & & & & \\
\hline S1 & 3 & 3.23 & 2 & 2.15 & 16 & 17.20 & 29 & 31.18 & 43 & 46.24 & 0 & 0.00 \\
\hline $\mathrm{S} 2$ & 3 & 3.23 & 7 & 7.53 & 12 & 12.90 & 34 & 36.56 & 36 & 38.71 & 1 & 1.08 \\
\hline S3 & 1 & 1.08 & 5 & 5.38 & 9 & 9.68 & 24 & 25.81 & 54 & 58.06 & 0 & 0.00 \\
\hline $\mathrm{S} 4$ & 5 & 5.38 & 4 & 4.30 & 14 & 15.05 & 26 & 27.96 & 44 & 47.31 & 0 & 0.00 \\
\hline S5 & 35 & 37.63 & 21 & 22.58 & 11 & 11.83 & 13 & 13.98 & 12 & 12.90 & 1 & 1.08 \\
\hline S6 & 30 & 32.26 & 21 & 22.58 & 18 & 19.35 & 19 & 20.43 & 5 & 5.38 & 0 & 0.00 \\
\hline S7 & 28 & 30.11 & 22 & 23.66 & 14 & 15.05 & 22 & 23.66 & 7 & 7.53 & 0 & 0.00 \\
\hline \multicolumn{13}{|c|}{$\underline{\text { Confidence }}$} \\
\hline \multicolumn{5}{|c|}{ Not Confident } & \multicolumn{5}{|c|}{ Somewhat Confident } & \multicolumn{3}{|c|}{ Very Confident } \\
\hline \multicolumn{5}{|l|}{5} & \multicolumn{5}{|l|}{45} & \multicolumn{3}{|l|}{40} \\
\hline
\end{tabular}

*Notes. $\mathrm{S}=$ Scale. $\mathrm{F}=$ False. $\mathrm{PF}=$ Probably False. $\mathrm{DK}=$ Don't Know. $\mathrm{PT}=$ Probably True. $\mathrm{T}=\mathrm{True} . \mathrm{NC}=$ Not Confident; Not Confident response counts are coded in red $. \mathrm{SC}=$ Somewhat Confident; Somewhat Confident responses are coded in yellow . VC $=$ Very Confident; Very Confident responses are coded in green . 


\section{Appendix Y}

\section{Study Four: Participant Confidence Comments}

NB: PCC $=$ Participant Confidence Comment

\section{Website/Browser Category}

\section{Question 1: What is a domain name?}

$\mathbf{P C C} \Rightarrow$ I'm pretty sure it's the name of the company or person that owns the website usually.

$\mathbf{P C C} \Rightarrow$ I've always thought that domain name is the name of the website.

$\mathbf{P C C} \Rightarrow$ took best guess

$\mathbf{P C C} \Rightarrow$ i know about my ability

PCC $\Rightarrow$ I don't believe it is simply the computer Web address, besides, http://www.walmart.com/index.gsp only points to the index page

PCC $\Rightarrow$ Look, I'm a senior network engineer and your answers are either all invalid, or so poorly written that it makes no sense.

$\mathbf{P C C} \Rightarrow$ i don't have much experience with buying domain names to know what they are $\mathbf{P C C} \Rightarrow$ I feel pretty sure of my answer as I have been told this information in the past.

$\mathbf{P C C} \Rightarrow$ This sounds like the most correct answer.

$\mathbf{P C C} \Rightarrow \mathrm{I}$ am a little confused because I think a domain name is the amazon part of amazon.com.

$\mathbf{P C C} \Rightarrow$ im just not sure

$\mathbf{P C C} \Rightarrow 10$ Years of working the I.T. field have taught me many things. I've managed domains and registered them before.

$\mathbf{P C C} \Rightarrow$ The domain name does verify and locate the particular website as its main purpose, even though it can also be used to identify the company that owns it, as in the case of Amazon. 
Question 2: Which of the following is a valid domain name? (Select all that apply)

$\mathbf{P C C} \Rightarrow$ These are just the base website.

$\mathbf{P C C} \Rightarrow$ I think it's pretty much anything with the .com or .edu or .org

$\mathbf{P C C} \Rightarrow$ only full site listed

$\mathbf{P C C} \Rightarrow$ I also think that it could be all except the first one.

$\mathbf{P C C} \Rightarrow$ Again, not index pages, so not domain names

$\mathbf{P C C} \Rightarrow$ Again - are you allowing sub-domains to be allowed here? You don't really state.

$\mathbf{P C C} \Rightarrow$ i have no idea what a domain name is

$\mathbf{P C C} \Rightarrow$ Using my knowledge these are the answers that tome look to be domain names

$\mathbf{P C C} \Rightarrow$ i'm not sure walmart.com/browse/computers/

$\mathbf{P C C} \Rightarrow$ All of those end in Top Level Domains with no further subdomains added.

$\mathbf{P C C} \Rightarrow$ I think the answers I selected describe what I chose as the answer for the first question

$\mathbf{P C C} \Rightarrow$ Im a little unsure of the .us extension on the end of one because I do not know why or when it is generally used.

$\mathbf{P C C} \Rightarrow$ The domain is simply the address followed by appendix (.com, .net, .org, etc.) Adding anything beyond that means you're requesting data from the server and adding anything before is a sub domain.

$\mathbf{P C C} \Rightarrow$ Domain names are valid with a basic web address that states no extra information. 
Question 3: What is a Web address?

$\mathbf{P C C} \Rightarrow$ It's a location to a specific page on a website.

$\mathbf{P C C} \Rightarrow$ I know a webpage is the entire portion of the website.

$\mathbf{P C C} \Rightarrow$ sounds most logical

PCC $\Rightarrow$ The 2nd and 4th answers are similar, but this answer as a little more information, using the term URL

PCC $\Rightarrow$ The URL also ends up pointing you to a specific document on the website, so you have two answers that are correct and mean the same thing.

$\mathbf{P C C} \Rightarrow$ it sounds like the right answer

$\mathbf{P C C} \Rightarrow$ Not sure that this is the best definition but out of the available choices, this is the one I feel is best.

$\mathbf{P C C} \Rightarrow$ i'm sure

$\mathbf{P C C} \Rightarrow$ I suppose internet terminology isn't in my wheel house.

$\mathbf{P C C} \Rightarrow$ A domain name could be used to identify a home web page on its own, but additional information will require other inputs. 
Question 4: Which of the following is a valid Web address? (Select all that apply)

$\mathbf{P C C} \Rightarrow$ Those are all pretty specific as to what page they are.

$\mathbf{P C C} \Rightarrow$ All of those display the full name.

$\mathbf{P C C} \Rightarrow$ several things seem to make sense for this question

$\mathbf{P C C} \Rightarrow$ I think http://localad.walmart/Walmart/Entry/Flash? is valid, but it looks like a flash element (no .swf), that requires a query string to display content

$\mathbf{P C C} \Rightarrow$ I do a lot of web programming and ALL of these can be valid addresses, depending on how you setup the server. I'm not sure if the people writing the question know that or not.

$\mathbf{P C C} \Rightarrow$ they look like address i have used before

$\mathbf{P C C} \Rightarrow$ This question does confuse me so I am not too sure that all of them are actually web addresses.

$\mathbf{P C C} \Rightarrow$ i'm sure it

PCC $\Rightarrow$ I feel that any link that gets you somewhere intended is the address for that somewhere.

$\mathbf{P C C} \Rightarrow$ The websites that I checked all have the necessary identifying information to constitute a valid web address. The two that are unchecked would result in errors due to a failure to have a complete URL. 
Question 5: In walmart.com, what does the .com mean?

$\mathbf{P C C} \Rightarrow$.com is generally used for commercial websites but it doesn't necessarily have to be a commercial site.

$\mathbf{P C C} \Rightarrow$ I'm not really sure.

$\mathbf{P C C} \Rightarrow$ no idea

PCC $\Rightarrow$ Already answers questions pertaining to the other answers

$\mathbf{P C C} \Rightarrow$ i've never thought about what .com means before

PCC $\Rightarrow$ My son, who is good with computers told me this a few years ago, so I am hoping he gave me good info.

PCC $\Rightarrow$ i'm not sure

$\mathbf{P C C} \Rightarrow$ Well, it's not any of the other ones. :)

$\mathbf{P C C} \Rightarrow$ I thought .com stood for communication or something, I don't think it is option 3.

PCC $\Rightarrow$.com is a top level domain that is used for commercial purposes and is commonly used for American websites. 
Question 6: In walmart.com, what does walmart mean?

$\mathbf{P C C} \Rightarrow$ It's usually the company or person that registered the site.

$\mathbf{P C C} \Rightarrow$ I'm guessing on this.

$\mathbf{P C C} \Rightarrow$ logial

$\mathbf{P C C} \Rightarrow$ I believe the domain extension is .com etc.

PCC $\Rightarrow$ No answer is valid. "walmart" could have been registered by John Doe. There's nothing that says it was registered by the official company name.

$\mathbf{P C C} \Rightarrow$ no comments

$\mathbf{P C C} \Rightarrow$ Just guessing here

$\mathbf{P C C} \Rightarrow$ i'm sure

$\mathbf{P C C} \Rightarrow$ It sounds like something my computer networking friend tried to teach me once. $\mathbf{P C C} \Rightarrow$ Walmart did not have to register its own name for the corporate website, but it is beneficial to do so. 
Question 7: In weeklyad.walmart.com/walmart, what does the weeklyad mean?

$\mathbf{P C C} \Rightarrow$ It's an extension of the walmart domain.

$\mathbf{P C C} \Rightarrow$ I'm not very sure.

$\mathrm{PCC} \Rightarrow$ guessed

$\mathbf{P C C} \Rightarrow$ Obvious, part of the domain, it's a subdomain

PCC $\Rightarrow$ It could be a sub-domain, or simply the hostname of a server on their site. We have no way of knowing if it's an entire sub-domain or not. We would have to look at the DNS zone file to determine if it's an A record or a whole other zone.

$\mathbf{P C C} \Rightarrow$ it makes the most sense to me

$\mathbf{P C C} \Rightarrow \mathrm{I}$ am guessing.

$\mathbf{P C C} \Rightarrow$ i'm not sure

$\mathbf{P C C} \Rightarrow$ Little to no background has informed this decision.

$\mathbf{P C C} \Rightarrow$ This subdomain references a specific location on the Walmart website. 
Question 8: Who is able to create the weeklyad in weeklyad.walmart.com/walmart?

$\mathbf{P C C} \Rightarrow$ I believe only the company that registers domains can add a sub domain.

$\mathbf{P C C} \Rightarrow$ I don't know.

$\mathbf{P C C} \Rightarrow$ guessed

$\mathbf{P C C} \Rightarrow$ i don't known

$\mathbf{P C C} \Rightarrow$ It is either the one I chose or the one right after.

PCC $\Rightarrow$ I believe the owner can do it, depends if they're hosting the content themselves I

think

PCC $\Rightarrow$ Technically, any of the root servers can add an address record for them. I doubt that's the answer you're looking for though. So I'll give the one you probably assume is correct.

$\mathbf{P C C} \Rightarrow \mathrm{i}$ think the owner is the only one who can make things on the site legally

$\mathbf{P C C} \Rightarrow$ This sounds logically like the right answer.

$\mathbf{P C C} \Rightarrow$ i'm not sure

$\mathbf{P C C} \Rightarrow$ I feel like that belongs to walmart from the way I've seen it used online e.g. mail.yahoo.com

$\mathbf{P C C} \Rightarrow$ A domain name can allow the company that uses it to have as many subdomains as they want. 
Question 9: In http://www.apple.com, what does the hyper-text transfer protocol (http) mean?

$\mathbf{P C C} \Rightarrow$ I'm pretty sure it means it's sent unsecured.

$\mathbf{P C C} \Rightarrow$ I'm not very sure.

$\mathbf{P C C} \Rightarrow$ https:// is secure

$\mathbf{P C C} \Rightarrow$ I know the difference between http and https

$\mathbf{P C C} \Rightarrow$ i think it would have to have an s at end for it to be secure

$\mathbf{P C C} \Rightarrow$ Actually this sound best but I am not too sure, just a guess here again.

$\mathbf{P C C} \Rightarrow$ i'm not sure

$\mathbf{P C C} \Rightarrow$ Process of elimination. If I read this long enough I will be able to reverse engineer a decent test. However, I feel that is not what you want so I will not.

$\mathbf{P C C} \Rightarrow$ Secured communication would use an https:// protocol. 
Question 10: In https://www.chase.com, what does the hyper-text transfer protocol secure (https) mean?

$\mathbf{P C C} \Rightarrow$ This means the communication is secured.

$\mathbf{P C C} \Rightarrow$ Not sure.

$\mathbf{P C C} \Rightarrow$ http:// is unsecure

$\mathbf{P C C} \Rightarrow$ i know

$\mathbf{P C C} \Rightarrow$ I know the difference between https and http

$\mathbf{P C C} \Rightarrow$ Again, this can be false. There can be data in the page, such as in an iframe, that uses insecure protocols. It's a widely known security issue.

$\mathbf{P C C} \Rightarrow$ no comments

$\mathbf{P C C} \Rightarrow \mathrm{I}$ am basing this on my previous answer as it makes the most sense to me.

$\mathbf{P C C} \Rightarrow$ i'm sure

$\mathbf{P C C} \Rightarrow$ Secure and encryption go hand in hand usually.

PCC $\Rightarrow$ The website is secured based upon the https protocol and an accompanying security certificate that explains what encryption is being used. 
Question 11: A company/organization may obtain a domain name via the following: (select all that apply).

$\mathbf{P C C} \Rightarrow$ I think that's the only way a domain can be registered but I could be wrong.

$\mathbf{P C C} \Rightarrow$ I don't really know.

$\mathbf{P C C} \Rightarrow$ all seem to be possible

$\mathbf{P C C} \Rightarrow$ maybe .gov has to register with government

$\mathbf{P C C} \Rightarrow$ I think you can register a domain name if you have your own web server, and it would depend on who you are to register with government departments

$\mathbf{P C C} \Rightarrow$ i'm not sure about government departments

$\mathbf{P C C} \Rightarrow$ I think I read this at one time.

$\mathbf{P C C} \Rightarrow$ i'm not sure

$\mathbf{P C C} \Rightarrow$ Until the government totally owns and controls the internet I feel that these 2 options make the most sense. I think ISPs just provide a 'road' between servers, or the infrastructure to the internet.

$\mathbf{P C C} \Rightarrow$ Domain name registration companies are the only groups that can assign a domain name to a particular company, who may then use it as they wish. 
Question 12: Once a company/organization obtains a domain name, who is in control of the domain name (i.e., is allowed to make changes, associate the domain name to a website, etc.)?

$\mathbf{P C C} \Rightarrow$ I believe the company that purchased it is in control of it.

$\mathbf{P C C} \Rightarrow$ I'm not sure.

$\mathrm{PCC} \Rightarrow$ guess

$\mathbf{P C C} \Rightarrow$ It may be the \#3 choice instead.

$\mathbf{P C C} \Rightarrow$ I guess it's really the domain name registration company that controls it, but the organization is paying the domain name registration company to control it, so I would think the organization paying ultimately controls it

$\mathbf{P C C} \Rightarrow$ no comments

$\mathbf{P C C} \Rightarrow$ Makes sense to me.

$\mathbf{P C C} \Rightarrow$ i'm not sure

PCC $\Rightarrow$ It sounds fair.

$\mathbf{P C C} \Rightarrow$ The company is allowed to use the domain name as it pleases for the given subscription period. 
Question 13: Please provide your opinion on the following statements.

$\mathbf{P C C} \Rightarrow$ I'm pretty confident about my answers as I've had several domains in the past.

$\mathbf{P C C} \Rightarrow$ Sounds about right to me.

$\mathbf{P C C} \Rightarrow$ True $=$ know these details Don't know $=$ not sure

$\mathbf{P C C} \Rightarrow$ I think I'm right about most things, and I stated my individual confidences in my answers in the previous questions

PCC $\Rightarrow$ Technically, you shouldn't buy TLDs that don't apply to you (such as an American buying an Austria TLD), but there are easy ways to get around that.

$\mathbf{P C C} \Rightarrow$ I am a newbie to this stuff. Sorry.

$\mathbf{P C C} \Rightarrow$ i'm not familiar with domain names

$\mathbf{P C C} \Rightarrow$ As far as I know these answers are correct but they are just my assumptions.

$\mathbf{P C C} \Rightarrow$ i'm sure it

$\mathbf{P C C} \Rightarrow$ Any company/organization or individual may obtain any of these extensions to use in conjunction with their website(s). Was there suppose to be a screenshot or additional information in conjunction with this question?

$\mathbf{P C C} \Rightarrow$ I am riding an 80\% confidence train because I feel I know a few answers.

$\mathbf{P C C} \Rightarrow$ I know about them even though I have no formal experience with computer security. 


\section{Question 14: What is an IP address?}

$\mathbf{P C C} \Rightarrow$ I'm pretty sure its assigned to the web address of a site.

$\mathbf{P C C} \Rightarrow$ Guessing

PCC $\Rightarrow$ guessed

$\mathbf{P C C} \Rightarrow$ It was a choice between the \#2 or \#3 answer.

$\mathbf{P C C} \Rightarrow$ At first I thought it was assigned to the domain name server (DNS) hosting the website at first, but it would be to any device utilizing Internet

PCC $\Rightarrow$ Technically, an IP address *is* assigned to the DNS server, so again, answers are pretty ambiguously worded.

$\mathbf{P C C} \Rightarrow$ no comments

$\mathbf{P C C} \Rightarrow$ This is true as I read it previously

$\mathbf{P C C} \Rightarrow$ i'm not sure

$\mathbf{P C C} \Rightarrow$ I know my computer has an IP address, so does my router I think.

PCC $\Rightarrow$ I have always associated IP Addresses with websites, but they can also be used to communicate with devices like my router or printer. 
Question 15: Can a domain name (for example: walmart.com) point to more than one IP address?

$\mathbf{P C C} \Rightarrow$ I believe it can point to multiple ip addresses.

$\mathbf{P C C} \Rightarrow$ I don't really know.

$\mathbf{P C C} \Rightarrow$ seems logical

PCC $\Rightarrow$ I would think one could do this, have a server depending on the region the client is connecting from or something

$\mathbf{P C C} \Rightarrow \mathrm{i}$ have no idea about this one at all

$\mathbf{P C C} \Rightarrow$ Not sure at all.

$\mathbf{P C C} \Rightarrow$ i'm not sure

PCC $\Rightarrow$ Safety nets like this seem likely.

$\mathbf{P C C} \Rightarrow \mathrm{I}$ am not certain, but Walmart is liable to have multiple servers to handle traffic from across the U.S. and would require such capabilities. 
Question 16: Can a fake website (i.e., a phishing website [which looks like the correct website but is not genuine]) have the same domain name (e.g., walmart.com) as the real website?

$\mathbf{P C C} \Rightarrow$ They can have similar domains but not the same exact one.

$\mathbf{P C C} \Rightarrow$ Guessing

$\mathbf{P C C} \Rightarrow$ hoping this is correct

$\mathbf{P C C} \Rightarrow$ I don't think a domain name can be acquired, but I think the browser could be spoofed into redirecting to another domain when walmart.com is loaded by the user $\mathbf{P C C} \Rightarrow$ There are a ton of DNS hijacking attacks out there. With DNSSEC, DNS is insecure.

$\mathbf{P C C} \Rightarrow \mathrm{i}$ think a fake site would have to have something different in the name

$\mathbf{P C C} \Rightarrow$ They are registered and can not be legally used by anyone else.

$\mathbf{P C C} \Rightarrow$ i'm not sure

$\mathbf{P C C} \Rightarrow$ It might take a little software affecting your browser but I see this as totally plausible.

PCC $\Rightarrow$ Phishing websites exist and can be used to fool users by having all of the same appearances as a real website. 


\section{Communications Category}

Question 1: Can your Internet Service Provider (e.g., Time Warner, Comcast, AT\&T) see the personal information you submit through online forms, as well as the information that is sent back to you by the website server?

PCC $\Rightarrow$ They're not supposed to be able to see what you do but after all the NSA spying controversy who knows what they can actually do and are doing.

$\mathbf{P C C} \Rightarrow$ Guessing

$\mathbf{P C C} \Rightarrow$ no idea

$\mathbf{P C C} \Rightarrow$ Not sure, they logs to turn over apparently

$\mathbf{P C C} \Rightarrow \mathrm{i}$ think that would be illegal if they could

$\mathbf{P C C} \Rightarrow$ I would hope so due to security issues.

$\mathbf{P C C} \Rightarrow$ i'm not sure

$\mathbf{P C C} \Rightarrow$ It makes sense they would see any information they are 'transporting.'

PCC $\Rightarrow$ My ISP has stated that it does not keep such records. 
Question 2: What are security certificates?

$\mathbf{P C C} \Rightarrow$ I'm not too terribly familiar with security certificate definitions.

$\mathbf{P C C} \Rightarrow$ Guessing

$\mathbf{P C C} \Rightarrow$ have experience with these

PCC $\Rightarrow$ The last answer just sounds the most concise, could be the other answers

$\mathbf{P C C} \Rightarrow$ no comments

$\mathbf{P C C} \Rightarrow$ I have been told this information in the past.

$\mathbf{P C C} \Rightarrow$ i'm sure

PCC $\Rightarrow$ The other options sound unlikely.

PCC $\Rightarrow$ The security certificates add an additional layer of security by describing a genuine website. 
Question 3: How does a company/organization or individual obtain a security certificate?

$\mathbf{P C C} \Rightarrow$ I'm not really sure if that's true but it sounds like it might be.

$\mathbf{P C C} \Rightarrow$ Guessing

$\mathbf{P C C} \Rightarrow$ no idea

$\mathbf{P C C} \Rightarrow$ I would assume for a security certificate to have any merit, it would have to be purchased

$\mathbf{P C C} \Rightarrow$ Again, you have multiple correct answers. You can buy a cert from CA (Verisign), or you can self-sign your certificate using x509.

$\mathbf{P C C} \Rightarrow$ It is the extension of a Web address found at the end of the address (e.g., .com, .net, .org, etc.).

$\mathbf{P C C} \Rightarrow$ i have no idea

$\mathbf{P C C} \Rightarrow$ I think this was part of what I learned in the past.

$\mathbf{P C C} \Rightarrow$ i'm sure

$\mathbf{P C C} \Rightarrow$ It is used for the sake of being able to show that the website is genuine and authorized by the particular company or organization in question. 
Question 4: What happens when you visit a website that has an expired security certificate?

$\mathbf{P C C} \Rightarrow$ I can't say for sure. I know it's happened to me before but I can't remember.

$\mathbf{P C C} \Rightarrow$ I know this is what happens with Firefox. I am not sure about other browsers.

$\mathbf{P C C} \Rightarrow$ I think this has happened to me before.

$\mathbf{P C C} \Rightarrow$ been warned before

$\mathbf{P C C} \Rightarrow$ Depends on the browser, some I believe would only remove the lock icon

PCC $\Rightarrow$ This completely depends on what security settings you have configured on your browser and the browser you are using. Some won't let you go to the site at all. Some just show a broken lock. Some notify you.

$\mathbf{P C C} \Rightarrow$ not sure if my browser or virus protection blocks access

$\mathbf{P C C} \Rightarrow$ I have seen this happen personally.

$\mathbf{P C C} \Rightarrow$ i'm sure

$\mathbf{P C C} \Rightarrow$ Depends on browser and settings.

$\mathbf{P C C} \Rightarrow$ I may have seen a notification like this before, not recently.

$\mathbf{P C C} \Rightarrow$ I have encountered such a problem while browsing the web once before. 
Question 5: Do expired security certificates indicate malicious intent in regards to the website?

$\mathbf{P C C} \Rightarrow$ I'm really not too sure about this one.

$\mathbf{P C C} \Rightarrow$ Not sure

$\mathbf{P C C} \Rightarrow$ unless they are forgetful, they are trying to trick people

PCC $\Rightarrow$ I'm thinking it's a situational thing again, and I'm just assuming the best. Probably not malicious

$\mathbf{P C C} \Rightarrow$ We cannot assume intent here. It can be either/or.

$\mathbf{P C C} \Rightarrow$ this can go both ways, sometimes it is just a forgotten issue and sometimes it is malicious intent

$\mathbf{P C C} \Rightarrow$ I think this is usually the case.

$\mathbf{P C C} \Rightarrow$ i'm sure

$\mathbf{P C C} \Rightarrow$ Keyword is "usually"

$\mathbf{P C C} \Rightarrow$ As pessimistic as I am I believe that browsers would likely attempt blocking you access to sites with expired security certs. if malicious intent was ALWAYS the case.

$\mathbf{P C C} \Rightarrow$ It can be because they forgot to renew the certificate, but it could also be because it is a malicious website. 
Question 6: What are security certificate authorities?

$\mathbf{P C C} \Rightarrow$ I've never heard of a certificate authority so I'm kind of clueless on this one.

$\mathbf{P C C} \Rightarrow$ Guessing

$\mathbf{P C C} \Rightarrow$ no idea

$\mathbf{P C C} \Rightarrow$ I believe it's commercial, like Verisign, not government owned

$\mathbf{P C C} \Rightarrow$ don't know this one

$\mathbf{P C C} \Rightarrow$ Guessing again.

$\mathbf{P C C} \Rightarrow$ i'm sure

PCC $\Rightarrow$ It seems a likely function and money maker of ISPs.

$\mathbf{P C C} \Rightarrow$ Most SCAs are unaffiliated with any particular national government or other web-based organization. 
Question 7: Who is responsible for the security mechanisms embedded into an Internet browser (e.g., Google Chrome, Mozilla Firefox, Microsoft Internet Explorer)?

$\mathbf{P C C} \Rightarrow$ I'm pretty sure the developers of the browser are responsible for the security measures

$\mathbf{P C C} \Rightarrow$ Guessing

$\mathbf{P C C} \Rightarrow$ guess, seems logical

$\mathbf{P C C} \Rightarrow$ Says in the question, "embedded into an Internet browser"

$\mathbf{P C C} \Rightarrow$ it is the only answer that makes sense

$\mathbf{P C C} \Rightarrow$ Sounds right.

PCC $\Rightarrow$ The company that created it is responsible for having them in place and available

- the user is responsible for keeping them up-to-date and enabled

$\mathbf{P C C} \Rightarrow$ i'm sure

$\mathbf{P C C} \Rightarrow$ The company that made the security mechanisms often updates the web browser to compensate for bugs and new changes. 
Question 8: Please provide your opinion on the following statements.

$\mathbf{P C C} \Rightarrow$ I'm not very confident with these security answers.

$\mathbf{P C C} \Rightarrow$ Assuming

$\mathbf{P C C} \Rightarrow$ some of these I know, some were hunches

PCC $\Rightarrow$ I don't know much about security certificates, except when they're expired. I think the cable company can see my records as well, don't see why not, just not sure $\mathbf{P C C} \Rightarrow$ The assumption is that https is completely secure is false. The server can use old crypto standards that are decipherable. If it's using AES, sure. It could be using DES. Who knows.

$\mathbf{P C C} \Rightarrow$ no comments

$\mathbf{P C C} \Rightarrow$ I am fairly sure of some of these but not too with others.

$\mathbf{P C C} \Rightarrow$ i'm sure

PCC $\Rightarrow$ I am very confident because most of my answers inherently show my lack of absolute knowledge.

$\mathbf{P C C} \Rightarrow$ Very confident

$\mathbf{P C C} \Rightarrow$ Much of these are up to personal experience. 


\section{Malware Category}

Question 1: How do malicious software applications get installed on your computer? (Select all that apply)

$\mathbf{P C C} \Rightarrow$ I'm pretty sure those are the most common ways of getting malicious software on your computer.

$\mathbf{P C C} \Rightarrow$ It comes through a lot of avenues.

$\mathrm{PCC} \Rightarrow$ all seem logical

$\mathbf{P C C} \Rightarrow$ Largely depends, some people have problems with pirated files. If you have a firewall or javascript disabled, dismissing pop ups by pressing OK may not do anything. Some reputable software applications do bundle spyware, some don't.

PCC $\Rightarrow$ I know you're going to mark my answers wrong; however, several times root kits have been found as part of reputable software company's product. At some point, even reading an email would launch some script (was it active-X crap?) that loaded a virus/trojan/whatever

$\mathbf{P C C} \Rightarrow$ no comments

$\mathbf{P C C} \Rightarrow$ This I have been told.

$\mathbf{P C C} \Rightarrow$ i'm sure

PCC $\Rightarrow$ Justification for selection of "physical store" and "reputable software downloads" I think the context of malicious you are going for might not include legitimate companies putting unwanted software on your computers, but often times, certain programs are bundled in with something you purchased, that you don't necessarily want.

$\mathbf{P C C} \Rightarrow$ I feel like I have gotten malicious software many of these ways. 
Question 2: What can malicious software applications do once installed on your computer? (Select all that apply)

$\mathbf{P C C} \Rightarrow$ I'm fairly confident about those answers.

$\mathbf{P C C} \Rightarrow$ Again, it comes by a lot of avenues.

$\mathbf{P C C} \Rightarrow$ all are possible

$\mathbf{P C C} \Rightarrow$ I'm pretty sure it can't fry a processor or shut down one's Internet connection permanently

$\mathbf{P C C} \Rightarrow$ The answer to this technically depends on who you were logged in as when you got the malicious software. If you're logged in as root/admin, then it runs as that user with full access. If it's just as a user, then there's a limited scope of damage.

$\mathbf{P C C} \Rightarrow$ no comments

$\mathbf{P C C} \Rightarrow$ I have been told of this.

PCC $\Rightarrow$ i'm sure

PCC $\Rightarrow$ Hackers the movie has me convinced. 
Question 3: Once a malicious software application is installed on your computer, is it able to do damage to your computer only after some time has passed, are the effects immediate, or are both scenarios possible?

$\mathbf{P C C} \Rightarrow$ Just depends on what the intent of the malicious software is.

$\mathbf{P C C} \Rightarrow$ It has happened to me I think.

$\mathbf{P C C} \Rightarrow$ experience

PCC $\Rightarrow$ Could have a user immediately remoting controlling one's PC, or just take up the computers resources over time

$\mathbf{P C C} \Rightarrow$ no comments

$\mathbf{P C C} \Rightarrow$ Common sense.

$\mathbf{P C C} \Rightarrow$ i'm sure

$\mathbf{P C C} \Rightarrow$ Sleeper cell. 
Question 4: How does Anti-Virus software help to protect your computer from malicious software applications? (Select all that apply)

$\mathbf{P C C} \Rightarrow$ I'm fairly confident of these answers.

$\mathbf{P C C} \Rightarrow$ Experience with

$\mathrm{PCC} \Rightarrow$ guessed

$\mathbf{P C C} \Rightarrow$ I believe Anti-Virus software can do all these things, I rarely use Anti-Virus software to know. I've been clean simply using NotScript in Chrome

$\mathbf{P C C} \Rightarrow$ This entirely depends on HOW you configure your AV software!!! This question is very specific to each person's setup!

$\mathbf{P C C} \Rightarrow$ no comments

$\mathbf{P C C} \Rightarrow$ From what I have read previously.

$\mathbf{P C C} \Rightarrow$ i'm sure

$\mathbf{P C C} \Rightarrow$ "All" malicious software is misleading, as AV Software is not all-knowing cure-all for everything. Some AV programs do a better job than others, while an AV program must be updated with the information to determine if a particular malware exists or not.

$\mathbf{P C C} \Rightarrow$ Ad-aware works in these ways. 
Question 5: How does Anti-Virus software determine whether there are malicious software applications installed on my computer?

PCC $\Rightarrow$ Not sure about those last 2 options of determining malicious software.

$\mathbf{P C C} \Rightarrow$ Experience with

PCC $\Rightarrow$ seems logical

PCC $\Rightarrow$ The phrase "look into" seemed weak, may be my limited understanding of Anti-Virus software, probably could "look into" programs as well. See what they hook in to

$\mathbf{P C C} \Rightarrow$ no comments

PCC $\Rightarrow$ Sounds about right.

$\mathbf{P C C} \Rightarrow$ i'm sure

$\mathbf{P C C} \Rightarrow$ I don't know but I have spent time speculating. 
Question 6: Please provide your opinion on the following statements.

$\mathbf{P C C} \Rightarrow$ Fairly confident about those answers.

$\mathbf{P C C} \Rightarrow$ Some assumptions I have

$\mathbf{P C C} \Rightarrow$ confident with most of the answers

$\mathbf{P C C} \Rightarrow$ I'm not sure as to their exact level of control/access. I'm not certain if they can obtain any file, as I would think critical OS files would be in use by the OS while running, and unless the virus was a rootkit that would boot up the computer with networking capabilities I don't think it has access to everything

$\mathbf{P C C} \Rightarrow$ Answers once again depend on who you are logged in as.

$\mathbf{P C C} \Rightarrow$ no comments

$\mathbf{P C C} \Rightarrow$ I thinks I read this.

PCC $\Rightarrow$ i'm sure

$\mathbf{P C C} \Rightarrow$ I am ignorant of malicious software. 


\section{Appendix Z}

\section{Study Five: Ethics Application (Internet Interface Features)}

\section{Purpose of Study}

Previous research has shown that people perceive the risks associated with specific activities and technologies differently than others, depending on their general knowledge and expertise regarding the activity or technology in question (Fischhoff et al, 1978; Starr, 1969). Research in risk communication stipulates that a better understanding of the population's beliefs about risks associated with a specific event is necessary in order to develop teaching tools to help them better understand these risks (Morgan et al, 2002).

Our current research will examine users' knowledge about the risks involved in Domain Name Server phishing and installing malicious software applications. The research will test users' knowledge about these facets of the Internet. We are also interested in their perception of risk associated with engaging in online activities.

This experiment will build upon the knowledge we accumulated from our latest studies, where we were able to determine that participants associate a high amount of risk to specific online activities (e.g., using a search engine, making an online purchase) that experts would normally perceive as being less risky (LeBlanc \& Biddle, 2012); we also determined that there are problematic areas in user understanding of the various threats and security mechanisms associated with various Internet activities (e.g., DNS phishing, downloading and installing malicious software).

By better understanding people's mental models of how the Internet works, and what risks they associate to engaging in online activities, we would be in a better position to propose the development of specific tools to help educate users on the many dangers prominent over the Internet. 


\section{Method}

Materials: We will use one demographics questionnaire created with the Limesurvey web survey system, running on our Hotsoft Lab secure web server. Participant answers on the demographics questionnaire will be kept on our secure server located within our HotSoft lab at Carleton University. This questionnaire can be found in Appendix Appendix AA.

Testing interface: Since we are testing users through the Amazon Mechanical Turk online marketplace, each user will use his or her computer in order to complete the tasks within the study.

The task presented to each participant will ask him or her to answer two types of questions: multiple choice questions and Likert rating scales. The questions can be found in Appendix Appendix AC

The questionnaire is divided into 5 parts: Risk perception of domain names; risk perception of malicious software applications; knowledge testing; online activities risk ratings; and, a post-test questionnaire that poses questions regarding users' various behaviours and attitudes regarding their time spent on the Internet.

Participants: 500 online participants will be recruited through the Amazon Mechanical Turk online marketplace (https://www.mturk.com/mturk/welcome). Participants will be recruited by way of a website posting on the Amazon Mechanical Turk online marketplace website. Each participant will receive a $\$ 1.50$ honorarium for his or her time. If, at any point during testing, should the Amazon Mechanical Turk online marketplace participant choose to quit the study before its completion, the participant will be redirected to a debriefing page.

Participants must be experienced Internet users (at least 2 years), and must use the Internet at least twice weekly. They must not be either experts or students in Internet security or computer security.

The Amazon mechanical Turk online marketplace participants will be limited to the United States. This is done in an effort to control for reliability within the test results. As such, the rating scale material has been created with American companies/organizations in 
mind.

Recruitment: We plan to post a recruitment notice on the Amazon Mechanical Turk online marketplace website in order to recruit participants.

Procedure: The tasks presented to each participant will ask him or her to answer multiple choice questions and Likert rating scales on various topics about the Internet. The multiple choice questions will offer users a choice of four answers, while the Likert rating scales will be on a 7-point scale. The task description can be found in Appendix . The list of questions and statements can be found in Appendix Appendix AC Each participant will be shown the tasks in the same order; the questions and rating scale statements will be randomly shown to the participant by the Limesurvey program.

Confidentiality: In our study, we do not ask participants for their name or email address. The Amazon Mechanical Turk online marketplace prohibits the collection of this information. Only the MTurk ID of each participant is shown to the researchers, in order to assign payment. The questionnaire data will be stored on our secure servers at Carleton University in the HotSoft lab.

Amazon Mechanical Turk online marketplace participants will be redirected to the debriefing form following the main task. 


\section{$\underline{\text { References }}$}

Fischhoff, B., Slovic, P., Lichtenstein, S., Read, S. \& Combs, B. (1978). How safe is safe enough? A psychometric study of attitudes towards technological risks and benefits. Policy Sciences, 9 (2), 127-152.

LeBlanc, D., \& Biddle, R. (2012). Risk perception of Internet-related activities. Twelfth Annual International Conference on Privacy, Security, and Trust (PST 2012), 88 95.

Morgan, M. G, Fischhoff, B., Bostrom, A., \& Atman, C. J. (Eds.). (2002). Risk Communication: A Mental Models Approach, (63-79). Cambridge, UK: Cambridge University Press.

Starr, C. (1969). Social benefits vs. technological risk. Science, 165, 1232 - 1238. 


\section{Informed Consent}

The purpose of an informed consent is to ensure that you understand the purpose of the study and the nature of your involvement. The informed consent must provide sufficient information such that you have the opportunity to determine whether you wish to participate in the study.

\section{Present Study: Internet Interface Features}

Research personnel: The following people are involved in this research project, and may be contacted at anytime if you have questions or concerns: Daniel LeBlanc (PhD Student in Psychology, danielleblanc3@cmail.carleton.ca, phone: 613-520-2600 ext. 1987), Dr. Robert Biddle (Faculty Sponsor in Computer Science, email: robert_biddle@carleton.ca, 613-5202600 ext. 6317).

Ethical concerns: Should you have any ethical concerns about this research, please contact Dr. Shelley Brown (Chair, Psychology Ethics Board, shelley.brown@carleton.ca, 613-5202600, ext. 1505).

Any other concerns: For any other concerns, please contact Dr. Joanna Pozzulo (Chair, Department of Psychology, 613-520-2600 ext. 1412, psychchair@carleton.ca).

Purpose: The purpose of this study is to investigate people's understanding of the interface features of the Internet.

Description: We are asking you to fill out a demographics questionnaire regarding your background. For the main task, we will ask you various questions in relation to online activities. These questions will be divided into five parts.

Duration: The session will last approximately 45 minutes.

Remuneration: You will receive a $\$ 1.50$ honorarium for your participation in the research. 
Potential Risk/Discomfort: There are no physical risks to participating in this study, beyond those involved in normal computer usage. If you feel any discomfort or distress, you may choose not to answer specific questions, and you will not be penalized in any way if you choose to do this. Please keep in mind that we are testing systems associated with the Internet, and not your personal abilities. The debriefing form given to you at the end of the study provides contact details that you may use if you wish to obtain additional information.

Anonymity/Confidentiality: The data collected in this experiment are strictly confidential. All data are coded such that your name is not associated with the responses you provide. Any identifying information associated with your code will be confined to a single page that will be separated from your questionnaire, and kept in a separate, secured file by the research investigators, who will keep this information confidential. To respect FIPPA (Freedom of Information and Protection of Privacy Act) requirements, we will keep your data in our secure records for no longer than 5 years, after which they will be deleted. Your online consent form and honorarium payment records will be kept in our Amazon Mechanical Turk online marketplace secured account for no longer than 5 years, after which they will be deleted. The data portion of the study will be kept on our secure servers during the aforementioned period of time.

Right to withdraw: Your participation in this study is entirely voluntary. At any point during the study, you have the right not to complete certain questions, or to withdraw from the study without penalty. Should you decide to withdraw during the testing session, you will still receive remuneration for your time. 


\section{Consent}

I have read the above form and understand the conditions of my participation. My participation in this study is voluntary, and I understand that if at anytime I wish to leave the experiment, I may do so without having to give an explanation and with no penalty whatsoever. Furthermore, I am also aware that the data gathered in this study are confidential and anonymous with respect to my personal identity. By clicking on the Agree button below, I agree to participate in this study.

[Button] I agree to participate in this study.

[Button] I do not wish to participate.

This study has received clearance by the Carleton University Psychology Research Ethics Board (Reference \#14-089). 


\section{$\underline{\text { Debriefing }}$}

\section{What are we trying to learn in this research?}

This research examines users' knowledge about the interface features of the Internet. We're hoping to determine whether users have complete and correct mental models of the Internet and its mechanisms. This experiment builds upon previously conducted studies, where we were able to determine that participants associate a high amount of risk to specific online activities (e.g., using a search engine, making an online purchase) that experts would normally perceive as being less risky.

\section{Why is this important to scientists or the general public?}

Previous research has shown that people perceive the risks associated with specific activities and technologies differently than others, depending on their general knowledge and expertise regarding the activity or technology in question. Our current research examines in greater detail people's perceptions of the threats that exist in conjunction with online activities, such as online banking and online purchasing. By better understanding how people perceive the threats associated with various online activities, we can develop educational tools to help them identify the more dangerous elements involved with interactions over the Internet.

\section{What are our hypotheses and predictions?}

We expect that users will have widely varying mental models of the interface features of the Internet. Some of these mental models will be more complete, while some will be less complete.

\section{Where can I learn more?}

In order to learn more about our research on security and trust in an online environment, please see our website at http://hotsoft.carleton.ca/security/ 


\section{What if I have questions later?}

If you have any remaining concerns, questions, or comments about the experiment, please feel free to contact Daniel LeBlanc (Principal Investigator) at: danielleblanc3@cmail.carleton.ca (613-520-2600 ext. 1987), Dr. Robert Biddle (Faculty Sponsor) at: robert_biddle@carleton.ca, 613-520-2600 ext. 6317. Should you have any ethical concerns about this research, please contact Dr. Shelley Brown (Chair, Psychology Ethics Board, shelley.brown@carleton.ca, 613-520-2600, ext. 1505). For any other concerns, please contact Dr. Joanna Pozzulo (Chair, Department of Psychology, 613-520-2600 ext. 1412, psychchair@carleton.ca).

Thank you for participating in this research! 


\section{$\underline{\text { Recruiting participants }}$}

Participants will be recruited by way of a website posting on the Amazon Mechanical Turk online marketplace (https://www.mturk.com/mturk/welcome).

\section{Announcement for recruiting participants (Amazon Mechanical Turk online marketplace)}

\section{Study Name: Internet Interface Features}

Description: This research examines users' knowledge about the interface features of the Internet. We're hoping to determine whether users have complete and correct mental models of the Internet and its mechanisms.

Eligibility Requirements: We are looking for adults 18 years of age or older, who possess a minimum of 2 years of experience with the Internet, and be regular users (i.e., use the Internet at least 2 times each week). We wish to exclude anyone who is an expert or student in computer or Internet security.

Duration: The session will last approximately 45 minutes.

Compensation: You will receive a $\$ 1.50$ USD honorarium for your time.

Contact: For more details, please contact Daniel LeBlanc at mmstudy@mvp.soft.carleton.ca

This study has received clearance by the Carleton University Psychology Research Ethics Board (Reference \#14-089). 


\section{Appendix AA}

\section{Study Five: Pre-Test Demographics Questionnaire}

In this questionnaire, we'll be asking some brief questions about your demographic information, as well as your level of Internet knowledge. All data is confidential and is coded for anonymity. You are not obliged to answer any of the questions.

There are 5 questions in this survey.

Question 1: What is your age?

Question 2: What is your gender?

Male

$\bigcirc$ Female

Prefer not to answer

Question 3: What is your occupation?

Question 4: What is your highest level of education?

$\bigcirc$ Primary

$\bigcirc$ Secondary or high school

$\bigcirc$ Trade or professional school

Some college or university

Bachelor's degree

Graduate degree

Other: 
Question 5: How often do you browse the Web?

Once a month

Once a week

Several times a week

$\bigcirc$ Daily

$\bigcirc$ Multiple times daily

Thank you for completing the demographics questionnaire. 


\section{Appendix AB}

\section{Study Five: Main Task Script}

You are now at the main task.

Over the next few pages, you'll be presented with a series of multiple choice and rating scale questions.

Please answer these questions to the best of your abilities. Keep in mind that you are not obliged to answer any of the questions present in the survey, but we hope that you will.

Main task begins. 


\section{Appendix AC}

\section{Study Five: Limesurvey Questionnaire - Part One: Domain Names}

Scenario: Imagine you go to each one of the domain names listed below with your Web browser. For each one, please rate the risk associated with the loss of personal, confidential, and/or financial information as a result of going to each of these domain names.

www.walmart.ebuying.com

$\bigcirc 0$ Not risky $\bigcirc 1 \quad \bigcirc 2 \quad \bigcirc 3 \quad \bigcirc 4 \quad \bigcirc 5 \quad \bigcirc 6$ Extremely risky

www.ebuying.walmart.com

$\bigcirc 0$ Not risky $\bigcirc 1 \quad \bigcirc 2 \quad \bigcirc 3 \quad \bigcirc 4 \quad \bigcirc 5 \quad \bigcirc 6$ Extremely risky

www.bankofamerica.us.com

$\bigcirc 0$ Not risky $\bigcirc 1 \quad \bigcirc 2 \quad \bigcirc 3 \quad \bigcirc 4 \quad \bigcirc 5 \quad \bigcirc 6$ Extremely risky

www.us.bankofamerica.com
$\bigcirc$ Not risky
$\bigcirc 1 \bigcirc 2$
$\bigcirc$
$\bigcirc 4$
5
6 Extremely risky

www.eapps.facebook.com
0 Not risky
$\bigcirc 1 \bigcirc 2$
$\bigcirc 3 \bigcirc 4$
5
Extremely risky

www.facebook.eapps.com
0 Not risky
$\bigcirc 1$
$\bigcirc 2$
$\bigcirc$
$\bigcirc 4$
$\bigcirc 5$
6 Extremely risky

www.books-amazon.com
0 Not risky
$\bigcirc 1$
$\bigcirc 2$
3
$\bigcirc$
$\bigcirc$
$\bigcirc$ Extremely risky 
www.books.amazon.com

$\bigcirc 0$ Not risky $\bigcirc 1 \quad \bigcirc 2 \quad \bigcirc 3 \quad \bigcirc 4 \quad \bigcirc 5 \quad \bigcirc 6$ Extremely risky www.shop-ebay.com

$\bigcirc 0$ Not risky $\bigcirc 1 \quad \bigcirc 2 \quad \bigcirc 3 \quad \bigcirc 4 \quad \bigcirc 5 \quad \bigcirc 6$ Extremely risky

www.shop.ebay.com

$\bigcirc 0$ Not risky $\bigcirc 1 \quad \bigcirc 2 \quad \bigcirc 3 \quad \bigcirc 4 \quad \bigcirc 5 \quad \bigcirc 6$ Extremely risky 


\section{Limesurvey Questionnaire - Section 2: Malware}

Scenario 1 - Photo Editing Software: You've obtained a software application in order to retouch a set of photographs you've recently transferred to your computer. Please rate the risk, in terms of likelihood, of the following events.

The software application you obtained posts some of your photos on the Internet without your permission.

$\bigcirc 0$ Low risk $\bigcirc 1 \quad \bigcirc 2 \quad \bigcirc 3$ Moderate risk $\bigcirc 4 \quad \bigcirc 5 \quad \bigcirc 6$ High risk

The software application you obtained deletes some of your existing photos from your computer.

$\bigcirc 0$ Low risk $\bigcirc 1 \bigcirc 2$ Moderate risk $\bigcirc 4$ M $\bigcirc \quad \bigcirc 6$ High risk

The software application you obtained changes some of your Word (.doc, .docx) documents.

$\bigcirc 0$ Low risk $\bigcirc 1 \quad \bigcirc 2 \quad \bigcirc 3$ Moderate risk $\bigcirc 4 \quad \bigcirc 5 \quad \bigcirc 6$ High risk

The software application you obtained stops you from using your Internet connection.

$\bigcirc 0$ Low risk $\bigcirc 1 \bigcirc 2$ Moderate risk $\bigcirc 4 \quad \bigcirc 5 \quad \bigcirc 6$ High risk 
The software application you obtained overlays advertising banners on your photos.
0 Low risk
$\bigcirc 1 \bigcirc 2$
3 Moderate risk
$\bigcirc 4$
$\bigcirc$
6 High risk

The software application you obtained deletes your email contacts list.

$\bigcirc 0$ Low risk $\bigcirc 1 \bigcirc 2$ Moderate risk $\bigcirc 4$ - 5 Pigh risk 
Scenario 2 - Game Software: You've obtained a game software for your computer. Please rate the risk, in terms of likelihood, of the following events.

The software application you obtained copies each of the persons on your contacts list, and sends the information to a third party.

$\bigcirc 0$ Low risk $\bigcirc 1 \quad \bigcirc 2 \bigcirc 3$ Moderate risk $\bigcirc 4 \quad \bigcirc 5 \bigcirc 6$ High risk

The software application you obtained downloads and installs several other games you don't want.

$\bigcirc 0$ Low risk $\bigcirc 1 \bigcirc 2$ Moderate risk $\bigcirc 4 \quad \bigcirc 5 \quad \bigcirc 6$ High risk

The software application you obtained installs a special application that allows another user to gain control of your computer remotely.

$\bigcirc 0$ Low risk $\bigcirc 1 \quad \bigcirc 2 \bigcirc 3$ Moderate risk $\bigcirc 4 \quad \bigcirc 5 \bigcirc 6$ High risk

The software application you obtained deletes all other games on your computer. $\bigcirc 0$ Low risk $\bigcirc 1 \bigcirc 2 \bigcirc 3$ Moderate risk $\bigcirc 4 \quad \bigcirc 5 \bigcirc 6$ High risk

The software application you obtained resets your high scores for all your other games.

$\bigcirc 0$ Low risk $\bigcirc 1 \quad \bigcirc 2 \bigcirc 3$ Moderate risk $\bigcirc 4 \quad \bigcirc 5 \quad \bigcirc 6$ High risk 
The software application you obtained deletes all of the music files on your computer.

$\bigcirc 0$ Low risk $\bigcirc 1 \quad \bigcirc 2 \quad \bigcirc 3$ Moderate risk $\bigcirc 4 \quad \bigcirc 5 \quad \bigcirc 6$ High risk 


\section{Limesurvey Questionnaire - Section 3: Knowledge Test}

1 - Which of the following is NOT a valid domain name?

Choose the best of the following answers

$\bigcirc$ www.domainname.com

www.domainname.org

www.subnet.domainname.net

www.subnet.domainnamecom

\section{2 - Which of the following is NOT a valid Web address?}

Choose the best of the following answers

http://www.webaddress.com/homepage/

$\bigcirc$ https://www.login.webaddress.com/idrecovery/

https://www.net.webaddress.com/login?[7844HTBRK]

$\bigcirc$ http://www.webaddress.com/login?>successful

3 - In walmart.com, what does the .com mean?

Choose the best of the following answers

.com is the top-level domain at the end of the domain name.

.com is the domain name extension at the end of the domain name, which is exclusive to the United States.

.com is the Web address of the domain name.

.com is the Uniform Resource Locator of the domain name. 
4 - In weeklyad.walmart.com/walmart/, what does the weeklyad mean?

Choose the best of the following answers

weeklyad is a subdomain of the walmart domain.

$\bigcirc$ weeklyad is a folder found on the walmart domain.

weeklyad is the Web address of the walmart website.

weeklyad is the Uniform Resource Locator of the walmart website.

5 - Who is able to create the weeklyad in weeklyad.walmart.com/walmart/?

Choose the best of the following answers

$\bigcirc$ Walmart.

The domain name registration company.

The government of the United States.

The Walmart company's Internet Service Provider.

6 - What does a keylogger software application do?

Choose the best of the following answers

A keylogger records the keystrokes typed on the user's computer.

A keylogger records a log of the emails sent to the user of a computer.

$\bigcirc$ A keylogger is a form of anti-virus application designed to detect malicious software applications on the user's computer.

A keylogger is a form of software that helps a user organize passwords. 
7 - Why do Anti-Virus software applications recommend periodic software updates?

Choose the best of the following answers

$\bigcirc$ They require updates in to refresh the list of malware identifiers in order to better detect malicious software applications.

They require updates in order to comply with changes in international law.

They require updates in order to obtain specific information about my computer, so that their Anti-Virus application can better protect me from malicious applications.

They require updates in order to update the list of malicious applications newly reported by the media (i.e., newspapers, television broadcasts, etc.).

\section{8 - What types of files can a malicious software application access?}

Choose the best of the following answers

It can access all files on the computer.

It can only access any type of password-protected file on the computer.

It can only access email files on the computer.

$\bigcirc$ It cannot access any files on the computer.

\section{9 - When can a malicious software application on my computer record informa-} tion submitted through online forms?

Choose the best of the following answers

If the information is submitted through an online form using the HTTP protocol.

If the information is submitted through an online form using the HTTPS protocol.

If the information is submitted through an online form on a website through a secure server.

All of the above. 
10 - How can a malicious software application be installed on my computer?

Choose the best of the following answers

$\bigcirc$ By opening an attachment found in an email message.

$\bigcirc$ By accepting a friend invite on Facebook.

By submitting my personal information through an online form on a website.

$\bigcirc$ By sending an email to an insecure mail server. 


\section{Limesurvey Questionnaire - Section 4: Perception of Risk}

Consider each of the activities below. Rate the risk involved in terms of the severity of consequences.

Typing the domain name www.bankofamerica.com into your browser's address bar could lead to the loss of personal information.

$\bigcirc 0$ Low severity $\bigcirc 1 \bigcirc 2 \bigcirc 3$ Moderate severity $\bigcirc 4 \bigcirc 5 \quad \bigcirc 6$ High severity

Clicking on a link found in an email, where the link displays the domain name www.bankofamerica.com

$\bigcirc 0$ Low severity $\bigcirc 1 \bigcirc 2 \bigcirc 3$ Moderate severity $\bigcirc 4 \bigcirc 5 \quad \bigcirc 6$ High severity

Typing the domain name www.paypal.us.com into your browser's address bar.

$\bigcirc 0$ Low severity $\bigcirc 1 \bigcirc 2 \bigcirc 3$ Moderate severity $\bigcirc 4 \bigcirc 5 \bigcirc 6$ High severity

Clicking on a link found in an email, where the link displays the domain name www.paypal.com

$\bigcirc 0$ Low severity $\bigcirc 1 \bigcirc 2 \bigcirc 3$ Moderate severity $\bigcirc 4 \bigcirc 5 \bigcirc 6$ High severity

Clicking on a link in a Google search result which brings you to the Web address https://www.bankofamerica.com/credit-cards/overview.go.

$\bigcirc 0$ Low severity $\bigcirc 1 \bigcirc 2 \bigcirc 3$ Moderate severity $\bigcirc 4 \bigcirc 5 \quad \bigcirc 6$ High severity

Installing software downloaded from an unknown source on the Internet.

$\bigcirc 0$ Low severity $\bigcirc 1 \bigcirc 2 \bigcirc 3$ Moderate severity $\bigcirc 4 \bigcirc 5 \quad \bigcirc 6$ High severity 
Downloading a PDF file containing a sales brochure for a new car, and viewing it in Adobe Reader.

$\bigcirc 0$ Low severity $\bigcirc 1 \bigcirc 2 \bigcirc 3$ Moderate severity $\bigcirc 4 \bigcirc 5 \quad \bigcirc 6$ High severity Reading an email from an unknown source and opening the attachment present with the email message.

$\bigcirc 0$ Low severity $\bigcirc 1 \bigcirc 2 \bigcirc 3$ Moderate severity $\bigcirc 4 \bigcirc 5 \quad \bigcirc 6$ High severity

Downloading and installing a new toolbar for your browser via the website http://www.github.com

$\bigcirc 0$ Low severity $\bigcirc 1 \bigcirc 2 \bigcirc 3$ Moderate severity $\bigcirc 4 \bigcirc 5 \quad \bigcirc 6$ High severity

Browse to a new concert ticket buying website by typing the Web address www.ticketsforyou.com

$\bigcirc 0$ Low severity $\bigcirc 1 \bigcirc 2 \bigcirc 3$ Moderate severity $\bigcirc 4 \quad \bigcirc 5 \bigcirc 6$ High severity 


\section{Limesurvey Questionnaire - Post-Test Questionnaire}

The survey is almost done. Please take a moment to answer the following post-test questions.

1 - How knowledgeable do you believe you are about:

How the Internet works?

$\bigcirc 0$ Not at all knowledgeable $\bigcirc 1 \bigcirc 2 \bigcirc 3 \bigcirc 4 \bigcirc 5 \bigcirc 6$ Extremely knowledgeable

How to protect your security and privacy on the Internet?

$\bigcirc 0$ Not at all knowledgeable $\bigcirc 1 \bigcirc 2 \bigcirc 3 \bigcirc 4 \bigcirc 5$ Extremely knowledgeable

How to avoid malicious software applications being installed on your computer?

$\bigcirc 0$ Not at all knowledgeable $\bigcirc 1 \bigcirc 2 \bigcirc 3 \bigcirc 4 \bigcirc 5$ Extremely knowledgeable

How to identify potentially harmful websites?

$\bigcirc 0$ Not at all knowledgeable $\bigcirc 1 \bigcirc 2 \bigcirc 3 \bigcirc 4 \bigcirc 5 \bigcirc 6$ Extremely knowledgeable

\section{2 - How capable do you believe you are of learning:}

How the Internet works?

$\bigcirc 0$ Not at all capable $\bigcirc 1 \quad \bigcirc 2 \quad \bigcirc 3 \quad \bigcirc 4 \quad \bigcirc 5 \quad \bigcirc 6$ Extremely capable 
How to protect your security and privacy on the Internet?

$\bigcirc 0$ Not at all capable $\bigcirc 1 \bigcirc 2 \bigcirc 3 \bigcirc 4 \bigcirc 5 \bigcirc 6$ Extremely capable

How to avoid malicious software applications being installed on your computer?

$\bigcirc 0$ Not at all capable $\bigcirc 1 \bigcirc 2 \bigcirc 3 \bigcirc 4 \bigcirc 5 \bigcirc 6$ Extremely capable

How to identify potentially harmful websites?

$\bigcirc 0$ Not at all capable $\bigcirc 1 \bigcirc 2 \bigcirc 3 \bigcirc 4 \bigcirc 5 \bigcirc 6$ Extremely capable

3 - How difficult do you believe it is to find information about:

How the Internet works?

$\bigcirc 0$ Not at all difficult $\bigcirc 1 \bigcirc 2 \bigcirc 3 \bigcirc 4 \bigcirc 5 \bigcirc 6$ Extremely difficult

How to protect your security and privacy on the Internet?

$\bigcirc 0$ Not at all difficult $\bigcirc 1 \bigcirc 2 \bigcirc 3 \bigcirc 4 \bigcirc 5 \bigcirc 6$ Extremely difficult

How to avoid malicious software applications being installed on your computer?

$\bigcirc 0$ Not at all difficult $\bigcirc 1 \bigcirc 2 \quad \bigcirc 3 \bigcirc 4 \quad \bigcirc 5 \bigcirc 6$ Extremely difficult

How to identify potentially harmful websites?

$\bigcirc 0$ Not at all difficult $\bigcirc 1 \bigcirc 2 \bigcirc 3 \bigcirc 4 \bigcirc 5 \bigcirc 6$ Extremely difficult

4 - How much time do you have to learn more about:

How the Internet works?

$\bigcirc 0$ No time at all $\bigcirc 1 \bigcirc 2 \bigcirc 3 \bigcirc 4 \bigcirc 5 \bigcirc 6$ A lot of time 
How to protect your security and privacy on the Internet?

$\bigcirc 0$ No time at all $\bigcirc 1 \bigcirc 2 \bigcirc 3 \bigcirc 4 \bigcirc 5 \bigcirc 6$ A lot of time

How to avoid malicious software applications being installed on your computer?

$\bigcirc 0$ No time at all $\bigcirc 1 \bigcirc 2 \bigcirc 3 \bigcirc 4 \bigcirc 5 \bigcirc 6$ A lot of time

How to identify potentially harmful websites?

$\bigcirc 0$ No time at all $\bigcirc 1 \bigcirc 2 \bigcirc 3 \bigcirc 4 \bigcirc 5 \bigcirc 6$ A lot of time

\section{5 - How motivated are you to learn more about:}

How the Internet works?

$\bigcirc 0$ Not at all motivated $\bigcirc 1 \bigcirc 2 \bigcirc 3 \bigcirc 4 \bigcirc 5 \bigcirc 6$ Extremely motivated

How to protect your security and privacy on the Internet?

$\bigcirc 0$ Not at all motivated $\bigcirc 1 \bigcirc 2 \bigcirc 3 \bigcirc 4 \bigcirc 5 \bigcirc 6$ Extremely motivated

How to avoid malicious software applications being installed on your computer?

$\bigcirc 0$ Not at all motivated $\bigcirc 1 \bigcirc 2 \bigcirc 3 \bigcirc 4 \bigcirc 5 \bigcirc 6$ Extremely motivated

How to identify potentially harmful websites?

$\bigcirc 0$ Not at all motivated $\bigcirc 1 \bigcirc 2 \bigcirc 3 \bigcirc 4 \bigcirc 5 \bigcirc 6$ Extremely motivated

6 - How responsible do you think the following entities are for protecting you from malicious software applications?

My Internet Service Provider (e.g., Comcast, Time Warner, etc.).

$\bigcirc 0$ Not at all responsible $\bigcirc 1 \bigcirc 2 \bigcirc 3 \bigcirc 4 \quad \bigcirc 5 \bigcirc 6$ Completely responsible 
My Anti-Virus software application provider (e.g., Norton, McAfee, etc.).

$\bigcirc 0$ Not at all responsible $\bigcirc 1 \bigcirc 2 \bigcirc 3 \bigcirc 4 \quad \bigcirc 5 \bigcirc 6$ Completely responsible My operating system provider (e.g., Microsoft, Apple, etc.).

$\bigcirc 0$ Not at all responsible $\bigcirc 1 \quad \bigcirc 2 \quad \bigcirc 3 \bigcirc 4 \quad \bigcirc 5 \quad \bigcirc 6$ Completely responsible

The police.

$\bigcirc 0$ Not at all responsible $\bigcirc 1 \quad \bigcirc 2 \quad \bigcirc 3 \bigcirc 4 \quad \bigcirc 5 \quad \bigcirc 6$ Completely responsible

The government of the United States.

$\bigcirc 0$ Not at all responsible $\bigcirc 1 \bigcirc 2 \quad \bigcirc 3 \bigcirc 4 \quad \bigcirc 5 \bigcirc 6$ Completely responsible

Myself.

$\bigcirc 0$ Not at all responsible $\bigcirc 1 \quad \bigcirc 2 \bigcirc 3 \bigcirc 4 \quad \bigcirc 5 \bigcirc 6$ Completely responsible

\section{7 - Please state your level of agreement or disagreement with the following statements:}

I have been the victim of a phishing website (which looks like the correct website but is not genuine).

$\bigcirc$-3 Strongly disagree $\bigcirc-2 \quad \bigcirc-1 \quad \bigcirc 0 \quad \bigcirc+1 \quad \bigcirc+2 \quad \bigcirc+3$ Strongly agree

I have had malicious software applications installed on my computer.

$\bigcirc$-3 Strongly disagree $\bigcirc-2 \quad \bigcirc-1 \quad \bigcirc 0 \quad \bigcirc+1 \quad \bigcirc+2 \quad \bigcirc+3$ Strongly agree 
One or more of my family members has been the victim of a phishing website (which looks like the correct website but is not genuine).

$\bigcirc-3$ Strongly disagree $\bigcirc-2 \quad \bigcirc-1 \quad \bigcirc 0 \quad \bigcirc+1 \quad \bigcirc+2 \quad \bigcirc+3$ Strongly agree

One or more of my family members has had malicious software applications installed on their computer.

$\bigcirc$-3 Strongly disagree $\bigcirc-2 \quad \bigcirc-1 \quad \bigcirc 0 \quad \bigcirc+1 \quad \bigcirc+2 \quad \bigcirc+3$ Strongly agree

When I browse the Internet, I am confident that I know how to avoid phishing websites (which look like the correct websites but are not genuine) and malicious software applications.

$\bigcirc$-3 Strongly disagree $\bigcirc-2 \quad \bigcirc-1 \quad \bigcirc 0 \quad \bigcirc+1 \quad \bigcirc+2 \quad \bigcirc+3$ Strongly agree 


\section{Appendix AD}

\section{Study Five: Results Part 2 - Knowledge and Risk}

Table 61

Part 2 - Knowledge Question 1: Which of the Following is Not a Valid Domain Name?

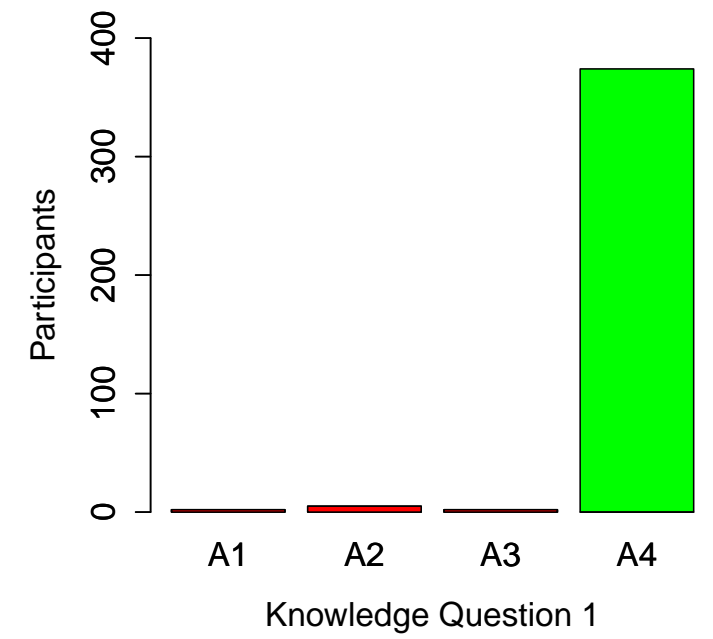

\begin{tabular}{|c|c|c|c|}
\hline Response Code & Possible Answer & Count & $\%$ \\
\hline A1 & www.domainname.com & 2 & 0.52 \\
\hline A 2 & www.domainname.org & 5 & 1.30 \\
\hline A3 & www.subnet.domainname.net & 2 & 0.52 \\
\hline A 4 & www.subnet.domainnamecom & 374 & 97.40 \\
\hline A5 & No response. & 1 & 0.26 \\
\hline
\end{tabular}

*Notes. The correct multiple choice answer is highlighted in pastel green . 
Table 62

Part 2 - Knowledge Question 2: Which of the following is NOT a valid Web address?

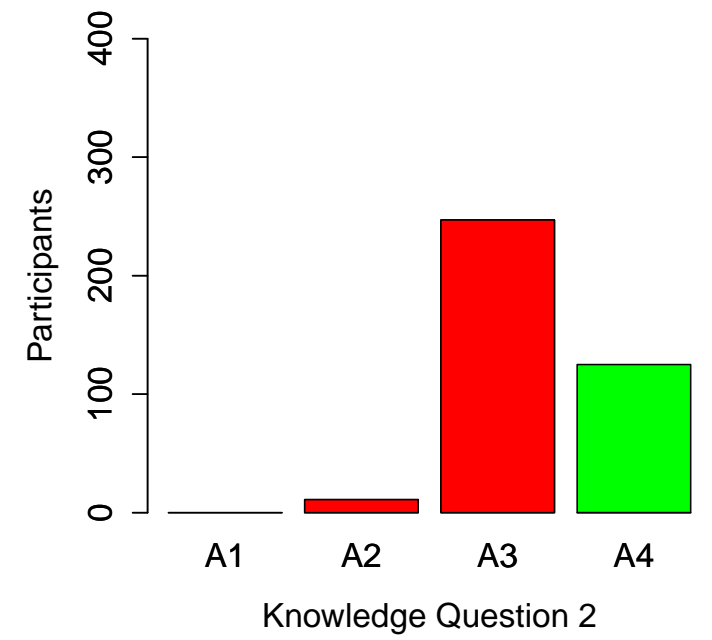

\begin{tabular}{|llrr|}
\hline Response Code & Possible Answer & Count & $\%$ \\
\hline A1 & http://www.webaddress.com/homepage/ & 0 & 0.00 \\
\hline A2 & https://www.login.webaddress.com/idrecovery/ & 11 & 2.86 \\
\hline A3 & https://www.net.webaddress.com/login?[7844HTBRK] & 247 & 64.32 \\
\hline A4 & http://www.webaddress.com/login?>successful & 125 & 32.55 \\
\hline A5 & No response. & 1 & 0.26 \\
\hline
\end{tabular}

*Notes. The correct multiple choice answer is highlighted in pastel green . 
Table 63

Part 2 - Knowledge Question 3: In walmart.com, What Does the .com Mean?

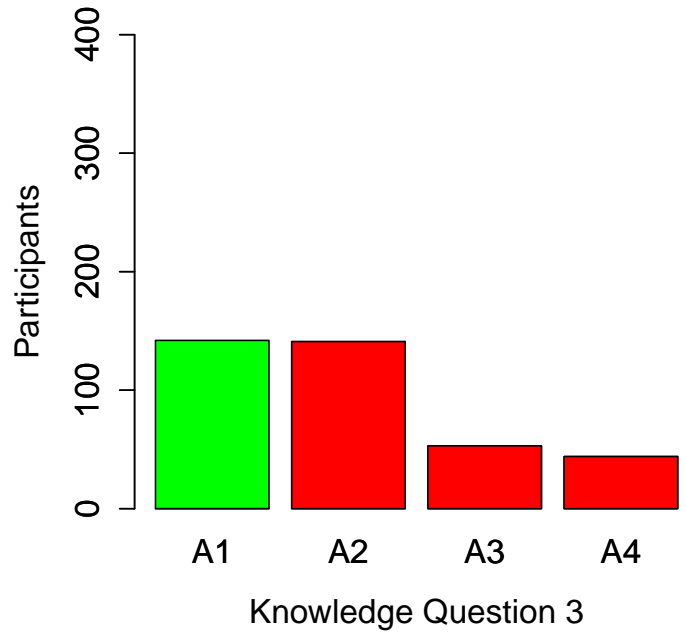

\begin{tabular}{|c|c|c|c|}
\hline Response Code & Possible Answer & Count & $\%$ \\
\hline A1 & .com is the top-level domain at the end of the domain name. & 142 & 36.98 \\
\hline A2 & $\begin{array}{l}\text {.com is the domain name extension at the end of the domain } \\
\text { name, which is exclusive to the United States. }\end{array}$ & 141 & 36.72 \\
\hline A3 & .com is the Web address of the domain name. & 53 & 13.80 \\
\hline A4 & .com is the Uniform Resource Locator of the domain name. & 44 & 11.46 \\
\hline A5 & No response. & 4 & 1.04 \\
\hline
\end{tabular}

*Notes. The correct multiple choice answer is highlighted in pastel green . 
Table 64

Part 2 - Knowledge Question 4: In weeklyad.walmart.com/walmart/, What Does the weeklyad Mean?

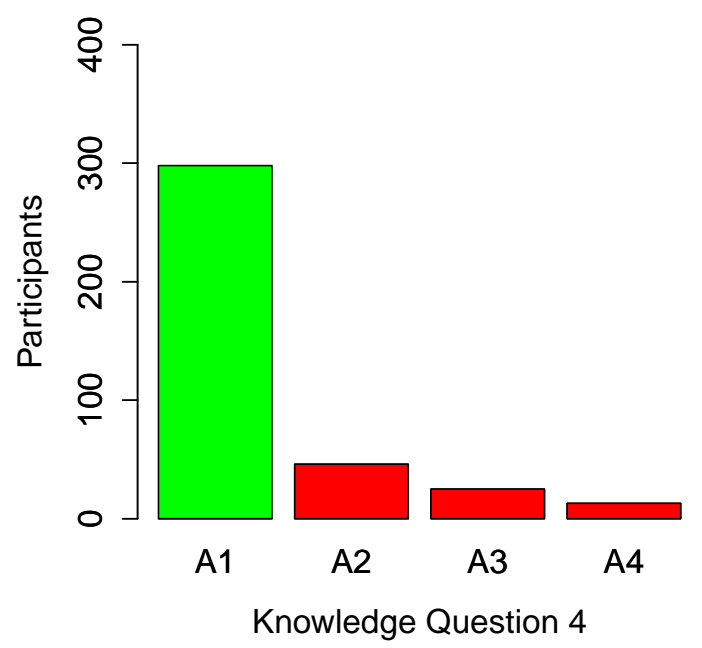

\begin{tabular}{|c|c|c|c|}
\hline Response Code & Possible Answer & Count & $\%$ \\
\hline A1 & weeklyad is a subdomain of the walmart domain. & 298 & 77.60 \\
\hline A 2 & weeklyad is a folder found on the walmart domain. & 46 & 11.98 \\
\hline A3 & weeklyad is the Web address of the walmart website. & 25 & 6.51 \\
\hline A 4 & $\begin{array}{l}\text { weeklyad is the Uniform Resource Locator of the walmart web- } \\
\text { site. }\end{array}$ & 13 & 3.39 \\
\hline A5 & No response. & 2 & 0.52 \\
\hline
\end{tabular}

*Notes. The correct multiple choice answer is highlighted in pastel green . 
Table 65

Part 2 - Knowledge Question 5: Who is Able to Create the weeklyad in weeklyad.walmart.com/walmart/?

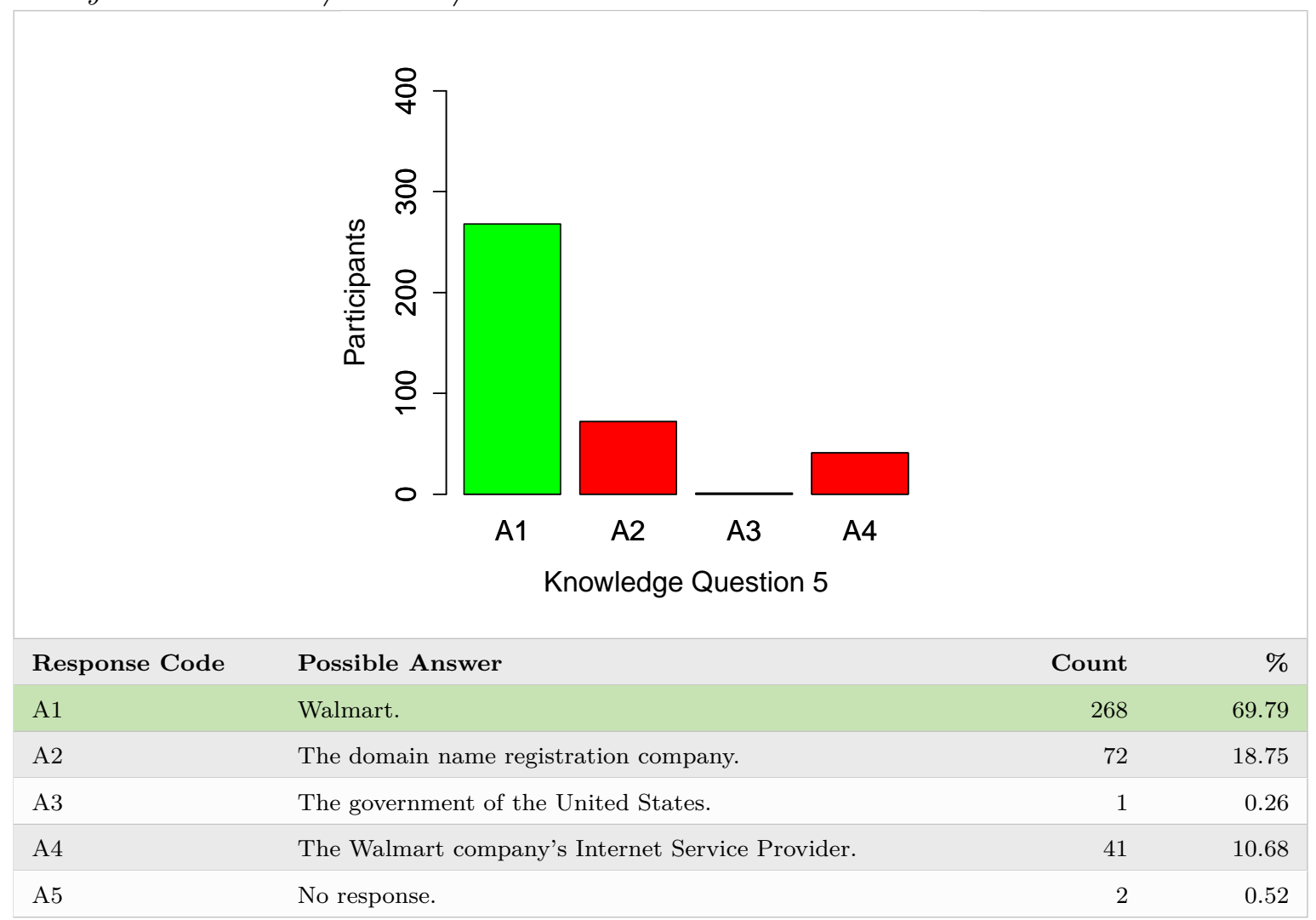

*Notes. The correct multiple choice answer is highlighted in pastel green . 
Table 66

Part 2 - Knowledge Question 6: What Does a Keylogger Software Application Do?

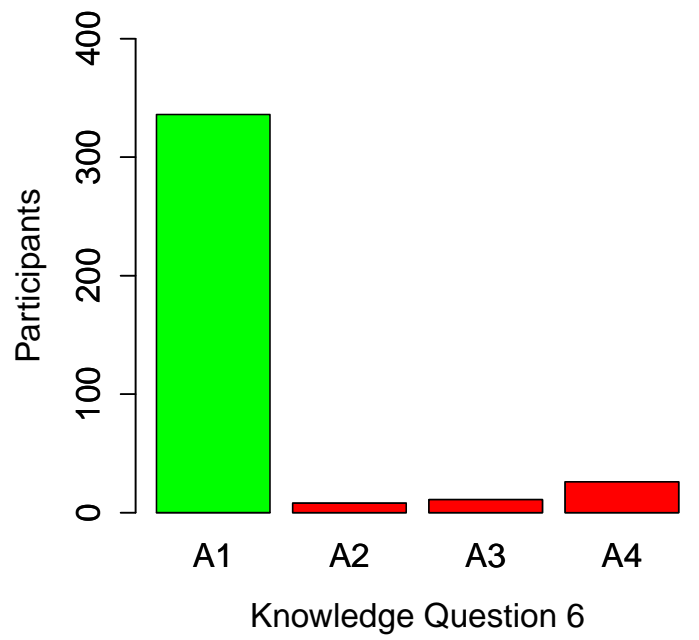

\begin{tabular}{|c|c|c|c|}
\hline Response Code & Possible Answer & Count & $\%$ \\
\hline A1 & $\begin{array}{l}\text { A keylogger records the keystrokes typed on the user's com- } \\
\text { puter. }\end{array}$ & 336 & 87.50 \\
\hline $\mathrm{A} 2$ & $\begin{array}{l}\text { A keylogger records a log of the emails sent to the user of a } \\
\text { computer. }\end{array}$ & 8 & 2.08 \\
\hline A3 & $\begin{array}{l}\text { A keylogger is a form of anti-virus application designed to } \\
\text { detect malicious software applications on the user's computer. }\end{array}$ & 11 & 2.86 \\
\hline A4 & $\begin{array}{l}\text { A keylogger is a form of software that helps a user organize } \\
\text { passwords. }\end{array}$ & 26 & 6.77 \\
\hline A5 & No response. & 3 & 0.78 \\
\hline
\end{tabular}

*Notes. The correct multiple choice answer is highlighted in pastel green . 


\section{Table 67}

Part 2 - Knowledge Question 7: Why Do Anti-Virus Software Applications Recommend Periodic Software Updates?

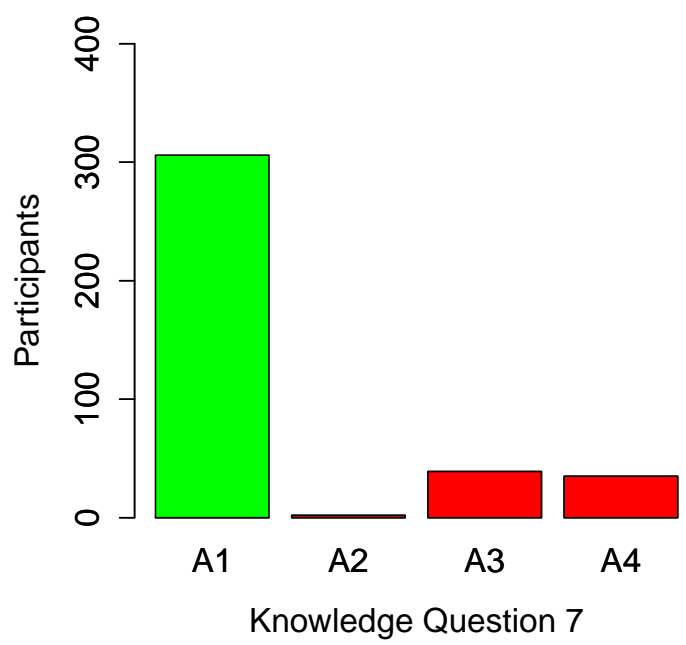

\begin{tabular}{|c|c|c|c|}
\hline Response Code & Possible Answer & Count & $\%$ \\
\hline $\mathrm{A} 1$ & $\begin{array}{l}\text { They require updates in to refresh the list of malware identi- } \\
\text { fiers in order to better detect malicious software applications. }\end{array}$ & 306 & 79.69 \\
\hline A 2 & $\begin{array}{l}\text { They require updates in order to comply with changes in in- } \\
\text { ternational law. }\end{array}$ & 2 & 0.52 \\
\hline A3 & $\begin{array}{l}\text { They require updates in order to obtain specific information } \\
\text { about my computer, so that their Anti-Virus application can } \\
\text { better protect me from malicious applications. }\end{array}$ & 39 & 10.16 \\
\hline $\mathrm{A} 4$ & $\begin{array}{l}\text { They require updates in order to update the list of malicious } \\
\text { applications newly reported by the media (i.e., newspapers, } \\
\text { television broadcasts, etc.). }\end{array}$ & 35 & 9.11 \\
\hline A5 & No response. & 2 & 0.52 \\
\hline
\end{tabular}

*Notes. The correct multiple choice answer is highlighted in pastel green . 
Table 68

Part 2 - Knowledge Question 8: What Types of Files Can a Malicious Software Application Access?

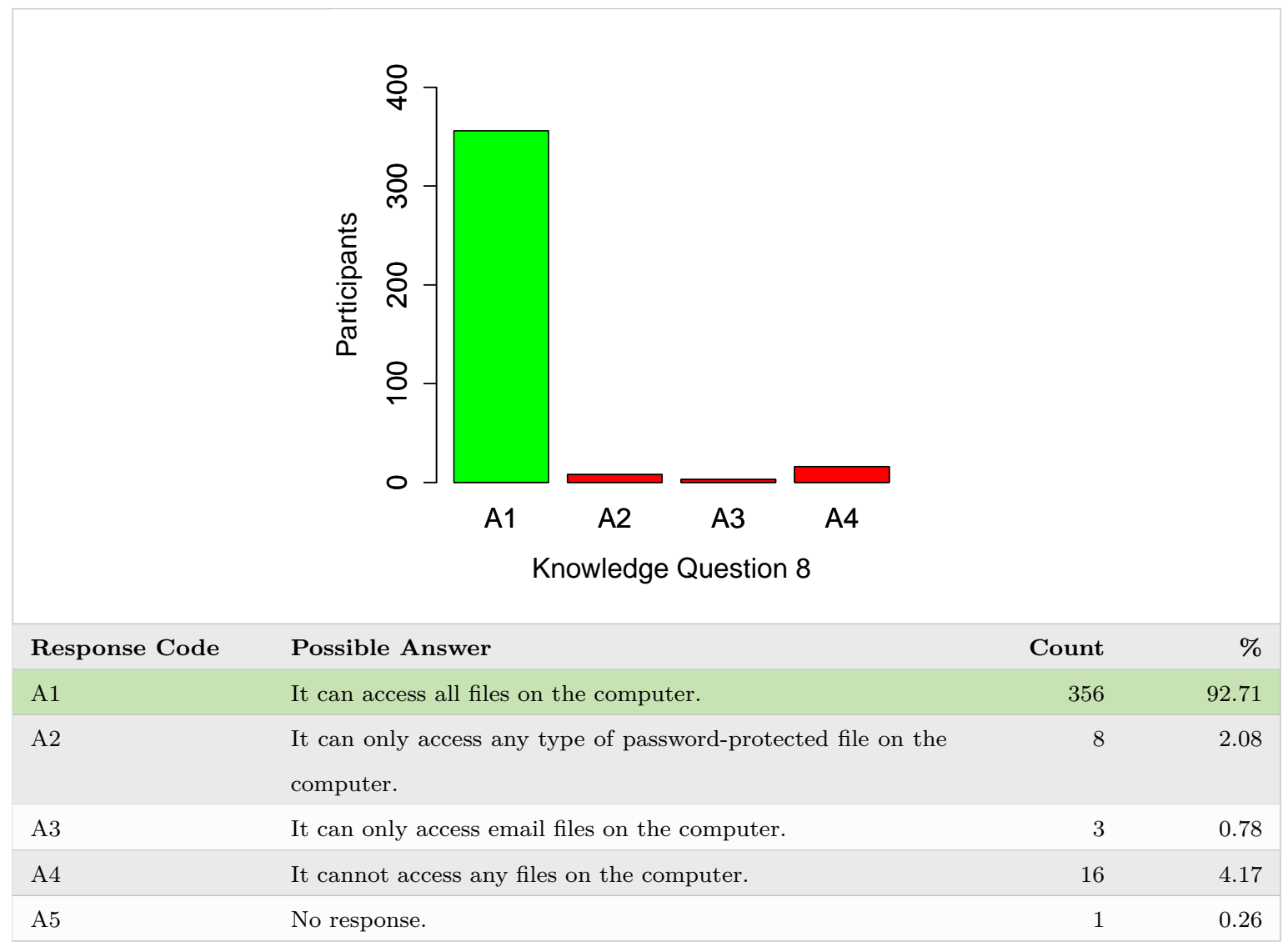

*Notes. The correct multiple choice answer is highlighted in pastel green . 
Table 69

Part 2 - Knowledge Question 9: When Can a Malicious Software Application on My Computer Record Information Submitted Through Online Forms?

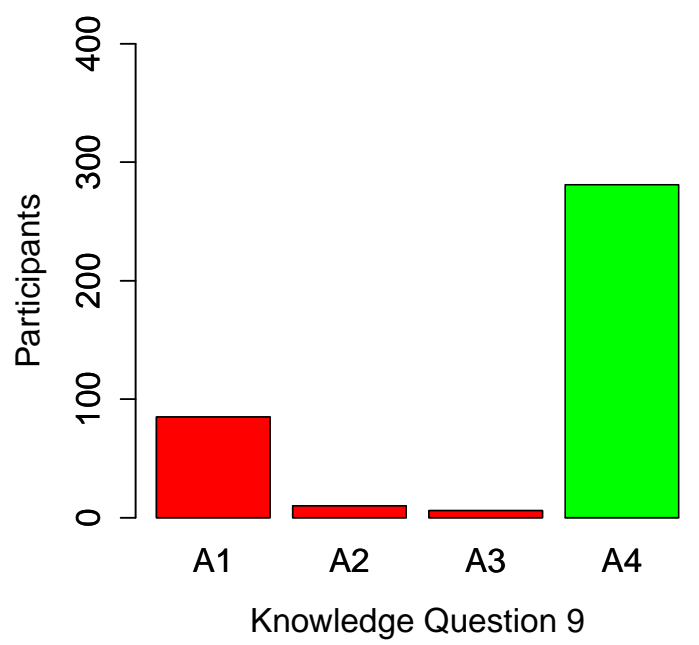

\begin{tabular}{|l|lr|r|}
\hline Response Code & Possible Answer & Count & $\%$ \\
\hline A1 & $\begin{array}{l}\text { If the information is submitted through an online form using } \\
\text { the HTTP protocol. }\end{array}$ & 85 & 22.14 \\
\hline A2 & $\begin{array}{l}\text { If the information is submitted through an online form using } \\
\text { the HTTPS protocol. }\end{array}$ & 10 & 2.60 \\
\hline A3 & If the information is submitted through an online form on a & 6 & 1.56 \\
\hline A4 & All of the above. & 281 & 73.18 \\
\hline A5 & No response. & 2 & 0.52 \\
\hline
\end{tabular}

*Notes. The correct multiple choice answer is highlighted in pastel green . 
Table 70

Part 2 - Knowledge Question 10: How Can a Malicious Software Application Be Installed on My Computer?

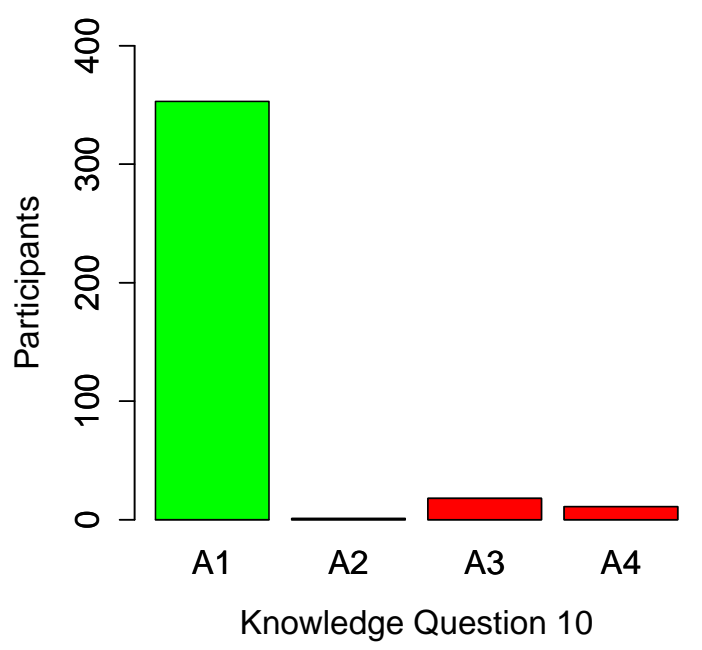

\begin{tabular}{|c|c|c|c|}
\hline Response Code & Possible Answer & Count & $\%$ \\
\hline A1 & By opening an attachment found in an email message. & 353 & 91.93 \\
\hline $\mathrm{A} 2$ & By accepting a friend invite on Facebook. & 1 & 0.26 \\
\hline A3 & $\begin{array}{l}\text { By submitting my personal information through an online } \\
\text { form on a website. }\end{array}$ & 18 & 4.69 \\
\hline A 4 & By sending an email to an insecure mail server. & 11 & 2.86 \\
\hline A5 & No response. & 1 & 0.26 \\
\hline
\end{tabular}

*Notes. The correct multiple choice answer is highlighted in pastel green . 


\section{Appendix AE}

\section{Study Five: Results Part 3 - Attitudes and Beliefs}

Table 71

Part 3 - Post Test Question 1: How Knowledgeable do You Believe You are About:

\begin{tabular}{|ll|}
\hline Code & Rating Scale Statement \\
S1 & How the Internet works? \\
S2 & How to protect your security and privacy on the Internet? \\
S3 & How to avoid malicious software applications being installed on your computer? \\
S4 & How to identify potentially harmful websites? \\
\hline
\end{tabular}

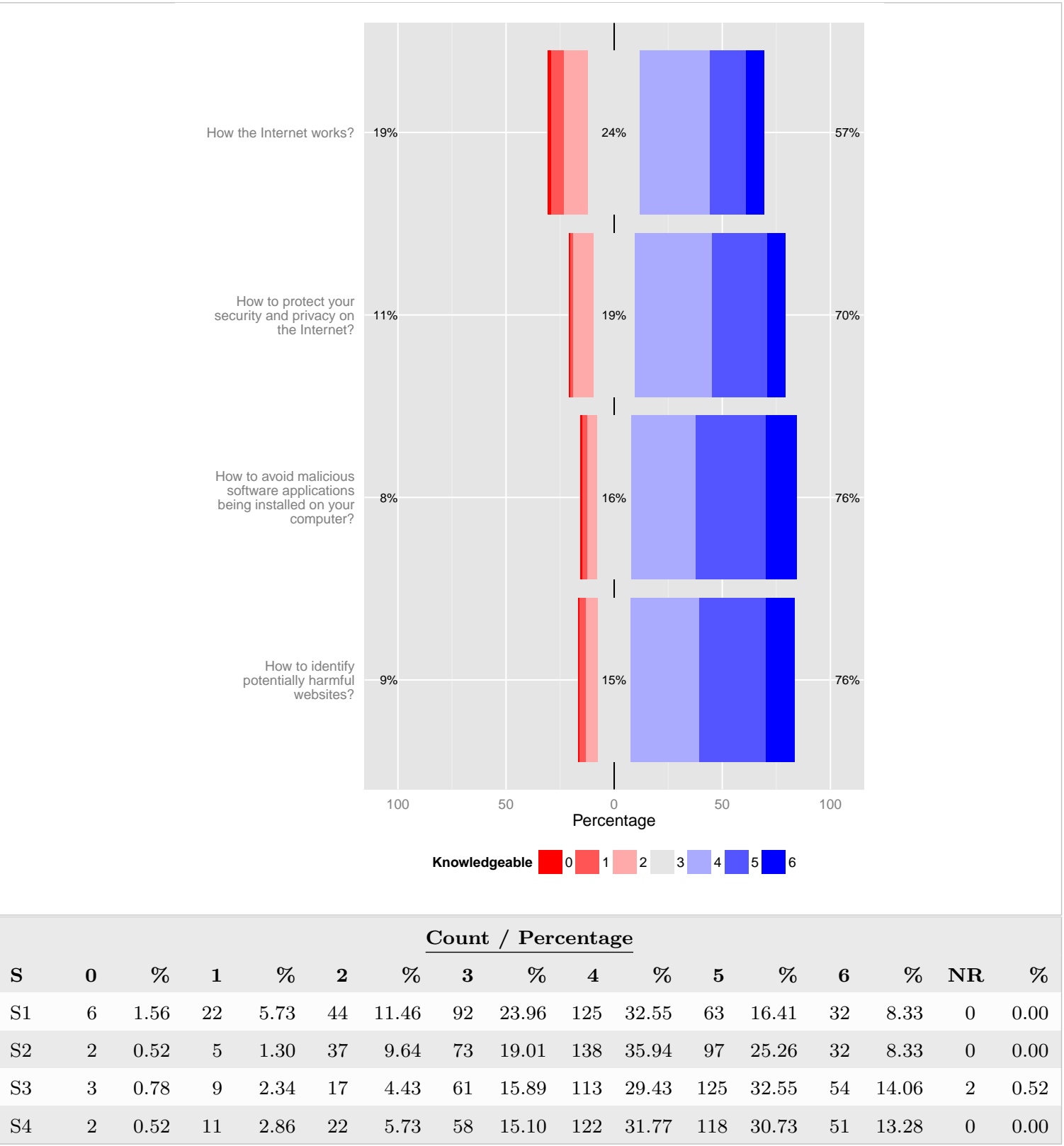

${ }^{*}$ Notes. $\mathrm{S}=$ Scale. $0=$ Not at all Knowledgeable. $6=$ Extremely Knowledgeable. $\mathrm{NR}=$ No Response. 
Table 72

Part 3 - Post Test Question 2: How Capable do You Believe You are of Learning:

$\begin{array}{ll}\text { Code } & \text { Rating Scale Statement } \\ \text { S1 } & \text { How the Internet works? } \\ \text { S2 } & \text { How to protect your security and privacy on the Internet? } \\ \text { S3 } & \text { How to avoid malicious software applications being installed on your computer? } \\ \text { S4 } & \text { How to identify potentially harmful websites? }\end{array}$

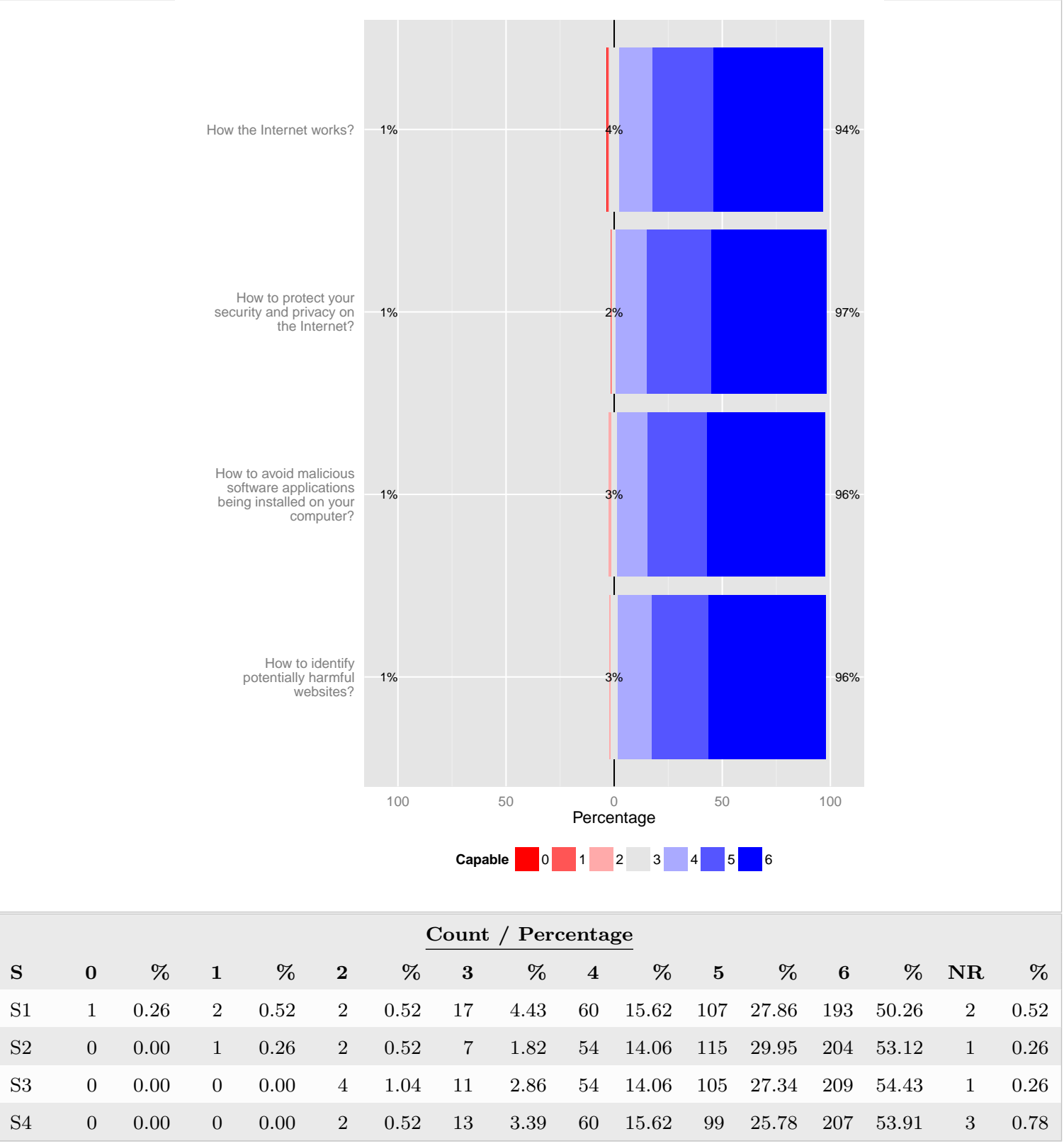

${ }^{*}$ Notes. $\mathrm{S}=$ Scale. $0=$ Not at all Capable. $6=$ Extremely Capable. NR $=$ No Response. 
Table 73

Part 3 - Post Test Question 3: How Difficult do You Believe it is to Find Information About:

$\begin{array}{ll}\text { Code } & \text { Rating Scale Statement } \\ \text { S1 } & \text { How the Internet works? } \\ \text { S2 } & \text { How to protect your security and privacy on the Internet? } \\ \text { S3 } & \text { How to avoid malicious software applications being installed on your computer? } \\ \text { S4 } & \text { How to identify potentially harmful websites? }\end{array}$

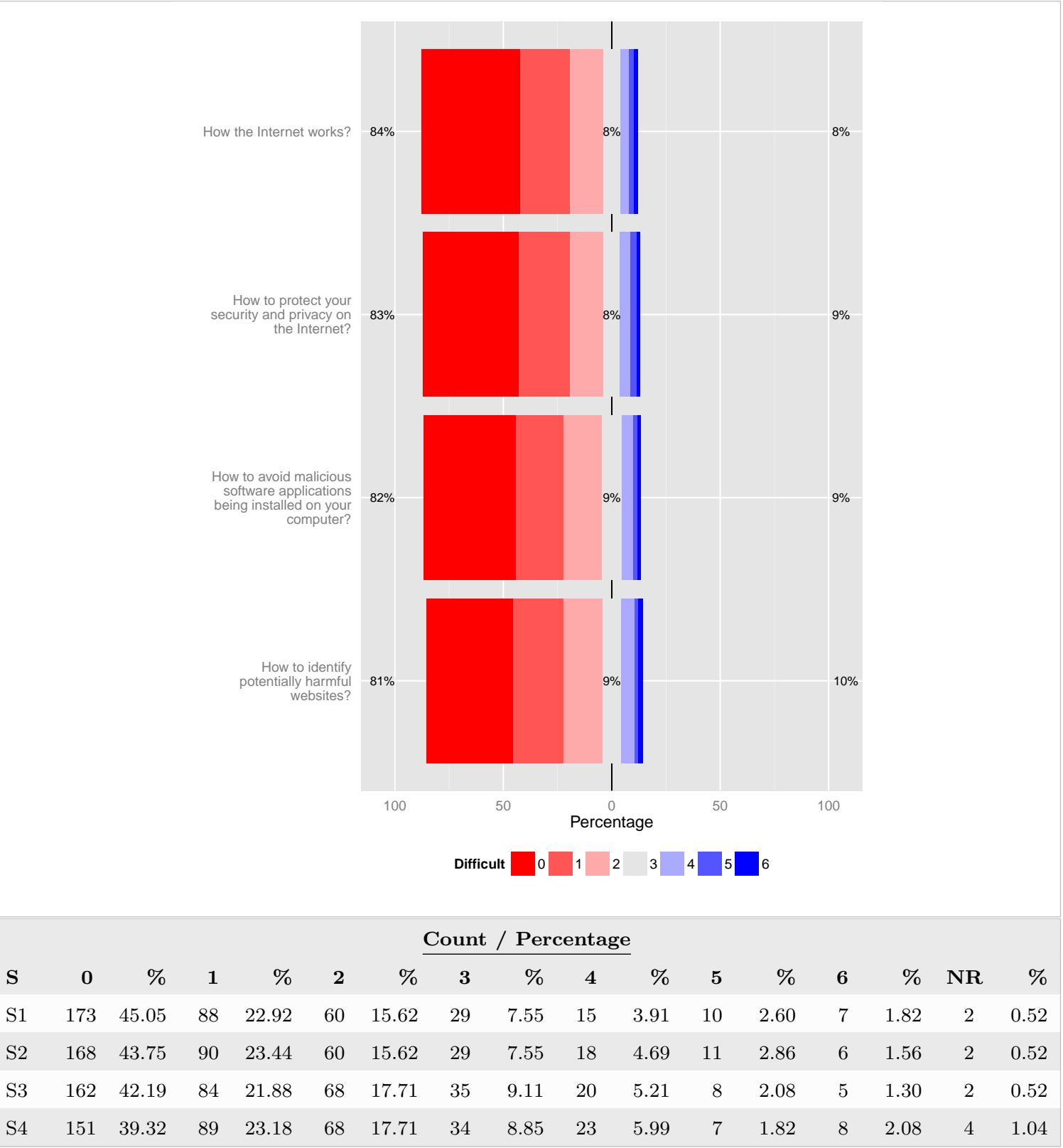

${ }^{*}$ Notes. $\mathrm{S}=$ Scale. $0=$ Not at all Difficult. $6=$ Extremely Difficult. NR $=$ No Response. 


\section{Table 74}

Part 3 - Post Test Question 4: How Much Time do You Have to Learn More About:

$\begin{array}{ll}\text { Code } & \text { Rating Scale Statement } \\ \text { S1 } & \text { How the Internet works? } \\ \text { S2 } & \text { How to protect your security and privacy on the Internet? } \\ \text { S3 } & \text { How to avoid malicious software applications being installed on your computer? } \\ \text { S4 } & \text { How to identify potentially harmful websites? }\end{array}$

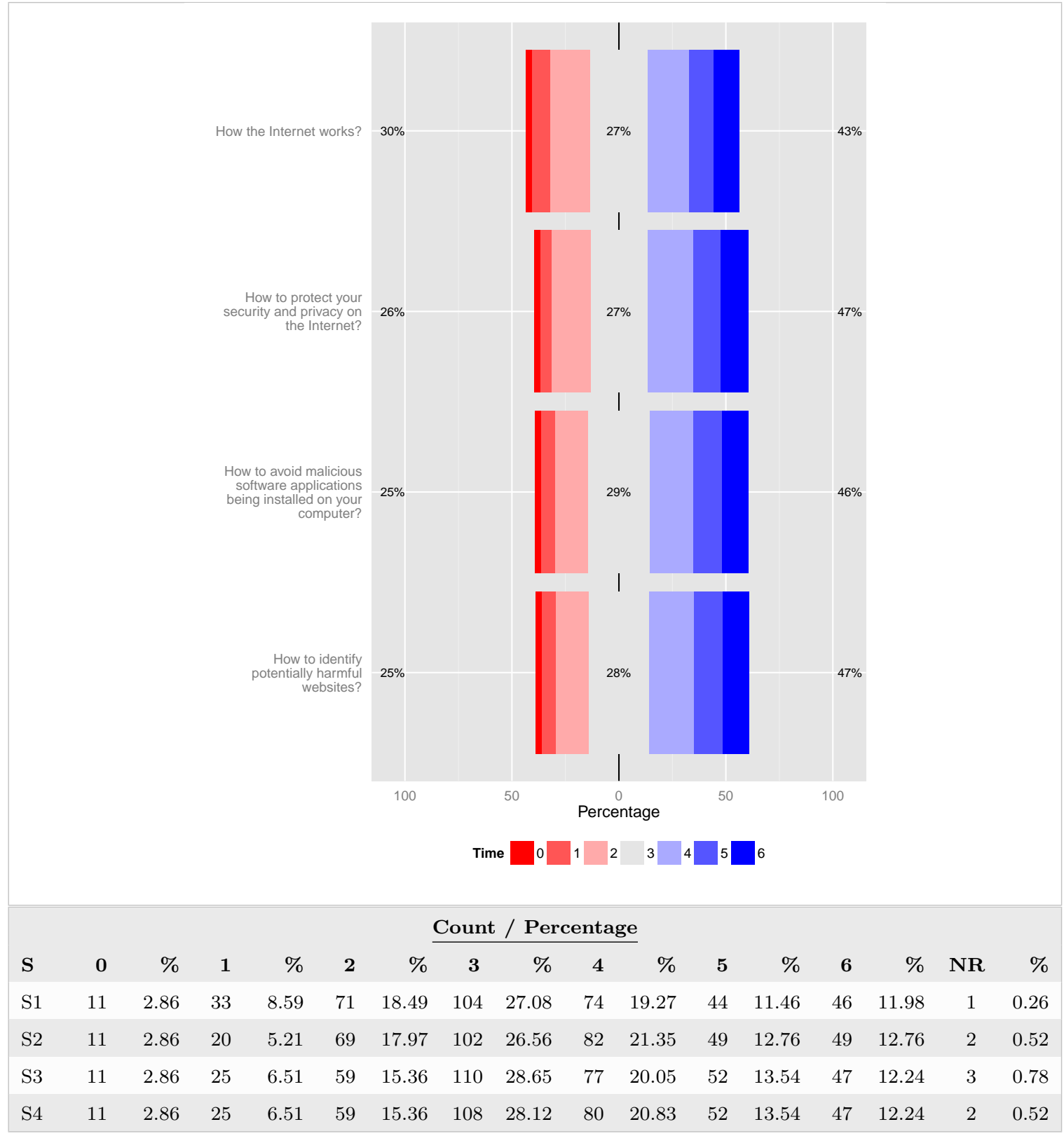

$*$ Notes. $\mathrm{S}=$ Scale. $0=$ No Time at All. $6=$ A Lot of Time. $\mathrm{NR}=$ No Response. 


\section{Table 75}

Part 3 - Post Test Question 5: How Motivated are You to Learn More About:

$\begin{array}{ll}\text { Code } & \text { Rating Scale Statement } \\ \text { S1 } & \text { How the Internet works? } \\ \text { S2 } & \text { How to protect your security and privacy on the Internet? } \\ \text { S3 } & \text { How to avoid malicious software applications being installed on your computer? } \\ \text { S4 } & \text { How to identify potentially harmful websites? }\end{array}$

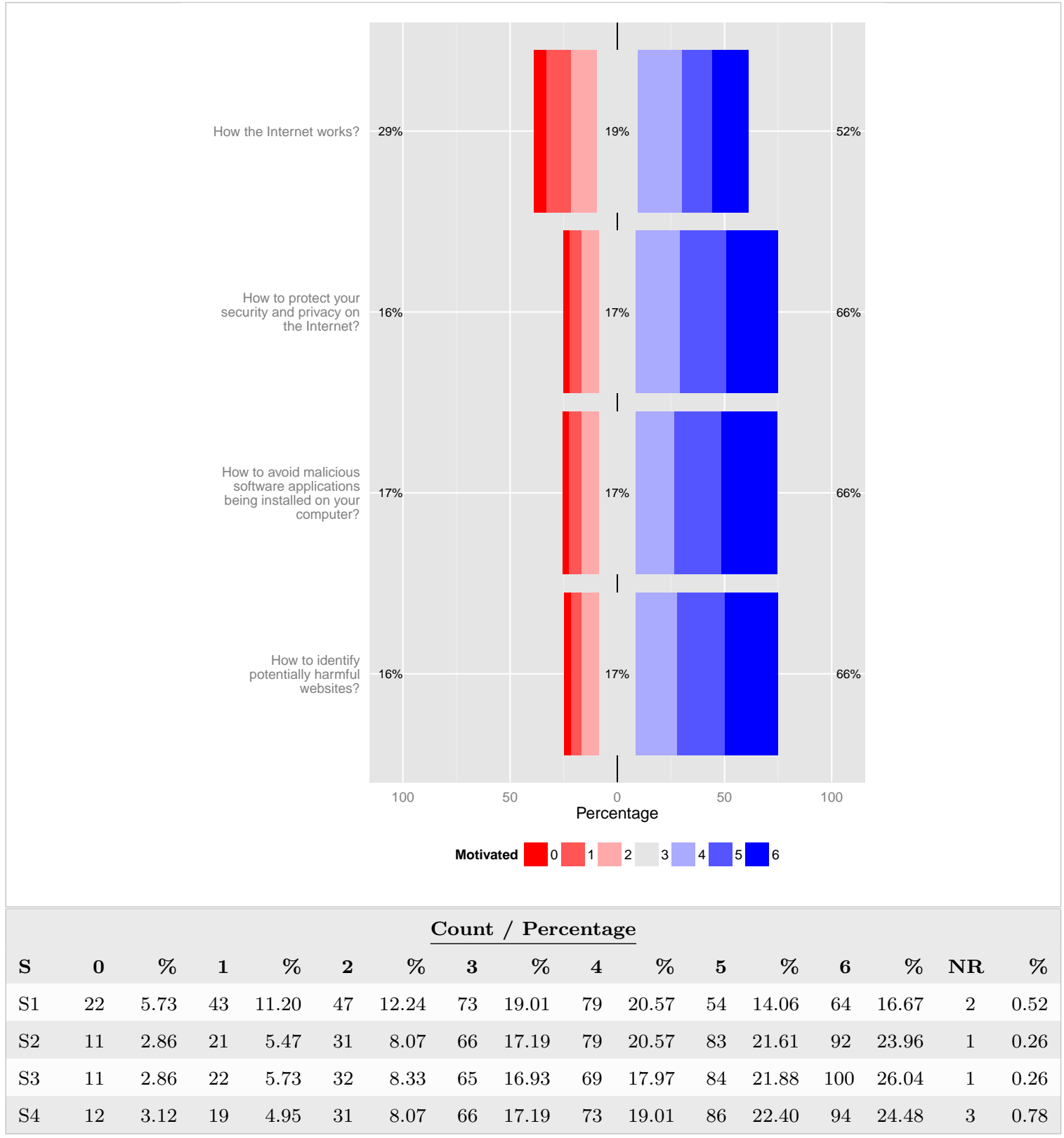

*Notes. $\mathrm{S}=$ Scale. $0=$ Not at all Motivated. $6=$ Extremely Motivated. NR $=$ No Response. 


\section{Self-rating of "Knowledgeable" scale}

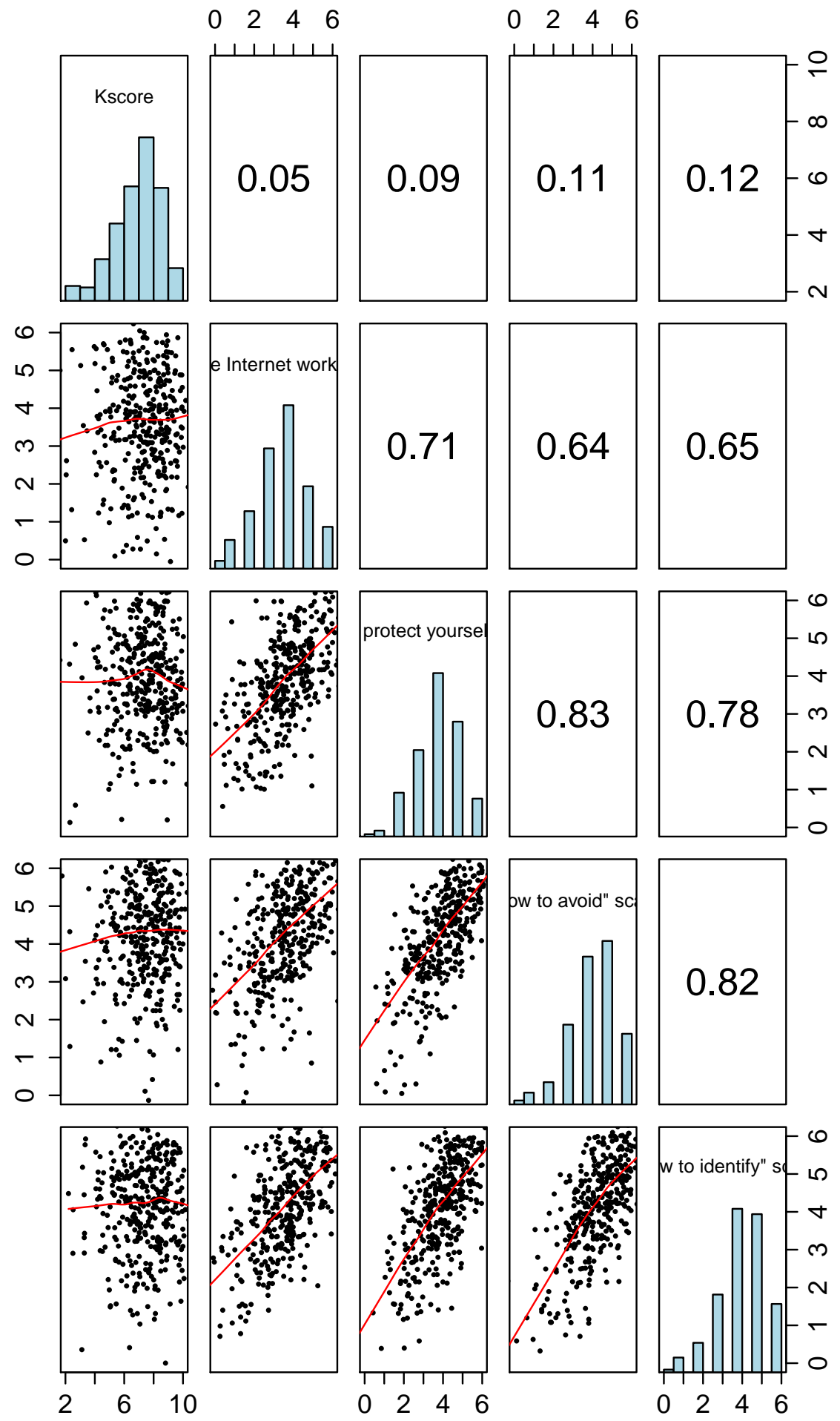

Figure 48. Correlation matrix of knowledge by knowledgeable dimension rating scales. 


\section{Self-rating of "Capable" scale}

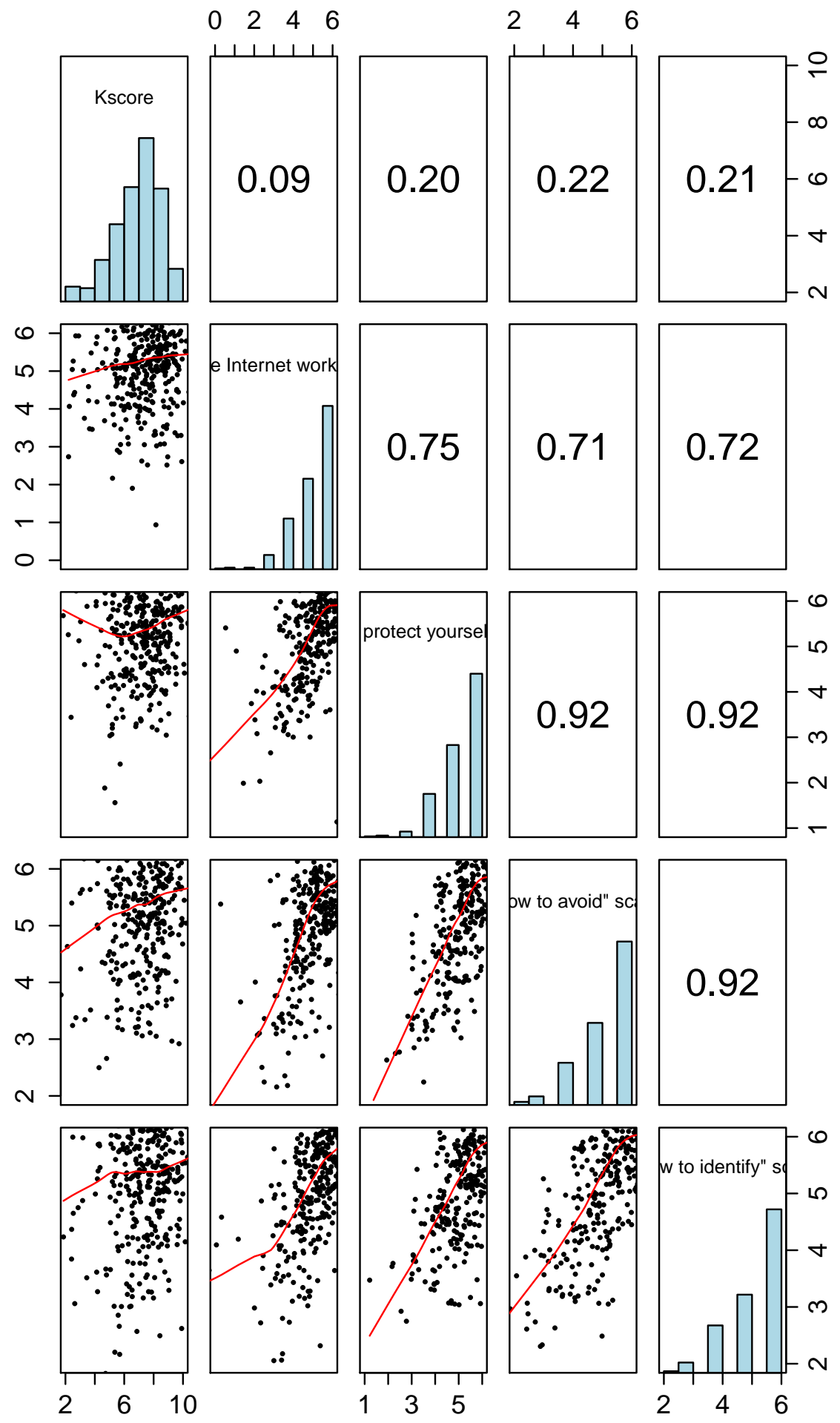

Figure 49. Correlation matrix of knowledge by capable dimension rating scales. 


\section{Self-rating of "Difficult" scale}

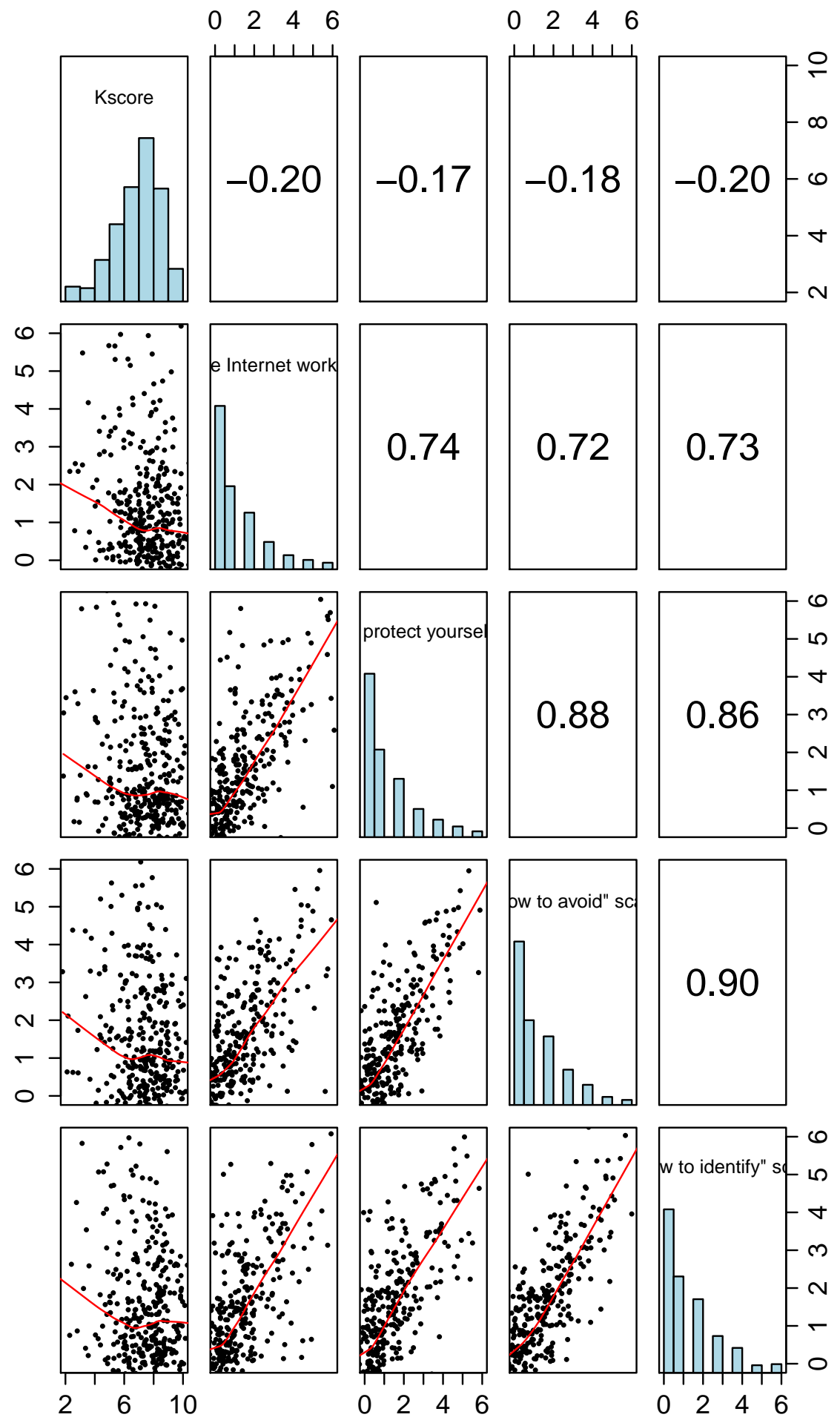

Figure 50. Correlation matrix of knowledge by difficult dimension rating scales. 


\section{Self-rating of "Time" scale}

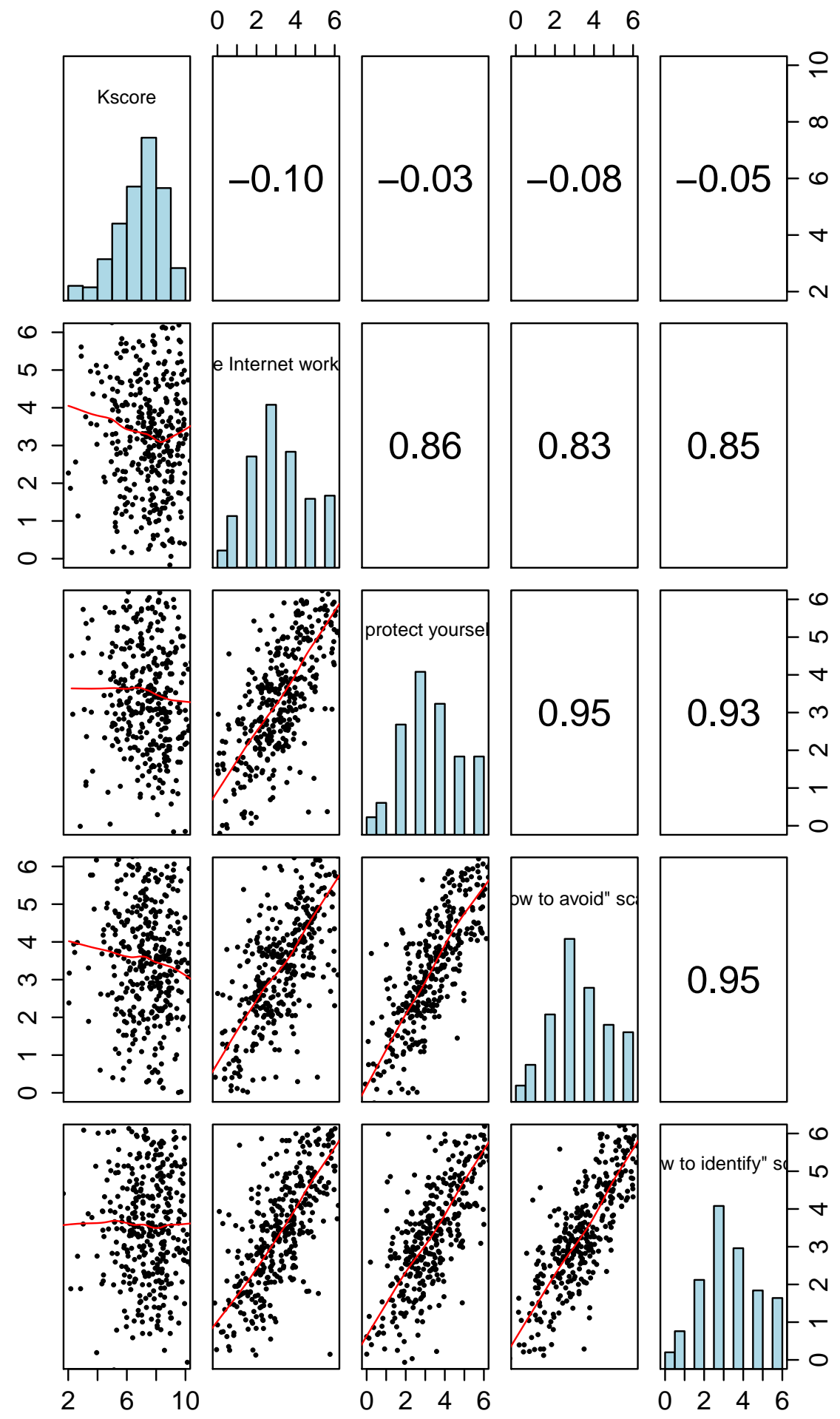

Figure 51. Correlation matrix of knowledge by time dimension rating scales. 
Self-rating of "Motivated" scale

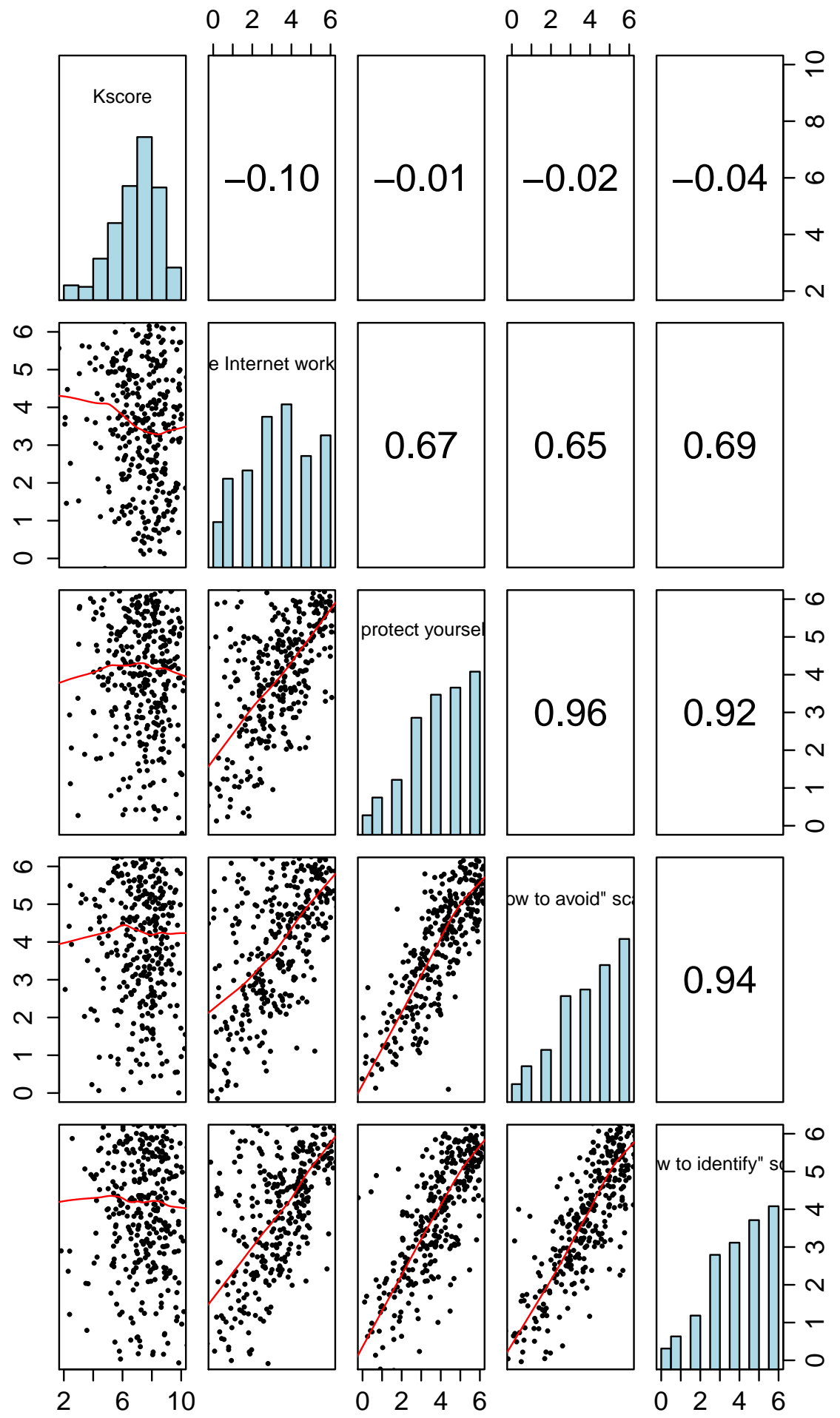

Figure 52. Correlation matrix of knowledge by motivated dimension rating scales. 


\section{"How the Internet works" scale}

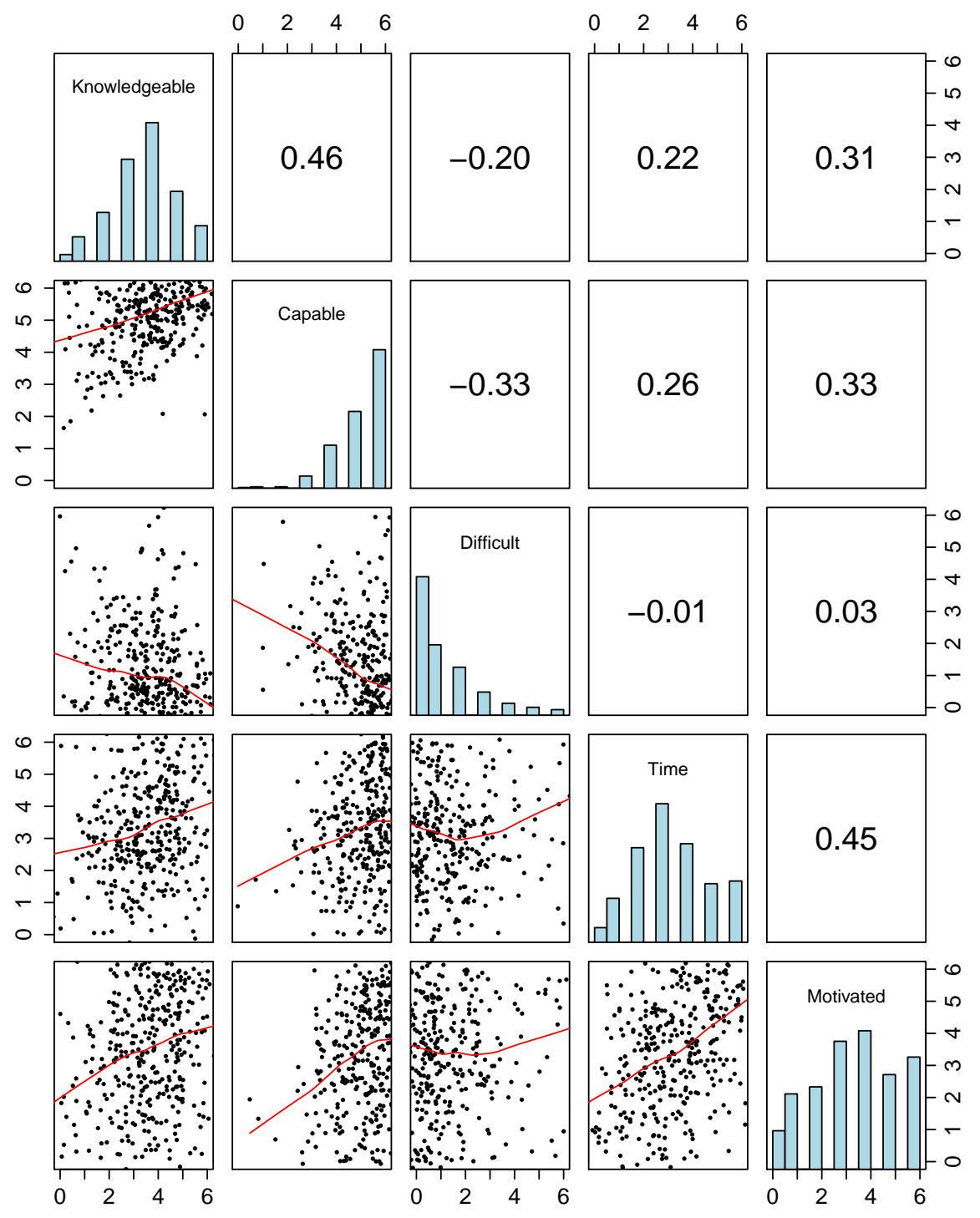

Figure 53. Correlation matrix of the "How the Internet Works" rating scale. 


\section{"How to protect yourself" scale}

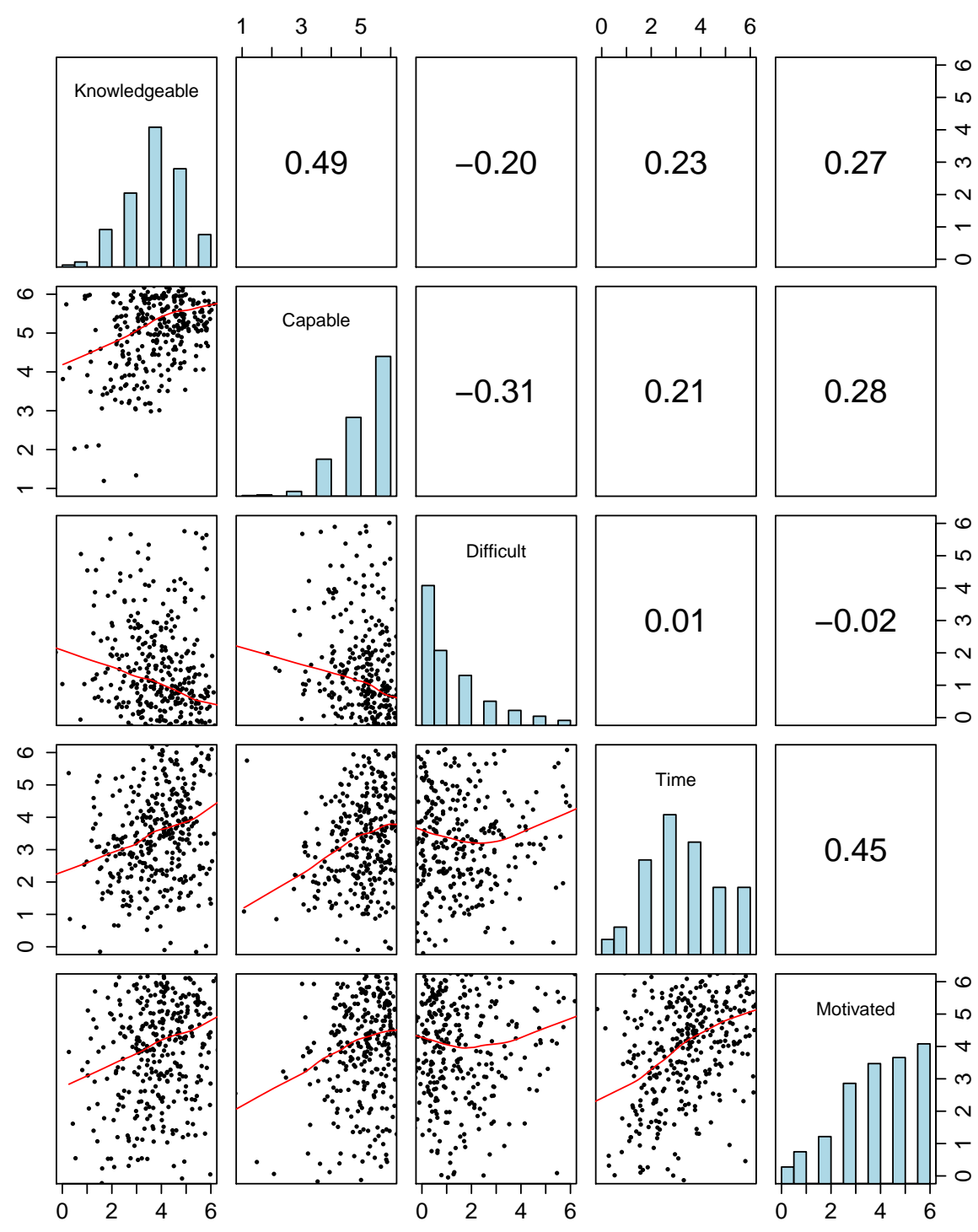

Figure 54. Correlation matrix of the "How to protect your security and privacy on the Internet" rating scale. 


\section{"How to avoid" scale}

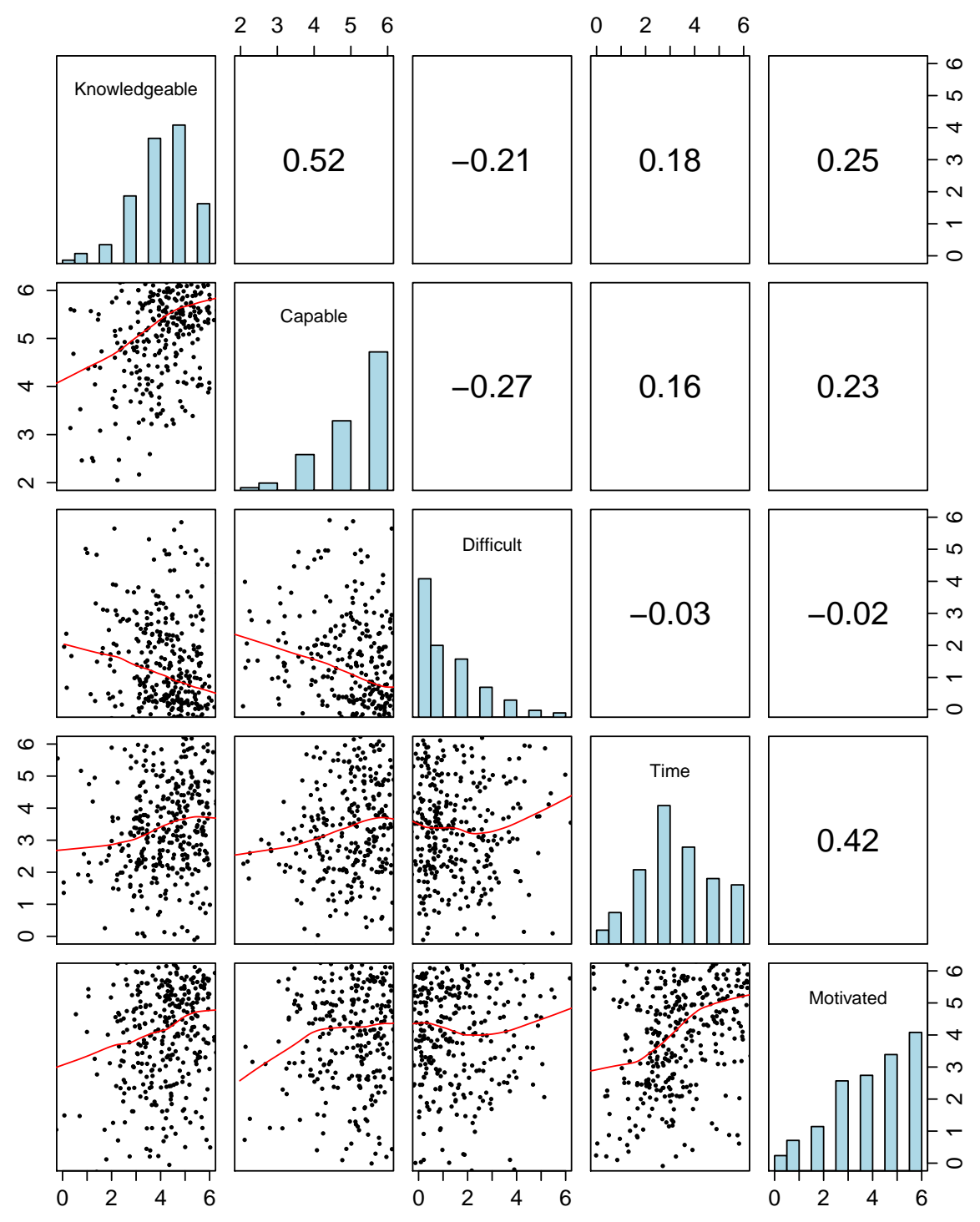

Figure 55. Correlation matrix of the "How to avoid malicious software applications being installed on your computer" rating scale. 


\section{"How to identify" scale}

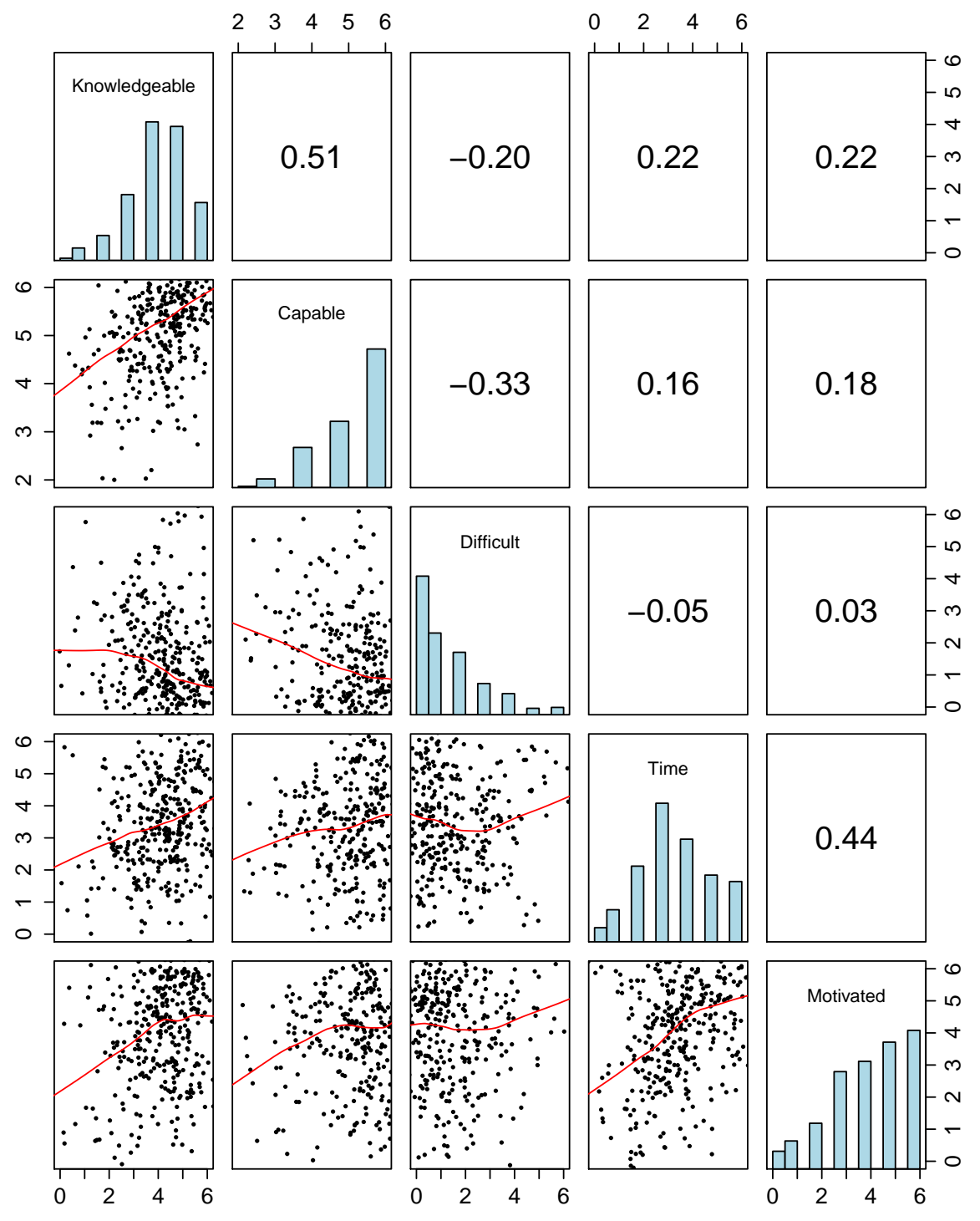

Figure 56. Correlation matrix of the "How to identify potentially harmful websites" rating scale. 